\title{
Le droit des aires protégées en Côte d'Ivoire
}

Citation for published version (APA):

Koffi, K. Y. (2016). Le droit des aires protégées en Côte d'Ivoire. [Doctoral Thesis, Maastricht University]. Maastricht University. https://doi.org/10.26481/dis.20161116kk

Document status and date:

Published: 01/01/2016

DOI:

10.26481/dis.20161116kk

Document Version:

Publisher's PDF, also known as Version of record

\section{Please check the document version of this publication:}

- A submitted manuscript is the version of the article upon submission and before peer-review. There can be important differences between the submitted version and the official published version of record.

People interested in the research are advised to contact the author for the final version of the publication, or visit the DOI to the publisher's website.

- The final author version and the galley proof are versions of the publication after peer review.

- The final published version features the final layout of the paper including the volume, issue and page numbers.

Link to publication

\footnotetext{
General rights rights.

- You may freely distribute the URL identifying the publication in the public portal. please follow below link for the End User Agreement:

www.umlib.nl/taverne-license

Take down policy

If you believe that this document breaches copyright please contact us at:

repository@maastrichtuniversity.nl

providing details and we will investigate your claim.
}

Copyright and moral rights for the publications made accessible in the public portal are retained by the authors and/or other copyright owners and it is a condition of accessing publications that users recognise and abide by the legal requirements associated with these

- Users may download and print one copy of any publication from the public portal for the purpose of private study or research.

- You may not further distribute the material or use it for any profit-making activity or commercial gain

If the publication is distributed under the terms of Article $25 \mathrm{fa}$ of the Dutch Copyright Act, indicated by the "Taverne" license above, 
Le droit des aires protégées en Côte d'Ivoire

Yves Kouassi Koffi 



\section{Le droit des aires protégées en Côte d'Ivoire}

\section{DISSERTATION}

To obtain the degree of Doctor at Maastricht University, on the authority of the Rector Magnificus, Prof. Dr. Rianne M. Letschert in accordance with the decision of the Board of Deans to be defend in public on Wednesday 16 November 2016, at 16 hours

by

\section{Yves Kouassi Koffi}




\section{Supervisors}

Prof. Dr. Michael G. Faure

Prof. Nicolas de Sadeleer (Université Saint-Louis-Bruxelles, Académie de Louvain, Royaume de Belgique)

\section{Assessment Committee}

Prof. mr. R. de Groot (Chairman)

Prof. mr. G. E. van Maanen

Prof. Dr. Marjan Peeters

Prof. Xavier Thunis (Université de Namur, Royaume de Belgique)

Dr. Yves Didier Tiebley (Université Félix Houphouët-Boigny de Cocody, République de Côte d'Ivoire) 


\section{LE DROIT DES AIRES PROTÉGÉES EN CôTE D'IVOIRE}

Yves K. Koffi

Directeurs de thèse :

Prof. Dr. Michael Faure

Prof. Nicolas de Sadeleer 
LISTE DES PRINCIPAUX SIGLES ET ABRÉVIATIONS .................................................................16

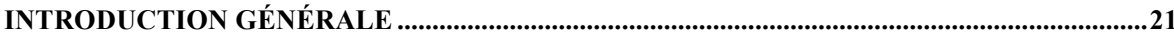

I- ENJEUX DE LA PROTECTION DES FORÊTS EN CÔTE D'IVOIRE ……………...................24

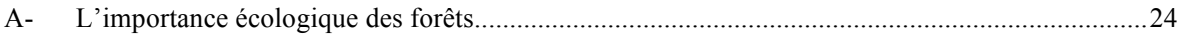

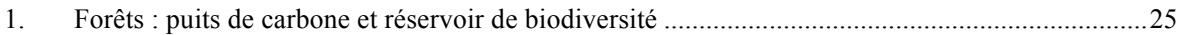

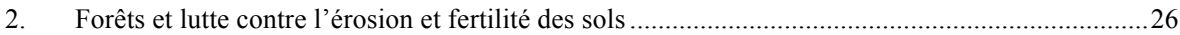

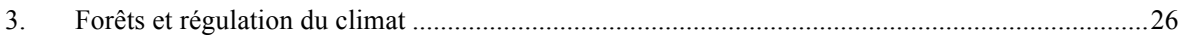

B- Les retombées socio-économiques de l'exploitation des forêts en Côte d'Ivoire ...........................27

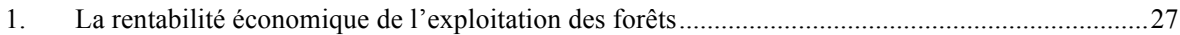

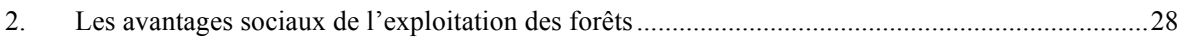

C- L'état des pressions anthropiques sur la biodiversité forestière en Côte d'Ivoire ............................30

1. L'érosion de la biodiversité forestière ivoirienne en général.........................................................30

2. Les pressions anthropiques sur les aires protégées en particulier .................................................31

II- APERÇU DE LA LÉGISLATION CONSACRÉE À LA PROTECTION DES

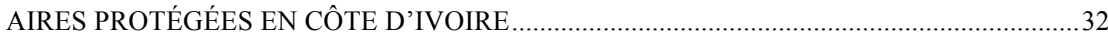

A- Les textes juridiques internationaux applicables aux aires protégées ............................................32

B- Les textes juridiques nationaux relatifs aux aires protégées..........................................................34

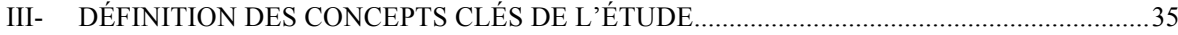

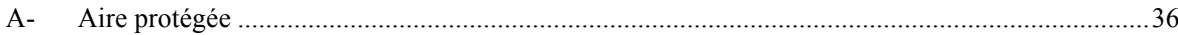

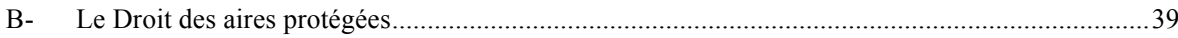

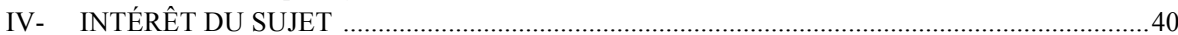

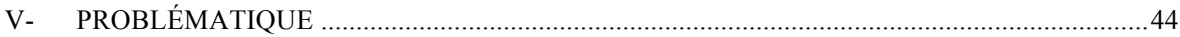

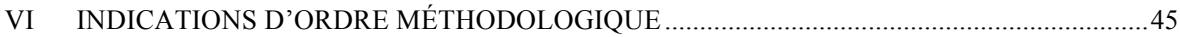

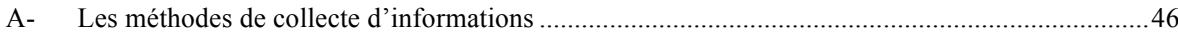

1. L'étude documentaire

2. L'enquête

B- Les techniques d'analyse des données collectées ou d'analyse de l'objet.....................................47

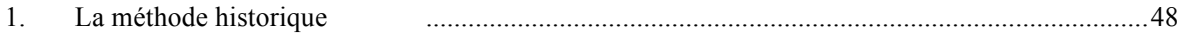

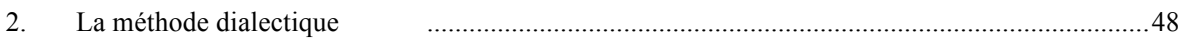

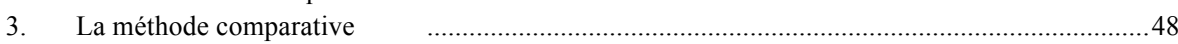

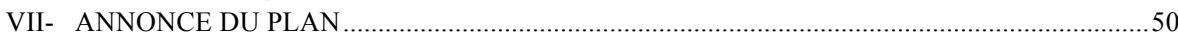

PREMIÈRE PARTIE : L'EXISTENCE AVÉRÉE D'UNE LÉGISLATION

CONSACRÉE AUX AIRES PROTÉGÉES .............................................................................5

TITRE I : LE CADRE JURIDIQUE ORIGINAIRE DES AIRES PROTÉGÉES .............................55

CHAPITRE I : LA PROTECTION JURIDIQUE DES AIRES PROTÉGÉES DE 1960 À 2002

SECTION I : Le cadre institutionnel de gestion des aires protégées

Paragraphe 1: Le service public de la conservation des aires protégées de 1960 à 2002, une

Administration centralisée

A- Les notions de « service public de la conservation des aires protégées » et de « centralisation » 57

1. La notion de service public de la conservation des aires protégées.............................................57

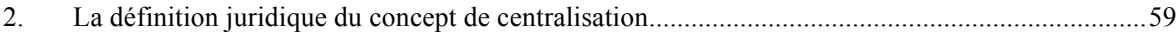

B- L'Administration en charge des aires protégées de 1960 à 2002, une Administration centralisée 59

1. La présentation de l'Administration en charge des aires protégées de 1960 à 2002 ....................59

2. L’organisation administrative des aires protégées de 1960 à 2002, un héritage colonial..............63 
Paragraphe 2 : Les ressources budgétaires de l'Administration en charge de la conservation des aires protégées 65

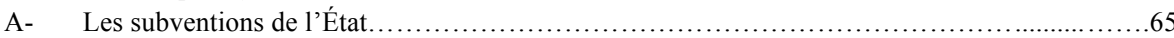

1. Les fonds accordés par l'État aux aires protégées au titre du Budget Général de fonctionnement de l'État de Côte d'Ivoire.

2. Les crédits budgétaires alloués aux aires protégées issus du Budget Spécial d'Investissement et d'Équipement de l'État de Côte d'Ivoire.

B- Le fonds spécial des Parcs Nationaux

SECTION II : Le cadre normatif des aires protégées

Paragraphe 1 : Les aires protégées, un élément constitutif du domaine privé de l'État de 1960 à $2002 . . .70$

A- $\quad$ Les lacunes des textes sur l'appartenance des aires protégées au domaine privé de l'État........70

B- $\quad$ Les aires protégées, un domaine privé de l'État par raisonnement déductif ...............................72

Paragraphe 2 : La répression des atteintes contre les aires protégées....................................................80

A- La définition des infractions contre les aires protégées...........................................................8

1. Le délit de chasse dans les Parcs Nationaux et Réserves naturelles ...........................................80

2. Les délits d'exploitation forestière et d'exploitation agricole dans les Parcs Nationaux et Réserves naturelles ............................................................................. 83

B- L'attribution de pouvoirs de répression au personnel en charge des aires protégées ..................82

\section{CHAPITRE II : LES INSUFFISANCES DU CADRE JURIDIQUE DES AIRES} PROTÉGÉES DE 1960 À 2002

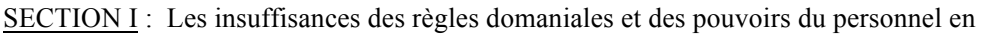
charge des aires protégée

Paragraphe $1:$ Les limites des règles domaniales des aires protégées ...............................................84

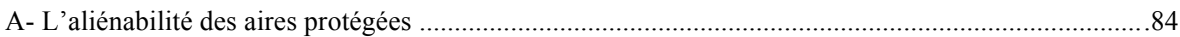

1. L'analyse juridique de l'aliénabilité des aires protégées ................................... 84

2 Des exemples pratiques d'aliénation de forêts issues du domaine forestier de l'État ivoirien .....85

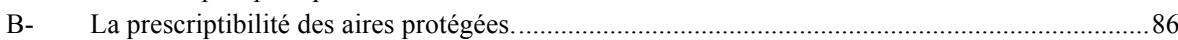

Paragraphe 2 : Les limites des pouvoirs du personnel en charge de la protection des aires protégées ....88

A- Les limites relatives aux pouvoirs d'administration des aires protégées..................................88

B- Les limites des pouvoirs de répression du personnel en charge des aires protégées.................89

$\underline{\text { SECTION II }}$ : Les carences des règles de financement de la protection des aires protégées..................90

Paragraphe 1 : L’Administration des aires protégées tributaire d'un financement étatique insuffisant

A- L'impossibilité juridique du recours au financement extra-étatique par

l'Administration des aires protégées.

1. L'absence de personnalité juridique de l'Administration en charge des aires protégées ............91

2. Les conséquences juridiques de l'absence de personnalité juridique de 1'Administration en charge des aires protégées sur le plan financier .......................................92

B- L'insuffisance des dotations budgétaires pour la conservation des aires protégées ..................92

Paragraphe 2 : La procédure de gestion des subventions étatiques, un obstacle à la conservation des aires protégées

A- $\quad$ Le circuit de l'exécution de la dépense publique ...............................................................94

B- Les conséquences des règles de gestion des subventions étatiques des aires protégées : les difficultés dans la mise à disposition des fonds

TITRE II : LES INNOVATIONS APPORTÉES AU CADRE JURIDIQUE 


\section{CHAPITRE I : LE RENFORCEMENT DE LA PROTECTION DE L'INTÉGRITÉ DES AIRES PROTÉGÉES SUR LE PLAN NORMATIF.}

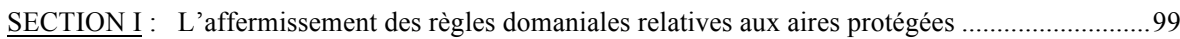

Paragraphe 1: L'incorporation des aires protégées au domaine public de l'État ................................99

A- Les raisons de l'incorporation des aires protégées au domaine public de l'État .......................100

B- Les modalités relatives à l'incorporation des aires protégées au domaine public de l'État .......100

Paragraphe 2 : Les implications de la domanialité publique sur la protection des aires Protégées ........ 101

A- L'inaliénabilité et l'imprescriptibilité des aires protégées...................................................... 101

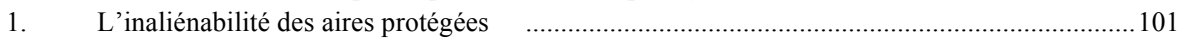

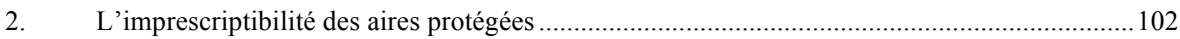

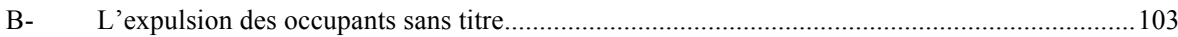

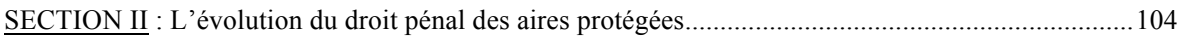

Paragraphe 1 : Le durcissement des textes de répression des atteintes contre les aires protégées .......... 105

A- L'élargissement du champ des éléments matériels et l'alourdissement des peines des infractions prévues par la législation initiale des aires protégées ............................................ 105

1. Le délit de chasse dans les Parcs Nationaux et Réserves naturelles .......................................107

2. Le délit d'exploitation forestière dans les Parcs Nationaux et Réserves naturelles...................108

3. Le délit d'exploitation agricole dans les Parcs Nationaux et Réserves naturelles ...................... 110

4. Le délit d'incendie de Parcs Nationaux et Réserves naturelles ............................................... 112

B- $\quad$ La définition de nouvelles infractions contre les aires protégées par le législateur....................112

1. Les infractions contre les aires protégées ........................................................................... 113

a. Les infractions relatives au sol et au sous-sol ...................................................................... 113

b. L'exploitation de bois d'œuvre et d'ébénisterie dans les Parcs Nationaux et

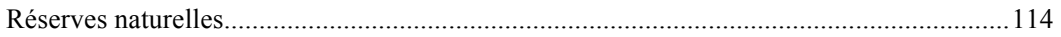

c. Les incriminations à caractère mixte..................................................................................... 115

2. Les infractions spécifiques aux zones périphériques des aires protégées..........................................117

Paragraphe 2 : Le renforcement des compétences pénales du personnel en charge des aires protégées

A- Le mécanisme du renforcement des pouvoirs de répression des gestionnaires des

Parcs et réserves : l'attribution de la qualité d'officier de Police judiciaire à certains personnels de l'OIPR

B- $\quad$ Les conséquences de l'élargissement des pouvoirs de répression du personnel en charge des aires protégées

\section{CHAPITRE II : L'ÉDICTION DE RÈGLES JURIDIQUES DE GESTION À} FINALITÉ PROTECTRICE DES PARCS NATIONAUX ET RÉSERVES NATURELLES .... 121

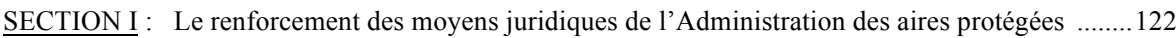

Paragraphe 1 : La délégation de la gestion des aires protégées à un établissement public national.......122

A- L'Office Ivoirien des Parcs et Réserves : l'Établissement délégataire de la gestion

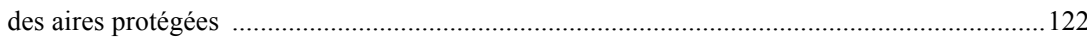

1. L'Office Ivoirien des Parcs et Réserves : un Établissement public national ...........................123

2. Les organes propres de l'Office Ivoirien des Parcs et Réserves ...............................................127

B- L'Autonomie d'administration des aires protégées par l'Office Ivoirien des Parcs et

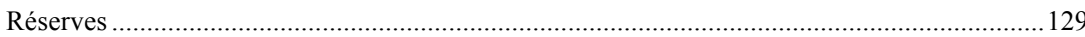

1. L'autonomie administrative et financière de la Direction Générale de l'Office Ivoirien des Parcs et Réserves

2. Les attributions administratives et financières des autorités locales déconcentrées de l'Office Ivoirien des Parcs et Réserves....

Paragraphe 2 : L'institution de mécanismes de financement extra-étatique pour la protection des aires protégées 
A- Les Fondations des Parcs et Réserves ......................................................................... 133

1. L'objet des Fondations pour les Parcs et Réserves .................................................................. 133

2. Les attributions des Fondations pour les Parcs et Réserves......................................................134

3. Les règles de gestion des Fondations pour les Parcs et Réserves ...........................................136

a. Les règles de gestion des financements mobilisés par les Fondations pour les Parcs et Réserves............................................................................................... 136

b. Les règles relatives au droit de contrôle des Fondations pour les

Parcs et Réserves par les donateurs ou bailleurs.................................................................... 137

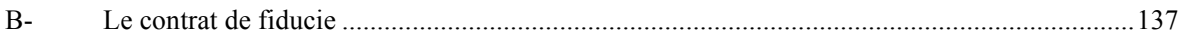

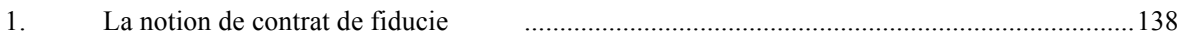

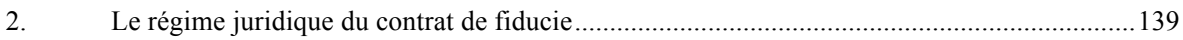

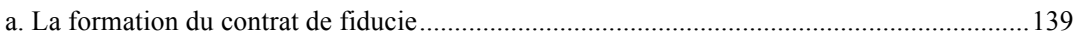

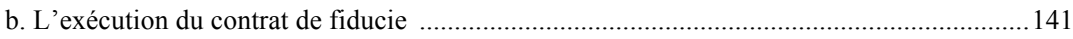

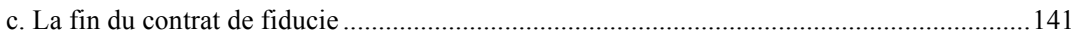

$\underline{\text { SECTION II }}$ : L'instauration d'un cadre participatif de gestion des aires protégées au niveau local...142

Paragraphe 1: L'abandon de la gestion exclusive des aires protégées par l'État ivoirien ....................143

A- L'importance de la gestion participative pour la conservation des aires protégées ...............143

1. Le recours à la gestion participative des aires protégées en Côte d'Ivoire, une conséquence de l'échec de l'approche protectionniste ................................................ 143

2. La gestion participative des ressources naturelles, un engouement planétaire ..................... 145

B- Les nouveaux acteurs associés à la gestion des aires protégées .......................................... 148

1. Les acteurs relevant de l'ordre administratif ................................................................... 148

a. Les autorités administratives déconcentrées associées à la gestion des aires protégées ....149

b. Les autorités de l'Administration décentralisée associées à la gestion des

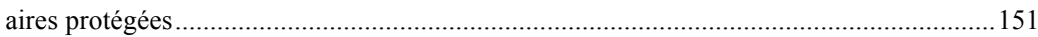

2. Les personnes morales de droit privé associées à la gestion des aires protégées ............................. 152

a. Les associations de populations locales .................................................................... 152

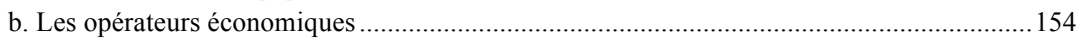

c. Les Organisations Non Gouvernementales ..................................................................... 156

Paragraphe 2 : Les modalités de mise en œuvre de la gestion participative des aires protégée ..............157

A- L'instauration de comités de gestion locale d'aire protégée .................................................. 157

1. Le comité de gestion locale d'aire protégée : une plate-forme de gestion multisectorielle des aires protégées... ................................................................................. 157

2. Le comité de gestion locale d'aire protégée : un cadre de concertation élargie

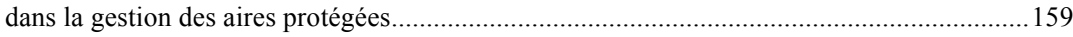

B- La contractualisation de la gestion des aires protégées ................................................... 160

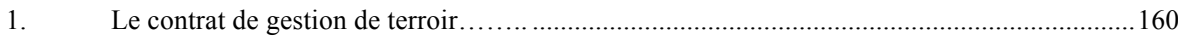

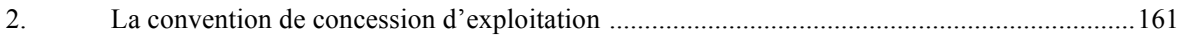

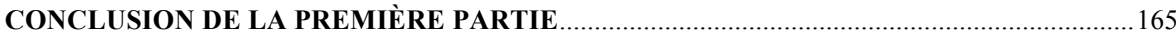

DEUXIÈME PARTIE : LA DIFFICILE APPLICATION DU DROIT DES AIRES PROTÉGÉES

TITRE I : LE CONSTAT AVÉRÉE D'UN DÉFAUT D'EFFECTIVITÉ DU DROIT DES AIRES PROTÉGÉES.

\section{CHAPITRE I : LE DÉFAUT D'ÉFFECTIVITÉ DU DROIT DES AIRES PROTÉGÉES,} UNE TARE CONGÉNITALE? 


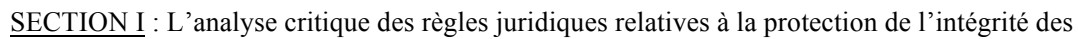
aires protégées

Paragraphe 1 : La réaffirmation de la protection de l'intégrité des aires protégées par l'évolution des règles domaniales

A- Le renforcement des règles procédurales de classement et de déclassement de catégories d'aires protégées

1. La loi, nouvel acte de classement et de déclassement de certaines catégories d'aires protégées

2. Les conséquences du classement et du déclassement des Parcs Nationaux et

Réserves intégrales par la loi sur leur protection

B- L'incorporation des aires protégées au domaine public de l'État, un remède aux mesures étatiques antérieures préjudiciables à leur conservation.

1. Exposé de quelques décisions du pouvoir exécutif préjudiciables à l'intégrité des aires protégées 174

a. Le déclassement d'une parcelle du Parc National de la Comoé en 1977............................... 174

b. Les modifications des limites du Parc National de Taï en 1973 et 1977 .............................. 175

2. L'inaliénabilité des aires protégées, un antidote juridique à leur distraction par l'autorité exécutive

Paragraphe 2 : La consolidation des dispositions pénales relatives à la protection de l'intégrité des aires protégées.

A- $\quad$ Le durcissement des textes d'incrimination et de pénalité ..................................................... 178

1. L'enrichissement des textes d'incrimination par le législateur...............................................178

2. Le constat de la sévérité des peines ...............................................................................180

B- $\quad$ L'impact de la sévérité des peines sur la baisse de la criminalité écologique dans les aires protégées, une déduction possible à partir de travaux antérieurs............................................ 181

1. Les résultats de l'étude de Kessler et Levitt .......................................................................... 182

2. Les résultats des travaux de Helland et Tabarrok ................................................................. 182

3. Les conclusions des recherches de Drago, Vertova et Galbiati .......................................... 182

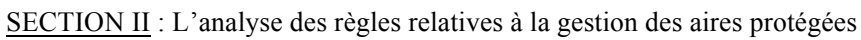

Paragraphe 1 : L'intérêt de la consécration légale du principe de gestion participative des aires protégées

A- L'impact de la délégation de la gestion des aires protégées à une structure autonome sur leur conservation

B- Les intérêts de l'implication des communautés locales à la gestion des aires protégées sur

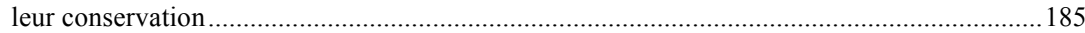

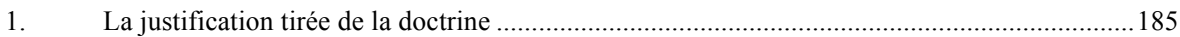

2. La justification basée sur les expériences pratiques de gestion de ressources naturelles ......... 186

Paragraphe 2 : L'élaboration de règles juridiques de financement à des fins de protection des aires protégées.

A- L'édiction de règles consacrant la diversification des sources de financement destiné aux aires protégées

1. Les Fondations pour les Parcs et Réserves de Côte d'Ivoire, un mécanisme incitatif de financement pour la protection des aires protégées.

2. Le fonctionnement du mécanisme fiduciaire, un moyen incitatif de mobilisation de financements pour la protection des aires protégées

B- La prescription de règles rigoureuses de mobilisation et de gestion des fonds destinés aux aires protégées

1. Les règles de gouvernance des Fondations des Parcs et Réserves

2. L'instauration d'une sécurité juridique autour des fonds fiduciaires alloués aux aires protégées 


\section{CHAPITRE II : LES PROBLÈMES PRATIQUES DE GESTION DES AIRES PROTÉGÉES, CAUSES DU DÉFAUT DE L'ÉFFECTIVITÉ DU DROIT DES AIRES PROTÉGÉES}

SECTION I : Les problèmes liés à l'exploitation du patrimoine naturel dans les aires protégées et leurs zones périphériques

Paragraphe 1: La forte dépendance des populations riveraines des aires protégées

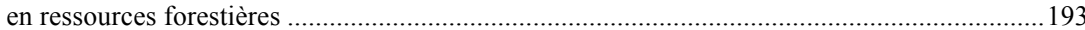

A- La pratique d'une agriculture écocide dans les zones périphériques des aires protégées .........194

1. L'agriculture, principale activité économique des populations riveraines des aires protégées enquêtées.

2. Les techniques agricoles utilisées dans les zones périphériques des aires protégées et leurs conséquences sur la forêt ....................................................................... 198

a. L'agriculture itinérante sur brûlis...................................................................................... 199

b. Les conséquences de l'agriculture itinérante sur brûlis dans les zones périphériques des aires protégées ..........................................................................200

B- $\quad$ La persistance de l'exploitation des aires protégées par les populations .................................201

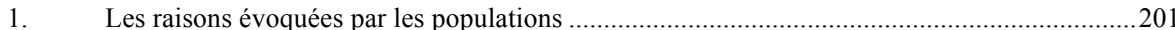

2. Illustrations des pressions anthropiques sur certaines aires protégées...................................203

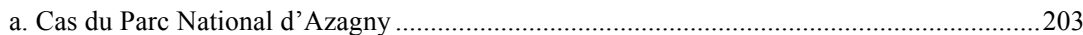

b. Cas du Parc National de Taï.................................................................................................204

c. Cas du Parc National de la Marahoué .............................................................................205

Paragraphe $2:$ Les problèmes relatifs aux aires protégées à proprement dit .......................................206

A- Les problèmes de gestion courante des aires protégées.........................................................206

1. L'insuffisance de financements et d'équipements dans la gestion des aires protégées ............206

2. L'insuffisance et le mauvais état des infrastructures de gestion des aires protégées................207

B- $\quad$ La survivance des problèmes relatifs au foncier dans les aires protégées ..............................209

1. La persistance des problèmes de revendications d'indemnisation et de contestation de limites d'aires protégées par les communautés locales ...................................209

2. Le laxisme dans les règlements des problèmes d'occupations illégales

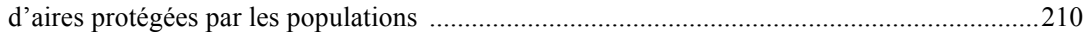

a. Le cas du Parc National de la Marahoué.......................................................................... 210

b. Le cas du Parc National de Taï ................................................................................. 213

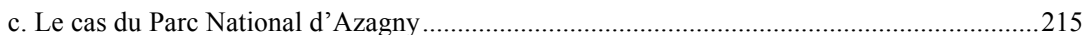

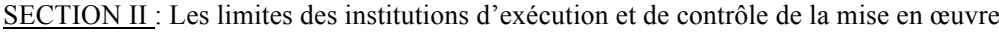

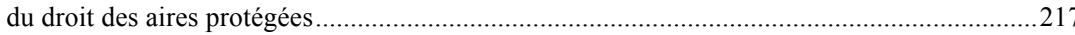

Paragraphe 1: Les limites structurelles à la mise en œuvre du droit des aires protégées .....................217

A- $\quad$ La récurrence du défaut de détection des violations du droit des aires protégées ....................218

B- La réaction inadéquate de l'Administration en charge des aires protégées en cas d'infraction au droit des aires protégées .......................................................................2 218

Paragraphe 2 : Les limites conjoncturelles à la mise en œuvre du droit des aires protégées.................219

A- $\quad$ Le retard dans la prise des textes réglementaires................................................................219

B- $\quad$ Le retard dans la mise en place et le fonctionnement des instruments et organes de gestion prévus par la loi de 2002 sur les PNR ..................................................................222

1. Le retard dans la conception et l'application des plans d'aménagement et de gestion d'aire protégée ...........................................................................................................222

2. Le retard dans la mise en place et le fonctionnement de certains organes

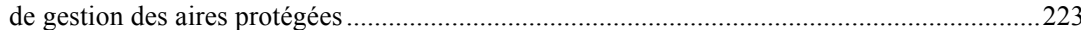

a. Le retard dans la mise en place du Conseil scientifique de l'OIPR ......................................223

b. Le retard dans la mise en place et le fonctionnement des comités de gestion locale d'aire protégée 


\section{TITRE II : LES REMÈDES POUR UNE EFFECTIVITÉ DU DROIT DES AIRES}

\section{CHAPITRE I : LA NÉCESSITÉ DE CONCILIER LA CONSERVATION DES AIRES} PROTÉGÉES ET LE DÉVELOPPEMENT LOCAL

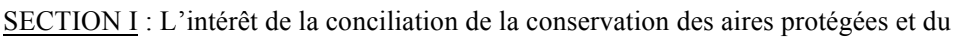
développement local

Paragraphe 1 : L'interdépendance de la conservation des ressources naturelles et du développement local

A- Conservation des ressources naturelles et développement local, deux facettes d'une même médaille.

1. Une recommandation des initiatives internationales de protection de l'environnement

a. Les recommandations issues des rencontres internationales des Nations Unies sur l'environnement

b. Les recommandations issues des Déclarations des congrès mondiaux sur les Parcs Nationaux

2. Une exigence des organismes de conservation et de développement

B- La justification de l'interdépendance fondée sur des résultats d'expériences de gestion de ressources naturelles

1. Des résultats de gestion d'aires protégées en Afrique de l'Est.................................................237

2. Des résultats de gestion d'aires protégées en Afrique australe ..................................................238

Paragraphe 2 : Les aires protégées, un moyen pour le développement des communautés locales.........239

A- Les aires protégées, un outil d'amélioration des moyens de subsistance des communautés locales ......................................................................................................240

1. L'exposé de quelques expériences de gestion d'aires protégées en Afrique de l'Est................240

2. La présentation d'exemples de gestion d'aires protégées en Afrique australe ..........................240

B- $\quad$ Les aires protégées, un moyen pour le développement des capacités d'organisation sociale des communautés locales.....

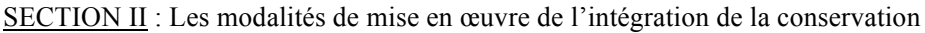
des aires protégées et du développement local

Paragraphe 1 : Les actions de conservation et de développement à entreprendre ..............................244

A- Les actions relevant de la responsabilité directe de l'État ....................................................244

1. Les actions urgentes et préalables de sauvegarde des aires protégées..................................245

2. La coordination des actions de développement autour de la conservation des aires protégées

B- $\quad$ L' encouragement des communautés locales à la mise en place de projets communautaires de gestion des ressources naturelles dans les zones périphériques des aires protégées

1. L'intérêt de la mise en place de projets communautaires de gestion des ressources naturelles dans les zones périphériques des aires protégées

2. Quelques exemples de projets communautaires de gestion des ressources naturelles à initier

3. Les recommandations relatives à la gestion des projets communautaires de gestion des ressources naturelles

a. Les recommandations relatives aux droits des communautés locales

b. Les recommandations portant sur la gestion courante des projets communautaires de gestion des ressources naturelles 
Paragraphe 2 : La nécessité de recourir au secteur privé pour la gestion durable des aires protégées ...259

A - L'intérêt du recours au secteur privé pour la mise en place de projets intégrant conservation et développement autour des aires protégées ....................................................259

1. L'importance du recours au secteur privé dans la gestion des aires protégées.........................259

2. L'existence en Côte d'Ivoire d'un cadre juridique favorable au recours au secteur privé pour la création et la gestion des aires protégées

B- La conclusion de contrats de partenariat public-privé, une solution de gestion durable des aires protégées en Côte d'Ivoire

1. L'intérêt de la conclusion de contrats de partenariat public-privé pour la gestion des aires protégées ivoiriennes ..........................................................................262

a. Les partenariats public-privé, un moyen pour la gestion durable des aires protégées ..........263

b. La présentation de partenariats public-privé de gestion d'aires protégées réussis ................264

2. Les précautions à prendre pour le succès des partenariats public-privé dans le cadre de la gestion des aires protégées ivoiriennes

\section{CHAPITRE II : LA VALORISATON TOURISTIQUE DES AIRES PROTÉGÉES ET DE LEURS ZONES PÉRIPHÉRIQUES}

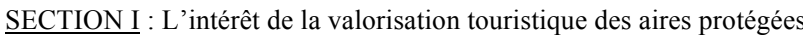

et de leurs zones périphériques

Paragraphe $1:$ Le tourisme, une activité financièrement rentable

A- Une source de financement pérenne pour la conservation des aires protégées ..........................2271

B- Un levier pour le développement des communautés riveraines des aires protégées .................272

1. L'exposé d'expériences de gestion d'aires protégées en Afrique de l'Ouest et en

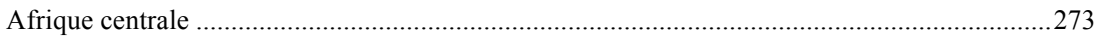

2. La présentation d'expériences de gestion d'aires protégées en Afrique de l'Est .....................274

3. Des exemples d'expériences de gestion d'aires protégées en Afrique australe.........................275

Paragraphe 2 : L'existence d'attraits naturels et culturels dans les aires protégées et leurs zones

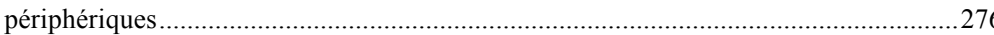

A- Les potentialités écotouristiques naturelles des aires protégées ..........................................276

1. Les atouts touristiques des aires protégées situées en zone forestière ..................................276

2. Les attraits écotouristiques des aires protégées localisées en zone de transition

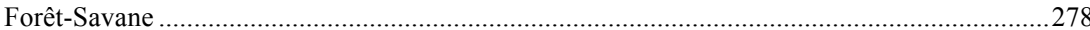

3. Les atouts écotouristiques des aires protégées situées en milieu urbain....................................280

4. Les attraits écotouristiques des aires protégées localisées sur la façade littorale atlantique

B- La diversité culturelle des communautés riveraines des aires protégées 282

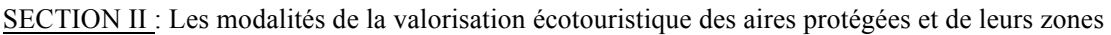
périphériques

Paragraphe 1 : La nécessité de concéder l'exploitation écotouristique des aires protégées à des opérateurs touristiques privés

A- De l'intérêt de la concession de l'exploitation écotouristique des aires protégées à des opérateurs touristiques privés

B- De l'environnement juridique de la concession de l'exploitation écotouristique des aires protégées à des opérateurs touristiques privés

1. De la forme juridique de l'accord de concession de l'exploitation écotouristique des aires protégées : le contrat de partenariat public-privé.

2. Des orientations relatives aux clauses du contrat de Partenariat

Public-Privé d'exploitation écotouristique des aires protégées 
Paragraphe 2 : La nécessité de développer le tourisme communautaire à l'intérieur et dans les zones périphériques des aires protégées

A- L'encouragement et l'encadrement des populations à la mise en place de projets touristiques

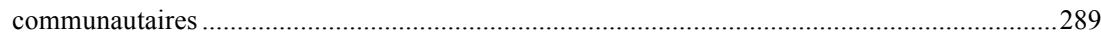

1. L'intérêt des projets touristiques communautaires ...........................................................290

2. De la conception à l'exécution des projets touristiques communautaires ................................291

3. Des activités touristiques à proposer dans le cadre du tourisme communautaire.......................293

B- La structuration juridique des projets touristiques communautaires ........................................294

1. De la forme juridique de l'entreprise touristique communautaire ............................................2294

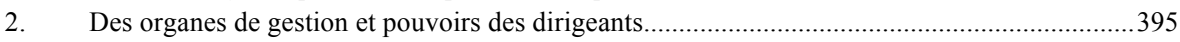

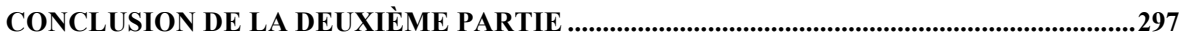

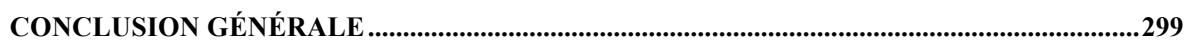

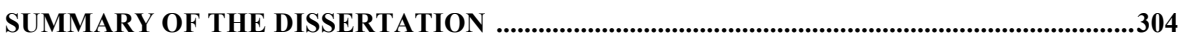

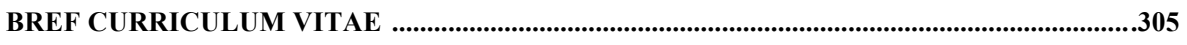

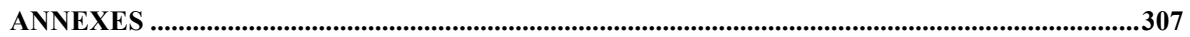

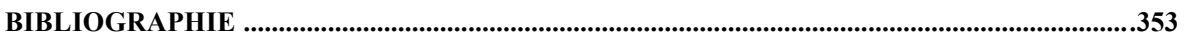




\section{LISTE DES PRINCIPAUX SIGLES ET ABRÉVIATIONS}

$\begin{array}{ll}\text { Art. : } & \text { Article } \\ \text { c. : } & \text { Contre }\end{array}$

Cass. Civ. : Cour de cassation chambre civile

Cass. Crim. : Cour de cassation chambre criminelle

Cf : Confère

Coll. : Collection

Concl. : Conclusion

D. : $\quad$ Dalloz

dir.: $\quad$ Sous la direction de

éd. : $\quad$ Éditions

ha : Hectare

hbts : habitants

km2 : Kilomètre carré

M. : $\quad$ Monsieur

no: $\quad$ Numéro

p. : $\quad$ Page

pp. : $\quad$ Pages

Rec. : $\quad$ Recueil

Rec. : Recommandation

s. : $\quad$ Suivant

S/p : $\quad$ Sous-préfectures

t. : $\quad$ tonne

AFD : $\quad$ Agence Française de Développement

AJDA : $\quad$ Actualité Juridique, Droit Administratif

ANDE : $\quad$ Agence Nationale de l'Environnement

ANGAP : Association Nationale de Gestion des Aires Protégées

ANPN : $\quad$ Agence Nationale des Parcs Nationaux du Gabon

BAD : $\quad$ Banque Africaine de Développement

BEI: $\quad$ Banque Européenne d'Investissement

BGF : $\quad$ Budget Général de Fonctionnement

BIRD : $\quad$ Banque Internationale pour la Reconstruction et le Développement

BM : $\quad$ Banque Mondiale

BOAD: $\quad$ Banque Ouest-Africaine de Développement

BSIE : $\quad$ Budget Spécial d'Investissement et d'Equipement

CA : $\quad$ Cour d'appel

CAMPFIRE :Communal Areas Management Programme for Indigenous Resources (en

français Programme de Gestion des Ressources Naturelles Communales)

CAPNR : Cellule d'Aménagement des Parcs Nationaux et Réserves

CCE : $\quad$ Commission des Communautés Européennes

CCNUCC : Convention-cadre des Nations Unies sur les changements climatiques

C2D : $\quad$ Contrat de désendettement et de développement

CEDA : $\quad$ Centre d'Edition et de Diffusion Africaines

CERAP : Centre de Recherche et d'Action pour la Paix

CGL: $\quad$ Comité de Gestion Locale

CHC : $\quad$ Compagnie Hévéïcole du Cavally

CICAD-ZP : Comité Interministériel de Coordination des Actions de Développement dans les Zones Périphériques des aires protégées 
CITES : Convention sur le commerce international des espèces de faune et de flore sauvages menacées de disparition

CNDJ : $\quad$ Centre Nationale de Documentation Juridique

CNO : $\quad$ Centre Nord-Ouest

CNPN : $\quad$ Conseil National des Parcs Nationaux

CNUEH : Conférence des Nations Unies sur l'Environnement Humain

CPP Code de Procédure Pénale

CSCA : $\quad$ Cour Suprême Chambre Administrative

CSSPPA : Caisse de Stabilisation et du Soutien des Prix des Produits Agricoles

DEA : $\quad$ Diplôme d'Études Approfondies

DESS : $\quad$ Diplôme d'Études Supérieures Spécialisées

DEF : $\quad$ Direction des Eaux et Forêts

DGEF: Direction Générale des Eaux et Forêts

DIGEBOS : en espagnol Dirección General de Bosques (en français Direction Générale des Forêts)

DPN : $\quad$ Direction de la Protection de la Nature

DZ: $\quad$ Direction de Zone

ECOFAC : Écosystèmes Forestiers d'Afrique Centrale

EDF : $\quad$ Électricité de France

EDICEF : Éditions Classiques d'Expression Française

EDUCI : Éditions Universitaires de Côte d'Ivoire

EPN : $\quad$ Établissement Public National

GCRN : $\quad$ Gestion Communautaire des Ressources Naturelles

GES : $\quad$ Gaz à Effet de Serre

GIEC Groupe d'experts Intergouvernemental sur l'évolution du Climat

FCFA : $\quad$ Franc de la Communauté Financière Africaine

FED : $\quad$ Fonds Européen de Développement

FEM : $\quad$ Fonds Mondial pour l'Environnement

FHB : $\quad$ Félix Houphouët-Boigny

FNDE : $\quad$ Fonds National de l'Environnement

FSAO: $\quad$ Fondation des Savanes Ouest Africaines

GTZ : Gesellschaft Technische Zusammnarbeit (CoopérationTechnique Allemande)

IBAP : $\quad$ Institut de la Biodiversité et des aires Protégées

ICCN: Institut Congolais pour la Conservation de la Nature

ICRAN: International Coral Reef Action Network (en français Réseau International pour les Récifs Coralliens)

IDH : $\quad$ Indice de développement humain

IGEF : Inspection Générale des Eaux et Forêts

IFDD : $\quad$ Institut de la Francophonie pour le Développement Durable

INAB : (en espagnol Instituto Nacional de Bosques, (en français Institut National des Forêts

INS : $\quad$ Institut National de la Statistique

IPNB : $\quad$ Institut des Parcs Nationaux du Congo belge

IRD : $\quad$ Institut de Recherche pour le Développement

IZCN : Institut Zaïrois pour la Conservation de la Nature

JO : $\quad$ Journal Officiel

JOAOF: Journal Officiel de l'Afrique Occidentale Française

JOCI : Journal Officiel de Côte d'Ivoire 
JORCI : Journal Officiel de la République de Côte d'Ivoire

JORF : Journal Officiel de la République Française

KWS : $\quad$ Kenyan Wildlife Service (en français Service de la Faune du Kenya)

LGDJ : $\quad$ Librairie Générale de Droit et de Jurisprudence

MINAGRA : Ministère de l'Agriculture et des Ressources Animales

MINEF : Ministère des Eaux et Forêts

MINEEF : Ministère de l'Environnement, des Eaux et Forêts

MINEFOR : Ministère des Eaux et Forêts

NEI : $\quad$ Nouvelles Éditions Ivoiriennes

NPWS : $\quad$ National Park and Wildlife Service (en français Département des Parcs

Nationaux et de la faune

OCDE : $\quad$ Organisation de Coopération et de Développement Économiques

OIF : $\quad$ Organisation Internationale de la Francophonie

OIPR : $\quad$ Office Ivoirien des Parcs et Réserves

ONG : $\quad$ Organisation Non Gouvernementale

ONU : $\quad$ Organisation des Nations Unies

OPJ : $\quad$ Officier de Police Judiciaire

ORSTOM : Office de Recherche Scientifique et Technique d'Outre

PAG : $\quad$ Plan d'Aménagement et de Gestion

PAPE : $\quad$ Programme d'Appui aux Parcs de l'Entente

PALMCI : Palmier à huile de Côte d'Ivoire

PCGAP : $\quad$ Programme Cadre de Gestion des Aires Protégées

PDF : $\quad$ Plan de Développement Forestier

PFBC : $\quad$ Partenariat pour les Forêts du Bassin du Congo

PFL : $\quad$ Produit Forestier Ligneux

PFNL : $\quad$ Produit Forestier Non Ligneux

PIB : $\quad$ Produit Intérieur Brut

PNA : $\quad$ Parc National d'Azagny

PNAE : $\quad$ Plan National d'Action Environnementale

PNB : $\quad$ Plan National du Banco

PNC : $\quad$ Plan National de la Comoé

PNIE : $\quad$ Parc National des Îles Ehotilé

PNM : $\quad$ Parc National de la Marahoué

PNMP : $\quad$ Plan National du Mont Péko

PNMS : $\quad$ Plan National du Mont Sangbé

PNT : $\quad$ Parc National de Taï

PNR : $\quad$ Parc National et Réserve

PPP : $\quad$ Partenariat Public-Privé

PUF : $\quad$ Presses Universitaires de France

RCI : $\quad$ République de Côte d'Ivoire

RFA : Réserve de Faune d'Abokouamékro

RFDA : $\quad$ Revue Française de Droit Administratif

RGPH : $\quad$ Recensement Général de la Population et de l'Habitat

RHPD : $\quad$ Rassemblement des Houphouétistes pour la Démocratie et la Paix

RNVC: $\quad$ Réserves naturelles volontaires communautaires

SAPH : $\quad$ Société Africaine des Plantations d'Hévéa

SCO : $\quad$ Service Central de l'Ordonnancement

S.E.M : $\quad$ Son Excellence Monsieur

SEPN/CAB : Secrétariat d'Etat des Parcs Nationaux/ Cabinet 
SGE : $\quad$ Sciences et Gestion de l'Environnement

SOGB : $\quad$ Société des Caoutchoucs de Grand-Béréby

TANAPA : Tanzania National Parks (en français Parcs Nationaux de Tanzanie)

UFR : $\quad$ Unité de Formation et de Recherche

UICN : $\quad$ Union Internationale pour la Conservation de la Nature (en anglais, IUCN : International Union for Conservation of Nature)

UNESCO : United Nations Educational, Scientific and Cultural Organization (Organisation des Nations unies pour l'éducation, la science et la Culture)

UNIDA : Association pour Unification du Droit en Afrique

UICN/BRAO : Union Mondiale pour la Nature/Bureau Régional d'Afrique de l'Ouest

UWA : $\quad$ Uganda Wildlife Authority (en français Autorité ougandaise pour la faune

WCF: Wild ChimpanzeeFoundation

WRI : $\quad$ World Resources Institute (en français Institut des Ressources Mondiales)

WWF: World WildlifeFund (littéralement, « Fonds mondial pour la vie sauvage»)

WWW: World Wide Web [littéralement la « toile mondiale »]

ZAF: $\quad$ Zone Agroforestière

ZAWA : Zambia Wildlife Authority (en français Autorité zambienne pour la faune

ZOC : $\quad$ Zone d'Occupation Contrôlée 
INTRODUCTION GÉNÉRALE 
«Selon la dernière évaluation des ressources forestières mondiales effectuée en l'an 2000, le couvert forestier continue de régresser de façon alarmante presque partout dans le monde, à un rythme moyen fort élevé. Ainsi, entre 1990 et 2000, la planète a perdu chaque année près de 10,7 millions d'hectares de forêts » ${ }^{1}$. Ces propos du Professeur Mohamed Ali MEKOUAR sont symptomatiques de ce que l'un des défis environnementaux mondiaux de notre époque demeure la conservation de la biodiversité forestière.

En effet, de nombreux auteurs soulignent dans leurs écrits et à divers égards le caractère alarmant de la perte de la biodiversité mondiale ${ }^{2}$. Si certains auteurs tels que Suzanne STOLL-KLEEMANN et Timothy O'RIORDAN ne se limitaient qu'à évaluer le rythme de cette perte inquiétante de la biodiversité de la planète (terre) ${ }^{3}$, d'autres par contre ont fait des projections encore alarmistes de cette perte. En guise d'exemples, Stuart PIMM et Peter RAVEN estiment qu'environ $18 \%$ des espèces contenues dans les "endroits les plus chauds du globe" qui concentrent entre $30-50 \%$ de toutes les espèces qui peuvent exister seront perdus en 2050, même si toutes ces zones sont pleinement protégées ${ }^{4}$. Pour Nicolas de SADELEER : "La dégradation de bon nombre d'écosystèmes est prête à atteindre un point de non-retour. Ainsi, la disparition particulièrement rapide de toute une série de milieux naturels irremplaçables, dont les milieux forestiers tropicaux sont les plus menacés, suscite une vive inquiétude chez les naturalistes...le nombre de forêts tropicales abattues ne fait que croître, à tel point qu'elles auront complètement disparu de la planète d'ici le milieu du siècle prochain. $)^{5}$.

De même, selon la $\mathrm{FAO}^{6}$, «Bien que l'on ait constaté ces dix dernières années un ralentissement du déboisement au niveau mondial, le taux de déboisement reste alarmant

\footnotetext{
${ }^{1}$ Voir MEKOUAR (Mohamed Ali), «Les forêts de Rio à Johannesburg et la perspective d'une convention forestière mondiale ", in PRIEUR (Michel) (dir.), Vers un nouveau droit de l' environnement ?, Limoges, Centre international de droit comparé de l'environnement, 2003/05, p. 69.

${ }^{2}$ Voir MYERS (Norman), MITTERMEIER (Russel A.), MITTERMEIER (Cristine G.), FONSECA (Gustavo A. B. da) et KENT (Jennifer), « Biodiversity hotspots for conservation priorities », Nature, Vol. 403, 2000, p. 855; STOLL-KLEEMANN (Suzanne) et O'RIORDAN (Timothy), « From Participation to Partnership in Biodiversity Protection : Experience from Germany and South Africa », Society and Natural Resources : An International Journal, Vol. 15, $\mathrm{n}^{\circ}$ 2, 2002, p. 161; MEKOUAR (Mohamed Ali), « Les forêts de Rio à Johannesburg et la perspective d'une convention forestière mondiale », in PRIEUR (Michel) (dir.), Vers un nouveau droit de $l^{\prime}$ environnement ?, op. cit., p. 69 ; VISSEREN-HAMAKERS (Ingrid J.) et GLASBERGEN (Pieter), «Partnerships in forest governance», Global Environmental Change, Vol. 17, n³-4, 2007, p. 408; VERMEULEN (Sonja), SHEIL (Douglas), « Partnerships for tropical conservation », Oryx, Vol. 41, $\mathrm{n}^{\circ} 4,2007$, p. 434.

${ }^{3}$ En effet, ils affirment : «De nos jours, d'un commun accord, nous assistons, et nous sommes partie à une extraordinaire perte d'espèces et leurs milieux écologiques ». Voir STOLL-KLEEMANN (Suzanne) et O'RIORDAN (Timothy), «From Participation to Partnership in Biodiversity Protection : Experience from Germany and South Africa », op.cit., p. 162.

${ }^{4}$ PIMM (Stuart L.) et RAVEN (Peter), « Extinction by numbers », Nature, Vol. 403, 2000, p. 844.

${ }^{5}$ Voir SADELEER (Nicolas de), «Les enjeux de la temporalité dans le droit de l'environnement », in GÉRARD (Philippe), OST (François) et KERCHOVE (Michel van de) (dir.), L'accélération du temps juridique, Bruxelles, Publications des Facultés universitaires Sant-Louis, 2000, p. 894.

${ }^{6}$ En Anglais Food and Agriculture Organization of the United Nations (FAO), FAO signifie, en français, Organisation des Nations Unies pour l'alimentation et l'agriculture. La FAO est une organisation intergouvernementale dont la mission est de contribuer à éliminer la faim, l'insécurité alimentaire et la malnutrition dans le monde. Ainsi, composée de 194 États-membres, de deux (2) membres associés, une
} 
dans de nombreuses régions $d u$ monde... ${ }^{7}$. Il s'en déduit la perte de la diversité biologique et particulièrement la dégradation des forêts du globe.

La situation est également la même en Côte d'Ivoire ${ }^{8}$. En effet, de plus 12 millions d'hectares de forêts en 1960, ce pays ne dispose que d'environ 2 millions d'hectares de nos jours (y compris les aires protégées qui font l'objet de notre étude) ${ }^{9}$. D'après Jacques GIROD, en Côte d'Ivoire, "La forêt est détruite au rythme de 2.500 hectares par an, surtout à cause de défrichements agricoles ${ }^{10}$.

Face à cette érosion de la biodiversité forestière dont l'Homme est le principale responsable, les pouvoirs publics ivoiriens entreprennent des initiatives de protection ${ }^{11}$. Aussi

organisation membre et l'Union européenne, elle intervient dans plus de 130 pays. Voir www.fao.org/about/what-we-do/fr/, consulté le 8 novembre 2015.

${ }^{7}$ Voir FAO, Situation des forêts du monde : Mieux tirer parti des avantages socioéconomiques des forêts, Rome, Organisation des Nations Unies pour l'Alimentation et l'Agriculture, 2014, p. 2.

${ }^{8}$ Ancienne colonie française, par l'effet du décret du 10 mars 1893 [voir LOUCOU (Jean-Noël), La Côte d'Ivoire coloniale 1893-1960, Abidjan, les Éditions FHB \& les Éditions du CERAP, 2012, p. 56], la République de Côte d'Ivoire est située dans la zone intertropicale de l'Afrique de l'Ouest (voir FAO, L'Etude prospective du secteur forestier en Afrique (FOSA) : Côte d'Ivoire, p. 4, disponible sur www.fao.org/docrep/003/X6780F/X6780F05.htm, consulté le 24 mai 2016).

Indépendante depuis le 7 août 1960 [voir FIERENS (Marie), “'Il était une fois l'indépendance de la Côte d'Ivoire...'. Récits médiatiques d'hier et d'aujourd'hui », Le Temps des Médias, Vol. 1, n²6, 2016, p. 144. ], la République de Côte d'Ivoire s'étend sur une superficie d'environ $322.462 \mathrm{~km}^{2}$ et de coordonnées de $2^{\circ} 30^{\prime}$ et $8^{\circ}$ $30^{\prime}$ de longitude ouest, $4^{\circ} 30^{\prime}$ et $10^{\circ} 30^{\prime}$ de latitude nord. Elle est limitée au Nord par le Mali et le Burkina-Faso, à l'Est par le Ghana, à l'Ouest par le Libéria et le Guinée, et au Sud par l'océan atlantique avec une façade littorale d'environ 550 kilomètres. Voir TIEBLEY (Yves-Didier), La Côte d'Ivoire et la gestion des ressources naturelles marines, Thèse unique, Droit public, Université de Maastricht, 20 mai 2010, p. 16.

Sa population est estimée à 23,2 millions habitants en 2015 [voir République de Côte d'Ivoire, Ministère du Plan et du Développement, Plan national de développement PND 2016-2020: Diagnostic stratégique de la Côte d'Ivoire sur la trajectoire de l'émergence (tome 1), p. 28], et la langue officielle est le français. Elle a pour capitale politique Yamoussoukro depuis 1983 en lieu et place d'Abidjan (ancienne capitale politique) qui est dorénavant la capitale économique (voir République de Côte d'Ivoire, Document Stratégique de Réduction de la Pauvreté, Stratégique de Relance du Développement et de Réduction de la Pauvreté, Janvier 2009, p.1.). Toutefois, le transfert de la capitale politique n'étant pas effectif, Abidjan rassemble encore la presque totalité des Administrations avant leur transfert effectif dans la capitale politique.

${ }^{9}$ Voir République de Côte d'Ivoire, Ministère d'État, Ministère du Plan et du Développement, Document stratégique de réduction de la pauvreté, Abidjan, 2009, p. 63.

${ }^{10}$ Voir GIROD (Jacques), L'énergie en Afrique : la situation énergétique de 34 pays de l'Afrique subsaharienne et $d u$ Nord, France, Karthala Éditions, 1994, p.146.

${ }^{11}$ D'abord en matière de législation, l'on peut noter, d'une part, le renforcement de la réglementation nationale destinée à la protection de l'environnement adoptée après les indépendances [voir GNANGUI (Adon), « Côte d'Ivoire : Évolution du droit de l'environnement depuis $1992 »$, in Michel PRIEUR (dir.), Vers un nouveau droit de l' environnement?, Limoges, Centre international de droit comparé de l'environnement, 2003/05, pp.361363.]. D'autre part, la Côte d'Ivoire a ratifié des conventions relatives à la protection de l'environnement ou y a adhéré (voir le point " II- Aperçu de la législation consacrée à la protection des aires protégées en Côte d'Ivoire" et précisément le point intitulé " A. Les textes juridiques internationaux applicables aux aires protégées"). En outre, la Côte d'Ivoire a défini des politiques et programmes de protection de l'environnement notamment le Plan National d'Action Environnemental (PNAE) élaboré en 1994. Selon GNANGUI (Adon), c'est un plan cohérent d'actions qui vise la protection de l'environnement. Voir GNANGUI (Adon), «Côte d'Ivoire : Évolution du droit de l'environnement depuis 1992 », in Michel PRIEUR (dir.), Vers un nouveau droit de $l^{\prime}$ environnement?, op.cit., p. 363. Il y a également le Programme Cadre de Gestion des Aires Protégées (PCGAP) mis en place en 2000 dans un but de gestion durable des aires protégées en proie à des pressions anthropiques qui menacent leur existence ( voir AFD, Note de communication publique d'opération. Côte d'Ivoire. C2D/ 
ces différentes initiatives témoignent-elles des enjeux de la conservation des forêts et notamment des aires protégées.

\section{I - ENJEUX DE LA PROTECTION DES FORÊTS EN CÔTE D'IVOIRE}

Selon la FAO, «La contribution des forêts au bien-être humain est considérable. Les forêts fournissent des ressources ligneuses précieuses, contribuent à la lutte contre la pauvreté rurale et à la sécurité alimentaire et aident à assurer des moyens de subsistance décents; elles offrent des occasions prometteuses de croissance verte à moyen terme et procurent des services environnementaux vitaux à long terme, comme l'air pur et l'eau propre, la biodiversité et l'atténuation des changements climatiques. ». Ces conclusions de la FAO relative à l'évaluation des ressources forestières mondiales réalisée dans 234 pays et territoires dont la Côte d'Ivoire ${ }^{12}$ attestent aussi que les enjeux de la protection des forêts sont multiples.

En Côte d'Ivoire, les enjeux de la protection des forêts sont d'ordre écologique (A) et socio-économique $^{13}(\mathrm{~B})$.

\section{A-L'importance écologique des forêts}

Les forêts sont des puits de carbone et des réservoirs de biodiversité (1). Elles contribuent à la fertilisation du sol, à la lutte contre l'érosion (2) ainsi qu'à la régulation du climat (3).

\footnotetext{
Conservation des ressources naturelles, pp. 2-3, disponible sur: www.afd.fr/base projets/downloadDocument.action?idDocument=1470 et consulté le 12 juin 2015).

Enfin, l'État de Côte d'Ivoire a créé, entre autres, des institutions spécialisées pour la protection de l'environnement que sont le Secrétariat national de l'ozone de la Côte d'Ivoire, l'Agence Nationale de l'Environnement (ANDE), le Fonds national de l'environnement (FNDE) pour le financement de la politique de protection de l'environnement et de la gestion des ressources naturelles (voir Adon GNANGUI, « Côte d'Ivoire : Évolution du droit de l'environnement depuis 1992 ", in Michel PRIEUR (dir.), Vers un nouveau droit de l' environnement ?, op.cit. p. 363). De même, l'Office Ivoirien des Parcs et Réserves a été créé pour la gestion et le financement des Parcs et Réserves de Côte d'Ivoire (voir Décret 2002-359 du 24 juillet 2002 portant création, organisation et fonctionnement de l'OIPR, JORCI n ${ }^{\circ} 40$ du 2 octobre 2003, p. 588).

${ }^{12} \mathrm{FAO}$, Évaluation des ressources forestières mondiales 2015. Répertoire des données de FRA 2015, Rome Organisation des Nations Unies pour l'Alimentation et l'Agriculture, 2015, p. 245.

${ }^{13} \mathrm{~A}$ propos de l'importance des forêts tropicales, LEROY (Maya) et al. écrivent: «La forêt, notamment tropicale, se trouve au ccur des questions de développement durable, aussi bien pour ses enjeux écologiques (conservation de la biodiversité végétale et animale, régulation des ressources en eau, stockage du carbone, etc.), sociaux (amélioration des conditions de vie des populations qui en dépendent, valorisation des connaissances traditionnelles, maintien de l'existence de ces écosystèmes pour les générations futures, etc.) qu'économiques (production et exploitation des ressources ligneuses, source de revenus pour les populations locales, etc.)» (souligné par nous). Voir LEROY (Maya), DERROIRE (Géraldine), VENDÉ (Jeremy) et LEMÉNAGER (Tiphaine), La gestion durable des forêts tropicales: De l'analyse critique du concept à l'évaluation environnementale des dispositifs de gestion, AFD, À savoir nº ${ }^{\circ} 8$, mai 2013, p. 25.
} 


\section{Forêts : puits de carbone et réservoir de biodiversité}

La Côte d'Ivoire compte environ deux (2) millions d'hectares de forêts dont les Parcs Nationaux et Réserves ${ }^{14}$. Ces forêts regorgent d'habitats, d'espèces de faune et de flore. Elles constituent leur refuge et assurent leur perpétuation à travers les processus écologiques naturels ${ }^{15}$.

Le rapport national sur la diversité biologique indique également que ces forêts contribuent à la régulation du stockage du carbone ${ }^{16}$. À titre d'illustration, une étude réalisée dans l'arboretum du Centre National de Floristique sis à Abidjan et crée en 1973 a montré que 41 ans après sa création, le stock total de carbone emmagasiné par «l'ensemble des tiges des espèces d'arbres est de 189,67tC/ha ${ }^{17}$. Il s'en déduit que les forêts et notamment les forêts tropicales sont très importantes pour la lutte contre les changements climatiques en ce sens qu'elles sont des puits de carbone ou de gaz à effet de serre ${ }^{18}$ responsables des changements climatiques $^{19}$.

Par ailleurs, les forêts contribuent à la lutte contre l'érosion et la fertilité des sols.

\footnotetext{
${ }^{14}$ Voir République de Côte d'Ivoire, Ministère d'État, Ministère du Plan et du Développement, Document stratégique de réduction de la pauvreté, op. cit.p. 63.

${ }^{15}$ Voir LAUGINIE (Francis), Conservation de la nature et des aires protégées en Côte d'Ivoire, Abidjan, NEI/Hachette et Afrique Nature, 2007, p. 43.

${ }^{16}$ République de Côte d'Ivoire, Cinquième rapport national sur la diversité biologique, mars 2014, 106 pages, p. 8.

${ }^{18}$ L'effet de serre «est un phénomène naturel qui permet à la Terre (comme toute autre planète) de retenir la chaleur solaire dans l'atmosphère et de maintenir une température acceptable pour entretenir la vie. Sans cela la température de la Terre serait d'environ $-18^{\circ} \mathrm{C}$. On appelle souvent "effet de serre" l'augmentation de l'effet de serre de notre époque, qui provoque le réchauffement climatique.» (voir

www.developpement-durable.gouv.fr/-Effet-de-serre-et-changement-.html, consulté le 26 mai 2016).

Ainsi, les gaz à effet de serre (GES) sont des composants gazeux généralement dues aux émissions de l'Homme [dont les principaux sont la vapeur d'eau $\left(\mathrm{H}_{2} \mathrm{O}\right)$, le dioxyde de carbone $\left(\mathrm{CO}_{2}\right)$; le méthane $\left(\mathrm{CH}_{4}\right)$, le protoxyde d'azote $\left(\mathrm{N}_{2} \mathrm{O}\right)$, l'ozone $\left(\mathrm{O}_{3}\right)$ ] qui absorbent la chaleur solaire émise par la surface terrestre et contribuent à réchauffer la planète terre à une température encore plus élevée qu'elle ne le devrait naturellement. Voir Protocole de Kyoto à la Convention-Cadre des Nations Unies sur les Changements Climatiques, 1998, Annexe A, disponible sur www.cop21.gouv.fr/quest-ce-que-les-gaz-a-effet-de-serre/ et consulté le 26 mai 2016.

${ }^{19}$ Selon le GIEC, on entend par changement climatique : «...toute évolution du climat dans le temps, qu'elle soit due à la variabilité naturelle ou aux activités humaines. ». Voir GIEC, " Résumé à l'intention des décideurs ", In : Changements climatiques 2007 : Les éléments scientifiques. Contribution du Groupe de travail I au quatrième Rapport d'évaluation du Groupe d'experts intergouvernemental sur l'évolution du climat. [SOLOMON (S.), QIN (D), MANNING (M), CHEN (Z), MARQUIS (M), AVERYT (K.B), TIGNOR (M) et MILLER (H.L) (éds.)]. Cambridge University Press, Cambridge, UK et New York, NY, USA, 2007, p. 2.

Cette définition du changement climatique formulée par le GIEC diffère de celle que donne l'article $1^{\text {er }}$ de la Convention-cadre sur le changement climatique en ce sens que cette dernière n'attribue qu'une origine anthropique au changement climatique (au contraire du GIEC qui retient qu'elle émane aussi bien de phénomènes naturels que des activités de l'Homme. En effet, l'article $1^{\text {er }}$ de la Convention-Cadre des Nations Unies sur les Changements Climatiques (CCNUCC) stipule que les changements climatiques sont «des changements de climat qui sont attribués directement ou indirectement à une activité humaine altérant la composition de l'atmosphère mondiale et qui viennent s'ajouter à la variabilité naturelle du climat observée au cours de périodes comparables. ".
} 


\section{Forêts et lutte contre l'érosion et fertilité des sols}

Les racines des arbres et autres végétaux contribuent à réduire ou à freiner l'érosion consécutive aux ruissellements, en retenant les sédiments et autres éléments minéraux qui enrichissent le sol. De même, les feuilles, les branches et autres parties des végétaux qui tombent et meurent constituent de la matière organique qui fertilise le $\mathrm{sol}^{20}$. En conséquence, cette matière organique participe au processus de régénérescence de la forêt qui régule le climat.

\section{Forêts et régulation du climat}

Les forêts participent à la régulation du climat pour deux (2) raisons principales. Elles garantissent une bonne pluviométrie et assurent une protection contre les évènements climatiques violents $^{21}$.

En effet, les travaux de Télesphore Y. BROU indiquent qu'entre 1955 et 1993, la superficie des zones forestières ivoiriennes non soumises à l'agriculture est passée de 8,3 à 1,5 millions d'hectares. Il s'agit d'une baisse de 6,5 millions d'hectares de forêts dévastées pour des raisons culturales ${ }^{22}$. Cette réduction accélérée du couvert forestier ivoirien a entraîné une diminution de la pluviométrie et favorisé la mise en place de milieux $\operatorname{secs}^{23}$.

Il en découle qu'il existe une corrélation très étroite entre l'existence de la forêt et la pluviométrie de sorte que la dégradation de celle-ci entraîne la diminution de celle-là. C'est cette réalité bioclimatique que Télesphore Y. BROU et al. traduisent de la manière suivante : «Parallèlement à la baisse enregistrée de la pluviométrie, on a observé que la part des forêts denses humides dans le Sud forestier ivoirien a considérablement diminuée de 1955 et 1990, au profit de la jachère et de cultures...» ${ }^{24}$.

$\mathrm{Au}$ surplus, d'après Célestin Y. AMANI, l'Organisation de Coopération et de Développement Économiques (OCDE) présente le climat comme facteur intégré dans l'ensemble des productions agricoles africaines et notamment celles de la Côte d'Ivoire dont l'économie repose sur l'agriculture ${ }^{25}$. Autrement dit, alors que la conservation de la forêt procure des avantages écologiques susmentionnés, son exploitation par les populations est une réalité qui s'analyse en termes d'enjeux socio-économiques.

\footnotetext{
${ }^{20}$ Pour ces développements concernant le rôle des forêts dans la lutte contre l'érosion et la fertilisation des sols, voir République de Côte d'Ivoire, Cinquième rapport national sur la diversité biologique, op.cit., p. 8.

${ }^{21}$ Voir République de Côte d'Ivoire, Cinquième rapport national sur la diversité biologique, op.cit., pp. 8-10.

${ }^{22}$ Voir BROU (Télesphore Yao), « Modification du régime des précipitations et risque de stress hydrique des formations végétales en zone de forêt dense humide ivoirienne », Cahiers Nantais, n51, 1999, pp. 113-114.

${ }^{23}$ Idem, p. 115.

${ }^{24}$ Voir BROU (Télesphore Yao), SERVAT (Eric), PATUREL (Jean-Emmanuel), « Contribution à l'analyse des interrelations entre activités humaines et variabilité climatique : cas du sud forestier ivoirien », Académie des sciences, Vol. 327, $\mathrm{n}^{\circ}$ 12, 1998, p. 838.

${ }^{25}$ Voir AMANI (Célestin Yao), "Production agricole et changement climatique: vers une tragédie des comportements paysans à Tiassalé?, European Scientific Journal, Vol. 8, n¹6, 2012, p. 229.
} 


\section{B - Les retombées socio-économiques de l'exploitation des forêts en Côte d'Ivoire}

L'exploitation des forêts en Côte d'Ivoire vise un objectif économique (1) et social (2).

\section{La rentabilité économique de l'exploitation des forêts}

«La forêt a toujours été au centre des préoccupations de la Côte d'Ivoire depuis la création de notre pays. Tout est parti de la forêt. Les premiers échanges entre notre pays et le monde extérieur étaient basés sur l'exploitation de nos bois. Et pendant longtemps, le bois a tenu la première place dans notre économie. C'est grâce à la forêt, à la richesse de son sol et à la pluviométrie maintenue par nos bois que la Côte d'Ivoire a pu devenir en Afrique et même dans le monde, un des pays les plus gros producteurs agricoles : premier producteur mondial de cacao, troisième producteur de café, deuxième pour l'huile de palme après la Malaisie; aujourd'hui, l'un des plus gros producteurs africains de caoutchouc, et nous nourrissons l'ambition de devenir en 1990 le premier producteur de caoutchouc en Afrique. Tout cela grâce à notre forêt. C'est une richesse que nous devons préserver $»^{26}$.

Cet extrait du discours du Président Félix HOUPHOUET-BOIGNY démontre l'importance et le rôle de la forêt dans le développement économique de la Côte d'Ivoire. Dès l'indépendance, le gouvernement ivoirien a choisi de développer une économie basée sur l'exploitation de la forêt ${ }^{27}$. Il s'agit d'une économie forestière et de plantation caractérisée par le développement de produits de rente tels que le cacao, le café, les oléagineux, le bois dont les conditions de production exigent la forêt ${ }^{28}$.

Ainsi, l'économie de la Côte d'Ivoire repose sur l'exploitation de la forêt, à travers l'agriculture de rente et l'exploitation des produits ligneux. En effet, le boom économique de la Côte d'Ivoire réalisée dans la décennie 70 à 80 est l'œuvre de l'agriculture ${ }^{29}$. Dans la décennie 80 à 90 , l'agriculture occupe toujours une place prépondérante dans l'économie nationale car les cultures industrielles d'exportation constituent le fer de lance de l'économie ivoirienne. L'agriculture représente à elle seule environ $40 \%$ du $\mathrm{PIB}^{30}$. Au cours de la décennie 90 à 2000, l'agriculture continue d'être la principale activité de la Côte d'Ivoire. Elle représente $33 \%$ du PIB, génère $66 \%$ des recettes d'exportations et deux (2) emplois sur trois (3). De l'an 2000 à nos jours, l'agriculture demeure toujours la base de l'économie ivoirienne.

\footnotetext{
${ }^{26}$ Extrait de l'allocution de S.E.M. Félix HOUPHOUET-BOIGNY (1 ${ }^{\text {er }}$ Président de la République de Côte d'Ivoire), prononcé, le 9 mai 1987, à l'issue du Séminaire des Eaux et Forêts de Yamoussoukro (voir République de Côte d'Ivoire, Ministère des Eaux et Forêts, Plan Directeur Forestier 1988-2015, Abidjan, décembre 1988, p. I).

${ }^{27}$ Voir AFD, Note de communication publique d'opération. Côte d'Ivoire. C2D/ Conservation des ressources naturelles, op.cit., p. 2.

${ }^{28}$ Ibidem.

${ }^{29}$ Voir ELDIN (Michel), MILLEVILLE (Pierre), Le risque en agriculture, IRD Éditons, 1989, p. 516.

${ }^{30}$ Voir République de Côte d'Ivoire, Ministère de l'Économie, des Finances et du Plan, La Côte d'Ivoire en chiffres, édition 86-87, Paris, Inter Afrique Presse, 1988, p. 92.
} 
Elle est la principale pourvoyeuse de l'industrie; emploie les $2 / 3$ de la population active et fournit avec le secteur agroindustriel $40 \%$ des recettes d'exportations ${ }^{31}$.

Par ailleurs, l'exploitation forestière est également d'un apport très appréciable dans le développement économique de la Côte d'Ivoire. Pendant longtemps, le bois a joué un rôle de premier plan dans l'économie ivoirienne. Par exemple, les exportations de bois (grumes et bois usinés) ont rapporté à la Côte d'Ivoire en moyenne 17,943 milliards de FCFA par an entre 1960 et $1971^{32}$; et 72,827 milliards de FCFA par an entre 1972 et $1980^{33}$. De 1981 à 1986, ces exportations ont fait gagner à la Côte d'Ivoire 92,660 milliards de FCFA par an ${ }^{34}$; et 121,338 millions par an, de 1991 à $1995^{35}$. En 1997, une mission de la banque mondiale créditait le secteur forestier ivoirien d'un chiffre d'affaires de 300 milliards de francs CFA et de 38 emplois $^{36}$. En 2011, une étude sur la filière bois révèle que pour les entrées en usine de $1.000 .786 \mathrm{~m}^{3}$, la filière bois à l'exploitation a généré plus de 97 milliards de FCFA, selon les douanes ivoiriennes. De plus, «...la valeur du marché informel de bois d'œuvre (plus 1,2 millions de $m^{3}$ de produits finis) s'élèverait à plus de 74 milliards de FCFA...» ${ }^{37}$. Selon cette étude, en novembre 2013, le marché de la filière bois est estimé à 94 milliards de FCFA ${ }^{38}$.

Au-delà de ces apports sur le plan économique, l'exploitation de la forêt a des avantages sur le plan social.

\section{Les avantages sociaux de l'exploitation des forêts}

En Côte d'Ivoire, les populations utilisent la forêt pour s'approvisionner en ressources alimentaires. Il s'agit de plantes alimentaires telles que les feuilles, les fruits, les tubercules consommées, entres autres, sous formes de condiments, d'épices, de boissons dont les principales sont: "Adansonia digitata (Baobab), Ceiba pentandra (Fromager), Irvingia gabonensis (Kplé en Guéré ${ }^{39}$ ), Hibiscus sabdariffa (Da), Lipia multiflora (Thé de Gambie),

\footnotetext{
${ }^{31}$ Voir République de Côte d'Ivoire, Ministère d'État, Ministère du Plan et du Développement, Document Stratégique de Réduction de la Pauvreté, op. cit., p. 39.

${ }^{32}$ Voir République de Côte d'Ivoire, Ministère du plan, La Côte d'Ivoire en chiffres, éd. 1975, Abidjan, 1975, p. 110 .

${ }^{33}$ Voir République de Côte d'Ivoire, Ministère de l'Économie et des Finances, Direction de la Programmation de la Budgétisation et du Contrôle de Gestion, Annuaire rétrospectif de statistiques agricoles et forestières 19001983, tome 1: L'agriculture et la forêt dans l'économie ivoirienne, Abidjan, 1985, pp. 248-251.

${ }^{34}$ Voir République de Côte d'Ivoire, Ministère de l'Économie et des Finances, La Côte d'Ivoire en chiffres, édition 86-87, op. cit., p.143.

${ }^{35}$ Voir République de Côte d'Ivoire, Ministère de l'Économie et des Finances, La Côte d'Ivoire en chiffres, édition 96-97, Abidjan, Dialogue Production, 1997, p. 54.

${ }^{36}$ Voir IBO (Guéhi Jonas), « Les nouvelles orientations de la gestion du patrimoine naturel en Côte d'Ivoire », in CORMIER-SALEM (Marie-Christine), JUHÉ-BEAULATON (Dominique), BOUTRAIS (Jean) et ROUSSEL (Bernard) (dir.), Patrimoine naturels au Sud: Territoires, identités et stratégies locales, IRD Éditions, $2005, \mathrm{p}$. 74.

${ }^{37}$ Voir LOUPPE (Dominique), OUATTARA (N'Klo), Étude sur l'exploitation forestière et les contraintes d'une gestion durable des forêts dans le domaine rural en Côte d'Ivoire, Abidjan, CIRAD, GTZ, décembre 2013, p. 41.

${ }^{38}$ Ibidem.

${ }^{39}$ Le Guéré est une ethnie de l'Ouest de la Côte d'Ivoire. Les peuples issus de cette sphère linguistique porte le même nom.
} 
Myrianthus arboreus (Tikriti en Bété), Piper guineense (Poivre noire), Ricinidendron heudelotii (Akpi), Zingiber officinale (Gingembre) $»^{40}$. En outre, la faune sauvage notamment les petits et grands mammifères (aulacodes, écureuils, biches, singes, mangoustes...) ainsi que les oiseaux sont prélevés dans la nature à des fins médicinales et de consommation ${ }^{41}$.

À l'instar de la Côte d'Ivoire, l'exploitation des aires protégées à but alimentaire est caractéristique des pays pauvres et en voie de développement ${ }^{42}$. En guise d'exemples, une étude menée au Swaziland atteste que plus de 200 plantes sauvages comestibles sont collectées par les populations dans les forêts ${ }^{43}$. Au Nigéria les communautés riveraines du Parc National Oban prélèvent plus de 150 espèces de plantes alimentaires sauvages des zones forestières ${ }^{44}$. Dans l'Ouest du Kenya, les communautés locales utilisent environ 100 espèces différentes de fruits et légumes provenant la nature ${ }^{45}$.

Aussi plus de 1500 espèces végétales sont-elles utilisées en Côte d'Ivoire à des fins médicinales, sous la forme de racines, écorces, tiges, fruits, feuilles et fleurs par les populations $^{46}$. Les ressources forestières telles que les produits ligneux et non ligneux ${ }^{47}$ sont

\footnotetext{
${ }^{40}$ Voir République de Côte d'Ivoire, Cinquième rapport national sur la diversité biologique, op. cit. p. 5.

${ }^{41}$ Ibidem.

${ }^{42}$ Une partie au moins des revenus d'environ $90 \%$ des pauvres dans le monde dépend des forêts (Voir USAID, Issues in Poverty Reduction and Natural Resource Management, United States Agency for International Development, Washington, DC., 2006, p. 3). Dans de nombreux pays africains, la population vit avec moins d'un dollar par jour (voir ANDERSON (J.) et al. cité par COUNSELL (Simon), Forest Governance in Africa, Johannesburg, South African Institute of International Affairs, Occasional paper n`50, Governance of Africa's Resources Programme, 2009, p. 6). De plus, $60 \%$ et $90 \%$ de la population active est dans le secteur rural (voir COUNSELL (Simon), Forest Governance in Africa, op. cit., p. 6. Il s'agit donc d'une population pauvre.

En Afrique australe, de l'Est et de l'Ouest, plus de $90 \%$ des ménages ruraux dépendent du bois de chauffe et du charbon de bois pour l'énergie (domestique). En outre, en Afrique subsaharienne, plus de $80 \%$ des populations recourent à la médecine naturelle par l'utilisation des produits issus des zones forestières. Voir COUNSELL (Simon), Forest Governance in Africa, op. cit., p. 6.

Selon la Banque mondiale, dans un pays comme la République démocratique du Congo, trois quarts de la population tirent ses moyens de subsistance des forêts. Voir DEBROUX (L.) et TOPA (G.) (eds), 2007 cité par COUNSELL (Simon), Forest Governance in Africa, op. cit., p. 6.

${ }^{43}$ Voir OGLE (Britta Mathilda) et GRIVETTI (Louis Evan), « Legacy of the Chameleon: Edible Wild Plants in the Kingdom of Swaziland, Southern Africa: A Cultural, Ecological, Nutritional Study, Part II -- Demographics, Species Availability and Dietary Use, Analysis by Ecological Zone.», Ecology of Food and Nutrition, Vol. 17, $\mathrm{n}^{\circ} 1,1985$, p. 1.

${ }^{44}$ Voir OKAFOR (J.C.), " Agroforestry Aspects, World Wide Fund for Nature », Surrey, UK., (extrait de bibliographie cité par SCOONES (Ian), MELNYK (Mary), PRETTY (Jules N.), The Hidden Harvest. Wild Foods Agricultural Systems. A literature Review and Annotated Bibliography, WWF/ IIED, 1992, p. 222.

${ }^{45}$ Voir MAKOMBE (Kudzai), Sharing the land: Wildlife, People, and Development in Africa, Harare, Zimbabwe, IUCN/SUWP? Washington, USA, IUCN/ROSA Environmental Issues Series ${ }^{\circ} 1$, IUCN/ROSA, 1994, p. 13.

${ }^{46}$ Voir République de Côte d'Ivoire, Cinquième rapport national sur la diversité biologique, op. cit., p.7.

${ }^{47}$ Les produits forestiers non ligneux (PFNL) sont des produits forestiers autres que le bois. Il s'agit par exemples des végétaux comestibles (champignons, des graines, des fruits, des plantes alimentaires), des plantes médicinales. Voir UICN/PACO, Retombées économiques des aires protégées d'Afrique de l'Ouest, Ouagadougou, BF, 2011, p.15; FAO, L'Etude prospective du secteur forestier en Afrique (FOSA) Côte d'Ivoire (disponible sur: www.fao.org/docrep/fao/003/X6780F/X6780F00.pdf, consulté le 4 juin 2016); QUEBEC, Ministère des Ressources Naturelles de la Faune et des Parcs, Direction du développement de l'industrie des produits forestiers, Direction du développement de l'industrie des produits forestiers, Aperçu des produits forestiers non ligneux (PFNL), juin 2003, p. 1.
} 
également utilisées par les populations pour des usages domestiques notamment la construction d'habitats, la fabrication d'objets divers (meubles, ustensiles de cuisine, objets de décoration etc.) et d'outils de travail (pirogues, houes...) $)^{48}$.

Par ailleurs, il est à noter que l'exploitation socio-économique des forêts en Côte d'Ivoire n'épargne pas les aires protégées objet de notre étude.

\section{C-L'état des pressions anthropiques sur la biodiversité forestière en Côte d'Ivoire}

Les pressions anthropiques sur la biodiversité concernent les forêts, en général (1) et les aires protégées, en particulier (2).

\section{L'érosion de la biodiversité forestière ivoirienne en général}

Il ressort du cinquième rapport national sur la diversité biologique que l'agriculture et l'exploitation forestière précédemment mentionnées sont les principales causes de la dégradation forestière en Côte $d^{\prime}$ Ivoire $^{49}$. D'après ce rapport, chaque année, les zones forestières et de savane sont parcourues par des feux de brousse utilisés surtout comme technique culturale (le brûlis) ou de chasse. Ces feux, tout en étant destructeurs, se le révèlent encore plus lorsqu'ils sont incontrôlés.

Il s'ensuit une dégradation de la flore et de la faune sauvage et d'autres éléments constitutifs de la biodiversité. En effet, le rapport précité indique que de 1960 à nos jours, 26 espèces de plantes vasculaires ont disparu ou ne se rencontrent qu'à titre exceptionnel. Soixante-dix (70) espèces (Alafia scandens, Anthostema senegalense, Chlorophora excelsa et Dioscorea lecardii ) sont en voie d'extinction ou devenues rares, en raison de leur surexploitation ou de la destruction de leur habitat.

En ce qui concerne la faune, quatre-vingt-deux (82) espèces de faune sont inscrites dans les catégories des espèces menacées de l'UICN. Il s'agit notamment de mammifères (22 espèces), d'oiseaux (59 espèces), du crapaud vivipare du mont Nimba (Nectophynoides occidentalis), et d'insectes tels que le Coléoptère Goliath (Golathus regius) ${ }^{50}$. Qu'en est-il des pressions sur les aires protégées?

\footnotetext{
Il s'en déduit que les produits forestiers ligneux (PFL) sont des produits forestiers faits de bois, c'est-à-dire des bois d'œuvre et de produits ligneux secondaires (bois de chauffe).

${ }^{48}$ Voir République de Côte d'Ivoire, Ministère de l'Environnement et des Eaux et Forêts/Office Ivoirien des Parcs et réserves, Plan d'aménagement du Parc National d'Azagny, 2008, p. 37.

${ }^{49}$ Voir République de Côte d'Ivoire, Cinquième rapport national sur la diversité biologique, op. cit., p.7.

${ }^{50}$ Pour ce développement sur les conséquences relatives à l'exploitation des forêts ivoiriennes évoquées dans le rapport national sur la diversité biologique de 2014, voir Idem, pp. 20-22.
} 


\section{Les pressions anthropiques sur les aires protégées en particulier}

Les aires protégées ivoiriennes, à savoir les Parcs Nationaux et les Réserves naturelles (au nombre de 14$)^{51}$ subissent toutes des pressions anthropiques à des degrés divers ${ }^{52}$. En 2008, une étude sur l'efficacité de la gestion des aires protégées en Côte d'Ivoire a montré que les populations locales pratiquent, dans l'ensemble des Parcs Nationaux et Réserves naturelles principalement et en violation de la loi, l'exploitation agricole, l'exploitation forestière, l'exploitation minière, le braconnage ${ }^{53}$. En outre, les populations riveraines des aires protégées prélèvent des produits forestiers non ligneux pour la pharmacopée et à des fins domestiques ainsi des produits ligneux (secondaires) ${ }^{54}$ dans la quasi-totalité des aires protégées $^{55}$. De plus, elles pratiquent la pêche dans les Parcs Nationaux d'Azagny et des Îles Ehotilé et exercent des pressions foncières dans les Parcs Nationaux du Banco, de la Marahoué, de Taï, et dans la Réserve de faune d'Abokouamékro ${ }^{56}$. Enfin, l'orpaillage et la transhumance sont des activités (illégales) menées respectivement dans les Parcs Nationaux de Taï et de la Comoé 57 .

En 2009, un rapport sur l'état de conservation des Parcs et Réserves de Côte d'Ivoire a essentiellement confirmé ces mêmes pressions ${ }^{58}$. Plus récemment en 2012, une étude socioéconomique menée dans le cadre du "Bilan de l'état de la biodiversité des aires protégées de Côte d'Ivoire" portant sur quatre aires protégées (Parcs Nationaux d'Azagny, de la Marahoué, de Mont Péko et du Mont Sangbé) a révélé encore que ces forêts subissent de fortes pressions anthropiques $^{59}$. Particulièrement pour les Parcs Nationaux de la Marahoué et du Mont Péko, ces pressions anthropiques menacent leur existence ${ }^{60}$. Et pourtant, ces aires protégées sont des forêts qui font l'objet d'une protection juridique.

\footnotetext{
${ }^{51}$ Voir République de Côte d'Ivoire, Ministère de l'Environnement et des Eaux et Forêts/OIPR, État de conservation des Parcs et Réserves de Côte d'Ivoire, Abidjan, 2009, p. 3.

${ }^{52}$ Voir UICN/BRAO, Évaluation de l'efficacité de la gestion des aires protégées : Parcs et Réserves de Côte d'Ivoire, 2008, p. 5 ; République de Côte d'Ivoire, Ministère de l'Environnement et des Eaux et Forêts/OIPR, État de conservation des Parcs et Réserves de Côte d'Ivoire, op.cit., pp. 6-9.

${ }^{53}$ Voir UICN/BRAO, Évaluation de l'efficacité de la gestion des aires protégées : Parcs et Réserves de Côte d'Ivoire, op.cit., pp. 20-27.

${ }^{54} \mathrm{Il}$ s'agit de produits forestiers fait de bois (bois de chauffe) à l'exception des bois d'œuvre (Voir www.evb.lacsq.org/fileadmin/user upload/microsites/eav-evb internet/documents/trousses-et-activites/foretsdu-monde/fiche 3 2.pdf, consulté le 15 juin 2015).

${ }^{55}$ Voir UICN/BRAO, Évaluation de l'efficacité de la gestion des aires protégées : Parcs et Réserves de Côte d'Ivoire, op.cit., p.28.

${ }^{56}$ Idem, p. 29.

${ }^{57}$ Idem, p. 28.

${ }^{58}$ Voir République de Côte d'Ivoire, Ministère de l'Environnement et des Eaux et Forêts/OIPR, État de conservation des parcs et réserves de Côte d'Ivoire, op.cit., pp. 6-9.

${ }^{59}$ Voir AKINDĖS (Francis), KOUAMÉ (Kra Walter), SÉDIA (Gisèle) et SÉZAN (Jules), Réalisation d'une étude sur l'état de la biodiversité des parcs nationaux et réserves de Côte d'Ivoire. Aspects socio-économiques (Rapport provisoire), Abidjan, août 2012, pp. 91-92.

${ }^{60}$ Idem, pp. 28 et $89-90$.
} 


\section{II - APERÇU DE LA LÉGISLATION CONSACRÉE À LA PROTECTION DES AIRES PROTÉGÉES EN CÔTE D'IVOIRE}

La législation relative à la protection des aires protégées en Côte d'Ivoire relève aussi bien de textes juridiques internationaux (A) que nationaux (B).

\section{A. Les textes juridiques internationaux applicables aux aires protégées}

Plusieurs textes juridiques internationaux régissent la conservation des aires protégées en Côte d'Ivoire. Toutefois, nous n'évoquerons que quelques-uns, en raison de leur lien direct avec la conservation des aires protégées et de leur biodiversité associée.

En premier lieu, il s'agit de la Convention sur la diversité biologique ${ }^{61}$ (ci-après CDB) dont l'un des objectifs est la conservation de la diversité biologique (art. $\left.1^{\mathrm{er}}\right)^{62}$ et notamment de la biodiversité forestière y compris les aires protégées. C'est d'ailleurs pour cette raison que cette convention encourage les États parties à la conservation in situ ${ }^{63}$ (telle que celle faite par la création d'aires protégées) pour conserver la diversité biologique. Elle encourage, de même, l'élaboration de lignes directrices de gestion en vue de la conservation et l'utilisation durable des espèces dans leurs milieux naturels (art. 8) ${ }^{64}$.

En deuxième lieu, il s'agit de la Convention relative aux zones humides d'importance internationale particulièrement comme habitats des oiseaux d'eau (ci- après Convention Ramsar ${ }^{65}$. Cette convention incite à protéger la flore et la faune des zones humides en créant

\footnotetext{
${ }^{61}$ La République de Côte d'Ivoire a ratifié la Convention sur la diversité biologique par le moyen du Décret $n^{\circ}$ 94-614 du 14 novembre 1994 portant ratification de la Convention sur la diversité biologique, faite à Rio de Janeiro, le 5 juin 1992 (JORCI n52 du jeudi 22 décembre 1994, p. 1004). Cette convention a été publiée au Journal Officiel de la République de Côte d'Ivoire par l'œuvre du Décret n $94-615$ du 14 novembre 1994 portant publication de la Convention sur la diversité biologique, faite à Rio de Janeiro, le 5 juin 1992 (JORCI n52 du jeudi 22 décembre 1994, p. 1004).

${ }^{62} \mathrm{~L}$ 'article $1^{\mathrm{er}}$ de la Convention sur la diversité biologique dispose : «Les objectifs de la présente convention... sont la conservation de la diversité biologique, l'utilisation durable de ses éléments et le partage juste et équitable des avantages découlant de l'exploitation des ressources génétiques... » (souligné par nous).

${ }^{63}$ Aux termes de l'article 2 de la $\mathrm{CDB}$, on entend par conservation in situ ... « la conservation des écosystèmes et des habitats naturels et le maintien et la reconstitution de populations viables d'espèces dans leur milieu naturel et, les espèces domestiquées et cultivées, dans leur milieu où se sont développés leurs caractères distinctifs ».

${ }^{64}$ L'article 8 de la CDB indique que chaque Partie Contractante... : « a) Établit un système de zones protégées où de zones où des mesures spéciales doivent être prises pour conserver la diversité biologique... ».

${ }^{65}$ La République de Côte d'Ivoire a adhéré à la Convention de Ramsar au moyen du Décret n94-450 du 25 août 1994 portant adhésion de la République de Côte d'Ivoire à la Convention relative aux zones humides d'importance internationale particulièrement comme habitats des oiseaux d'eau, adoptée à Ramsar (Iran), le 2 février 1971 (JORCI n48 du jeudi 24 novembre 1994, p. 913). Elle a été amendée à Paris par le Protocole du 3 décembre 1982. La Convention de Ramsar a été publiée au Journal Officiel de la République de Côte d'Ivoire par l'œuvre du Décret $n^{\circ} 94-451$ du 25 août 1994 portant publication de la Convention relative aux zones humides d'importance internationale particulièrement comme habitats des oiseaux d'eau (JORCI $\mathrm{n}^{\circ} 48$ du jeudi 24 novembre 1994, p. 913).
} 
des aires protégées (Réserves naturelles) dans les zones humides (art. 4) ${ }^{66}$. Ainsi, la Côte d'Ivoire possède six (6) zones humides d'importance internationale ${ }^{67}$ aux nombre desquelles l'on a deux (2) aires protégées, à savoir le Parc National d'Azagny (PNA) et le Parc National des Îles Éhotilé (PNIE). La Convention Ramsar régit, par conséquent, les aires protégées ivoiriennes.

En troisième lieu, l'on a la Convention-cadre des Nations Unies sur les changements climatiques (ci-après CCNUCC). L'objectif de cette convention est de stabiliser les concentrations des gaz à effet de serre dans l'atmosphère à «un niveau qui empêche toute perturbation dangereuse du système climatique» (art. 2). La CCNUCC que la Côte d'Ivoire a ratifiée ${ }^{68}$ reconnaît, entre autres, le rôle et l'importance des écosystèmes terrestres tels que les forêts et partant les aires protégées comme des puits et réservoirs qui permettent de réduire et de maîtriser les émissions de gaz à effet de serre (GES). C'est pourquoi elle met à la charge des États contractants l'obligation de protéger ces écosystèmes forestiers (art. 4) ${ }^{69}$.

En quatrième et dernier lieu, il y a la Convention sur le commerce international des espèces de faune et de flore sauvages menacées de disparition (ci-après CITES) qui est applicable à la protection des forêts ivoiriennes ${ }^{70}$. En effet, la CITES a pour objet de protéger la flore et la faune sauvage dont elle reconnaît l'importance écologique et socio-économique en réglementant le commerce international dont elle est l'objet ${ }^{71}$. À ce titre, la flore et la faune des forêts ivoiriennes y compris celles des aires protégées ${ }^{72}$ est concernée.

\footnotetext{
${ }^{66}$ Selon cet article, "Chaque Partie contractante favorise la conservation des zones humides et des oiseaux d'eau en créant des réserves naturelles dans les zones humides...et pourvoit de façon adéquate à leur surveillance ».

${ }^{67}$ Il s'agit du Parc National d'Azagny inscrit sur la liste des zones humides d'importance internationale le 27/20/1996 et des Îles Ehotilé-Essouman, du N'Ganda N'Ganda, du Complexe Sassandra-Dagbego, de GrandBassam et de Fresco inscrits sur la liste ramsar le 18/10/2005. Ces sites Ramsar couvre une superficie de 127,344 hectares. Voir TIEBLEY (Yves-Didier), La Côte d'Ivoire et la gestion des ressources naturelles marines, Thèse unique, op. cit., p. 60.

${ }^{68}$ Voir le Décret n ${ }^{\circ} 94-616$ du 14 novembre 1994 portant ratification de la Convention sur les changements climatiques, faite à New York, le 9 mai 1992 (JORCI n51 du jeudi 15 décembre 1994, p. 980); et le Décret $\mathrm{n}^{\circ}$ 94-617 du 14 novembre 1994 portant publication de la Convention sur les changements climatiques, faite à New York, le 9 mai 1992 (JORCI n51 du jeudi 15 décembre 1994, p. 980).

${ }^{69}$ L'article 4 de la CCNUCC stipule : «Toutes Parties (...) Encouragent la gestion rationnelle et encouragent et soutiennent par leur coopération la conservation et le cas échéant, le renforcement des puits et réservoirs de tous les gaz à effet de serre non réglementés par le Protocole de Montréal, notamment la biomasse, les forêts et les océans de même que les autres écosystèmes terrestres, côtier et marins... ».

${ }^{70}$ La Côte d'Ivoire a adhéré à la CITES par l'œuvre du Décret n94-448 du 25 août 1994 portant adhésion de la République de Côte d'Ivoire à la Convention sur le commerce international des espèces de faune et de flore sauvages menacées de disparition, signée à Washington le 3 mars 1973 (JORCI n 47 du jeudi 17 novembre 1994, p. 892). La CITES a été publiée au Journal officiel de la République de Côte d'Ivoire (JORCI) par l'oeuvre du Décret n94-449 du 14 novembre 1994 portant publication de la Convention sur le commerce international des espèces de faune et de flore sauvages menacées de disparition, signée à Washington le 3 mars 1973 (JORCI n47 du jeudi 17 novembre 1994, p. 892).

${ }^{71}$ www.cites.org/fra/disc/what.php (consulté le 14 juin 2015).

${ }^{72}$ En effet, le dernier rapport sur la diversité biologique mentionne que ces forêts contiennent des espèces inscrites sur la liste CITES. Il s'agit, entres autres, de végétaux tels que Cycas revoluta, Euphorbia spp. et d'animaux tels que Varanus spp.,Chamaeleo spp. Python spp. Crocodylus cataphractus et Crocodylus niloticus, Gyps africanus, Cercopithecus spp. Hetero branchus spp., Loxodonta africana, Loxodonta cyclotis.
} 
Ces différents textes juridiques internationaux qui régissent les aires protégées ivoiriennes sont complétés sur le plan interne par des textes juridiques nationaux.

\section{B. Les textes juridiques nationaux relatifs aux aires protégées}

La législation nationale ivoirienne relative à la protection des aires protégées a évolué avec le temps. D'abord, après l'accession de la Côte d'ivoire à l'indépendance, les aires protégées ont été créées et régies à la fois par la loi nº5-255 du 4 août 1965 relative à la protection de la faune et à l'exercice de la chasse ${ }^{73}$ et la loi portant Code forestier $^{74}$. Ces deux (2) lois ont été complétées par un décret spécialement consacrée à la gestion des aires protégées. Il s'agit du décret $\mathrm{n}^{\circ} 66-433$ du 15 septembre 1966 portant statut et réglementation de la procédure de classement des Réserves naturelles intégrales ou partielles et des Parcs Nationaux ${ }^{75}$.

Ensuite, tout ce dispositif légal a été complété par la loi n96-766 du 3 octobre 1996 portant Code de l'Environnement ${ }^{76}$. Le Code de l'Environnement s'applique à l'environnement, en général, et aux aires protégées, en particulier. En effet, certaines dispositions dudit code traitent spécifiquement des aires protégées (voir les articles arts. 87 et $\left.89^{77}\right)$.

Enfin, depuis 2002, la législation ivoirienne en matière de conservation des aires protégées a été enrichie par l'œuvre de la loi n²002-102 du 11 février 2002 relative à la création, à la gestion et au financement des Parcs Nationaux et Réserves naturelles (ci-après loi de 2002 sur les PNR) ${ }^{78}$. Cette loi consacrée spécialement aux aires protégées a

\footnotetext{
${ }^{73}$ JORCI n41 du 26 août 1965, p. 898. La loi précitée a été modifiée par la loi n94-442 du 16 août 1994 portant modification de la loi $\mathrm{n}^{\circ} 65-255$ du 4 août 1965 relative à la protection de la faune et à l'exercice de la chasse (JORCI n42 du 13 octobre 1994, p. 778).

${ }^{74}$ Il s'agit de la loi $n^{\circ} 65-425$ du 20 décembre 1965 portant Code forestier (JORCI n ${ }^{\circ} 3$ du 13 janvier 1966, p. 38). Cette loi qui a pendant longtemps régit les aires protégées vient d'être abrogée par un nouveau Code forestier par l'œuvre de la loi n²014-427 du 14 juillet 2014 portant Code forestier, JORCI n ${ }^{\circ} 2$ spécial du Vendredi 20 mars 2015, page 17. Ce nouveau Code forestier ne s'applique pas à la faune et aux Parcs Nationaux et Réserves naturelles, ainsi que le dispose son article 3. Ces aires protégées sont régies par la loi n²002-102 du 11 février 2002 relative à la création, à la gestion et au financement des Parcs Nationaux et Réserves naturelles, JORCI $\mathrm{n}^{\circ} 20 \mathrm{du} 16$ mai 2002, p. 338.

${ }^{75}$ JORCI n ${ }^{\circ} 50$ du 27 octobre 1966, p. 1419.

${ }^{76}$ JORCI n $^{\circ} 6$ du 6 février 1997, p. 114.

${ }^{77}$ L'article 87 du Code de l'environnement ivoirien dispose : « Il est interdit de:

-Tuer, blesser ou capturer les animaux appartenant aux espèces protégées ;

- Détruire ou endommager les habitats, les larves et les jeunes espèces protégées ;

- Faire périr, endommager les végétaux protégés, en cueillir tout en partie ;

- Transporter ou mettre en vente tout ou partie d'un animal ou d'un végétal protégé ;

- Procéder à l'abattage d'arbres dans les forêts classées, aires protégées et parcs nationaux. ».

L'article 89 suivant indique : "Est puni d'un emprisonnement de deux mois à deux ans et d'une amende de 5.000.000 de francs, quiconque procède ou fait procéder à l'abattage d'arbres ou d'animaux dans les forêts classées, les aires protégées et les parcs nationaux». Ces parties des libellés des articles 87 et 89 ci-dessus ont été mises en relief par nous.

${ }^{78}$ JORCI n $^{\circ} 20$ du 16 mai 2002, p. 338.
} 
essentiellement renforcé leur protection juridique ${ }^{79}$, les pouvoirs de répression de certains personnels de gestion ${ }^{80}$. Elle confère également l'autonomie administrative et financière à la structure délégataire de leur gestion ${ }^{81}$ et prévoit la participation des communautés locales à leur gestion ${ }^{82}$.

Par ailleurs, pour comprendre les développements à venir, il sied de définir au préalable les concepts clés de notre étude.

\section{III - DÉFINITION DES CONCEPTS CLÉS DE L'ÉTUDE}

Les concepts que nous définirons sont: l'Aire protégée (A) et le Droit des aires protégées $(\mathrm{B})$.

\footnotetext{
${ }^{79}$ Anciennement biens du domaine privé de l'État, les Parcs Nationaux et Réserves font partie du domaine public de l'État. Sur la question, voir respectivement la Première partie de cette thèse, le Titre I, le Chapitre I, la Section II, et précisément le "Paragraphe 1 : Les aires protégées, un élément constitutif du domaine privé de l'État de 1960 à 2002" et le Titre II de la première partie de cette étude, le Chapitre I, la Section I et précisément le "Paragraphe 1 : L’incorporation des aires protégées au domaine public de l'État".

${ }^{80}$ En effet, tandis que les pouvoirs de répression de certains membres du personnel en charge des aires protégées (qui avaient la qualité d'agent assermenté) ne se limitaient qu'à constater les infractions contre les aires protégées par procès-verbal, la loi de 2002 a élargi ces pouvoirs en conférant la qualité d'officier de Police judiciaire à une catégorie du personnel en charge des aires protégées. Sur la question, le Chapitre 4 de la loi de 2002 sur les PNR qui traite du Pouvoir de Police dispose en son article 38 : "Le Directeur de l'Établissement, les directeurs des parcs et réserves ou groupements de parcs et réserves, ainsi que les agents des Eaux et Forêts appartenant au corps des ingénieurs, détachés auprès d'un parc ou d'une réserve, ont la qualité d'officier de Police judiciaire ». Le décret $\mathrm{n}^{\circ}$ 2013-22 du 9 janvier 2013 portant modalités d'habilitation, en qualité d'officiers de Police Judiciaire, des fonctionnaires et agents des Parcs Nationaux, Réserves naturelles et des Eaux et Forêts, (JORCI $\mathrm{n}^{\circ} 13$ du jeudi 28 mars 2013, p.226) fixe les modalités de leur habilitation. Pour plus d'informations sur le renforcement des pouvoirs de répression du personnel en charge de la gestion des aires protégées, voir la Première partie de cette thèse, le Titre II, le Chapitre I, la Section 2 et précisément le "Paragraphe 2 : Le renforcement des compétences pénales du personnel en charge des aires protégées".

${ }^{81}$ Aux termes de l'article 19 de la loi de 2002 sur les PNR : «La gestion des parcs et réserves relève d'un Établissement public national de type particulier, doté de la personnalité morale et de l'autonomie financière dont les missions et les principes d'organisation et de fonctionnement sont définis par la présente loi ». Le décret n²002-359 du 24 juillet 2002 portant création, organisation et fonctionnement de l'Office Ivoirien des Parcs et Réserves (JORCI $n^{\circ} 40$ du 2 octobre 2002, p. 588) a créé cette structure.

${ }^{82}$ La participation des communautés locales à la gestion des aires protégées est d'abord prévue par l'article 21 de la loi 2002 sur les PNR qui consacre l'institution d'un comité de gestion (locale) au niveau de chaque Parc et Réserve qui participe " à l'élaboration et à la mise en ouvre des plans de gestion durable des parcs et réserves ». Ensuite, cette participation est déduite des dispositions de l'article 32 de ladite loi qui prescrit que «Tout ou partie des missions d'exploitation d'un parc ou d'une réserve peuvent être concédées » par l'Établissement c'est-à-dire l'Administration en charge des aires protégées à une personne morale de droit privé à travers une Convention de concession d'exploitation. Enfin, l'article 33 de la même loi dispose : «les contrats de gestion de terroirs sont passés au bénéfice réciproque d'un parc ou d'une réserve et de ses populations environnantes. Ces contrats ont pour objet de définir les conditions et modalités de l'association de ces populations à la conservation du parc ou de la réserve et de favoriser ainsi les retombées économiques ». Ces parties des libellés des articles ci-dessus cités ont été mises en gras par nous.

Par ailleurs, pour plus d'informations sur le contrat de gestion de terroir, voir la Première partie de la présente étude, le Titre II, le Chapitre II, la Section II, le Paragraphe 2, le point B et précisément le sous-point "1. Le contrat de gestion de terroir".
} 


\section{A. Aire protégée}

L'Union mondiale pour la nature (UICN) ${ }^{83}$, définit l'aire protégée comme étant : « une portion de terre et/ou de mer vouée spécialement à la protection et au maintien de la diversité biologique, ainsi que des ressources naturelles et culturelles associées, et gérées par des moyens efficaces et juridiques ou autres $\gg{ }^{84}$.

Toutefois, la définition qu'en donne le législateur ivoirien, à travers l'article $1^{\mathrm{er}}$ de la loi de 2002 sur les PNR précitée, nous semble plus complète, en ce sens qu'elle tient compte de la diversité des écosystèmes de la Côte d'Ivoire. Ainsi, aux termes de cet article, l'aire protégée désigne: «...une portion de terre, de mer, de rivière et/ou de la lagune géographiquement délimitée et gérée pour la protection et le maintien du patrimoine naturel et culturel ». Il en découle que le législateur ivoirien s'est voulu plus large que l'UICN dans l'énonciation de la définition de l'aire protégée relativement au patrimoine naturelle à protéger. En effet, au-delà de la mer, la prise en compte de milieux aquatiques tels que les rivières et les lagunes enrichissent cette définition.

Mieux, la définition de l'aire protégée que formule le législateur ivoirien semble plus explicite que celle que propose la doctrine. En effet, reprenant C. de KLEMM, le Professeur Maurice KAMTO définit juridiquement l'aire protégée comme étant : « un espace soumis à un régime spécial, exorbitant du droit commun, qui permet d'interdire ou de réglementer les activités humaines susceptibles de porter atteinte au milieu naturel ${ }^{85}$. Si cette définition doctrinale traduit dans l'idée celle du législateur ivoirien, elle est de portée générale. C'est la raison pour laquelle la définition de l'aire protégée qu'énonce le législateur ivoirien retient davantage notre attention.

Pour les besoins de notre analyse, il est utile de souligner qu'en Côte d'Ivoire, la loi de 2002 sur les PNR prévoit quatre (4) types d'aires protégées. Ce sont: le Parc National, la Réserve naturelle intégrale, la Réserve naturelle partielle ${ }^{86}$; et la Réserve naturelle volontaire $^{87}$.

\footnotetext{
${ }^{83}$ Créée en 1948, à la suite d'une conférence internationale tenue à Fontainebleu, l'UICN, ONG internationale, est le plus vaste réseau mondial de protection de la nature. C'est elle qui a élaboré une définition de référence du concept d'aire protégée et fait figure d'autorité en la matière. Voir www.uicn.fr/-Aires-protégées-.html (consulté le 12 octobre 2012). En effet, cette définition de référence est constamment reprise par nombre d'auteurs contemporains qui traitent de la question. Il s'agit notamment de MARTINEZ (Carole), TRIPLET (Patrick), «Définir les aires protégées », in TRIPLET (Patrick) (dir.), Manuel de gestion des aires protégées d'Afrique francophone, Paris, Awely, 2009, p. 2 ; DIALLO (Mamadou Saïdou), Évolution de la gestion des aires protégées en Guinée: La difficile cohabitation des politiques publiques et des systèmes traditionnels. Cas du Parc National du Haut Niger, Thèse unique, Géographie, Université du Maine, 14 avril 2011, pp. 23 et s.

${ }^{84}$ Voir le site de 1'UICN : www.uicn.fr/-Aires-protégées-.html (consulté le 12 octobre 2012). Pour une définition similaire, lire également BINOT (Aurélie), La conservation de la nature en Afrique centrale entre théorie et pratique. Des espaces protégées à géométrie variable, Thèse unique, Géographie, Université Paris 1 PanthéonSorbonne, 9 août 2010 , p. 61.

${ }^{85}$ Voir KAMTO (Maurice), Droit de l'environnement en Afrique, Vanves, AUPELF/UREF, 1996, p. 202.

${ }^{86}$ Le Parc National est une aire protégée créée dans le but de la conservation des espèces animales, végétales, de paysages ou de formations géologiques d'une valeur particulière ; dans l'intérêt et la récréation du public (art. 1,
} 
Cependant, les aires protégées dont traite cette étude sont celles que gère l'Office Ivoirien des Parcs et Réserves (OIPR) ${ }^{88}$. Il s'agit fondamentalement des Parcs Nationaux, des Réserves naturelles intégrales et partielles. En d'autres termes, les Réserves naturelles volontaires ont été exclues de notre champ d'étude ; et ce pour plusieurs raisons. En effet, elles sont de création récente ${ }^{89}$. C'est ainsi qu'il convient d'attendre encore des années pour évaluer l'observation ou le non-respect des règles qui les régissent par le public; de même que les conséquences qui peuvent en résulter. De plus, l'une des difficultés réelles qu'aurait soulevé leur prise en compte dans la réalisation de cette étude est la collecte d'informations relatives à chacune de ces Réserves (naturelles volontaires), en raison de la diversité des organes de gestion ${ }^{90}$. Mieux, les aires protégées (étatiques) gérées actuellement par l'Administration en charge des aires protégées constituent essentiellement le réseau d'aires protégées en Côte d'Ivoire. La figure ci-dessous renseigne sur la localisation géographique des Parcs Nationaux et Réserves naturelles de Côte d'Ivoire.

\footnotetext{
loi de 2002 sur les PNR). À la différence du Parc National, la Réserve naturelle intégrale est une aire protégée qui n'est pas ouverte au public (art. $1^{\mathrm{er}}$, loi de 2002 sur les PNR). Enfin, en ce qui concerne la Réserve naturelle partielle, c'est une aire protégée dont la création est motivée par la conservation d'espèces animales ou végétales spécifiques; et la récréation du public (art. 1er, loi de 2002 sur les PNR). Ainsi, l'on distingue des Réserves partielles de faune ou de flore.

${ }^{87}$ Les articles $1^{\text {er }}$ et 13 de la loi de 2002 sur les PNR disposent respectivement que «Une réserve naturelle volontaire désigne la Réserve naturelle partielle créée à l'initiative d'une collectivité territoriale, d'un établissement public ou d'une personne de droit privé, sur un terrain lui appartenant et pour la préservation d'un écosystème ou d'un paysage remarquable " et que "Les réserves naturelles volontaires sont gérées par la collectivité territoriale, l'établissement public ou la personne morale de droit privé, propriétaire du terrain constituant la réserve naturelle avec l'appui le cas échéant, de l'autorité chargée de la surveillance des Parcs et Réserves ». Il découle de ce qui précède que la particularité des Réserves naturelles volontaires est qu'elles peuvent être créées par une entité juridique infra étatique voire par une personne de droit privé.

${ }^{88}$ L'Office Ivoirien des Parcs et Réserves (OIPR) est l'Établissement public national qui a la charge la gestion des Parcs et Réserves relevant du domaine de l'État en Côte d'Ivoire, depuis l'année 2002. Cette institution est l'œuvre du décret 2002-359 du 24 juillet 2002 portant création, organisation et fonctionnement de l'OIPR (JORCI n ${ }^{\circ} 40$ du 2 octobre 2002, p. 588).

${ }^{89}$ Les premières Réserves naturelles volontaires de Côte d'Ivoire ont été créées en décembre 2012.

${ }^{90}$ Les Réserves naturelles volontaires sont créées par des personnes de droit privé ou des collectivités propriétaires et administrées par celles-ci ; le cas échéant, avec l'appui de l'Administration en charge des aires protégées (art. 13 de loi de 2002 sur les PNR ci-dessus cité ).
} 
Figure 1 : Répartition des Parcs Nationaux et Réserves naturelles de Côte d'Ivoire

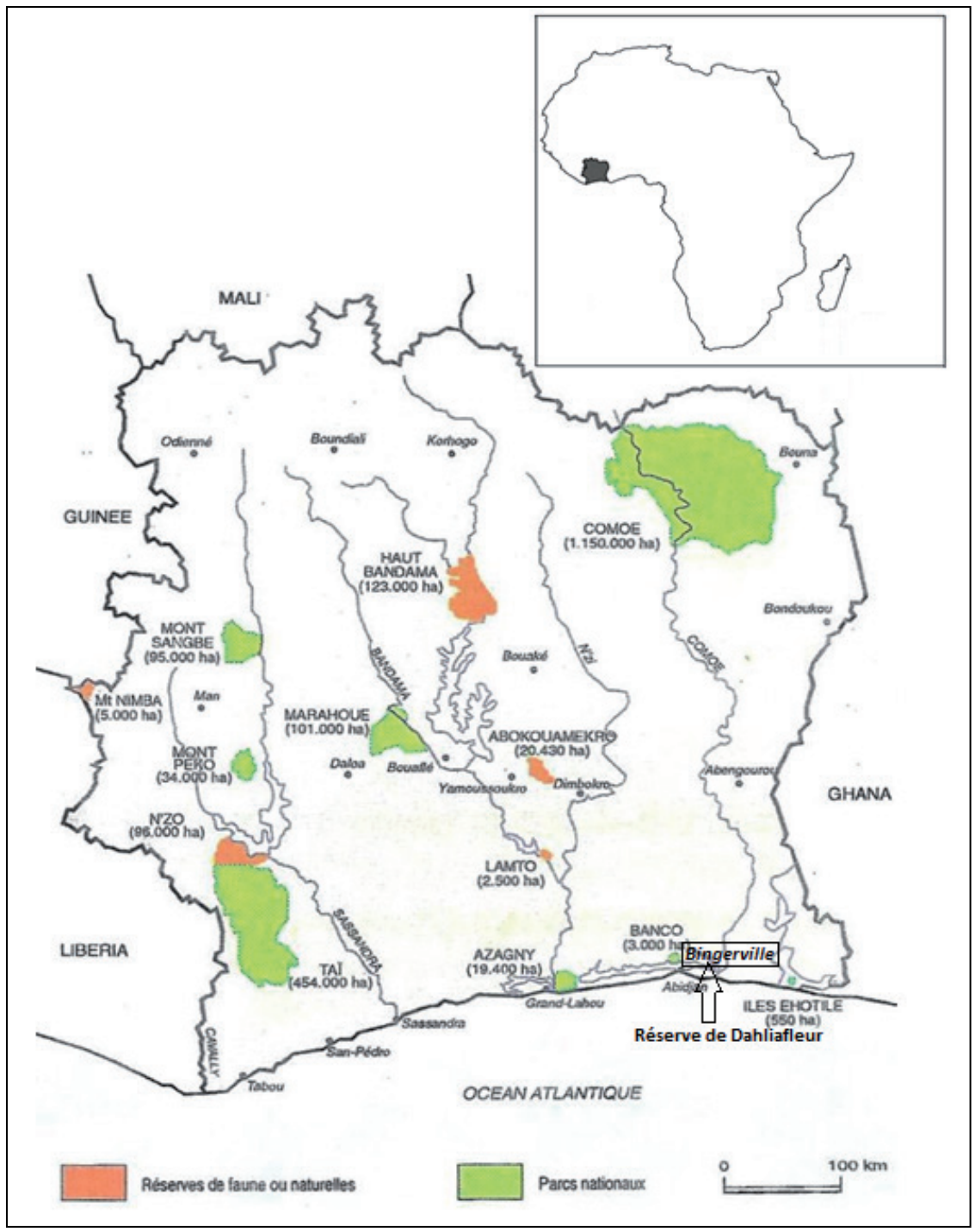

Source : Office Ivoirien des Parcs et Réserves. 


\section{Commentaires}

À la lecture de cette carte, nous constatons qu'il existe huit (8) Parcs Nationaux (Azagny, Banco, Comoé, Iles Ehotilé, Marahoué, Mont Péko, Mont Sangbé, Taî1 ${ }^{91}$ et six (6) Réserves naturelles (Abokouamékro, Dalhia Fleur, Haut Bandama, Lamto, Mont Nimba, N'zo). La superficie de ces aires protégées représente un total de $6,5 \%$ du territoire national soit 2.270 .148 ha $^{92}$.

Aussi la définition de l'aire protégée rend-elle plus aisée celle du droit des aires protégées.

\section{B. Le Droit des aires protégées}

En l'absence de définition légale, on peut cependant définir le Droit des aires protégées à partir du sens du mot « Droit» (qui est polysémique ${ }^{93}$ ) et notamment de celui du Droit objectif $^{94}$. Ainsi, le droit des aires protégées pourrait être appréhendé comme l'ensemble des règles de droit qui régissent la conservation des aires protégées et dont la violation est sanctionnée par les pouvoirs publics.

À ce titre, d'une part, ces règles organisent la gestion et la valorisation de ces milieux naturels. D'autre part, elles prescrivent des restrictions pour certaines activités ${ }^{95}$ dont la réalisation dans les zones périphériques des aires protégées est susceptible de menacer leur conservation.

Ces clarifications conceptuelles étant faites, il convient de présenter l'intérêt du sujet de cette étude.

\footnotetext{
${ }^{91}$ Il est à préciser que le Parc National de Taï absorbera la Réserve de N'Zo par fusion. Sous la réserve d'une telle consécration par le droit des aires protégées, cela l'est dans la pratique pour une efficacité de la gestion. Ces informations nous ont été fournies au cours d'un entretien que nous a accordé le Directeur technique de l'OIPR (M. N'GORAN DJE), le jeudi 11 décembre 2014, au siège de l'OIPR (Abidjan, République de Côte d'Ivoire).

${ }^{92}$ Voir UNESCO/UICN, Rapport de mission, Suivi de l'état de la conservation du Parc National de la Comoé en Côte d'Ivoire, site du Patrimoine Mondial, 10-23 juin 2006, p. 8.

${ }^{93}$ Selon Xavier THUNIS, «Le droit est une notion polysémique : prérogative ou pouvoir d'agir reconnu à un individu ou un groupement (intérêt ou droit subjection), ensemble des règles en vigueur dans une société donnée à une époque donnée (droit positif ou objectif), idéal supérieur de justice s'imposant aux règles (droit naturel) et enfin discipline intellectuelle s'efforçant de tenir un discours ordonné et critique sur le phénomène juridique et d'en améliorer la connaissance ou le fonctionnement.». Voir THUNIS (Xavier), « La recherche juridique à contretemps ", in GÉRARD (Philippe), OST (François) et KERCHOVE (Michel van de) (dir.), L'accélération du temps juridique, op. cit., p. 911-912.

${ }^{94}$ Le Droit objectif est également défini par GUINCHARD (Serges) et DEBARD (Thierry) comme étant l'ensemble des règles destinées à régir les rapports sociaux et dont la violation est sanctionnée par les pouvoirs publics. Voir GUINCHARD (Serges) et DEBARD (Thierry) (dir.), Lexiques des termes juridiques, $19^{\mathrm{e}}$ édition, Paris, Dalloz, 2012, p. 327.

${ }^{95} \mathrm{La}$ réalisation de projets industriel, minier, de carrière, de lotissement ou d'équipement touristique, de fouille archéologique, d'infrastructures linéaires (routes, oléoducs, gazoducs, lignes électriques) sont soumis à l'avis préalable de l'OIPR et font l'objet d'une étude d'impact environnemental (art. 33, loi de 2002 sur les PNR).
} 


\section{IV - INTÉRÊT DU SUJET}

La conservation des forêts dans les pays tropicaux tels que la Côte d'Ivoire ${ }^{96}$ est une préoccupation mondiale ${ }^{97}$. Selon l'AFD, «Les ressources forestières fournissent l'essentiel des revenus des pays tropicaux et contribuent à des besoins légitimes et souvent cruciaux de développement. Or, en dépit des efforts des institutions internationales et des ONG, les surfaces forestières reculent, et avec elles, des ressources précieuses en biodiversité. $)^{98}$. La question est d'autant plus préoccupante que malgré des accords internationaux en matière de protection de la biodiversité, la déforestation et la dégradation des forêts continuent ${ }^{99}$.

Les causes de dégradation de la biodiversité mondiale dont le constat est fait ci-dessus sont diverses. Elles relèvent aussi bien de phénomènes tels que les changements climatiques $^{100}$ que de causes socio-économiques dont les plus importantes sont l'exploitation forestière illégale et la pauvreté ${ }^{101}$. Traitant de la question dans le cadre des pays en voie de développement tels que la Côte d'Ivoire ${ }^{102}$, Faure et al. notent que la meilleure façon d'améliorer la qualité de l'environnement dans ces pays est de réduire la pauvreté ${ }^{103}$.

S'agissant particulièrement des aires protégées, les pressions sur ces milieux naturels sont dues au fait qu'elles regorgent d'importantes ressources (nourriture, eau douce, bois de

\footnotetext{
${ }^{96}$ Compris entre $4^{\circ}$ et $10^{\circ}$ de latitude nord, le territoire de la Côte d'Ivoire est partagé entre le climat équatorial et le climat tropical. Voir HALLE (Birgit), Dr. BRUZON (Véronique), Profil Environnemental de la Côte d'Ivoire, Rapport Final, Août 2006, p. 16 ; FAO, L'Etude prospective du secteur forestier en Afrique, op. cit., p. 4.

${ }^{97}$ Voir VERMEULEN (Sonja), SHEIL (Douglas), " Partnerships for tropical conservation », art. cit.,p. 434.

${ }^{98}$ Voir AFD, Gestion durable de la forêt et lutte contre la désertification, Paroles d'acteurs, n 1, 2002, p. 9.

${ }^{99}$ Voir VISSEREN-HAMAKERS (Ingrid J.) et GLASBERGEN (Peter), « Partnerships in forest governance », art. cit., p. 408.

${ }^{100}$ Sur la question, reprenant Watson et al., VISSEREN-HAMAKERS (Ingrid J.) et GLASBERGEN (Peter) indiquent que l'une des principales menaces en matière dégradation des forêts reste les changements climatiques. Voir VISSEREN-HAMAKERS (Ingrid J.) et GLASBERGEN (Peter), " Partnerships in forest governance », art. cit., p. 408.

${ }^{101}$ Plusieurs auteurs ont établissent un lien la pauvreté et la dégradation des ressources naturelles. Sur la question, voir BARRETT (Christopher B.), TRAVIS (Alexander J.), DASGUPTAD (Partha), « On biodiversity conservation and poverty traps », Proceedings of the National Academy of Sciences, Vol. 108, n³4, 2011, pp. 13907 et 13909 ; NAUGHTON-TREVESA (Lisa), ALIX-GARCIAB (Jennifer), et CHAPMAN ( Colin A.),

«Lessons about parks and poverty from a decade of forest loss and economic growth around Kibale National Park, Uganda », Proceedings of the National Academy of Sciences, Vol. 108, n³4, 2011, p. 13919 ; COOMES (Oliver T.), TAKASAKI (Yoshito) et RHEMTULLA (Jeanine M.), Land-use poverty traps identified in shifting cultivation systems shape long-term tropical forest cover, Proceedings of the National Academy of Sciences, Vol. 108, n³4, 2011, p. 19325.

${ }^{102}$ Selon la Banque mondiale, la Côte d'Ivoire est un pays en voie de développement. Son indice de développement humain (IDH) qui est de 0,457 en 2015 , lui donne lui confère le rang de $143^{\text {ème }}$ pays sur 187 selon leur IDH. Voir www.donnees.banquemondiale.org/pays/cote-d'ivoire (consulté le 25 mai 2016). Pour PAULAIS (Thierry) également, la Côte d'Ivoire est un pays en voie de développement. Voir PAULAIS (Thierry), Le développement urbain en Côte d'Ivoire, 1979-1990: les projets de la Banque mondiale, Paris, KARTHALA, 1995, p. 11.

${ }^{103}$ Voir FAURE (Michaël), GOODWIN (Morag) et WEBER (Franziska), « Bucking the Kuznets Curve: Designing Effective Environmental Regulation in Developing Countries ", Virginia journal of international law, Vol. 51, 2010, p.154
} 
chauffe, plantes médicinales...) qui servent aux populations pauvres ${ }^{104}$. Mieux, dans les pays en développement, les populations riveraines des aires protégées sont souvent pauvres ${ }^{105}$. Selon Lea M. SCHERL et al., hormis le service d'approvisionnement des aires protégées en ressources, les autres services écosystémiques, à savoir les services de régulation (séquestration du carbone, purification de l'eau, régulation du climat), les services culturels (éducation, tourisme, valeurs religieuses) sont tous aussi importants ${ }^{106}$.

Cependant, les populations (pauvres) ne les considèrent pas comme tels parce qu'ils ont peu d'impact sur la réduction de la pauvreté au sein des communautés vivant dans et autour des aires protégées ${ }^{107}$. À titre illustratif, en Afrique subsaharienne, les principales menaces à la conservation des sites du patrimoine mondial sont liées à la pauvreté. Celle-ci entraîne leur exploitation par les populations à travers des activités telles que braconnage, le ramassage du bois de chauffe, la pêche illicite qui affectent $71 \%$ de ces sites contre $38 \%$ pour l'exploitation agricole et l'élevage ${ }^{108}$.

En plus de la pauvreté, l'augmentation de la croissance démographique qui accroît la demande des populations en produits forestiers ligneux et non ligneux est une cause de la surexploitation, de la dégradation et de la destruction des forêts ${ }^{109}$. Par conséquent, la croissance démographique s'analyse comme une cause aggravante de la dégradation des forêts dans les pays en voie de développement.

Selon Suzanne STOLL-KLEEMANN et Timothy O'RIORDAN, "pour inverser la tendance, il convient d'accorder une priorité à la protection des espèces et des habitats (notre traduction)» ${ }^{110}$. C'est cet objectif de protection qu'a toujours poursuivi la législation des aires protégées adoptée en Côte d'Ivoire ${ }^{111}$. Autrement dit, depuis l'accession de la Côte d'Ivoire à l'indépendance en 1960, ce pays s'est dotée de règles juridiques (successives) destinées à

\footnotetext{
${ }^{104}$ Voir SCHERL (Lea M.) et al., Can Protected Areas Contribute to Poverty Reduction? Opportunities and Limitations, Gland, Switzerland and Cambridge, UK, IUCN, 2004, p. 20.

${ }^{105}$ Idem, p. 25; PIMBERT (Michel P.) et PRETTY (Jules N.), Parks, People and Professionals: Putting 'Participation' into Protected Area Management, UNRISD/IIED/WWF, Discussion Paper n 57, February 1995. UNRISD, Geneva, p.15.

${ }^{106}$ Voir SCHERL (Lea) et al., Can Protected Areas Contribute to Poverty Reduction? Opportunities and Limitations, op.cit., p. 21.

${ }^{107}$ Ibidem.

${ }^{108}$ Voir WILSON (A.C.) et WILSON (E.B.), A Review of Threats to World Heritage Sites 1993-2002. Unpublished paper for World Commission on Protected Areas, 2004 cités par SCHERL (Lea) et al., Can Protected Areas Contribute to Poverty Reduction? Opportunities and Limitations, op.cit., p. 26.

${ }^{109}$ Voir SZELL (Andrea B.), HALLETT (Lucius F.), «Attitudes and Perceptions of Local Residents and Tourists toward the Protected Area of Retezat National Park, Romania», International Journal of Humanities and Social Science, Vol. 3, n 4 (numéro special), 2013, p. 18.

${ }^{110}$ Voir STOLL-KLEEMANN (Suzanne) et O'RIORDAN (Timothy), « From Participation to Partnership in Biodiversity Protection: Experience from Germany and South Africa », Society and Natural Resources: An International Journal, Vol. 15, n², 2002, p. 161.

${ }^{111}$ À propos de la législation relative à la conservation des aires protégées en Côte d'Ivoire, voir en Introduction (de la présente étude), le point "II- Aperçu de la législation consacrée à la protection des aires protégées en Côte d'Ivoire".
} 
régir la préservation des ressources naturelles et notamment la conservation de ses aires protégées, à savoir les Parcs Nationaux et les Réserves naturelles (PNR) ${ }^{112}$.

Malgré cet arsenal juridique, la conservation des aires protégées reste compromise, eu égard à leur exploitation continue par l'homme. À bien constater, le droit des aires protégées n'assure pas sa fonction de protection des Parcs Nationaux et Réserves naturelles, dans la mesure où soit c'est l'État qui viole les dispositions dudit droit soit il peine à le faire respecter par les populations.

C'est en cela que réside le sens de ces réflexions sur le droit des aires protégées en Côte d'Ivoire. En effet, l'État crée des aires protégées pour éviter que toutes les forêts de la Côte d'Ivoire soient exploitées par les populations ou les entreprises opérant dans le secteur du bois. Autrement dit, les aires protégées sont créées en vue de la conservation des espèces animales et végétales dans leur habitat originel (conservation in situ). Par conséquent, elles sont créées dans un but de la conservation de la nature ${ }^{113}$.

On comprend dès lors que l'article 3 de la loi de 2002 sur les PNR dispose : «Les parcs et réserves sont créés aux fins de permettre la conservation des milieux naturels, de la faune et de la flore terrestres et aquatiques, ainsi que le maintien de la diversité biologique et des processus écologiques contre toutes les causes de dégradation qui les menacent. Ils ont également pour vocation de participer, par la récréation et l'éducation du public, à l'équilibre harmonieux des populations...». Ainsi, dès leur création, le droit des aires protégées consacre l'interdiction des droits d'usage qui s'exerçaient auparavant sur ces forêts $^{114}$.

\footnotetext{
${ }^{112} \mathrm{Ces}$ initiatives de protection des ressources naturelles interviennent dans un contexte international favorable à la protection des forêts. En effet, depuis les années 1960, les grandes rencontres internationales relatives à la protection de l'environnement mondial consacrent à la conservation durable des forêts, une place de choix. Que ce soit à la Conférence des Nations Unies sur l'environnement de Stockholm(SUĖDE) de 1972, à celle de Rio (BRÉSIL) sur l'environnement et le développement de 1992, de Johannesburg (AFRIQUE du SUD) ${ }^{\circ}$ de 2002 et de Rio + 20 de 2012, la conservation durable des forêts est retenue comme étant une des solutions aux problèmes environnementaux mondiaux, tels que les changements climatiques et l'avancée du désert.

${ }^{113}$ Les aires protégées sont à conserver car ils constituent un moyen de conservation de la biodiversité. Voir SCHERL (Lea) et al., Can Protected Areas Contribute to Poverty Reduction? Opportunities and Limitations, op. cit., p. 47; HATCHWELL (Matthew), « Public-private partnerships as a management option for protected areas», Animal Conservation, Vol. 17, $\mathrm{n}^{\circ} 1,2014$, p. 3.

${ }^{114}$ En effet, aux termes de l'article $1^{\text {er }}$ de la loi de 2002 sur les PNR qui reprend l'esprit de la législation ancienne sur les aires protégées qui les affranchissaient de tous droits d'usage, le Parc National désigne une aire «...exclusivement destinée à la propagation, la protection, la conservation et l'aménagement de la végétation et les populations d'animaux sauvages, ainsi qu'à la protection des sites, des paysages ou des formations géologiques d'une valeur scientifique ou esthétique particulière, dans l'intérêt et pour la récréation du public...Dans laquelle l'abattage, la chasse, la capture d'animaux et la destruction ou la collecte des plantes sont interdits...». Quant à la Réserve naturelle intégrale, ledit article dispose qu'elle s'entend d'une aire «...sur l'étendue de laquelle toute espèce de chasse ou de pêche, toute exploitation forestière, agricole ou minière, tout pâturage, toute fouille ou prospection, tout sondage ou tout terrassement, toute construction, tous travaux tendant à modifier l'aspect du terrain ou de la végétation ...tout acte de nature à apporter des perturbations à la faune ou à la flore...seront strictement interdits... ». Pour ce qui est de la Réserve naturelle partielle, le même article la définit comme étant "une aire protégée gérée dans un but de conservation in situ des écosystèmes naturels ou d'espèces ou de biotopes spécifiques au profit et à l'avantage et pour l'utilisation durable, la récréation et l'éducation du public». Enfin, la Réserve partielle de faune est une aire : «...Mise à
} 
Cependant, cette interdiction n'est pas observée par le public alors qu'il semble indispensable qu'elle le soit. En effet, ces forêts sont les seules affranchies de tout droit d'usage, au contraire des autres types de forêts ${ }^{115}$.

Aussi dans ce contexte de dégradation des aires protégées en dépit de leur importance et la protection juridique dont elles sont l'objet, avons-nous choisi de réfléchir sur le sujet "Le droit des aires protégées en Côte d'Ivoire". Ce sujet revêt un intérêt scientifique et social.

L'intérêt scientifique de cette recherche ${ }^{116}$ est d'étudier le droit des aires protégées en vue de savoir si les difficultés liées à son effectivité résultent d'insuffisances intrinsèques ou de facteurs externes. En effet, le droit des aires protégées prescrit des règles qui assurent la conservation des aires protégées car il les affranchit de tout droit d'usage. De ce fait, les aires protégées ne devraient pas présenter en Côte d'Ivoire une dégradation si avancée, en raison de l'action de l'Homme.

Certes, des résultats de travaux de recherches sont disponibles, en ce qui concerne les problèmes de conservation de ces aires protégées. Cependant, rares sont les recherches qui abordent principalement, à travers une étude analytique et critique, le droit des aires protégées, en vue de connaître les raisons pour lesquelles il peine à remplir sa fonction de conservation de ces milieux naturels. En définitive, c'est en cela que réside l'intérêt scientifique de cette étude. Qu'en est-il de l'intérêt social?

L'intérêt social de cette étude ${ }^{117}$ consiste à faire des propositions en vue de l'amélioration de la conservation des aires protégées, dans une perspective de conciliation des différents intérêts en présence ${ }^{118}$; de sorte à réduire les conflits récurrents entre gestionnaires

part pour la conservation, l'aménagement et la propagation de la vie animale sauvage, ainsi que pour la protection et l'aménagement de son habitat...Dans laquelle la chasse, l'abattage ou la capture de la faune sont interdits... 》 (Ces parties de l'article $1^{\mathrm{er}}$ précité ont été mises en gras ont été faites par nous).

${ }^{115} \mathrm{Il}$ s'agit, d'une part, des forêts du domaine rural encore appelé forêt protégée (art. $1^{\mathrm{er}}$, nouveau Code Forestier de 2014) qui sont exploitables par les populations selon les textes en vigueur. D'autre part, l'on a les forêts classées qui le sont également, selon une planification définie par les autorités publiques qui ont en charge leur gestion. En effet, si selon l'article 23 du nouveau Code forestier précité, le domaine forestier classé se compose de forêts classées, à savoir : les forêts de protection, les forêts de production, les forêts de récréation et les forêts d'expérimentation, selon les objectifs principaux fixés, l'article 26 suivant précise que "Les forêts classées sont susceptibles de déclassement partiel ou total dans les mêmes procédures et formes que leur classement » (Les mises en gras des différentes parties de ces textes ont été faires par nous).

${ }^{116}$ Selon N'DA (Paul), l'intérêt scientifique d'un sujet renvoie à sa contribution à l'avancement des connaissances dans un domaine du savoir. Il montre l'actualité et l'originalité d'une étude. Le but recherché ici est essentiellement théorique. Voir N'DA (Paul), Méthodologie de la recherche: de la problématique à la discussion des résultats. Comment réaliser un mémoire, une thèse en Sciences Sociales et en Éducation, $2^{\text {ème }}$ éd., Abidjan : EDUCI, 2002, p.31.

${ }^{117}$ Contrairement à l'intérêt scientifique, l'intérêt social d'une recherche a un but utilitaire. Il réside dans la réponse qu'il apporte aux préoccupations de la société. Voir N'DA (Paul), Méthodologie de la recherche: de la problématique à la discussion des résultats. Comment réaliser un mémoire, une thèse en Sciences Sociales et en Éducation, op. cit., p. 31).

${ }^{118}$ Il s'agit de la conservation des aires protégées, d'une part; et du développement des communautés locales, d'autre part. 
et communautés ${ }^{119}$. Pour atteindre les objectifs qui découlent de l'intérêt de la présente étude, celle-ci nous inspire plusieurs interrogations que nous soulèverons dans la problématique du sujet.

\section{V - PROBLÉMATIQUE}

La dégradation continue des aires protégées en Côte d'Ivoire du fait de l'Homme malgré l'adoption successive de lois (lato sensu) qui assurent et même renforcent leur protection depuis le début des années $1960^{120}$ suscite une interrogation fondamentale. La législation des aires protégées adoptée en Côte d'Ivoire depuis les années 1960 accorde-t-elle une protection juridique adéquate à ces milieux naturels ?

Pour répondre à cette interrogation, il convient de se poser plusieurs autres questions qui permettront d'investir le sujet. En effet, nous avons mentionné à la page précédente que le droit des aires protégées de la Côte d'Ivoire (souveraine) s'est formé (historiquement) sur deux (2) périodes. Celle qui part de 1960 à 2002 a consacré une première législation des aires protégées. Cette législation été profondément remaniée depuis 2002 par l'intervention de la loi de 2002 sur les PNR précitée ainsi que ses décrets d'application. Fort de cela, nous sommes tentés de nous poser les questions suivantes.

Quelles sont les raisons qui ont amené les pouvoirs publics ivoiriens à adopter une nouvelle législation à partir de 2002 en vue de la conservation des aires protégées ? Autrement dit, la législation relative aux aires protégées en vigueur avant l'année 2002 présentait-elle des insuffisances? De la sorte, l'analyse des dispositions juridiques qui ont régi les aires protégées de 1960 à 2002 permettra de savoir si l'intervention du législateur ivoirien en 2002, par la prise d'une nouvelle loi pour la gestion des aires protégées, est effectivement consécutive à une insuffisance des dispositions juridiques antérieurement en vigueur.

Par ailleurs, malgré l'adoption d'une nouvelle législation des aires protégées depuis 2002, ces milieux naturels sont toujours sujets aux pressions anthropiques qui les dégradent davantage. Ainsi, la législation des aires protégées, en l'état actuel, leur assure-t-elle une protection juridique suffisante dans le sens de leur conservation durable ? En d'autres termes,

\footnotetext{
${ }^{119}$ À titre illustratif, au cours de nos entretiens avec les gestionnaires du Parc National d'Azagny, nous avons été informé qu'en 2006, un jeune a reçu une décharge d'arme automatique, suite à des échanges de coup de feu avec les éléments de la brigade mobile sud de l'OIPR. De plus, les gestionnaires du Parc National de la Marahoué nous ont instruit de ce que le 5 août 2004, l'arrestation d'un "délinquant" dans la Zone Agroforestière du Parc National de la Marahoué (Gbangbokouadiokro) a causé la mort d'un jeune planteur. Environ une décennie avant, le Caporal Diarrasouba et le Sergent Kouakou K. Zéphirin ont été abattus par des braconniers respectivement le 07 juillet 1986 et 19 mars 1994. En outre, les gestionnaires des aires protégées nous ont révélé qu'il s'est souvent produit des cas de confiscation ou la destruction de véhicules de patrouille ; la séquestration ou la bastonnade d'agents. Sur ce dernier point, voir AKOUÉ (Yao Claude), Les aspects conflictuels liés à la conservation des aires protégées de Côte d'Ivoire: le cas du Parc National de la Marahoué à Bouaflé, Mémoire de DEA en Sciences et Gestion de l'Environnement option Politique en Environnement, Université d'Abobo-Adjamé, août 2004 , p. 63.

${ }^{120}$ Voir en Introduction (de cette étude), le point intitulé "II- Aperçu de la législation consacrée à la protection des aires protégées en Côte d'Ivoire".
} 
si l'une des causes principales des pressions que les populations exercent sur les aires protégées ivoiriennes résulte des problèmes de développement des communautés locales ${ }^{121}$, le droit des aires protégées concilie-t-il protection des aires protégées et développement des communautés riveraines ? C'est ainsi que l'analyse du droit positif des aires protégées dont la loi de 2002 sur les aires protégées constitue l'épine dorsale mérite d'être faite (au fond). Ce faisant, il serait possible de savoir si, tout en prônant leur conservation, le droit des aires protégées intègre les questions de développement des communautés locales.

De plus, étant donné que les pressions sur les aires protégées vont grandissantes, en dépit l'évolution des règles de protection de ces forêts, on pourrait se poser la question suivante : le droit positif des aires protégées comporte t-il des insuffisances qui entravent son application ? Ou encore, sont-ce, au contraire, des problèmes pratiques de gestion qui rendent difficile son application ? La réponse à ces interrogations ci-dessus se fera sur le fondement d'une hypothèse de départ. Celle-ci postule que si le droit positif des aires protégées n'est pas effectif, c'est parce qu'il recèle d'insuffisances qui entravent son application. Aussi quelles pourraient-être les solutions pour remédier à cette situation?

La résolution de ces différentes interrogations se fera selon la méthodologie cidessous.

\section{VI - INDICATIONS D'ORDRE MÉTHODOLOGIQUE}

La méthodologie désigne les méthodes utilisées dans un domaine particulier ${ }^{122}$. En l'espèce, il s'agit du droit de l'environnement. Pour traiter les problématiques relatives au droit de l'environnement, l'on ne saurait se limiter à la seule réflexion ou théorie juridique. En effet, la mise en œuvre dudit droit se produit dans un milieu social qui est susceptible de l'influencer fortement ${ }^{123}$. C'est à juste titre que dans le cadre de cette étude, au-delà des techniques de raisonnement juridique (théorique), nous avons eu recours à certains nombre de

\footnotetext{
${ }^{121}$ Sur la question et relativement au Parc National de Taï (en Côte d'Ivoire), GOH (Denis) écrit : «Au plan socioéconomique, l'enjeu de la gestion participative du Parc National de Taï est le développement social, économique et culturel des zones riveraines du Parc National de Taï. Il s'agit de mettre en œuvre un mécanisme concerté de gestion du parc qui, tout en sauvegardant la biodiversité du site, satisfait les besoins de développement des populations riveraines. ».Voir GOH (Denis), Les approches participatives dans la gestion des Aires Protégées en Côte d'Ivoire : l'expérience du Projet Autonome pour la Conservation du Parc National de Taï (PACPNT), Thèse unique, Environnement, Université d'Abobo-Adjamé, 21 mai 2005, p. 256.

$\mathrm{Au}$ Burkina-Faso, la pauvreté des populations (donc le déficit de développement duquel elle résulte) est l'une des causes explicatives des pressions anthropiques sur les ressources naturelles pour les besoins alimentaires. Voir GRANIER (Laurent), "Introduction », in GRANIER (Laurent) (dir.), Aspects contemporains du droit de l'environnement en Afrique de l'Ouest et centrale, UICN, Gland, Suisse, 2008, p. 25.

${ }^{122}$ Voir THEUX (Axel de), KOVALOVSKY (Imre), BERNARD (Nicolas), Précis de méthodologie juridique : les sources documentaires du droit ( $2^{\text {ème }}$ édition revue et mise à jour), Bruxelles, Publications des Facultés universitaires Saintes-Louis, 2000, p. 639.

${ }^{123}$ En guise d'exemple, traitant du faible degré d'effectivité du droit de l'environnement au Burkina-Faso, ZAKANÉ (Vincent) cité par GRANIER (Laurent) stigmatise, entres autres causes, le « défaut d'adéquation [des règles] avec le contexte social et culturel de la société dans laquelle elles sont appelées à s'appliquer (cette partie du texte est mise en gras par nous) ».Voir GRANIER (Laurent), " Introduction », in GRANIER (Laurent) (dir), Aspects contemporains du droit de l'environnement en Afrique de l'Ouest et centrale, op. cit., p. 2.
} 
techniques généralement usitées dans les sciences sociales ${ }^{124}$ telles que la sociologie, l'anthropologie, la géographie, la psychologie etc. Il s'agit des méthodes de collectes de données (A) et de techniques d'analyse pour l'exploitation des informations récoltées (B).

\section{A - Les méthodes de collecte d'informations}

Les méthodes de collecte d'information utilisées pour cette étude sont l'étude documentaire (1) et l'enquête (2).

\section{L'étude documentaire}

L'étude documentaire se définit comme l'exploitation de sources de renseignements déjà existants ${ }^{125}$. L'intérêt de cette démarche s'explique pour plusieurs raisons.

Premièrement, étant donné que cette étude porte sur l'analyse du droit des aires protégées en Côte d'Ivoire, elle exigeait nécessairement de consulter les lois (lato sensu) sur les aires protégées et de recourir à la doctrine juridique, en général, et celle relative au droit à l'environnement, en particulier. En effet, la doctrine juridique (en général) sert de base aux réflexions menée en l'espèce sur le droit ivoirien des aires protégées, d'autant plus que la littérature juridique (spécifique aux aires protégées en Côte d'Ivoire) est quasi-inexistante.

Dans un deuxième temps, la consultation de rapports et autres documents de travail relatifs à la gestion des aires protégées ont contribué à appréhender les problèmes de conservation de ces milieux naturels. De cette manière, les recommandations faites en fin d'étude seront plus pratiques que théoriques parce qu'elles tiennent compte (au mieux) des réalités de leur gestion.

Dans un troisième temps, l'étude documentaire a permis de comprendre des expériences de conservation des aires protégées de divers pays du monde, en général, et

\footnotetext{
${ }^{124}$ La notion de sciences sociales ne fait pas encore l'objet d'une définition unanime aux contours précis, même si les auteurs s'accordent à les définir comme étant des disciplines qui étudient les phénomènes sociaux, les faits sociaux ou tout simplement l'homme en société. Voir GRAVITZ (Madeleine), Lexique des sciences sociales, $8^{\text {ème }}$ édition, Paris, Dalloz, 2004, p. 365.

Pour BAYLE (Jean-Louis Loubel del), les sciences sociales : «constituent une branche des sciences humaines, c'est-à-dire des sciences qui ont l'homme et ses activités pour objet d'étude...Parmi les sciences humaines, les sciences sociales se distinguent des disciplines qui étudient les aspects physiques de l'homme, comme la médecine, ou qui envisagent les hommes en tant qu'individus, comme la psychologie. Les sciences sociales étudient une catégorie particulière de phénomènes humains : les phénomènes sociaux, les faits sociaux. ». Voir BAYLE (Jean-Louis Loubel del), Initiation aux méthodes des sciences sociales, Paris, Le Harmattan, 2000 , p. 7. Selon GRAVITZ (Madeleine) : "À l'heure actuelle, on considère que les principales sciences sociales sont la sociologie, l'anthropologie, la psychologie sociale, l'histoire, la géographie, la démographie, l'économie politique, la science politique et la linguistique. Cette liste n'est pas limitative. ». Voir GRAVITZ (Madeleine), Méthodes des sciences sociales, $11^{\mathrm{ème}}$ édition, Paris, Dalloz, 2001, p. 77.

${ }^{125} \mathrm{Ces}$ sources de renseignements peuvent être des documents écrits (textes), sonores (disques), visuels (dessins). Pour plus de détails sur la question, Voir N'DA (Paul), Méthodologie de la recherche : de la problématique à la discussion des résultats. Comment réaliser un mémoire, une thèse en Sciences Sociales et en Éducation, op. cit., pp. 75-76.
} 
surtout de pays africains dont le contexte socioéconomique des populations à des similitudes avec celles de la Côte d'Ivoire ${ }^{126}$. Ceci dans l'optique de tirer les leçons des échecs et des succès enregistrés. Grâce à cette méthode, des propositions pour la gestion durable des aires protégées ivoiriennes, inspirées de ces expériences de gestion d'aires protégées, ont pu être faites.

Enfin, l'étude documentaire a permis d'obtenir des informations (préalables) sur les aires protégées et les zones périphériques à enquêter, avant l'étape de l'enquête de terrain. C'est ainsi que dans un second temps, une enquête (de terrain) a été réalisée.

\section{L'enquête}

L'enquête a eu une place de choix dans le cadre de la présente étude. En plus des informations recueillies durant la recherche documentaire, il nous a paru nécessaire de rencontrer, d'échanger avec les gestionnaires d'aires protégées et autorités de tutelle ainsi que les communautés locales. Ces entretiens ou échanges ont eu pour objectif de confirmer, infirmer ou actualiser certaines informations obtenues grâce à l'étude documentaire.

L'enquête a consisté à nous rendre dans les services publics dont relève les aires protégées et dans certaines localités de la Côte d'Ivoire où sont situées des aires protégées. Ce faisant, nous avons collecté des informations auprès des communautés et gestionnaires locaux; et observé des réalités de la gestion de ces milieux naturels ${ }^{127}$. Ces informations ont été croisées avec celles recueillies auprès des autorités de gestion et de tutelle des aires protégées dans les services publics visités.

Les résultats de ces investigations ont servi essentiellement à comprendre, à expliquer et à illustrer nos développements sur l'analyse de l'effectivité du droit des aires protégées dans la deuxième partie de ce travail.

Aussi les informations obtenues ont-elles été exploitées à l'aide de certaines techniques d'analyse.

\section{B - Les techniques d'analyse des données collectées ou d'analyse de l'objet}

Trois (3) techniques d'analyse ont été utilisées dans cette étude. Il s'agit de la méthode historique (1), de la méthode dialectique (2) et de la méthode comparative (3).

\footnotetext{
${ }^{126}$ Ces pays africains sont notamment l'Afrique du Sud, la Namibie, la Zambie, le Kenya, l’Éthiopie, le Botswana, l'Ouganda, la Tanzanie, le Zimbabwe, le Rwanda, le Cameroun, le Gabon, la République démocratique du Congo, la Centrafrique, le Sénégal, la Guinée-Bissau, le Bénin, le Burkina-Faso, le Nigéria, le Ghana et Madagascar etc. En ce qui concerne les autres pays du monde, ces études d'expériences de gestion d'aires protégées ont porté, entre autres, sur le Guatemala, la Serbie, la Roumanie, la Nouvelle-Zélande, le Royaume-Uni, la Norvège, l'Australie et l'Inde.

${ }^{127}$ Les informations relatives au déroulement de l'enquête et les résultats figurent à l'Annexe 3.
} 


\section{La méthode historique}

Selon P. ROGERE cité par Gilles A. KRAGBÉ, la méthode historique est définie comme étant: "la genèse des situations étudiées pour rendre compte de la situation actuelle ${ }^{128}$. Dans l'analyse du cadre juridique de protection des aires protégées en Côte d'Ivoire qui se veut évolutif, la méthode historique a permis de connaître et d'étudier les règles originaires de protection ainsi que les raisons qui ont motivé leur réécriture successive. Autrement dit, cette méthode a aidé à étudier le droit qui a régi successivement les aires protégées de 1960 à nos jours.

De plus, cette méthode a servi à comprendre la genèse de la création des aires protégées et des conflits qui, bien souvent, opposent les gestionnaires et les communautés locales. Qu'en est-il de la méthode dialectique?

\section{La méthode dialectique}

Selon Paul N'DA, la méthode dialectique contribue à expliquer des faits sociaux en recherchant les contradictions qui constituent souvent l'essence d'une réalité ${ }^{129}$. La méthode dialectique a contribué à expliquer les entraves à la conservation des aires protégées résultant des contradictions qui la caractérisent. En effet, certaines communautés riveraines des aires protégées et les pouvoirs publics, en général ${ }^{130}$ ainsi que les gestionnaires des aires protégées, en particulier, affirment reconnaître la nécessité de la conservation des aires protégées ${ }^{131}$.

Cependant, en bien des cas, elles posent des actions qui contrarient les efforts de leur conservation. Autrement dit, les communautés locales exercent des pressions sur ces milieux naturels avec ou sans la complicité de certains gestionnaires corrompus qui les exploitent également ${ }^{132}$.

Pire, l'État ivoirien qui définit la conservation des aires protégées déclasse, dans certains cas, des superficies au profit des populations. Également, les pouvoirs publics de la

\footnotetext{
${ }^{128}$ Voir KRAGBÉ (Gilles A.), Les politiques de gestion de l'environnement à l'aune du droit traditionnel africain : cas des peuples des régions forestières de la Côte d'Ivoire, Thèse unique, Environnement, Université d'Abobo-Adjamé, 17 février 2011, p. 35.

${ }^{129}$ Voir N'DA (Paul), Méthodologie de la recherche: de la problématique à la discussion des résultats. Comment réaliser un mémoire, une thèse en Sciences Sociales et en Éducation, op cit., p. 55.

${ }^{130} \mathrm{Il}$ s'agit de toute personne investie d'un pouvoir administratif représentant l'État de Côte d'Ivoire [autorités ministérielles en charge de la tutelle (technique) des aires protégées, de représentants de l'Administration étatique déconcentrée etc.].

${ }^{131}$ Il ressort de l'enquête de terrain effectuée auprès des communautés locales riveraines des aires protégées enquêtées au cours de cette étude que $\mathbf{2 0} \%$ d'entre elles reconnaissent l'importance (écologique) de protéger les Parcs Nationaux et Réserves. Pour ce qui est des pouvoirs publics, l'importance de la conservation des aires se déduit de leur mission de protection et de la solidarité gouvernementale dans la réalisation des missions de l’État.

${ }^{132}$ Voir UICN/BRAO, Évaluation de l'efficacité de la gestion des aires protégées : Parcs et Réserves de Côte d'Ivoire, 2008, op.cit., p. 32.
} 
République de Côte d'Ivoire tolèrent l'occupation de certaines aires protégées, en violation de la $10 i^{133}$.

De plus, la méthode comparative a été mise à contribution au cours de cette étude.

\section{La méthode comparative}

C'est une méthode qui permet de comparer des faits et des théories en rapprochant des réalités différentes, en vue d'établir une vérité scientifique ${ }^{134}$. Le recours à cette méthode s'explique par le fait qu'elle est conseillée par la doctrine en droit de l'environnement ${ }^{135}$.

\footnotetext{
${ }^{133}$ En Côte d'Ivoire, les cas de légitimation des occupations des aires protégées par l'État sont légions. Par exemple, suite à la forte infiltration du Parc National de la Marahoué (PNM), le gouvernement a délimité les espaces fortement dégradés dans ledit Parc. L'ensemble de ces espaces qui représentent environ 9.000 ha a été baptisé "zone agroforestière", aux fins de la réinstallation (sur ces sites) de tous les planteurs et familles qui l'ont infiltrée. C'est la note circulaire n¹075/MINEFOR/IGEF du 21 juin 1988 du Ministre LOKROU (Vincent Pierre), adressée aux autorités administratives déconcentrées, aux services des Eaux et Forêts et, ayant pour objet la «Réhabilitation des Forêts classées, Parcs Nationaux et Réserve » qui sert de fondement à cette nouvelle zonation du PNM effectuée par la SODEFOR. En effet, il peut être lu sur la deuxième page de cette note circulaire, entre autres: "...Ce plan vise notamment à soustraire une plus grande superficie possible du Domaine Permanent de l'État aux agressions de toute sorte tout en maintenant intact le potentiel agricole productif généré dans ces domaines.
}

À cet égard, je tiens à vous confirmer les dispositions ci-après :

- interdiction formelle d'installer des habitations à l'intérieur des Forêts classées, des Parcs Nationaux et Réserves;

- autorisation d'accéder aux plantations en production pour les entretenir et en cueillir les produits sur une période non renouvelable de trois ans, sans toutefois procéder à de nouvelles extensions de cultures ;

- relocalisation des plantations en un seul domaine qui proviendrait en priorité de récupérations de jachères inexploitées et le cas échéant, d'un déclassement partiel du Domaine Permanent. ».

Dans le Parc National de Taï (PNT) l'exploitation à but agricole de certaines parties de cette aire protégée (dans la zone d'ADK dans le secteur de gestion V6 ; dans la partie Est du secteur de gestion Soubré ; dans le secteur Dapadji) opérée par les populations a été également légitimée par l'État, à travers les gestionnaires, sous la forme d'une zone dite zone d'occupation contrôlée prévu dans le plan de gestion (voir UICN/BRAO, Évaluation de l'efficacité de la gestion des aires protégées : Parcs et Réserves de Côte d'Ivoire, 2008, op.cit., p. 27).

À la création du Parc National d'Azagny (PNA) en 1981, dans la zone centrale de protection et la zone périphérique existaient des plantations de café, cacao, palmiers à huile etc. Un délai d'exploitation de trois (3) ans fut accordé aux paysans qui en étaient propriétaires. Ils avaient été recasés dans la forêt classée du Gôdé déclassée partiellement à cet effet. Cependant, à l'expiration de ce délai, ces paysans excipaient de problèmes d'installation pour plaider la prorogation du délai d'autorisation de récolter les plantations de la zone centrale de protection et de la zone périphérique du PNA. En effet, ils évoquaient des problèmes de production et de destructions de cultures par les éléphants. Ce faisant, les exploitations agricoles se poursuivaient de fait dans la zone centrale de protection et dans la zone périphérique, jusqu'en 1997 où intervient un arrêté ministériel qui autorise officiellement l'entretien des plantations dans ces zones. Les paysans en ont profité pour agrandir leurs plantations. En 2006, suite à un homicide perpétré par un élément de la brigade mobile en patrouille de surveillance sur un "délinquant", l'Administration en charge de la gestion des aires protégées [1'Office Ivoirien des Parcs et Réserves (OIPR) et subséquemment sa Direction Générale] a autorisé la récolte des plantations des cultures pérennes dans ces zones, sous certaines conditions dont la principale est leur non entretien. Cela fit l'objet d'un contrat d'autorisation d'exploitation passé entre l'OIPR et les paysans concernés, de 2006 à 2008. Cette zone du PNA exploitée, nonobstant son statut qu'il l'affranchit de tout droit d'usage, était appelée «Zone d'Occupation Contrôlée ". Cette exploitation a duré jusqu'en 30 septembre 2008, date d'échéance des contrats d'autorisation d'exploitation dans le PNA. Ainsi, depuis cette date, il n'existe plus officiellement de ZOC dans le PNA. Pour ces développements sur le Parc National d'Azagny, voir République de Côte d'Ivoire, Ministère de l'Environnement et des Eaux et Forêts/OIPR, Plan d'aménagement et de gestion du parc national d'Azagny, Abidjan, 2008, (inédit), pp. 70 et 71. 
Dans la présente étude, la méthode comparative a servi à étudier les expériences de gestion des aires protégées dans plusieurs pays africains en vue d'établir une vérité : identifier les facteurs de succès ou d'échec des politiques de conservation des aires protégées. Plusieurs expériences de gestion d'aires protégées de pays en voie de développement, à l'instar de la Côte d'Ivoire, ont été analysées en général.

Toutefois, la gestion des aires protégées des pays africains anglophones a particulièrement retenu notre attention, en raison de leurs expériences réussies de conservation des aires protégées. De cette manière, des propositions ont pu être faites dans la mesure où la Côte d'Ivoire qui possède des richesses naturelles similaires pourrait s'en servir pour arriver à une conservation durable de ses aires protégées.

Au surplus, le contenu de cette étude sera présenté selon le plan ci-dessous.

\section{VII - ANNONCE DU PLAN}

La présente étude est conduite sur la base de l'hypothèse de travail selon laquelle le défaut de l'effectivité du droit des aires protégées en Côte d'Ivoire depuis 1960 s'explique par des insuffisances qui le caractérisent. Pour vérifier cette hypothèse de travail, cette étude sera menée en deux (2) temps.

La première partie de ce travail exposera la manière dont la Côte d'Ivoire assure la protection juridique des aires protégées depuis son accession à l'indépendance en 1960. Pour ce faire, vu que ledit droit procède d'une évolution historique qui pourrait être scindée en deux (2) périodes, à savoir de 1960 à 2002 et de 2002 à nos jours, il sera fait, d'une part, l'analyse de la protection juridique des aires protégées de 1960 à 2002. D'autre part, les innovations que le législateur a apportées au droit des aires protégées depuis 2002 seront étudiées. De la sorte, les insuffisances du cadre juridique des aires protégées qui ont conduit le législateur à le réécrire en 2002 permettront de mieux comprendre le sens ces réformes juridiques opérées depuis 2002.

\footnotetext{
${ }^{134}$ Voir GRAWITZ (Madeleine), Lexique des sciences sociales, $8{ }^{\text {ème }}$ édition, Paris, Dalloz, 2004, p.74. En effet, en règle générale, les problèmes environnementaux dépassent le cadre des frontières des États. C'est cette réalité que le Professeur SADELEER (Nicolas de) traduit en ces termes : "Ni les espèces végétales, ni les espèces animales, ni leurs biotopes ne connaissent les frontières, surtout lorsque celles-ci ne sont pas délimitées par des éléments géographiques. À quelques exceptions près, ces problèmes transnationaux appellent des réponses communes. ». SADELEER (Nicolas de), «Particularités de la subsidiarité dans le domaine de l'environnement», Droit et Société, Vol. 1, n80, p. 76.

${ }^{135}$ En effet, pour le Professeur KAMTO (Maurice) il est utile de recourir à l'étude comparative pour l'analyse des problématiques environnementales car : "l'environnement ignore les frontières ainsi que les barrières de toutes sortes ». Voir KAMTO (Maurice), Droit de l'environnement en Afrique, Vanves, AUPELF/UREF, 1996, op. cit., p.22.

Ce point de vue est confirmé par le Professeur PRIEUR (Michel) en ces termes : «L'apparition concomitante de droits de l'environnement dans les divers pays du monde rend indispensable l'étude comparative de ces droits. Ces réflexions comparées doivent permettre la détermination de tendances générales de développement du droit de l'environnement et une harmonisation ou une unification future de ce droit ». Voir PRIEUR (Michel), Droit de l'environnement, 4ème édition, Paris, éditions Dalloz, 2001, p.13.
} 
La deuxième partie de cette étude sera consacrée à l'effectivité de la protection juridique des aires protégées. La dégradation continue des aires protégées sous-entend que de toute évidence le droit des aires protégées connaît des difficultés d'application. C'est la raison pour laquelle le droit des aires protégées fera l'objet d'une analyse critique. Cette manière de procéder permettra de savoir si ledit droit porte en lui-même les germes de son inobservation ou si celle-ci est exclusivement justifiée par des facteurs externes. Dès-lors, des recommandations seront formulées selon que l'effectivité du droit des aires protégées requiert des réformes juridiques en vue de corriger ses insuffisances et/ou des solutions aux problèmes pratiques de gestion. 


\title{
PREMIÈRE PARTIE :
}

\author{
L'EXISTENCE AVÉRÉE D'UNE LÉGISLATION \\ CONSACRÉE À LA PROTECTION DES PROTÉGÉES
}


À l'instar des forêts tropicales du monde, en Côte d'Ivoire, la conservation des forêts notamment des aires protégées revêt un enjeu à la fois écologique et économique ${ }^{136}$. En effet, d'une part, la forêt constitue un réservoir de biodiversité et un puits de carbone ainsi qu'un régulateur du climat qui conditionne la pratique des activités agricoles. D'autre part, l'exploitation de la forêt créée des activités économiques agricoles. Ces activités économiques agricoles qui occupent la majorité des populations ivoiriennes, surtout en milieu rural, constituent la base de l'économie ivoirienne.

Conscients de ces enjeux et des risques de dégradation du patrimoine forestier ivoirien consécutifs à son utilisation socio-économique, les pouvoirs publics ivoiriens ont mis en défens des forêts telles que les aires protégées. Elles sont affranchies de tous droits d'usage ${ }^{137}$. Ainsi, les aires protégées créées par l'État ivoirien font l'objet d'une protection juridique. Cette protection est l'œuvre de plusieurs lois (lato sensu) successives qui traduisent le caractère évolutif de la législation des aires protégées. Sur le plan historique, la protection juridique des aires protégées pourraient se subdiviser en deux (2) grandes périodes.

La première s'inscrit dans la borne chronologique 1960-2002. Elle représente la période dans laquelle ces aires protégées sont régies par des lois éparses qui les dotent d'une protection juridique dont l'application est faite à travers une gestion exclusivement étatique aux moyens insuffisants. Il s'agit du cadre juridique originaire des aires protégées (Titre I).

Malgré l'existence de ce cadre juridique, les aires protégées de Côte d'Ivoire ont fait l'objet de pressions anthropiques qui ont parfois constitué des menaces pour leur existence. Ces pressions ont été justifiées par les insuffisances qui ont caractérisé le cadre juridique des aires protégées de 1960 à 2002. C'est ainsi que depuis l'année 2002, les autorités compétentes ivoiriennes ont pris des textes en vue d'assurer une meilleure conservation des aires protégées. Ces différentes lois (lato sensu) adoptées au cours de cette deuxième période de l'histoire de la protection juridique de ces espaces naturels visent à apporter des innovations au cadre juridique originaire des aires protégées (Titre II).

\footnotetext{
${ }^{136}$ Pour plus de précisions sur les enjeux écologiques et économiques de la protection des forêts en Côte d'Ivoire, voir les développements faits (antérieurement) dans l'Introduction de la présente thèse notamment le point intitulé "I-Enjeux de la protection des forêts en Côte d'Ivoire".

${ }^{137}$ En effet, aux termes de l'article $1^{\text {er }}$ de la loi de 2002 sur les PNR, le Parc National désigne une aire «...exclusivement destinée à la propagation, la protection, la conservation et l'aménagement de la végétation et les populations d'animaux sauvages, ainsi qu'à la protection des sites, des paysages ou des formations géologiques d'une valeur scientifique ou esthétique particulière, dans l'intérêt et pour la récréation du public...dans laquelle l'abattage, la chasse, la capture d'animaux et la destruction ou la collecte des plantes sont interdits...». Quant à la Réserve naturelle intégrale, l'article précité dispose qu'elle est une aire : «...sur l'étendue de laquelle toute espèce de chasse ou de pêche, toute exploitation forestière, agricole ou minière, tout pâturage, toute fouille ou prospection, tout sondage ou tout terrassement, toute construction, tous travaux tendant à modifier l'aspect du terrain ou de la végétation...tout acte de nature à apporter des perturbations à la faune ou à la flore...seront strictement interdits... (souligné par nous) ». En ce qui concerne la Réserve naturelle partielle, le même article la définit comme "une aire protégée gérée dans un but de conservation in situ des écosystèmes naturels ou d'espèces ou de biotopes spécifiques au profit et à l'avantage et pour l'utilisation durable, la récréation et l'éducation du public». Enfin, la Réserve partielle de faune est une aire «...Mise à part pour la conservation, l'aménagement et la propagation de la vie animale sauvage, ainsi que pour la protection et l'aménagement de son habitat...dans laquelle la chasse, l'abattage ou la capture de la faune sont interdits... » (Ces parties de l'article $1^{\mathrm{er}}$ précité ont été mises en gras par nous).
} 


\section{TITRE I :}

LE CADRE JURIDIQUE ORIGINAIRE DES AIRES PROTÉGÉES 
De 1960 à 2002, l'État ivoirien met progressivement en place un cadre juridique (originaire) pour assurer la conservation de ses aires protégées. D’une part, ce cadre juridique institue un service public qui a la charge de la gestion des aires protégées. D’autre part, il consacre des dispositions qui protègent l'intégrité des aires protégées et organisent la répression des infractions contre ces milieux naturels. Autrement dit, il s'agit d'un ensemble de textes destinés à assurer la protection juridique des aires protégées de 1960 à 2002 (Chapitre I).

Cependant, durant cette période, les pressions auxquelles étaient sujettes les aires protégées conduisent à la conclusion selon laquelle ces normes juridiques se sont révélées insuffisantes pour leur protection durable (Chapitre II). 


\section{CHAPITRE I : LA PROTECTION JURIDIQUE DES AIRES PROTÉGÉES DE 1960 À 2002}

En Côte d'Ivoire, de 1960 à 2002, la protection juridique des Parcs Nationaux et Réserves a fait l'objet d'un cadre juridique élaboré par l'État ivoirien. Ce cadre juridique comporte deux (2) volets, à savoir un cadre institutionnel (Section I) et un cadre normatif (Section II).

\section{SECTION I : Le cadre institutionnel de gestion des aires protégées}

Au plan institutionnel, la gestion et notamment la conservation des aires protégées (pour tenir compte de la destination de ces milieux naturels) est assurée par un service public (Paragraphe 1) qui dispose de ressources budgétaires à cet effet (Paragraphe 2).

\section{Paragraphe 1 : Le service public de la conservation des aires protégées de 1960 à 2002, une Administration centralisée}

Affirmer que le service public de la conservation des aires protégées a été une Administration centralisée de 1960 à 2002 requiert qu'il soit présenté, en vue de savoir si son fonctionnement était calqué sur celui de la centralisation administrative (B). Pour ce faire, il est indiqué d'appréhender au préalable les notions de «service public de la conservation des aires protégées » et de « centralisation » (A).

\section{A- Les notions de « service public de la conservation des aires protégées » et de «centralisation »}

Il s'agira, à travers les lignes qui suivent, de traiter successivement des notions de « service public de la conservation des aires protégées » et de « centralisation ».

\section{La notion de service public de la conservation des aires protégées}

Le service public a toujours été une notion difficile à définir ${ }^{138}$ en ce sens que les éléments (le but : la mission d'intérêt général et le moyen : mission assurée par une personne publique) qui servent à la définir sont en constante recomposition ${ }^{139}$. Toutefois, dans leurs

\footnotetext{
${ }^{138} \mathrm{C}$ 'est à juste titre qu'évoquant cette difficulté, le Professeur DÉGNI-SÉGUI (René) affirme : « Le service public est une notion essentielle du droit administratif...Et pourtant, ni le législateur, ni la jurisprudence n'en donnent une définition. Aussi ne peut-on que faire appel à quelques éléments constitutifs, ou à tout le moins d'identification du service public, pour tenter d'en dégager la notion » (souligné par nous). Voir DÉGNISÉGUI (René), Droit administratif général : L'action administrative (t. 2), 4 ème édition, Abidjan, NEI-CEDA, 2012, p. 114). Sur la difficulté de la définition du service public, voir également RIVERO (Jean), WALINE (Jean), Droit administratif, 20 ${ }^{\text {ème }}$ édition, Paris, Dalloz, 2004, p. 301; GUGLIELMI (Gilles J.), KOUBI (Geneviève), Droit du service public, Paris, Montchrestien, 2000, p. 62 ; PEISER (Gustave), Droit administratif général, 21 édition, Paris, Dalloz-Sirey, 2002, p. 175.

${ }^{139} \mathrm{Au}$ sujet de cette difficulté à définir la notion de service public en raison de l'incertitude qui caractérise la notion d'intérêt général, GEORGES (Phillipe) écrit : «c'est l'autorité publique compétente qui apprécie les
} 
essais de définition du service public, les auteurs sont unanimes que cette notion a une double définition : une définition organique et une définition matérielle ${ }^{140}$.

Sur le plan matériel, le service public est une activité dont le but est de satisfaire une

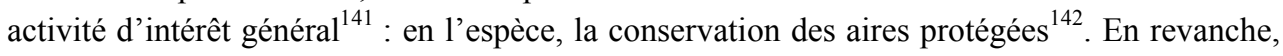
sur le plan organique, le service public est «un organe de gestion...un organisme ou un appareil administratif $»^{143}$ dont la mission est d'accomplir une activité d'intérêt général.

Aussi convient-il de noter qu'en utilisant les critères organique et matériel ci-dessus, la doctrine parvient à une définition de synthèse du service public. Elle entend comme étant une activité ou mission d'intérêt général assurée ou assumée par une personne morale de droit $\operatorname{public}^{144}$.

Il en résulte que le service public de la conservation des aires protégées pourrait se définir comme étant l'activité de conservation des aires protégées assurée par l'appareil administratif ou l'Administration en charge des aires protégées.

La notion de « service public de la conservation des aires protégées » ainsi définie, il convient d'en faire autant pour celle de « centralisation ».

exigences de l'intérêt général, exigences variables dans le temps et dans l'espace ». Voir GEORGES (Phillipe), Droit public: concours administratifs, 8 édition, Paris, Sirey, 1992, p. 301.

${ }^{140}$ Voir RIVERO (Jean), WALINE (Jean), Droit administratif, 20 eme édition, op. cit., pp. 301-302 ; GEORGES (Phillipe), Droit public: concours administratifs, 8 édition, op. cit., p. 300 ; DÉGNI-SÉGUI (René), Droit

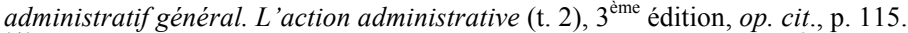

${ }^{141}$ Voir RIVERO (Jean), WALINE (Jean), Droit administratif, 20 eme édition, op. cit., p. 301 ; GEORGES (Phillipe), Droit public: concours administratifs, 8 édition, op. cit., p. 300 ; DÉGNI-SÉGUI (René), Droit administratif général. L'action administrative(t. 2), $3^{\text {ème }}$ édition, op. cit., p. 115.

${ }^{142}$ Selon l'article 4 de la loi de 2002 sur les PNR, la conservation des aires protégées est une mission d'intérêt général.

${ }^{143}$ Voir GEORGES (Phillipe), Droit public: concours administratifs, 8 édition, op. cit., p. 300 ;René DÉGNISÉGUI, Droit administratif général. L'action administrative, op. cit., p.115 ; RIVERO (Jean), WALINE (Jean), Droit administratif, 20 ème édition,op. cit., pp. 301-302.

${ }^{144}$ Cette définition de synthèse est formulée pour la première fois par René CHAPUS qui à propos des éléments de définition du service public affirmait ceci : «Les meilleurs esprits se rejoignent pour professer que la notion de service public est indéfinissable. En réalité, il n'en est rien; et il serait d'ailleurs bien étonnant que l'édifice $d u$ droit administratif ait pu être construit sur quelque chose d'indéfinissable. Si on veut bien ne pas confondre ce qui est définition et ce qui est diagnostic, on doit reconnaître qu'il est parfaitement possible de définir notre notion, à condition que deux données soient de façon égale prises en considération : l'une (souvent négligée ou insuffisamment retenue) est le rattachement organique de l'activité à qualifier; l'autre est la raison d'être de cette activité, l'objectif en vue duquel elle est exercée. La définition s'énonce simplement : une activité constitue un service public quand elle est assurée ou assumée par une personne publique en vue d'un intérêt public. Toutes autres données ou considérations sont indifférentes (la partie de cette citation mise en gras l'a été par nous) » Voir CHAPUS (René), Droit administratif général (t. 1), $11^{\text {ème }}$ édition, Paris, Montchrestien, 1997, p. $520, \mathrm{n}^{\circ} 748$ ). Cette définition générale du service public donnée par René CHAPUS est reprise par plusieurs auteurs qui ne manquent pas de souligner la difficulté à définir le service public. Il s'agit, entre autres, de GEORGES (Phillipe), Droit public: concours administratifs, 8 édition, op.cit.,p. 301 ; GUGLIELMI (Gilles J.), KOUBI (Geneviève), Droit du service public, op.cit.,p. 62 ; PEISER (Gustave), Droit administratif général, 21 édition, op.cit., p. 175 . 


\section{La définition juridique du concept de centralisation}

La centralisation pourrait se définir comme un système d'organisation administrative dans lequel le pouvoir de décision est dévolu exclusivement à une autorité administrative étatique dont les collaborateurs sont tenus dans les liens de la subordination hiérarchique ${ }^{145}$. C'est ce qui a fait dire au Professeur René DÉGNI-SÉGUI que la centralisation : " s'entend, de ce point de vue, du procédé technique, qui consiste à reconnaître l'État comme la seule personne publique compétente pour régler tous les problèmes de la Nation ${ }^{146}$.

L'Administration centralisée se caractérise, par conséquent, par sa forte hiérarchisation. En effet, la totalité des activités du service public est assurée par les services administratifs centraux. Ces services règlent les affaires d'intérêt général tandis que les services administratifs locaux assurent l'exécution des affaires d'intérêt local ; mais sous les ordres des premiers cités.

Les services administratifs locaux sont donc le prolongement des services administratifs centraux. Cette modalité de la centralisation administrative qu'est la déconcentration permet à l'État d'être la seule personne à gérer les affaires de la nation. Il existe, dans ce cas, un centre unique de décision qui émet les ordres et coordonne l'exécution de toutes les activités administratives.

Par ailleurs, l'analyse du fonctionnement de l'Administration en charge des aires protégées de 1960 à 2002 révèle qu'elle est organisée sur le modèle de la centralisation administrative.

\section{B - L'Administration en charge des aires protégées de 1960 à 2002, une Administration centralisée}

Dès son accession à l'indépendance, la Côte d'Ivoire met en place une Administration centralisée en vue de la gestion des affaires de l'État notamment la conservation des aires protégées. Ce choix pour une Administration centralisée en charge des aires protégées qui sera présentée (1) repose certainement sur des raisons historiques (2).

\section{La présentation de l'Administration en charge des aires protégées de} 1960 à 2002

De 1960 à 2002, l’Administration en charge des aires protégées se présentait schématiquement comme suit :

\footnotetext{
${ }^{145}$ Voir GUINCHARD (Serges), DEBARD (Thierry) (dir.), Lexiques des termes juridiques, $19^{\mathrm{e}}$ édition, Paris, Dalloz, 2012, p. 136.

${ }^{146}$ Voir DÉGNI-SÉGUI (René), Droit administratif général : L'organisation administrative (t. 1), $4^{\text {ème }}$ édition, Abidjan, éditions Omniprésence, 2013, p. 62.
} 
Figure 2 : Organisation du service public de la conservation des aires protégées de 1960 à 2002

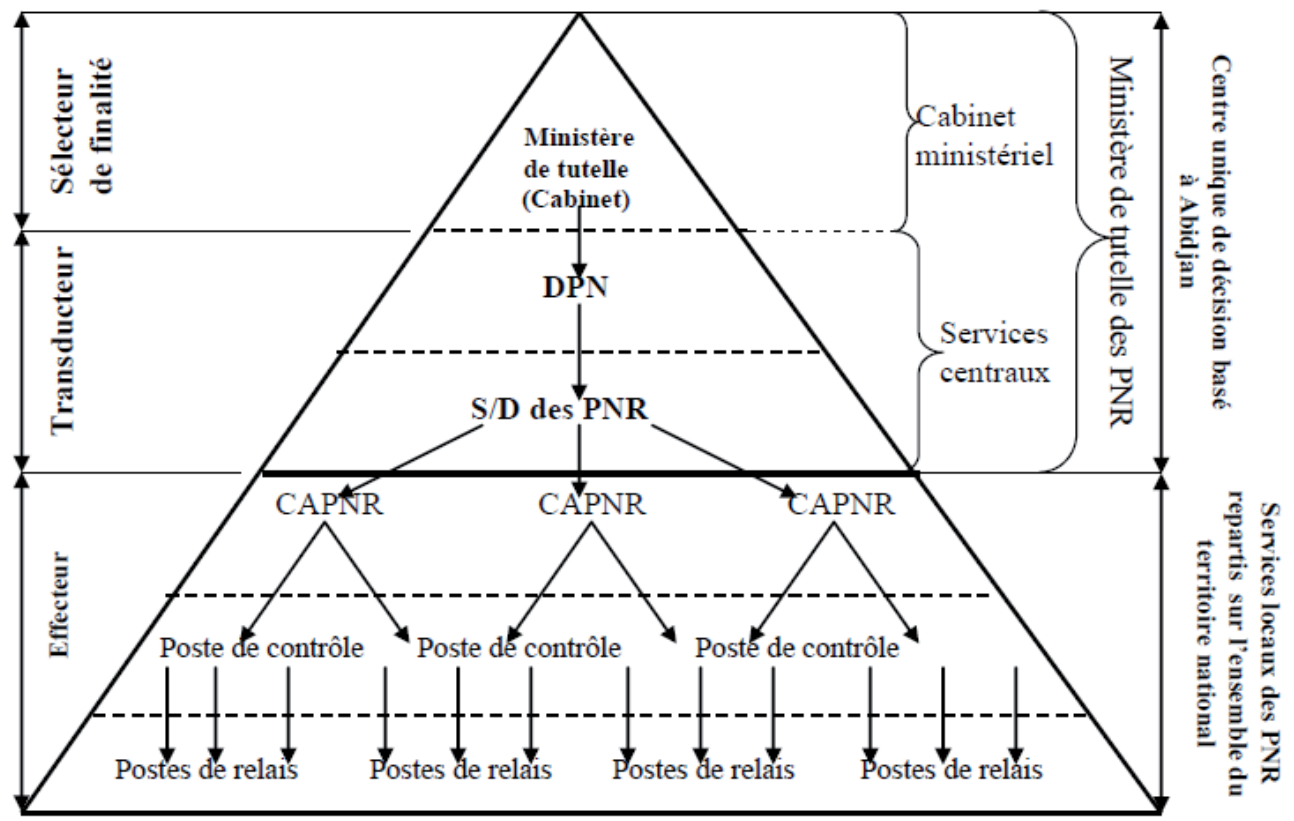

Source : Enquêtes, 2009

\section{Commentaire}

De 1960 à 2002, le service public de la conservation des aires protégées présente une structure pyramidale à trois (3) niveaux, fortement hiérarchisée. Au sommet, se trouve le ministère de tutelle des aires protégées. Il définit la politique sectorielle du gouvernement en matière de conservation des aires protégées et veille à sa mise en œuvre.

À un niveau intermédiaire se trouvent les services centraux du ministère en charge des aires protégées, à savoir la Direction de la Protection de la Nature (DPN) et la Sous-Direction des Parcs et Réserves. En effet, les services centraux exercent les activités de conception, de planification et émettent des ordres précis qui rendent possible la réalisation de la politique de conservation des aires protégées.

L'ensemble des deux échelons de cette organisation administrative (décrits ci-dessus) représente le centre unique de décision. Ce centre unique de décision est basé à Abidjan ${ }^{147}$ alors que les aires protégées sont reparties sur tout l'ensemble du territoire ivoirien $^{148}$.

\footnotetext{
${ }^{147}$ Abidjan, ville située au Sud de la Côte d'Ivoire, est la capitale économique de la République de Côte d'Ivoire. Autrefois, elle fut la capitale politico-économique. Elle concentre sur son territoire tous les ministères et services centraux de l'État de Côte d'Ivoire. En 1983, le transfert de la capitale politique à Yamoussoukro, ville située au centre du pays, ne s'est pas fait avec les infrastructures symbolisant le pouvoir politique de l'État. Toutefois, cela
} 
Au bas de cette pyramide administrative, l'on a un ensemble de services qui exécutent matériellement les ordres émis par les services centraux. Ce sont les Cellules d'Aménagement des Parcs Nationaux et Réserves (CAPNR), les Postes de contrôle et les Postes de contrôle avancés ou de relais qui assurent la gestion pratique des aires protégées.

Ces services d'exécution sont hiérarchisés et assurent la responsabilité technique ainsi que la coordination de toutes les activités de conservation des aires protégées. En effet, au niveau de chaque aire protégée, il y a une cellule d'aménagement qui gère plusieurs postes de contrôle ; lesquels possèdent plusieurs postes de contrôle avancés ou de relais ${ }^{149}$.

En somme, le fonctionnement de l'Administration des aires protégées montre que pour assurer la protection des aires protégées, l'État ivoirien s'est posé comme le seul gestionnaire de ces milieux naturels, de 1960 à 2002.

À l'analyse, la gestion centralisée des aires protégées par l'État n'est pas spécifique à la Côte d'Ivoire. Elle est commune à tous les États, en général. Cette approche de gestion des aires protégées a une explication historique.

En effet, l'histoire nous enseigne que le premier Parc National crée (en 1872) qui est celui de Yellowstone (États-Unis) ${ }^{150}$, l'a été selon l'approche préservationniste de la conservation des aires protégées ${ }^{151}$. Par la suite, les aires protégées que les États ont créées l'ont également été sous ce modèle ${ }^{152}$. Ce courant préservationniste de la conservation des

est en cours de réalisation. Voir République de Côte d'Ivoire, Ministère d'État, Ministère du Plan et du Développement, Document Stratégique de Réduction de la Pauvreté, Stratégique de Relance du Développement et de Réduction de la Pauvreté, Janvier 2009, p.1.

${ }^{148} \mathrm{La}$ carte de répartition des aires protégées de Côte d'Ivoire (Figure 1: Répartition des Parcs Nationaux et Réserves naturelles de Côte d'Ivoire) peut être consultée dans l'Introduction de la présente étude au point intitulé "III-Définition des concepts clés de l'étude".

${ }^{149}$ Ces développements relatifs au commentaire sur le fonctionnement du service public de la conservation des aires protégées de 1960 à 2002 s'inspire du fonctionnement de l'Administration ivoirienne centralisée que décrit le Professeur DÉGNI-SÉGUI (René). Voir DÉGNI-SÉGUI (René), Droit administratif général : L'organisation administrative (t. 1), $4^{\text {ème }}$ édition, op.cit., pp. 62-63.

${ }^{150}$ Crée en 1872 , le Parc National de Yellowstone l'a été par la mise à l'écart des populations et partant une gestion exclusive des autorités publiques américaines. Voir PIMBERT (Michel P.) et PRETTY (Jules N.), Parks, People and Professionals : Putting 'Participation' into Protected Area Management, UNRISD/IIED/WWF, Discussion Paper n 57, February 1995, UNRISD, Geneva, p. 6.

${ }^{151}$ Selon l'approche préservationniste des aires protégées qui est celle des écologistes qui sont à l'origine de la création de ces types de forêts, pour conserver les aires protégées, il faut tenir l'homme à l'écart de ces milieux naturels et de leur gestion. Voir PIMBERT (Michel P.) et PRETTY (Jules N.), Parks, People and Professionals : Putting 'Participation' into Protected Area Management, op. cit.,p. 3 ; AGRAWA (Arun), VARUGHESE (George), Conservation's Visions: Poverty, Participation, and Protected Area Management in Nepal's Terai, Conference Paper: Constituting the Commons : Crafting Sustainable Commons in the New Millennium, the Eighth Biennial Conference of the International Association for the Study of Common Property, (May 31-June 4) 2000 , p. 3 .

${ }^{152}$ Selon ANDRADE (Gustavo S. M.) et RHODES (Jonathan. R.) : «De nombreuses aires protégées (AP) ont suivi l'approche conventionnelle et d'exclusion appliquée à Yellowstone en 1872. En tant que tel, de nombreux parcs ont pas réussi à intégrer pleinement les autres facteurs importants, tels que les questions sociales, culturelles et politiques ». Voir ANDRADE (Gustavo S. M.) et RHODES (Jonathan. R.), « Protected areas and local communities: an inevitable partnership toward successful conservation strategies ? ", Ecology and Society, Vol. $17, \mathrm{n}^{\circ} 4,2012$, p.1. 
aires protégées encourage la centralisation de leur gestion par l'autorité étatique ${ }^{153}$. C'est ainsi que dans la plupart des pays en voie de développement, en général, et africains, en particulier, la gestion des forêts étatiques et notamment des aires protégées a d'abord été faite exclusivement par l'État lui-même en tant que collectivité territoriale à travers ses ministères.

$\mathrm{Au}$ Guatemala ${ }^{154}$ par exemple, avant la création de l'Institut National des Forêts en 1996 (en espagnol Instituto Nacional de Bosques, en Abrégé INAB), la gestion des forêts était confiée à la Direction de la Gestion des Forêts (DIGEBOS) qui était une Direction du ministère de l'Agriculture ${ }^{155}$. En Zambie ${ }^{156}$, les aires protégées ont été, dans un premier temps, gérées par l'État par le truchement d'un département ministériel. Il s'agit de celui en charge des Parcs Nationaux et de la faune et partant des aires protégées c'est-à-dire le National Park and Wildlife Service(NPWS) ${ }^{157}$.

Pour ce qui est particulièrement de l'Afrique, cette manière de gérer les aires protégées remonte à la période coloniale ${ }^{158}$. En effet, en Afrique subsaharienne, la plupart des zones protégées ont d'abord été créées pendant la période coloniale comme réserve de chasse ou comme Parc National pour les élites européennes ${ }^{159}$.

\footnotetext{
${ }^{153}$ Voir RUTAGARAMA (Eugène), et MARTIN (Adrian), «Partnerships for protected area conservation in Rwanda », The Geographical Journal, Vol. 172, n 4, December 2006, p. 291.

${ }^{154}$ Le Guatemala est un État d'Amérique centrale. Avec une population de 15468000 d'habitants en 2013 et une superficie de $109000 \mathrm{~km}^{2}$, sa capitale est Guatemala. Il est bordé par la mer des caraïbes, l'océan Pacifique, le Mexique, le Belize, le Honduras et le Salvador. Voir www.larousse.fr/encyclopedie/pays/Guatemala/122493 (Consulté le 24 mai 2016).

${ }^{155}$ Voir BIRNER (Regina) et WITTMER (Heidi), « Better public sector governance through partnership with the private sector and civil society: the case of Guatemala's forest administration ", International Review of Administrative Sciences, Vol 72, $\mathrm{n}^{\circ} 4,2006$, p. 461.

${ }^{156} \mathrm{La}$ Zambie est un pays de l'Afrique australe. Avec une superficie de $746000 \mathrm{~km}^{2}$, elle fait frontière avec l'Angola, la République démocratique du Congo, le Mozambique, le Malawi, la Tanzanie, la Namibie et le Zimbabwe. Elle a pour capitale Lusaka et sa population est estimée à 14539000 d'habitants en 2013. Voir www.larousse.fr/encyclopedie/pays/Zambie/150467

(Consulté le 15 juin 2015).

${ }^{157}$ Voir POPE (Adam), A Preliminary Examination of Public Private Partnerships in National Park Management in Zambia, 2006, WHYDAH CONSULTING LTD. Development and Natural Resource Consultants, p. 3.

${ }^{158}$ Voir GBADEGESIN (Adeniyi) et AYILEKA (Olatubosum), « Avoiding the mistakes of the past: Towards a community oriented management strategy for the proposed National Park in Abuja-Nigeria », Land Use Policy, Vol. 17, n², 2000, p. 89. Il en est de même pour PIMBERT (Michel P.) et PRETTY (Jules N.) qui affirment que les modèles de conservation de l'Occident ont été transposés en Afrique et en Asie par les autorités coloniales. Voir PIMBERT (Michel P.) et PRETTY (Jules N.), Parks, People and Professionals: Putting 'Participation' into Protected Area Management, op. cit., p. 5.

${ }^{159}$ Voir ANDERSON (David) et GROVE (Richard H.), « The scramble for Eden: Past, present and future in African conservation » In ANDERSON and Richard H. GROVE, Conservation in Africa: People, Policies and Practice. Cambridge University Press, 1988, pp.1-12 ; MENGUE-MEDOU (Célestine), « Les aires protégées en Afrique: perspectives pour leur conservation ", VertigO - la revue électronique en sciences de l'environnement [En ligne], Vol. 3, $\mathrm{n}^{\circ}$ 1, 2002, mis en ligne le 01 avril 2002, consulté le 12 février 2016. URL : www.vertigo.revues.org/4126 (lire "La situation des aires forestières protégées : nature et étendue »; ADAMS (Williams M.), « Nature and the colonial mind "In Williams M. ADAMS and Martin MULLIGAN (eds), Decolonizing Nature: Strategies for Conservation in the Postcolonial Era. , London: Earthscan, 2003, pp.16-50; TRIPLET (Patrick), LANGRAND (Olivier), «Définir les aires protégées d'Afrique » in TRIPLET (Patrick) (dir.), Manuel de gestion des aires protégées d'Afrique francophone, Paris, Awely, 2009, pp. 18-19.
} 
En Côte d'Ivoire par exemple, le Parc National d'Azagny était à l'origine une Réserve de chasse en 1954. Il est érigé en Réserve partielle de faune de 30.000 ha, par l'arrêté n536 du 25 juin $1960^{160}$, avant de devenir un Parc National en $1981^{161}$. Il en est de même pour le Parc National de la Comoé qui a d'abord été une Réserve de faune avant d'être érigé en Parc National, après l'accession de la Côte d'Ivoire à la souveraineté internationale ${ }^{162}$.

Certains auteurs affirment que la gestion exclusive et dirigiste des aires protégées par l'État a toujours cours dans des pays en voie de développement ${ }^{163}$. Par conséquent, cette forme de gestion des aires protégées qui procède d'un héritage colonial a été perpétuée après les indépendances des pays africains, en général, et en Côte d'Ivoire, en particulier.

\section{L'organisation administrative des aires protégées de 1960 à 2002, un héritage colonial}

En Côte d'Ivoire, la monopolisation de la gestion des aires protégées par l'État, au lendemain de l'indépendance, s'inscrit dans le contexte général d'exercice de la souveraineté politique par l'État. En effet, la souveraineté politique obtenue, la capacité pour le jeune État à s'assumer sur les plans politique, économique, social et culturel reste un défi que les autorités ivoiriennes se doivent de relever.

C'est ainsi que, par l'œuvre de l'article 76 de la Constitution ivoirienne du 3 novembre $1960^{164}$, la Côte d'Ivoire (ancienne colonie française) ${ }^{165}$ reconduit la législation coloniale ${ }^{166}$

\footnotetext{
${ }^{160}$ Voir LAUGINIE (Francis), Conservation de la nature et aires protégées en Côte d'Ivoire, op. cit., p. 81.

${ }^{161}$ Voir Décret n'81-218 du 2 avril 1981 portant création du parc national d'Azagny avec une zone de protection périphérique, JORCI ${ }^{\circ} 21$ du Jeudi 14 mai 1981, p. 241.

${ }^{162}$ En 1926, un arrêté définit les limites Nord de l'actuel Parc National de la Comoé comme un Refuge qui devient par l'effet de l'arrêté n²2 du 3 novembre 1942 la Réserve de faune de Bouna dont la partie occidentale bénéficie d'un statut de Réserve partielle, puis de Réserve totale de faune en 1953, avant d'être classée Parc National de la Comoé en 1968 par le Décret 68-81 du 9 février 1968.Voir LAUGINIE (Francis), Conservation de la nature et aires protégées en Côte d'Ivoire, p.121.

${ }^{163}$ Voir GBADEGESIN (Adeniyi) et AYILEKA (Olatubosum), « Avoiding the mistakes of the past: Towards a community oriented management strategy for the proposed National Park in Abuja-Nigeria », art. cit., p. 90 ; NELSON (John) et GAMI (Norbert), Enhancing equity in the relationship between protected areas and indigenous and local communities in Central Africa, in the context of global change, CEESP-WCPA-IUCN Theme on Indigenous and Local Communities, Equity and Protected Areas, (Final Report), 2003, pp. 4 et 11. Selon John NELSON (John) et GAMI (Norbert), la gestion exclusive et autoritaire des aires protégées en Afrique est un héritage colonial. Voir NELSON (John) et GAMI (Norbert), Enhancing equity in the relationship between protected areas and indigenous and local communities in Central Africa, in the context of global change, op. cit. p. 4.

${ }^{164}$ Loi n ${ }^{\circ} 60-356$ du 3 novembre 1960 portant Constitution de la Côte d'Ivoire de la République de Côte d'Ivoire, JORCI n58 Numéro Spécial du 4 novembre 1960, p. 1271.

${ }^{165}$ La Côte d'Ivoire a été une colonie française de 1893 à 1960 (c'est-à-dire le 7 août 1960, date de son indépendance). En effet, l'historien ivoirien LOUCOU (Jean-Noël) affirme: «La colonie française de Côte d'Ivoire fut officiellement créée par le décret du 10 mars 1893 » (Voir LOUCOU (Jean-Noël), La Côte d'Ivoire coloniale. 1893-1960, Abidjan, Les Éditions FHB \& Les Éditions du CERAP, 2012, p. 56). À ce titre, elle fait partie des colonies françaises d'Afrique noire qui sont reparties en deux grands ensembles fédéraux. Il s'agit de l'Afrique occidentale française (AOF) et de l'Afrique orientale française (AEF). Selon LOUCOU (JeanNoël), l'Afrique occidentale française est créée en 1895 et réorganisée en 1904. Elle comprenait au départ cinq (5) colonies, à savoir les colonies de la Côte d’Ivoire, du Sénégal, du Dahomey, de la Guinée, du Haut-SénégalNiger, avant d'être étoffé de trois (3) nouvelles colonies : la Haute-Volta, le Niger détaché du Haut-Sénégal-
} 
qui du reste consacrait la centralisation administrative ${ }^{167}$. En effet, la gestion des affaires publiques de la colonie était rigoureusement hiérarchisée et fortement centralisée, à l'instar de la colonie elle-même qui était très stratifiée sur le plan territorial et administratif ${ }^{168}$. Elle se décomposait graduellement en cercles, subdivisions, cantons et villages ${ }^{169}$.

À la tête de la colonie, se trouve un gouverneur ${ }^{170}$ qui est assisté d'un Secrétaire Général et d'un Conseil d'administration. Chef unique de la colonie, les compétences du gouverneur s'étendaient sur tous les secteurs de la colonie, à savoir : politique, économique, financier, administratif, judiciaire et militaire ${ }^{171}$. Le gouverneur dirigeait une Administration fortement centralisée $^{172}$. En effet, seul responsable des finances dans la colonie et ordonnateur des dépenses, le gouverneur donnait des instructions aux commandants de cercles qui les répercutaient aux chefs de cantons et de villages. Ceux-ci rendent compte aux commandants de cercles qui à leur tour font de même pour le gouverneur ${ }^{173}$. C'est sans doute la savante organisation de cette Administration coloniale parfaitement centralisée que le Professeur Guéhi J. IBO désigne par le groupe de mots « machine administrative ${ }^{174}$.

Niger, en 1919 et en 1922, et la Mauritanie détaché du Sénégal en 1920 (Voir LOUCOU (Jean-Noël), La Côte d'Ivoire coloniale 1893-1960, op. cit., p.100). Sur l'appartenance de la Côte d'Ivoire aux groupes de colonies de l'Afrique occidentale française lire également KIPRE (Pierre), Côte d'Ivoire : La formation d'un peuple, Paris, SIDES-IMA, Collection L'AFRIQUE " dans tous ses états", 2005, p. 221.

${ }^{166} \mathrm{~L}$ 'article 76 de la Constitution précitée dispose : "La législation actuellement en vigueur en Côte d'Ivoire reste applicable, sauf l'intervention de textes nouveaux en ce qu'elle n'a rien de contraire à la présente Constitution. ».

${ }^{167}$ Sur la question de l'organisation administrative des pays africains anciennement sous domination coloniale, ANDRÉ (Pierre) et al. écrivent : «Les pays ont hérité de l'administration coloniale, un système de gestion centralisée qu'ils ont maintenu. ». Voir ANDRÉ (Pierre), BEAUDET (René), BERNIER (Michel), CÔTÉ (Louis) et LANMAFANKPOTIN (Georges), SAMOURA (Karim), La participation publique dans l'évaluation environnementale en Afrique francophone, Québec, IFDD, Points de repère 23, 2013, p. 14.

${ }^{168}$ À propos de l'organisation administrative et territoriale de la colonie de la Côte d'Ivoire, Pierre KIPRE écrit: «Au plan institutionnel...est mis en place un système pyramidal, sous l'autorité du Gouverneur de la colonie. Le territoire est divisé en cercles dirigés par les administrateurs de cercle; les cercles regroupent à leur tour un nombre variable de subdivisions que commandent des administrateurs de subdivision qui, comme les administrateurs de cercle, sont tous français. Chaque subdivision comprend un certain nombre de villages...» (voir KIPRE (Pierre), Côte d'Ivoire : La formation d'un peuple, op.cit., p. 122). Ainsi, sous le haut contrôle du Gouverneur général, le Gouverneur de la colonie qui détient l'essentiel des pouvoirs a une autorité qui s'étend sur les administrateurs français placés à la tête des circonscriptions territoriales et sur les chefs indigènes locaux [voir LOUCOU (Jean-Noël), La Côte d'Ivoire coloniale 1893-1960, op. cit., p. 102].

${ }^{169}$ Voir IBO (Guéhi Jonas), « La politique coloniale de protection de la nature en Côte d'Ivoire (1900-1958) », Revue Française d'Histoire d'Outre-mer, Vol. 80, n²98,1993, p. 85 ; LOUCOU (Jean-Noël), La Côte d'Ivoire coloniale 1893-1960, op. cit., pp. 102-103 ; KIPRE (Pierre), Côte d'Ivoire : La formation d'un peuple, op.cit., p.122.

${ }^{170}$ Il est appelé Lieutenant-gouverneur jusqu'en 1937 (voir LOUCOU (Jean-Noël), La Côte d'Ivoire coloniale 1893-1960, op. cit., p. 102.

${ }^{171}$ Idem, p. 103.

${ }^{172}$ À titre d'exemple, le commandant de cercle, qui est nommé par le gouverneur, «dirige tous les services publics, contrôle la perception de l'impôt, rend la justice, fait la police, gère les prisons...» [voir LOUCOU (Jean-Noë1), La Côte d'Ivoire coloniale 1893-1960, op. cit., p. 102.].

${ }^{173}$ IBO (Guéhi Jonas), « La politique coloniale de protection de la nature en Côte d'Ivoire (1900-1958)», art. cit., p. 85.

${ }^{174}$ Ibidem 
La reconduction de la législation coloniale en matière environnementale a été une pratique commune aux États colonisés tels que la Côte d'Ivoire. Selon le Professeur Michaël FAURE et al., la formation du droit et partant du droit de l'environnement dans les anciennes colonies tant anglophones que francophones procède d'un mimétisme juridique. En effet, ces auteurs font remarquer que la législation du pays colonisateur est reconduite et/ou le droit élaboré par les autorités des anciennes colonies s'inspire largement de celui en vigueur dans les anciennes puissances coloniales ${ }^{175}$. Cette opinion est partagée par plusieurs auteurs. Pour Moustapha NGAIDO cité par Pierre ANDRÉ et al., « En Afrique et dans l'océan Indien, la moitié des pays ont un système juridique basé entièrement ou en grande partie sur la tradition française. C'est ainsi que beaucoup de codes de l'environnement adoptés dans ces pays ont subi l'influence de la loi française $n^{\circ} 95-101$ du 2 février 1995 relative au renforcement de la protection de l'environnement communément appelée loi Barnier. ${ }^{176}$. Selon John NELSON et Norbert GAMI qui traitent particulièrement de la législation de la conservation de la nature en Afrique centrale, la plupart des pays de cette partie du continent (africain) ont reconduit la législation coloniale en matière de conservation de la nature, au lendemain de leur accession à l'indépendance. Cette législation avait une tendance à la centralisation de la gestion des ressources naturelles. En dépit des différentes réformes juridiques opérées dans les pays de l'Afrique centrale, l'approche centralisée de gestion des ressources naturelles persiste, dans la pratique $^{177}$.

En plus de la mise en place d'une Administration centralisée des aires protégées en vue de la protection de ces forêts, l'État ivoirien la dote de ressources budgétaires à cet effet.

\section{Paragraphe 2 : Les ressources budgétaires de l'Administration en charge de la conservation des aires protégées}

Les ressources budgétaires que la loi définit pour l'accomplissement des missions de l'Administration en charge des aires protégées et notamment la protection de ces milieux naturels de 1960 à 2002 sont de deux (2) ordres. Il s'agit des subventions de l'État (A) et du Fonds spécial des Parcs Nationaux (B).

\section{A - Les subventions de l'État}

Pour la protection des aires protégées de 1960 à 2002, l'État ivoirien a mis à la disposition du service public en charge de la conservation des aires protégées des ressources budgétaires (encore appelées subventions). Ces subventions émanent du Budget Général de

\footnotetext{
${ }^{175}$ Voir FAURE (Michaël), GOODWIN (Morag) et WEBER (Franziska), « Bucking the Kuznets Curve: Designing Effective Environmental Regulation in Developing Countries », art. cit., pp. 100-102.

${ }^{176}$ Voir ANDRÉ (Pierre), BEAUDET (René), BERNIER (Michel), CÔTÉ (Louis) et LANMAFANKPOTIN (Georges), SAMOURA (Karim), La participation publique dans l'évaluation environnementale en Afrique francophone, op. cit, p. 19.

${ }^{177}$ Voir NELSON (John), GAMI (Norbert), Enhancing equity in the relationship between protected areas and indigenous and local communities in Central Africa, in the context of global change, op. cit., p. 4.
} 
Fonctionnement (1) et du Budget Spécial d'Investissement et d'Équipement (2) de l'État de Côte d'Ivoire de 1960 à $1998^{178}$.

\section{Les fonds accordés par l'État aux aires protégées au titre du Budget Général de Fonctionnement de l'État de Côte d'Ivoire}

Le budget général de fonctionnement (BGF) de l'État prévoit les dépenses en matériel et en personnel pour les principales institutions ivoiriennes. Il s'agit des pouvoirs publics et les différents ministères ainsi que les recettes correspondant aux ressources fiscales ordinaires ${ }^{179}$.

Le budget général de fonctionnement sert à faire les dépenses de fonctionnement qui sont des dépenses courantes que commandent la bonne marche de l'État et des services publics. En guise d'exemples, il s'agit du traitement des fonctionnaires et agents de l'État tels que ceux en charge de la protection des aires protégées, des dépenses relatives au matériel ${ }^{180}$ et notamment des dépenses nécessaires à la conservation de ces milieux naturels.

Ainsi, le ministère en charge des aires protégées bénéficie de ressources budgétaires au titre du budget général de fonctionnement pour le fonctionnement de ses services centraux et d'exécution compétents pour la protection des aires. La part du budget général de fonctionnement de l'État alloué aux Parcs et Réserves était directement administrée par le Directeur de chaque cellule d'aménagement de Parc National ou de Réserve naturelle. Ce budget (BGF) était destiné à couvrir les frais de fonctionnement de l'Administration en charge de la conservation des aires protégées.

Outre le BGF, un autre budget était destiné à la conservation des aires protégées. Il s'agit du Budget Spécial d'Investissement et d'Équipement de l'État (BSIE).

\section{Les crédits budgétaires alloués aux aires protégées issus du Budget Spécial d'Investissement et d'Équipement de l'État de Côte d'Ivoire}

Le Budget Spécial d'Investissement et d'Équipement (BSIE) détermine les recettes et les dépenses liées à l'investissement ${ }^{181}$. Selon l'article 18 nouveau alinéa $1^{\text {er }}$ de la loi 59-249

\footnotetext{
${ }^{178}$ En effet, le Document-cadre du Programme cadre de gestion des Aires Protégées mentionne que jusqu'en 1998 chaque Parc National ou Réserve naturelle (PNR) disposait de deux (2) budgets, à savoir le budget général de fonctionnement (BGF) et le budget spécial d'investissement et d'équipement (BSIE). Il précise également que de 1999 à 2002, ces deux budgets (BGF et BSIE) sont regroupés en un budget unique géré par le Directeur de la protection de la nature. Voir République de Côte d'Ivoire, Ministère de l'Environnement et des Eaux et Forêts /Direction de la Protection de la Nature, Programme Cadre de Gestion des Aires Protégées. Document-cadre, Abidjan, Janvier 2001, p. 31.

${ }^{179}$ Voir MAHIEU (François Régis), Introduction aux finances publiques de la Côte d'Ivoire, Abidjan, Les Nouvelles Éditions Africaines, 1983, p. 23.

${ }^{180}$ Ibidem.

${ }^{181}$ Ibidem.
} 
du 31 décembre 1959 organisant les finances publiques en Côte d'Ivoire ${ }^{182}$, les dépenses d'investissement comprennent, entre autres, les dépenses destinées à la création et à la modernisation du patrimoine de l'État ainsi qu'à l'exécution des plans de développement économique et social. L'alinéa 2 suivant indique que le BSIE détermine chaque année les investissements qui doivent être réalisés dans le but d'atteindre les objectifs de développement économique et social fixés par le Gouvernement.

Il en résulte que ces investissements peuvent porter sur tous les domaines de l'État et notamment la protection durable des aires protégées. En effet, la conservation d'aires protégées, qui fait même l'objet de plusieurs textes de loi adoptés par l'État ivoirien ${ }^{183}$, poursuit un objectif de développement économique et social de la Côte d'Ivoire. C'est ainsi que pour les investissements à réaliser en matière de protection des aires protégées, il est alloué au chapitre du BSIE des ressources financières aux services en charge de la protection des aires protégées.

La part du BSIE réservée au secteur des Parcs Nationaux et Réserves naturelles était gérée par le Directeur de la Protection de la Nature. L'octroi desdits fonds a contribué à la réalisation d'actions de protection des aires protégées.

À titre d'exemples, citons les investissements réalisés dans le Parc National d'Azagny et la Réserve de Faune d'Abokouamékro. Dans le cadre de l'aménagement du Parc National d'Azagny à travers le Projet IVC 1998, un cofinancement de la Banque mondiale et de l'État de Côte d'Ivoire a permis, entre autres, la réalisation d'importantes infrastructures de surveillance et l'acquisition de plusieurs équipements ${ }^{184}$. En outre, pour la création et l'aménagement de la Réserve de faune d'Abokouamékro, l'État de Côte d'Ivoire a dépensé des sommes qui s'élèvent à plus de 3 milliards FCFA. Il en va de même pour les importants équipements acquis ${ }^{185}$. Ces financements débloqués dans ces deux (2) cas et qui sont relatifs aux investissements dans les PNR et à l'acquisition d'équipements nécessaires à leur gestion, en général, et à leur protection, en particulier, le sont au chapitre du BSIE.

Le financement exclusif des aires protégées sous forme de subvention étatique n'est pas l'apanage de la Côte d'Ivoire (de 1960 à 2002). Ce mode de financement de la conservation des Parcs Nationaux et Réserves a été également commun aux pays africains. En effet, si des auteurs affirment qu'en Afrique australe les financements des aires protégées ont

\footnotetext{
${ }^{182}$ Cette loi est modifiée par les loi ${ }^{\circ} 62-53$ du 12 janvier 1962 organisant la gestion des Finances publiques (JORCI n 9 Numéro Spécial du 23 février 1962, p. 213); 67-58 du 31 décembre 1967 ; abrogée et remplacée par la loi 70-214 du 24 mars 1970.

${ }^{183} \mathrm{Il}$ s'agit de lois (lato sensu) qui transposent dans l'ordonnancement juridique interne des traités internationaux relatifs à la protection de l'environnement et également de lois internes en la matière. Pour plus de détails sur la protection juridique des aires protégées en Côte d'Ivoire, voir "Aperçu de la législation consacrée à la protection des aires protégées en Côte d'Ivoire", dans la partie introductive de la présente thèse.

${ }^{84}$ Voir République de Côte d'Ivoire, Ministère de l'Environnement et des Eaux et Forêts, Programme Cadre de Gestion des Aires Protégées, op.cit., p. 22.

${ }^{185}$ Ibidem.
} 
d'abord été l'œuvre des États ${ }^{186}$, pour Anne Borge JOHANNESEN c'est le cas de tous les pays africains, en général ${ }^{187}$.

À la réflexion, le financement de la conservation des aires protégées en Afrique par les États sous la forme de subvention est une conséquence directe de la gestion exclusive de ces espaces naturels par ceux-ci. Ils devaient subséquemment fournir les moyens financiers pour les conserver.

En plus du BGF et du BSIE, l'État ivoirien met en place un fonds spécial dénommé le Fonds spécial des Parcs Nationaux pour la protection des aires protégées.

\section{B - Le Fonds spécial des Parcs Nationaux}

Crée par décret ${ }^{188}$, le Fonds spécial des Parcs Nationaux, même ainsi dénommé, est destiné à la fois au financement de la protection des Parcs Nationaux à proprement dit et des Réserves naturelles. En effet, l'article $1^{\mathrm{er}}$ du décret précité qui traite de l'objet dudit fonds dispose : «Le fonds spécial des Parcs Nationaux est destiné à assurer le financement :

- Des plans de développement des parcs nationaux et réserves ;

- Des actions nécessaires à la protection de la faune ;

- Des actions de promotion du tourisme cynégétique. » (souligné par nous).

À l'analyse de cette disposition, le développement des Parcs Nationaux et Réserves (dont le fonds est censé assurer le financement) sous-entend la protection de l'intégrité des aires protégées ou du moins celles déjà existantes au moment de la création du fonds. C'est à juste titre que le même article $1^{\text {er }}$ du décret ci-dessus visé libelle également que le Fonds spécial des Parcs Nationaux assure le financement des actions nécessaires à la protection de la faune c'est-à-dire celle des Parcs Nationaux et Réserves naturelles.

L'objectif de protection des Parcs Nationaux et Réserves que poursuit le Fonds spécial des Parcs Nationaux découle de ce qu'il est destiné, toujours selon l'article premier susmentionné, à financer des actions de promotion du tourisme cynégétique. Autrement dit, pour que cette activité puisse avoir lieu, il faudrait que la faune soit protégée à l'intérieur de son habitat originel, à savoir les Parcs Nationaux et les Réserves naturelles; lequel habitat doit aussi être préservé.

\footnotetext{
${ }^{186}$ Voir KRUG (Wolf), Private Supply of Protected Land in Southern Africa: A Review of Markets, Approaches, Barriers and Issues, Workshop Paper, World Bank / OECD International, Workshop on Market Creation for Biodiversity Products and Services, Paris, 25 and 26 January 2001, p. 9; POPE (Adam), A Preliminary Examination of Public Private Partnerships in National Park Management in Zambia, op. cit., p. 1.

${ }^{187}$ Voir JOHANNESEN (Anne Borge), Protected areas, wildlife conservation and local welfare, Working Paper Series, No. 13/2005 (Department of Economics, Norwegian University of Science and Technology), p. 3.

${ }^{188}$ Voir Décret $\mathrm{n}^{\circ} 73-27$ du 17 janvier 1973, fixant les conditions de fonctionnement et de gestion du Fonds spécial des Parcs Nationaux, JORCI n8 du 8 février 1973, p. 245.
} 
En plus du cadre institutionnel que l'État ivoirien met en place pour la protection des aires protégées de 1960 à 2002, il institue aussi un cadre normatif en vue de la réalisation de cet objectif.

\section{SECTION II : Le cadre normatif des aires protégées}

Au plan normatif, de 1960 à 2002, la protection des aires protégées en Côte d'Ivoire relève de plusieurs textes de loi (entendu au sens large : lois, décrets, arrêtés etc.). À titre principal, les aires protégées sont réglementées par le décret de 1966 portant statut et réglementation de la procédure de classement et de déclassement des Parcs et Réserves ${ }^{189}$. Il affranchit les aires protégées de tout droit d'usage ${ }^{190}$ et les définit comme étant des biens du domaine de l'État ${ }^{191}$.

Subsidiairement, les aires protégées de Côte d'Ivoire sont régies par le Code forestier $^{192}$ et la loi sur la protection de la faune et l'exercice de la chasse ${ }^{193}$ pour deux (2)

\footnotetext{
${ }^{189}$ Voir Décret $\mathrm{n}^{\circ}$ 66-433 du 15 septembre 1966 portant statut et réglementation de la procédure de classement des réserves naturelles intégrales ou partielles et des Parcs Nationaux, JORCI n50 du 27 octobre 1966, p. 1419.

${ }^{190}$ Aux termes de l'article $1^{\mathrm{er}}$ alinéa 3 du décret de 1966 portant statut et réglementation de la procédure de classement des Réserves naturelles intégrales ou partielles et des Parcs Nationaux: "Les Réserves naturelles intégrales sont affranchies de tout droit d'usage ». Également, l'article 3 suivant dispose en son alinéa 3 : «Les Parcs Nationaux sont affranchis de tout droit d'usage ». En ce qui concerne les Réserves naturelles partielles, bien que le décret ci-dessus ne mentionne pas expressément qu'elles sont affranchies de tout droit d'usage, les décrets qui les créent interdisent aux communautés locales d'y exercer des droits d'usage. C'est ainsi que par exemple, l'article 2 du décret portant création de la Réserve partielle de faune du N'Zo (Décret n $72-545$ du 28 août 1972 portant création de la Réserve partielle de faune du N’Zo, JORCI n43 du 14 septembre 1972, p. 1452) dispose : "Dans la Réserve de Faune ainsi définie y compris le lit des rivières et marigots, l'emprise des routes et pistes formant limites, tout acte de chasse, de poursuite et de capture et de provocation du gibier qu'elle qu'en soit la nature, est interdit ainsi que les défrichements et feux de brousse aux fins de cultures agricoles ». Il en est de même pour la Réserve de Faune et de Flore du Haut-Bandama créée par le Décret 73 133 portant création de la Réserve de Faune et de Flore du Haut-Bandama (JORCI n 18 du 10 avril 1973, p. 603). En effet, aux termes de l'article 2 de ce décret, "La Réserve de Faune et de Flore du Haut-Bandama est constituée en vue de la propagation, la protection et la conservation de la vie animale sauvage et de la végétation naturelle dans un intérêt scientifique et éducatif au profit, à l'avantage et pour la récréation du public ». C'est donc à juste titre que les articles 4,5 et 6 suivants mentionnent respectivement «Dans la Réserve de Faune et de Flore ainsi délimitée y compris le lit des rivières, l'emprise des routes ou pistes formant limites, tout acte de chasse, et toute provocation du gibier quelle qu'en soit la nature sont interdits de même que tout abattage ou manipulation d'arbres. » et que «Tout acte de pêche dans les rivières et mares situées à l'intérieur ou en limite de la réserve de Faune et de Flore est interdite. » et «La récolte de miel, cire, plantes médicinales, est interdite. ».

${ }^{191} \mathrm{Si}$ l'article $1^{\text {er }}$ alinéa $1^{\text {er }}$ du décret de 1966 portant statut et réglementation de la procédure de classement et de déclassement des Parcs et Réserves prescrit: "Les Réserves naturelles intégrales font partie du domaine forestier classé de la République de Côte d'Ivoire », les articles 2 et 3 disposent respectivement que les Réserves naturelles partielles et les Parcs Nationaux font partie du domaine forestier classé (allusion faite de la République de Côte d'Ivoire).

${ }^{192}$ Voir loi n 65-425 du 20 décembre 1965 portant Code forestier, JORCI n³ du 13 janvier 1966, p. 38. Il est à préciser que même si depuis 2014, la Côte d'Ivoire s'est dotée d'un nouveau Code forestier par l'effet de la loi $\mathrm{n}^{\circ} 2014-427$ du 14 juillet 2014 portant Code forestier (JORCI n² Numéro Spécial du vendredi 20 mars 2015 , p. 17), l'article 3 de ladite loi exclut les Parcs Nationaux et Réserves de son champ d'application. En effet, l'article 3 du nouveau Code forestier ivoirien de 2014 dispose : «La présente loi s'applique aux forêts et aux arbres hors forêts sur le territoire national. La présente loi ne s'applique pas à la faune; aux Parcs Nationaux et Réserves naturelles. (souligné par nous) ».
} 
raisons. D'une part, les aires protégées sont des forêts et partant concentrent une diversité d'espèces animales (faune) sauvages. D'autre part, ce sont ces deux (2) lois qui prévoient la répression des infractions à la législation des aires protégées.

Ces textes affranchissent les aires protégées de tout droit d'usage. Ils les définissent comme biens du domaine de l'État et répriment par conséquent leur violation (Paragraphe 2). Cependant, ils restent muets sur la catégorie de domaine (public ou privé) à laquelle appartiennent ces forêts alors que cette précision a une conséquence notable sur leur protection. Toutefois, il semble que les aires protégées ont appartenu au domaine privé de l’État de 1960 à 2002 (Paragraphe 1).

\section{Paragraphe 1 : Les aires protégées, un élément constitutif du domaine privé de l'État de 1960 à 2002}

En Côte d'Ivoire, les textes qui régissent les aires protégées sont lacunaires sur leur appartenance au domaine privé de l'État (A). Cependant, cette appartenance peut être déduite (B).

\section{A - Les lacunes des textes sur l'appartenance des aires protégées au domaine privé de l'État}

En droit, on appelle lacune un point sur lequel la loi est muette ou insuffisante de sorte qu'elle a besoin d'être complétée par celui qui l'applique ou l'interprète ${ }^{194}$. Dès lors, on entend par l'expression lacune de l'appartenance des aires protégées au domaine privé de l'État, le fait que les textes de lois (lato sensu) soient muets sur l'appartenance des aires protégées au domaine privé de l'État. Cependant, qu'entend-on par la notion de domaine privé de l'État ou plus globalement de celle de domaine de l'État?

À l'instar des particuliers qui sont propriétaires de biens, les personnes publiques telles que l'État sont également propriétaires de biens dont l'ensemble constitue leur domaine ${ }^{195}$. Le domaine de l'État est, par conséquent, l'ensemble des biens de l'État. Ces biens sont corporels ou incorporels, mobiliers ou immobiliers ${ }^{196}$ tels que les aires protégées.

\footnotetext{
${ }^{193}$ Voir loi n ${ }^{\circ}$ 65-255 du 4 août 1965 relative à la protection de la faune et à l'exercice de la chasse, JORCI $n^{\circ} 41$ du 26 août 1965, p. 898. Cette loi est modifiée par la loi nº94-442 du 16 août 1994 portant modification de la loi $n^{\circ}$ 65-255 du 4 août 1965 relative à la protection de la faune et à l'exercice de la chasse, JORCI n42 du 13 octobre 1994, p. 778.

${ }^{194}$ Voir CORNU (Gérard) (dir.),Vocabulaire juridique, $4{ }^{\mathrm{ème}}$ éd. (Association Henri Capitant), Paris, PUF, 2003, p. 513 .

${ }^{195}$ En la matière, on peut citer MORAND-DEVILLER (Jacqueline) pour qui : «le domaine est l'ensemble des biens et des droits mobiliers et immobiliers appartenant aux personnes publiques $»$. Voir Jacqueline MORANDDEVILLER, Droit administratif des biens, Paris, Montchrestien, 1999, p.11.

${ }^{196}$ Voir LAUBADÈRE (André de), VENEZIA (Jean-Claude), GAUDEMET (Yves), Traité de droit administratif, 10 éd., t. 2, Paris, LGDJ, 1995, pp. 219-220.
} 
Le domaine de l'État peut être subdivisé en deux (2) catégories, à savoir le domaine public et le domaine privé ${ }^{197}$. Le domaine privé de l'État est l'ensemble des biens appartenant à l'État mais qui ne sont ni affectés à l'usage du public ni au service public ${ }^{198}$. Il se constitue de diverses façons.

Pour qu'un bien entre dans le domaine privé de l'État, il peut être acquis à titre gratuit (dons, legs) ou onéreux (vente, échange) ${ }^{199}$. En plus de ces modes d'acquisition de droit commun, le domaine privé de l'État peut être acquis par des modes exorbitants du droit commun (expropriation, réquisition, nationalisation) ${ }^{200}$. Le domaine privé de l'État peut aussi se constituer par détermination de la $1 \mathrm{oi}^{201}$.

En ce qui concerne le domaine public de l'État, c'est l'ensemble des biens dont l'État est propriétaire et qui sont affectés soit à l'usage du public, soit au service public; et spécialement aménagés à cet effet ${ }^{202}$.

L'entrée d'un bien dans le domaine public de l'État peut se faire par détermination de la loi ${ }^{203}$. Hormis cette hypothèse, l'incorporation d'un bien dans le domaine public de l'État diffère, selon qu'il s'agisse du domaine public naturel ${ }^{204}$ ou artificiel $^{205}$. En effet, l'entrée d'un bien dans le domaine public naturel est automatique, dans la mesure où elle résulte de phénomènes naturels ${ }^{206}$. A contrario, la sortie d'un bien du domaine public naturel sera automatique lorsque les phénomènes naturels qui ont provoqué l'incorporation auront disparu. S'agissant de la sortie d'un bien du domaine public artificiel de l'État, elle nécessite une désaffectation (déclassement) juridique et de fait. Ainsi, le bien tombe dans le domaine privé de l'État; et bénéficie d'une protection moindre que celle du domaine public de l'État.

\footnotetext{
${ }^{197}$ Voir idem p. 187 ; CHAPUS (René), Droit administratif général, $11^{\text {ème }}$ éd., t. 3, Paris, Montchrestien, 1998 , p. 352 et s.; ROUGEVIN-BAVILLE (Michel), DENOIX de SAINTMARC (Renaud) et LABETOULLE (Daniel), Leçons de droit administratif, Paris, Éditions Hachette, 1989, p. 539 ; DUPUIS (Georges), GUEDON

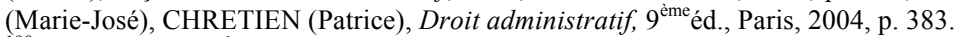

${ }^{198}$ Voir LAUBADÈRE (André de), VENEZIA (Jean-Claude) et GAUDEMET (Yves), Traité de droit administratif, 10 éd., t. 2, op.cit., p. 218 ; PEISER (Gustave), Droit administratif général, 21 édition, op.cit., p. 63 ; CHAPUS (René), Droit administratif général (t. 1), $11^{\text {ème }}$ édition, op. cit.,p. 503.

${ }^{199}$ Voir Voir PEISER (Gustave), Droit administratif général, 21 édition, op.cit. p. 64 ; LAUBADÈRE (André de), VENEZIA (Jean-Claude) et GAUDEMET (Yves), Traité de droit administratif, 10 éd., t. 2,op. cit., p. 341.

${ }^{200}$ Voir Gustave PEISER (Gustave), Droit administratif général, 21 édition, op.cit. p. 64.

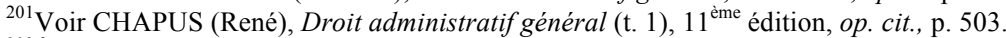

${ }^{202}$ À propos, en doctrine lire René CHAPUS, op. cit., p. 358 ; Gustave PEISER, op. cit., p. 9 ; LAUBADÈRE (André de), VENEZIA (Jean-Claude), et GAUDEMET (Yves), Traité de droit administratif, 10 éd., t. 2, op. cit., p. 192 ; Gustave PEISER, op. cit., p. 63.

${ }^{203}$ Voir PEISER (Gustave), Droit administratif général, 21 édition, op. cit., pp. 7-8.

${ }^{204} \mathrm{Il}$ s'agit de biens dont l'entrée dans le domaine public résulte d'un fait naturel; et que l'autorité administrative ne fait que délimiter en vue de déterminer la contenance. Voir LAUBADÈRE (André de), VENEZIA (JeanClaude) et GAUDEMET (Yves), Traité de droit administratif, 10 éd., t. 2,op. cit., p. 242.

${ }^{205}$ À l'opposé des biens du domaine public naturel, les biens du domaine public artificiel résultent d'un acte exprès d'affectation appelée classement (Idem, pp. 242-243).

${ }^{206} \grave{A}$ titre d'exemple, l'extension du rivage de la mer à une propriété privée voisine. En ce qui concerne l'incorporation d'un bien au domaine public artificiel, elle requiert l'affectation (classement) dudit bien à une destination par un acte juridique (acte administratif : une décision de classement), en principe; et une affectation de fait. Voir LAUBADÈRE (André de), VENEZIA (Jean-Claude) et GAUDEMET (Yves), Traité de droit administratif, 10 éd., t. 2, op. cit., p. 242.
} 
Ces précisions notionnelles faites, à quelle catégorie du domaine de l'État (domaine public/domaine privé) appartiennent les aires protégées de Côte d'Ivoire ? La réponse à cette question nécessite une analyse, une interprétation de textes de lois et de doctrine, dans la mesure où les articles $1^{\mathrm{er}}, 2,3$ du décret de 1966 portant statut et réglementation de la procédure de classement et de déclassement des Parcs et Réserves ne se résument qu'à indiquer que les aires protégées de Côte d'Ivoire font partie du domaine forestier classé de l'État. En effet, les articles $1^{\mathrm{er}}, 2,3$ du décret ci-dessus cité disposent respectivement : «les réserves naturelles intégrales font partie du domaine forestier classé de la République de Côte d'Ivoire » et aussi que "les réserves partielles... font partie du domaine forestier classé », et enfin « les parcs nationaux font partie du domaine forestier classé ».

En tant que biens du domaine forestier classé de l'État, les aires protégées sont, d'abord et avant tout, des biens du domaine de l'État. En conséquence, elles doivent appartenir soit à son domaine public soit à son domaine privé.

Cependant, le décret précité ne détermine pas expressément la nature du domaine de l’État (public ou privé) à laquelle appartiennent les aires protégées. C'est ainsi que la détermination de l'appartenance des aires protégées au domaine privé de l'État de 1960 à 2002 résulte d'un raisonnement déductif.

\section{B - Les aires protégées, un domaine privé de l'État par raisonnement déductif}

En Côte d'Ivoire, en dehors de la distinction entre domaine public et domaine privé de l'État par détermination de la loi, cette distinction pourrait se faire sur la base de la définition du domaine public énoncée par le décret du 29 septembre 1928 portant réglementation du domaine public et des servitudes d'utilité publique ${ }^{207}$. Ce décret procède de deux (2) manières pour classer un bien dans le domaine public.

D'une part, la définition du domaine public est faite par la technique de l'énumération. En effet, aux termes de l'article $1^{\text {er }}$ du décret du 29 septembre 1928 ci-dessus cité : «font partie du domaine public:

- les rivages de la mer jusqu'à la limite des plus hautes marées ainsi qu'une zone de $100 \mathrm{~m}$ à partir de cette limite;

- les cours d'eau navigables ou flottables ainsi qu'une zone de passage de 25 m de large sur chaque rive;

- les sources et cours d'eau non navigables ni flottables;

- les lacs, étangs et lagunes ainsi qu'une zone de passage de 25 m de large sur chaque rive;

- les nappes aquifères quelle que soit leur provenance, leur nature et leur profondeur;

\footnotetext{
${ }^{207}$ Voir Décret du 29 septembre 1928 modifiant le décret du 9 juillet 1900 portant réglementation du domaine public et des servitudes d'utilité publique en Afrique Occidentale Française, Bulletin Officiel des Colonies $n^{\circ}$ 10,1928. Ce décret est, jusqu'à ce jour, applicable en Côte d'Ivoire..
} 
- les canaux de navigation, les canaux d'irrigation, les canaux d'assèchement exécutés dans l'intérêt du public ainsi que les dépendances de ces ouvrages;

- les chemins de fer, les routes, les voies de communication de toute nature et les dispositifs de protection de ces voies ;

- les conduits d'eau, les conduites d'égouts, les ports, les digues maritimes et fluviales, les ouvrages d'éclairage et de balisage et leurs dépendances;

- les lignes télégraphiques, téléphoniques et leurs dépendances ainsi que les aériens des stations radioélectriques;

- les ouvrages déclarés d'utilité publique en vue de l'utilisation des forces hydrauliques et du transport de l'énergie électrique;

- les ouvrages de fortification des places de guerre ou les postes militaires ainsi qu'une zone large de 250 m autour de ces ouvrages. ».

D'autre part, selon ce même article $1^{\text {er }}$ in fine, font partie du domaine public : «...les biens de toute nature que le Code civil et les lois déclarent non susceptibles de propriété privée. ».

Les aires protégées de Côte d'Ivoire ne font pas partie de la liste ci-dessus qui énumère un ensemble de biens appartenant au domaine public, en droit ivoirien. Elles ne sont non plus désignées par le Code civil ${ }^{208}$ ou toute autre loi comme biens du domaine public.

Dans ces conditions et en l'absence de jurisprudence (ivoirienne), le recours à la doctrine peut permettre de déduire que ces aires protégées faisaient partie du domaine privé de l’État de 1960 à 2002. En la matière, Albert LEY qui a écrit sur le régime domanial et foncier de la Côte d'Ivoire affirmait que les biens de l'État (entendons de l'État de Côte d'Ivoire) qui ne relèvent pas de son domaine public font subséquemment partie de son domaine privé ${ }^{209}$.

\footnotetext{
${ }^{208}$ Il s'agit du Code civil français qui régissait la colonie de Côte d'Ivoire ; et rendu applicable en République de Côte d'Ivoire au moyen de l'article 76 de la loi $n^{\circ} 60-356$ du 3 novembre 1960 portant Constitution de la République de Côte d'Ivoire (JORCI n58 Numéro Spécial du 4 novembre 1960, p. 1271), au lendemain de l'indépendance (proclamée le 7 août 1960). Si la Côte d'Ivoire a pris des lois en matière civile et notamment en ce qui concerne le droit des personnes et la famille en 1964, les dispositions relatives au droit des biens et des obligations restent inchangées. Autrement dit, il s'agit de celles figurant dans le Code civil français (de 1804) rendues applicables en Côte d'Ivoire par l'effet de l'article 76 de la Constitution du 3 novembre 1960. Ainsi, le Code civil, en ses articles 538 à 541, donne une liste de biens appartenant au domaine public de l'État. Ce sont : les chemins, routes et rues à la charge de l'État, les fleuves et rivières navigables ou flottables, les rivages, lais et relais de la mer, les ports, les havres, les rades et généralement toutes les portions du territoire ivoirien qui ne sont pas susceptibles d'une propriété privée (art 538); tous les biens vacants et sans maître, et ceux des personnes qui décèdent sans héritiers, ou dont les successions sont abandonnées (art. 539); les portes, murs, fossés, remparts des places de guerre et de forteresses (art. 540); les terrains, les fortifications et remparts des places qui ne sont plus places de guerre et n'ont pas été aliénés ou dont l'État n'a pas perdu la propriété par prescription (art. 541).

${ }^{209}$ Selon LEY (Albert), il faut définir le domaine privé «...par élimination à partir de la définition du domaine public. Tout ce qui n'est pas domaine public est domaine privé » (Voir LEY (Albert), Le régime domanial et foncier et le développement économique de la Côte d'Ivoire, Tome XVIII, Paris, LGDJ, 1972, p. 87). À l'instar de LEY (Albert), plusieurs auteurs soutiennent que les biens des personnes publiques qui ne font pas partie de leur domaine public font partie de leur domaine privé. Ces auteurs sont, entres autres, CHAPUS (René), Droit
} 
De plus, pour la doctrine française et partant la France dont le droit ivoirien s'inspire largement ${ }^{210}$, les forêts domaniales ont, bien souvent, été classées comme des biens appartenant au domaine privé de l'État ${ }^{211}$. Ce recours à la doctrine (juridique) française pour établir que de 1960 à 2002 les aires protégées de Côte d'Ivoire ont appartenu au domaine privé de l'État relève de plusieurs constats.

En effet, en premier lieu, il ressort des travaux du Professeur René DÉGNI-SÉGUI relatifs au processus de formation du droit ivoirien que ce processus participe du phénomène de transfert de législation ${ }^{212}$. C'est ce qu'il traduit lorsqu'il écrit: «Le colonisateur a d'abord transplanté sa législation et maintenu les droits traditionnels comme loi d'exception $»^{213}$. Ce transfert de législation qui a été imposé (durant la colonisation française) a d'abord concerné le droit foncier et par la suite le droit du travail ${ }^{214}$ et le droit civil ${ }^{215}$.

Ensuite, le transfert de législation a été volontaire lorsque l'État de Côte d'Ivoire a succédé au droit colonial par l'effet de la reconduction du droit colonial français à travers l'article 76 de sa Constitution. En effet, cet article dispose : «La législation actuellement applicable en Côte d'Ivoire reste applicable, sauf intervention de textes nouveaux en ce qu'elle n'a rien de contraire à la présente Constitution ${ }^{216}$. En outre, la succession au droit colonial par la Côte d'Ivoire procède d'accords de coopération conclus entre la Côte d'Ivoire et la France. D'une part, il s'agit d'accords signés avant l'indépendance (en 1958) ; mais au moment où la Côte d'Ivoire jouissait d'une autonomie relative. D'autre part, ces accords sont ceux conclus après l'indépendance (7 août 1960), notamment les accords du 21 avril 1961. Ces accords reconduisent la législation coloniale en matière judiciaire, budgétaire, monétaire, économique ou financière ou introduisent la législation métropolitaine en droit ivoirien. Pour ce second cas, il est introduit dans l'ordonnancement juridique ivoirien les lois et règlements

administratif général (t. 1), $11^{\text {ème }}$ édition, op. cit., p. 503 ; PEISER (Gustave), Droit administratif général, 21 édition, op. cit., p. 9.

${ }^{210}$ Voir DÉGNI-SÉGUI (René), Introduction au Droit, Abidjan, EDUCI, Collections Sciences Juridiques, 2009, pp. 267-342.

${ }^{211}$ Sur la question, le Professeur PEISER (Gustave) écrit que les forêts domaniales « sont traditionnellement classées dans le domaine privé » (PEISER (Gustave), Droit administratif général, 21 édition, op. cit., p. 9.) ; également, les Professeurs LAUBADERE (André de), VENEZIA (Jean-Claude) et Yves GAUDEMET soutiennent que «Le régime très spécial» dont bénéficie les forêts domaniales en France «ne soustrait pas les forêts de l'État au domaine privé, ni leur contentieux à la juridiction judiciaire » [Voir LAUBADERE (André de), VENEZIA (Jean-Claude) et Yves GAUDEMET, Traité de droit administratif, 10 éd., t. 2, op.cit., p. 338). De même, LAUBADÈRE (André de) et GAUDEMET (Yves) affirment qu'il est de conception traditionnelle que «les forêts domaniales appartiennent au domaine privé » et que cette conception est toujours maintenue ( voir LAUBADÈRE (André de) et GAUDEMET (Yves), Traité de Droit Administratif, Droit Administratif des Biens. Les domaines administratifs. L'expropriation : la réquisition, les travaux publics (t. 2), $11^{\text {ème }}$ édition, Paris, LGDJ, 1998,p. 223).

${ }^{212}$ Voir DÉGNI-SÉGUI (René), Introduction au Droit, op. cit., pp. 267-342.

${ }^{213}$ Idem, p. 270.

${ }^{214}$ Idem, p. 275.

${ }^{215}$ À titre d'exemple, en droit de la famille, le mariage en colonie de Côte d'Ivoire était régi par le décret du 15 juin 1939 portant réglementation des mariages entre indigènes en Afrique Occidentale française ou décret Mandel (JOAOF, $35^{\text {ème }}$ année, $n^{\circ} 1837$ du 24 juin 1939, p. 842).

${ }^{216}$ Voir loi 60-356 du 3 novembre 1960 portant Constitution de la République de Côte d'Ivoire, JORCI n58 Numéro Spécial du 4 novembre 1960, p. 1271. 
français relatifs à l'Enseignement supérieur, l'organisation des services du Trésor et les règles relatives à la responsabilité des comptables du Trésor français. Au surplus, l'État ivoirien codifie le droit métropolitain (droit constitutionnel, droit administratif, droit social, droit

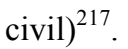

Enfin, la jurisprudence française est reconduite en Côte d'Ivoire, à l'instar de la législation. Toutefois, contrairement à la reconduction de la législation qui s'est opérée par la Constitution ivoirienne, la reconduction de la jurisprudence française en Côte d'Ivoire est l'œuvre du juge ivoirien ${ }^{218}$. En effet, le juge ivoirien soutien que la jurisprudence française étant indissociable de la loi française qui s'applique en Côte d'Ivoire par l'oeuvre de l'article 76 de la Constitution précité du 3 novembre 1960, la jurisprudence française s'applique également en Côte d'Ivoire ${ }^{219}$.

De même, pour ce qui est de la législation environnementale, en général, et spécifiquement celle relative aux aires protégées, l'élaboration de la réglementation n'échappe pas à ce contexte de reproduction du droit métropolitain. Autrement dit, les aires protégées dénommées Parc National et Réserve intégrale que la Côte d'Ivoire met aussi en place (à partir des années 1965) sont également inspirées par le droit français. En effet, l'article $1^{\mathrm{er}}$ alinéa $1^{\text {er }}$ de la loi française $n^{\circ} 60-708$ du 22 juillet 1960 relative à la création de Parcs Nationaux ${ }^{220}$ dispose : "Le territoire de tout ou partie d'une ou de plusieurs communes peut être classé par décret pris en conseil d'État en "parc national" lorsque la conservation de la faune, de la flore, du sol, du sous-sol, de l'atmosphère, des eaux et, en général, d'un milieu naturel présente un intérêt spécial et qu'il importe de préserver ce milieu contre tout effet de dégradation naturelle et de le soustraire à toute intervention artificielle susceptible d'en altérer l'aspect, la composition et l'évolution.». De plus, aux termes de l'article 2 alinéas $1^{\text {er }}$

\footnotetext{
${ }^{217}$ Voir DÉGNI-SÉGUI (René), Introduction au Droit, op. cit., pp. 306-307.

${ }^{218}$ Dans l'affaire François-Xavier SANTUCCI c/ l'Université d'Abidjan (Côte d'Ivoire) en date du 28 avril 1976 , la Cour Suprême Chambre Administrative (CSCA) a posé le principe que la jurisprudence française étant indissociable de la loi française, celle-là est applicable en Côte d'Ivoire dans la mesure où celle-ci à été reconduite en Côte d'Ivoire. En effet, dans cet arrêt le juge ivoirien affirme :

«Considérant ...que l'accord de coopération franco-ivoirien en matière d'enseignement supérieur du 24 avril 1961 portant transfert à la Côte d'Ivoire du Centre d'enseignement supérieur créé à ABIDJAN par décision du président de la communauté en date du 31 juillet 1959, a, en son article 12, confirmé l'arrêté ministériel du 11 septembre 1959 sur l'applicabilité à cet organisme de l'ensemble des dispositions françaises métropolitaines alors applicables à l'Enseignement supérieur ;

Considérant par conséquent que l'Université d'ABIDJAN (nom donné au Centre d'enseignement supérieur par décret $n^{\circ} 64-42$ du 9 janvier 1964) reste, sur le plan législatif, régie par les textes d'origine française ainsi incorporés au droit positif de la République dès lors que, de par leur objet, ils traitent des matières que l'article 41 de la Constitution réserve à la loi ;

Considérant qu'il n'est pas possible de dissocier des règles législatives de droit positif ainsi rendues applicables, la jurisprudence constante du Conseil d'État français affirmant le caractère de juridiction des organismes disciplinaires d'instance et d'appel dont les lois françaises ont doté l'Université (souligné par nous) ».Voir CSCA, 28 avril 1976 : François-Xavier SANTUCCI c/ l'Université d'Abidjan in BLÉOU (Djézou Martin), Les grands arrêts de la jurisprudence administrative ivoirienne, Abidjan, CNDJ/GIZ, 2012, pp. 90-93.

${ }^{219}$ Idem, pp. 339-340. C'est également la raison pour laquelle en l'absence de jurisprudence ivoirienne relativement à certaines matières, nous avons eu recours à la jurisprudence française, dans le cadre de cette étude.

${ }^{220}$ JORF du 23 juillet 1960, p. 6751.
} 
et 2 suivants de la loi française de 1960 précitée relative à la création de Parcs Nationaux : "Le décret créant un Parc National, qui est pris après une enquête publique...peut soumettre à un régime particulier et, le cas échéant, interdire à l'intérieur du parc la chasse et la pêche, les activités industrielles, publicitaires et commerciales, l'exécution des travaux publics et privés, l'extraction des matériaux concessibles ou non, l'utilisation des eaux, la circulation $d u$ public quel que soit le moyen emprunté, toute action susceptible de nuire au développement naturel de la faune et de la flore et, plus généralement d'altérer le caractère du parc national.

Ce décret réglementera, en outre, l'exercice des activités agricoles, pastorales et forestières. $»$.

Il découle de ces deux dispositions de la loi française de 1960 relative à la création de Parcs Nationaux qu'une portion de territoire de la France peut être classée en Parc National, à l'issue d'une enquête publique. Dans ce Parc National, il ne peut être mené des activités qui pourraient compromettre la conservation, en raison de l'intérêt à le conserver.

L'État de Côte d'Ivoire en consacrant également un type d'aire protégée dénommé Parc National, à l'instar de la France, reprend l'esprit des articles $1^{\text {er }}$ et 2 ci-dessus de la loi française précitée relative à la création de Parcs Nationaux. En effet, l'article 3 du Décret (ivoirien) précité de 1966 portant statut et réglementation de la procédure de classement et de déclassement des Réserves naturelles intégrales ou partielles et des Parcs Nationaux dispose : «Les Parcs nationaux...sont consacrés à la propagation, à la protection de la vie animale et de la végétation sauvage, à la conservation d'objets d'intérêt esthétique, géologique, historique ou scientifique au profit du public, pour son éducation et sa recréation.». Il indique aussi : "Les Parcs Nationaux sont affranchis de tout droit d'usage. Y sont strictement prohibés la chasse, la pêche ou la capture de tous animaux, le prélèvement d'espèces végétales ou d'objets quelconques, l'exploitation ou la prospection minière. »

L'alinéa 3 de l'article 2 de la loi française de 1960 relative à la création de Parcs Nationaux mentionne que «Des sujétions particulières à des zones dites "Réserves intégrales" peuvent être édictées par décret afin d'assurer, dans un but scientifique, sur une ou plusieurs parties d'un Parc National, une protection plus grande de certains éléments de la faune et de la flore... ». Autrement dit, la législation française consacre un type d'aire protégée dénommé "Réserves intégrales" (à l'intérieur du Parc National) dans lequel la protection de la nature est une priorité. De la même manière, il est à noter que, d'une part, la législation ivoirienne, à savoir l'article 4 de la loi de 1965 sur la protection de la faune et l'exercice de la chasse dispose : « La protection de la faune est assurée... » par la constitution et l'entretien de «Réserves naturelles intégrales ou partielles, et de Parcs Nationaux... ». D'autre part, l'article $1^{\text {er }}$ du Décret précité de 1966 portant statut et réglementation de la procédure de classement et de déclassement des Réserves naturelles intégrales ou partielles et des Parcs Nationaux prévoit que dans les Réserves naturelles intégrales :

«...Sont strictement interdits... :

- Tout acte de chasse ou de pêche ; 
-Toute exploitation forestière, agricole ou minière ;

-Toutes fouilles ou prospections, sondages, terrassement ou constructions et généralement tous travaux tendant à modifier l'aspect du terrain ou de la végétation;

Tout acte de nature à nuire ou à apporter des perturbations à la Faune ou à la Flore.

Les Réserves intégrales sont affranchies de tout droit d'usage. ».

Il en résulte que la réglementation des aires protégées élaborée aux premières heures après l'indépendance de l'État de Côte d'Ivoire (1965) s'inspire fortement de celle qui était en vigueur en France quelques années auparavant, c'est-à-dire en 1960. Subséquemment, les forêts domaniales qui sont considérées en France comme des biens appartenant au domaine privé (de l'État français) le sont également (par raisonnement déductif) en Côte d'Ivoire ; d'autant plus que ce pays s'est posée comme héritier du droit français. Par conséquent, on peut conclure que de 1960 à 2002, les aires protégées de l'État de Côte d'Ivoire ont appartenu à son domaine privé, par raisonnement déductif.

En deuxième et dernier lieu, la création des aires protégées en Côte d'Ivoire (ancienne colonie française ${ }^{221}$ ) remonte à la période coloniale. Au total, sur les 14 aires protégées, huit $(8)^{222}$ ont été créées à partir de massifs forestiers qui étaient sous statut de protection durant la colonisation.

Ces aires protégées dont l'origine de la création remonte à la période coloniale peuvent être réparties en trois (3) catégories. Une première catégorie se compose de celles qui ont été créées, sous leur forme actuelle, pendant la colonisation. Il s'agit du Parc National du Banco $^{223}$ et de la Réserve intégrale du Mont Nimba ${ }^{224}$. Les deux (2) autres catégories renferment des aires protégées qui avaient à cette époque soit le statut de Réserve ou de forêt classée avant d'être créées et désignées sous leur appellation actuelle, après l'indépendance de la Côte d'Ivoire le 7 août 1960.

\footnotetext{
${ }^{221}$ La Côte d'Ivoire a été une colonie française, par l'effet du décret du 10 mars 1893. Voir LOUCOU (JeanNoël), La Côte d'Ivoire coloniale 1893-1960, Abidjan, les Éditions FHB \& les Éditions du CERAP, 2012, p. 56. La colonie de Côte d'Ivoire acquiert son indépendance et devient République de Côte d'Ivoire le 7 août 1960. Voir KIPRE (Pierre), Côte d'Ivoire : La formation d'un peuple, Paris, SIDES-IMA, Collection L'AFRIQUE " dans tous ses états", 2005, pp. 203-204.

${ }^{222}$ Il s'agit des Parcs Nationaux d'Azagny, du Banco, de la Comoé, de la Marahoué du Mont Sangbé, deTaï, de la Réserve de flore et de faune du Haut-Bandama et de la Réserve intégrale du Mont Nimba.

${ }^{223}$ Le Parc National du Banco à l'origine était une réserve forestière (1.600 ha) en 1926 qui s'est étendue en 1929 à 3.700 ha avec le statut de forêt classée. Réduite à 2.800 ha en 1935, elle est érigée en Parc National (du Banco) par décret du 31 mars 1953. Voir LAUGINIE (Francis), Conservation de la nature et des aires protégées en Côte d'Ivoire, op. cit., p.99.

${ }^{224}$ Cette aire protégée qui a été une forêt de 19.500 ha repartie entre le territoire de la Guinée-Conakry (14.500 ha) et le territoire de la République de Côte d'Ivoire (5.000 ha) est devenue une forêt classée en 1943. Elle est érigée comme Réserve intégrale du Mont Nimba par le décret du 5 juillet 1944 (Idem, p. 319).
} 
Parmi les aires protégées actuelles, celles qui ont été des Réserves avant l'indépendance de la Côte d'Ivoire sont les Parcs Nationaux de Taî ${ }^{225}$, de la Marahoué ${ }^{226}$, de la Comoé $^{227}$, d'Azagny ${ }^{228}$. En ce qui concerne celles qui étaient anciennement des forêts classées, l'on a le Parc National du Mont Sangbé ${ }^{229}$ et la Réserve de flore et de faune du HautBandama $^{230}$.

Il suit de là que durant la période coloniale, ces aires protégées (forêts) étaient régies par la législation coloniale française alors en vigueur, à l'instar des autres domaines de la vie coloniale dont nous avons traité ${ }^{231}$. En la matière, les forêts et partant les aires protégées de la colonie de Côte d'Ivoire ont été régies successivement par le Décret du 20 juillet 1900 organisant le régime forestier de la Côte d'Ivoire ${ }^{232}$; le Décret du 18 juin 1912 réglementant le régime forestier de la Côte d'Ivoire ${ }^{233}$ et le Décret du 4 juillet 1935 sur le régime forestier de l'Afrique occidentale française ${ }^{234}$.

C'est ainsi qu'à l'indépendance de la Côte d'Ivoire en 1960 et jusqu'à l'adoption du Code forestier de $1965^{235}$ et de la loi de 1965 sur la chasse et la protection de la faune et l'exercice de la chasse ${ }^{236}$ desquels émane le décret de 1966 relatif au statut et à la procédure

\footnotetext{
${ }^{225}$ Le Parc National de Taï était en 1926 un Parc-Refuge (560.300 ha) ; en 1955, la forêt classée Taï-Hana, puis la Réserve intégrale de faune et de flore de Taï, avant d'être crée par le décret 72-544 du 28 août 1972 portant création du Parc National de Taï, JORCI n 43 du 14 septembre 1972, p. 1451 (Idem, p. 239).

${ }^{226}$ Le Parc National de la Marahoué crée par le décret 68-80 du 9 février 1968 (JORCI n 11 du 22 février 1968 , p. 309) a d'abord été une Réserve de faune en 1956 (Idem, p. 177).

${ }^{227}$ Crée par le décret 68-81 du 9 février 1968 (JORCI n¹1 du 22 février 1968, p. 310), le Parc National de la Comoé a été en 1942 la Réserve de faune de Bouna par l'effet de l'Arrêté n²2 du 3 novembre 1942. En 1953, elle acquiert le statut de Réserve totale de faune (Idem, p. 121).

${ }^{228}$ En 1954, le Parc National d'Azagny (crée par Décret n ${ }^{\circ}$ 81-218 du 2 avril 1981 portant création du Parc National d'Azagny avec une zone de protection périphérique, JORCI n²1 du Jeudi 14 mai 1981, p. 241) a été une réserve de chasse interdite à toute activité cynégétique en 1954. En 1960, il est classé Réserve partielle de faune par l'Arrêté n`536 du 25 mai 1960 (Idem, p. 81).

${ }^{229}$ Le Parc National du Mont Sangbé est créé par le Décret n76-215 du 19 février 1976 portant création du Parc National du Mont Sangbé (JORCI n 13 du jeudi 18 mars 1976, p. 515), à partir de deux massifs forestiers qui ont chacun eu le statut de forêt classée dont le $1^{\text {er }}$ de 105.000 ha le fut par l'Arrêté n ${ }^{\circ} 82 / S E . F$ du 10 janvier 1945 ; et le $2^{\text {nd }}$ de 40.000 ha par l'Arrêté n8889/SE.F du 27 décembre 1954 (Idem, p. 211).

${ }^{230}$ Pour ce qui est de la Réserve de flore et de faune du Haut-Bandama (123.000 ha), elle est créée par le Décret 73-133 du 21 mars 1973 (JORCI n 18 du jeudi 19 avril 1973, p. 603), à partir de la Forêt classée du Haut Bandama (56.000 ha) créée par l'Arrêté n806/SE/F du 14 mars 1942 ; et de la Forêt classée du Bandama blanc (24.000 ha) par l'Arrêté n5895/SE/F du 13/08/1954. Situées respectivement sur les rive gauche et droite du fleuve Bandama, ces deux Forêts classées sont complétées par 42.000 ha de savanes et forêts claires du domaine rural lors de la création de la Réserve de flore et de faune du Haut-Bandama (Idem, p. 285).

${ }^{231}$ Il s'agit, par exemple, du mariage avec le décret Mandel précédemment cité; des affaires pénales, financières et administratives dont nous avons fait mention dans les analyses ci-dessus.

${ }^{232}$ JOCI du 15 octobre 1900 , p. 1.

${ }^{233}$ JOCI du 31 août 1912, p. 457.

${ }^{234}$ JOAOF du 3 août 1935 , p. 611.

${ }^{235}$ Voir loi n ${ }^{\circ} 65-425$ du 20 décembre 1965 portant Code forestier, JORCI n³ du 13 janvier 1966, p.38.

${ }^{236}$ Voir loi $\mathrm{n}^{\circ} 65-255 \mathrm{du} 4$ août 1965 relative à la protection de la faune et à l'exercice de la chasse, JORCI ${ }^{\circ} 41$ du 26 août 1965, p. 898 modifiée par la loi n $94-442$ du 16 août 1994 portant modification de la loi $n^{\circ} 65-255$ du 4 août 1965 relative à la protection de la faune et à l'exercice de la chasse, JORCI n42 du 13 octobre 1994, p. 778 .
} 
de classement des Parcs Nationaux et Réserves Naturelles ${ }^{237}$, les aires protégées de Côte d'Ivoire ont été régies par les dispositions du Décret du 4 juillet 1935 sur le régime forestier de l'Afrique occidentale française précité. Cette succession au droit colonial par l'État de Côte d'Ivoire a été rendue possible par le mécanisme du transfert de législation consacré par l'article 76 de la Constitution ivoirienne du 3 novembre $1960^{238}$.

À l'article $1^{\text {er }}$ du décret du 4 juillet 1935 précité, il est écrit : «Les forêts vacantes et sans maître en Afrique occidentale française, ainsi que les périmètres de reboisement définis à l'article 6 appartiennent à l'État.». Il s'agit de l'État français dont la Côte d'Ivoire était l'une des colonies à cette époque ${ }^{239}$. En plus, les articles 3 et 4 disposent respectivement que : «Les forêts domaniales sont réparties en deux catégories:

$1^{\circ}$ Forêts classées constituant le Domaine forestier protégé ;

$2^{\circ}$ Forêts protégées constituant le Domaine forestier protégé 》 et que "Sont considérées comme forêts classées :

- Les forêts réservées, avant la date de promulgation du présent décret par arrêté du Gouverneur et des Lieutenant-gouverneurs, c'est-à-dire qui ont déjà été soumises à un régime spécial restrictif concernant l'exercice des droits d'usage des indigènes et les exploitations. Des arrêtés du Gouverneur général placeront définitivement ces forêts dans le domaine sous les conditions que les actes constitutifs de mise en réserve déterminent leurs limites d'une façon précise, qu'elle soit libres de tout droit d'usage, ou que ces droits d'usage aient fait l'objet d'un règlement d'aménagement;

- Les forêts classées par voie d'arrêté du Gouverneur général conformément aux dispositions $d u$ titre présent. ». Quant à l'article 5, il précise que "Sont considérées comme forêts protégées toutes les autres forêts du Domaine n'ayant pas encore fait l'objet d'un arrêté de classement. ».

Il ressort de l'analyse conjointe des articles 3, 4 et 5 précités que les forêts classées par voie d'arrêté ou les forêts réservées avant l'entrée en vigueur du Décret du 4 juillet 1935 précité sont des forêts mises en réserve ou qui font l'objet de restrictions telles que les aires protégées, créées durant la période coloniale soit sous la forme de Parc National soit comme Réserve naturelle ou forêt classée. Autrement dit, si ces forêts sont dites domaniales, c'est dire qu'il s'agit du domaine de l'État français. Par conséquent, ces aires protégées qui sont des forêts classées (mises en défens) et partant des forêts domaniales au sens de l'article 3 précité du Décret du 4 juillet 1935 sur le régime forestier de l'Afrique occidentale

\footnotetext{
${ }^{237}$ Voir Décret 66-433 du 15 septembre 1966 portant statut et réglementation de la procédure de classement des Réserves naturelles intégrales ou partielles et des Parcs Nationaux, JORCI n50 du 27 octobre 1966, p. 1419.

${ }^{238}$ En la matière, il peut être lu à l'article 76 de la Constitution ivoirienne du 3 novembre 1960 « La législation actuellement applicable en Côte d'Ivoire reste applicable, sauf intervention de textes nouveaux en ce qu'elle n'a rien de contraire à la présente Constitution ». Voir loi 60-356 du 3 novembre 1960 portant Constitution de la République de Côte d'Ivoire, JORCI n58 Numéro Spécial du 4 novembre 1960, p. 1271.

${ }^{239} \mathrm{La}$ Côte d'Ivoire a été une colonie française, par l'effet du décret du 10 mars 1893. Voir LOUCOU (JeanNoël), La Côte d'Ivoire coloniale 1893-1960, Abidjan, les Éditions FHB \& les Éditions du CERAP, 2012, p. 56.
} 
française ${ }^{240}$, appartiennent à l'État français autant que les forêts vacantes et sans maître dont traite ce même décret, en son article $1^{\mathrm{er}}$ précité.

Il en résulte que les aires protégées de Côte d'Ivoire sont régies par une partie de ce qui constitue le bloc légal de la métropole de France ou droit métropolitain (français). Il s'agit du droit qui est édicté par les autorités publiques françaises mais applicable aux colonies de la France dont la colonie de Côte d'Ivoire. En d'autres termes, les aires protégées ivoiriennes sont régies par la partie du droit français qui est seulement applicable aux colonies de l'Afrique occidentale française.

En conséquence, étant donné que le droit (français) applicable aux colonies régissait les aires protégées de la colonie de Cote d'Ivoire (forêts du domaine de la France) et que les forêts situées sur le territoire français appartiennent au domaine privé de l'État (français), les aires protégées établies sur le territoire de la colonie de Côte d'Ivoire appartiennent également au domaine privé de l'État (français). On peut donc conclure que lorsque la Côte d'Ivoire souveraine reconduit le droit colonial français par l'effet de l'article 76 de la Constitution ivoirienne du 3 novembre 1960, les aires protégées de cette époque appartiennent toujours au domaine privé de l'État. Cependant, cette fois-ci, il s'agit de l'État de Côte d'Ivoire.

Par ailleurs, dans l'optique d'assurer la protection des aires protégées, le législateur ivoirien réprime les atteintes contre les aires protégées.

\section{Paragraphe 2 : La répression des atteintes contre les aires protégées}

La répression des atteintes contre les aires protégées se fait en droit ivoirien par la définition d'infractions contre ces milieux naturels (A) ainsi que l'attribution de pouvoirs de répression au personnel en charge des aires protégées $(\mathrm{B})$.

\section{A - La définition des infractions contre les aires protégées}

De 1960 à 2002, les infractions contre les aires protégées sont le délit de chasse dans les Parcs Nationaux et Réserves naturelles (1), les délits d'exploitation forestière et agricole dans les Parcs Nationaux et Réserves naturelles (3).

\section{Le délit de chasse dans les Parcs Nationaux et Réserves naturelles}

Selon le dictionnaire Larousse français "chasser" signifie poursuivre un animal en vue de le tuer ou de le capturer vivant ${ }^{241}$. Quant à l'article 7 alinéa $1^{\mathrm{er}}$

\footnotetext{
${ }^{240} \mathrm{Ce}$ décret est signé à Paris, le 4 juillet successivement par le Président de la République (française) en la personne d'Albert LEBRUN, le Ministre des colonies, Louis ROLLIN et le Garde des Sceaux, Ministre de la Justice, Léon BERARD. Voir IBO (Guéhi Jonas), Histoire de la protection de la nature en Côte d'Ivoire par les textes, IRD/UAA, 2004, pp. 29-34.
} 
de la loi sur la protection de la faune et l'exercice de la chasse, il définit la "chasse" comme tout acte tendant soit à blesser ou à tuer un animal sauvage, pour s'approprier ou non tout ou partie de sa dépouille ${ }^{242}$... soit à détruire les œufs des oiseaux ou des reptiles. L'alinéa 2 suivant définit l'"acte de capture" comme tout acte tendant à priver un animal sauvage de sa liberté ou à récolter et retirer hors de leur lieu naturel d'éclosion, les œufs des oiseaux ou des reptiles $^{243}$.

Il découle de ce qui précède que la loi de 1965 sur la protection de la faune et l'exercice de la chasse fait une distinction entre la chasse et la capture des animaux sauvages. En effet, elle définit la chasse comme une activité qui consiste à une atteinte à l'intégrité physique (blesser, tuer,) d'espèces animales sauvages ou la destruction de leurs œufs. En ce qui concerne la capture, elle renvoie à la privation de liberté de ces animaux ou à la soustraction de leurs œufs de leur biotope.

La loi de 1965 sur la protection de la faune et l'exercice de la chasse incrimine la chasse dans les aires protégées sous la forme de délit et partant punit les auteurs. En effet, il ressort de la lecture combinée des articles 33 et 34 de la loi sur la chasse ${ }^{244}$ que les auteurs de délit de chasse dans les Parcs Nationaux et Réserves s'exposent à un emprisonnement de quatre mois à deux ans et d'une amende de 6.000 à 600.000 FCFA.

Ce délit expose également les auteurs à la confiscation des animaux blessés ou capturés ou de la dépouille des animaux tués ou encore une condamnation au paiement d'une somme égale à leur valeur s'ils ne peuvent être commodément saisis. En outre, ces peines peuvent

${ }^{241}$ Voir JEUGE-MAYNART (Isabelle) (dir.), Le Petit Larousse illustré, édition 2013, Paris, Larousse, 2013, p. 186.

${ }^{242} \mathrm{Au}$ sens de l'article $1^{\mathrm{er}}$ de la loi de 1965 sur la protection de la faune et l'exercice de la chasse, la faune est constituée de tous les animaux sauvages vivant en liberté dans leur milieu naturel, classés parmi les mammifères (exception faite des rats, souris, et des chauves-souris), et parmi les oiseaux, les crocodiles, les varans et les pythons.

${ }^{243}$ Pour plus de précisions sur ces espèces animales, se référer à l'article $1^{\text {er }}$ de la loi précitée de 1965 sur la protection de la faune et l'exercice de la chasse.

${ }^{244}$ Ces deux articles, à savoir les articles 33 et 34 disposent respectivement: « Les infractions à la présente loi et à ses décrets d'application sont punies :

1. D'une amende de 3.000 à 300.000 FCFA et d'un emprisonnement de deux mois à un an ou de l'une de ces deux peines seulement ;

2. De la confiscation des animaux blessés ou capturés ou de la dépouille des animaux tués, ou d'une condamnation au paiement d'une somme égale à leur valeur, s'ils ne peuvent être commodément saisis.

Ces peines peuvent être assorties en outre:

1. De la confiscation des armes, minutions, engins, matériaux ayant servi à commettre le délit. Le véhicule automobile ou tout autre, ayant été utilisé délibérément à des fins cynégétiques est considéré comme matériel susceptible de confiscation notamment lorsqu'il a été utilisé comme moyen de poursuite du gibier, comme engin éblouissant par ses phares ou pour transporter des chasseurs délinquants à l'intérieur d'une réserve ou d'un parc national.

2. De la déchéance du permis et éventuellement, de la privation temporaire ou définitive d'octroi de tout autre permis ou licence de chasse ou de capture. » et que "Les peines d'amende ou d'emprisonnement prévues à l'article précédent sont portées au double lorsque l'une des trois conditions est réalisée:

1. Délit commis dans une réserve ou un parc national ;

2. Délit commis de nuit avec un engin éclairant ;

Récidive. ». 
être assorties de la confiscation des armes, munitions, engins, matériaux qui ont servi à commettre le délit. Outre la chasse, l'exploitation forestière et agricole dans les Parcs Nationaux et Réserves constituent un délit, au regard des dispositions du Code forestier ivoirien.

\section{Les délits d'exploitation forestière et d'exploitation agricole dans les} Parcs Nationaux et Réserves naturelles

Ces délits sont prévus par les l'article 50 du Code forestier de 1965 en ses alinéa $1^{\text {er }}$ et $2^{245}$. Ainsi, sous l'empire de l'article 50 alinéa $1^{\text {er }}$ du Code forestier de 1965, l'élément matériel du délit d'exploitation forestière dans les aires protégées consistait à couper, enlever, mutiler, écorcher, arracher des arbres ou d'exploiter des produits forestiers accessoires. En ce qui concerne l'alinéa 2 dudit article, l'exploitation agricole consiste au défrichement portant sur une partie des Parcs Nationaux et Réserves en vue d'une mise en culture ${ }^{246}$.

Avant 2002, ces deux (2) délits étaient passibles des mêmes peines. Il s'agit d'un emprisonnement de deux mois à deux ans et/ou une amende de 10.000 à 1.000.000 de FCFA. En plus, les auteurs de ces délits s'exposent à la confiscation du matériel ayant servi à la commission de l'infraction; à la restitution des ressources forestières prélevées; à la remise en état des lieux ainsi qu'à des dommages et intérêts ${ }^{247}$.

De plus, pour assurer la protection des aires protégées, il était reconnu (jusqu'en 2002) des compétences pénales à certains fonctionnaires des Eaux et Forêts en charge des aires protégées.

\section{B - L'attribution de pouvoirs de répression au personnel en charge des aires protégées}

De 1965 à 2002, la loi attribue des compétences pénales à certains fonctionnaires des Eaux et Forêts en charge des aires protégées. Il s'agit exclusivement des inspecteurs et agents assermentés des Eaux et forêts. En effet, l'article 22 du Code de procédure pénale (CPP) ivoirien ${ }^{248}$ dispose : «Les inspecteurs et agents assermentés des Eaux et Forêts recherchent et

\footnotetext{
${ }^{245}$ En effet, cette article dispose: «Sans préjudice des confiscations, restitutions, remise en état des lieux et dommages et intérêts, sont passibles d'une peine deux mois à un an d'emprisonnement et d'une amende de 10.000 à 1.000 .000 de francs ou de l'une de ces deux peines seulement, sauf les cas prévus aux articles 14, 15, et 18 :

1. Quiconque coupe, enlève, mutile, écorche ou arrache des arbres ou exploite des produits forestiers accessoires, dans un but commercial ou non, sans y être autorisé ;

2. Quiconque, en vue d'une mise en culture ou non, effectue, sans autorisation un défrichement portant sur une partie du domaine forestier classé, des périmètres de protection et des reboisements...».

${ }^{246}$ Article 50 alinéa 2, Code forestier de 1965.

${ }^{247}$ Article 50, alinéa $1^{\text {er }}$, Code forestier de 1965.

${ }^{248}$ Il s'agit de la loi $n^{\circ} 60-366$ du 14 novembre 1960 portant code de procédure pénale, JORCI n 12 Numéro Spécial du 24 février 1961, p. 207.
} 
constatent par procès-verbaux les infractions à la réglementation des Eaux et Forêts et de la Chasse ».

C'est ainsi que se juchant sur l'article 22 du Code de procédure pénale précité, les lois forestières de 1965, à savoir le Code forestier et la loi sur la protection de la faune et l'exercice de la chasse, prévoient respectivement en leur article 28 et 37 que les infractions contre ces milieux naturels sont recherchées et constatées (par procès-verbal) par des agents habilités ${ }^{249}$.

Il en résulte qu'en cas d'infraction à la législation des aires protégées (délit de chasse, délits d'exploitation agricole ou d'exploitation forestière), les fonctions de police judiciaire des agents sus-nommés se limitent à la recherche et à la constatation de l'infraction par procès-verbal. Autrement dit, pour toutes les autres fonctions de police judiciaire (enquêtes, auditions, arrestations), le personnel des Eaux et Forêts en charge de la répression des infractions contre les aires protégées doit nécessairement recourir à un officier de Police judiciaire.

C'est à juste titre que si l'article 23 alinéa $1^{\text {er }}$ du CCP donne compétence aux inspecteurs et agents assermentés des Eaux et Forêts de suivre les choses enlevés (en l'espèce, des ressources naturelles des aires protégées) où elles sont transportées et de les mettre sous séquestre, l'alinéa 2 suivant dispose qu'ils : " ne peuvent cependant pénétrer dans les maisons, ateliers, bâtiments, cours adjacentes et enclos qu'en présence d'un officier de police judiciaire... ». Aussi l'article 24 du CPP dispose-t-il qu'en cas de flagrant délit, les agents assermentés et les inspecteurs des Eaux et Forêts ont recours à un Officier de police judiciaire (OPJ).

En somme, de 1960 à 2002, le législateur ivoirien a procédé à la protection pénale des aires protégées de deux façons. D'une part, il a incriminé les atteintes contre les aires protégées en définissant des infractions que sont les délits de chasse, d'exploitation agricole et forestière. D'autre part, il reconnaît aux inspecteurs et agents assermentés des services des Eaux et Forêts des compétences pénales en vue de la répression des infractions contre les aires protégées.

Toutefois, cette armature juridique destinée à la protection des aires protégées de 1960 à 2002 comportait des insuffisances.

\footnotetext{
${ }^{249}$ Aux termes de l'article 28 de la loi de 1965 relative à la protection de la faune et à l'exercice de la chasse précédemment citée, «Tout individu trouvé en infraction à la présente loi par un agent habilité mais n'ayant pas qualité d'officier de police judiciaire, sera, s'il ne peut justifier valablement de son identité et de sa résidence, conduit immédiatement devant l'officier de Police judiciaire le plus proche ou le cas échéant devant le procureur de la République ou le juge de la section de tribunal». Quant à l'article 37 de la loi portant Code forestier, il prescrit : "les agents habilités par l'Administration doivent être assermentés en vue de la recherche et de la constatation des infractions en matières de forêts ».
} 


\section{CHAPITRE II : LES INSUFFISANCES DU CADRE JURIDIQUE DES AIRES PROTÉGÉES DE 1960 À 2002}

Les insuffisances du cadre juridique des aires protégées seront étudiées, selon qu'elles ont trait aux règles domaniales qui régissent ces forêts et aux pouvoirs du personnel en charge de leur conservation (Section I) ainsi qu'à celles relatives à leur financement (Section II).

\section{SECTION I : LES INSUFFISANCES DES RÈGLES DOMANIALES ET DES POUVOIRS DU PERSONNEL EN CHARGE DES AIRES PROTÉGÉES}

Les insuffisances des règles domaniales qui régissent les aires protégées seront abordées (Paragraphe 1) avant celles relatives aux pouvoirs du personnel en charge de ces milieux naturels (Paragraphe 2).

\section{Paragraphe 1 : Les limites des règles domaniales des aires protégées}

L'appartenance des aires protégées au domaine privé de l'État les expose à deux (2) conséquences juridiques. Elles sont aliénables (A) et prescriptibles (B).

\section{A - L'aliénabilité des aires protégées}

L'aliénabilité des aires protégées, de 1960 à 2002, sera démontrée par une analyse sur le plan juridique (1); laquelle sera soutenue par des exemples pratiques d'aliénation de portions du domaine forestier de l'État (2).

\section{L'analyse juridique de l'aliénabilité des aires protégées}

Dire des biens du domaine privé de l'État qu'ils sont en principe aliénables signifie que des tiers peuvent, sauf dispositions législatives contraires, en devenir propriétaires. En d'autres termes, ces tiers ont le droit de jouir du bien (une portion d'aire protégée) en l'utilisant (usus), en jouissant de ses fruits (fructus) et en disposer (abusus) ou d'être titulaires de quelques-unes de ces prérogatives sur ceux-ci ${ }^{250}$. En effet, les règles applicables au domaine privé sont essentiellement celles du droit privé et partant du droit civil en ce qui concerne l'aliénation du domaine privé de l'État ${ }^{251}$. Dans ces conditions, le bien du domaine privé de l'État et qui est régi par le droit civil pourra être aliéné comme tout bien avec pour conséquence de transférer le droit de propriété ou un démembrement du droit de propriété au profit d'un tiers. C'est ainsi que l'État peut aliéner volontairement des biens de son domaine

\footnotetext{
${ }^{250}$ En droit civil, le droit de propriété englobe l'usus, le fructus et l'abusus qui pris séparément constituent les démembrements du droit de propriété. Voir SEUBE (Jean-Baptiste), Droit des biens, $3^{\text {ème }}$ édition, Paris,

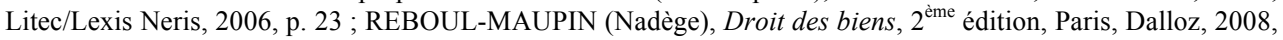
pp. 133-138.

${ }^{251}$ Voir LAUBADÈRE (André de), VENEZIA (Jean-Claude), GAUDEMET (Yves), Traité de droit administratif, 10 éd., t. 2, op.cit., pp. 342-343.
} 
privé, soit par l'échange, la vente ou les cessions à des particuliers comme c'est le cas avec ses réserves foncières ${ }^{252}$.

En conséquence, l'aliénabilité des aires protégées sous-entend que ces milieux naturels pouvaient entrer dans le patrimoine d'un tiers, soit en totalité, soit en partie. Ce tiers aurait exercé les droits réels principaux et accessoires afférents sur l'aire protégée ou la portion d'aire protégée à lui octroyée.

En Côte d'Ivoire, les aires protégées ont toujours été convoitées par les populations ; d'où leur infiltration massive pour l'agriculture ${ }^{253}$. Cette situation a parfois amené l'État à prendre une décision (un déclassement ${ }^{254}$ ) pour autoriser l'exploitation des portions de forêts appartenant au domaine forestier de l'État (biens de son domaine privé), à des fins agricoles $^{255}$. Ces décisions ont été certainement facilitées par le caractère aliénable de ces milieux naturels. C'est ainsi qu'en Côte d'Ivoire, les exemples d'aliénation de forêts relevant du domaine forestier de l'État sont légions.

\section{Des exemples pratiques d'aliénation de forêts issues du domaine forestier de l'État ivoirien}

En Côte d'Ivoire, plusieurs forêts appartenant au domaine forestier de l'État, à savoir les aires protégées (Parcs Nationaux et Réserves) et les forêts classées ont été mises à la

\footnotetext{
${ }^{252}$ En revanche, l'État ne peut se voir priver de dépendances de son domaine privé par des aliénations forcées. En effet, il est à noter que c'est l'État lui-même qui est exclusivement titulaire du droit d'exproprier et donc ne peut être contraint à l'expropriation. Voir KOBO (Pierre-Claver), Droit Administratif des Biens (D.A.B.), Abidjan, A.B.C édition, 2004, p. 158 et s. De plus, les autres formes d'aliénation forcée telles que la saisie et l'hypothèque ne sont pas en principe possible à l'égard de l'État, en ce sens qu'en règle générale les voies d'exécution ne s'appliquent pas aux biens publics. Voir ASSI-ESSO (Anne-Marie Hortense), DIOUF (N'diaw), OHADA. Recouvrement des créances, Bruxelles, Bruylant, [Collection UNIDA], 2002, p. 41 et s. En effet, selon l'article 30 alinéa 2 de l'Acte Uniforme portant organisation des procédures simplifiées de recouvrement et des voies d'exécution, les personnes morales de droit public et les entreprises publiques bénéficient d'une immunité d'exécution («Acte Uniforme portant organisation des procédures simplifiées de recouvrement et des voies d'exécution» [JO OHADA, ${ }^{\circ} 6$, du $1^{\text {er }}$ juin 1998] in OHADA, Traités et Actes Uniformes commentés et annotés, Juriscope, 2011, 1072 pages + annexes). Voir également Cass. civ. 21 déc. 1987 Bureau de recherches géologiques et minières c./ Société Lloyd Continental, Bull. civ. I, n 348, p. 249.

${ }^{253}$ Une évaluation de l'efficacité de la gestion des Parcs et Réserves ivoiriennes montrent que la quasi-totalité de ces aires protégées est infiltrée à des fins d'exploitations agricoles. En effet, cette évaluation indiquait que le Parc National de la Marahoué est occupé à plus de 60\% de sa superficie par environ entre 5.000 à 20.000 hommes qui pratiquent l'agriculture. Le rapport indique que la situation est aussi problématique au Parc National du Mont Péko pour les mêmes raisons. Il en est de même (et dans une moindre mesure) pour le Parc National d'Azagny, le Parc National de Taï et la Réserve de faune d'Abokouamékro etc. Sur tous ces différents points, voir UICN/BRAO, Évaluation de l'efficacité de la gestion des aires protégées : Parcs et Réserves de Côte d'Ivoire, 2008, p. 22.

${ }^{254}$ Déclasser ici (en matière de conservation de la nature) signifie simplement que l'État prend une décision pour autoriser l'exploitation de forêts par les populations dans un but agricole ou autre. Cela n'a aucun lien avec le déclassement (notion du droit administratif des biens) qui fait sortir un bien du domaine public de l'État.

${ }^{255}$ Pour des exemples d'aliénation de forêts appartenant au domaine forestier de l'État ivoirien, voir les développements faits au point 2 ci-dessus intitulé "2. Des exemples pratiques d'aliénation de forêts issues du domaine forestier de l'État ivoirien".
} 
disposition des populations pour la pratique de l'agriculture. Les autorisations données par les autorités administratives dans ces cas l'ont été, bien souvent, suite à des occupations anarchiques de forêts appartenant au domaine forestier de l'État par les populations à des fins agricoles.

En guise d'exemples, il peut être souligné que des portions de Parcs Nationaux et Réserves ont été déclassés en vue de l'installation de paysans sur ces terres. En effet, en 1977, une partie du Parc National de la Comoé qui a été créée en $1968^{256}$ a été déclassée au profit des populations (des agriculteurs) qui l'avaient infiltrée et fortement dégradée ${ }^{257}$.

De même, en 1981, le décret de création du Parc National d'Azagny ${ }^{258}$ déclasse 8.150 ha de la Réserve partielle de faune de 30.000 ha qu'elle fut à sa création en $1960^{259}$, au profit des populations. Une telle décision a été prise par les pouvoirs publics parce que la portion de forêt déclassée a été dégradée par les populations du fait de la pratique de l'agriculture ${ }^{260}$. Par ailleurs, dans la région de Grand-Lahou, la forêt classée du Gô a été partiellement déclassée à deux (2) reprises (1982 et 1987) pour la réinstallation de populations infiltrées dans le Parc National d'Azagny ${ }^{261}$.

Ces décisions de déclassement de ces espaces forestiers en faveur des populations paysannes ont été certainement facilitées par le caractère aliénable de ces milieux naturels, qui étaient aussi prescriptibles.

\section{B - La prescriptibilité des aires protégées}

Aux termes de l'article 2219 du Code civil: «la prescription est un moyen d'acquérir ou de se libérer par un certain laps de temps ${ }^{262}$, et sous les conditions déterminées par la loi». Ces conditions qu'énonce l'article 2229 dudit code sont que «pour pouvoir prescrire, il faut une possession continue et non interrompue, paisible, publique et non équivoque, et à titre de propriétaire ».

Ainsi, la prescriptibilité d'un bien qui fait partie du domaine privé de l'État signifierait que le tiers qui en a la possession peut acquérir un droit réel ${ }^{263}$ sur ledit domaine, par

\footnotetext{
${ }^{256}$ Voir Décret 68-81 du 9 février 1968 portant création du Parc National de la Comoé, JORCI n 11 du 22 février 1968, p. 556.

${ }^{257}$ Voir Décret 77-116 du 25 février 1977 portant déclassement d'une parcelle du Parc National de la Comoé, JORCI n 13 du 31 mars 1977 p. 521.

${ }^{258}$ Voir Décret $n^{\circ} 81-218$ du 2 avril 1981 portant création du Parc National d'Azagny avec une zone de protection périphérique, JORCI n²1 du Jeudi 14 mai 1981, p. 241.

${ }^{259}$ Voir LAUGINIE (Francis), Conservation de la nature et des aires protégées en Côte d'Tvoire, op. cit., p.41.

${ }^{260}$ Ibidem.

${ }^{261}$ Voir successivement l'Arrêté n ${ }^{\circ}$ 63/Minefor/DGEF du 22/11/1982 portant déclassement partiel de la forêt du Gô et le Décret n॰87-759 du 22 juillet 1987 portant déclassement partiel de la forêt du Gô (JORCI n 32 du 20 août 1987, p. 310).

${ }^{262}$ Ce délai est de trente ans, selon les termes de l'article 2229 du Code civil.

${ }^{263}$ On appelle droit réel, le droit qui porte directement sur une chose et procure à son titulaire tout ou partie de l'utilité économique de cette chose. Il s'agit, par exemple, du droit de propriété (droit réel le plus complet) et de
} 
l'écoulement d'un délai de trente ans. Il s'agit de la prescription acquisitive ${ }^{264}$. Ce faisant, l'État perd ce droit réel du fait de son inaction prolongée à l'exercer (prescription extinctive). Cette possession pourra être opposée à l'État par des actions possessoires ${ }^{265}$ exercées devant le juge.

L'analyse de la prescriptibilité des aires protégées se fera sous l'angle des prétentions éventuelles des populations riveraines de ces forêts. La prescriptibilité des aires protégées traduit l'idée que si ces espaces avaient été accaparés en partie ou en totalité par un tiers, celui-ci aurait pu valablement en revendiquer la propriété, après l'écoulement d'un délai de 30 ans. Toutefois, cette revendication se ferait sous la réserve des conditions de l'article 2219 du Code civil précitées. La situation de ce tiers serait alors consolidée par l'inactivité ou la négligence de l'État qui n'aurait pas agi dans ce délai légal pour revendiquer sa propriété sur l'aire protégée en cause. C'est ce que l'on désigne sous l'appellation de prescription acquisitive ou usucapion $^{266}$.

C'est ainsi que nous nous posons la question suivante. En Côte d'Ivoire, les populations qui, ont depuis des lustres, occupé des terres dans les aires protégées (Azagny, Marahoué, Taï...) pouvaient-elles prétendre à une action possessoire ? Nous répondons par l'affirmative, dans la mesure où, d'une part, le régime juridique de la domanialité privée auquel était soumis les aires protégées les exposait à la prescription ${ }^{267}$. D'autre part, dans la pratique, le risque pour l'État ivoirien de perdre des portions d'aires protégées par le jeu de la prescription acquisitive était probable. En effet, la Côte d'Ivoire a érigé de vastes étendues de forêts en aire protégée ${ }^{268}$ alors que les personnels en charge de la conservation de ces massifs forestiers ne disposent pas de moyens suffisants pour assurer cette mission ${ }^{269}$. Selon Anne B. JOHANNESEN, l'érection de vastes étendues de forêts en aires protégées nécessitait des

ses démembrements tels que la servitude, l'hypothèque, le droit d'usage etc. Voir CORNU (Gérard) (dir.), Vocabulaire juridique, $4{ }^{\mathrm{èm}}$ éd. (Association Henri Capitant), op. cit., p. 749.

${ }^{264}$ À l'instar du législateur ivoirien, le Professeur René CHAPUS affirme dans son ouvrage de Droit administratif général que les dépendances du domaine privé peuvent: "être usucapées par le jeu de la prescription trentenaire ». Voir CHAPUS (René), Droit administratif général(t. 1), $11^{\mathrm{e} m e}$ édition, op. cit., p. 505 ; voir aussi PEISER (Gustave), Droit administratif général, 21 édition, op. cit., p. 64.

${ }^{265}$ L'action possessoire est une action en justice ouverte au possesseur d'un bien de demander au juge d'assurer le respect de cette possession. Voir CORNU (Gérard) (dir.), Vocabulaire juridique, $4^{\text {ème }}$ éd. (Association Henri Capitant), op. cit., p. 669.

${ }^{266}$ Sur la question, consulter CHAPUS (René), Droit administratif général(t. 1), 11 ème édition, op.cit., p. 505 ; LAUBADÈRE (André de), VENEZIA (Jean-Claude), GAUDEMET (Yves), Traité de droit administratif, 10 éd., t. 2, op.cit., p. 341 et s. Ces auteurs écrivent, à propos du domaine privé, que la Cour de cassation juge que «toute personne physique ou morale apte à avoir un patrimoine est admise à se prévaloir des effets de la prescription acquisitive ou à se les voir opposer y compris les personnes publiques » (ibidem).

${ }^{267}$ Toutefois, les conditions de la prescription acquisitive auraient été analysées (au fond) par le juge saisi de cette prétention; ce qui suppose tout de même la recevabilité de l'action sur la forme.

${ }^{268}$ Par exemple, le Parc National de la Comoé qui a une superficie de 1.149 .150 ha est le plus vaste Parc d'Afrique de l'Ouest. Le Parc National de Taï et la Réserve de Faune du N'zo qui sont contigus couvrent une superficie de 536.000 ha. En ce qui concerne les Parcs Nationaux de la Marahoué, du Mont Sangbé, du Mont Péko et d'Azagny s'étendent respectivement sur 101.000 ha, $95.000,34.000$ ha et 21.850 ha. Voir UICN/BRAO, Évaluation de l'efficacité de la gestion des aires protégées : Parcs et Réserves de Côte d'Ivoire, 2008, pp. 9-11.

${ }^{269}$ Voir République de Côte d'Ivoire, Ministère de l'Environnement et des Eaux et Forêts /Direction de la Protection de la Nature, Programme Cadre de Gestion des Aires Protégées, op. cit., p. 44. 
moyens conséquents pour leur conservation mais dont les États ne disposaient pas a été un fait commun à toute l'Afrique ${ }^{270}$.

Par conséquent, compte tenu de l'insuffisance des moyens de l'Administration en charge de la conservation des aires protégées, une possession paisible et de longue durée (30 ans) aurait été possible. Dès lors, une pareille possession aurait pu exposer des portions d'aire protégée à l'usucapion, en raison de la prescriptibilité des aires protégées.

$\mathrm{Au}$ regard des analyses précédentes, il est à remarquer que l'appartenance des aires protégées au domaine privé de l’État (de 1960 à 2002) ne leur assurait pas une protection durable quant à leur consistance vis-à-vis de la convoitise ou des accaparements des tiers. En d'autres termes, en dehors de toute transmission volontaire dans le patrimoine d'autres personnes, l'État aurait pu perdre la propriété de certaines parcelles d'aires protégées du fait de la possession prolongée de celles-ci par autrui. Cette situation aurait résulté de sa négligence ou se serait produite à son insu, en raison des limites des pouvoirs du personnel en charge de la protection des aires protégées.

\section{Paragraphe 2 : Les limites des pouvoirs du personnel en charge de la protection des aires protégées}

Les limites des pouvoirs du personnel en charge de la protection des aires protégées se rapportent aux pouvoirs d'administration de ces forêts (A) ainsi qu'aux pouvoirs de répression des infractions contre les aires protégées reconnues audit personnel (B).

\section{A - Les limites relatives aux pouvoirs d'administration des aires protégées}

La centralisation de la gestion des aires protégées présentait des inconvénients sur le plan administratif. Le pouvoir de décision relevait d'un organe unique c'est-à-dire la Direction de la Protection de la Nature (DPN) qui est basée à Abidjan (dans l'ancienne la capitale politique de la Côte d'Ivoire ${ }^{271}$ ) alors que les aires protégées sont reparties sur l'ensemble du territoire ivoirien.

\footnotetext{
${ }^{270}$ Voir JOHANNESEN (Anne Borge), Protected areas, wildlife conservation and local welfare, op. cit., p.3.

${ }^{271}$ Située en Afrique de $1^{\prime}$ Ouest sur une superficie d'environ $322.462 \mathrm{~km} 2$ et de coordonnées de $2^{\circ} 30^{\prime}$ et $8^{\circ} 30^{\prime}$ de longitude ouest, $4^{\circ} 30^{\prime}$ et $10^{\circ} 30^{\prime}$ de latitude nord, la République de Côte d'Ivoire est limitée au Nord par le Mali et le Burkina-Faso, à l'Est par le Ghana et à l'Ouest par le Libéria et le Guinée, et au Sud par l'océan atlantique avec au Sud, une façade littorale d'environ 550 kilomètres. Voir TIEBLEY (Yves-Didier), La Côte d'Ivoire et la gestion des ressources naturelles marines, Thèse unique, Droit public, Université de Maastricht, 20 mai 2010, p. 16.

Elle a pour capitale politique Yamoussoukro depuis 1983 en lieu et place d'Abidjan (ancienne capitale politique) qui est dorénavant la capitale économique. Toutefois, le transfert de la capitale politique n'étant pas effectif, Abidjan rassemble encore la presque totalité des Administrations avant leur transfert effectif dans la capitale politique. Voir République de Côte d'Ivoire, Ministère d'État, Ministère du Plan et du Développement, Document Stratégique de Réduction de la Pauvreté, Stratégique de Relance du Développement et de Réduction de la Pauvreté, op. cit., p.1.
} 
Dans ces conditions, le danger est que ce centre de décision n'a pas toujours les informations réelles concernant les nécessités ou besoins locaux qui diffèrent d'une aire protégée, en fonction des réalités locales. Et pourtant, leur prise en compte dans les décisions aurait permis de mieux conserver les aires protégées qui restent fortement infiltrées et dégradées $^{272}$. En effet, en l'absence de connaissance des nécessités ou besoins locaux relatifs à la conservation durable des aires protégées, il est difficile pour l'autorité détentrice du pouvoir de décision de les adapter aux différentes situations.

Dès lors, le risque pour les Administrations locales (déconcentrées) des aires protégées de ne pas pouvoir appliquer les décisions prises par le centre unique de décision basé à Abidjan (et coupé de certaines réalités locales de gestion des aires protégées) ou celui de leur violation par les populations locales est réel.

Par ailleurs, les limites des pouvoirs du personnel en charge des aires protégées s'étendent à leurs pouvoirs de répression des infractions à la législation des aires protégées.

\section{B - Les limites des pouvoirs de répression du personnel en charge des aires protégées}

De 1965 à 2002, la loi reconnaît quelques fonctions de Police judiciaire aux inspecteurs et agents assermentés des Eaux et Forêts en vue de la répression des infractions contre les aires protégées ${ }^{273}$. En effet, ils ne pouvaient que rechercher et constater par procèsverbal les infractions à la législation sur les aires protégées.

Ainsi, de par leurs attributions, ils ne peuvent interroger des personnes qu'ils suspectent d'avoir commis des infractions contre les aires protégées. Ils ne peuvent non plus faire des perquisitions, c'est-à-dire effectuer une recherche d'éléments de preuve d'une infraction au domicile ${ }^{274}$ d'un particulier ou sur la personne d'un individu, aux termes de l'article 23 alinéa 2 du CPP. En effet, l'article 23 alinéa $1^{\text {er }}$ du CCP donne compétence aux inspecteurs et agents assermentés des Eaux et Forêts de suivre les choses enlevées (en l'espèce, des ressources naturelles des aires protégées) où elles sont transportées et de les mettre sous séquestre. Cependant, selon l'alinéa $2:$ "ne peuvent cependant pénétrer dans les maisons, ateliers, bâtiments, cours adjacentes et enclos qu'en présence d'un officier de police judiciaire... ».

\footnotetext{
${ }^{272}$ À propos de l'état des pressions anthropiques sur les aires protégées ivoiriennes, voir l'introduction de la présente étude au point "I. Enjeux de la protection des forêts en Côte d'Ivoire" et plus précisément le sous-point "2. Les pressions anthropiques sur les aires protégées en particulier".

${ }^{273}$ Pour plus d'informations sur les compétences de ces inspecteurs agents et agents assermentés des Eaux et Forêts ainsi que les infractions contre les aires protégées de 1960 à 2002, voir le Chapitre I du Titre I de la Première partie de la présente étude, la Section 2, le Paragraphe 2 intitulé "La répression des atteintes contre les aires protégées".

${ }^{274}$ Aux termes de l'article 102 du Code civil : «Le domicile est le lieu où une personne a son principal établissement. ».
} 
De même, en cas de flagrant délit, l'article 24 du CPP ivoirien dispose que les agents assermentés et les inspecteurs des Eaux et Forêts ont recours à un officier de Police judiciaire (OPJ). Cette prescription de l'article 24 alinéa $1^{\mathrm{er}}$ du CPP s'explique par le fait que les agents assermentés et les inspecteurs des Eaux et Forêts n'ont pas la qualité d'OPJ. En effet, dans l'hypothèse du flagrant délit, en dehors de la concomitance entre la constatation de l'infraction et sa commission, le prévenu ne présentera que des traces ou indices qui établiraient sa culpabilité. Le doute créé par cette situation ne pourrait être dissipé que par l'intervention d'un officier de Police judiciaire (OPJ) ${ }^{275}$. C'est la raison pour laquelle n'ayant pas cette qualité, les agents assermentés et les inspecteurs des Eaux et Forêts conduisent le prévenu devant un OPJ, ainsi que le prescrit l'article 24 alinéa $1^{\mathrm{er}}$ précité du CPP.

À l'analyse, le recours à un OPJ limitait les agents assermentés et inspecteurs des Eaux et Forêts dans leur mission de protection des aires protégées. En effet, en matière d'infraction contre les aires protégées, les cas de flagrants délits sont fréquents. Ainsi, le temps qui sépare la sollicitation d'un OPJ (par les agents assermentés ou les inspecteurs des Eaux et Forêts) dans un temps voisin d'une infraction et l'intervention de celui-ci aurait pu contribuer à dissiper des éléments de preuve. En effet, les personnels en charge de la conservation des aires protégées ont toujours été jugés insuffisants et ne disposent pas de moyens adéquats pour l'accomplissement de leurs missions ${ }^{276}$.

Par conséquent, il eut fallu que les agents assermentés et inspecteurs des Eaux et Forêts aient la qualité d'OPJ pour être plus efficace dans l'exercice de leur mission de protection des aires protégées.

$\mathrm{Au}$ surplus, les insuffisances du cadre juridique de protection des aires protégées concernent également les mécanismes de financement de leur protection.

\section{SECTION II : Les carences des règles de financement de la protection des aires protégées}

Le financement de la protection des aires protégées obéit à des règles de fonctionnement de l'Administration publique ivoirienne qui ne sont pas toujours adaptées en la matière. D'une part, sur le plan juridique, l'Administration en charge des aires protégées est astreinte au financement étatique ou celui qui est obtenu par l'État pour son compte (Paragraphe 1). D'autre part, la procédure de gestion des subventions étatiques accordées aux aires protégées est un obstacle à leur protection (Paragraphe 2).

\footnotetext{
${ }^{275} \mathrm{Ce}$ dernier pourra conduire des investigations qui peuvent être, entre autres, la vérification de l'identité du prévenu, son audition ou de la prise de mesures de sauvegarde. Cette analyse résulte de l'interprétation de l'article 61 alinéa 2 du Code de procédure pénale qui dispose : «Toute personne dont il apparaît nécessaire, au cours des recherches judiciaires, d'établir ou de vérifier l'identité, doit à la demande de l'officier de Police judiciaire, se prêter aux opérations qu'exige cette mesure. ».

${ }^{276}$ Voir République de Côte d'Ivoire, Ministère de l'Environnement et des Eaux et Forêts /Direction de la Protection de la Nature, Programme Cadre de Gestion des Aires Protégées, op. cit.,p. 44.
} 


\section{Paragraphe 1 : L'Administration des aires protégées tributaire d'un financement étatique insuffisant}

L'impossibilité juridique pour l'Administration des aires protégées de rechercher des fonds autres que ceux alloués par l'État $(\mathrm{A})$ pose un problème pratique en matière de gestion des aires protégées. Il s'agit de l'insuffisance des subventions étatiques pour la conservation des aires protégées $(\mathrm{B})$.

\section{A - L'impossibilité juridique du recours au financement extra-étatique par l'Administration des aires protégées}

En Côte d'Ivoire, de 1960 à 2002, l'Administration en charge des aires protégées ne disposait pas de la personnalité juridique (1). Ce défaut de personnalité juridique de l'Administration en charge des aires protégées a eu des conséquences d'ordre juridique en matière de financement des aires protégées (2).

\section{L'absence de personnalité juridique de l'Administration en charge des aires protégées}

La personnalité juridique se définit comme l'aptitude pour une personne (physique ou morale) à être titulaire de droits et tenue d'obligations ${ }^{277}$. En ce sens, c'est la personnalité juridique qui permet d'agir sur la scène juridique et d'entretenir des relations juridiques avec toute autre personne.

Dès lors, pourquoi peut-on soutenir que l'Administration en charge des aires protégées n'a pas de personnalité juridique propre ? La réponse à cette question exige une analyse des règles qui régissent le fonctionnement de l'Administration, en général, et de celle des aires protégées, en particulier.

Dans l'organisation administrative de la Côte d'Ivoire, on distingue l'Administration d'État et l'Administration décentralisée ${ }^{278}$. L'Administration d'État est composée de la présidence de la République, de la primature, des ministères et leurs services centraux ainsi que des différents autres services rattachés aux ministères, à la primature ou encore à la présidence de la République ${ }^{279}$. Toutes ces composantes de l'Administration d'État n'ont pas la personnalité juridique car seul l'État en est titulaire ${ }^{280}$. Par conséquent, les personnes habilitées à représenter ces services publics agissent au nom de l'État qui est le titulaire (exclusif) de la personnalité juridique.

\footnotetext{
${ }^{277}$ Voir DÉGNI-SÉGUI (René), Introduction au Droit, op. cit., p 195.

${ }^{278}$ Voir DÉGNI-SÉGUI (René), Droit administratif général. L'organisation administrative (t.1), $4{ }^{\text {ème }}$ édition, Abidjan, Édition Omniprésence, 2013, p. 99.

${ }^{279}$ Idem, p. 100.

${ }^{280}$ Idem, p. 125.
} 
Il en résulte que le service public ou l'Administration en charge des aires protégées qui, de 1960 à 2002, était géré par les services centraux des différents ministères de tutelles, n'a pu avoir de personnalité juridique. Aussi cette absence de personnalité juridique de l'Administration en charge des aires protégées a-t-elle des conséquences sur plan financier.

\section{Les conséquences juridiques de l'absence de personnalité juridique de l'Administration en charge des aires protégées sur le plan financier}

Sur le plan financier, les conséquences de l'absence de personnalité juridique de l'Administration des aires protégées sont de deux (2) ordres. D'une part, n'ayant pas la personnalité juridique, l'Administration en charge des aires protégées ne peut, par elle-même, rechercher et autogérer des financements extra-étatiques. D'autre part, elle n'est pas non plus habilitée à recevoir (directement) des financements de personnes autres que l'État et à les affecter aux chapitres de ses activités de protection des aires protégées. Or, les bailleurs de fonds sont quelques fois méfiants à remettre ces fonds à l'État, en raison des doutes qu'ils ont sur la gestion rigoureuse de ces financements ${ }^{281}$.

Dès lors, l'Administration en charge des aires protégées n'a essentiellement pour ressources financières que les fonds d'origine étatique ${ }^{282}$. Ces fonds ont été jugés insuffisants pour les besoins de conservation des aires protégées ${ }^{283}$.

\section{B - L'insuffisance des dotations budgétaires pour la conservation des aires protégées}

Il ressort du Document-cadre du Programme Cadre de Gestion des Aires Protégées (PCGAP) de Côte d'Ivoire que l'une des difficultés de leur protection et partant de leur conservation est l'insuffisance des subventions de l'État qui leur sont allouées ${ }^{284}$.

Les raisons de l'insuffisance du financement des actions de protection des aires protégées sont au nombre de deux (2). En effet, d'une part, le PCGAP précité révèle que la crise économique qu'a traversée la Côte d'Ivoire durant les années 1980, à cause de la chute des cours ses matières premières, a entraîné la réduction des budgets accordés à la

\footnotetext{
${ }^{281}$ Voir République de Côte d'Ivoire, Ministère de l'Environnement et des Eaux et Forêts /Direction de la Protection de la Nature, Programme Cadre de Gestion des Aires Protégées, op. cit., p. 31.

${ }^{282} \mathrm{Il}$ peut arriver que les aires protégées bénéficient d'aides financières dans le cadre de la coopération bilatérale. Autrement dit, ce sont des soutiens financiers dont la Côte d'Ivoire a pu souvent bénéficier. À titre d'exemples, dans le cadre d'un projet de conservation entre le Parc régional du Haut-Jura et le Parc National du Mont Sangbé initié en 1995, le Parc National du Mont Sangbé a bénéficié d'un appui financier de la Commission européenne d'un montant de 1,8 milliard de francs CFA. De même, jusqu'à la fin de l'année 2001, la KFW a accordé un financement d'un montant de 4 milliards pour le Projet Autonome pour la Conservation du Parc National de Taï (PACPNT), dans le cadre de la coopération ivoiro-allemande. Pour ces différents exemples d'aides financières issues de la coopération bilatérale et biens d'autres, voir République de Côte d'Ivoire, Ministère de l'Environnement et des Eaux et Forêts/Direction de la Protection de la Nature, Programme Cadre de Gestion des Aires Protégées, op. cit., p. 23.

${ }^{283}$ Idem, p. 31.

${ }^{284}$ Ibidem.
} 
conservation des aires protégées $^{285}$. À titre d'exemple, en 1999, alors que le budget nécessaire au secteur des Parcs Nationaux est estimé à 4 milliards, l'État ivoirien n'alloue seulement que 1,5 milliards à la gestion de ces sites ${ }^{286}$.

Dans l'histoire de la protection des aires protégées, l'insuffisance des budgets affectés à leur protection n'est pas spécifique à la Côte d'Ivoire. En effet, dans les pays en voie de développement, en général, et africains, en particulier, ces financements d'origine étatique sont le plus souvent insuffisants ${ }^{287}$. En la matière et à partir d'études réalisées dans le bassin du Congo, au Cameroun, au Ghana et en Bolivie, BRUNER et al. affirment : "Les dépenses courantes moyennes mentionnées par ces études varient de 0,05\$à 3,00\$ par ha, alors que les besoins réels varient de $\$ 0,90$ à $9,00 \$$ par ha $»{ }^{288}$ (notre traduction).

Cette situation s'explique par le fait que les gouvernements ont souvent des contraintes budgétaires $^{289}$. Dès lors, ces budgets varient d'années en années ${ }^{290}$, non pas qualitativement, alors qu'en règle générale les aires protégées à gérer s'étalent sur de vastes étendues ${ }^{291}$. Il en découle qu'il faut des moyens financiers conséquents pour leur conservation que les gestionnaires ne reçoivent pas des gouvernements ${ }^{292}$.

À titre illustratif, en Afrique australe, les structures en charge de la gestion des aires protégées ont eu très souvent des budgets insuffisants ${ }^{293}$ pour l'accomplissement de leur mission, en raison des problèmes macro-économiques et de la demande sociale pour la réduction de la pauvretée ${ }^{294}$. C'est également la même situation en Europe de l'Est, plus précisément en Roumanie. En effet, le financement du Parc National de Retezat (qui est

\footnotetext{
${ }^{285}$ Ibidem.

${ }^{286}$ Ibidem.

${ }^{287}$ Voir KRUG (Wolf), Private Supply of Protected Land in Southern Africa: A Review of Markets, Approaches, Barriers and Issues, op. cit.,p. 9; BRUNER (Aaron G.), GULLISON (Raymond E.) et BALMFORD (Andrew), «Financial cost and shortfalls managing and expanding protected-area systems in developing countries»,BioScience, Vol. 54, $\mathrm{n}^{\circ} 12,2004$, p. 1121.

${ }^{288}$ Ibidem.

${ }^{289}$ Voir LEADER-WILLIAMS (N.) et ALBON (S. D.), « Allocation of resources for conservation », Nature, Vol. 336, n 6199, 1988, p. 533.

${ }^{290}$ Voir KRUG (Wolf), Private Supply of Protected Land in Southern Africa: A Review of Markets, Approaches, op. cit.,p. 10.

${ }^{291}$ Voir JOHANNESEN (Anne Borge), Protected areas, wildlife conservation and local welfare, op. cit., p. 4.

Par exemple, en Côte d'Ivoire, il existe des aires protégées qui occupent d'importantes superficies. En effet, le Parc National de la Comoé qui s'étend sur une superficie de 1.149.000 ha est le plus vaste Parc d'Afrique de l'Ouest. De plus, le Parc National de Taï et la Réserve de Faune du N'zo qui sont contigus (et qui ont été unifiés dans la pratique pour des raisons d'efficacité de gestion) couvrent une superficie de 536.000 ha. En ce qui concerne les Parcs Nationaux de la Marahoué, du Mont Sangbé, du Mont Péko et d'Azagny s'étendent respectivement sur 101.000 ha, $95.000,34.000$ ha et 21.850 ha. Voir UICN/BRAO, Évaluation de l'efficacité de la gestion des aires protégées: Parcs et Réserves de Côte d'Ivoire, 2008, pp. 9-11.

${ }^{292}$ Voir ANDRADE (Gustavo S. M.) et RHODES (Jonathan. R.), « Protected areas and local communities: an inevitable partnership toward successful conservation strategies ? »,op. cit. , p. 2.

${ }^{293}$ Voir KRUG (Wolf), Private Supply of Protected Land in Southern Africa: A Review of Markets, Approaches, op. cit. p. 9.

${ }^{294}$ En Zambie par exemple, l'on constate au début des années 1970 une baisse significative des contributions de l'État au secteur de la faune. Voir POPE (Adam), A Preliminary Examination of Public Private Partnerships in National Park Management in Zambia, op. cit., p.1.
} 
étatique) a connu une diminution à cause des conséquences fâcheuses des problèmes économiques mondiaux sur les économies des pays en développement. ${ }^{295}$ De même, dans ce pays, un grand nombre d'aires protégées sont confrontés aux problèmes de ressources financières limitées ${ }^{296}$. C'est aussi le cas pour la Serbie où un gestionnaire d'aire protégée notamment celui de la Réserve Naturelle Spéciale ZASAVICA (en anglais Special Nature Reserve Zasavica ou SNR ZASAVICA) affirme que l'un des principaux problèmes qu'il rencontre dans la gestion de cette Réserve est le déficit de financement ${ }^{297}$.

$\mathrm{Au}$ surplus, l'insuffisance du financement pour la protection des aires protégées ivoiriennes est due au fait que le Fonds spécial des Parcs Nationaux qui était destiné à compléter les dotations budgétaires de l'État n'a fait l'objet d'aucun apport ${ }^{298}$.

Par ailleurs, l'autre carence des règles de financement de la protection des aires protégées réside en ce qu'elles imposent une procédure de gestion desdits fonds qui n'est pas adaptée à la protection de ces forêts.

\section{Paragraphe 2 : La procédure de gestion des subventions étatiques, un obstacle à la conservation des aires protégées}

La gestion des subventions que l'État accorde au secteur des aires protégées est gérée selon les règles de la comptabilité publique. Ainsi, l'exécution de dépenses relatives à la protection des aires protégées suit un circuit (A) qui, bien souvent, a entraîné des difficultés pour la protection des aires protégées (B).

\section{A - Le circuit de l'exécution de la dépense publique}

Les dépenses publiques ivoiriennes sont celles inscrites comme telles dans les budgets de l'État, et ceux des collectivités et des organismes parapublics ${ }^{299}$. Ces dépenses qui permettent l'existence de l'État ivoirien sont mises en œuvre selon une procédure d'exécution qui comporte quatre (4) phases ${ }^{300}$. En effet, en Côte d'Ivoire, le circuit de la dépense publique a trois (3) phases administratives et une phase comptable. Ce sont l'engagement, la liquidation, l'ordonnancement de la dépense et une quatrième phase purement comptable qui est le paiement.

\footnotetext{
${ }^{295}$ Voir SZELL (Andrea B.), HALLETT (Lucius F.), « Attitudes and Perceptions of Local Residents and Tourists toward the Protected Area of Retezat National Park, Romania », art. cit., p. 18.

${ }^{296}$ Idem, p. 22.

${ }^{297}$ Voir TOMIĆEVIĆ (Jelena), BJEDOV (Ivana), SHANNON (Margaret A.)et OBRATOV-PETKOVIĆ (Dragica), « Understanding Linkages Between Public Participation and Management of Protected Areas - Case Study of Serbia», in Dr. ISHWARAN (Natarajan) (Eds), The Biosphere, mars 2012, Intech, p. 135.

${ }^{298}$ En l'absence d'indications provenant de documents, nos enquêtes ne nous ont pas permis de trouver des personnes ressources pouvant nous donner les raisons pour lesquels ce fonds est resté virtuel. C'est la raison pour laquelle nous n'avons pu que faire ce constat.

${ }^{299}$ Voir MAHIEU (François Régis), Introduction aux finances publiques de la Côte d'Ivoire, op.cit. p. 45.

${ }^{300}$ Idem, p. 79.
} 
On appelle Engagement l'acte qui fait naître une dépense à la charge de l'État. En effet, pour initier l'exécution d'une dépense publique, la phase d'engagement comprend les étapes suivantes. L'administrateur des crédits d'un service, en l'occurrence celui du service public en charge des aires protégées délivre un bon d'engagement. Ce bon d'engagement est soumis au contrôleur financier auprès du ministère en charge des aires protégées. Celui-ci a le droit de juger aussi bien de l'utilité que du coût de la dépense. Après cette étape, le bon d'engagement est soumis à l'agence comptable centrale du Trésor, au sein duquel le Service Central de l'Ordonnancement (S.C.O) vérifie, entre autres, qu'il existe des crédits correspondants sur le chapitre budgétaire intéressé ${ }^{301}$.

Ensuite, intervient la phase de liquidation (de la dépense) qui consiste à déterminer le montant exact de la dépense engagée. La liquidation s'effectue en deux (2) étapes que sont la constatation du service fait et la liquidation proprement dite. En effet, il est un principe que l'État ne paie pas d'avance, il ne paie que la prestation qui est effectivement accomplie et que l'on peut constater. Dès lors, intervient la liquidation proprement dite qui a pour but de déterminer le montant exact de la dette due par l'État par la vérification des factures. Enfin, suivent les phases d'ordonnancement et de paiement qui consistent successivement pour l'ordonnateur de la dépense à émettre l'ordre de payer au comptable payeur qui, à son tour, libère l'État de sa créance.

Le circuit de la dépense publique qui a été brièvement décrit ci-haut, se fait à travers une Administration caractérisée par la lourdeur et la lenteur ${ }^{302}$. Par conséquent, l'Administration en charge des aires protégées éprouve par moment des difficultés à utiliser les budgets alloués à la protection des aires protégées. En pareil cas, elle ne peut mener des actions de protection en temps opportun ${ }^{303}$.

\footnotetext{
${ }^{301}$ En effet, il ressort des attributions du Service Central de l'Ordonnancement (S.C.I.) qu'en matière d'engagement des dépenses, ce service :

- centralise les propositions d'engagement faites par les administrateurs de crédits et vérifie leur qualité d'administrateurs de crédits ;

- contrôle la régularité des dossiers en ce qui concerne l'imputation de la dépense selon sa nature ou son objet, de la disponibilité et du rythme de consommation des crédits ;

- émet les titres de créances qui confirment les engagements ;

- à la compétence exclusive pour tenir la comptabilité des dépenses (voir MAHIEU (François Régis), Introduction aux finances publiques de la Côte d'Ivoire, op.cit., p. 80).

${ }^{302} \mathrm{C}$ 'est à juste titre que dans son diagnostic relatif aux maux qui minent l'Administration ivoirienne, M. DIABY (Maméry), Directeur des études de la Réforme administrative, stigmatise la lenteur et la lourdeur de l'Administration ivoirienne. En effet, il écrit : « elle est lente, tatillonne, opaque, gangrenée par la corruption, la fraude, l'absentéisme, le laxisme et la désinvolture.». Voir M. DIABY (Maméry) cité par DÉGNI-SÉGUI (René), Droit administratif général : L'organisation administrative (t. 1), $4^{\text {ème }}$ édition, op. cit., p. 25.

${ }^{303}$ Voir République de Côte d'Ivoire, Ministère de l'Environnement et des Eaux et Forêts /Direction de la Protection de la Nature, Programme Cadre de Gestion des Aires Protégées, op. cit., p. 32.
} 


\section{$B$ - Les conséquences des règles de gestion des subventions étatiques des aires protégées : les difficultés dans la mise à disposition des fonds}

La difficulté de la mise à disposition des fonds alloués à la protection des aires protégées a résulté de deux (2) faits. Premièrement, Il s'agit de la lenteur et de la lourdeur qui ont longtemps caractérisé l'Administration ivoirienne et par conséquent de l'Administration financière ivoirienne ${ }^{304}$. Deuxièmement, l'État a souvent eu des difficultés pour mobiliser son budget $^{305}$. En effet, la structure du budget de l'État ivoirien montre que les recettes des budgets ${ }^{306}$ de l'État sont respectivement constituées de recettes fiscales et domaniales, de recettes de services, de recettes diverses ${ }^{307}$ et d'emprunts ${ }^{308}$.

Pour le budget général de fonctionnement (BGF), les recettes fiscales et domaniales sont : les revenus du domaine, les droits d'enregistrements et de timbre ; les impôts directs, à savoir les impôts sur le revenu, les impôts fonciers, les patentes, les licences; et les impôts indirects que sont les taxes intérieurs, les droits et taxes à l'importation ainsi que les droits et taxes à l'exportation. Les impôts indirects sont une part importante du BGF dans la mesure où elle représente $70 \%$ de ce budget ${ }^{309}$. Quant aux recettes de services du BGF, elles proviennent, par exemple, du domaine public, de la vente automobile, du journal officiel. Les recettes diverses du BGF sont les produits des amendes en Douane, les ristournes, les recettes d'ordre...).

En ce qui concerne le budget spécial d'investissement et d'équipement (BSIE), la structure des recettes se présentait comme suit ${ }^{310}$. Premièrement les recettes du BSIE/Trésor sont constituées des recettes fiscales qui provenaient de certains impôts extérieurs affectés à des taux variables d'une année à une autre; les recettes diverses et les emprunts extérieurs. Deuxièmement, les recettes du BSIE proviennent des Organisations Internationales (FMI, BIRD...), des Organismes Européens (FED, BEI...); des Organisations africaines telles que la $\mathrm{BOAD}$ et la BAD. Ces ressources provenaient également des aides accordées par des gouvernements tels que la France, le Canada, les États-Unis, le Brésil ; des Établissements financiers privés ; et de sociétés privées en général. Enfin, les ressources du BSIE provenaient de la caisse de stabilisation et du soutien des prix des produits agricoles (CSSPPA).

Analysant les structures budgétaires du BGF et du BSIE, François Régis MAHIEU ne manquent pas de souligner que l'un des problèmes liés à la gestion des recettes fiscales est la

\footnotetext{
${ }^{304}$ Ibidem.

${ }^{305}$ Ibidem.

${ }^{306}$ Nous faisons allusion au (Budget Général de Fonctionnement de l'État (BGF) et au Budget Spécial d'Investissement et d'Équipement (BSIE).

${ }^{307}$ Voir MAHIEU (François Régis), Introduction aux finances publiques de la Côte d'Ivoire, pp. 26-27.

${ }^{308}$ Idem, pp. 30-31.

${ }^{309}$ Idem, p. 27.

${ }^{310}$ Les développements suivants et se rapportant à la structure du BSIE sont largement inspirés de MAHIEU (François Régis), Introduction aux finances publiques de la Côte d'Ivoire, op. cit., pp. 30-31.
} 
fraude fiscale, en raison de la complexité des impôts ${ }^{311}$. En Côte d'Ivoire, le secteur informel est beaucoup développé et les acteurs de ce secteur ne sont pas toujours imposés. En effet, il y a une difficulté réelle des agents de l'État à identifier les acteurs du secteur informel comme des contribuables $^{312}$. Il en est de même pour l'impôt foncier. Dans ces conditions, la fraude fiscale fait perdre à l'État d'importantes ressources financières. C'est ainsi que l'État a eu, bien souvent, des difficultés à mobiliser les budgets requis pour son bon fonctionnement ${ }^{313}$.

Ces contraintes budgétaires ont impacté négativement la protection des aires protégées. En effet, les budgets alloués aux aires protégées (et qui sont d'ailleurs insuffisants) ne sont pas toujours débloqués à temps et fluctuent d'une année à l'autre. Ce sont ces entraves à la protection des aires protégées que le document-cadre du PCGAP souligne en ces termes : "L'assujettissement des dotations budgétaires aux règles de la comptabilité publique entraîne une lourdeur dans les procédures d'engagement et des difficultés dans la liquidation des dépenses. Le choix de l'année calendaire comme référence budgétaire est, par ailleurs, inadapté à une gestion efficace des aires protégées. Par exemple, la saison sèche coïncide avec une période de moindre disponibilité en financements alors que c'est à cette saison de moindre pluviosité et d'harmattan que les activités de lutte contre le braconnage et d'entretien des infrastructures devraient s'intensifier sur le terrain, eu égard aux conditions favorables pour la chasse, les défrichements et les feux de brousse.»1 ${ }^{314}$.

Au total, ces différentes insuffisances de la législation applicable aux aires protégées depuis le début des années 1960 ont amené le législateur ivoirien à apporter des innovations au cadre juridique des aires protégées en 2002.

\footnotetext{
${ }^{311}$ Idem, p. 61.

${ }^{312}$ Voir LOUPPE (Dominique), OUATTARA (N'Klo), Étude sur l'exploitation forestière et les contraintes d'une gestion durable des forêts dans le domaine rural en Côte d'Ivoire, op. cit., p. 11.

${ }^{313}$ Voir République de Côte d'Ivoire, Ministère de l'Environnement et des Eaux et Forêts /Direction de la Protection de la Nature, Programme Cadre de Gestion des Aires Protégées, op. cit., p. 32.

${ }^{314}$ Ibidem.
} 


\section{TITRE II : LES INNOVATIONS APPORTÉES AU CADRE JURIDIQUE DES AIRES PROTÉGÉES DEPUIS 2002}

Le cadre juridique des aires protégées de 1960 à 2002 présentait des insuffisances qui ne permettaient pas de réaliser une protection durable de ces milieux naturels. C'est ainsi que le législateur ivoirien a pris des textes dans l'optique de corriger ces insuffisances.

Il s'agit essentiellement de la loi de 2002-102 du 11 février 2002 relative à la création, à la gestion et au financement des Parcs Nationaux et Réserves naturelles ${ }^{315}$. En outre, des décrets d'application prévus par la loi de 2002 sur les PNR ont été pris ${ }^{316}$ en vue de la mise en œuvre de la loi de 2002 sur les PNR. Autrement dit, il s'agit de permettre la protection des Parcs Nationaux et Réserves naturelles.

Ces différents textes apportent des innovations au cadre juridique de protection des aires protégées. D'une part, ces textes renforcent la protection de l'intégrité des aires protégées sur le plan normatif (Chapitre I). D'autre part, ils consacrent des règles juridiques de gestion à finalité protectrice des aires protégées (Chapitre II).

\footnotetext{
${ }^{315}$ JORCI $n^{\circ} 20$ du 16 mai 2002, p. 338. Cette loi est modifiée par la loi n²013-864 du 23 décembre 2013 modifiant l'alinéa 2 de l'article 9 de la loi n²002-102 du 11 février 2002 relative à la création, à la gestion et au financement des Parcs Nationaux et Réserves naturelles, JORCI n²0 du 16 mai 2002, p. 338.

${ }^{316}$ Il s'agit du décret $\mathrm{n}^{\circ} 2002-359$ du 24 juillet 2002 portant création, organisation et fonctionnement de l'OIPR, JORCI n ${ }^{\circ} 2002-40$ du 2 octobre 2002, p. 588 ; du décret n²012-163 du 09 février 2012 déterminant les procédures de classement des Parcs Nationaux et Réserves naturelles (JORCI n ${ }^{\circ} 7$ du 16 février 2012, p.93.); du décret $\mathrm{n}^{\circ}$ 2013-22 du 9 janvier 2013 portant modalités d'habilitation, en qualité d'officiers de Police Judiciaire, des fonctionnaires et agents des Parcs nationaux, Réserves naturelles et des Eaux et Forêts, JORCI n ${ }^{\circ} 13$ du jeudi 28 mars 2013, p. 226.
} 


\section{CHAPITRE I : LE RENFORCEMENT DE LA PROTECTION DE L'INTÉGRITÉ DES AIRES PROTEGÉES SUR LE PLAN NORMATIF}

Les aires protégées de Côte d'Ivoire ont fait l'objet de pressions anthropiques diverses malgré les règles qui les régissait ${ }^{317}$. L'analyse de la législation applicable aux aires protégées ivoiriennes de 1960 à 2002 a révélé que le droit des aires protégées n'accordait pas une protection juridique suffisante à ces forêts ${ }^{318}$. C'est ce qu'atteste également les conclusions du séminaire de mars 1995 sur l'audit de l'organisation des services des Parcs et Réserves; lequel a abouti en 1996 à la préparation du Programme Cadre de Gestion des Aires Protégées $(\text { PCGAP) })^{319}$.

C'est donc fort de cette insuffisance des règles juridiques de protection des aires protégées que le législateur ivoirien à renforcer les dispositions domaniales (Section 1) et pénales (Section 2) qui les régissent.

\section{SECTION I : L'affermissement des règles domaniales relatives aux aires protégées}

Anciennement bien du domaine privé de l'État, le renforcement des règles domaniales relatives aux aires protégées résulte de leur incorporation au domaine public de l'État (Paragraphe 1). Ce passage des aires protégées de bien du domaine privé de l'État à son domaine public a des conséquences juridiques sur leur protection (Paragraphe 2).

\section{Paragraphe 1 : L’incorporation des aires protégées au domaine public de l'État}

Selon le Vocabulaire juridique, en droit administratif, l'incorporation est: "une opération consistant à faire entrer une personne ou un bien dans une catégorie soumise à un régime déterminé $\gg{ }^{320}$. Ce faisant, l'incorporation des aires protégées au domaine public de l'État pourrait s'entendre de ce qu'elles entrent désormais (depuis 2002) dans le domaine public de l'État. Quelles sont les raisons (A) et les modalités (B) de l'incorporation des aires protégées au domaine public de l'État?

\footnotetext{
${ }^{317}$ Voir la Première partie de cette étude, le Titre I, le Chapitre II et précisément la Section I intitulé " Les insuffisances des règles domaniales et des pouvoirs du personnel en charge des aires protégées.".

${ }^{318}$ Sur la question, voir la Première partie de cette la présente thèse, le Titre I et précisément " Chapitre II : Les insuffisances du cadre juridique des aires protégées de 1960 à $2002 "$.

${ }^{319}$ Voir République de Côte d'Ivoire, Ministère de l'Environnement et des Eaux et Forêts/Direction de la Protection de la Nature, Programme Cadre de Gestion des Aires Protégées, op.cit., p. 8.

${ }^{320}$ Voir CORNU (Gérard) (dir.), Vocabulaire juridique, $4{ }^{\text {ème }}$ éd. (Association Henri Capitant), op.cit., p.458 et s.
} 


\section{A - Les raisons de l'incorporation des aires protégées au domaine public de l'État}

Plusieurs raisons pourraient expliquer l'incorporation des aires protégées au domaine public de l'État. Ces raisons émanent principalement d'un constat fait à partir des réalités de la gestion des aires protégées. En effet, dans l'ensemble, les aires protégées de Côte d'Ivoire sont soumises à de fortes pressions anthropiques ${ }^{321}$ alors qu'elles sont affranchies de tous droits d'usage ${ }^{322}$.

Elles sont l'objet d'occupations à des fins d'habitation, agricoles et de toutes autres formes d'exploitation (exploitation forestière, orpaillage, pêche illégale, braconnage...) ${ }^{323}$. En outre, certains périmètres d'aires protégées font l'objet de revendications par les populations qui contestent leur propriété à l'État ${ }^{324}$.

Dès lors, l'incorporation des aires protégées au domaine public de l'État rendrait compte du souci de conférer à ces aires protégées une meilleure protection juridique, à travers les règles d'inaliénabilité et d'imprescriptibilité ${ }^{325}$. Cependant, comment s'effectue le passage des aires protégées, de biens du domaine privé à biens du domaine public de l'État?

\section{B - Les modalités relatives à l'incorporation des aires protégées au domaine public de l'État}

L'incorporation des aires protégées au domaine public de l'État est l'œuvre du législateur ivoirien. En effet, l'article 7 de la loi précitée de 2002 sur les PNR dispose que les Parcs Nationaux et Réserves naturelles intégrales «font partie du domaine public et inaliénable de l'État...».

L'article 13 suivant prescrit que les Réserves naturelles partielles «peuvent appartenir indifféremment au domaine public ou privé de l'État...». Il ressort de la lecture de ces articles que l'incorporation des aires protégées ivoiriennes au domaine de l'État depuis 2002 s'est faite par détermination de la loi.

Aussi nous parait-il que ce serait les conséquences juridiques de l'incorporation des aires protégées au domaine public sur leur protection qui amène le législateur ivoirien à conférer la domanialité publique à ces espaces naturels.

\footnotetext{
${ }^{321}$ Voir UICN/BRAO, Évaluation de l'efficacité de la gestion des aires protégées : Parcs et Réserves de Côte d'Ivoire, 2008, op.cit., p. 5.

${ }^{322}$ Voir en Introduction (de la présente étude), le point intitulé "IV- Intérêt du sujet".

${ }^{323}$ Voir en Introduction (de cette étude), les points "I- Enjeux de la protection des forêts en Côte d'Ivoire", " CL'état des pressions anthropiques sur la biodiversité en Côte d'Ivoire", et précisément le point intitulé "2. Les pressions anthropiques sur les aires protégées en particulier ".

${ }^{324}$ Une étude de l'UICN sur l'efficacité de la gestion des aires protégées en Côte d'Ivoire a révélé que dans les Parcs Nationaux de la Marahoué, de Taï, du Mont Péko ainsi qu'à la Réserve de Faune d'Abobokouamékro, il y a des contestations de limites (desdites aires protégées) par les populations locales. Voir UICN/BRAO, Évaluation de l'efficacité de la gestion des aires protégées : Parcs et Réserves de Côte d'Ivoire, 2008, op.cit., p. 20.

${ }^{325}$ À propos de l'inaliénabilité et de l'imprescriptibilité des aires protégées, voir le paragraphe 2 suivant intitulé
} "Les implications de la domanialité publique sur la protection des aires protégées". 


\section{Paragraphe 2 : Les implications de la domanialité publique sur la protection des aires protégées}

Les conséquences juridiques de l'appartenance des aires protégées au domaine public de l'État sont diverses. D'une part, l'incorporation des aires protégées au domaine de l'État les rend désormais inaliénables et imprescriptibles (A). D'autre part, les personnes qui les occupent sans disposer d'un titre s'exposent à l'expulsion (B).

\section{A - L'inaliénabilité et l'imprescriptibilité des aires protégées}

L'inaliénabilité et l'imprescriptibilité des aires protégées seront successivement analysées.

\section{L'inaliénabilité des aires protégées}

En droit administratif des biens, l'inaliénabilité est l'impossibilité pour les biens faisant partie du domaine public à être cédés à des tiers avant d'avoir fait l'objet d'une mesure de désaffectation ou de déclassement ${ }^{326}$. Par conséquent, l'inaliénabilité des aires protégées signifie que ces espaces naturels ne peuvent entrer dans le patrimoine de tiers, tant qu'ils n'ont pas reçu une désaffectation.

L'inaliénabilité des aires protégées (biens du domaine public de l'État) induit plusieurs conséquences juridiques qui renforcent davantage leur protection. La première est que la transmission de droit de propriété sur une ou portion d'aire protégée à un tiers est nulle ${ }^{327}$, quelle que soit l'opération juridique (aliénation volontaire) ${ }^{328}$ dont elle est issue et le mode de l'aliénation ${ }^{329}$. Il est courant que des terres situées dans les limites de ces aires protégées soient l'objet de pressions foncières ${ }^{330}$ de la part des populations locales. Cette situation se justifierait par le manque de terres cultivables dans le domaine rural et/ou les revendications de propriété coutumière des populations sur ces terres. Dans tous les cas, de telles transactions encourent nullité ${ }^{331}$.

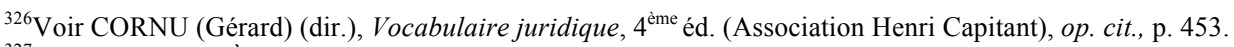

${ }^{327}$ Voir LAUBADÈRE (André de), VENEZIA (Jean-Claude), GAUDEMET (Yves), Traité de droit

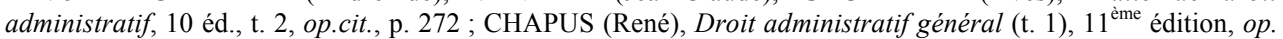
cit., p. 405 et s. ; Cass civ. $1^{\text {er }}, 2$ avril 1963, Montagne c/ Réunion des musées de France, A.J.D.A., 1963, p. 486, note Jean DUFAU ; C.E. français $1^{\mathrm{er}}$ mars 1989, Béro, D. adm. 1989, nº 244 ; Cass. civ. $3^{\mathrm{e}}$, 12 fév. 1986, Société Notre Dame des Fleurs c/ S.A.R.L. Montlaur-Grasse, A.J.D.A. 1986, p. 391, observ. J.C. ; Cass. civ. $1^{\text {er }} 3$ mai 1988, Consorts Renaults c/ E.D.F., A.J.D.A. 1988, p. 676, note Jean DUFAU; et supra, p.12, note 30.

${ }^{328}$ Vente, don, legs etc.

${ }^{329}$ Il s'agit de l'aliénation à titre gratuit, onéreux, d'échanges.

${ }^{330}$ Voir en Introduction (de cette étude), les points "I- Enjeux de la protection des forêts en Côte d'Ivoire", " Cl'état des pressions anthropiques sur la biodiversité en Côte d'Ivoire", et précisément le point intitulé "2. Les pressions anthropiques sur les aires protégées en particulier ".

${ }^{331}$ La Cour de cassation française, dans l'arrêt Montagne contre Musée de France du 13 avril 1963 (A.J.D.A., 1963, note de Dufau p. 486) a confirmé la nullité de la vente d'un bien du domaine public.
} 
La seconde conséquence de l'inaliénabilité des aires protégées est l'impossibilité de les exproprier ${ }^{332}$. Enfin, l'inaliénabilité des aires protégées emporte en principe l'interdiction de constituer des droits réels ${ }^{333}$ sur ceux-ci au profit des tiers ${ }^{334}$. Cette interdiction s'explique en ce que la création de droits réels sur les aires protégées constitue un démembrement de la propriété. Dès lors, les parcelles ainsi grevées pourraient être aliénées alors que le domaine public est par essence inaliénable ${ }^{335}$.

S'il est vrai que l'inaliénabilité instaure un régime juridique davantage protecteur du domaine public, elle n'est pas la seule conséquence de l'appartenance des aires protégées au domaine public. On a également l'imprescriptibilité de ces milieux naturels.

\section{L'imprescriptibilité des aires protégées}

En droit administratif des biens, l'imprescriptibilité signifie que les tiers ne peuvent être propriétaires de biens composant le domaine public ou être titulaires de droit réels sur lesdits biens par l'écoulement d'un délai ${ }^{336}$. En conséquence, l'imprescriptibilité des aires protégées a pour effet de paralyser la prescription acquisitive ${ }^{337}$. L'État ne court donc plus le risque d'être dépossédé de portions d'aire protégée, à son insu ou du fait de sa négligence ${ }^{338}$.

En effet, l'imprescriptibilité des aires protégées engendre trois (3) conséquences juridiques. Premièrement, il y a impossibilité pour toute personne d'acquérir une portion de

\footnotetext{
${ }^{332} \mathrm{~L}$ 'expropriation est une aliénation forcée qui est imposée au propriétaire d'un bien immeuble moyennant une

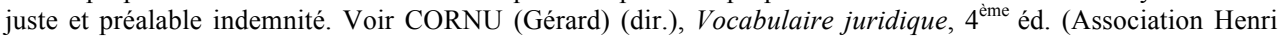
Capitant), op. cit. p. 378.

${ }^{333}$ On appelle droit réel, le droit qui porte directement sur une chose et procure à son titulaire tout ou partie de l'utilité économique de cette chose. Voir CORNU (Gérard) (dir.), Vocabulaire juridique, $4{ }^{\mathrm{ème}}$ éd. (Association Henri Capitant), op. cit., p. 749. Il s'agit par exemple du droit de propriété (droit réel le plus complet) et de ses démembrements (servitude, hypothèque, droit d'usage...).

${ }^{334}$ Il ressort de l'arrêt Commune de Champigny (C.E. français. 10 déc. 1954 Commune de Champigny, Rec. p. 658) qu'il n'y a pas de droits réels civils sur le domaine public au profit des tiers ; voir également C.E. français 6 mai 1985, Assoc. Eurolat, R.F.D.A. 1986, p. 21, concl. B. Genevois.

${ }^{335}$ Cependant, on pourrait voir dans le droit des aires protégées une limite (exception) légale au principe de l'interdiction de constituer des droits réels sur les aires protégées. Elle s'expliquerait par des objectifs de valorisation de ces milieux ; lesquels contribuent de manière oblique à leur protection. En effet, aux termes de l'article 32 alinéa $1^{\mathrm{er}}$ de la loi de 2002 sur les PNR «tout ou partie des missions d'exploitation d'un parc ou d'une réserve, selon la nature de ce parc ou de cette réserve, peuvent être concédées par l'Etablissement à une personne morale de Droit privé dans le cadre d'une Convention de concession d'exploitation ». C'est à juste titre que dans le cadre des concessions d'exploitation, le concessionnaire pourra valablement construire des infrastructures touristiques sur le territoire des aires protégées. Par conséquent, il pourrait légitimement avoir sur ceux-ci des droits réels ; du moins temporairement. Pour CHAPUS (René), la constitution de droit réels sur le domaine public découle de ce qu'ils participent à inciter les sociétés à faire des «investissements lourds» sur le domaine public ; lesquels sont «aussi profitables à l'intérêt général ». Sur la question, voir CHAPUS (René),

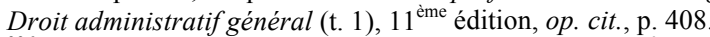

${ }^{336}$ Voir CORNU (Gérard) (dir.), Vocabulaire juridique, $4{ }^{\text {ème }}$ éd. (Association Henri Capitant), op.cit., 451.

${ }^{337}$ La prescription acquisitive se définit comme étant : "l'acquisition d'un droit réel principal (ex. propriété), surtout en matière immobilière, par la possession prolongée pendant trente ans...ou pendant une durée inférieure déterminée par la loi... ». Voir CORNU (Gérard) (dir.), Vocabulaire juridique, $4^{\text {ème }}$ éd. (Association Henri Capitant), op.cit., 681.

${ }^{338}$ Voir LAVIALLE (Christian), "L’imprescriptibilité du domaine public », Revue Française de Droit, Administratif, $\mathrm{n}^{\circ} 1,1985$, p. 27.
} 
terres dans les limites des aires protégées ou des droits réels immobiliers sur celles-ci par la prescription trentenaire.

Deuxièmement, l'imprescriptibilité des aires protégées implique que les actions en revendication et l'action domaniale ne peuvent s'éteindre par la prescription (extinctive). En effet, les actions en revendication et en réparation à l'encontre des occupants illégaux demeurent pour l'État jusqu'à ce qu'il l'exerce ${ }^{339}$.

Par conséquent, l'accaparement de superficies dans les limites des aires protégées n'est plus (juridiquement) possible. Ce régime de protection est de nature à décourager les initiatives des populations qui s'infiltrent dans les espaces mis en défens; s'y sédentarisent et espèrent qu'elles s'en verront reconnaître la propriété.

Par ailleurs, l'expulsion des occupants sans titre des aires protégées est une autre conséquence de leur appartenance au domaine public de l'État.

\section{B - L'expulsion des occupants sans titre}

En matière d'occupation d'aires protégées, l'occupation sans titre pourrait se concevoir de deux (2) manières. D'abord, elle consiste à y mener une activité illicite à caractère permanent, sans y habiter. Il peut s'agir par exemple de la création et/ou l'entretien de plantations ${ }^{340}$, comme c'est communément le cas avec les populations riveraines; ou à une échelle moindre, de l'installation d'équipements pour la pratique d'activités minières ${ }^{341}$. Ensuite, l'occupation sans titre des aires protégées peut résulter du fait que les populations riveraines des aires protégées en plus d'y mener des activités agricoles s'y établissent. C'est principalement le cas dans le Parc National de la Marahoué ${ }^{342}$.

\footnotetext{
${ }^{339}$ Sur ce point, voir AUBY (Jean-Marie), « L'action domaniale », A.J.D.A, octobre 1983, p.507 pages ; KOBO (Pierre-Claver), Droit Administratif des Biens (D.A.B.), Abidjan, A.B.C édition, 2004, 162 pages. p. 70.

${ }^{340}$ Une étude de l'Union mondiale pour la nature (UICN) montre que les Parcs Nationaux de la Marahoué, du Mont péko, d'Azagny, de Taï, et la Réserve d'Abokouamékro font objet d'exploitation agricole de la part des communautés locales. Voir UICN/BRAO, Évaluation de l'efficacité de la gestion des aires protégées : Parcs et Réserves de Côte d'Ivoire, 2008, op. cit., p. 22.

${ }^{341}$ Les activités minières dans les aires protégées sont essentiellement l'orpaillage. Au Parc National de Taï (PNT), il représente la deuxième menace, après le braconnage. Voir République de Côte d'Ivoire, Ministère de l'Environnement et des Eaux et Forêts/Office Ivoirien des Parcs et Réserves, Plan d'aménagement et de gestion du parc national de Tä̈, Abidjan, mars 2006, p. 30. L'orpaillage est aussi pratiqué dans le Parc National de la Comoé (voir République de Côte d'Ivoire, Ministère de l'Environnement et des Eaux et Forêts/Office Ivoirien des Parcs et Réserves, État de conservation des parcs et réserves de Côte d'Ivoire, op.cit., p.11) ainsi que dans le Parc National du Mont Sangbé (voir OIPR/AFD, Réalisation d'une étude sur l'état de la biodiversité de quatre Parcs Nationaux de Côte d'Ivoire. Azagny, Marahoué, Péko, Sangbé, Afrique Nature International, 2012 (Rapport final), p. 44).

${ }^{342}$ L'étude ci-dessus citée de l'UICN fait état de ce qu'environ 20.000 personnes vivent dans ce Parc. Voir UICN/BRAO, Évaluation de l'efficacité de la gestion des aires protégées: Parcs et Réserves de Côte d'Ivoire, 2008, op. cit., p. 22.
} 
De quels moyens dispose l'OIPR pour faire cesser ces troubles ? Peut-elle user du privilège de l'exécution d'office ${ }^{343}$ ? Tous les Parcs Nationaux et Réserves naturelles de Côte d'Ivoire sont infiltrés par les populations pour la pratique d'activités diverses alors que ces forêts (en principe affranchis de tous droits d'usage) sont les derniers vestiges de forêts primaires $^{344}$. La menace et le dommage écologique qui en résulte sont préoccupants. En effet, les aires protégées constituent les espaces naturels dans lesquels on retrouve les espèces en voie de disparition ${ }^{345}$. Le caractère urgent de leur protection est manifeste. Par conséquent, nous sommes en présence d'une situation où l'exécution d'office pourrait être envisagée par l'OIPR.

Toutefois, des conditions cumulatives (à savoir la légalité d'une telle décision, la résistance des occupants illégaux et la proportionnalité des moyens utilisés) doivent être réunies. L'OIPR dispose également de l'action répressive ${ }^{346}$ devant le juge ou de l'action civile en expulsion des occupants sans titre des aires protégées.

En somme, le législateur ivoirien fait passer les aires protégées du domaine privé de l'État à son domaine public en vue de leur assurer une meilleure protection juridique. C'est également ce souci de protection des aires protégées qui justifient le renforcement du droit pénal des aires protégées.

\section{SECTION II : L'évolution du droit pénal des aires protégées}

Le droit pénal est l'ensemble des règles de droit ayant pour but de sanctionner des infractions, c'est-à-dire des comportements qu'il interdit et réprime par des peines prévues à cet effet, s'ils sont imputables à leurs auteurs ${ }^{347}$. C'est ainsi qu'en l'absence de textes, on pourrait toutefois définir le droit pénal des aires protégées comme l'ensemble des règles qui prévoient les infractions contre les aires protégées et les sanctions correspondantes. Il a donc

\footnotetext{
${ }^{343}$ L'exécution d'office est: "la faculté qu'a l'Administration d'accomplir elle-même, par la contrainte, les actes d'exécution de la décision administrative ». L'exécution d'office s'applique dans trois (3) cas, à savoir lorsqu'elle est expressément autorisée par la loi ou lorsqu'il y a urgence ou encore lorsqu'il n'y a pas d'autres voies de droit. Quant à l'exercice de cette prérogative de l'Administration elle-même, elle nécessite trois conditions cumulatives. D'abord, l'acte à exécuter doit avoir sa source dans un texte précis. Ensuite, son inexécution doit résulter de la résistance de l'administré. Enfin, les mesures utilisées par l'Administration pour vaincre la résistance de l'administré doivent être proportionnelles à sa résistance. Sur ces différents points relatifs à l'exécution d'office, voir DÉGNI-SÉGUI (René), Droit administratif général : L'organisation administrative (t. 1), $4^{\text {ème }}$ édition, op.cit., pp. 334-336.

${ }^{344}$ Selon LAUGINIE (Francis), "Le Parc National de Taï est l'un des derniers vestiges du bloc originel des forêts denses d'Afrique occidentale ». Voir LAUGINIE (Francis), Conservation de la nature et des aires protégées en Côte d'Ivoire, op. cit., p. 241.

${ }^{345} \mathrm{Au}$ Parc National d'Azagny (PNA), des espèces animales telles que le Sitatunga Tragelaphus spekii, la Panthère noire Panthera pardus, le Lamantin Trichechus senegalensis se raréfient (voir Idem, p. 90. De même, au Parc National de la Marahoué, les populations de singes, hormis les babouins se font de plus en plus rares en raison de leur braconnage intensif (voir Idem, p. 182).

${ }^{346}$ En effet, la loi de 2002 sur les PNR prévoit des infractions relatives aires protégées que nous développerons dans la Section 2 du présent chapitre (ci-dessous).

${ }^{347}$ Voir GUINCHARD (Serges), DEBARD (Thierry) (dir.), Lexiques des termes juridiques, $19^{\mathrm{e}}$ édition, op.cit., p. 31 ; DESPORTES (Frédéric), GUNEHEC (Francis Le), Droit pénal général, 14 édition, Paris, Economica, 2007, p. 3 ; MERLE (Roger), VITU (André), Traité de droit criminel (t. 1), $7^{\mathrm{e}}$ édition, Paris, Cujas, 1997, p. 211.
} 
pour objet de protéger les aires protégées contre toutes menaces ou troubles d'origine anthropique.

L'évolution du droit pénal des aires protégées veut dire en l'espèce que les anciennes dispositions dudit droit se sont renforcées ou qu'il s'est enrichi de nouvelles règles de répression. La lecture conjointe de la loi de 2002 sur les PNR et de l'ancienne législation régissant ces milieux naturels ${ }^{348}$ renseigne sur l'évolution du droit pénal des aires protégées.

Celle-ci se traduit par le durcissement des textes de répression des atteintes contre les aires protégées (Paragraphe 1). De même, le renforcement des pouvoirs de répression du personnel en charge des aires protégées est expressif de l'évolution du droit pénal des aires protégées (Paragraphe 2).

\section{Paragraphe 1 : Le durcissement des textes de répression des atteintes contre les aires protégées}

Par l'expression "durcissement des textes de répression", il faudrait entendre le fait de rendre davantage sévères les règles relatives aux infractions contre les aires protégées. En l'espèce, la loi de 2002 sur les PNR élargit le champ des éléments matériels et/ou alourdit les peines des infractions contre les aires protégées prévues par la législation en vigueur de 1960 à $2002^{349}$ (A). Elle définit également de nouvelles infractions contre les aires protégées (B).

\section{A - L'élargissement du champ des éléments matériels et l'alourdissement des peines des infractions prévues par la législation initiale des aires protégées}

Pour une meilleure compréhension des développements à venir, il convient de présenter au préalable ces infractions dont les textes de répression ont été renforcés par la loi de 2002 sur les PNR, à travers un tableau comparatif qui sera assorti d'un commentaire.

\footnotetext{
${ }^{348}$ Il s'agit de la loi ${ }^{\circ}$ 65-255 du 4 août 1965 relative à la protection de la faune et à l'exercice de la chasse (JORCI n ${ }^{\circ} 41$ du 26 août 1965, p. 898); de la loi n $65-425$ du 20 décembre 1965 portant Code forestier (JORCI $n^{\circ} 3$ du 13 janvier 1966, p. 38) et du Décret 66-433 du 15 septembre 1966 portant statut et réglementation de la procédure de classement des Réserves naturelles intégrales ou partielles et des Parcs Nationaux (JORCI n50 du 27 octobre 1966, p. 1419).

${ }^{349}$ Nous désignons ainsi les infractions courantes dans les aires protégées que sont: les délits de chasse, d'exploitation agricole et d'exploitation forestière.
} 


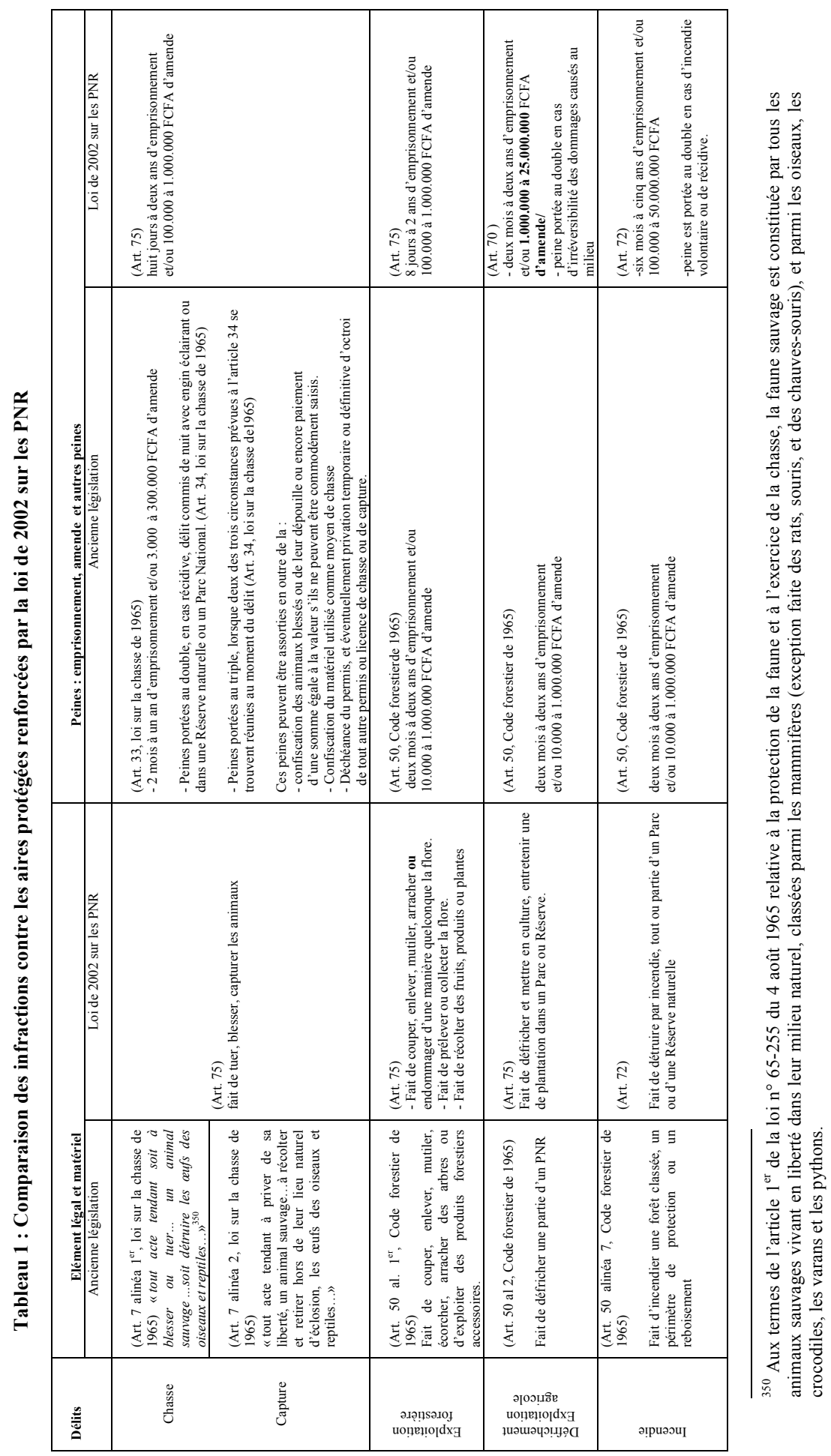




\section{$\underline{\text { Commentaires }}$}

Elaboré sur la base des dispositions pénales de loi précitée de 1965 portant Code forestier ; de la loi précitée de 1965 relative à la protection de la faune et l'exercice de la chasse (ancienne législation); et de la loi précitée de 2002 sur les PNR, le tableau 2 ci-dessus traite des infractions relatives aux aires protégées. Exclusivement des délits ${ }^{351}$, ces infractions renforcées par le législateur sont présentées dans le tableau 2 par comparaison avec les anciennes formulations des dispositions qui les consacraient.

Il s'agit du délit de chasse (1), du délit d'exploitation agricole (2) et du délit d'exploitation forestière (3) et enfin du délit d'incendie commis dans les Parcs Nationaux et Réserves naturelles (4).

\section{Le délit de chasse dans les Parcs Nationaux et Réserves naturelles}

Selon le dictionnaire Larousse français, "chasser" signifie poursuivre un animal en vue de le tuer ou de le capturer vivant ${ }^{352}$. Si les éléments matériels du délit de chasse dans les aires protégées demeurent les mêmes, tant dans l'ancienne législation que dans la nouvelle (loi de 2002 sur les PNR) ${ }^{353}$, il reste qu'il en va différemment des peines (emprisonnement et amende) qui, elles, sont renforcées. En effet, la peine d'emprisonnement qui était de deux mois à un $\mathrm{an}^{354}$ passe de huit jours à deux ans ${ }^{355}$. Autrement dit, le maximum légal de la peine

\footnotetext{
${ }^{351}$ Selon l'article 3 de loi n81-640 du 31 juillet 1981 instituant le Code pénal (JORCI n 1 Numéro Spécial du lundi 4 janvier 1982, p. 1), modifiée notamment par la loi nº5-522 du 6 juillet 1995 et la loi n 2015-134 du 9 mars 2015), il y a trois (3) catégories d'infractions, à l'instar de celles classiquement admises. Ce sont les crimes, les délits, et les contraventions. En effet, selon cet article, est qualifiée crime toute infraction passible d'une peine privative de liberté perpétuelle ou d'une peine d'emprisonnement supérieure à 10 ans. S'agissant des contraventions, ce sont des infractions passibles d'une peine privative de liberté inférieure ou égale à deux mois, d'une amende inférieure ou égale à 360.000 francs CFA ou l'une de ces deux (2) peines seulement. Les délits sont des infractions passibles d'une peine privative de liberté ou d'amende autre que les précédentes.

${ }^{352}$ Voir JEUGE-MAYNART (Isabelle) (dir.), Le Petit Larousse illustré, op. cit., p. 186.

${ }^{353} \mathrm{Il}$ ressort aussi bien de l'ancienne législation sur les aires protégées (art. 7, loi sur la chasse, 1965) que de la nouvelle (art. 75, loi de 2002 sur les PNR) que la chasse consiste à une atteinte à l'intégrité physique (blesser, tuer,) ou à la capture d'espèces animales sauvages ; ainsi qu'à la destruction ou la destruction de leurs œufs de leur biotope.

${ }^{354}$ Selon l'article 33 de la loi précitée de 1965 sur la protection de la faune et la législation de la chasse qui était applicable au délit de chasse dans les Parcs Nationaux et Réserves, cette infraction est punie : «D'une amende de 3.000 à 300.000 Francs $\boldsymbol{C F A}$ et d'un emprisonnement de deux mois à un an ou de l'une de ces deux peines seulement...» (souligné par nous). L'article 34 poursuit en mentionnant: "Les peines d'amende ou d'emprisonnement prévues à l'article précédent sont portées au double lorsque l'une des trois conditions suivantes est réalisées:

$1^{\circ}$ Délit commis dans une Réserve ou Parc National;

$2^{\circ}$ Délit commis de nuit avec engin éclairant;

$3^{\circ}$ Récidive. $\gg$.

${ }^{355}$ L'article 75 de la loi précitée de 2002 sur les PNR dispose : «Est puni d'une peine d'emprisonnement de 100.000 à 1.000.000 de francs CFA et d'un emprisonnement de huit jours à deux ans ou de l'une de ces deux peines seulement, quiconque, dans un Parc National ou une Réserve naturelle, sans l'autorisation préalable du directeur du Parc ou de la Réserve naturelle:

-Prélève ou collecte la flore ;

-Récolte des plantes, fruits ou produits;

-Coupe, arrache, enlève ou endommage d'une manière quelconque la flore;
} 
d'emprisonnement a été relevé d'un an de plus. Quant à la peine d'amende qui était de 3.000 à 300.000 francs CFA, sous l'empire de l'article 33 la de loi de 1965 sur la protection de la nature et l'exercice de la chasse, elle va dorénavant de 100.000 à 1.000.000 francs CFA, selon l'article 75 la loi de 2002 sur les PNR ci-dessous mentionné. En d'autres termes, pour fixer le minimum légal de l'amende de ce délit (100.000 francs CFA), le législateur a multiplié plus de 33 fois l'ancienne amende (3.000 francs CFA) tandis que le maximum légal de cette amende (1.000.000 francs CFA) représente plus de 3 fois celui prévu par l'ancienne législation (300.000 francs CFA).

Le constat qui en résulte est que ces peines relatives au délit de chasse dans les aires protégées que prévoit la loi de 2002 sur les PNR sont plus sévères que celles anciennement en vigueur sous l'empire de la loi précité de 1965 sur l'exercice de la chasse. Il nous semble que les raisons qui ont motivé le législateur à renforcer les peines relatives au délit de chasse dans les Parcs Nationaux se résument à dissuader les braconniers. En effet, en dépit de la fermeture de la chasse en Côte d'Ivoire depuis $1974^{356}$, le braconnage est une infraction courante en Côte d'Ivoire, en général et particulièrement dans les aires protégées. Une étude sur l'évaluation de l'efficacité de la gestion des aires protégées montrent que le braconnage est une infraction courante qui constitue une menace pour la biodiversité animale dans l'ensemble des aires protégées de Côte d'Ivoire ${ }^{357}$. De même, un rapport de 2009 sur l'état de la conservation des Parcs Nationaux et Réserves de Côte d'Ivoire révèle que « Le braconnage constitue la principale pression dans tous les Parcs. Il s'intensifie car la viande de brousse est très prisée par les populations rurales et urbaines. Il est le fait des allogènes, des allochtones, des populations autochtones et des braconniers professionnels. ${ }^{358}$.

Par ailleurs, il apparaît que c'est pour atteindre le même objectif de dissuasion des délinquants que le législateur renforce les dispositions pénales applicables au délit d'exploitation forestière dans les aires protégées.

\section{Le délit d'exploitation forestière dans les Parcs Nationaux et Réserves naturelles}

Les aires protégées font couramment l'objet d'exploitation forestière malgré le fait qu'elle soit constitutive d'infraction ${ }^{359}$. L'étude sur l'évaluation de l'efficacité de la gestion des aires protégées ivoiriennes conduite par l'UICN en 2008 a montré que les Parcs Nationaux et Réserves de Côte sont l'objet d'exploitation forestière dans l'ensemble. Par exemple au Parc National du Mont Péko, l'exploitation forestière est un véritable problème.

\footnotetext{
-Tue, blesse, pêche ou capture les animaux. » (souligné par nous).

${ }^{356}$ Voir l'arrêté n`003/ SEPN/ CAB du 20 février 1974 portant fermeture de la chasse (JORCI n ${ }^{\circ} 13$ du 28 mars 1974, p. 487).

${ }^{357}$ Voir UICN/BRAO, Évaluation de l'efficacité de la gestion des aires protégées : Parcs et Réserves de Côte d'Ivoire, 2008, p. 21.

${ }^{358}$ Voir République de Côte d'Ivoire, Ministère de l'Environnement et des Eaux et Forêts/Office Ivoirien des Parcs et Réserves, État de conservation des Parcs et Réserves de Côte d'Ivoire, op. cit., p. 6.

${ }^{359}$ Voir UICN/BRAO, Évaluation de l'efficacité de la gestion des aires protégées : Parcs et Réserves de Côte d'Ivoire, 2008, p.27
} 
La situation est pareille dans les Parcs Nationaux de la Marahoué, de la Comoé, du Mont Sangbé, les Réserves naturelles du Mont Nimba, la Réserve de faune d'Abokouamékro, la Réserve de Lamto où l'exploitation forestière représente une menace ${ }^{360}$.

C'est à juste titre que le législateur ivoirien a toujours incriminé ce fait. En effet, sous l'empire de l'article 50 alinéa $1^{\text {er }}$ du Code forestier de $1965^{361}$, l'élément matériel du délit d'exploitation forestière dans les aires protégées consistait à couper, enlever, mutiler, écorcher, arracher des arbres ou à exploiter des produits forestiers accessoires ${ }^{362}$. Aussi dans le souci de renforcer la protection juridique de ces aires protégées, le législateur ivoirien a-t-il élargi le champ des éléments matériels de ce délit. En effet, en plus de reconduire les éléments matériels "fait de couper, enlever, mutiler, arracher" (la flore), l'article 75 de la loi de 2002 sur les $\mathrm{PNR}^{363}$ fait mention de nouveaux éléments matériels. Ce sont le "fait d'endommager d'une manière quelconque la flore, de prélever ou collecter la flore, de récolter des fruits, produits ou plantes" dans les aires protégées. Ces éléments matériels viennent s'ajouter à la liste de ceux précédemment définis par le Code forestier de 1965 et les préciser davantage. En effet, "prélever ou collecter la flore, récolter des fruits, produits ou plantes" explicite la notion d'exploitation des produits forestiers accessoires, prévue à l'article 50 alinéa $1^{\text {er }}$ du Code forestier de 1965.

À l'analyse, pour élargir le champ des éléments matériels du délit d'exploitation forestière dans les Parcs Nationaux et Réserves, le législateur procède de diverses manières. Premièrement, il est à noter qu'il fait usage du vocable "flore" qui est plus globalisant que la notion d'arbre et de produits forestiers accessoires, en ce sens qu'il désigne l'ensemble des espèces végétales qui croissent dans un milieu ou une région donnée ${ }^{364}$. Deuxièmement, le

\footnotetext{
${ }^{360}$ Pour ces exemples, voir UICN/BRAO, Évaluation de l'efficacité de la gestion des aires protégées : Parcs et Réserves de Côte d'Ivoire, 2008, p.27. D'autres travaux relativement plus récents confirment l'évaluation de l'UICN en ce qui concerne l'exploitation forestière dans les Parcs Nationaux de la Comoé (Voir République de Côte d'Ivoire, Ministère de l'Environnement et des Eaux et Forêts/Office Ivoirien des Parcs et Réserves, État de conservation des Parcs et Réserves de Côte d'Ivoire, op. cit., pp.11-12); du Mont Péko et du Mont Sangbé (voir et OIPR/ AFD, Réalisation d'une étude sur l'état de la biodiversité de quatre Parcs Nationaux de Côte d'Ivoire. Azagny, Marahoué, Péko, Sangbé,, op. cit. p. 44).

${ }^{361}$ Aux termes de cet article, est puni «...d'une peine de deux mois à deux ans d'emprisonnement et d'une amende de 10.000 à 1.000.000 de francs CFA ou de l'une de ces deux peines seulement...Quiconque coupe, enlève, mutile, écorche ou arrache des arbres ou exploite des produits forestiers accessoires, dans un but commercial ou non, sans y être autorisé...".

${ }^{362}$ Les produits forestiers accessoires désignent les produits forestiers autres que les arbres (lianes, raphias, fruits...).Voir QUEBEC, Ministère des Ressources Naturelles de la Faune et des Parcs, Direction du développement de l'industrie des produits forestiers, Direction du développement de l'industrie des produits forestiers, Aperçu des produits forestiers non ligneux, op. cit., p. 1 ; UICN/PACO, Retombées économiques des aires protégées d'Afrique de l'Ouest, op. cit., p. 15.

${ }^{363} \mathrm{~L}$ 'article 75 de la loi précitée de 2002 sur les PNR dispose : «Est puni d'une peine d'emprisonnement de 100.000 à 1.000.000 de francs CFA et d'un emprisonnement de huit jours à deux ans ou de l'une de ces deux peines seulement, quiconque, dans un Parc National ou une Réserve naturelle, sans l'autorisation préalable du directeur du Parc ou de la Réserve naturelle :

-Prélève ou collecte la flore ;

-Récolte des plantes, fruits ou produits ;

-Coupe, arrache, enlève ou endommage d'une manière quelconque la flore ;

-Tue, blesse, pêche ou capture les animaux. » (souligné par nous).

${ }^{364}$ Voir JEUGE-MAYNART (Isabelle) (dir.), Le Petit Larousse illustré, op.cit., p. 426.
} 
législateur termine l'énumération des éléments matériels du délit d'exploitation forestière dans les Parcs Nationaux et Réserves naturelles par l'emploi de l'expression "endommager d'une manière quelconque la flore", en vue d'incriminer toutes les formes ou techniques d'atteintes possibles à la végétation des aires protégées.

Outre, l'extension de la liste des éléments matériels du délit d'exploitation dans les Parcs Nationaux et Réserves naturelles, le législateur renforce les peines relatives à ce délit. En effet, bien que le maximum légal de la peine d'emprisonnement demeure de deux ans, tant sous l'empire du Code forestier de 1965 (art. 50) que celle de la loi de 2002 sur les PNR (art. 75), les peines d'amende qui étaient de 10.000 à 1.000 .000 francs CFA sont désormais fixées de 100.000 à 1.000.000 FCFA. Autrement dit, le minimum de l'amende est relevé à 100.000 francs CFA, soit dix (10) fois celui du montant du minimum de l'ancienne amende. Par cet acte, le législateur entendrait dissuader les auteurs de cette infraction.

Par ailleurs, le délit d'exploitation agricole dans les aires protégées a été renforcé par la loi de 2002 sur les PNR.

\section{Le délit d'exploitation agricole dans les Parcs Nationaux et Réserves naturelles}

Le droit positif des aires protégées évoque le délit d'exploitation agricole ${ }^{365}$ dans les Parcs Nationaux et Réserves sans le définir. Toutefois, l'exploitation agricole dans les aires protégées pourrait s'entendre du fait de défricher et mettre en culture ou d'entretenir une plantation en ces lieux ${ }^{366}$.

Le renforcement des textes de répression relatifs au délit d'exploitation agricole dans les Parcs Nationaux et Réserves naturelles intervient à deux (2) niveaux, à savoir la qualité de l'auteur de l'infraction et la peine d'amende. En effet, tandis que l'article 50 du Code forestier de 1965 ne punissait que l'auteur matériel ${ }^{367}$ du délit de défrichement en vue d'une culture ou

\footnotetext{
${ }^{365}$ Art. 70 alinéa 1 er de la loi de 2002 sur les PNR.

${ }^{366}$ Cette infraction est reconduite par la loi de 2002 sur les PNR, dans la mesure où elle est une des plus courantes commises dans les aires protégées. En effet, ces forêts sont convoitées par les populations riveraines, essentiellement paysannes, en raison de la saturation foncière dans le domaine rural et de la fertilité de leurs sols propices à l'agriculture. Si sous l'empire du Code forestier l'incrimination de l'exploitation à but agricole dans les Parcs Nationaux et Réserves opérait une distinction entre le fait de défricher simplement des terres et celui de les défricher en vue d'une mise en culture (art. 50), la loi de 2002 sur les PNR ne fait plus cette distinction. Mieux, elle incrimine directement l'exploitation agricole. En effet, de façon présomptive et dans la pratique, le défrichement dans les aires protégées est fait pour être suivi d'une mise en culture.

${ }^{367}$ L'auteur d'une infraction est la personne à qui peut être imputée la commission ou la tentative de celle-ci, pour avoir personnellement réalisé ses éléments constitutifs (voir CORNU (Gérard) (dir.), Vocabulaire juridique, $4^{\text {ème }}$ éd. (Association Henri Capitant), op. cit., p. 90). La doctrine s'accorde à nommer celui qui commet matériellement l'infraction "auteur matériel" et "auteur moral" celui qui incite un tiers à la commettre. Voir notamment DESPORTES (Frédéric), GUNEHEC (Francis Le), Droit pénal général, $14^{\mathrm{e}}$ édition, op. cit., p.473 ; BOULOC (Bernard), Droit pénal général, $19^{\mathrm{e}}$ édition, Paris, Dalloz, 2005, p. 264-267.
} 
non ${ }^{368}$, l'article 70 alinéa $1^{\text {er }}$ de la loi de 2002 sur les PNR prévoit et punit deux (2) sortes d'auteurs. Il s'agit de l'auteur matériel du délit d'exploitation agricole dans les Parcs Nationaux et Réserves naturelles et aussi de celui sur instruction duquel agit l'auteur matériel de cette infraction. C'est ainsi que cet article dispose : " est puni d'une amende de 1.000.000 à 25.000.000 francs et d'un emprisonnement de deux mois à deux ans ou de l'une de ces deux peines seulement, quiconque entreprend ou fait entreprendre...des exploitations agricoles dans les Parcs Nationaux et les Réserves naturelles. » (souligné par nous).

En élargissant le champ de la qualité d'auteur, le législateur entend sanctionner à la fois celui qui exploite par lui-même une aire protégée à but agricole et celui sur instruction duquel l'auteur matériel agit. Cette attitude du législateur pourrait s'expliquer par le fait que, en règle générale, les exploitants agricoles engagent une main d'œuvre.

En outre, le législateur relève le minimum et le maximum légal de l'amende du délit d'exploitation agricole qui passe respectivement de 100.000 à 1.000.000 francs CFA, sous l'empire du Code forestier de $1965^{369}$ à 1.000 .000 à 25.000.000 francs CFA actuellement ${ }^{370}$. La peine est double « si les dommages causés au milieu naturel sont irréversibles ${ }^{371} »$. En d'autres termes, le minimum et le maximum légal de ces amendes sont relevées respectivement à 10 fois et 25 fois de leur valeur en francs CFA par rapport au montant prévu sous l'empire du Code forestier de 1965.

Le relèvement du minimum et du maximum légal de l'amende de ce délit pourrait s'expliquer par le fait que ces espaces, qui ont pour vocation de conserver les ressources naturelles dans leur habitat originel, sont sujets à des infiltrations massives à but agricole ${ }^{372}$. Par conséquent, il semble qu'à travers ce durcissement des textes de répression, le législateur entendrait dissuader les auteurs de cette infraction. Qu'en est-il du délit d'incendie de Parcs Nationaux et Réserves naturelles?

\footnotetext{
${ }^{368}$ L'article 50 alinéa 2 du Code forestier de 1965 déclare qu'est puni «...d'une peine de deux mois à deux ans d'emprisonnement et d'une amende de 10.000 à 1.000 .000 de francs CFA ou de l'une de ces deux peines seulement...Quiconque, en vue d'une mise en culture ou non, effectue sans autorisation un défrichement portant sur une partie du domaine forestier classé, des périmètres de protection et des reboisements... ».

${ }^{369}$ Art. 50 alinéa 2 du Code forestier de 1965.

${ }^{370}$ Aux termes de 1'article 70 alinéa alinéa $1^{\mathrm{er}}$ de la loi de 2002 sur les PNR, «Est puni d'une amende de 1.000.000 à 25.000.000 de francs CFA et d'un emprisonnement de deux mois à deux ans ou de l'une de ces deux peines seulement, quiconque entreprend ou fait entreprendre des constructions ou des travaux de fouille, prospection, sondage ou terrassement ou des exploitations agricoles dans les Parcs Nationaux et les Réserves naturelles.

La peine est portée au double si les dommages causés au milieu naturel sont irréversibles. " (souligné par nous).

${ }^{371}$ Art. 70 alinéa 2, loi de 2002 sur les PNR.

${ }^{372}$ Voir l'Introduction de la présente thèse, le point "I- Enjeux de la protection des forêts en Côte d'Ivoire", " Cl'état des pressions anthropiques sur la biodiversité en Côte d'Ivoire", et précisément le point intitulé "2. Les pressions anthropiques sur les aires protégées en particulier ".
} 


\section{Le délit d'incendie de Parcs Nationaux et Réserves naturelles}

L'incendie se définit comme un feu violent qui consume un édifice, une forêt etc. ${ }^{373}$. En Côte d'Ivoire, les feux de brousse constituent une menace pour la conservation des aires protégées $^{374}$. Cette situation pourrait expliquer le fait que l'article 72 de la loi de 2002 sur les PNR renforce les peines applicables aux auteurs d'incendie dans les aires protégées. En effet, sous l'empire de l'ancienne législation des aires protégées, l'article 50 du Code forestier sanctionnait d'une peine d'emprisonnement de deux mois à deux ans et d'une amende de 10.000 à 1.000.000 francs CFA ou de l'une de ses deux peines seulement, l'incendie d'une aire protégée ${ }^{375}$. Depuis 2002, cette infraction est punie d'un emprisonnement de six mois à cinq ans et d'une amende de 100.000 à 50.000 .000 francs CFA ou l'une de ces deux (2) peines seulement ${ }^{376}$. Ces peines sont portées au double lorsque l'incendie est volontaire ou que l'auteur est un récidiviste ${ }^{377}$.

En plus de ces infractions, à savoir les délits de chasse, d'exploitation agricole, forestière et d'incendie dont les textes de répression sont renforcés, la loi de 2002 sur les PNR prévoit de nouvelles infractions.

\section{B - La définition de nouvelles infractions contre les aires protégées par le législateur}

Les nouvelles infractions prévues par la loi de 2002 sur les PNR sont définies à partir des différentes formes de pressions que connaissent ces milieux ou qu'ils sont susceptibles de subir, en fonction de l'évolution de la criminalité écologique. Ces pressions représentent directement ou indirectement un danger pour la conservation des aires protégées.

Ces nouvelles infractions peuvent être classées en deux (2) catégories. Il s'agit d'infractions contre les aires protégées (1) et de celles commises dans leurs zones périphériques (2).

\footnotetext{
${ }^{373}$ Voir www.larousse.fr/encyclopedie/rechercher? $q=$ incendie $\& \mathrm{t}=$ (consulté le 4 juin 2016).

${ }^{374}$ C'est par exemple le cas dans le Parc National de la Comoé et d'Abokouamékro. En effet, il ressort du rapport d'évaluation de l'efficacité de la gestion des aires protégées que les feux de brousse sont récurrents dans ces deux (2) aires protégées; et qu'ils sont le fait des populations riveraines, des transhumants et des braconniers. Voir UICN/BRAO, Évaluation de l'efficacité de la gestion des aires protégées : Parcs et Réserves de Côte d'Ivoire, 2008, op.cit., p. 28).

${ }^{375}$ Aux termes, de l'article 50 alinéa 7 du Code forestier précité de 1965, est passible : «...d'une peine de deux mois à deux ans d'emprisonnement et d'une amende de 10.000 à 1.000 .000 de francs CFA ou de l'une de ces deux peines seulement ...Quiconque, par imprudence, négligence, inattention, inobservation des règlements, cause un incendie dans une forêt classée, un périmètre de protection ou un reboisement, ou ne se conforme pas à la réglementation sur les feux... ».

${ }^{376}$ En effet, l'article 72 alinéa de la loi précitée de 2002 sur les PNR dispose : «Est puni d'une amende de 100.000 à 50.000 .000 de francs CFA et un emprisonnement de six mois à cinq ans ou de l'une de ces deux peines seulement, quiconque détruit par incendie, tout ou partie d'un Parc ou d'une Réserve naturelle. La peine est portée au double s'il s'agit d'un acte volontaire ou en cas de récidive. ").

${ }^{377}$ Art. 72 alinéa 2, loi de 2002 sur les PNR.
} 


\section{Les infractions contre les aires protégées}

Les nouvelles incriminations contre les aires protégées consacrées par la loi de 2002 sur les PNR, peuvent être classées en trois (3) catégories. Il s'agit d'infractions relatives au sol et au sous-sol des aires protégées (a), à la flore des aires protégées (b) et d'incriminations à caractère mixte $(\mathrm{c})$.

\section{a. Les infractions relatives au sol et au sous-sol}

Cette catégorie d'infractions inclut un ensemble de faits ou activités de l'Homme qui ont des effets nocifs sur le sol et le sous-sol des aires protégées. Aux termes de l'article 70 alinéa $1^{\mathrm{er}}$ de la loi précitée, les faits incriminés sont les constructions ou travaux de fouilles, prospection, sondage ou terrassement dans les aires protégées. En règle générale, ces travaux et constructions sont ceux réalisés dans le cadre de l'exploitation minière.

Leur incrimination par le législateur pourrait s'expliquer par le fait qu'étant nocifs et incompatibles avec la conservation des aires protégées, elles se développent pourtant, de plus en plus, dans ces milieux naturels ${ }^{378}$. On comprend dès lors que le législateur punisse sévèrement ces faits. Selon l'article 70 alinéa $1^{\text {er }}$ de la loi précitée de 2002 sur les PNR, les auteurs de ces faits sont passibles d'une peine d'emprisonnement allant de deux mois à deux ans et d'une amende de 1.000 .000 à 25.000.000 francs $\mathrm{CFA}^{379}$. En outre, en cas de circonstances aggravantes, c'est-à-dire lorsque les dommages causés aux aires protégées sont irréversibles, l'article 70 alinéa 2 portent au double ces peines.

La loi de 2002 sur les PNR consacre également une nouvelle infraction relative à la flore. Il s'agit de l'exploitation de bois d'œuvre et d'ébénisterie dans les Parcs Nationaux et Réserves naturelles.

\footnotetext{
${ }^{378}$ À titre d'exemples, le délit d'orpaillage occupe le $2^{\text {nd }}$ rang au nombre des délits commis dans le Parc National de Taï, après le délit de braconnage. Voir République de Côte d'Ivoire, Ministère de l'Environnement et des Eaux et Forêts/Office Ivoirien des Parcs et Réserves, Plan d'aménagement et de gestion du Parc National de Taï, Plan d'aménagement et de gestion du Parc National de Taï, op. cit.,p. 30. ; UICN/BRAO, Évaluation de l'efficacité de la gestion des aires protégées : Parcs et Réserves de Côte d'Ivoire, 2008, op. cit., p. 28.

L'orpaillage est également pratiqué dans le Parc National de la Comoé car l'un des permis de prospection minière accordée à sa périphérie « couvre les sites des Monts tingui et Gorowi et empiète sur le Parc d'environ $40 \mathrm{~km}^{2}$ ». Voir République de Côte d'Ivoire, Ministère de l'Environnement et des Eaux et Forêts/Office Ivoirien des Parcs et Réserves, État de conservation des parcs et réserves de Côte d'Ivoire, op.cit., p.11. De plus, l'orpaillage est une activité qui est exercée dans le Parc National du Mont Sangbé. En ce qui concerne ce dernier exemple, voir OIPR/AFD, Réalisation d'une étude sur l'état de la biodiversité de quatre Parcs Nationaux de Côte d'Ivoire. Azagny, Marahoué, Péko, Sangbé, Afrique Nature International, 2012 (Rapport final), p. 44.

${ }^{379}$ Aux termes de l'article 70 alinéa de la loi de 2002 sur les PNR : "Est puni d'une amende de 1.000.000 à 25.000.000 de francs CFA et d'un emprisonnement de deux mois à deux ans ou de l'une de ces deux peines seulement, quiconque entreprend ou fait entreprendre des constructions ou des travaux de fouille, prospection, sondage ou terrassement ou des exploitations agricoles dans les Parcs Nationaux et les Réserves naturelles.
}

La peine est portée au double si les dommages causés au milieu naturel sont irréversibles. " (souligné par nous). 


\section{b. L'exploitation de bois d'œuvre et d'ébénisterie dans les Parcs Nationaux et Réserves naturelles}

Les bois d'œuvre et d'ébénisterie ont permis à la Côte d'Ivoire de réaliser des performances économiques considérables ${ }^{380}$. Cependant, l'exploitation irrationnelle qui s'en

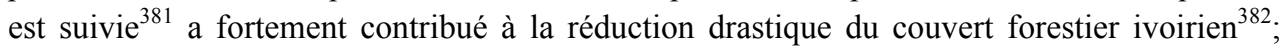
faisant des aires protégées, les derniers lieux où l'on trouve ces essences de qualité. Subséquemment, certaines populations et/ou exploitants forestiers vont les rechercher dans les aires protégées telles que les Parcs Nationaux de la Comoé ${ }^{383}$, du Mont Péko, du Mont Sangbé ${ }^{384}$. L'incompatibilité de pareilles pratiques avec les objectifs de conservation de ces milieux naturels a amené le législateur à faire de l'exploitation de bois d'œuvre et d'ébénisterie dans les aires protégées une infraction spéciale, distincte de l'exploitation forestière ${ }^{385}$.

C'est ainsi qu'il sanctionne sévèrement les auteurs du délit d'exploitation de bois d'œuvre et d'ébénisterie dans les Parcs Nationaux et Réserves naturelles. En effet, les auteurs de cette infraction s'exposent à des peines d'emprisonnement et surtout d'amende plus élevées que celles du délit d'exploitation forestière dans les Parcs Nationaux et Réserves naturelles. Tandis que l'article 75 de la loi de 2002 sur les PNR sanctionne l'exploitation forestière dans les PNR à une peine d'emprisonnement allant de 8 jours à deux ans et une amende de 100.000 à 1.000 .000 francs CFA, l'article 71 alinéa $1^{\text {er }}$ de ladite $10{ }^{386}$ punit le délit d'exploitation de bois d'œuvre et d'ébénisterie à une peine d'emprisonnement de 6 mois à 5 ans et à une amende de 5.000.000 à 50.000.000 francs CFA. L'article 71 alinéa 2 suivant

\footnotetext{
${ }^{380}$ Sur le rôle du bois dans l'économie de la Côte d'Ivoire, se référer à l'Introduction de cette étude, notamment les points "I- Enjeux de la protection des forêts en Côte d'Ivoire", " B- Les retombées socio-économiques de l'exploitation des forêts en Côte d'Ivoire", et précisément le point intitulé "1. La rentabilité économique de l'exploitation des forêts ".

${ }^{381}$ Sur la question, LAUGINIE (Francis) en parlant de la Côte d'Ivoire écrit " C'est avec l'accession à l'indépendance, en 1960, que l'activité forestière, encouragée par le faible niveau de la taxe d'abattage et des redevances d'exploitation, s'est intensément développée... L'exploitation s'est exercée selon un type minier, sans la moindre parcimonie...» Voir LAUGINIE (Francis), Conservation de la nature et des aires protégées en Côte d'Ivoire, op. cit., p.21.

${ }^{382}$ De plus 12 millions d'hectares de forêts en 1960, la Côte d'Ivoire ne dispose que d'environ deux millions d'hectares de forêts (voir Ministère d'État, Ministère du Plan et du Développement, Document stratégique de réduction de la pauvreté, op. cit., p. 63). En effet la forêt ivoirienne disparaît à un rythme de 2.500 ha par an. Voir GIROD (Jacques), L'énergie en Afrique : la situation énergétique de 34 pays de l'Afrique subsaharienne et du Nord, op. cit., p. 146.

${ }^{383}$ Voir République de Côte d'Ivoire, Ministère de 1'Environnement et des Eaux et Forêts/Office Ivoirien des Parcs et Réserves, État de conservation des parcs et réserves de Côte d'Ivoire, op. cit., pp. 11-12.

${ }^{384}$ Voir OIPR/AFD, Réalisation d'une étude sur l'état de la biodiversité de quatre Parcs Nationaux de Côte d'Ivoire. Azagny, Marahoué, Péko, Sangbé,, op. cit. p. 44.

${ }^{385} \mathrm{Si}$ l'exploitation forestière peut désigner celle des produits forestiers primaires, les grumes et notamment les bois d'œuvre et d'ébénisterie, elle renvoie également à l'exploitation des produits forestiers secondaires, plantes alimentaires et médicinales, les fruits, les écorces etc.

${ }^{386} \mathrm{~L}$ 'article 71 de la loi précitée de 2002 sur les PNR prescrit, "Est puni d'une amende de 5.000 .000 à 50.000.000 de francs CFA et d'un emprisonnement de deux mois à cinq ans ou de l'une de ces deux peines seulement, quiconque entreprend l'exploitation de bois d'œuvre et d'ébénisterie à l'intérieur d'un Parc National ou d'une Réserve naturelle.

La peine est portée au double s'il s'agit d'un acte volontaire ou en cas de récidive.
} 
porte au double les peines de cette infraction, si elle est commise volontairement et que son auteur est un récidiviste.

Par ailleurs, certaines nouvelles infractions prévues par la loi de 2002 sur les PNR sont des incriminations à caractère mixte.

\section{c. Les incriminations à caractère mixte}

À travers l'expression incrimination à caractère mixte, nous voudrions signifier que les différents faits qu'incrimine le législateur portent aussi bien sur le sol et le sous-sol ainsi que sur la flore des aires protégées. En d'autres termes, ces faits incriminés sont à la fois des facteurs de dégradation du sol, du sous-sol et de la flore. Il s'agit de la pollution de Parcs Nationaux et Réserves naturelles ou du fait de s'introduire frauduleusement dans ces aires protégées.

\section{- La pollution de Parc National et Réserve naturelle}

Le Lexique des termes juridiques définit la pollution comme étant tout « effet sur les terres, les eaux, l'atmosphère, des déversements de déchets, des produits résiduaires solides, liquides ou gazeux et l'utilisation systématique de substances chimiques qui, au-delà d'une limite de quantité...» détruisent la fertilité des sols et les espèces animales et végétales ${ }^{387}$. À l'analyse, sont constitutifs de pollution, les déversements, écoulements, rejets et dépôts de substances de toute nature ${ }^{388}$ faits soit directement ou indirectement dans les aires protégées $^{389}$. En effet, ces faits sont susceptibles de porter atteinte à l'équilibre biologique de ces milieux ${ }^{390}$ ou aux activités relatives à leur exploitation écotouristique.

Par conséquent, ils peuvent être qualifiés (de délit) de pollution de Parc National et Réserve, en raison de la classification tripartite (crime délit et contravention) des infractions précédemment évoquées $^{391}$. C'est ce qui explique que ces faits exposent leurs auteurs à des

\footnotetext{
${ }^{387}$ Voir GUINCHARD (Serges), DEBARD (Thierry) (dir.), Lexiques des termes juridiques, 19 ème édition, op.cit., p. 651.

${ }^{388}$ Pour ce qui est particulièrement des dépôts de substances de toute nature, en l'absence d'une énumération expresse, ces substances peuvent être des hydrocarbures, des déchets industriels ou encore des substances nocives d'origine chimique et bactériologique et autres, susceptibles de dégrader fortement l'écosystème aires protégées.

${ }^{389} \mathrm{C}$ 'est ce que révèle l'article 73 de la loi précitée de 2002 sur les PNR en ces termes: «...est puni d'une amende de 500.000 à 100.000 .000 de francs CFA et d'un emprisonnement de deux mois à deux ans ou de l'une de ces deux peines seulement, quiconque procède directement ou indirectement à des déversements, écoulements, rejets et dépôts de substance de toute nature susceptible de porter atteinte à l'intégrité du Parc National ou d'une Réserve naturelle ou aux activités de leur exploitation écotouristique.

La peine est portée au double s'il s'agit de substances toxiques ou en cas de récidive. ».

${ }^{390}$ Elles peuvent changer les facteurs physiques du milieu en causant une destruction des zones fragiles tels que les frayères, les niches écologiques etc.

${ }^{391}$ Selon l'article 3 nouveau du Code pénal (loi nº $81-640$ du 31 juillet 1981 instituant le Code pénal, JORCI n 1 Numéro Spécial du lundi 4 janvier 1982, p. 1, modifiée notamment par les lois n95-522 du 6 juillet 1995 et n²015-134 du 9 mars 2015):

«L'infraction est qualifiée:
} 
sanctions pénales. Aux termes de l'article 73 alinéa $1^{\text {er }}$ de la loi de 2002 sur les PNR, ce délit est puni d'une peine d'emprisonnement de deux mois à deux ans et surtout d'une peine d'amende allant de 500.000 à 100.000 .000 francs CFA, sans préjudice de l'article 98 du Code de l'environnement ${ }^{392}$. L'alinéa 2 suivant porte ces peines au double si l'auteur de l'infraction est un récidiviste ou si les substances sont toxiques.

En outre, la seconde incrimination à caractère mixte prévue par le droit des aires protégées est le fait pour une personne de s'introduire frauduleusement dans un Parc National ou une Réserve naturelle.

\section{- Le fait de s'introduire frauduleusement dans un Parc National ou une Réserve naturelle}

Toute personne qui souhaite mener des activités légales dans les aires protégées, doit satisfaire à la procédure d'accès requise, au lieu de s'y introduire frauduleusement. À l'inverse, l'intention coupable de celui qui s'introduit frauduleusement dans les aires protégées est sans équivoque. C'est ainsi que cette infraction est punie de huit jours à un an d'emprisonnement et/ou d'une amende de 20.000 à 250.000 francs CFA, aux termes de l'article 74 de la loi de 2002 sur les $\mathrm{PNR}^{393}$.

Par ailleurs, au-delà des aires protégées, les nouvelles infractions définies par le droit des aires protégées s'étendent à leurs zones périphériques.

$1^{\circ}$ ) crime ; si elle est passible d'une peine privative de liberté perpétuelle ou supérieure à 10 ans ;

$2^{\circ}$ ) contravention : si elle est passible d'une peine privative de liberté inférieure ou égale à 2 mois et d'une peine d'amende inférieure ou égale à 360.000 francs ou de l'une de ces deux peines seulement;

$\left.3^{\circ}\right)$ délit: si elle est passible d'une peine privative de liberté ou d'une peine d'amende autre que les précédentes. ». En côte d'Ivoire la monnaie nationale utilisée est le francs CFA.

${ }^{392} \mathrm{~L}$ 'article $98 \mathrm{du}$ Code de l'environnement ivoirien indique : « Est puni d'une amende de 100.000.000 de francs à 1.000.000.000 de francs et d'un emprisonnement d'un à cinq ans ou de l'une des deux peines seulement sans préjudice des sanctions administratives en vigueur, quiconque, nonobstant les dispositions spéciales des conventions internationales, procède à des déversements, immersion et incinération dans les eaux maritimes sous juridiction ivoirienne, des substances de toutes natures susceptibles :

- de porter atteinte à la santé publique et aux ressources maritimes biologiques;

- de nuire aux activités maritimes y compris la navigation et la pêche ;

- d'altérer la qualité des eaux maritimes;

- de dégrader les valeurs d'agrément et le potentiel touristique de la mer et du littoral.

L'Administration maritime peut arraisonner tout navire surpris en flagrant délit de déversement de contaminants, y compris les hydrocarbures en mer.

En cas de récidive, l'amende est portée au double et l'Administration se réserve le droit de procéder à la saisie du navire. ».

${ }^{393}$ Cet article dispose : «Est puni d'une amende de 20.000 à 250.000 francs CFA et d'un emprisonnement de huit jours à deux ans ou de l'une de ces deux peines seulement, quiconque nuit ou apporte des perturbations graves à la faune ou à la flore ou s'introduit de manière frauduleuse dans un parc national ou une réserve naturelle. ». 


\section{Les infractions spécifiques aux zones périphériques des aires protégées}

Les principales causes de dégradation des aires protégées résultent des pressions que les communautés locales exercent directement sur ces forêts ${ }^{394}$. De plus, il est possible que certains aménagements opérés dans leur cadre de vie constituent également une menace pour la conservation durable des aires protégées.

Il semble que ce soit la raison pour laquelle la loi de 2002 sur les PNR prévoit des restrictions relatives à la réalisation de certains travaux dans les zones périphériques des aires protégées. En effet, l'article 17 de la loi sus-citée, en ses alinéas 2 et 3, soumet à une étude d'impact environnemental, tous les travaux dans la zone périphérique des aires protégées dont la réalisation requiert l'autorisation préalable de l'OIPR. Ce sont :

- les travaux miniers, industriels, de carrière, de lotissement ou d'équipement touristique, de fouille archéologique ;

- les réalisations d'infrastructures linéaires que sont les oléoducs, gazoducs, lignes électriques, routes.

La transgression de cette prescription légale est un délit, au sens de l'article 76 de la loi de 2002 sur les PNR. Les sanctions y relatives sont prévues par l'article 88 de la loi portant Code de l'environnement ${ }^{395}$. Il s'agit de la suspension ou de l'arrêt de ces travaux par la fermeture des établissements qui les réalisent, sans préjudice des mesures de réparation des dommages causés.

Les menaces que représentent ces différentes atteintes contre les aires protégées ont amené le législateur à renforcer les compétences pénales du personnel en charge de ces milieux naturels.

\section{Paragraphe 2 : Le renforcement des compétences pénales du personnel en charge des aires protégées}

Le renforcement des compétences pénales du personnel en charge des aires protégées s'opère par l'attribution de la qualité d'officiers de Police judiciaire (OPJ) à certains membres dudit personnel (A). Ce statut d'officiers de Police judiciaire élargit les pouvoirs de répression des personnels des aires protégées qui en dotés (B).

\footnotetext{
${ }^{394}$ Voir en Introduction (de cette étude), le point "I- Enjeux de la protection des forêts en Côte d'Ivoire", " Cl'état des pressions anthropiques sur la biodiversité en Côte d'Ivoire", et précisément le point intitulé "2. Les pressions anthropiques sur les aires protégées en particulier ".

${ }^{395}$ En effet, l'article 76 de la loi précitée fait un renvoi à l'article 88 du Code de l'environnement en ce qui concerne la sanction de ce délit.
} 


\section{A - Le mécanisme du renforcement des pouvoirs de répression des gestionnaires des Parcs et Réserves : l'attribution de la qualité d'officier de Police judiciaire à certains personnels de l'OIPR}

L'officier de Police judiciaire (OPJ) est un auxiliaire du procès pénal. Il a pour mission de contribuer à l'administration de la justice ${ }^{396}$. La qualité d'OPJ est, par conséquent, l'ensemble des compétences que la loi reconnaît à un individu qui est chargé de constater les infractions à la loi pénale, d'en rassembler les preuves et de rechercher les auteurs, tant qu'une information n'est pas ouverte ${ }^{397}$. Le cas échéant, l'OPJ exécute les délégations des juridictions d'instruction et défère à leurs réquisitions.

Dans le droit des aires protégées, c'est l'article 38 de la loi de 2002 sur les PNR qui attribue la qualité d'OPJ à certains membres de l'Office Ivoirien des Parcs et Réserves (OIPR). Cet article allonge la liste des OPJ énumérées à l'article 16 du Code de procédure pénale ivoirien $(\mathrm{CPP})^{398}$. Ainsi, ont qualité d'OPJ à l'OIPR :

- le Directeur Général de l'OIPR ;

- les Directeurs des Parcs et Réserves ou groupement de Parcs et Réserves ;

- les agents des Eaux et Forêts appartenant au corps des ingénieurs détachés auprès d'un Parc ou d'une Réserve.

L'attribution de la qualité d'OPJ à certains membres du personnel de l'OIPR a une conséquence sur leur protection.

\footnotetext{
${ }^{396}$ Voir CORNU (Gérard) (dir.), Vocabulaire juridique, $4{ }^{\text {ème }}$ éd. (Association Henri Capitant), op. cit., p. 608.

${ }^{397} \mathrm{~L}$ 'information judiciaire est la phase de la procédure pénale qui précède celle jugement et au cours de laquelle le juge d'instruction, en personne, procède à plusieurs actes d'enquête judiciaire en vue de la manifestation de la vérité ou lorsque, dans le même but, la police judiciaire exécute ses délégations et défère à ses réquisitions, après que le procureur ait jugé opportun de mettre l'affaire en instruction, c'est-à-dire de rechercher davantage d'éléments à la manifestation de la vérité.

${ }^{398}$ En droit ivoirien, c'est l'article 16 du CPP qui énumère la liste des officiers de Police judiciaire. En effet, cet article dispose que sont OPJ :

«- les procureurs de la République et leurs substituts;

- les juges d'instruction;

- les juges de section;

- les maires et leurs adjoints;

- les directeurs de police;

- les commissaires de police;

- les officiers de police;

- les inspecteurs nommés OPJ par arrêté du Garde des Sceaux, Ministre de la Justice, sur proposition du Ministre de l'intérieur et du procureur général ;

- les officiers et gradés de la Gendarmerie;

- les sous-officiers de la Gendarmerie, commandants de brigade ou chef de poste;

- les sous-officiers de la Gendarmerie ayant satisfait aux épreuves de l'examen d'OPJ et nominativement désignés dans les conditions déterminées par décret;

- les officiers de la garde républicaine:

- les sous-officiers de la garde républicaine nommés par arrêté du Garde des Sceaux, sur proposition individuelle du directeur des forces publiques nationales et exerçant les fonctions de commandant de peloton ou d'adjoint à un commandant de peloton de la garde républicaine ».
} 


\section{B - Les conséquences de l'élargissement des pouvoirs de répression du personnel en charge des aires protégées sur leur protection}

L'attribution de la qualité d'OPJ à ces membres du personnel de l'OIPR a élargi les compétences pénales du personnel en charge de la répression des infractions contre les aires protégées. Dorénavant, en plus de rechercher et de constater les infractions contre les aires protégées par procès-verbal, le personnel de l'OIPR en charge de la répression des infractions contre les aires protégées a le pouvoir de mener des enquêtes de police judiciaire. Cela signifie qu'en cas de commission d'une infraction, ce personnel a compétence pour rassembler les preuves et rechercher les auteurs en vue de les mettre à la disposition de la justice, tant qu'une information n'est pas ouverte (art. 53 et 74 du CCP). Le cas échéant, il exécute les délégations du juge d'instruction et défèrent à ses réquisitions.

À l'analyse, le statut d'OPJ conféré à certains membres du personnel de l'OIPR a des conséquences notables sur la protection des aires protégées. En effet, suivant que l'infraction commise sur la territorialité des aires protégées est actuelle ou éloignée dans le temps, l'OPJ de l'OIPR pourra soit procéder à une enquête de flagrant délit, soit à une enquête préliminaire.

Le flagrant délit est une infraction constatée pendant sa commission où dans un temps très voisin de l'infraction ${ }^{399}$. L'enquête de flagrant délit est donc l'ensemble des opérations menées par un OPJ lorsqu'une infraction est en train de se commettre ou dans un temps voisin de l'action en vue de la constater, d'en rassembler les preuves et arrêter les auteurs ${ }^{400}$.

Dans le cas contraire, c'est une enquête préliminaire que mène l'OPJ en cas de commission d'une infraction. Prévue par les articles 74,75 et 76 du CPP, l'enquête préliminaire est une enquête diligentée par un OPJ soit d'office soit sur instruction du parquet, en vue d'obtenir les premiers éléments sur une infraction commise dans un Parc ou une Réserve. Elle permet au procureur de la République de décider de l'opportunité de la poursuite. Elle a donc lieu lorsqu'une information n'est pas ouverte. Au cours de l'accomplissement de l'enquête préliminaire, les OPJ de l'OIPR peuvent se transporter sur les lieux de l'infraction dont ils ont connaissance afin de la constater ${ }^{401}$.

\footnotetext{
${ }^{399}$ Art. 53 du CPP ivoirien.

${ }^{400} \mathrm{~L}$ 'analyse interprétative de l'article 53 du CPP nous amène à conclure qu'il y a infractions flagrantes dans les aires protégées, dans plusieurs hypothèses :

- lorsque l'infraction se commet actuellement c'est-à-dire que le délinquant est surpris dans son action illégale par les agents de l'OIPR, ou toute personne (dont la présence y est justifiée) qui se trouve à l'intérieur d'un Parc ou d'une Réserve ;

- lorsque l'infraction vient de se commettre c'est-à-dire que le délinquant est dans les limites d'un Parc National ou d'une Réserve naturelle géré par l'OIPR et a en sa possession la ressource animale ou végétale qu'il a prélevée ; ou encore vient de quitter les lieux lorsqu'on découvre le fait délictueux ;

- lorsque dans un temps très voisin de l'action, la personne soupçonnée est poursuivie par la clameur publique et présente des indices qui laissent croire qu'elle a participé à une infraction contre les aires protégées.

${ }^{401}$ Ces lieux sont les aires protégées pour les OPJ de l'OIPR, par interprétation de l'article 18 alinéa $1^{\mathrm{er}}$ du CPP. En effet, cet article dispose : "les officiers de police judiciaire ont compétence dans les limites territoriales où ils exercent leurs fonctions habituelles. ».
} 
En somme, l'extension de la compétence des pouvoirs en matière pénale du personnel en charge de la répression des aires protégées répond au souci de renforcer leur efficacité dans la mission de protection des aires protégées.

Outre ces innovations que le législateur ivoirien apporte au droit des aires protégées en vue de renforcer la protection de l'intégrité de ces forêts, il a édicté d'autres dispositions qui ont également pour finalité d'assurer leur protection. 


\section{CHAPITRE II : L'ÉDICTION DE RÈGLES JURIDIQUES DE GESTION À FINALITÉ PROTECTRICE DES PARCS NATIONAUX ET RÉSERVES NATURELLES}

La loi de 2002 sur les PNR et ses instruments réglementaires de mise en œuvre contiennent, en plus de celles préalablement évoquées, d'autres dispositions qui ont pour finalité la protection des aires protégées. En effet, premièrement, ces dispositions instaurent dorénavant la décentralisation technique ${ }^{402}$ comme mode d'organisation administrative du service public des aires protégées. En procédant ainsi, le législateur ivoirien entend créer un cadre de gestion de ces milieux naturels à travers lequel il est plus facile d'impliquer les autorités locales; de connaître l'opinion des populations sur leurs préoccupations locales en lien avec la conservation des aires protégées. Ainsi, les décisions prises pour la conservation des aires protégées pourraient être le mieux adaptées à cet effet. Le législateur a, par conséquent, voulu corriger les inconvénients de la centralisation administrative (qui avait cours jusqu'en 2002) en la matière.

Deuxièmement, la loi de 2002 sur les PNR permet au service public en charge de la gestion des aires protégées de mobiliser des financements extra-étatiques qui sont indispensables à la conservation de ces milieux naturels. En effet, sous l'empire de l'ancienne législation, le service public en charge des aires protégées était astreint aux subventions de l'État qui étaient insuffisants pour la conservation des aires protégées ${ }^{403}$. Cette insuffisance de la législation des aires protégées d'alors, qui a pendant longtemps compromis leur conservation $^{404}$, est dorénavant corrigée par la loi de 2002 sur les PNR.

Enfin, la loi de 2002 sur les aires protégées consacre désormais un cadre de gestion participative des aires protégées. En effet, avant l'année 2002, la gestion qui était exclusivement le fait des personnels du service des Eaux et Forêts a montré ses limites pour ce qui est de leur conservation ${ }^{405}$.

\footnotetext{
${ }^{402} \mathrm{La}$ décentralisation est le procédé technique qui consiste à conférer des pouvoirs de décision à des organes locaux, autonomes, distincts de ceux de l'État...À l'instar de la déconcentration, la décentralisation peut revêtir deux formes ou deux modalités : la décentralisation territoriale ou horizontale et la décentralisation technique ou verticale. La décentralisation territoriale : «...repose sur une base géographique. Elle consiste à conférer l'autonomie administrative, c'est-à-dire "l'autonomie dans l'organisation administrative" à une circonscription locale en lui octroyant la personnalité juridique». Quant à la décentralisation technique : "Elle consiste à conférer l'autonomie administrative à un service public en lui octroyant la personnalité juridique. Le service public ainsi personnifié devient un établissement public. " (souligné par nous). Pour ces définitions sur la décentralisation, voir DÉGNI-SÉGUI (René), Droit administratif général : L'organisation administrative (t. 1), $4^{\text {ème }}$ édition, op. cit., pp. 72-76.

${ }^{403}$ À ce sujet, voir le Titre I de la Première partie de cette étude, le Chapitre II, la Section 2 et précisément le "Paragraphe 1: L'astreinte de l'Administration des aires protégées à un financement étatique insuffisant".

${ }^{404} \hat{A}$ propos de l'insuffisance des financements pour la conservation des aires protégées, voir nos analyses antérieures, aux mêmes références que celles indiquées ci-dessus et précisément le point " B- L'insuffisance des dotations budgétaires pour la conservation des aires protégées ".

${ }^{405}$ Les analyses sur les limites des personnels (des Eaux et Forêts) à qui la charge de la gestion des aires protégées était exclusivement confiées (de 1960 à 2002) peuvent être consultées à la Première partie de cette
} 
Aussi ces nouvelles règles de gestion des aires protégées peuvent-elles être réparties en deux (2) catégories. D'une part, il y a les règles qui opèrent le renforcement des moyens juridiques de l'Administration des aires protégées (Section I). D'autre part, l'on a celles qui définissent un cadre participatif de gestion des aires protégées (Section II).

\section{SECTION I : Le renforcement des moyens juridiques de l'Administration des aires protégées}

Le renforcement des moyens juridiques de l'Administration des aires protégées s'opère, d'une part, par la réorganisation du service public en charge des aires protégées et plus précisément par la délégation de la gestion de ces sites naturels à un Établissement public national (Paragraphe 1). D'autre part, il procède de la possibilité dudit service public de recourir à des mécanismes de financements extra-étatiques en vue de leur conservation (Paragraphe 2).

\section{Paragraphe 1 : La délégation de la gestion des aires protégées à un Établissement public national}

En droit, la délégation est une opération qui consiste pour le titulaire d'une fonction à transférer son exercice à une autre ${ }^{406}$. En l'espèce, biens du domaine de l'État ${ }^{407}$, qui par conséquent est titulaire du droit de leur administration, la gestion des aires protégées est déléguée à un Établissement public national (EPN) par la loi de 2002 sur les PNR.

L'établissement public délégataire de la gestion des aires protégées est l'Office Ivoirien des Parcs et Réserves (A). Cette manière de procéder du législateur a une conséquence sur leur gestion durable qui s'analyse en termes d'autonomie d'administration de ces forêts par l'OIPR (B).

\section{A - L'Office Ivoirien des Parcs et Réserves : l’Établissement public délégataire de la gestion des aires protégées}

La délégation de la gestion des aires protégées de Côte d'Ivoire à un Établissement public national, à savoir l'Office Ivoirien des Parcs et Réserves (1), lui permet de disposer d'organes propres pour l'exercice de cette mission (2).

thèse, le Titre I, le Chapitre II, la Section I, et précisément au " Paragraphe 2 : Les limites des pouvoirs du personnel en charge de la protection des aires protégées ".

${ }^{406}$ Voir CORNU (Gérard) (dir.), Vocabulaire juridique, 4 ème éd. (Association Henri Capitant), op. cit., p. 271.

${ }^{407}$ Les aires protégées de Côte d'Ivoire ont successivement appartenu au domaine privé de l'État de 1960 à 2002 (voir le Titre I de la Première partie de cette étude, le Chapitre I, la Section II, le point intitulé " Paragraphe 1 : Les aires protégées, un élément constitutif du domaine privé de l'État de 1960 à 2002" ) et à son domaine public de depuis 2002 (voir le Titre II de la Première partie de cette thèse, le Chapitre I, la Section I, le point intitulé " Paragraphe 1 : L'incorporation des aires protégées au domaine public de l'État "). 


\section{L'Office Ivoirien des Parcs et Réserves : un établissement public national}

En droit ivoirien, c'est la loi n98-338 du 2 juillet 1998 fixant les règles générales relatives aux Établissements publics nationaux et portant création de catégories d'Établissements publics et abrogeant la loi n80-1070 du 13 septembre $1980^{408}$ qui fixe le droit commun des EPN. Aux termes de l'article $1^{\text {er }}$ de ladite loi, l'EPN est une "personne morale de droit public créée par l'État, disposant de l'autonomie financière, dont l'objet exclusif et spécialisé est de remplir une mission de service public en suivant des règles adaptées à sa mission et comportant des contraintes et prérogatives de droit public ».

Aussi l'article 3 alinéa $1^{\text {er }}$ suivant prévoit-il que pour l'accomplissement de missions spécialisées de service public ou d'intérêt général, relevant de la compétence exclusive de l'État telles que la conservation durable des aires protégées ${ }^{409}$, il peut être crée des services des Établissements publics nationaux. C'est fort de cela que le législateur délègue, en 2002, la gestion des aires protégées à un Établissement public national qu'est l'Office Ivoirien des Parcs et Réserves (OIPR). En effet, si le législateur affirme, à travers l'article 19 de la loi de 2002 sur les PNR, que la gestion des Parcs et Réserves relève d'un Établissement public national, ce sont les articles $1^{\text {er }}$ et 3 du décret portant création de l'OIPR qui précisent que cet EPN est l'OIPR ${ }^{410}$.

À l'instar de la Côte d'Ivoire, la délégation de la gestion des aires protégées par les États à des structures de gestion autonome sur les plans administratif et financier est une pratique répandue dans le monde. Au Guatemala ${ }^{411}$, avant la création de l'Institut National des Forêts (en espagnol Instituto Nacional de Bosques, en Abrégé INAB), la gestion des forêts était confiée à la Direction de la Gestion des Forêts (en espagnol Dirección General de Bosques, en abrégé DIGEBOS) qui était une Direction du ministère de l'Agriculture ${ }^{412}$. Les politiques

\footnotetext{
${ }^{408}$ JORCI, n³0, du jeudi 23 juillet 1998, p. 707.

${ }^{409} \mathrm{~L}$ 'article 2 alinéa 1 er de la loi de 2002 sur les PNR dispose ce qui suit : «L'objectif général de la présente loi est de marquer la volonté de l'État de Côte d'Ivoire d'agir dans le secteur des parcs et réserves, et permettre le renforcement de la politique globale de conservation de la nature. Les objectifs spécifiques sont de :

- Adapter le service public en charge des Parcs et Réserves aux impératifs actuels d'une gestion rationnelle;

- Conférer aux biens fonciers des parcs nationaux et réserves naturelles la domanialité publique afin de les rendre inaliénables ;

- Définir la catégorie d'établissement la mieux adaptée pour gérer, les parcs nationaux et réserves ;

- Préciser les modalités d'intervention contractuelle du Secteur privé dans la gestion des parcs et réserves ;

- Définir un mécanisme de financement à long terme des parcs et réserves. ". Si ces les objectifs de l'article 2 précité se résument à la conservation des aires protégées, l'alinéa 2 suivant précisent que ces objectifs sont d'intérêt général lorsqu'il énonce que «La réalisation de ces objectifs et d'une façon générale, la protection du patrimoine naturel sont d'intérêt général » (souligné par nous).

${ }^{410}$ En effet, ces deux derniers articles disposent respectivement : "il est créé un Établissement public de type particulier dénommé Office Ivoirien des Parcs et Réserves » qui « a pour mission, sur l'ensemble des parcs et réserves... la gestion de la faune, de la flore, et de leur biotope qui en constitue le fondement... ».

${ }^{411}$ Le Guatemala est un État de d'Amérique centrale dont la population est estimée à 4.341 .000 habitants. Sa capitale est Guatemala. Il est bordé par la mer des caraïbes, l'océan Pacifique, le Mexique, le Belize, le Honduras et le Salvador. www.larousse.fr/encyclopedie/pays/Guatemala/122493, (consulté le 2 juin 2016).

${ }^{412}$ Voir BIRNER (Regina) et WITTMER (Heidi), « Better public sector governance through partnership with the private sector and civil society: the case of Guatemala's forest administration », art. cit., p. 461.
} 
d'ajustement structurel auquel était soumis ce pays ont considérablement réduit les capacités de DIGEBOS en matière de gestion des forêts (du Guatemala). En effet, les ressources financières de cette structure en proie à la corruption étaient limitées. C'est ainsi que pour opérer une gestion durable des forêts du Guatemala, la gestion des forêts a été déléguée l'INAB qui est une structure dotée de ressources financières conséquentes et d'un fonds spécial. Créée en 1996, l'INAB a également des pouvoirs considérables sur le plan administratif et financier ${ }^{413}$.

En d'autres termes, à l'instar de l'OIPR qui est composé de membres de l'Administration du secteur public, privé et de la société civile pour la conservation des aires protégées ivoiriennes, l'INAB est un partenariat que le secteur public a noué avec le secteur privé et la société civile pour la gestion durable des forêts du Guatemala ${ }^{414}$. À ce titre, son conseil d'administration est composé d'un représentant d'entités composant le secteur public c'est-à-dire les ministères de l'Agriculture, des Finances, de l'Enseignement supérieur, des Collectivités décentralisées. Le secteur privé y est également représenté à travers un représentant de l'industrie du bois et un représentant du secteur technique. Il en est de même pour la société civile par le truchement des ONG environnementales. À travers ce partenariat, le secteur privé et la société civile participe à la prise de décision à la gestion des forêts à un haut niveau ${ }^{415}$. C'est ainsi que l'INAB constitue un cadre de gestion des forêts où les différents intérêts des parties prenantes sont équilibrés, les connaissances scientifiques partagées dans le sens de la gestion durable des forêts du Guatemala ${ }^{416}$.

En Afrique également, l'on constate de nos jours que les États délèguent la gestion des aires protégées à des structures (personnes morales) diverses autonomes sur les plans administratif et financier pour optimiser la gestion des aires protégées ${ }^{417}$. Cette délégation peut avoir différents modes.

D'une part, elle peut être faite à des collectivités publiques et plus précisément des Établissements publics comme c'est le cas en Côte d'Ivoire avec l'OIPR. Il en est de même pour l'Institut congolais pour la conservation de la nature en République démocratique du Congo (ICCN) ${ }^{418}$, l'Autorité ougandaise pour la faune (en anglais Uganda Wildlife Authority

\footnotetext{
${ }^{413}$ Idem, pp. 462-463.

${ }^{414}$ Idem, p. 461.

${ }^{415}$ Ibidem.

${ }^{416}$ Idem, pp. 465.

${ }^{417}$ Voir HATCHWELL (Matthew), « Public-private partnerships as a management option for protected areas », art. cit., p. 3 .

${ }^{418}$ Depuis l'année 2010, l'Institut Congolais pour la Conservation de la Nature (ICCN), alors en charge de la gestion des aires protégées au Congo-Kinshasa, est transformé en établissement public par l'œuvre du Décret $n^{\circ}$ 10/15 du 10 avril 2010 fixant les statuts d'un établissement public dénommé Institut Congolais pour la Conservation de la Nature en sigle « I.C.C.N. » (Journal officiel de la République démocratique du Congo ${ }^{\circ} 11$ du $1^{\text {er }}$ juin 2010). En effet, aux termes de l'article $1^{\text {er }}$ du décret sus-indiqué: «L'Institut Congolais pour la Conservation de la Nature, en sigle «ICCN », créé par la Loi n 75-023 du 22 juillet 1975, est transformé en établissement public à caractère scientifique et technique, doté de la personnalité juridique...».
} 
[UWA $]^{419}$ et de l'Autorité zambienne pour la faune en Zambie (en anglais Zambia Wildlife Authority $[\mathrm{ZAWA}]^{420}$ ).

Pour ce dernier cas (en Zambie ${ }^{421}$ ) par exemple, les aires protégées ont d'abord été gérées par l'État grâce au département ministériel en charge des aires protégées, à savoir le National Park and Wildlife Service (NPWS). Ce mode de gestion a eu l'inconvénient de voir le budget des aires protégées diminuer au cours des années 1970, en raison de la baisse de l'enveloppe budgétaire de ce département ministériel en charge des Parcs Nationaux et de la faune. Une autre insuffisance de cette gestion a été la lourdeur des procédures en matière de gestion des aires protégées de la Zambie. Pour corriger cette situation, la gestion des aires protégées a été déléguée à un organisme de gestion doté de l'autonomie administrative et financière dénommé la Zambia Wildlife Authority (ZAWA). Dans l'entendement des autorités zambiennes, l'autonomie administrative et financière de la ZAWA lui permettrait d'être plus efficace. En effet, la ZAWA est désormais juridiquement apte à adopter des règles de gestion plus souples, rapides à mettre en œuvre et dispose d'une gestion financière autonome qui permettrait une bonne conservation des aires protégées ${ }^{422}$.

En Guinée-Bissau ${ }^{423}$, la gestion des aires protégées a été déléguée, tout comme en Côte d'Ivoire, à un Établissement public doté de l'autonomie administrative et financière en vue de leur gestion durable. Il s'agit de l'Institut de la Biodiversité et des Aires Protégées (IBAP) ${ }^{424}$. Au Kenya, la gestion des aires protégées a été confiée depuis janvier 1991 à la Kenyan Wildlife Service (KWS). C'est également une structure parapublique qui a une autonomie administrative financière ${ }^{425}$. De même, au Gabon, la gestion des Parcs Nationaux et Réserves

\footnotetext{
${ }^{419}$ L'Uganda Wildlife Authority (UWA) est une structure parapublique (semi-autonome) en charge de la gestion de la faune à l'intérieur des Parcs Nationaux et des Réserves (y compris de jeu) ainsi que du suivi des activités relatives à la faune en dehors des aires protégées (notre traduction). Cette structure a été créée en 1996. Avant cette date, cette mission incombait à Uganda National Parks and the Game Department (en français Département ougandais en charge des Parcs Nationaux et des Jeux -notre traduction). Voir www.ugandawildlife.org/.

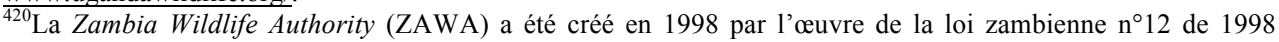
relative à la gestion et la conservation de la faune sauvage (notre traduction). Cette structure a pour mission la gestion durable des aires protégées de la Zambie en intégrant les politiques de conservation de la faune et les politiques économiques en vue du développement économique durable de ce pays. Voir Republic of Zambia, Ministry of Tourism, Environment and Natural Resources, Reclassification and effective management of protected areas system project: Current protected area categories and the institutions responsible for their management in Zambia, UNDP/GEF, pp. 14 et 9.

${ }^{421} \mathrm{La}$ Zambie est un pays de l'Afrique australe qui est limitrophe de l'Angola, la République démocratique du Congo, le Mozambique, le Malawi, la Tanzanie, la Namibie et le Zimbabwe. Elle a pour capitale Lusaka et sa population est estimée à 14.539 .000 millions d'habitants en 2013. Voir www.larousse.fr/encyclopedie/pays/Zambie/150467 (consulté le 4 juin 2015).

${ }^{422}$ Pour ces développements relatifs à l'exemple zambien de gestion des aires protégées, voir POPE (Adam), $A$ Preliminary Examination of Public Private Partnerships in National Park Management in Zambia, op. cit., p. 3.

${ }^{423} \mathrm{La}$ Guinée-Bissau est un pays de 1'Afrique de 1'Ouest qui, en 2003, comptait une population estimée à 1.704.000 d'habitants. La Guinée-Bissau est limitée au Nord par le Sénégal, au Sud et à l'Est par la Guinée et à l'Ouest par l'océan atlantique. La capitale de ce pays lusophone est Bissau. Voir www.larousse.fr/encyclopedie/pays/Guinée-Bissau/122719.

${ }^{424}$ Voir UICN, Renforcer la conservation des aires protégées d'Afrique... : synthèse de la rencontre Weotenga, UICN-PAPACO - Ouagadougou, janvier 2012, p. 16.

${ }^{425}$ Idem, pp. 17-18.
} 
est déléguée à un Établissement publique à caractère scientifique et environnementale qui dispose de l'autonomie administrative et financière, après avoir été géré par une structure interministérielle, à savoir le Conseil National des Parcs Nationaux ${ }^{426}(\mathrm{CNPN})^{427}$. Il s'agit de l'Agence Nationale des Parcs Nationaux du Gabon (ANPN).

D'autre part, dans le cadre de partenariat public-privé, les structures délégataires de la gestion des aires protégées peuvent être des personnes morales de droit privé avec des formes juridiques variées (ONG, Fondations, associations...). Par exemple, à travers des partenariats public-privé, en République démocratique du Congo, l'Institut congolais pour la conservation de la nature a délégué la gestion du Parc National de la Virunga à l'ONG African Conservation. De même, en République du Congo, la gestion du Parc National OdzalaKokoua a été déléguée au Réseau des Parcs Africains quand la Wildlife Conservation Society s'est vue confier la même responsabilité pour le Parc National Nouabalé-Ndoki.

À Madagascar, avant la création de l'Association Nationale de Gestion des Aires Protégées (ANGAP), la gestion de ces milieux naturels incombait à l'État malgache, par le truchement de la Direction des Eaux et Forêts (DEF) ${ }^{428}$. L'ANGAP est une ONG de droit privé reconnue d'utilité publique et devenue depuis le 14 novembre 2008 Madagascar National Parks (en français Les Parcs Nationaux de Madagascar-notre traduction) ${ }^{429}$. Créé en 1990, Madagascar National Parks (à la réalité, il s'agit de l'ANGAP qui a été rebaptisée ainsi le 14 novembre 2008) assure la conservation et la gestion durable du réseau national de Parcs Nationaux et Réserves de Madagascar. Les instances de gestion de Madagascar National Parks (MNP) sont: l'Assemblée générale, le Conseil d'administration et la Direction Générale. L'Assemblée générale définit les orientations de Madagascar National Parks (MNP) et le Conseil d'administration décide des projets à réaliser. Quant à la Direction Générale, elle est l'organe exécutif ${ }^{430}$. Il en résulte que c'est une structure autonome qui a la gestion du réseau d'aires protégées de Madagascar.

Au demeurant, la délégation de la gestion des aires protégées à des personnes morales distinctes de l'État s'explique par le fait qu'elles disposent de capacité de gestion et de crédit pour mobiliser les financements nécessaires à leur gestion ${ }^{431}$. C'est ainsi que pour revenir à

\footnotetext{
${ }^{426}$ Le Conseil National des Parcs Nationaux est créé par l'œuvre de l'Ordonnance n6/2002 du 22 août 2002 portant modification de certaines dispositions de la loi $n^{\circ} 16 / 2001$ du 31 décembre 2001 portant Code forestier de la République gabonaise.

${ }^{427}$ Voir www.parcsgabon.org/l-anpn/l-agence, consulté le 16 novembre 2015.

${ }^{428}$ Voir www.cons-dev.org/consdev/madagascar/MANANARA/Mananet/TEXTE/annexes/angap.html (consulté le 2 juin 2015).

429 Cette information émane d'un extrait de l'article "ANGAP devient Madagascar National Parks" de RATSIAOZO (Léa ), publié le 15 novembre 2008 et lu sur www.madagascar-tribune.com/Angap-devientMadagascar-National,9937.html et consulté le 28 aout 2015.

${ }^{430}$ Voir www.parcs-madagascar.com/madagascar-national-parks.php?Navigation=25 (consulté le 28 août 2015).

${ }^{431}$ Sur ces points, voir nos analyses afférentes dans la deuxième partie de cette étude, le Titre I, le Chapitre I, la Section II, le Paragraphe 1 et précisément le point "A- L'impact de la délégation de la gestion des aires protégées à une structure autonome sur leur conservation".
} 
l'expérience ivoirienne, l'OIPR (Établissement public national) délégataire de la gestion des aires protégées (OIPR) dispose d'organes propres à cet effet.

\section{Les organes propres de l'Office Ivoirien des Parcs et Réserves}

En déléguant en 2002 la gestion des aires protégées à un Établissement public national (Office Ivoirien des Parcs et Réserves), le législateur ivoirien a reconfiguré l'organisation du service public en charge des aires protégées comme l'atteste la figure 3 ci-dessous.

Figure 3 : Organisation du service public de la conservation des aires protégées depuis 2002

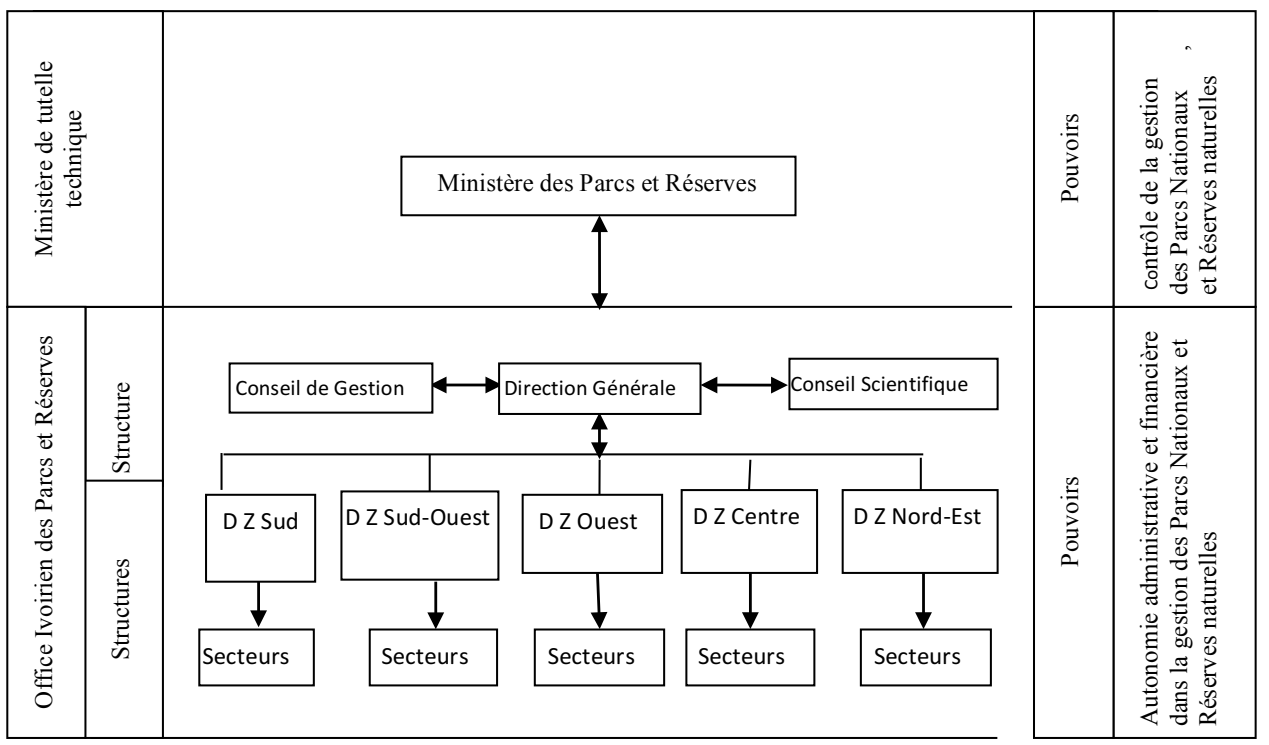

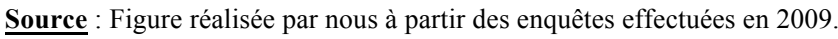

\section{Commentaires}

Depuis 2002, le service public en charge des aires protégées présente deux (2) structures indépendantes l'une de l'autre. D'une part, il y a le ministère des Parcs et Réserves à qui est dévolu dorénavant la tutelle technique de la gestion des aires protégées. D'autre part, l'on a l'Office Ivoirien des Parcs et Réserves (OIPR) qui, composé d'une structure centrale (Conseil de Gestion, Direction Générale, Conseil Scientifique) et de structures déconcentrées (Direction de Zones de PNR et Secteurs), assure la gestion des aires protégées.

En effet, selon l'article 5 du décret précité de 2002 portant création de 1'OIPR, cet EPN est doté de trois (3) organes : un Conseil de gestion, un Conseil scientifique et une Direction Générale. Aux termes de l'article 6 du décret précédemment cité, l'OIPR est 
administré par un Conseil de gestion qui est investi des pouvoirs les plus étendus ${ }^{432}$ pour agir en toutes circonstances en son nom, sous réserve des attributions du Directeur Général et des règles de tutelle. Ce conseil est composé de douze (12) membres, nommés pour une durée de trois (3) ans renouvelable une fois ${ }^{433}$. Ceux-ci proviennent aussi bien de l'Administration publique $^{434}$ qu'en dehors de ladite Administration. Il s'agit, dans ce dernier cas, de personnalités extérieures ${ }^{435}$ nommées par leur organisation respective; et dont ils assurent la représentation d'intérêts collectifs, sectoriels, locaux, financiers ou techniques ${ }^{436}$.

S'agissant du Conseil scientifique de l'OIPR, prévu par les articles 34 et s. de la loi de 2002 sur les PNR, c'est un organe consultatif à caractère scientifique. La mission du Conseil scientifique de l'OIPR est de définir avec le Conseil de gestion de ladite structure et sa Direction Générale :

- l'orientation générale des activités spécifiques de l'OIPR;

- l'élaboration et la mise en œuvre des programmes de recherches ou des objectifs scientifiques et techniques qui lui sont confiés.

Sous la réserve de sa composition ${ }^{437}$, l'article 36 de la loi de 2002 sur les PNR précise que « le Conseil scientifique est constitué de personnalités issues des milieux scientifiques et de la recherche, de nationalité ivoirienne ou étrangère, choisies pour leur compétence et leur expérience, ainsi que leur complémentarité en matière de conservation de la nature ».

\footnotetext{
432 Il s'agit, notamment :

- du vote du budget de l'OIPR et de l'arrêt de ses comptes annuels ;

- de l'approbation de la nomination et de la révision des Directeurs des Parcs et Réserves proposées par le Directeur Général de l'OIPR ;

- de l'approbation des mesures de gestion des Parcs et Réserves proposées par le Directeur Général pour la conservation durable de l'écosystème naturel et, notamment l'aménagement des Parcs et Réserves et leur ouverture au public ;

- de l'approbation du règlement intérieur des Parcs et Réserves proposé par le Directeur Général de l'OIPR ainsi que des règles de gestion du personnel qu'il élabore ;

- de l'approbation de projet de concession d'exploitation d'un Parc ou d'une Réserve.

${ }^{433}$ Art. 7, Décret de 2002 portant création de l'OIPR.

${ }^{434}$ Nommés par arrêté ministériel, les membres du Conseil de gestion issus de l'Administration publique se présentent comme suit :

- un représentant du Ministère chargé des Parcs et Réserves, Président du Conseil de gestion ;

- un représentant du Ministère chargé de l'Agriculture et des Ressources Animales ;

- un représentant du Ministère chargé de l'Economie et des Finances ;

- un représentant du Ministère chargé des Eaux et Forêts ;

- un représentant du Ministère chargé de l'Enseignement Supérieur et de la Recherche Scientifique ;

- un représentant du Ministère chargé du Tourisme et de l'Artisanat ;

- un représentant du Ministère chargé de l'Intérieur, de la Décentralisation et de l'Administration territoriale. Voir art. 8 du Décret précité de 2002 portant création de l'OIPR.

${ }^{435}$ Ce sont :

- un représentant des Fondations ayant pour objet le financement des Parcs et Réserves ;

- trois représentants des populations des zones périphériques des Parcs et Réserves ;

- un représentant des Organisations Non Gouvernementales nationales de protection de la nature.

${ }^{436}$ Art. 8, Décret précité de 2002 portant création de l'OIPR.

${ }^{437}$ Aux termes de l'article 23 du décret précité de 2002 portant création de l'OIPR : «la composition, les attributions et le fonctionnement du Conseil Scientifique sont définis par arrêté du Ministre chargé des parcs et réserves. ».
} 
En ce qui concerne la Direction Générale de l'OIPR, c'est un organe d'exécution qui a pour mission la gestion courante des aires protégées ${ }^{438}$. Elle dispose de Directions centrales ${ }^{439}$ et de structures déconcentrées, à savoir les Directions de Zones (5) subdivisées chacune en Secteurs $^{440}$.

De plus, la délégation de la gestion des aires protégées à l'OIPR conduit à leur autonomie d'administration par celui-ci.

\section{B - L'autonomie d'administration des aires protégées par l'Office Ivoirien des Parcs et Réserves}

Dans le droit des aires protégées, l'autonomie d'administration des aires protégées par l'OIPR se manifeste, d'une part, par l'autonomie administrative et financière de l'OIPR, en tant qu'Administration centrale (1). D'autre part, certains pouvoirs en matière administrative et financière reconnus aux autorités locales déconcentrées en charge de la gestion des aires protégées sont évocateurs de l'idée d'une autonomie de gestion de ces forêts (2).

\section{L'autonomie administrative et financière de la Direction Générale de l'Office Ivoirien des Parcs et Réserves}

L'Office Ivoirien des Parcs et Réserves est un Établissement public national qui gère les aires protégées depuis 2002. À ce titre, il est doté de la personnalité juridique et de l'autonomie financière.

En sa qualité de service public spécialisée, l'autonomie administrative de l'OIPR lui permet de gérer en toute autonomie les aires protégées, par le truchement de son organe d'exécution : la Direction Générale de l'OIPR. Ainsi, à travers la décentralisation technique de la gestion des aires protégées, la Direction Générale de l'OIPR est dorénavant à même de prendre des décisions adaptées aux réalités locales de gestion qu'elle est censée savoir par l'œuvre de ses services déconcentrés (les Directions de Zones et leurs secteurs).

De plus, l'autonomie administrative de l'OIPR ne le serait que de façade si elle ne reposait pas sur une autonomie financière qui lui permet de matérialiser ou d'affirmer cette

\footnotetext{
${ }^{438}$ Voir UICN/BRAO, Évaluation de l'efficacité de la gestion des aires protégées : Parcs et Réserves de Côte d'Ivoire, op. cit., p. 16.

${ }^{439}$ Les articles 11 et s. du décret de 2002 portant création de l'OIPR indique que la Direction Générale de l'OIPR se compose de :

- une Direction Technique chargée de la programmation, la coordination et du suivi des activités et de recherche de I'OIPR ;

- une Direction de l'Administration et des Ressources Humaines ;

- une Direction des Finances et de la Comptabilité ;

- deux cellules techniques autonomes, à savoir la cellule du développement informatique et du contrôle et de la planification.

${ }^{440} \mathrm{Au}$ nombre de quatorze (14) au total, les secteurs sont des entités opérationnelles chargées de la mise en œuvre des activités de conservation sur le terrain. Voir UICN/BRAO, Évaluation de l'efficacité de la gestion des aires protégées : Parcs et Réserves de Côte d'Ivoire, op. cit., p. 16.
} 
autonomie administrative. En conséquence, l'OIPR dispose désormais d'un patrimoine propre qu'il peut rentabiliser, en vue de mener à bien ses missions. En effet, contrairement au passé où l'Administration des Parcs et Réserves ne pouvait que recevoir des fonds de l'État, dorénavant l'OIPR et notamment sa Direction Générale peut, en plus des subventions de l'État, rechercher des financements extra-étatiques et recevoir directement des fonds pour l'accomplissement de ses missions ${ }^{441}$.

À l'analyse, l'autonomie administrative et financière reconnu à l'OIPR, permet de purger les lourdeurs qui caractérisent le circuit de la gestion centralisée ${ }^{442}$; lesquelles entravaient la conservation et la valorisation durable des aires protégées ${ }^{443}$. Dorénavant le service public en charge des aires protégées (OIPR) peut prendre des décisions adaptées à la conservation durable des aires protégées et mener les actions de terrain avec la célérité requise.

Par ailleurs, le décret de 2002 portant création de l'OIPR reconnaît un certain nombre de pouvoirs aux gestionnaires des aires protégées, à l'échelle locale.

\section{Les attributions administratives et financières des autorités locales déconcentrées de l'Office Ivoirien des Parcs et Réserves}

L'article 32 du décret de 2002 portant création de l'OIPR investit le Directeur d'aire protégée de pouvoirs de gestion. Ces pouvoirs de gestion revêtent des aspects financiers, d'une part; et d'administration de l'écosystème de ces aires protégées et leurs zones périphériques, d'autre part.

\footnotetext{
${ }^{441}$ Sur la question, l'article 26 de la loi de 2002 sur les PNR atteste que les ressources financières de l'OIPR sont constituées par :

- les subventions de l'État;

- les taxes parafiscales affectées ;

- les transferts des Fondations ;

- les subventions d'organismes publics autres que l'État ou privés nationaux ou internationaux ;

- les produits de l'exploitation du domaine qui lui est affecté ;

- les produits de l'exploitation légalement autorisée de la faune ou de la flore ;

- les produits de ses prestations de services;

- les redevances des activités concédées ;

- le produit des amendes et confiscations affecté par l'État ;

- les dons et legs.

${ }^{442}$ En effet, traitant des conditions de la décentralisation (La reconnaissance d'intérêts propres, l'existence d'organes propres, l'octroi de la personnalité juridique), le Professeur DÉGNI-SÉGUI (René) écrit relativement à la finalité de la dernière condition : «La personnalité juridique permet à la collectivité décentralisée d'exister juridiquement et de prendre effectivement en charge, de manière autonome, les "affaires locales"... La nouvelle personne juridique peut ainsi, en toute liberté et sans "instruction" de l'État, gérer les affaires locales par le truchement de ses propres organes. ». Voir DÉGNI-SÉGUI (René), Droit administratif général : L'organisation administrative (t. 1), $4^{\text {ème }}$ édition, op.cit., 74.

${ }^{443}$ Voir Sur la question, voir nos analyses antérieures à la Première partie de cette thèse, le Titre I, le Chapitre II, la Section 2, et précisément le "Paragraphe 2: La procédure de gestion des subventions étatiques, un obstacle à la conservation des aires protégées".
} 
Sur le plan de l'administration de l'écosystème des aires protégées et de leurs zones périphériques, l'article 32 précité mentionne que le Directeur d'aire protégée met en œuvre toutes les mesures nécessaires pour faire respecter les interdictions prévues par les articles 10, 11, 12 et 15 de la loi de 2002 sur les PNR. Il s'agit de l'interdiction de la destruction ou du prélèvement d'espèces animales, végétales; de toute exploitation minière, forestière à but agricole ou de pâturage ; et de tout acte de nature à apporter des perturbations à la faune et à la flore des aires protégées. Il en résulte que le Directeur d'aire protégée apprécie souverainement les mesures à prendre ainsi que leur opportunité, en fonction des réalités locales, pour faire observer les interdictions ci-dessus citées. Autrement dit, ce pouvoir d'appréciation souveraine du Directeur d'aire protégée (autorité déconcentrée) traduit l'idée d'une autonomie (fut-elle relative) tant dans le choix des mesures à prendre que de leur opportunité.

En outre, le même article 32 indique que dans le cadre des activités de gestion, de récréation ou d'éducation de l'aire protégée dont il a la charge, le Directeur d'aire protégée assure la préparation du plan d'aménagement et de gestion; élabore le règlement intérieur ; les règles générales régissant l'exploitation touristique et fixe les conditions et modalités de circulation, d'hébergement, de survol ${ }^{444}$.

Il est à remarquer que cette deuxième catégorie de prérogatives du Directeur d'aire protégée est exercée sous la forme de propositions ou projets adressés au Directeur Général de l'OIPR, ainsi que le dispose l'article 32 de la loi précitée. Ces propositions du Directeur d'aire protégée au Directeur Général de l'OIPR devraient tenir compte autant que possible de celles du Comité de gestion locale d'aire protégée. En effet, ce comité émet des avis consultatifs sur les aspects de la gestion des aires protégées à lui soumis par le Directeur d'aire protégée ${ }^{445}$.

\footnotetext{
${ }^{444}$ En effet, aux termes de l'article 32 du Décret précité de 2002 sur les PNR, «le Directeur du parc, de la réserve met en æuvre toutes les mesures nécessaires pour faire observer les interdictions prévues par la loi. $\dot{A}$ cet effet, le Directeur du parc ou de la réserve fait ordonner, par application des articles 10, 11, 12 et 15 de la loi $n^{\circ}$ 2002-102 du 11 février 2002, l'arrachage des plantations ainsi que la destruction des installations et impenses privées, non autorisées, à l'intérieur des limites du parc ou de la réserve considérées. Le Directeur du parc ou de la réserve:

- élabore le règlement intérieur du parc ou de la réserve dont il assure la gestion et le soumet, préalablement à son entrée en vigueur, au Directeur Général pour avis ;

- fixe les conditions et modalités de circulation, d'hébergement et, le cas échéant, de survol au-delà de 200 mètres d'altitude nonobstant la réglementation de l'aviation civile, dans le cadre des activités de gestion ou de récréation ou d'éducation ;

- surveille toute l'étendue du parc ou de la réserve, dans des conditions fixées par décret, de toute forme de capture de la faune, de destruction ou de collection de la flore, de récolte de plantes, fruits ou produits ;

- soumet à la Direction Générale, les règles générales de l'exploitation touristique du parc ou de la réserve dont il a la charge. Le Directeur d'un parc, d'une réserve, ou d'un groupement de parcs et réserves :

- assure la préparation et l'exécution du ou des plan (s) d'aménagement et de gestion des parcs ou réserves dont il a la charge ;

- négocie les contrats de terroir ;

-met en cuvre la Stratégie de gestion des populations infiltrées dans les parcs ou réserves ;

- sélectionne les concessionnaires et suit les contrats de concession. » (souligné par nous).

${ }^{445} \mathrm{~L}$ 'article $38 \mathrm{du}$ décret précité portant création de l'Office Ivoirien des Parcs et Réserves (OIPR) dispose : «Le comité de gestion locale se réunit au moins une fois par trimestre. Le comité de gestion locale donne son avis sur toutes les questions d'ordre technique, culturel ou budgétaires qui lui sont soumises, à titre consultatif, par
} 
C'est sans doute pour cette raison que ses avis sont annexés aux propositions de gestion faites par lui au Directeur Général de l'OIPR ainsi que le prescrit l'article 38 du décret précité portant création de l'OIPR. Ce dernier doit à son tour faire des propositions au Conseil de gestion de l'OIPR, dans l'esprit de celles faites par ses collaborateurs. Il en découle qu'en principe et par effet de chaîne, les avis du Directeur d'aire protégée et du Comité de gestion locale de l'aire protégée sont également analysés par le Conseil de gestion de l'OIPR.

Par ailleurs, les pouvoirs reconnus au Directeur d'aire protégée s'étendent aux aspects contractuels et budgétaires de la gestion des aires protégées. En effet, d'une part, selon l'article 32 du décret portant création de l'OIPR, le Directeur d'aire protégée est compétent pour négocier les contrats de gestion terroirs ${ }^{446}$ et sélectionner les concessionnaires et assurer le suivi des contrats de concession ${ }^{447}$. D'autre part, en matière budgétaire, il a la qualité d'ordonnateur délégué en ce qui concerne le budget secondaire de chaque Parc et Réserve $^{448}$. En d'autres termes, le Directeur d'aire protégée (autorité déconcentrée qui représente l'OIPR) a, au niveau local, la liberté de négocier les contrats de gestion de terroir et les contrats de concession d'exploitation. Ainsi, il apprécie toute de même souverainement l'objet et détermine l'autre partie contractante future. De plus, en sa qualité d'ordonnateur délégué du budget secondaire, il apprécie la liquidation et l'ordonnancement des dépenses inscrites au budget de chaque Parc et Réserve. Ces différents pouvoirs d'appréciation de l'autorité locale déconcentrée en charge de la gestion des aires protégées traduisent également une certaine autonomie dans l'appréciation des choix à faire ou des mesures à prendre pour la conservation durable des aires protégées.

Une autre innovation de la loi de 2002 sur les PNR dont l'objet est de permettre la conservation durable des aires protégées est l'institution de mécanismes de financement extraétatiques pour la protection des aires protégées.

\footnotetext{
le Directeur du parc ou de la réserve considérée. Les avis du comité de gestion locale sont joints aux rapports transmis par le Directeur du parc ou de la réserve concerné au Directeur Général de l'Office. ».

${ }^{446}$ Aux termes de l'article $1{ }^{\text {er }}$ de la loi de 2002 sur les PNR le contrat de gestion de terroir est un «contrat passé entre le gestionnaire d'un Parc ou d'une Réserve et les populations rurales de la zone périphérique représentées par des structures associatives, privées ou administratives. Ce contrat définit, notamment, les modalités d'intervention des populations contractantes dans la surveillance, la gestion, l'entretien et, le cas échéant, l'animation culturelle et touristique d'un parc, 'une réserve ou de leur zone périphérique. ». Pour plus d'informations sur ce contrat, voir la première partie de cette étude, le Titre II, le Chapitre II, la Section II, le Paragraphe 2, le point B (La contractualisation de la gestion des aires protégées et précisément le sous-point "1. Le contrat de gestion de terroir".

${ }^{447}$ Pour une définition de la convention de concession d'exploitation dans le cadre de la gestion des aires protégées de la Côte d'Ivoire et pour plus d'informations en la matière, voir les développements effectués sur ledit contrat dans la première partie de cette thèse, le Titre II, le Chapitre II, la Section II, le Paragraphe 2, le point B (La contractualisation de la gestion des aires protégées et précisément le sous-point "2. La convention de concession d'exploitation".

${ }^{448}$ Art. 31, Décret précité de 2002 sur les PNR.
} 


\section{Paragraphe 2 : L’institution de mécanismes de financement extra-étatique pour la protection des aires protégées}

Pour trouver une solution aux problèmes de financement exclusif des aires protégées par l'État y compris les difficultés liées à la mise à disposition des subventions étatiques allouées à la protection aires protégées, le législateur a institué dans la loi de 2002 sur les PNR d'autres mécanismes de financement additionnels au financement d'origine étatique. Il s'agit de mécanismes de financement extra-étatiques des aires protégées que sont les Fondations des Parcs et Réserves (A) et le contrat de fiducie (B).

\section{A - Les Fondations pour les Parcs et Réserves}

Les Fondations des Parcs et Réserves seront étudiées à travers leur objet (1) et les attributions qui leur sont dévolues par la loi (2).

\section{L'objet des Fondations pour les Parcs et Réserves}

Il ressort de la lecture conjointe des articles $1^{\text {er }}$ et 42 alinéa $1^{\text {er }}$ de la loi de 2002 sur les PNR que la Fondation est une personne morale (association) créée en vue de financer exclusivement certaines charges récurrentes et des investissements relatifs à la gestion des Parcs Nationaux et Réserves naturelles ${ }^{449}$. De plus, plutôt que de parler de Fondation au singulier, la loi précitée opte pour le pluriel. D'où l'emploi du vocable "Fondations" en ses articles $1^{\text {er }}, 42$ alinéa $1^{\text {er }}$ et suivants. Cela voudrait dire que si jusqu'à ce jour il n'y a qu'une seule Fondation, à savoir la Fondation pour les Parcs et Réserves de Côte d'Ivoire ${ }^{450}$, il reste que d'autres pourraient voir le jour.

Quoiqu'il en soit, le financement des charges récurrentes et des investissements dont il est fait mention dans l'article 42 alinéa $1^{\text {er }}$ renvoient à toutes les fonctions de gestion des aires protégées. Il s'agit essentiellement du management, du suivi écologique, de la valorisation touristique, de l'acquisition de matériels et équipements nécessaires à la gestion.

\footnotetext{
${ }^{449}$ En effet, l'article $1{ }^{\text {er }}$ de la loi de 2002 sur les PNR dispose, entre autres, que par «fondations » il faut entendre « une personne morale de créée en vue de la réaliser un but d'intérêt général. L'affectation de biens, droits ou ressources, à la réalisation de ce but d'intérêt général est irrévocable. Dans le cas d'espèce, les Fondations désigneront des associations dont l'objet et les principes d'organisation sont définis au titre IV de la présente loi...» (souligné par nous). Le titre IV dont il est fait référence ici contient l'article 42 alinéa $1{ }^{\mathrm{er}}$ sus-cité qui dispose que "le financement durable de certaines charges récurrentes des parcs et réserves et de la réalisation de certains investissements est assuré par le revenu des placements des Fondations exclusivement destinées au financement des parcs et réserves... " (souligné par nous). Par ailleurs, il convient de préciser que l'article 2 de la loi de 2002 sur les PNR indique, en faisant allusion aux Parcs nationaux et Réserves naturelles, que la réalisation des objectifs de ladite loi et la sauvegarde de ce patrimoine naturel sont «d'intérêt général...» (souligné par nous).

${ }^{450}$ Créée le 20 novembre 2003, et déclarée d'utilité publique par le Décret n 2009-05 du 08 janvier 2009, la Fondation pour les Parcs et Réserves de Côte d'Ivoire a pour mission de mobiliser et de gérer de manière efficiente des fonds destinés à assurer le financement durable des actions de protection des Parcs Nationaux et Réserves naturelles, en complément de celles réalisées par l'État ou qui l'incombe. Voir www.fondationparc.ci, consulté le 12/08/2015.
} 
À titre d'exemples, le 14 avril 2015, la Fondation pour les Parcs et Réserves a octroyé 700 millions de francs CFA à l'OIPR pour le financement d'activités de gestion et de préservation des Parcs Nationaux de la Comoé et de Taï. De même, dans le dernier trimestre de l'année 2014, l'OIPR a reçu 270 millions de francs CFA de la Fondation pour les Parcs et Réserves $^{451}$.

Pour ce faire, la loi de 2002 sur les PNR reconnaît certaines compétences aux Fondations pour les Parcs et Réserves.

\section{Les attributions des Fondations pour les Parcs et Réserves}

Selon l'article 45 alinéa $1^{\text {er }}$ de la loi précitée de 2002 sur les PNR, les Fondations sont habilitées à recevoir des financements de nature et de provenance diverses, à l'effet de la réalisation de leur objet ${ }^{452}$. Il s'agit du financement de certaines charges récurrentes et d'investissements relatifs la gestion des aires protégées. De plus, dotée de la personnalité morale, si les Fondations pour les Parcs et Réserves ont compétence pour recevoir des financements, cela suppose qu'elles sont également habilitées à rechercher des financements. Par conséquent les Fondations ont compétences aussi bien pour rechercher que recevoir des fonds en vue du financement de la conservation des aires protégées. Également, selon l'article 45 alinéa 2 suivant, les Fondations pour les Parcs et Réserves peuvent recueillir des fonds spécialement et exclusivement affectés à un Parc, une Réserve, une activité ou un objet particulier en rapport avec la conservation des aires protégées.

Ce mécanisme de financement des aires protégées (les Fondations pour les Parcs et Réserves) est de nature extra-étatique quelle que soit l'origine étatique ou privée du financement ${ }^{453}$. En effet, les Fondations sont par nature des associations de droit privé et partant distinctes de l'État.

Au surplus, l'un des aspects de la mobilisation de financements par les Fondations pour les Parcs et Réserves demeure les facilités fiscales qui leur sont faites. En effet, l'article 46 de la loi précitée de 2002 sur les PNR dispose que les fonds recueillis par les fondations sont exonérés de tous impôts et taxes. Partant, ces impôts et taxes ne représentant plus un surcoût de frais pour les donateurs, il en découle qu'il sera plus aisé pour eux de consentir des donations aux Fondations pour les Parcs et Réserves en vue de la protection de ces milieux.

\footnotetext{
${ }^{451}$ Pour ces exemples de financement des activités de l'OIPR par la Fondation pour les Parcs et Réserves, voir Www.gouv.ci/actualite 1.php?recordID $=5525$ (consulté le 12/08/2015).

${ }^{452}$ Aux termes de l'article 45 alinéa $1^{\text {er }}$ de la loi de 2002 sur les PNR : "À l'effet de la réalisation de leur objet, les Fondations reçoivent, notamment, des dons et legs de personnes physiques ou morales, nationales ou internationales, des dotations, subventions et contreparties financières de l'État, les produits du mécénat en faveur de l'environnement, les affectations de taxes parafiscales, la conversion de dettes, le produit de campagnes publicitaires et médiatiques organisées pour leur compte, ainsi que tout autre produit financier $»$.

${ }^{453}$ Il s'agit respectivement des dotations, des subventions et contreparties financières de l'État, d'affectations de taxes parafiscales et des dons, legs, des produits du mécénat, de conversion de dettes, de produits de campagnes publicitaires et médiatiques et de tout produit financier (Art. 45 alinéa $1^{\mathrm{er}}$ de la loi de 2002 sur les PNR).
} 
Par ailleurs, l'article 47 suivant pose que les Fondations pour les Parcs et Réserves ont le droit de faire des placements qui leur génèrent des revenus ; lesquels sont exonérés de tous impôts et taxes. Ce faisant, elles peuvent mobiliser davantage de fonds destinées à assurer la protection des aires protégées.

À l'instar de la Côte d'Ivoire, d'autres pays africains ont eu recours aux Fondations dans leur politique de conservation des aires protégées. À Madagascar ${ }^{454}$, dans un contexte de pressions anthropiques sur la diversité biologique, en général, et des aires protégées, en particulier, ainsi que de faiblesse des ressources étatiques allouées à leur protection ${ }^{455}$, il a été créé le 6 janvier 2005 la Fondation pour les aires protégées et la biodiversité de Madagascar $^{456}$. La Fondation a donc pour mission le financement pérenne de la protection des aires protégées y compris la recherche et l'extension du réseau des aires protégées. Elle a également pour mission leur valorisation écotouristique, la sensibilisation et l'éducation du public à leur conservation durable ${ }^{457}$.

En Afrique de l'Ouest, un projet régional de conservation des aires protégées formant le complexe $\mathrm{WAPO}^{458}$ et dénommé Programme d'Appui aux Parcs de l'Entente (PAPE) ${ }^{459}$ qui regroupe les États que sont Burkina-Faso, le Togo, le Niger et le Bénin, a mis en place en 2008 la Fondation des Savanes Ouest Africaines (FSOA). Cette Fondation a pour mission le financement pérenne tant des actions de conservation des aires protégées dans ces pays que d'entreprendre des actions de développement local ; qui du reste permettent d'atteindre cet objectif de conservation $^{460}$.

\footnotetext{
${ }^{454}$ Madagascar «est État insulaire constitué par une grande partie de l'île de l'océan Indien que le canal de Mozambique sépare de l'Afrique (distant de $400 \mathrm{~km}$ environ) ». Ce pays dont la population était estimée en 2013 à 22925000 d'habitants a pour capitale Antananarivo. Il est entouré par d'autre îles et archipels tels que La Réunion, les Comores, les Seychelles, l'Île Maurice. Voir www.larousse.fr/encyclopedie/pays/Madagascar/130983 (consulté le 24 mai 2016).

${ }^{455}$ En effet, à Madagascar, $70 \%$ de la population vie sous le seuil de la pauvreté. La pratique de défrichement à but agricole constitue une menace pour la biodiversité alors que les ressources subventions de l'État pour leur protection sont faibles. Voir AFD, Gestion durable de la biodiversité, Paroles d'acteurs n5, 2005, p. 36.

${ }^{456}$ Ibidem.

${ }^{457}$ Idem., p. 38

${ }^{458}$ Le complexe WAPO est un regroupement d'aires protégées qui fait l'objet d'un projet régional de conservation en Afrique de l'Ouest (Programme d'Appui aux Parcs de l'Entente, en abrégé PAPE). Il s'agit, en premier lieu, du Parc National W qui est une aire protégée transfrontalière située sur les territoires de trois (3) pays de l'Afrique de l'Ouest que sont le Burkina-Faso, le Bénin et le Niger. En deuxième lieu, l'on a les Parcs Nationaux de la Pendjari au Bénin et de Oti-kéran-Mandouri au Togo. UICN-PACO, Bâtir l'avenir par des solutions naturelles et novatrices : Rapport annuel 2013, Ouagadougou, Burkina Faso : UICN, 2014, p. 7.

${ }^{459}$ Placé sous la coordination générale de l'Union Économique et Monétaire de l'Afrique de l'Ouest (UEMOA), le projet Programme d'Appui aux Parcs de l'Entente (PAPE) initié par quatre pays, à savoir le Burkina-Faso, le Bénin, le Niger et le Togo a pour objectif la conservation des aires protégées dans ces États (www.bf.undp.org/content/burkina faso/fr/home/operations/projects/poverty reduction/PAPE.html). $\quad \mathrm{Ce}$ programme de conservation a été élaboré en raison des pressions anthropiques exercées sur les ressources naturelles dans un contexte d'instabilité climatique et de fragilité des écosystèmes. Voir UICN-PACO, Bâtir l'avenir par des solutions naturelles et novatrices : Rapport annuel 2013, op. cit., p. 7.

${ }^{460}$ Idem, p. 10.
} 
$\mathrm{Au}$ surplus, les Fondations sont un mécanisme de financement (extra-étatique) si important pour la gestion durable des Parcs et Réserves que le législateur ivoirien a prévu des règles de gestion pour leur permettre d'atteindre cet objectif.

\section{Les règles de gestion des Fondations pour les Parcs et Réserves}

Les règles de gestion des Fondations pour les Parcs et Réserves dont nous traiterons sont, d'une part, relatives à celles qui ont trait à la gestion des financements mobilisés par les Fondations (a). D'autre part, il s'agira d'analyser celles qui se rapportent aux droits des donateurs ou bailleurs de ces Fondations (b).

\section{a. Les règles de gestion des financements mobilisés par les Fondations pour les Parcs et Réserves}

L'article 49 alinéa $1^{\text {er }}$ de loi de 2002 sur les PNR donne la possibilité aux Fondations pour les Parcs et Réserves de confier les fonds qu'elles détiennent à des spécialistes de gestion de patrimoine en vue de les faire fructifier ${ }^{461}$. Ainsi, elles pourront disposer davantage de ressources financières pour le financement de la conservation des aires protégées.

C'est certainement pour réaliser ces objectifs de rentabilité financière que l'article 50 de la loi de 2002 sur les PNR indique que «Nul ne peut être dirigeant ou administrateur, s'il a fait l'objet d'une mesure d'interdiction de diriger, de gérer ou de contrôler une entreprise ou d'une mesure de faillite personnelle, ou s'il a subi une condamnation pénale ou une sanction professionnelle pour des faits contraires à l'honneur, à la probité ou aux bonnes mœurs ». On comprend dès lors que pour une gestion optimale des fonds dont disposent les Fondations pour les Parcs et Réserves, le législateur requiert, des dirigeants ou administrateurs, des qualités professionnelles en matière de gestion et des valeurs morales. Ils doivent faire preuve de probité et d'exemplarité dans l'exercice de fonctions similaires.

C'est également pour ces mêmes exigences de rentabilité financière qu'ils pèsent sur les Fondations des Parcs et Réserves une obligation de gestion transparente. En effet, elles sont astreintes à la tenue de comptes annuels de leur gestion financière ${ }^{462}$.

\footnotetext{
${ }^{461}$ Aux termes de 1'article 49 alinéa $1^{\text {er }}$ de la loi de 2002 sur les PNR : «Les Fondations peuvent, par une décision du conseil d'administration prise conformément à leurs statuts et après avis des commissaires aux Comptes, confier la gestion de tout ou partie de leurs fonds, et de ceux qui leur sont confiés par application d'un contrat de fiducie, à une ou plusieurs personnes morales tierces, nationales ou étrangères, présentant toutes les garanties professionnelles en la matière, spécialisées dans la gestion de patrimoine, sélectionnées par appel d'offres, à l'effet d'en retirer un revenu optimal» (souligné par nous). Il ressort de cet article que les fonds des Fondations ainsi que ceux qui leur sont confiés, dans le cadre d'un contrat de fiducie, peuvent être confiés à une ou plusieurs personnes morales nationales ou étrangères.

${ }^{462}$ Selon l'article 53 de la loi de 2002 : «Les comptes annuels des Fondations comprennent, outre le bilan, le compte de résultats et les annexes prévues par les dispositions législatives et réglementaires en vigueur...Les comptes des Fondations sont soumis annuellement au contrôle d'un ou plusieurs commissaires aux Comptes désignés par l'assemblée générale » (souligné par nous).
} 
Aussi la tenue de ces comptes permet-elle aux donateurs d'exercer leur droit de contrôle sur la gestion des fonds effectuée par les Fondations d'aires protégées.

\section{b. Les règles relatives au droit de contrôle des Fondations pour les Parcs et Réserves par les donateurs ou bailleurs}

L'article 45 de la loi de 2002 sur les PNR reconnaît aux donateurs ou bailleurs de Fondations de Parcs et Réserves un droit de contrôle sur la gestion des fonds qu'ils mettent à leur disposition ${ }^{463}$. Ce contrôle s'effectue à un double niveau. Il s'agit d'un contrôle a priori et d'un contrôle a posteriori.

S'agissant du premier type de contrôle (contrôle a priori), il est rendu possible par la participation majoritaire des intérêts privés à la composition du conseil d'administration des Fondations ${ }^{464}$. En conséquence, en amont, le conseil d'administration d'une Fondation d'aire protégée oriente les actions, les activités de ladite fondation, en définissant celles qui doivent être menées dans l'intérêt du financement de la protection des aires protégées. Cette possibilité d'orientation des actions des Fondations d'aire protégée est une forme de contrôle des fonds desdites Fondations. En effet, l'orientation des actions et activités de la Fondation permet au conseil d'administration (dominé majoritairement par des intérêts privés) de faire respecter la destination des fonds alloués au financement des aires protégées.

Concernant le contrôle a posteriori, il s'entend comme étant celui qui s'effectue après l'exercice de la mission de chaque Fondation de Parc et Réserve. Il vise à établir une conformité ou non entre les actions entreprises par l'organe exécutif d'une Fondation et les décisions du conseil d'administration. Ce faisant, ces donateurs ou bailleurs de fonds ont la possibilité de s'assurer de la destination des fonds alloués aux aires protégées et de la qualité de la gestion financière qui en est faite.

Par ailleurs, le deuxième mécanisme de financement extra-étatique destiné à la protection des aires protégées qu'a institué le législateur 2002 est le contrat de fiducie.

\section{B - Le contrat de fiducie}

Dans le cadre de la gestion des aires protégées ivoiriennes, l'analyse du contrat de fiducie comme mécanisme de financement extra-étatique de ces milieux naturels se fera à

\footnotetext{
${ }^{463}$ Les alinéas 4 et 5 de l'article 45 de la loi précitée de 2002 sur les PNR disposent respectivement : "Tous les fonds recueillis par les Fondations, ainsi que les revenus des placements, sont des deniers privés. » et que «Les dispositions de l'alinéa précédent ne font pas obstacle à l'exercice, par les donateurs des Fondations, des contrôles qu'ils doivent exercer conformément à leur statut, aux dispositions qui les régissent... » (souligné par nous).

${ }^{464}$ Aux termes de l'article 44 alinéa 2 de loi 2002 sur les PNR : «Les statuts des Fondations fixent, notamment, les modalités de détermination de la composition du conseil d'administration, de telle sorte que l'expression de la représentation des intérêts privés soit toujours majoritaire...».
} 
travers l'étude du régime juridique dudit contrat (2). Toutefois, il importe au préalable d'aborder cette analyse par l'étude de la notion de contrat de fiducie (1).

\section{La notion de contrat de fiducie}

Aux termes de l'article 1101 du Code civil, "Le contrat est une convention par laquelle une ou plusieurs personnes s'obligent, envers une ou plusieurs autres, à donner, à faire ou à ne pas faire quelque chose. ». Quant à la fiducie, elle désigne une opération dans laquelle un constituant transfère au fiduciaire des biens ou des droits au profit d'un ou de plusieurs bénéficiaires :

- soit pour constituer une sûreté (fiducie à titre de garantie d'une créance), sous l'obligation de rétrocéder le bien au constituant lorsque celle-ci n'a plus lieu de jouer ;

- soit à des fins de libéralité (fiducie à fins de libéralité), sous l'obligation de transférer le bien à un tiers bénéficiaire, après l'avoir géré dans l'intérêt de celui-ci ou d'une autre personne, pendant un certain temps ;

- soit à des fins de gestion (gérer le bien dans l'intérêt du bénéficiaire), sous l'obligation de le rétrocéder au fiduciant, à une certaine date ${ }^{465}$.

Fort de ces deux définitions, le contrat de fiducie peut être défini comme une convention par laquelle une personne, le fiduciant (constituant), transfère à une autre, le fiduciaire (gestionnaire des biens et droits mis en fiducie) ${ }^{466}$ tout ou partie de ses biens ou droits au profit de un ou plusieurs bénéficiaires :

- soit pour constituer une sûreté, sous l'obligation de rétrocéder le bien au constituant lorsque l'obligation garantie est éteinte ;

- soit à des fins de libéralité, sous l'obligation de transférer le bien au bénéficiaire, après l'avoir géré dans l'intérêt de celui-ci ou d'une autre personne, à l'échéance du terme de la convention ;

- soit à des fins de gestion (gérer le bien dans l'intérêt du bénéficiaire), sous l'obligation de le rétrocéder au fiduciant, le terme du contrat échu ${ }^{467}$.

Si cette définition du contrat de fiducie que nous avons formulée est dans le fond similaire à celle que propose la loi de 2002 sur les $\mathrm{PNR}^{468}$, il faut toutefois noter qu'elle a l'avantage d'être encore plus explicite. De la sorte, elle permettra de comprendre les

\footnotetext{
${ }^{465}$ Voir CORNU (Gérard) (dir.), Vocabulaire juridique, $4{ }^{\text {ème }}$ éd. (Association Henri Capitant), op. cit., p. 396.

${ }^{466}$ Dans le contrat de fiducie, le fiduciant ou le fiduciaire peut être toute personne morale (ONG, association de conservation de la nature, fondation, une structure publique, un État, etc.) ou physique qui justifie d'un intérêt pour la conservation des aires protégées.

${ }^{467}$ Cette définition formulée est à partir de celle donnée par CORNU (Gérard), [voir CORNU (Gérard) (dir.), Vocabulaire juridique, $4^{\text {eme }}$ éd. (Association Henri Capitant), op. cit., p. 396] et l'article $1^{\text {er }}$ de la loi de 2002 sur les PNR.

${ }^{468} \mathrm{~L}$ 'article $1^{\text {er }}$ de la loi de 2002 sur les PNR définit le contrat de fiducie comme étant une «...Convention par laquelle un constituant ou mandant transfère tout ou partie de ses biens et droits à un gestionnaire qui, tenant ces biens et droits séparés de son patrimoine personnel, agit dans un but déterminé au profit d'un ou de plusieurs bénéficiaires. ».
} 
développements ultérieurs sur le fonctionnement du mécanisme fiduciaire à travers l'étude du régime juridique du contrat de fiducie.

\section{Le régime juridique du contrat de fiducie}

Dans le cadre de la gestion des aires protégées ivoiriennes, le contrat de fiducie est, à titre principal, régi par les dispositions de la loi de 2002 sur les PNR y relatives et subsidiairement par le Code civil $^{469}$. Ces règles seront étudiées à travers celles qui se rapportent à sa formation (a), son exécution (b) et sa fin (c).

\section{a. La formation du contrat de fiducie}

À l'instar de tout contrat, la formation du contrat de fiducie obéit aussi bien à des conditions de forme qu'à des conditions de fond. En ce qui concerne les conditions de forme, le contrat de fiducie est un contrat solennel, c'est-à-dire que sa conclusion oblige les parties au respect de certaines mentions énumérées par l'article 56 alinéa $1^{\mathrm{er}}$ de la loi de 2002, à peine de nullité absolue ${ }^{470}$. D'une part, ce sont :

- la détermination de biens et droits qui en sont l'objet; la définition de la mission du fiduciaire et l'étendue de ses pouvoirs d'administration et de disposition ;

- l'indication des conditions dans lesquelles les biens et droits sont représentés ou transmis par au bénéficiaire ; et de la durée du contrat de fiducie qui ne peut excéder quatre-vingt-dix-neuf ans.

D'autre part, la solennité du contrat de fiducie relève de ce qu'il est, à peine de nullité, passé devant notaire, lorsqu'il :

- est conclu à des fins de transmission à titre gratuit ou porte sur un bien immobilier ;

- porte sur une somme ou sur un bien d'un montant supérieur à un montant fixé par décret.

C'est sans doute pour cette raison que l'article 56 alinéa 2 dispose que le contrat de fiducie est passé par écrit ${ }^{471}$; d'autant plus que la volonté des parties doit être expresse (art. 56 alinéa 4). Il découle de l'article 56 précité que la formation du contrat de fiducie procède d'une exception au principe du consensualisme consacré en droit (français) de formation des contrats $^{472}$.

\footnotetext{
${ }^{469}$ En effet, l'article 55 alinéa 3 de la loi de 2002 sur les PNR dispose : «Le contrat de fiducie est soumis aux règles ci-après énoncées et, à titre subsidiaire, aux dispositions du Code civil.».

${ }^{470}$ Voir CORNU (Gérard) (dir.), Vocabulaire juridique, $4^{\text {eme }}$ éd. (Association Henri Capitant), op.cit., p. 842 ; TOULET (Valérie), Droit civil : Obligations, Responsabilité civile, Paris, Centre de publication universitaires,

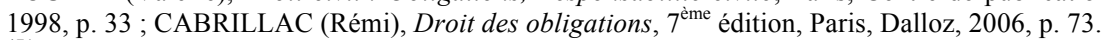

${ }^{471} \mathrm{D}$ 'une part cet écrit peut être un acte sous seing privé, c'est-à-dire établi par les parties elles-mêmes sous leur seule signature sans l'intervention d'un officier public. D'autre part, il peut s'agir d'un acte notarié (acte authentique).

${ }^{472}$ La Côte d'Ivoire indépendante hérite également de ce droit. Sur la formation du droit ivoirien, lire nos analyses effectuées au Titre I de la Première partie de cette thèse, le Chapitre I, la Section 2, le Paragraphe 1 et précisément le point intitulé " B- Les aires protégées, un domaine privé de l'État par raisonnement déductif" ; voir également DÉGNI-SÉGUI (René), Introduction au Droit, op. cit., pp. 306-307. À titre illustratif, les dispositions du Droit ivoirien des contrats applicables en Côte d'Ivoire sont une reprise textuelle des
} 
Formelle par essence, le contrat de fiducie peut-être un acte sous seing privé ou un acte authentique. Dans ce dernier cas, l'article 56 alinéa 3 prescrit que le contrat de fiducie doit avoir pour objet soit la transmission à titre gratuit soit un bien immobilier ou encore soit une somme d'un montant supérieur à un montant fixé par décret ${ }^{473}$. L'article 56 alinéa 3 réaffirme la solennité du contrat de fiducie. En effet, l'inobservation des formalités qui confèrent au contrat son caractère authentique, dans les conditions qu'il énumère, emporte nullité.

Pour ce qui est des conditions de fond, la formation contrat de fiducie doit respecter les dispositions des articles 6 et 1108 du Code civil qui posent les conditions générales de formation de tout contrat. En effet, l'article 6 dispose que «on ne peut déroger, par des conventions particulières, aux lois qui intéressent l'ordre public et les bonnes mœurs ». Quant à l'article 1108, il requiert pour la formation de tout contrat, le consentement (libre) des parties, leur capacité à contracter, la licéité de la cause du contrat et enfin l'existence de l'objet du contrat dans le commerce.

Ainsi, même si le contrat de fiducie met en présence trois (3) personnes, à savoir, le constituant ou fiduciant, le gestionnaire ou fiduciaire et le bénéficiaire, les parties au contrat de fiducie sont le constituant et le gestionnaire. Le constituant peut être une personne physique ou morale (un individu, une fondation ${ }^{474}$, une ONG de conservation de la nature...), qui transfère tout ou partie de ses biens et droits aux fins du contrat de fiducie. En ce qui concerne le gestionnaire, il est la personne physique ou morale (un individu, une fondation, une ONG de conservation de la nature...) qui reçoit les biens et droits transférés par le constituant et les administre conformément aux stipulations du contrat de fiducie. Le bénéficiaire (qui peut être une Fondation) est tiers au contrat de fiducie.

S'agissant de son objet, le contrat de fiducie est destiné au financement d'une activité ou d'un investissement relatif à la conservation ou à la valorisation des aires protégées. En effet, l'article 55 alinéa $1^{\text {er }}$ de la loi de 2002 sur les PNR dispose : «En vue du financement d'une activité ou d'un investissement à réaliser conformément aux objectifs définis par la présente loi, par ou pour le compte de l'Établissement, il peut être conclu un contrat de fiducie... ».

\footnotetext{
dispositions du Droit français des contrats. En effet, les articles 6 et 1108 du Code civil (ivoirien) disposent respectivement et de même que les articles 6 et 1108 du Code civil français que «On ne peut déroger, par des conventions particulières, aux lois qui intéressent l'ordre public et les bonnes mours.»; et que "Quatre conditions sont essentielles pour la validité d'une convention:

- le consentement de la partie qui s'oblige;

- sa capacité de contracter;

- un objet certain qui forme la matière de l'engagement;

- une cause licite dans l'obligation. ».

${ }^{473}$ Il est à noter que jusque-là ce décret n'est pas encore pris et partant ce montant n'est pas encore fixé.

${ }^{474}$ Selon l'article 55 alinéa 2 de la loi de 2002 sur les PNR qui traite de la qualité que peut avoir une Fondation dans un contrat de fiducie, "une Fondation peut être constituant, bénéficiaire ou gestionnaire de tels contrats. ». (souligné par nous).
} 
L'importance de l'objectif de financement des aires protégées ivoiriennes que poursuit le législateur à travers la mise en place de ce mécanisme de financement (contrat de fiducie) l'a amené à prévoir des sanctions en cas d'inobservation des conditions de formation dudit contrat. C'est ainsi que le non-respect des règles de formation du contrat de fiducie entraîne sa nullité. Outre la nullité ${ }^{475}$, une autre sanction de l'inobservation des règles de fond du contrat de fiducie est la réduction de libéralités excessives lorsque le contrat de fiducie porte atteinte aux droits des héritiers réservataires ${ }^{476}$.

Par contre, le contrat de fiducie qui est formé dans les conditions susmentionnées est exécuté selon les stipulations dudit contrat et des lois en vigueur.

\section{b. L'exécution du contrat de fiducie}

À la lecture des dispositions de la loi de 2002 sur les PNR qui traitent de l'exécution du contrat de fiducie, le fiduciant ou le bénéficiaire a le droit de demander en justice que soit remplacer le fiduciaire, en cas de défaillance dans la gestion des biens fiduciaires. En effet, aux termes de l'article 59 de la loi de 2002 sur les PNR, si le fiduciaire « manque gravement à ses devoirs ou met en péril les intérêts qui lui sont confiés, le constituant ou le bénéficiaire peut demander en justice, la nomination d'un administrateur provisoire ou le remplacement du gestionnaire contesté ».

De plus, l'article 60 suivant indique que le fiduciant doit prendre toutes les mesures, en vue d'éviter la confusion entre les biens fiduciaires et ses biens propres. Enfin, l'article 61 de la loi précitée prévoit que le contrat de fiducie ne doit pas porter atteinte aux droits des héritiers réserves.

Par ailleurs, comme tout contrat, le contrat de fiducie à une fin.

\section{c. La fin du contrat de fiducie}

Il ressort de la lecture de l'article 68 de la loi de 2002 sur les PNR que le contrat de fiducie prend fin de deux (2) façons. Le contrat de fiducie prend fin selon les stipulations dudit contrat ou par une décision de justice.

La fin conventionnelle du contrat de fiducie renvoie à deux (2) réalités. En effet, d'une part, le terme du contrat de fiducie est l'échéance que se sont fixés le constituant et le gestionnaire pour être libéré de leur obligation contractuelle. À cette échéance, ils sont libérés de tout engagement relatif au contrat de fiducie, s'il a été exécuté conformément à la loi et de bonne foi. Elle intervient dans une durée légale inférieure à quatre-vingt-dix-neuf (99) ans, à

\footnotetext{
${ }^{475}$ Art. 56 alinéa 2 de la loi précitée de 2002 sur les PNR.

476 C'est ce que dispose l'article 61 de la loi de 2002 sur les PNR en ces termes: «Si, lors du décès du constituant, la valeur des biens et droits transférés au gestionnaire excède la quotité disponible, le contrat de fiducie est réductible suivant les règles applicables aux donations entre vifs, conformément aux dispositions législatives et réglementaires en vigueur.».
} 
compter de la conclusion du contrat de fiducie. D'autre part, le contrat de fiducie prend fin par la réalisation du but poursuivi par les parties. En effet, le but poursuivi une fois réalisé, le contrat n'a plus de raison d'exister.

En outre, la fin du contrat de fiducie peut-être prononcée par voie judiciaire. En effet, selon l'article 68 alinéa $1^{\text {er }}$ de la loi précitée de 2002 sur les PNR, le contrat de fiducie peut également prendre fin par une décision de justice. La résiliation judiciaire du contrat de fiducie a lieu sous deux (2) conditions cumulatives. La première est l'absence de stipulations prévoyant sa poursuite. La seconde renvoie à la survenance de l'un des événements ci-après : - la renonciation du bénéficiaire à tout ou partie des biens transmis ;

- la dissolution de la Fondation bénéficiaire, le contrat pouvant cependant se poursuivre jusqu'à la clôture des opérations de liquidation ;

- la liquidation judiciaire de la Fondation bénéficiaire ;

- la disparition de la Fondation, par suite d'une absorption ou d'une session prononcée dans le cadre d'un redressement judiciaire.

À l'analyse des évènements ci-dessus énumérés par l'article 68 alinéa $1^{\mathrm{er}}$ précité, la résiliation judiciaire du contrat intervient lorsque la destination de l'actif fiduciaire ne peut plus être assurée. Toutefois, le principe de l'extinction du contrat de fiducie, prévu à l'article 68 alinéa $1^{\mathrm{er}}$ sus-cité, connaît une dérogation judiciaire. En effet, à l'initiative du constituant ou du bénéficiaire, le juge, suite à une appréciation discrétionnaire de l'intérêt, peut décider de la poursuite du contrat de fiducie.

En somme, d'une part, les règles de mobilisation de financements extra-étatiques des aires protégées qui régissent les Fondations et le contrat de fiducie permettent de mobiliser des fonds pour le financement pérenne de la conservation de ces forêts. D'autre part, ces règles permettent de les gérer rigoureusement de sorte à réaliser cet objectif.

Au surplus, une autre innovation caractéristique du droit des aires protégées depuis 2002 demeure la consécration d'un cadre participatif de gestion des aires protégées.

\section{SECTION II : L'instauration d'un cadre participatif de gestion des aires protégées au niveau local}

Depuis 2002, la gestion des aires protégées ivoiriennes n'est plus l'exclusivité de l'État de Côte d'Ivoire, par le truchement de ses services (déconcentrés) techniquement compétents en la matière ${ }^{477}$. En d'autres termes, la législation ivoirienne relative aux aires protégées consacre désormais l'approche de gestion participative de ces milieux naturels (Paragraphe 1). Aussi la mise en œuvre de cette nouvelle approche de gestion est-elle rendue possible par des mécanismes qu'a prévu le législateur ivoirien (Paragraphe 2).

${ }^{477}$ Il s'agit des services de l'Administration des Eaux et Forêts. 


\section{Paragraphe 1 : L'abandon de la gestion exclusive des aires protégées par l'État ivoirien}

Depuis l'année 2002, le législateur ivoirien a abandonné la gestion exclusive des aires protégées au profit de leur gestion participative. Cette attitude législative qui témoigne certainement de l'importance de la gestion participative pour la conservation des aires protégées $(\mathrm{A})$ a eu pour conséquence d'associer de nouveaux acteurs à la gestion de ces forêts (B).

\section{A - L'importance de la gestion participative pour la conservation des aires protégées}

Terme couramment employé en matière de gestion des ressources naturelles, la gestion participative ou gestion conjointe ou encore gestion collaborative ${ }^{478}$ est un concept polysémique. Toutefois, en règle générale, elle peut être entendue comme étant une gestion desdites ressources opérée de concert par l'État et les communautés ${ }^{479}$ qui promeut une démocratie participative ainsi que des incitations au niveau local pour leur conservation durable $^{480}$. Selon des auteurs, la conservation des aires protégées implique nécessairement la participation des populations locales ${ }^{481}$. Ainsi, si le législateur ivoirien opte pour cette approche de gestion expérimentée partout dans le monde (2), son choix pourrait se justifier par le fait qu'elle est une conséquence des limites de l'approche préservationniste de la conservation de la biodiversité (1).

\section{Le recours à la gestion participative des aires protégées en Côte d'Ivoire, une conséquence de l'échec de l'approche préservationniste}

De 1960 à 2002, la Côte d'Ivoire opte pour la gestion dirigiste des aires protégées ${ }^{482}$. Or, la gestion des aires protégées par l'État sans la participation des communautés locales et plus largement du public a été fortement critiquée ${ }^{483}$. En effet, elle a fondamentalement abouti à l'échec des politiques de conservation de la nature ${ }^{484}$. En Côte d'Ivoire et au Ghana ${ }^{485}$ par

\footnotetext{
${ }^{478}$ Voir KEPE (Thembela), « Land claims and co-management of protected areas in South Africa: Exploring the challenges », Environmental management, Vol. 41, n³, 2008, p. 314.

${ }^{479}$ Voir MASHALE (Christopher Modise), Theresa MOYO, Olivier MTAPURI, « Evaluation of the PublicPrivate Partnership in the Lekgalameetse Nature Reserve in South Africa ", Mediterranean Journal of Social Sciences, Vol. 5, n²0, 2014, p. 858.

${ }^{480}$ Voir KEPE (Thembela), « Land claims and co-management of protected areas in South Africa: Exploring the challenges », art. cit. p. 311.

${ }^{481}$ En effet, ces auteurs affirment: "Réaliser une conservation efficace dans les pays tropicaux est une préoccupation mondiale, mais implique les populations » (notre traduction). Voir VERMEULEN (Sonja) SHEIL (Douglas), «Partnerships for tropical conservation», Oryx, Vol. 41, n4, 2007, p. 434.

${ }^{482}$ À propos de la gestion dirigiste des aires protégées par l'État ivoirien de 1960 à 2002, voir la Première partie de cette étude, le Titre I, le Chapitre I, la Section I, et précisément le "Paragraphe 1 : Le service public de la conservation des aires protégées de 1960 à 2002, une Administration centralisée".

${ }^{483}$ Voir INAMDAR (Amar), JODE (Helen de), LINDSAY (Keith) et COBB (Stephen), «Capitalizing on Nature Protected Area Management», Science, Vol. 283, n5409, 1999, pp. 1856-1857.

${ }^{484}$ Voir STOLL-KLEEMANN (Suzanne) et O'RIORDAN (Timothy), «From Participation to Partnership in Biodiversity Protection: Experience from Germany and South Africa», art. cit., p. 163; ANDRADE (Gustavo S.
} 
exemple, les aires protégées restent toujours sujettes à des pressions de la part des populations locales, en dépit de leur protection par la loi ${ }^{486}$. Par conséquent, il est de plus en plus courant que la gestion des milieux naturels tels que les aires protégées soit ouverte à des personnes autres que l'État. En la matière, des études montrent que ces dernières années, l'on assiste en Afrique à une tendance du recours à la gestion participative des ressources naturelles, en lieu et place de l'approche dirigiste anciennement vulgarisée ${ }^{487}$.

Ce recours à la participation pour la gestion des milieux naturelles et notamment des aires protégées pourrait s'expliquer par l'importance que représente cette approche de gestion pour leur conservation durable. C'est ce que soulignent des auteurs tels que PIMBERT et PRETTY qui affirment que sans la participation locale, il y a peu de chance de protection de la faune ${ }^{488}$; et par voie de conséquence leurs habitats que sont les aires protégées. Selon ces auteurs, les aires protégées s'étendent sur de vastes superficies alors que les moyens humains et matériels pour la mise en œuvre des politiques de conservation sont limités. Ainsi, la gestion participative peut permettre de réduire les coûts de gestion ${ }^{489}$ (patrouilles et autres coûts) et partant réduire les activités illégales dans ces milieux naturels ${ }^{490}$. De même, pour ANDRADE et RHODES, le fait d'impliquer les populations locales dans la prise de décision relative à la gestion des aires protégées contribue à leur bonne conservation ${ }^{491}$. C'est dans ce contexte que confrontés à la dégradation des aires protégées du fait des pressions anthropiques, les pouvoirs publics ivoiriens font également le choix d'ouvrir la gestion des

M.) et RHODES (Jonathan. R.), « Protected areas and local communities: an inevitable partnership toward successful conservation strategies? », art. cit., p. 14.

${ }^{485}$ Situé en Afrique occidentale au bord du Golfe de Guinée, le Ghana est un pays est frontalier à la Côte d'Ivoire à l'Ouest, au Togo à l'Est et au Burkina-Faso au Nord. Ayant pour capitale Accra, sa population est estimée en 2013 à 25, 9 millions d'habitants en 2013. Voir www.larousse.fr/encyclopedie/pays/Ghana/121314 (consulté le 24 mai 2016).

${ }^{486}$ Pour des exemples de pressions sur les aires protégées ivoiriennes, voir l'Introduction de cette thèse, le point "I- Enjeux de la protection des forêts en Côte d'Ivoire", " C- l'état des pressions anthropiques sur la biodiversité en Côte d'Ivoire", et précisément le point intitulé "2. Les pressions anthropiques sur les aires protégées en particulier ".

En ce qui concerne le Ghana, une étude d'évaluation de la gestion des aires protégées de ce pays montrent qu'elles sont aussi en proie à des pressions anthropiques. En effet, caractérisées par la richesse de leur diversité biologique, les aires protégées du Ghana font l'objet, entre autres, de braconnage, de feux de brousse, de pâturage, d'exploitation agricole et forestière. Voir UICN/PACO, Parks and reserves of Ghana: Management effectiveness assessment of protected areas. Ouagadougou, Burkina Faso: UICN/PACO, 2010, p. 9.

${ }^{487}$ Voir AGRAWAL (Arun), GIBSON (Clark C.), « Enchantment and Disenchantment: The Role of Community in Natural Resource Conservation », World Development, Vol. 27, n 4, 1999, p. 629 ; MBURU (John) et BIRNER (Regina), « Emergence, Adoption, and Implementation of Collaborative Wildlife Management or Wildlife Partnerships in Kenya: A Look at Conditions for Success», Society and Natural Resources, Vol. 20, $\mathrm{n}^{\circ} 5,2007$, p. 379.

${ }^{488}$ Voir PIMBERT (Michel P.) et PRETTY (Jules N.), Parks, People and Professionals: Putting 'Participation' into Protected Area Management, op. cit., p. 29.

${ }^{489}$ Ibidem.

${ }^{490}$ Voir HOROWITZ (Leah Sophie), « Integrating indigenous resource management with wildlife conservation: a case study of Batang Ai National Park, Sarawak, Malaysia », Human Ecology, Vol. 26, n³, 1998, p. 371; PRETTY (Jules) et SMITH (David), " Social capital in biodiversity conservation and management ", Conservation Biology, Vol. 18, n³, 2004, p. 632.

${ }^{491}$ Voir ANDRADE (Gustavo S. M.) et RHODES (Jonathan R.), « Protected areas and local communities: an inevitable partnership toward successful conservation strategies? », art. cit., pp. 19-20. 
aires protégées à des acteurs autres que l'État, en général, et principalement aux communautés locales.

La participation des communautés à la gestion des aires protégées est si importante que le IVème Congrès mondial sur les Parcs Nationaux et les aires protégées a recommandé la participation accrue des communautés locales et l'équité dans les prises de décision relatives à la gestion des aires protégées ${ }^{492}$. C'est donc à juste titre que de nos jours, les politiques étatiques de protection de la nature cherchent à associer les communautés locales et plus globalement le public dans la gestion desdites ressources ${ }^{493}$.

\section{La gestion participative des ressources naturelles, un engouement planétaire}

Le recours à l'approche de gestion participative des ressources naturelles et notamment des aires protégées est expressif d'un élan mondial. En Europe, la gestion participative des ressources naturelles est généralement prônée par les pays. En effet, dans l'Union européenne par exemple, et au Royaume-Uni ${ }^{494}$, la participation du public et le travail en partenariat caractérise les stratégies des gouvernements ${ }^{495}$. En Norvège ${ }^{496}$, la gestion exclusive des aires protégées par l'État a rencontré des résistances au sein des populations. En effet, les sites à conserver étaient désignés par la « Direction de la gestion de la nature (notre traduction) » qui est une des Directions du ministère de l'Environnement. Ainsi, dans une localité telle que Snavoy ${ }^{497}$, la désignation de sites à conserver se fait d'accord partie entre les propriétaires fonciers et les autorités publiques. Ces dernières prennent en compte leurs intérêts tels que la pratique de l'agriculture. Une telle approche a permis de conclure des partenariats public-privé réussis qui ont contribué à la conservation de la nature ${ }^{498}$.

\footnotetext{
${ }^{492}$ Voir IUCN, Parks for Life: Report of the IVth World Congress on National Parks and Protected Areas, Gland, Switzerland, , IUCN, 1993, pp. 10-11 et 14-16.

${ }^{493}$ Voir CHAPMAN (Duane) et BARKER (Randolph), « Environmental protection, resource depletion, and the sustainability of developing country agriculture », Economic Development and Cultural Change, Vol. 39, $\mathrm{n}^{\circ} 4$, 1991, p. 723 ; THOMAS-SLAYTER (Barbara), « Implementing Effective Local Management of Natural Resources: New Roles for NGOs in Africa », Human Organization, Vol. 51, n²,1992, p. 136.

${ }^{494}$ Le Royaume-Uni (en anglais United Kingdom) est un pays situé dans la partie nord de l'Europe de l'Ouest. Composé de quatre nations constitutive que sont l'Angleterre, l'Écosse, l'Irlande du Nord et le pays de Galles, ce pays fait frontière avec la République d'Irlande et est entouré de mers que sont la mer d'Irlande à l'Ouest, la Manche au Sud, la mer du Nord à l'Est et de l'océan Atlantique (à l'est). Le Royaume-Uni a une superficie de $253500 \mathrm{~km}^{2}$ et une population estimée en 2013 à 63,13 millions d'habitants. Sa capitale est Londres. Voir www.larousse.fr/encyclopedie/pays/Royaume-Uni de GrandeBretagne et dIrlande du Nord institutions/122086 (consulté le 24 mai 2016).

${ }^{495}$ Voir PIPER (Jake M.), « Partnership and Participation in Planning and Management of River Corridors », Planning, Practice and Research, Vol. 20 (1), 2005, p. 1.

${ }^{496}$ Frontalière à la Suède, la Finlande et à la Russie, la Norvège est un pays situé en Europe du Nord sur une superficie de $325.000 \mathrm{~km}^{2}$ et qui a une population estimée à 5,04 millions d'habitants en 2013. Voir www.larousse.fr/encyclopedie/pays/Norvège/135583 (consulté le 24 mai 2016).

${ }^{497}$ L'île de Svanøy est située en Scandinavie sur la côte de la Norvège. Voir HEGLAND (Joar Stein), RYDGREN Knut, SELDAL (Tarald), "The response of Vaccinium to variations in grazing intensity in a Scandinavian pine forest on the island of Svanøy », Canadian journal of botany, vol. 83, n¹2, 2005, p. 1638.

${ }^{498}$ Voir HOVIK (Sissel), EDVARDSEN (Morten), « Private-public partnership: An exceptional solution in nature conservation in Norway », Local Environment, Vol. 11, n²4, 2006, pp. 368-370.
} 
En Serbie ${ }^{499}$ également, l'on a d'abord assisté à une gestion des aires protégées selon une approche centralisée et exclusive opérée par le gouvernement. Cependant, les insuffisances de cette approche de gestion ont conduit les autorités de ce pays à impliquer désormais le public dans la gestion de ces forêts. Une étude de cas menée dans le cadre de la gestion de l'aire protégée de Zasavica montre que le public participe à la planification, la prise de décision et la gestion de cette aire protégée ${ }^{500}$.

$\mathrm{Au} \mathrm{Népal}^{501}$, la participation des communautés locales à la gestion des ressources naturelles, en général, et aux aires protégées, en particulier, a cours depuis les années $1980^{502}$. Cette situation est consécutive à l'échec des politiques de gestion exclusive des ressources naturelles par les autorités de ce pays ${ }^{503}$. En Australie ${ }^{504}$, des études de cas relatives à la gestion des aires protégées concluent que la conservation de la biodiversité requiert un engagement des États qui devraient faciliter la participation du public, du secteur de l'industrie et de l'ensemble de la communautét ${ }^{505}$.

En Afrique également, la gestion participative des ressources naturelles et notamment des aires protégées est une approche qui a été adoptée suite à l'échec des politiques de gestion exclusive préalablement en cours dans les États ${ }^{506}$. C'est ainsi qu'en Afrique australe et notamment au Botswana ${ }^{507}$, la gestion participative des ressources naturelles a été

\footnotetext{
${ }^{499}$ La Serbie est un État de l'Europe du Sud qui fait partie de l'Europe centrale ainsi que des Balkans occidentaux. Ayant pour capitale Belgrade et une superficie de $253.500 \mathrm{~km}^{2}$ et une population estimée à 7.777 . 128 d'habitants, la Serbie fait frontière à la Bosnie-Herzégovine, la Hongrie, la Bulgarie, la Roumaine, la République de Macédoine, au Kosovo et au Monténégro. Voir www.larousse.fr/encyclopedie/pays/Serbie/143964 (consulté le 24 mai 2016).

${ }^{500}$ Voir TOMIĆEVIĆ (Jelena), BJEDOV (Ivana), SHANNON (Margaret A.) et OBRATOV-PETKOVIĆ (Dragica), op. cit., p. 140.

${ }^{501}$ La République démocratique fédérale du Népal est un pays enclavé de l'Himalaya. Elle a pour frontières la Chine au Nord et l'Inde à l'Est, à l'Ouest et au Sud. Avec une superficie de $140000 \mathrm{~km}^{2}$, la population du Népal est estimée à 27.797.000 d'habitants en 2013. Voir www.larousse.fr/encyclopedie/pays/Népal/134965 (consulté le 24 mai 2016).

${ }^{502}$ Voir AGRAWAL (Arun), VARUGHESE (George), Conservation's Visions : Poverty, Participation, and Protected Area Management in Nepal's Terai, 2000, 44 pages, (extrait du résumé, page non numérotée). Document disponible sur www.dlc.dlib.indiana.edu/dlc/bitstream/handle/10535/1106/agrawala041000.pdf?sequence=1 (consulté le 2juin 2015)

${ }^{503}$ Ibidem

${ }^{504}$ L'Australie est un pays de l'hémisphère du Sud dont superficie couvre la plus grande partie de l'Océanie. Il s'étend sur une superficie de $253.500 \mathrm{~km}^{2}$ et une population estimée à 7.777 .128 d'habitants (consulté le 24 mai 2016).

${ }^{505}$ Voir THACKWAY (R.), OLSSON (K.), «Public/private partnerships and protected areas : Selected Australian case studies», Landscape and urban planning, vol. 44, n²-3, 1999, p. 87.

${ }^{506}$ Voir STOLL-KLEEMANN (Suzanne) et O'RIORDAN (Timothy), «From Participation to Partnership in Biodiversity Protection : Experience from Germany and South Africa », art. cit., p. 163; ANDRADE (Gustavo S. M.) et RHODES (Jonathan R.), «Protected areas and local communities: an inevitable partnership toward successful conservation strategies? », art. cit., p. 14 ; PATRICK (Triplet), VERMEULEN (Cédric), « Améliorer la participation des populations à la gestion des aires protégées » in TRIPLET (Patrick) (dir.), Manuel de gestion des aires protégées d'Afrique francophone, Paris, Awely, 2009, p. 228.

${ }^{507}$ Le Botswana est un pays d'Afrique australe entouré de la Zambie au Nord, du Zimbabwe au Nord-Est, de l'Afrique du Sud au Sud et de la Namibie à l'Ouest. Avec une superficie de $570.000 \mathrm{~km}^{2}$, la capitale du Botswana est Gaborone. En ce qui concerne sa population, elle est estimée à 2,021 millions en 2013. Voir www.larousse.fr/encyclopedie/pays/Botswana/109606 (consulté le 24 mai 2016).
} 
expérimentée sous la forme de gestion communautaire de ces ressources ${ }^{508}$. En Afrique du $\mathrm{Sud}^{509}$, précisément dans le Kwazulu Natal, les populations ont été associées à la gestion du Hhuhuwe-Umfolozi Park (Parc Hhuhuwe-Umfolozi). En effet, elles ont participé à la planification des projets d'écotourisme et de conservation de ce $\operatorname{Parc}^{510}$. Il en est de même pour la Lekgalameetse Nature Reserve (Réserve naturelle de Lekgalameetse) ${ }^{511}$. Également en $Z_{\text {Zambie }}{ }^{512}$, la gestion participative des aires protégées est mise en œuvre par les autorités publiques sous la forme de partenariat public-privé ${ }^{513}$.

En Afrique centrale, par exemple, il est développé depuis les années 1990 des programmes de gestion participative des aires protégées, dans le cadre des activités de l'ECOFAC $^{514}$. Au Rwanda ${ }^{515}$ des travaux de recherches attestent que la gestion participative des aires protégées a été adoptée, même si cette approche de gestion a eu un succès relatif; malgré la volonté des différents acteurs de s'impliquer dans la protection des aires protégées $^{516}$.

\footnotetext{
${ }^{508}$ Voir MBAIWA (Joseph E.), STRONZA (Amanda L.), « Changes in resident attitudes towards tourism development and conservation in the Okavango Delta », Journal of Environmental Management, 92, 2011, p. 1950.

${ }^{509}$ Situé à l'extrémité de la partie australe du continent africain, l'Afrique du Sud est un pays frontalier de la Namibie, du Botswana et du Zimbabwe au Nord, du Mozambique et du Swaziland au Nord-Est. Il fait également frontière avec l'État du Lesotho qui est enclavé par le territoire sud-africain. L'Afrique du Sud a pour capitale administrative Pretoria, tandis que Le Cap et Bloemfontein sont respectivement les capitales législative et judiciaire. Sa superficie est de $1.221 .000 \mathrm{~km}^{2}$, et sa population est estimée à 52.776.000 d'habitants, en 2013 . Voir www.larousse.fr/encyclopedie/pays/Afrique du Sud/104193 (consulté le 24 mai 2016).

${ }^{510}$ Voir ADELEKE (Bola Olusola), NZAMA (Thandi), « Assessment on Community Participation in Ecotourism and Conservation at Hhuhuwe-Umfolozi Park », Journal of Environment and Earth Science, Vol. 3, n³, 3013, p. 27.

${ }^{511}$ Voir MASHALE (Christopher Modise), MOYO (Theresa) et MTAPURI (Olivier), « Evaluation of the PublicPrivate Partnership in the Lekgalameetse Nature Reserve in South Africa ", Mediterranean Journal of Social Sciences ,Vol. 5, n²0, 2014, p. 855

${ }^{512}$ Avec une superficie de $746.000 \mathrm{~km}^{2}$ et Lusaka pour capitale, la Zambie est un pays d'Afrique australe entouré de la République démocratique du Congo, du Malawi, du Mozambique, de la Namibie, de la Tanzanie et du Zimbabwe. Sa population est estimée à 14.539.000 d'habitants en 2013. Voir www.larousse.fr/encyclopedie/pays/Zambie/150467 (consulté le 24 mai 2016).

${ }^{513}$ Nous pouvons citer, entre autres, POPE (Adam), A Preliminary Examination of Public Private Partnerships in National Park Management in Zambia, 2006, op. cit., 24 pages + Annexes ; NSHIMBI (Muleba) et VINYA (Royd), "Impacts of Public-Private Partnership on Local Livelihoods and Natural Resource Dynamics: Perceptions from Eastern Zambia », Resources, Vol. 3, n², 2014, pp. 471-487.

${ }^{514}$ Initié en 1992 par la Commission européenne, le programme Écosystèmes Forestiers d'Afrique Centrale (ECOFAC) est un programme régional qui couvre six (6) pays d'Afrique centrale. Il est destiné à assurer la conservation des forêts tropicales, par l'aménagement des aires protégées (voir www.riddac.org/content/view/51/66/, consulté le 2 septembre 2015).
}

À travers ce programme, des sites sont retenus pour la conservation et la protection durable des forêts par le truchement de l'implication des populations locales, dans une approche intégrant conservation des ressources naturelles et développement local. Voir

www.europarl.europa.eu/workingpapers/agri/s5-7-2 fr.htm, consulté le 2 septembre 2015.

${ }^{515}$ Le Rwanda est un pays situé en Afrique centrale, dans la région des Grands Lacs, qui s'étend sur une superficie de $26.338 \mathrm{~km}^{2}$. Il est limitrophe de la République démocratique du Congo, à l'Ouest, du Burundi, au Sud; de la Tanzanie, à l'Est et de l'Ouganda au Nord. Le Rwanda a pour capitale Kigali et sa population est estimée en 2013 à 11,77 millions d'habitants (voir www.larousse.fr/encyclopedie/pays/Rwanda/141732).

${ }^{516}$ Voir RUTAGARAMA (Eugène) et MARTIN (Adrian), " Partnerships for protected area conservation in Rwanda », op. cit., p. 303. 
En Afrique de 1'Est, dans un pays tel que le Kenya ${ }^{517}$, la gestion participative des ressources naturelles et notamment de la faune est mise en œuvre par la Kenya Wildlife Service (KWS). En effet, la gestion participative de la conservation de la faune se fait à travers des projets de partenariats conclus entre la KWS et des aires de conservation privées telles que Kimana Community Wildlife Sanctuary (Kimanas Sanctuary) et Golini-Mwaluganje Elephant Sanctuary (GM Sanctuary) ${ }^{518}$. En Ouganda ${ }^{519}$, la gestion participative des forêts a été aussi entreprise. Une étude relative à la gestion de la Forêt impénétrable de Bwindi confirme par exemple qu'un partenariat a été conclu entre un Écolodge touristique et la Forêt impénétrable de Bwindi pour la conservation durable de ladite forêt ${ }^{520}$.

En faisant le choix d'adopter la gestion participative des aires protégées, à l'instar de ces pays précédemment cités, le législateur ivoirien associe des acteurs autres que le personnel des services des Eaux et Forêts à la gestion des aires protégées.

\section{B - Les nouveaux acteurs associés à la gestion des aires protégées}

Les nouveaux acteurs qui, depuis 2002, participent à la gestion des aires protégées avec les fonctionnaires de l'Administration des Eaux et Forêts peuvent être classés en deux (2) catégories. Il s'agit d'acteurs relevant de l'ordre administratif (1) et d'autres catégories d'acteurs que sont les personnes morales de droit privé (2).

\section{Les acteurs relevant de l'ordre administratif}

L'article $1^{\text {er }}$ de loi $n^{\circ} 2014-451$ du 05 août 2014 portant orientation de l'organisation générale de l'Administration territoriale ${ }^{521}$ dispose qu'en Côte d'Ivoire " L'Administration territoriale est structurée selon les principes de la déconcentration, de la décentralisation et l'entité territoriale particulière qu'est le District Autonome.». Selon l'article 2 suivant, l'Administration territoriale déconcentrée est réalisée dans le cadre de circonscriptions

\footnotetext{
${ }^{517}$ Le Kenya est un pays de l'Afrique de l'Est dont la superficie s'étend sur $583000 \mathrm{~km}^{2}$. Baigné au Sud-Est par l'océan Indien, le Kenya a en partage des frontières avec l'Éthiopie et le Soudan du Sud, au Nord; la Somalie, à l'Est; l'Ouganda, à l'Ouest; et la Tanzanie, au Sud-Ouest. Le Kenya a pour capitale Nairobi et sa population est estimée en 2013 à 44,35 millions d'habitants. Voir www.larousse.fr/encyclopedie/pays/Kenya/127291 (consulté le 4 juin 2016).

${ }^{518}$ Voir MBURU (John ) et BIRNER (Regina), « Emergence, Adoption, and Implementation of Collaborative Wildlife Management or Wildlife Partnerships in Kenya: A Look at Conditions for Success ", Society and Natural Resources, Vol. 20, n5, 2007, p. 393

${ }^{519}$ L'Ouganda est un pays de l'Afrique de l'Est. Avec une superficie est de $237.000 \mathrm{~km}^{2}$, ce pays est entouré par le Kenya, la République démocratique du Congo, le Rwanda, le Soudan du Sud et la Tanzanie. Sa capitale est Kampala et sa population est estimée en 2013 à 37,58 millions d'habitants. Voir www.larousse.fr/encyclopedie/pays/Ouganda/136566 (consulté le 4 juin 2016).

${ }^{520}$ Voir AHEBWA (Wilber Manyisa), DUIM (V. René Van der) et SANDBROOK (Chris G.), « Privatecommunity Partnerships: Investigating a New Approach to Conservation and Development in Uganda », Conservation and Society, Vol. 10, ${ }^{\circ} 4,2012$, p. 305.

${ }^{521}$ (JORCI n 39 du 21 août 2014, p. 877). Cette loi abroge l'ordonnance n ${ }^{\circ}$ 2011-262 du 28 septembre 2011 portant orientation de l'organisation générale de l'Administration Territoriale de l'Etat (JORCI n 3 du 20 octobre 2011, p. 33) qui elle-même a remplacé la loi n²001-476 du 9 août 2001 d'orientation sur l'organisation générale de l'Administration territoriale (JORCI n³6 du jeudi 6 septembre 2001, p. 746).
} 
administratives hiérarchisées, à savoir les Régions, les départements, les sous-préfectures, les villages. Quant à l'article 32 de la même loi, il indique que l'Administration décentralisée est assurée dans le cadre de collectivités territoriales qui sont les Régions et les Communes.

Les aires protégées objet de cette étude se trouvent réparties, sur le plan spatial, dans cette architecture administrative. C'est ainsi qu'en plus des fonctionnaires des Eaux et Forêts et agents de l'État ayant traditionnellement la charge de la gestion des aires protégées, depuis 2002, la législation sur les aires protégées associe également d'autres autorités publiques à la gestion des Parcs Nationaux et Réserves naturelles. Ces autorités publiques sont issues aussi bien de l'Administration déconcentrée (a) que de l'Administration décentralisée (b).

\section{a. Les autorités administratives déconcentrées associées à la gestion des aires protégées}

Dans le droit des aires protégées, c'est l'article 35 du décret portant création de l'OIPR qui prévoit que les autorités administratives déconcentrées associées à la gestion des aires protégées sont le préfet de Région ou de Département dont dépend la plus grande superficie du Parc ou de la Réserve ${ }^{522}$.

À l'analyse, l'association des premiers responsables de ces collectivités territoriales (préfet de Région, préfet de Département) trouverait sa justification dans les attributions qui leur sont dévolues. En effet, l'article 6 alinéa $1^{\mathrm{er}}$ de la loi précitée de 2014 portant orientation de l'organisation générale de l'Administration Territoriale dispose : «La Région constitue l'échelon de conception, de programmation, d'harmonisation, de soutien, de coordination et de contrôle des actions et des opérations de développement économique, social et culturel qui s'y réalisent à l'intervention de l'ensemble des services des administrations civiles de l'État. ». Il en résulte que le préfet de Région et à une échelle inférieure le préfet de département ${ }^{523}$ ont une mission de développement et d'administration de la Région ou du département ${ }^{524}$.

$\mathrm{Au}$ regard de leurs missions, nous pouvons affirmer que l'association de ces entités administratives à la gestion des aires protégées pourrait s'expliquer par plusieurs raisons. D'une part, la protection de l'environnement et notamment la conservation des aires protégées ainsi que la gestion de secteurs qui lui sont liées (loisirs, promotion touristique...) sont des

\footnotetext{
${ }^{522}$ Aux termes de l'article 35 du décret portant création de l'OIPR « Chaque parc et chaque réserve sont dotés d'un Comité de Gestion locale présidé par le Préfet de Département ou de Région dont dépend la plus grande superficie du parc et de la réserve... » (souligné par nous).

${ }_{523}$ Si l'article 18 de la loi précitée de 2014 portant orientation de l'organisation générale de l'Administration tterritoriale dispose : «Le département est administré par un préfet », en l'occurrence le préfet de département, l'article 17 qui le précède indique que «Le département constitue l'échelon de relais entre la Région et la souspréfecture. ".

${ }_{524}$ En effet, les articles 9 et 19 de loi précitée de 2014 portant orientation de l'organisation générale de l'Administration tterritoriale mentionnent respectivement: "Le préfet de Région est chargé d'une mission générale de développement et d'administration de la Région» et : le préfet de département est "responsable du suivi des actions de développement du département...Dirige, anime, coordonne et contrôle les activités des services administratifs et techniques du département... » (souligné par nous).
} 
responsabilités (administratives) réparties entre plusieurs autorités administratives. Celles-ci relèvent du Préfet de Région et à une échelle moindre du préfet de département, dans leur ressort territorial respectif. Par conséquent, l'exercice des missions de coordination et d'harmonisation dévolues au Préfet de Région et au préfet de département pourrait contribuer à une gestion multisectorielle ${ }^{525}$ des aires protégées, au contraire de la gestion sectorielle ${ }^{526}$ qui a montré ses limites ${ }^{527}$. De la sorte, la gestion des aires protégées n'est plus uniquement pensée dans une approche restrictive de protection de l'intégrité de ces massifs forestiers. Audelà de cette dimension, elle intègre la valorisation en vue du développement local et national. Subséquemment, les problèmes de gestion que rencontrent les aires protégées pourraient être abordés et trouver des solutions dans un cadre de gestion plus large.

À titre d'exemple, l'un des problèmes de gestion des aires protégées ivoiriennes a, pendant longtemps, été les difficultés de décaissement des subventions de l'État, en raison des lourdeurs administratives caractéristiques de l'Administration ivoirienne ${ }^{528}$. Ce faisant, le fait pour les préfets de Région et de département qui ont des attributions relatives au budget de l'État ${ }^{529}$ dans leur ressort territorial d'être associés à la gestion des aires protégées pourrait être une solution pour la mise à disposition, à temps opportun, des fonds destinés à leur gestion durable.

L'association des préfets de Région et de département à la gestion des aires protégées pourrait s'expliquer par deux (2) autres raisons. Premièrement, ces autorités administratives sont investies d'une mission de développement au niveau local. Deuxièmement, la valorisation des aires protégées pourrait contribuer à la réalisation cette mission (de développement local ${ }^{530}$ ) assignée à la région et partant au département et réduire les pressions sur les aires protégées. En effet, certaines études montrent que les pressions anthropiques sur

\footnotetext{
${ }^{525} \mathrm{La}$ gestion multisectorielle des aires protégées pourrait s'entendre de celle à laquelle participent plusieurs Administrations publiques (par exemple celles des loisirs, du transport, du tourisme, de la sécurité) et autres personnes de droit privé aux côtés du personnel des Eaux et Forêts en charge de la gestion des aires protégées.

${ }_{526}$ À travers l'expression gestion sectorielle des aires protégées, il faut entendre celle qui par le passé était exclusivement opérée par les fonctionnaires du corps des Eaux et Forêts ayant la charge de la gestion des aires protégées.

${ }^{527}$ À ce sujet, voir la Première partie de cette étude, le Titre I, le Chapitre II, la Section 1, le "Paragraphe 2 : Les limites des pouvoirs du personnel en charge des aires protégées".

${ }^{528}$ Le Professeur DÉGNI-SÉGUI (René) souligne cette lourdeur qui caractérise l'Administration ivoirienne dans ces travaux. En effet, reprenant M. DIABY (Maméry) et traitant de 1'Administration ivoirienne, il écrit : «elle est lente, tatillonne, opaque, gangrenée par la corruption, la fraude, l'absentéisme, le laxisme et la désinvolture.». Voir DÉGNI-SÉGUI (René), Droit administratif général : L'organisation administrative (t. 1), $4^{\text {ème }}$ édition, op. cit., p. 25.

${ }^{529}$ En effet, aux termes de l'article 10 de la loi précitée de 2014 portant orientation de l'organisation générale de l'Administration territoriale : «Le Préfet de Région est ordonnateur secondaire des crédits délégués du budget de l'État, y compris les crédits délégués par les Ministres au bénéfice des services régionaux de leur ministère. Il ordonnance et liquide les dépenses sur proposition des Directeurs Régionaux. ».

En outre, selon l'article 19 suivant le préfet de département : «...est ordonnateur secondaire des crédits délégués du budget de l'État, y compris les crédits délégués par les Ministres au bénéfice des services départementaux de leur Ministère ; ... ».

${ }^{530}$ En ce qui concerne le développement des communautés locales par le moyen de la valorisation des aires protégées, voir nos analyses afférentes dans la deuxième partie de cette étude, le Titre II, le Chapitre II, la Section I, Le Paragraphe 1 (Le tourisme, une activité financièrement rentable) et précisément le point "B- Un levier pour le développement des communautés riveraines des aires protégées".
} 
les aires protégées s'expliquent, entres autres, soit par le dysfonctionnement du processus de développement, soit par le déficit de développement des zones dans lesquelles ces aires protégées sont situées ${ }^{531}$.

Au surplus, la deuxième catégorie d'autorités publiques associées à la gestion des aires protégées, depuis 2002, sont celles relevant de l'Administration décentralisée.

\section{b. Les autorités de l'Administration décentralisée associées à la gestion des aires protégées}

L'article 32 de la loi précitée de 2014 portant orientation de l'organisation générale de l'Administration territoriale indique que l'Administration décentralisée est assurée dans le cadre de Collectivités territoriales que sont les Régions et les Communes ${ }^{532}$.

Il est à préciser qu'avant l'entrée en vigueur de la loi précitée de 2014 portant orientation de l'organisation générale de l'Administration territoriale, c'est-à-dire sous l'empire de la loi d'orientation sur l'organisation de l'Administration précitée de 2001, les autorités publiques relevant de l'Administration décentralisée associées à la gestion des aires protégées ivoiriennes étaient le président de Conseil régional et le président du Conseil général $^{533}$. Toutefois, avec la nouvelle nomenclature de l'Administration décentralisée, la seule autorité décentralisée qui participe à la gestion des aires protégées est le président du Conseil régional. À l'instar des autorités administratives déconcentrées associées à la gestion des aires protégées, le président de Conseil régional à une mission de développement et d'encadrement des populations. Mieux, il a la particularité d'avoir une mission de gestion des terroirs et de l'environnement ${ }^{534}$.

\footnotetext{
${ }^{531}$ Voir SANDIA (Mohamed), Rapport de l'Exercice de Consultation des Populations Riveraines aux Aires Protégées, Abidjan, PCGAP/BM, mai 1998, pp. 10-14 et 23 ; GTZ/OIPR, Le profil de la Zone Périphérique du Parc National de Taï, Quel potentiel pour la conservation du Parc ?, Abidjan, mars 2006, pp. 20-24.

${ }^{532} \mathrm{C}$ 'est ce que dispose cet article en ces termes : "L'Administration décentralisée est assurée dans le cadre de collectivités territoriales que sont:

- les Régions;

- les Communes. ».

Cette formulation de l'article 32 ramène à deux (2) les différents types de collectivités territoriales, à savoir la Commune et la Région, au contraire du libellé de l'article 32 de la loi n 2001-476 du 9 août 2001 d'orientation sur l'organisation générale de l'Administration territoriale (JORCI n³6 du 6 septembre 200, p. 746) qui prévoyait quatre (4) types de collectivités territoriales que sont: le Département, le District, la Ville et la Commune.

${ }^{533}$ Selon l'article 38 de la loi d'orientation sur l'organisation de l'Administration précitée de 2001, «La Région est une Collectivité territoriale. ». L'article 39 de cette loi dote la Région de quatre (4) organes qui sont le Conseil régional, le président du Conseil régional, le bureau du Conseil régional, le Comité économique et social régional. À l'instar de la Région, le département est une Collectivité territoriale (art. 41, loi d'orientation sur l'organisation de l'Administration précitée de 2001) qui est dotée également de quatre (4) organes qui sont le Conseil général, le président du Conseil général, le bureau du Conseil général, le Comité économique et social général (art. 42, loi d'orientation sur l'organisation de l'Administration précitée de 2001).

${ }_{534}$ Ces missions découlent des termes de l'article 32 de loi précitée de 2014 portant orientation de l'organisation générale de l'Administration territoriale selon lesquels : "L'Administration décentralisée est assurée dans le cadre de collectivités territoriales que sont:

- les Régions;
} 
Par conséquent, le fait d'associer le président du Conseil régional à la gestion des aires protégées pourraient contribuer à l'effectivité de la mise en œuvre de la politique étatique de conservation de ces forêts et au développement local. En effet, les aires protégées représentent un patrimoine naturel dont la valorisation pourrait contribuer de manière significative au développement local ${ }^{535}$.

Par ailleurs, en dehors de ces autorités administratives susmentionnées, la loi de 2002 sur les PNR associe des personnes morales de droit privé à la gestion des aires protégées.

\section{Les personnes morales de droit privé associées à la gestion des aires protégées}

Les personnes morales de droit privé associées à la gestion des aires protégées depuis l'année 2002 sont les associations de populations (a), les opérateurs économiques (b) et les Organisations Non Gouvernementales (c).

\section{a. Les associations de populations locales}

La participation des populations à la gestion des aires protégées en Côte d'Ivoire est l'œuvre de la loi de 2002 sur les PNR. En effet, aux termes de l'article 17 de ladite loi : «Les conditions et modalités d'association, d'une part, des populations des zones périphériques à la gestion d'un parc national et d'une réserve naturelle...font l'objet d'un contrat de gestion de terroir...» (souligné par nous). En d'autres termes, depuis l'année 2002, la réglementation de la gestion des aires protégées consacre le principe de participation des populations locales à la gestion des aires protégées.

Cette innovation opérée dans le droit des aires protégées par le législateur semble être motivée par plusieurs raisons. En effet, la gestion exclusive des aires protégées par l'État en tant que collectivité territoriale a montré ses limites ${ }^{536}$. De plus, les aires protégées sont des espaces autour desquels sont établies des communautés humaines. L'existence de ces communautés en ces lieux préexiste, bien souvent, à leur érection en aire protégée par l'État. Pendant longtemps, elles y ont longtemps mené des activités diverses (agriculture, pêche, chasse, cueillette...) à des fins de subsistance ou économique.

\footnotetext{
- les Communes.

Les collectivités territoriales ont pour missions, dans la limite de leurs compétences :

- l'organisation de la vie collective dans la collectivité territoriale;

- la participation des populations à la gestion des affaires locales;

- la promotion et la réalisation du développement local;

- la modernisation du monde rural;

- l'amélioration du cadre de vie;

- la gestion des terroirs et de l'environnement. (souligné par nous) ».

${ }^{535}$ Voir nos développements sur la question dans la deuxième partie de cette thèse, le Titre II, le Chapitre II, la Section I, le Paragraphe 1 (Le tourisme, une activité financièrement rentable) et précisément "Un levier pour le développement des communautés riveraines des aires protégées".

${ }^{536}$ À propos de ces limites, lire la Première partie de la présente thèse, le Titre I, le Chapitre II, la Section 1 et précisément le point intitulé "Paragraphe 2 : Les limites des pouvoirs du personnel en charge des aires protégées".
} 
Cependant, le classement de ces espaces naturels sur lesquels elles disposaient d'un droit de propriété coutumière contrevient à leurs intérêts en raison de la suppression de leurs droits d'usage sur ces espaces ${ }^{537}$. Autrement dit, les populations riveraines des aires protégées doivent cesser de les exploiter à des fins économiques (exploitations agricole et commerciale, confection d'outils de travail) et de subsistance (alimentation, santé et construction d'habitats...), par l'arrêt des prélèvements de ressources animales et végétales.

Le constat qui en résulte est que les pressions diverses que subissent ces aires protégées et qui constituent une entrave à leur conservation durable sont largement d'origine anthropique $^{538}$. Cette situation pourrait traduire l'idée que l'interdiction des droits d'usage des populations sur les aires protégées, en vue de leur conservation, n'est pas, en général, bien comprise et appréciée par les populations locales qui sont essentiellement rurales ${ }^{539}$. En plus, elle pourrait faire penser que les populations n'ont pas la (pleine) conscience des implications juridiques du classement de certaines portions de terres en aire protégée.

Quoiqu'il en soit, l'on assiste souvent à une opposition d'intérêts qui se pose en termes d'exigence de protection de la nature pour l'État (représenté par les gestionnaires des aires protégées), et d'exploitation à but économique et de subsistance pour les populations ${ }^{540}$. Cette opposition d'intérêt aboutit, en général, à des conflits violents ${ }^{541}$.

C'est peut-être pour trouver une solution, en vue de la conservation durable des aires protégées, que la loi de 2002 sur les PNR introduit, pour la première fois, dans le droit ivoirien des aires protégées, le principe de participation des populations à la gestion des aires protégées. De la sorte, la participation des populations à la gestion des aires protégées permettrait de les sensibiliser sur le bien-fondé des objectifs et enjeux de la politique de conservation des aires protégées. De même, les populations pourraient faire savoir leurs contraintes en lien avec la conservation des aires protégées et le développement local de sorte que des solutions consensuelles soient adoptées en vue de la conservation durable de ces forêts $^{542}$.

\footnotetext{
${ }^{537} \mathrm{Au}$ sujet de la suppression des droits d'usage des communautés locales sur les aires protégées, voir en Introduction (de cette étude), le point intitulé "IV- Intérêt du sujet", la note 114.

${ }^{538}$ Il s'agit du braconnage familial qui est le fait des populations ou de type commercial pratiqué par des braconniers professionnels, d'une part. D'autre part, l'on a le prélèvement de produits forestiers secondaires : bois de chauffe, plante médicinales, curent-dent, vin de palme ; et de la mise en culture de certains superficies de ces aires protégées etc. Sur ce point, lire Ministère de l'Environnement et des Eaux et Forêts/Office Ivoirien des Parcs et Réserves, État de conservation des parcs et réserves de Côte d'Ivoire, op . cit., p.6.

${ }^{539}$ En Côte d'Ivoire, $59 \%$ de la population totale (estimée à 20,8 millions d'habitants dont $26 \%$ d'immigrés) est rurale. Voir Ministère d'État, ministère du Plan et du Développement, Document stratégique de réduction de la pauvreté, op.cit., p. 37 et s.

${ }^{540}$ Sur la question, voir GOH (Denis), Les approches participatives dans la gestion des Aires Protégées en Côte d'Ivoire : l'expérience du Projet Autonome pour la Conservation du Parc National de Taï (PACPNT), Thèse unique, Environnement, Université d'Abobo-Adjamé, 21 mai 2005, p. 2 et s.

${ }^{541}$ Voir AKOUÉ (Yao Claude), Les aspects conflictuels liés à la conservation des aires protégées de Côte d'Ivoire: le cas du Parc National de la Marahoué à Bouaflé, Mémoire de DEA en Sciences et Gestion de l'Environnement option Politique en Environnement, Université d'Abobo-Adjamé, août 2004, p. 63.

${ }^{542} \mathrm{Il}$ est vrai que des mécanismes de gestion participative avec le grand public et surtout les communautés locales existent. Cependant, il ressort de nos enquêtes sur le terrain que leur mise en place tardive et les difficultés de
} 
En associant dorénavant les populations à la gestion des aires protégées, le législateur précise que cette participation doit se faire sous forme associative. En effet, à l'article $1^{\mathrm{er}}$ de la loi de 2002 sur les PNR, le contrat de gestion de terroir «désigne le contrat passé entre le gestionnaire d'un parc ou réserve et les populations rurales de la zone périphérique représentées par des structures associatives, privées ou administratives. Ce contrat définit, notamment, les modalités d'intervention des populations contractantes dans leur surveillance, la gestion, l'entretien et, le cas échéant, l'animation culturelle et touristique d'un parc, d'une réserve ou de leur zone périphérique. » (souligné par nous).

En plus des populations, les opérateurs économiques sont une autre catégorie de personnes morales de droit privé associées dorénavant à la gestion des aires protégées.

\section{b. Les opérateurs économiques}

Par vocation, les opérateurs économiques sont des acteurs essentiels du développement économique, tant à l'échelle nationale que locale. Les effets de leurs actions sur le développement s'apprécient en termes de réduction de la pauvreté. En effet, ils contribuent à la création d'emplois, grâce aux investissements et à la mobilisation de capitaux.

En Côte d'Ivoire, l'une des principales causes des pressions anthropiques sur les aires protégées, évoquées par les populations, les ONG et les pouvoirs publics, est la pauvreté des populations rurales qui est une résultante du déficit de développement des zones périphériques des aires protégées ${ }^{543}$. Également, le constat est que les aires protégées ivoiriennes sont insuffisamment valorisées ${ }^{544}$. Cette situation pourrait résulter du fait de l'insuffisance des moyens financiers que l'État accorde pour la gestion des aires protégées vu que le développement d'une industrie touristique autour des aires protégée requiert des moyens financiers conséquents. Or, les aires protégées ont un fort potentiel d'attractivité en matière touristique qui pourrait générer d'importantes richesses ${ }^{545}$. De ce fait, elles pourraient contribuer au développement économique des localités où elles sont établies.

\footnotetext{
fonctionnement qui les caractérisent, dans l'ensemble, les ont rendu pendant longtemps virtuel ou théorique. Voir l'Annexe 3 de la présente étude intitulé "Enquête sur les réalités de la gestion des aires protégées en Côte d'Ivoire", le "III- Résultats de l'enquête auprès des gestionnaires d'aires" et précisément le point "5- L'état de la mise en place des outils de gestion participative".

${ }^{543} \mathrm{Il}$ ressort des résultats des enquêtes réalisées dans le cadre de cette thèse que $66 \%$ des personnes enquêtées pensent que les pressions sur les aires protégées sont dues à la pauvreté. Voir l'Annexe 3 de la présente thèse.

${ }^{544}$ Voir MONSAN (Ignace), Stratégie de développement de l'écotourisme : le cas du Parc National d'Azagny, Mémoire de DEA en Sciences et Gestion de l'Environnement option Politique en Environnement, Université d'Abobo-Adjamé, 17 août 2004, pp. 28, 30.

${ }^{545}$ Sur ce point, voir nos analyses dans la Deuxième partie de cette thèse, le Titre II, le Chapitre I, la Section I et précisément le point intitulé "Paragraphe 2: L'existence d'attraits naturels et culturels dans les aires protégées et leurs zones périphériques".
} 
Dans la pratique, c'est le secteur privé qui détient plus d'expertise et investit des moyens financiers importants pour la valorisation des aires protégées ${ }^{546}$. Il nous semble que c'est pour cette raison que depuis 2002, la législation relative aux aires protégées associe les opérateurs économiques à la gestion de ces forêts. De la sorte, les opérateurs économiques pourraient contribuer à la valorisation (touristique) des aires protégées.

C'est à juste titre que l'article 36 du décret de 2002 portant création de l'OIPR, tout en instituant un comité de gestion locale pour chaque Parc ou Réserve, définit des opérateurs économiques comme membres dudit comité. Il s'agit précisément de deux (2) opérateurs économiques intervenant dans le secteur du tourisme et de l'artisanat désignés par leurs organisations respectives $^{547}$.

Le choix de ces deux (2) secteurs d'activités pourrait se justifier par le fait qu'ils sont un moyen de valorisation des aires protégées et des cultures des communautés locales. En d'autres termes, ces secteurs d'activités peuvent générer des ressources financières importantes, à la fois, pour la conservation de ces forêts et pour le développement local ${ }^{548}$.

De plus, la Côte d'Ivoire présente une diversité de cultures dont la richesse témoigne d'un savoir-faire ${ }^{549}$. Ce potentiel est adapté à la demande touristique. Ce faisant, le développement de l'industrie touristique et artisanale pourrait constituer un levier pour le développement des zones périphériques des aires protégées. De la sorte, cette activité aiderait à contribuer à la réduction de la pauvreté. Par contrecoup, les pressions anthropiques dont font l'objet ces aires protégées pourraient diminuer, ainsi que le démontrent des expériences de gestion d'aires protégées en Afrique de l'Est et en Afrique australe ${ }^{550}$.

$\mathrm{Au}$ surplus, les Organisations Non Gouvernementales (ONG) sont la dernière catégorie de personne morale de droit privé associée à la gestion des aires protégées.

\footnotetext{
${ }^{546}$ Voir nos développements nos développement sur la question dans la deuxième partie de cette étude, le Titre II, le Chapitre II, la Section II, le Paragraphe 1, et précisément le point intitulé "De l'intérêt de la concession de l'exploitation écotouristique des aires protégées à des opérateurs touristiques privés".

${ }^{547}$ Sur ce point, il peut être lu à l'article $37 \mathrm{du}$ décret de 2002 portant création de l'OIPR : « Les membres du comité de gestion locale sont choisis selon les modalités définies par les organisations qu'ils représentent. ».

${ }^{548}$ Des expériences de gestion d'aires protégées en Afrique de l'Ouest, en Afrique de l'Est et en Afrique australe montrent que leur valorisation touristique y compris celles de leurs zones périphériques a un impact positif important en matière de développement local. Voir nos développements afférents dans la deuxième partie de cette étude, le Titre II, le Chapitre II, la Section I, Le Paragraphe 1 (Le tourisme, une activité financièrement rentable) et précisément le point "B- Un levier pour le développement des communautés riveraines des aires protégées".

${ }^{549}$ Voir la deuxième partie de cette thèse, le Titre II, le Chapitre II, la Section I, Le Paragraphe 2 et précisément le point "B- La diversité culturelle des communautés riveraines des aires protégées".

${ }^{550}$ En la matière, pour des informations sur ces expériences de gestion d'aires protégées, voir la deuxième partie de la présente étude, le Titre II, le Chapitre I, la Section I, le Paragraphe 1 (L'interdépendance de la conservation des ressources naturelles et du développement local), et précisément le point intitulé " B- La justification de l’interdépendance fondée sur des expériences de gestion de ressources naturelles".
} 


\section{c. Les Organisations Non Gouvernementales}

Selon le Professeur Maurice KAMTO, on entend par Organisations Non Gouvernementales (ONG) : «tout groupement, association constitué de façon durable -sur la base d'un acte juridique généralement appelé statut-par des individus ou des personnes morales appartenant à un même Etat ou à des Etats différents, en vue de la poursuite de buts non lucratifs $»^{551}$. Aux termes de l'article 36 du décret de 2002 portant création de l'OIPR, deux (2) représentants élus des ONG intervenant dans une aire protégée ou sa zone périphérique de protection sont membres du comité de gestion locale de ladite aire protégée.

L'association de telles ONG à la gestion des aires protégées se justifierait par diverses raisons. En effet, les ONG, qu'elles soient nationales ou internationales, se posent comme des acteurs de développement ${ }^{552}$, quel que soit leur domaine d'intervention. Elles accompagnent le processus de développement ou l'impulsent, selon le contexte politico-économique dans lequel elles interviennent.

Spécifiquement, en matière d'environnement, après la conférence de Rio, l'importance des $\mathrm{ONG}$ est de plus en plus manifeste et réaffirmée ${ }^{553}$. En effet, elles contribuent à la mise en œuvre des objectifs de gestion durable de l'environnement. Les ONG sensibilisent les populations (principaux auteurs des pressions sur les aires protégées) sur les risques environnementaux de leurs comportements écocides, d'une part. D'autre part, disposant d'une expertise (avérée) et d'une puissance financière (ce dernier cas concerne les ONG internationales), elles participent à la gestion durable de l'environnement. En conséquence, elles sont susceptibles de proposer des solutions appropriées pour les problèmes environnementaux qui se posent.

Il est à noter qu'en Côte d'Ivoire, la loi n'autorise pas les ONG et notamment les ONG environnementales à initier des actions en justice ou à intervenir dans des procédures pour la protection des forêts. En effet, si l'article $1^{\text {er }}$ alinéa $1^{\text {er }}$ du Code de procédure civile ivoirien dispose que «Toute personne, physique ou morale, peut agir devant les juridictions de la République de Côte d'Ivoire, en vue d'obtenir la reconnaissance, la protection ou la sanction de son droit. ", l'article 3 suivant qui définit les conditions d'exercice du droit d'ester en justice ajoute, entre autres, que l'intérêt défendue soit légitime et juridiquement protégé, direct et personnel ${ }^{554}$. Ainsi, les ONG peuvent saisir la Justice pour obtenir le respect d'un droit,

\footnotetext{
${ }^{551}$ Voir KAMTO (Maurice), Droit de l'environnement en Afrique, op. cit., p. 381.

${ }^{552}$ Le chapitre 27 de l'Agenda 21 mentionne que les ONG sont des partenaires du développement durable.

${ }^{553}$ Entres autres textes, les chapitres 21 (paragraphe 27-10) et 27 de l'Agenda 21 (renforcement du rôle des ONG partenaires pour un développement durable) traitent de l'importance des ONG et de la nécessité pour les États de les impliquer davantage dans la protection de l'environnement. De même, dans le préambule de la Convention de Rio sur la diversité biologique, le point 14 recommande la coopération entre les États et le secteur non gouvernemental en vue de la conservation de la diversité biologique.

${ }^{554}$ En effet, l'article 3 du Code de procédure civile ivoirien précité est libellé comme suit: «L'action n'est recevable que si le demandeur:

$\left.1^{\circ}\right)$ justifie d'un intérêt légitime juridiquement protégé direct et personnel ;

$2^{\circ}$ ) a la qualité pour agir en justice;
} 
c'est-à-dire empêcher ou faire cesser sa violation en ce que le tort qui leur est fait les atteint directement et de façon personnel en tant que personne morale. Cependant, elles ne peuvent ester en justice pour la protection des forêts dans la mesure où elles ne justifieraient pas d'un intérêt direct et personnel.

Par ailleurs, pour permettre la mise en œuvre de la gestion participative des aires protégées, le législateur ivoirien a prévu des modalités à cette effet.

\section{Paragraphe 2 : Les modalités de mise en œuvre de la gestion participative des aires protégées}

Les modalités de mise en œuvre de la gestion participative des aires protégées ivoiriennes prévues par la loi sont le comité de gestion locale d'aire protégée (A) et aussi la contractualisation de la gestion de ces milieux naturels (B).

\section{A- L'instauration de comités de gestion locale d'aire protégée}

L'idée que le comité de gestion locale de Parc et Réserve est un cadre de gestion participative des aires protégées découle de ce qu'il constitue une plate-forme de gestion multisectorielle des aires protégées (1) et par contrecoup de concertation pour leur gestion (2).

\section{Le comité de gestion locale d'aire protégée : une plate-forme de gestion multisectorielle des aires protégées}

Dans le droit positif des aires protégées, c'est l'article 35 du décret précité de 2002 portant création, organisation et fonctionnement de l'OIPR qui prescrit dorénavant que «chaque parc et chaque réserve sont dotés d'un comité de gestion locale...». Prévu par l'article 31 de la loi précitée de 2002 sur les PNR, le comité de gestion locale de Parc ou Réserve ${ }^{555}$ n'est pas défini par ladite loi. Il n'est non plus défini par le décret précité de 2002 portant création, organisation et fonctionnement de l'OIPR qui à travers ses articles 35 et suivants en définit la composition et les attributions.

Toutefois, la composition dudit comité pourrait être utilisée comme un critère pour le définir. Il ressort de la lecture de l'article 36 du décret de 2002 portant création de l'OIPR que le comité de gestion locale d'un Parc ou d'une Réserve est composé de quinze (15) membres au maximum. Il s'agit :

\footnotetext{
$\left.3^{\circ}\right)$ possède la capacité d'agir en justice.

${ }^{555}$ L'article 31 de la loi précitée de 2002 sur les PNR dispose que : «Les modalités de la gestion décentralisée des parcs Nationaux et des réserves naturelles et, notamment, les attributions et la composition des comités de gestion, ainsi que les attributions des directeurs, sont définies par décret pris en conseil des ministres ». Si le présent article 31 n'utilise que le groupe de mots "comité de gestion" sans y adjoindre l'épithète "locale" c'est le décret portant création de l'OIPR précité qui utilise l'expression "comité de gestion locale" conformément aux attributions que les rédacteurs dudit décret donnent au Comité de gestion.

Par ailleurs, cette appellation est également déductible de l'article 21 de la loi précitée sur les PNR qui prescrit que : « À l'effet de l'exécution de ses missions, l'Établissement est chargé de... Veiller avec l'appui du Comité de gestion de chaque parc et réserve... ».
} 
- d'autorités administratives que sont les Préfets de Départements ou de Régions concernés ; - de responsables de l'OIPR, à savoir le Directeur du Parc ou de la Réserve, un membre du Conseil scientifique ainsi que le Directeur Général de l'OIPR ou son représentant ;

- de deux (2) représentants élus des ONG intervenant dans le Parc ou la Réserve concernée, ou dans sa zone périphérique de protection ;

- de deux (2) représentants des opérateurs économiques exerçant dans le secteur du tourisme et de l'artisanat en vue de la valorisation des Parcs Nationaux et Réserves ;

- de représentants des collectivités territoriales et des terroirs constituant la zone périphérique de protection que sont, entre autres, les Présidents des Conseils régionaux et généraux ${ }^{556}$ et les représentants des organisations villageoises ${ }^{557}$.

Autrement dit, d'une part, le comité de gestion locale de Parc ou Réserve comprend : des autorités relevant de l'Administration (déconcentrée et décentralisée), des responsables de l'OIPR. D'autre part, il comprend des représentants d'ONG de conservation de l'environnement et de développement, les populations, des opérateurs économiques. Par conséquent, le comité de gestion locale de Parc ou Réserve pourrait s'entendre comme un regroupement de plusieurs entités de nature diverse qui composent l'environnement d'un Parc ou d'une Réserve et qui participent à sa gestion. Quant à la gestion multisectorielle des aires protégées pourrait se définir comme une gestion des aires protégées opérée aussi bien par les agents des Eaux et Forêts et d'autres pouvoirs publics ainsi que des compétences du secteur privé.

La composition du comité de gestion locale étant diversifiée, la gestion des aires protégées par ce comité n'est plus sectorielle telle qu'elle l'a été par le personnel des Eaux et Forêts (de 1960 à 2002). Bien au contraire, cette approche de gestion forestière (sectorielle) s'enrichit désormais de l'expertise technique, scientifique et de l'aide financière des ONG. En outre, elle intègre les aspects de développement des zones périphériques, par la mise en

\footnotetext{
${ }^{556}$ La nouvelle organisation administrative de la Côte d'Ivoire ne retient plus les départements comme étant des collectivités décentralisées. Par conséquent, il n'existe plus de Président de Conseil général qui, par le passé, dirigeait la collectivité territoriale qu'est le département. En effet, l'article 32 de la loi précitée de 2014 portant orientation de l'organisation générale de l'Administration territoriale dispose : "L'Administration décentralisée est assurée dans le cadre de collectivités territoriales que sont:

- les Régions;

- les Communes. ».

${ }^{557}$ Aux termes de l'article 36 du décret de 2002 portant création de 1'OIPR, « La composition et les modalités de fonctionnement de chaque Comité de gestion locale sont fixées par arrêté conjoint du Ministre chargé des Parcs et Réserves et du Ministre chargé de l'Intérieur, de la Décentralisation et des Collectivités Territoriales, dans la limite de 15 membres au maximum dont notamment:

- les Préfets des Départements ou des Régions concernées ;

- le Directeur du parc ou de la réserve ;

- deux représentants élus des organisations non gouvernementales intervenant dans le parc ou la réserve concernée, ou dans sa zone périphérique de protection;

- les représentants des collectivités territoriales et des terroirs constituant la zone périphérique de protection (Présidents des Conseils Régionaux, Présidents des Conseils Généraux, Représentants des Organisations Villageoises ...) ;

- deux représentants des opérateurs économiques dans le secteur du tourisme et de l'artisanat;

- le Directeur Général de l'Office ou son représentant ;

- un membre du Conseil Scientifique. ».
} 
place de projets d'écotourisme et de développement. Ceci se fait par le truchement d'opérateurs économiques, des autorités de l'Administration déconcentrée ainsi que les autorités des collectivités territoriales en vue du bien-être des populations. C'est cette approche globale de la gestion des aires protégées que nous qualifions de multisectorielle.

Par ailleurs, le comité de gestion locale d'aire protégée est un instrument de conservation durable desdits espaces parce qu'il représente un cadre de concertation élargie pour leur gestion.

\section{Le comité de gestion locale d'aire protégée : un cadre de concertation élargie pour la gestion des aires protégées}

Organe collégial au vu de sa composition, le comité de gestion locale d'aire protégée est par excellence un cadre de concertation. En effet, l'article 38 du décret de 2002 portant création de l'OIPR dispose : "Le comité de gestion locale se réunit au moins une fois par trimestre. Le comité de gestion locale donne son avis sur toutes les questions d'ordre technique, culturel ou budgétaires qui lui sont soumises, à titre consultatif, par le Directeur du parc ou de la réserve considérée. " (souligné par nous). C'est ainsi que le comité de gestion locale est une plateforme d'échanges, de discussions. Mieux, c'est un cadre d'expression de la démocratie participative en matière de gestion d'aire protégée.

Cette démocratie participative dans la gestion des aires protégées est consolidée par le fait que les membres du comité de gestion locale sont désignés par leur organisation respective $^{558}$. Par conséquent, ils sont autonomes et libres de prendre des décisions requises pour la conservation durable des aires protégées.

De plus, la composition diversifiée du comité de gestion locale donne un caractère qualitatif à la concertation qui la fonde en ce sens que ce comité met en présence plusieurs acteurs aux compétences diverses. D'une part, il s'agit des gestionnaires dont la mission essentielle est de protéger l'intégrité de ces aires protégées; et des populations qui sont en grande partie responsables de la dégradation des aires protégées. D'autre part, ces acteurs sont des représentants de l'Administration déconcentrée, des collectivités territoriales et des ONG dont la mission est d'accompagner le développement local et de protéger l'environnement.

Par conséquent, les problèmes ou toutes autres questions liées à la gestion des aires protégées sont abordés dans un cadre global (comité de gestion locale) qui promeut la concertation. Ce cadre de concertation pourrait être un atout pour la conservation durable des aires protégées. En effet, les décisions de gestion du comité de gestion locale étant consensuelles, elles favorisent la prise en compte des intérêts des populations riveraines des aires protégées. Dans ces conditions, elles seraient plus disposées à respecter le droit des aires protégées.

\footnotetext{
${ }^{558}$ Art. 37 et 38 du décret de 2002 portant création de l'OIPR.
} 
C'est également dans cet élan de gestion participative des aires protégées que le législateur ivoirien a envisagé la contractualisation de la gestion des aires protégées.

\section{B - La contractualisation de la gestion des aires protégées}

La gestion participative des aires protégées en Côte d'Ivoire se réalise également sous la forme contractuelle à travers le contrat de gestion de terroir (1) et la convention de concession d'exploitation (2).

\section{Le contrat de gestion de terroir}

Prévu par la loi de 2002 précitée sur les PNR, le contrat de gestion de terroir ${ }^{559}$ est défini à l'article $1^{\text {er }}$ de ladite loi comme «le contrat passé entre le gestionnaire d'un parc ou d'une réserve et les populations rurales de la zone périphérique représentées par des structures associatives, privées ou administratives. Ce contrat définit, notamment, les modalités d'intervention des populations contractantes dans la surveillance, la gestion, l'entretien et, le cas échéant, l'animation culturelle et touristique d'un parc, d'une réserve ou de leur zone périphérique... » (souligné par nous). De plus, l'article 33 alinéa 2 suivant qui traite de l'objet dudit contrat, dispose «...Ces contrats ont pour objet de définir les conditions et modalités de l'association de ces populations à la conservation du Parc ou de la Réserve et de favoriser ainsi les retombées économiques pour ces populations. ».

Il est en découle que le contrat de gestion de terroir peut être défini comme étant un contrat conclu à l'échelle de la zone périphérique d'une aire protégée ${ }^{560}$, dans l'intérêt de sa conservation et (du développement) des communautés locales. Autrement dit, le caractère synallagmatique du contrat de gestion de terroir découle de ce qu'il est destiné à préserver les aires protégées des pressions anthropiques dont elles sont en règle générale l'objet et aussi d'œuvrer au développement économique des communautés locales.

Cette innovation législative semble se justifier. En effet, le constat est que les dégradations que subissent les aires protégées ivoiriennes sont pour la plupart d'origine anthropique ${ }^{561}$. Ce constat amène donc les rédacteurs de la loi précitée de 2002 sur les PNR à reconnaitre l'importance et la nécessité de l'implication des populations riveraines des aires protégées à leur conservation durable. Dès lors, l'institution d'une zone périphérique des aires

\footnotetext{
${ }^{559}$ Les articles 17 alinéa $1^{\text {er }}$ et 33 alinéa $1^{\text {er }}$ de la loi précitée de 2002 sur les PNR prescrivent respectivement que : «Les conditions et modalités d'association, d'une part, des populations des zones périphériques à la gestion d'un parc national ou d'une Réserve naturelle et, d'autre part, de l'autorité chargée de la gestion d'une telle aire protégée au développement de la zone périphérique, font l'objet d'un contrat de gestion de terroir mentionné à l'article 33 ci-dessous. » et que "Les contrats de gestion de terroir sont passés au bénéfice réciproque d'un parc ou d'une réserve et de ses populations environnantes. ».

${ }^{560}$ L'article $1^{\text {er }}$ de la loi de 2002 sur les PNR définit la zone périphérique d'un Parc National ou d'une Réserve naturelle comme étant celle géographique environnante des parcs et réserves, constituée de l'entière superficie des terroirs, des sous-préfectures et, le cas échéant, des forêts classées.

${ }^{561}$ Voir en Introduction (de cette étude), le point "I- Enjeux de la protection des forêts en Côte d'Ivoire", " Cl'état des pressions anthropiques sur la biodiversité en Côte d'Ivoire", et précisément le point intitulé "2. Les pressions anthropiques sur les aires protégées en particulier ".
} 
protégées permet de circonscrire une communauté humaine avec laquelle l'OIPR peut échanger, établir et formaliser des relations en vue de la conservation durable de ladite aire protégée ${ }^{\mathbf{5 6 2}}$ notamment dans le cadre de contrats de gestion de terroir.

Aux termes de l'article 33 alinéa 2 de la loi de 2002 sur les PNR qui traite de l'objet $\mathrm{du}$ contrat de gestion de terroir, ces contrats «peuvent porter, notamment, selon qu'il s'agit d'un parc ou d'une réserve et en considération des espaces concernés de la zone périphérique, sur la gestion des ressources naturelles, sur les activités d'éducation, de loisir, de formation de guides, d'hôtellerie et d'aménagement. » (souligné par nous). Il résulte des termes de l'article 33 alinéa 2 de la loi de 2002 que le contrat de gestion de terroir peut avoir pour objet, d'une part, la gestion durable des aires protégées. D'autre part, il pourrait s'agir d'activités destinées à l'exploitation écotouristique ${ }^{563}$ (activités d'éducation, de loisir, de formation de guides, d'hôtellerie et d'aménagement) qui, tout en permettant sa conservation, concourent au développement des communautés locales.

En conséquence, les parties au contrat de gestion de terroir sont, d'une part, l'autorité en charge de la gestion des aires protégées, à savoir l'Office Ivoirien des Parcs et Réserves (OIPR). D'autre part, ce sont les représentants des populations environnantes de l'aire protégée objet du contrat. À titre illustratif, l'OIPR et une collectivité locale telle que le Conseil régional du Bounkani ${ }^{564}$ ont signé, le vendredi 22 août 2014 à Bondoukou, un contrat de gestion de terroir pour la gestion durable du Parc National de la Comoé ${ }^{565}$.

En plus du contrat de gestion de terroir, la gestion participative des aires protégées sous forme contractuelle se réalise également à travers la convention de concession d'exploitation.

\section{La convention de concession d'exploitation}

Dans le cadre de la gestion des aires protégées, c'est l'article 32 alinéa $1^{\text {er }}$ de la loi de 2002 sur les PNR qui prévoit la convention de concession d'exploitation, sans pour autant que ladite loi la définisse. En effet, aux termes de cet article: «Tout ou partie des missions d'exploitation d'un parc ou d'une réserve, selon la nature de ce parc ou de cette réserve, peuvent être concédées par l'Établissement à une personne morale de Droit privé dans le cadre d'une Convention de concession d'exploitation. » (souligné par nous).

\footnotetext{
${ }^{562}$ Art. 1er, loi de 2002 sur les PNR.

563 Selon la Société Internationale de l'Écotourisme, "L'écotourisme est un voyage responsable dans des environnements naturels où les ressources et le bien-être des populations sont préservés». Voir www.ecotourism.org, consulté le 25 août 2015.

${ }^{564} \mathrm{La}$ région du Bounkani est située à l'extrémité nord-est de la Côte-d'Ivoire. Elle est frontalière de la République du Ghana à l'Est et du Burkina Faso au Nord. La région du Bounkani est limitée à l'Ouest par la région du Tchologo et au Sud par la région du Gontougo. Voir www.ardci-rd.org/index.php/vie-desregions/bounkani, consulté le 2 août 2015.

${ }^{565}$ Cette information nous a été fournie au cours d'un entretien réalisé avec le Directeur technique de l'Office Ivoirien des Parcs et Réserves (M. N'Goran DJÈ), le 11 décembre 2014.
} 
Toutefois, la convention de concession d'exploitation, en l'espèce, pourrait être définie à partir des apports de la doctrine en la matière et de certaines dispositions de la loi précitée de 2002 sur les PNR. En effet, selon le Professeur René DÉGNI-SÉGUI : «La concession de service public se définit comme le procédé par lequel une personne publique, appelée autorité concédante ou concédant, confie à une personne privée, dénommée concessionnaire, l'exploitation d'un service public, moyennant rémunération perçue sur les usagers. ${ }^{566}$. En ce qui concerne la convention de concession, cet auteur note que c'est un document succinct dans lequel les parties à une concession de service public expriment leur $\operatorname{accord}^{567}$. Quant à l'exploitation (sous-entendue l'exploitation des Parcs et Réserves) dont il est fait allusion en l'espèce, elle renvoie à une des missions de l'OIPR. En effet, à l'article 20 de la loi précitée de 2002 sur les PNR qui traite des missions de l'OIPR désigné sous le vocable de «l'Établissement ${ }^{568}$, il peut être lu, entre autres: " Les missions de l'Établissement, sur l'ensemble des parcs et réserves sur lesquels il exerce son autorité, comprennent, notamment et obligatoirement : ...La mise en cuvre d'une politique de gestion durable par la promotion des activités légalement permises en fonction de la nature juridique du parc ou de la réserve considérée et de sa zone périphérique... » (souligné par nous).

De plus, hormis la conservation des aires protégées et de leur diversité biologique associée, les aires protégées sont gérées dans l'optique de participer à la récréation du public. C'est ce qui découle de l'article 3 de la loi de 2002 sur les PNR ainsi libellé : "Les parcs et réserves sont créés et gérés aux fins de permettre la conservation de ses milieux naturels, de la faune et de la flore terrestre et aquatique, ainsi que le maintien de la diversité biologique et des processus écologiques contre toutes les causes de dégradations qui les menacent. Ils ont également pour vocation de participer, par la récréation et l'éducation du public à l'équilibre harmonieux des populations, qu'elles soient rurales ou urbaines. » (souligné par nous). En pratique, dans le cadre de l'exploitation des aires protégées, la fonction de recréation du public, à travers la promotion d'activités légalement permises conformément à leur statut, se réalise par le truchement du tourisme de nature ou l'écotourisme ${ }^{569}$ ou encore du

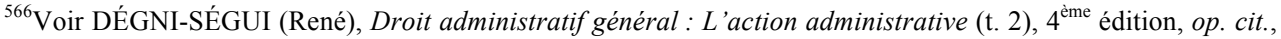
p. 172.

${ }^{567} \mathrm{C}$ 'est cette idée que traduit le Professeur DÉGNI-SÉGUI (René) lorsqu'il affirme que « L'acte de concession, comprend en fait deux parties : la convention de concession et un cahier de charges, qui y est annexé. La première est un document assez bref, qui exprime l'accord des parties. Le second texte, très long et détaillé, détermine les droits et obligations des parties, y compris les principes d'organisation et de fonctionnement du service public. Il se réfère à un cahier de charges-type élaboré par l'Administration.».Voir Idem, p. 173.

${ }^{568}$ En effet, si aux termes de l'article $1^{\text {er }}$ de la loi de 2002 sur les PNR, l'Établissement « désigne une personne morale de Droit public gérant un service public» et que l'article 19 suivant dispose que «La gestion des Parcs et Réserves relève d'un Établissement public national de type particulier, doté de la personnalité morale et de l'autonomie financière...», ce sont les articles $1^{\text {er }}$ et 3 du décret précité de 2002 portant création de l'OIPR qui indiquent respectivement que cet Établissement public de type particulier est dénommé «Office Ivoirien des Parcs et Réserves » et que l'Office Ivoirien des Parcs et Réserves (OIPR) a "pour mission, sur l'ensemble des parcs et réserves sur lesquels il exerce son autorité, notamment :

1. la gestion de la faune, de la flore, et de leur biotope qui en constitue le fondement... ».

${ }^{569}$ Selon le document de travail de l'Office Ivoirien des Parcs et Réserves relatif aux Procédures de concession de l'exploitation touristique des Parcs Nationaux et Réserves, "l'écotourisme" désigne toutes les formes de tourisme-nature, qui de façon responsable, veulent minimiser tant les effets négatifs sur l'environnement que les
} 
tourisme, en général. Par conséquent, l'exploitation des Parcs et Réserves au moyen de la convention de concession d'exploitation renvoie essentiellement à leur exploitation à but touristique y compris les offres culturelles des terroirs riverains.

Cette mission d'exploitation touristique des aires protégées et de manière incidente du patrimoine culturel des communautés locales accomplie par l'OIPR est une mission de service public. En effet, l'activité à réaliser (exploitation des Parcs et Réserves) est d'intérêt général $^{570}$ et l'Administration (Office Ivoirien des Parcs et Réserves-OIPR) qui l'assure est à l'Établissement public national ${ }^{571}$. En tant que telle, l'exercice de cette mission de service public peut être confié à une personne morale de droit privé ${ }^{572}$ par le truchement d'une convention de concession de service public.

Ce faisant, dans le cadre de la gestion des aires protégées en Côte d'Ivoire, la convention de concession d'exploitation pourrait se concevoir comme un document qui formalise l'accord de l'OIPR, autorité concédante, de concéder à une personne morale de Droit privé, concessionnaire, une des missions de service public dont il a la charge. En l'espèce, il s'agit de l'exploitation touristique des Parcs Nationaux et Réserves naturelles. Il découle de cette définition de la convention de concession d'exploitation d'aire protégée que les parties à cette convention sont, d'une part, le concédant, c'est-à-dire l'Office Ivoirien des Parcs et Réserves (OIPR) ${ }^{573}$ et d'autre part, le concessionnaire qui est une personne morale de Droit privé.

\footnotetext{
changements socioculturels. C'est un tourisme qui prend en compte le bien-être de l'environnement naturel et de ses habitants dans une activité d'agrément, de loisir ou de découverte, en s'efforçant au mieux de préserver les espaces qu'il utilise pour ses activités. Le tourisme-nature regroupe l'ensemble des activités axées sur la nature : les activités scientifiques, sportives, l'aventure, l'observation et la photographie de la flore et de la faune, les activités prédatrices comme la pêche et la chasse. ». Pour ces définitions, voir République de Côte d'Ivoire, Ministère de l'Environnement, des Eaux et Forêts/ Office Ivoirien des Parcs et Réserves, Procédures de concession de l'exploitation touristique des Parcs Nationaux et Réserves, Abidjan, avril 2010, p. 3.

${ }^{570}$ L'article 2 de la loi de 2002 sur les PNR qui traite des objectifs de ladite loi dispose, entre autres, « $L a$ réalisation de ces objectifs et d'une façon générale, la sauvegarde du patrimoine naturel sont d'intérêt général et constituent des objectifs prioritaires de la nation. "».

${ }^{571}$ En effet, le service public est une activité destinée à satisfaire un besoin d'intérêt général (définition matérielle) assurée ou assumée par une personne morale de droit public: État, Collectivité locale ou Établissement public (définition organique). Voir GEORGES (Phillipe), Droit public: concours administratifs, 8 édition, op. cit., pp. 300-301 ; GUGLIELMI (Gilles J.), KOUBI (Geneviève), Droit du service public, op. cit.,

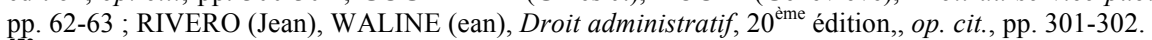

${ }^{572}$ Sur la question, traitant de la primauté de la définition matérielle du service public (sur sa définition organique), le Professeur DÉGNI-SÉGUI (René ) note : "Cette primauté procède de ce que le critère matériel est, à lui seul, suffisant pour définir le service public. Il n'est pas nécessaire de prendre en considération le caractère administratif de l'organisme qui exécute l'activité. Ainsi, un service public peut être géré par des personnes morales de droit privé... ». DÉGNI-SÉGUI (René), Droit administratif général : L'action administrative (t. 2), $4^{\text {ème }}$ édition, op. cit., pp. 115-116.

${ }^{573}$ En effet, si aux termes de l'article $1^{\text {er }}$ de la loi de 2002 sur les PNR, l'Établissement « désigne une personne morale de Droit public gérant un service public. Dans le cas d'espèce, si l'Établissement est chargé de la gestion des parcs et réserves » et que l'article 19 suivant dispose que "La gestion des Parcs et Réserves relève d'un Établissement public national de type particulier, doté de la personnalité morale et de l'autonomie financière...», ce sont les articles $1^{\mathrm{er}}$ et 3 du décret précité de 2002 portant création de l'OIPR qui indiquent respectivement que cet Établissement public de type particulier est dénommé «Office Ivoirien des Parcs et Réserves» et que l'Office Ivoirien des Parcs et Réserves (OIPR). Il a "pour mission, sur l'ensemble des parcs et réserves sur lesquels il exerce son autorité, notamment :
} 
Selon le document de travail de l'OIPR intitulé Procédures de concession de l'exploitation touristique des Parcs Nationaux et Réserves, l'objet de la convention de concession d'exploitation est l'exploitation touristique d'une ou partie d'aire protégée. Ainsi, les activités touristiques concédées sont celles qui relèvent de la mission de service public et devrait en principe être accomplies par l'Administration. Il s'agit d'activités ayant un rapport direct avec l'écologie et le développement durable menées dans un Parc National ou une Réserve naturelle partielle. Elles préservent l'équilibre biologique de ces milieux naturels et valorisent les traditions et cultures des populations locales ${ }^{574}$.

En guise d'exemple, l'OIPR a conclu avec la SODERTOUR LACS ${ }^{575}$ une convention de concession de l'exploitation touristique de la Réserve de faune d'Abokouamékro, le Vendredi 16 mai 2014 ${ }^{576}$. Traitant de l'expérience de l'Australie, Erica WILSON et al. affirment qu'à New South Wales ${ }^{577}$, des partenariats public-privé touristique ont été conclus. Ces partenariats formés entre l'autorité en charge des aires protégée (New South Wales National Parks and Wildlife Service) et les opérateurs touristiques privés ont pour objet l'exploitation touristique des aires protégées ${ }^{578}$. Ces auteurs écrivaient qu'en 40 ans, plusieurs accords de concession en la matière avaient été conclus ${ }^{579}$.

\footnotetext{
1. la gestion de la faune, de la flore, et de leur biotope qui en constitue le fondement... ».

${ }^{574} \mathrm{Ces}$ différents développements sur l'objet des conventions de concession d'exploitation touristique sont essentiellement tirés du document référencé comme suit: "République de Côte d'Ivoire, Ministère de l'Environnement, des Eaux et Forêts/Office Ivoirien des Parcs et Réserves, Procédures de concession de l'exploitation touristique des Parcs Nationaux et Réserves, Abidjan, avril 2010, p. 3".

${ }^{575}$ Les articles $1^{\text {er }}$ et 2 du décret 2008-137 du 14 avril 2008 portant création d'une Société d'État dénommée Société de Développement Touristique de la Région des Lacs en agrégé «SODETOUR-LACS » (JORCI n 4 du jeudi 27 mars 2011, p. 38) indiquent successivement que la SODETOUR-LACS «est une Société d'État régie par les dispositions de l'Acte Uniforme de l'OHADA ", de la loi 97-519 du 4 septembre 1997 portant définition et organisation des sociétés d'État; et que l'objet de cette société est d' " assurer toutes les prestations relatives à la promotion et au développement du tourisme...». Régie par l'Acte Uniforme OHADA, la SODETOURLACS (Société d'État) est une personne morale de droit Privé, d'autant plus que l'article 4 de la loi précitée de 1997 sur les Société d'État dispose : «La société d'État est une personne morale de Droit privé, commerciale par sa forme.».

${ }_{576}$ Cette information nous a été également fournie au cours d'un entretien réalisé avec le Directeur technique de l’Office Ivoirien des Parcs et Réserves (OIPR), le 14 décembre 2014.

${ }^{577}$ (en Français, le Sud de la Nouvelles Galles -notre traduction).

${ }^{578}$ Voir WILSON (Erica), NIELSEN (Noah)et BUULTJENS (Jeremy), «From lessees to partners: exploring tourism public-private partnerships within the New South Wales national parks and wildlife service », Journal of Sustainable Tourism, Vol. 17, n², 2009, p. 278.

${ }^{579} I d e m$, p. 269.
} 


\section{CONCLUSION DE LA PREMIÈRE PARTIE}

En somme, les analyses précédentes renseignent sur la volonté de l'État de Côte d'Ivoire d'assurer une conservation durable de ses aires protégées (Parcs Nationaux et Réserves). Celle-ci se manifeste par l'élaboration de textes juridiques qui réglementent la gestion durable des aires protégées dans une perspective de conservation desdites forêts et de développement économique. Ces textes juridiques se caractérisent par une évolution tendant à adapter la législation relative aux aires protégées aux réalités de leur gestion.

Premièrement, de son accession à l'indépendance en 1960 jusqu'à 2002, la volonté de l'État ivoirien de protéger la nature et partant de préserver les aires protégées se traduit par la définition d'un cadre institutionnel à cet effet. Il s'agit de la mise en place du service public en charge de la conservation des aires protégées. En outre, des subventions étatiques (exclusivement) sont allouées pour la conservation des aires protégées.

Deuxièmement, sur le plan normatif, la protection des aires protégées, biens du domaine privé de l'État de 1960 à 2002, est assurée par les règles de protection domaniale afférentes. De plus, durant cette période, les atteintes contre ces milieux naturels, qui étaient affranchies de tous droits d'usage, ont fait l'objet d'incrimination sous la forme de délit (délits d'exploitation agricole, forestière, et de chasse). Ainsi, la répression de ces délits relevait de la compétence des inspecteurs et agents assermentés des Eaux et Forêts qui, n'ayant pas la qualité d'Officier de police judiciaire, n'en exerçaient que quelques fonctions. Il s'agit de la recherche de ces infractions et leur constat par procès-verbal.

Cependant, les insuffisances cette législation originaire destinée à la conservation des aires protégées et le constat de leur dégradation continue de 1960 à 2002, ont conduit le législateur à apporter des innovations au droit des aires protégées en 2002. En la matière, cet enrichissement porte sur les règles relatives à la protection de l'intégrité des aires protégées ainsi que celles afférentes à leur gestion.

Concernant la protection de l'intégrité des aires protégées, le législateur les incorpore au domaine public de l'État. Ainsi, ces forêts sont désormais inaliénables et imprescriptibles et, par conséquent, insusceptibles d'être déclassées par l'autorité exécutive, comme ce fut le cas, dans la pratique, avant l'année 2002. Également, il durcit les textes réprimant les délits contre les aires protégées prévus par la législation des aires protégées en vigueur avant l'année 2002 et définit de nouvelles infractions contre ces forêts. Par ailleurs, le législateur confère la qualité d'officier de Police judiciaire au personnel de l'Office Ivoirien des Parcs et Réserves (OIPR) en charge la répression des infractions contre les aires protégées

Quant aux innovations relatives aux règles de gestion des aires protégées, le législateur opère la délégation de la gestion de ces milieux naturels à un Établissement public national : l'Office Ivoirien des Parcs et Réserves. Ainsi, le service public de la conservation des aires protégées peut désormais recourir à des financements extra-étatiques et mener des actions de 
conservation avec plus de célérité. Au surplus, la loi précitée de 2002 sur les PNR instaure un cadre participatif de gestion mettant en relation des autorités administratives, des acteurs du secteur privé et des organisations de la société civile en vue de la gestion durable des aires protégées.

En dépit de l'existence d'une législation consacrée aux aires protégées, la dégradation continuelle de ces milieux naturels témoigne de la difficile application de ladite législation. 


\section{DEUXIÈME PARTIE :}

LA DIFFICILE APPLICATION DU DROIT DES AIRES PROTÉGÉES 
L'application de la règle de droit par la société renvoie à son effectivité. En droit civil, l'effectivité est le "caractère réel et concret d'un droit, au-delà de sa reconnaissance abstraite dans les textes de loi. ${ }^{580}$. Fort de cette définition, l'effectivité du droit des aires protégées s'entend de son application par le corps social, et partant du respect de ses différentes prescriptions telles que l'interdiction des droits d'usage sur ces types de forêts.

En Côte d'Ivoire, le constat est que le problème de l'effectivité de l'application des lois, en général, se pose avec une acuité réelle. Par conséquent, le droit de l'environnement souffre aussi d'un problème d'effectivité quant à son application. C'est ce qu'affirme Adon GNANGUI à travers les propos suivants: "Si le progrès enregistré par le droit de l'environnement en Côte d'Ivoire paraît satisfaisant, il faut dire qu'il se pose par ailleurs, le problème de l'effectivité de son application. Or, l'efficacité du droit de l'environnement ou du droit tout simplement, résulte dans son application effective. En Côte d'Ivoire, comme dans la plupart des pays en développement, pour des raisons socioéconomiques et politiques l'application du droit est très difficile. ${ }^{581}$.

Pour ce qui est particulièrement des aires protégées, malgré la réécriture des textes juridiques qui les régissent en 2002, ces milieux naturels continuent de subir des pressions anthropiques. À la réflexion, si le droit est censé réglementer les rapports sociaux, les aires protégées qui sont régies par des règles qui organisent leur conservation durable ne devraient pas en principe connaître un tel niveau de dégradation ${ }^{582}$. En d'autres termes, le droit des aires protégées connaît de réelles difficultés d'application (Titre 1) dont l'analyse servira à faire des recommandations (Titre 2) en vue d'inverser cette tendance.

\footnotetext{
${ }^{580}$ Voir GUINCHARD (Serge) (dir.), Lexique des termes juridiques, $19^{\text {ème }}$ édition, Paris, Dalloz, Coll. Campus LMD, 2012, p.351.

${ }^{581}$ Voir GNANGUI (Adon), « Côte d'Ivoire : Évolution du droit de l'environnement depuis 1992 » in PRIEUR (Michel) (dir.), Vers un nouveau droit de l' environnement?, Limoges, Centre international de droit comparé de l'environnement, 2003/05, p. 364.

${ }^{582}$ En effet, en Côte d'Ivoire, si dans l'ensemble les aires protégées subissent des dégradations, il reste que certaines d'entre elles sont fortement dégradées. En la matière, voir nos développements antérieurs faits dans la partie introductive de cette thèse, les points "I- Enjeux de la protection des forêts en Côte d'Ivoire", " C- l'état des pressions anthropiques sur la biodiversité en Côte d'Ivoire", et précisément le point intitulé "2. Les pressions anthropiques sur les aires protégées en particulier".
} 


\section{TITRE I : LE CONSTAT AVÉRÉ D'UN DÉFAUT D'EFFECTIVITÉ DU DROIT DES AIRES PROTÉGÉES}

Le droit des aires protégées a pour finalité la conservation durable de ces milieux naturels. Ce faisant, l'application effective dudit droit aboutit inéluctablement à la conservation des aires protégées.

Or, dans la pratique, les aires protégées sont fortement agressées par les populations. Il s'ensuit que le droit des aires protégées n'est pas observé par celles-ci. Ainsi, le problème de l'effectivité de l'application du droit des aires protégées pourrait conduire à s'interroger sur les raisons de la persistance de cette application difficile dudit droit malgré sa réécriture en 2002.

L'élucidation de cette question pourrait être faite sous deux (2) angles. D'une part, on pourrait penser que si le droit des aires protégées connaît des difficultés d'application, c'est peut-être parce qu'il comporte des insuffisances. La vérification de cette hypothèse impose une évaluation dudit droit (Chapitre 1). D'autre part, les raisons de ce défaut d'effectivité du droit des aires protégées pourrait être le fait de problèmes pratiques de gestion des aires protégées (Chapitre 2). 


\section{CHAPITRE I : LE DÉFAUT D'EFFECTIVITÉ DU DROIT DES AIRES PROTÉGÉES, UNE TARE CONGÉNITALE?}

Le droit positif des aires protégées a pour finalité la conservation durable de ces milieux naturels. En conséquence, procéder à une évaluation dudit droit revient à analyser ses dispositions dans l'optique de savoir si elles permettent d'atteindre cet objectif.

Le droit positif des aires protégées en Côte d'Ivoire est l'œuvre d'une réécriture opérée par le législateur. Cette réécriture est révélatrice de plusieurs réformes destinées à la conservation de ces milieux naturels. Dans cette entreprise, plusieurs points importants pour la conservation de ces forêts ont essentiellement retenu l'attention du législateur ivoirien. $\mathrm{Ce}$ sont notamment le financement et la gestion participative des aires protégées ; le renforcement des règles domaniales de protection de l'intégrité des aires protégées ainsi que les dispositions pénales qui les régissent.

L'analyse critique de ces différents apports aura plusieurs avantages. D'une part, elle permettra de savoir si la législation applicable aux aires protégées leur accorde une protection juridique suffisante. D'autre part, elle révèlera si ledit droit recèle toujours des insuffisances qui entravent et/ou expliquent les problèmes de conservation de ces espaces naturels.

Aussi analyserons-nous les règles juridiques relatives à la protection de l'intégrité des aires protégées (Section I) et celles qui ont trait à leur gestion (Section II).

\section{SECTION I : L'analyse critique des règles juridiques relatives à la protection de l'intégrité des aires protégées}

L'étude critique du droit positif des aires protégées fait apparaître, d'une part, la réaffirmation de la protection de l'intégrité de ces milieux naturels par l'évolution des règles domaniales qui leur sont applicables (Paragraphe 1). D'autre part, elle atteste que le législateur a consolidé les dispositions pénales relatives à la protection de l'intégrité des aires protégées (Paragraphe 2).

\section{Paragraphe 1 : La réaffirmation de la protection de l'intégrité des aires protégées par l'évolution des règles domaniales}

Tel que conçu par le législateur ivoirien depuis 2002, le droit des aires protégées accorde toujours une place d'honneur à la protection de ces forêts naturelles. Celle-ci est manifeste à travers l'évolution des dispositions domaniales que la loi précitée de 2002 sur les PNR consacre à cet effet.

Aussi l'évolution du droit domanial applicable aux aires protégées témoigne-t-elle du renforcement des règles procédurales de classement et de déclassement de catégories d'aires 
protégées (A). De même, l'incorporation des aires protégées au domaine public de l'État est un obstacle aux mesures étatiques antérieures préjudiciables à leur conservation (B).

\section{A - Le renforcement des règles procédurales de classement et de déclassement de catégories d'aires protégées}

Avec l'intervention de la loi précitée de 2002 sur les PNR, le déclassement de certaines catégories d'aires protégées relève du pouvoir législatif (1). Cette innovation d'ordre procédural a des incidences sur la protection de l'intégrité des aires protégées (2).

\section{La loi, nouvel acte de classement et de déclassement de certaines catégories d'aires protégées}

En Côte d'Ivoire, les règles procédurales en matière de classement et de déclassement des aires protégées connaissent des évolutions depuis 2002. Toutefois, notre analyse ne s'appesantira que sur le changement de l'acte de classement et de déclassement de certaines aires protégées, en ce qu'il a un lien direct avec les développements à venir.

Avant 2002, les aires protégées étaient classées ou déclassées par l'autorité exécutive. En effet, l'article 5 de la loi $65-255$ du 4 août 1965 relative à la protection de la faune et l'exercice de la chasse ${ }^{583}$ dispose : «Le classement des réserves naturelles intégrales ou partielles et des parcs nationaux est décidé par décrets. ». Si l'acte classement est désigné de façon expresse par la loi, il n'en est pas de même pour le déclassement. Néanmoins, le constat que les déclassements d'aires protégées faits en Côte d'Ivoire l'ont été par décret, avant l'adoption de la loi de 2002 sur les PNR.

À la réflexion, cette pratique pourrait s'expliquer par le principe du parallélisme des formes ${ }^{584}$. Cette position se justifie par le fait que le classement des aires protégées se faisant par décret, à l'opposé, le déclassement devrait se faire par décret. C'est certainement la raison pour laquelle les déclassements des aires protégées l'ont été par décret.

Dorénavant, le classement et le déclassement total ou partiel des Réserves naturelles intégrales et des Parcs Nationaux est l'œuvre de la loi. En effet, aux termes de l'article 8 de la loi de 2002 sur les PNR: "la loi porte classement et déclassement total ou partiel des Réserves naturelles intégrales et des Parcs Nationaux ».

\footnotetext{
${ }^{583}$ JORCI n $^{\circ} 41$ du 26 août 1965 , p. 898.

${ }^{584} \mathrm{Le}$ parallélisme des formes est un principe selon lequel « une décision administrative prise sous une certaine forme ne peut être annulée ou modifiée qu'en respectant les mêmes formes ". Voir www.dictionnaire.reverso.net/francaisdefinition/principeduparallismedesformes. Pour BECQUE-ICKOWICZ (Solange): " Le parallélisme des formes est un principe consacré en droit romain et en droit public...Le parallélisme des formes désigne l'extension d'une forme d'un acte juridique à un autre acte juridique. Cette forme peut être une formalité imposée par la loi -il s'agit d'un parallélisme des formalités-ou une forme d'extériorisation de la volonté -on peut alors parler d'un parallélisme des compétences.». Voir BECQUEICKOWICZ (Solange), Le parallélisme des formes en droit privé, Paris, Éditions Panthéon Assas, Coll. "Thèses", 490 pages (extrait de la préface faite par Yves Gautier).
} 
Il nous semble que cette volonté législative de changer l'acte de classement et de déclassement des Parcs Nationaux et Réserves naturelles intégrales (c'est-à-dire la "Loi" en lieu et place du "Décret") est justifiée par ses conséquences sur la protection des aires protégées.

\section{Les conséquences du classement et du déclassement des Parcs Nationaux et Réserves intégrales par la loi sur leur protection}

Entendue au sens strict, la loi désigne l'acte voté par le parlement et promulgué par le chef de l'État. En Côte d'Ivoire, le vote de la loi est l'exclusivité de l'Assemblée nationale qui détient le pouvoir législatif ${ }^{585}$. Avant l'adoption de la loi, son élaboration suit une procédure qui pourrait être résumée en six (6) étapes. Le projet de loi ou la proposition de loi ${ }^{586}$ est présenté à l'Assemblée nationale. Il s'ensuit l'examen du projet ou de la proposition par la commission de l'Assemblée nationale en séance non publique pour des amendements éventuels. Le texte examiné en commission est alors présenté à l'ensemble des députés et discuté en plénière. Ensuite, la loi est adoptée par un vote, à la majorité des députés et promulguée par le président de la République. Enfin, elle est publiée au Journal Officiel de la République de Côte d'Ivoire (JORCI) pour être opposable aux citoyens ${ }^{587}$.

Contrairement à la loi, le projet de décret est préparé par les services compétents du ministère de tutelle technique du domaine concerné. Il est transmis au Secrétariat Général du gouvernement en vue d'un examen préalable. Ensuite, le projet de décret passe en conseil des ministres où il est fait exposé par le ministre compétent. Le décret est pris par le président de la République ${ }^{588}$.

${ }^{585}$ C'est ce que mentionne l'article 71 de la Constitution ivoirienne du $1^{\text {er }}$ août 2000 en ces termes: «L'Assemblée nationale détient le pouvoir législatif. Elle vote seule la loi. La loi fixe les règles concernant:

- La citoyenneté, les droits civiques et les garanties fondamentales accordées aux citoyens pour l'exercice des libertés publiques ;

- La nationalité, l'état et la capacité des personnes, les régimes matrimoniaux, les successions et les libéralités;

- La procédure selon laquelle les coutumes sont constatées et mises en harmonie avec les principes fondamentaux de la Constitution;

- La détermination des crimes et délits ainsi que des peines qui leur sont applicables, par procédure pénale, l'amnistie ;

- L'organisation des tribunaux judiciaires et administratifs et la procédure devant ces interdictions ;

- Le statut des magistrats, des officiers ministériels et des auxiliaires de justice ; (...)

- De l'aliénation et de la gestion du domaine de l'État; (...)

- De la protection de l'environnement... » (souligné par nous).

${ }^{586}$ En Côte d'Ivoire, les articles 42 alinéa $1^{\text {er }}$ et 58 alinéa $1^{\text {er }}$ de la Constitution précitée du $1^{\text {er }}$ août 2000 dispose que «Le Président de la République a l'initiative de la loi, concurremment avec les membres de l'Assemblée nationale. » et que «Le Parlement est constitué par une Chambre unique dite Assemblée nationale...». Lorsque l'initiative de la loi émane du Président de la République, elle est dénommée projet de loi. Par contre, dans l'hypothèse où elle est présentée par l'Assemblée nationale, on parle de proposition de loi. Voir GUINCHARD (Serges), DEBARD (Thierry) (dir.), Lexiques des termes juridiques, $19^{\text {ème }}$ édition, op. cit., et respectivement les pages 692 et 694 .

${ }^{587}$ Voir DÉGNI-SÉGUI (René), Introduction au Droit, op. cit., p. 89 et s.

${ }^{588}$ Ces informations sur les étapes de la prise du décret ont été obtenus auprès de M. AKA K. Richard, Conseiller technique au Secrétariat Général du gouvernement, au cours d'un entretien réalisé le 26 juin 2015. 
Une analyse comparative des deux (2) procédures conduit aux remarques suivantes. Dans un premier temps, tandis que le projet ou la proposition de loi est examiné par deux (2) instances $^{589}$, le décret n'est soumis qu'au seul examen des membres du conseil des ministres. Dans un deuxième temps, le Parlement étant en principe multicolore, l'examen de projets ou de propositions de loi y fait l'objet de véritables débats caractéristiques d'un système politique multipartite.

Contrairement à la loi, l'élaboration du décret n'est pas le fruit de la même intensité de débats. Au sein de l'exécutif, la conduite des affaires de l'État, en l'occurrence les échanges précédant la prise des décrets, sont guidés par le principe de la solidarité gouvernementale.

Enfin, la loi et notamment celle relative aux aires protégées est adoptée par une instance ou formation collégiale (la majorité des députés) au contraire du décret qui est pris par une seule autorité (le président de la République). En conséquence, une des difficultés majeures de l'adoption de la loi demeure le quorum de la majorité qui doit être atteint.

Il résulte de ces deux (2) procédures que celle dont émane la loi est relativement plus longue et plus complexe que celle dont est issu le décret. Par conséquent, la protection des Parcs Nationaux et Réserves naturelles intégrales est renforcée par le fait que leur déclassement relève du Parlement et non plus de l'autorité exécutive qui a procédé à plusieurs déclassements par le passé.

Outre ces règles d'ordre procédural, la domanialité publique conférée aux aires protégées par la loi de 2002 sur les PNR constitue un rempart à la distraction de superficies forestières dans ces milieux naturels.

\section{B - L'incorporation des aires protégées au domaine public de l'État, un remède aux mesures étatiques antérieures préjudiciables à leur conservation}

La loi précitée sur les PNR de 2002 incorpore les aires protégées (Parc National, Réserve naturelle intégrale ou partielle) au domaine public de l'État; même si la dernière citée peut aussi appartenir à son domaine privé ${ }^{590}$. Cependant, avant l'année 2002, les aires protégées appartenaient toutes au domaine privé de l'État ${ }^{591}$.

C'est ainsi que le régime juridique auquel elles étaient soumises (de 1960 à 2002) a permis aux autorités exécutives ou administratives d'alors de distraire certaines de leur

\footnotetext{
${ }^{589}$ Il s'agit de la commission parlementaire en charge des questions environnementales et de l'ensemble des députés.

${ }^{590}$ Ces différents points ont été développés dans la Première partie de cette étude, au Titre II, le Chapitre I, la Section I, et précisément le "Paragraphe 1: L'incorporation des aires protégées au domaine public de l'État".

${ }^{591}$ À propos de l'appartenance des aires protégées au domaine privé de l'État, voir les développements afférents dans la Première partie de cette thèse, le Titre I, le Chapitre I, la Section II, et précisément le "Paragraphe 1 : Les aires protégées, un élément constitutif du domaine privé de l'État de 1960 à 2002".
} 
superficie (1). Dorénavant, l'inaliénabilité de catégories d'aires protégées constitue un obstacle juridique à la distraction de parcelles dans ces forêts par l'autorité administrative (2).

\section{Exposé de quelques décisions du pouvoir exécutif préjudiciables à l'intégrité des aires protégées}

Nous présenterons un cas de déclassement de parcelle dans le Parc National de la Comoé (a) et des cas de modification des limites du Parc National de Taï (b).

\section{a. Le déclassement d'une parcelle du Parc National de la Comoé en 1977}

Le Parc National de la Comoé a été classé en 1968 par le décret n68-81 du 9 février 1968 portant création du Parc National de la Comoé ${ }^{592}$. Aux termes de l'article $1^{\text {er }}$ dudit décret : "Est constitué en Parc National...et dénommé Parc National de la Comoé une zone de 1.150.000 hectares environ.». L'article 2 suivant indique que le Parc National de la Comoé a été érigé en aire protégée dans un objectif de conservation de la nature (propagation, protection et conservation de la vie animale sauvage et de la végétation naturelle) et "dans un intérêt scientifique et éducatif au profit, à l'avantage et pour la récréation du public ». C'est d'ailleurs à juste titre qu'il est affranchi de tout droit d'usage, c'est-à-dire protégé contre toute atteinte humaine qui compromettrait sa conservation, par les articles 4,5 et 6 du décret précité portant création du Parc National de la Comoé. Il ressort de la lecture de ces articles que sont respectivement interdits dans le Parc National de la Comoé, la chasse, l'exploitation forestière et les feux de brousse ${ }^{593}$; la pêche ${ }^{594}$; la récolte de produits alimentaires (miel), domestiques à usage multiple (cire) et de plantes médicinales ${ }^{595}$.

Cependant, contre toute attente, cinq années après sa création, l'autorité exécutive ampute de cette aire de conservation, placée sous protection stricte, une superficie de 850 hectares au profit d'agriculteurs et d'éleveurs pour la pratique d'activités agro-pastorales. En effet, si l'article 1er du décret n77-116 du 19 février 1977 portant déclassement d'une parcelle du Parc National de la Comoé ${ }^{596}$ dispose : «Est distraite du Parc national dit de la Comoé, la parcelle de terrain dont les limites sont définies comme suit.." et précise ses limites physiques, ce sont les articles 2 et 3 suivants qui précisent respectivement qu'il s'agit d'une superficie de 850 hectares ${ }^{597}$ déclassée pour des activités agro-pastorales ${ }^{598}$.

\footnotetext{
${ }^{592}$ JORCI n $^{\circ} 11$ du 22 février 1968, p. 310.

${ }^{593}$ Aux termes de l'article 4 du décret précité de 1968 portant création du Parc National de la Comoé : «Dans le Parc national ainsi délimité y compris le lit des rivières, l'emprise des routes et pistes formant limites, tout acte de chasse, de poursuite, de capture et toute provocation du gibier quelle qu'en soit la nature, sont interdits; de même tout abattage ou mutilation d'arbres ainsi que les feux de brousse sont interdits. ".

${ }^{594} \mathrm{~L}$ 'article $5 \mathrm{du}$ décret précité dispose : «Toute action de pêche quelle qu'en soit la nature, dans les rivières et mares situées tant à l'intérieur qu'en limite du Parc National, est interdite. ».

${ }^{595}$ L'article 6 du décret portant création du Parc National de la Comoé indique : «La récolte du miel, de la cire, des plantes médicinales ou alimentaires est interdite. " .

${ }^{596}$ JORCI n ${ }^{\circ} 13$ du 31 mars 1977 , p. 556.

${ }^{597}$ L'article 2 du décret précité de 1977 portant déclassement d'une parcelle du Parc National de la Comoé indique : "La parcelle de terrain définie à l'article $1^{e r}$, d'une superficie de 850 hectares environ constitue une enclave de cultures au profit des agriculteurs et éleveurs de la sous-préfecture de Téhini. ».
} 
À l'instar du Parc National de la Comoé, le Parc National de Taï a aussi fait l'objet de déclassements qui méritent d'être étudiés.

\section{b. Les modifications des limites du Parc National de Taï en 1973 et 1977}

Crée par décret ${ }^{599}$, le Parc National de Taï (PNT) s'étend à l'origine sur une superficie estimée à 350.000 hectares. C'est ce que révèle l'article $1^{\mathrm{er}}$ dudit décret en ces termes : «Est constitué en Parc National, dénommé Parc National de Taï, le périmètre du domaine forestier de l'État, couvrant environ 350.000 hectares. ».

Tout comme le Parc National de la Comoé dont nous avons traité au point précédent, le Parc National de Taï a été créé dans un but de conservation de la nature et dans l'intérêt du public $^{600}$. On comprend dès lors qu'il soit, à l'instar du Parc National de la Comoé, affranchi, dans les mêmes termes, de tout droit d'usage par les articles 3, 4 et 5 du décret qui le créé ${ }^{601}$.

Également, dans le présent cas et malgré les objectifs de conservation poursuivis par la création de cette aire protégée, l'autorité exécutive a, à plusieurs reprises, réduit sa superficie. La première modification de limite du PNT faite à son détriment est l'œuvre du décret $\mathrm{n}^{\circ} 73$ 132 du 21 mars 1973 portant modification de la limite séparant le Parc National de Taï de la Réserve partielle de Faune du N'Zo ${ }^{602}$. L'article $1^{\text {er }}$ du décret sus-cité précise les nouvelles limites du Parc National de Taï en termes de coordonnées géographiques, sans donner une estimation numérique de la superficie en hectares ${ }^{603}$. Fort heureusement, le plan d'aménagement dudit Parc précise qu'il est amputée de 20.000 hectares au profit de la Réserve partielle de Faune du N'Zo ${ }^{604}$.

\footnotetext{
${ }^{598}$ Selon l'article 3 du décret précité de 1977 portant déclassement d'une parcelle du Parc National de la Comoé, «...aucune construction temporaire ou définitive ne pourra être érigée à l'intérieur de cette enclave qui est réservée aux activités agro-pastorales. ".

${ }^{599}$ Il s'agit du décret n ${ }^{\circ} 72-544$ du 28 août 1972 portant création du Parc National de Taï et création d'une zone périphérique de protection, JORCI n 43 du 14 septembre 1972, p. 1451.

${ }^{600}$ En effet, l'article 2 du décret précité de 1972 portant création du Parc National de Taï prescrit : «Le Parc National de Taï est constitué en vue de la propagation, la protection et la conservation de la vie animale sauvage et de la végétation naturelle dans un intérêt scientifique et éducatif au profit, à l'avantage et pour la récréation du public ».

${ }^{601}$ Selon l'article 3 du décret précité de 1972 portant création du Parc National de Taï : Dans le Parc National ainsi délimité y compris le lit des rivières, l'emprise des routes et pistes formant limites, tout acte de chasse, de poursuite, de capture et toute provocation du gibier quelle qu'en soit la nature, sont interdits; de même tout abattage ou mutilation d'arbres ainsi que les feux de brousse. ». L'article 4 dudit décret indique : "Toute action de pêche quelle qu'en soit la nature, dans les rivières et mares situées tant à l'intérieur qu'en limite du Parc National, est interdite. ». Quant à l'article 5 suivant, il dispose : «La récolte du miel, de la cire, des plantes médicinales ou alimentaires est interdite. ».

${ }^{602}$ JORCI n ${ }^{\circ} 18$ du 19 avril 1973, p. 603.

${ }^{603}$ Cet article dispose : «La limite séparant la Réserve partielle de Faune du N’Zo du Parc national de Taï précédemment définie par le parallèle $6^{\circ} 10^{\circ}$ (ligne XY des décrets décret $n^{\circ} 72-544$ du 28 août 1972, portant création du Parc national de Taï et $n^{\circ} 72-544$ du 28 août 1972, portant création Réserve partielle de Faune du $\left.N^{\prime} Z o\right)$, est dorénavant définie par une droite est-ouest géographique parallèle à la ligne XY précédente et située à $5 \mathrm{~km}$ au Sud de celle-ci. ».

${ }^{604}$ Voir République de Côte d'Ivoire, Ministère de l'Environnement et des Eaux et Forêts/Office Ivoirien des Parcs et Réserves, Plan d'aménagement et de gestion du Parc National de Taï, Abidjan, mars 2006, p. 5.
} 
Or, dans la Réserve partielle de Faune du N'Zo, l'exploitation forestière est exceptionnellement admise par un décret ${ }^{605}$ autre que celui qui créé cette aire protégée ${ }^{606}$. En effet, le décret de création de la Réserve du N'Zo interdit les droits d'usage traditionnellement exercés par les populations sur les forêts (chasse, agriculture, pêche, coupe de bois de chauffe, ramassage de produits et cueillette de plantes...) ${ }^{607}$. Nonobstant ces interdictions, l'exploitation de la Réserve de Faune du N'Zo est admise pour les exploitants forestiers qui détiennent une autorisation délivrée à cet effet ${ }^{608}$.

Il s'en déduit que contrairement aux exploitants forestiers, les communautés locales n'y sont pas autorisées à exploiter des produits forestiers. Cette situation pourrait s'expliquer par le fait que c'est pour des raisons économiques que l'autorité exécutive a exceptionnellement autorisé (par décret) la coupe de bois dans la Réserve de Faune du N'Zo dès sa création; et même a augmenté sa superficie au détriment du PNT, en 1973. En effet, le bois a joué un rôle indéniable dans les performances économiques de la Cote d'Ivoire surtout dans la décennie 1970. Il continue de jouer ce rôle, même à un degré moindre, en raison de la réduction de la couverture forestière ${ }^{609}$.

De plus, en 1977, les limites du PNT sont encore modifiées et sa superficie est revue à la baisse par l'autorité exécutive. En effet, si l'article $1^{\text {er }}$ du décret 77-348 du 3 juin 1977 portant redéfinition des limites du Parc National de Taï et création d'une zone périphérique de protection ${ }^{610}$ prescrit qu'il abroge le décret précité de 1972 portant création du Parc National de Taï, l'article 2 suivant mentionne que la superficie du Parc National de Taï «couvre environ 340.000 hectares ». Une fois de plus, la superficie du PNT est réduite, d'autant plus que sous l'empire du décret de création de ce Parc en 1972, sa superficie était estimée à 350.000 hectares. Enfin, l'article 8 supprime tout droit d'usage dans la zone périphérique de protection du Parc National de Taï, exception faite de la poursuite des activités d'exploitation forestière en cours dans ces zones jusqu'à leur échéance ${ }^{611}$. À l'analyse, les hectares amputés

${ }^{605}$ Il s'agit du décret $\mathrm{n}^{\circ} 72-546$ du 28 août 1972, portant autorisation exceptionnelle de coupes de bois dans la Réserve de Faune du N’Zo (JORCI n43 du 14 septembre 1972, p. 1452) dont l'article $1^{\text {er }}$ dispose: " L'exploitation forestière est autorisée temporairement dans Réserve partielle de Faune du N'Zo ».

${ }^{606}$ Voir le décret n ${ }^{\circ} 72-545$ du 28 août 1972, portant création de la Réserve partielle de Faune du N'Zo (JORCI $\mathrm{n}^{\circ} 43 \mathrm{du} 14$ septembre 1972, p. 1451).

${ }^{607} \mathrm{C}$ 'est ce que dispose l'article 2 dudit décret en ces termes : «Dans la Réserve de Faune ainsi définie y compris le lit des rivières et marigots, l'emprise des routes et pistes formant limites, tout acte de chasse, de poursuite, de capture et toute provocation du gibier quelle qu'en soit la nature, est interdit ainsi que les défrichements et feux de brousse aux fins de cultures agricoles. ".

${ }^{608}$ Sur la question, l'article $1^{\text {er }}$ du décret $n^{\circ} 72-546$ du 28 août 1972, portant autorisation exceptionnelle de coupes de bois dans la Réserve de Faune du N'Zo dispose : "L L'exploitation forestière est autorisée temporairement dans Réserve partielle de Faune $d u N^{\prime} Z o$ ». En outre, à , l'article 3 suivant il peut être lu : «Les exploitants forestiers qui auront bénéficié d'autorisation de coupes dans cette zone devront détruire enfin d'exploitation les bâtiments qu'ils auront été amenés à construire pour les besoins de leur exploitation. » (souligné par nous).

${ }^{609}$ Sur les performances du bois dans l'économie ivoirienne, voir nos analyses antérieures dans l'Introduction de cette thèse, les points "I- Enjeux de la protection des forêts en Côte d'Ivoire", " B- Les retombées socioéconomiques de 1'exploitation des forêts en Côte d'Ivoire", et précisément le point intitulé "1. La rentabilité économique de l'exploitation des forêts ".

${ }^{610}$ JORCI n$^{\circ} 30$ du 28 juillet 1977 , p. 1438.

${ }^{611}$ L'article 7 du décret 77-348 du 3 juin 1977, portant redéfinition des limites du Parc National de Taï et création d'une zone périphérique de protection mentionne : "Est érigé en " zone périphérique de protection du Parc 
à la superficie initiale du PNT l'ont été en vue de créer sa zone périphérique de protection qui pourtant fait l'objet d'exploitation forestière.

Il ressort de ces exemples d'aliénations de parcelles d'aires protégées opérées par l'autorité exécutive que celles-ci ont été possibles parce que la loi le permettait avant l'année 2002. En d'autres termes, à cette époque, les aires protégées appartenaient au domaine privé de l'État et par conséquent étaient aliénables.

Toutefois, sur le plan juridique, l'inaliénabilité des aires protégées consacrée par les dispositions de loi de 2002 sur les PNR constituent désormais un obstacle à de telles pratiques qui sont néfastes pour la conservation des aires protégées.

\section{L'inaliénabilité des aires protégées, un antidote juridique à leur} distraction par l'autorité exécutive

Aux termes de l'article 7 de la loi précitée de 2002 sur les PNR: «Les réserves naturelles intégrales et les parcs nationaux font partie du domaine public inaliénable de l'État, à compter de leur classement dans l'une ou l'autre de ces catégories. ». Pour ce qui est particulièrement des Réserves naturelles partielles, l'article 13 de ladite loi déclare : «Les réserves naturelles partielles sont classées par décret pris en Conseil des ministres. » et «...peuvent appartenir indifféremment au domaine public ou privé de l'État ou des Collectivités territoriales, ou au domaine des particuliers. » (souligné par nous).

Il ressort de ces dispositions que deux (2) catégories d'aires protégées (Parcs Nationaux et Réserves naturelles intégrales) appartiennent systématiquement au domaine public de l'État; et que les Réserves naturelles partielles peuvent appartenir également à son domaine public. Ce faisant, les aires protégées ivoiriennes sont inaliénables ${ }^{612}$. De la sorte, elles ne peuvent être amputées de certaines superficies qui sortiraient du patrimoine de l'État pour entrer dans le patrimoine d'une autre personne. En conséquence, aucune autorité exécutive, sous peine de nullité, ne peut dorénavant prendre un acte destiné à soustraire des superficies de forêts aux Réserves naturelles intégrales et aux Parcs Nationaux, contrairement au passé. Il en est ainsi pour les Réserves naturelles partielles qui appartiennent au domaine

\footnotetext{
National de Tä̈" avec statut de réserve partielle de faune, la zone d'environ 66.000 hectares. ». L'article 8 suivant dispose : «Dans la zone de protection ainsi définie, sont interdits :

-Tout acte de chasse, de poursuite, de provocation et de capture des animaux quelle qu'en soit la nature;

-Tout défrichement et feu de brousse aux fins de cultures agricoles ou autres;

-Toute installation de campements et villages, colons ou autochtones, quelle que soit la raison motivant cette installation ;

-Toute exploitation forestière; néanmoins les chantiers en cours pourront poursuivre leurs activités jusqu'à leur échéance ;

Tout droit d'usage coutumier. » (souligné par nous).

${ }^{612}$ Sur ce point, voir les développements faits sur la conséquence juridique de l'appartenance des aires protégées au domaine public de l'État qu'est l'inaliénabilité dans la Première partie de cette étude, au Titre II, le Chapitre I, la Section I, le Paragraphe 2, et précisément le point intitulé "A- L'inaliénabilité et l'imprescriptibilité des aires protégées ".
} 
public de l'État. Par conséquent, la domanialité publique conférée aux aires protégées ivoiriennes depuis 2002 renforce leur protection.

Il résulte des développements précédents que l'évolution des règles domaniales de laquelle découle une conséquence telle que l'inaliénabilité des aires protégées, d'une part, et le classement ou le déclassement de catégories d'aires protégées (Parcs Nationaux, Réserves naturelles intégrale) par la loi, d'autre part, renforce la protection de ces forêts.

Outre ces règles domaniales sus-évoquées destinées à protéger l'intégrité des aires protégées, le législateur a également procédé à la consolidation des dispositions pénales qui régissent ces forêts, avec l'adoption de la loi de 2002 sur les PNR.

\section{Paragraphe 2 : La consolidation des dispositions pénales relatives à la protection de l'intégrité des aires protégées}

Le renforcement de la protection pénale de l'intégrité des aires protégées par le législateur se traduit par le durcissement des textes d'incrimination et de pénalité (A) qui a un impact sur la protection de ces types de forêts (B).

\section{A - Le durcissement des textes d'incrimination et de pénalité}

Dans le but de lutter davantage contre la criminalité écologique dans les aires protégées ivoiriennes, le législateur a procédé à l'enrichissement des textes d'incrimination (1) et alourdi les peines relatives aux infractions qui y sont commises (2), en 2002.

\section{L'enrichissement des textes d'incrimination par le législateur}

L'analyse des dispositions pénales des aires protégées montre que le droit pénal des aires protégées connaît une évolution qui se traduit notamment par un enrichissement des textes d'incrimination. D'une part, l'enrichissement des textes d'incrimination s'est opéré par l'extension du champ des éléments matériels de certaines infractions qui existaient sous l'empire de l'ancienne législation des aires protégées (avant l'année 2002). Il s'agit des délits d'exploitation forestière et d'exploitation agricole.

En effet, en reconduisant les éléments du délit d'exploitation agricole prévu par l'article 50 alinéa $1^{\mathrm{er}}$ du Code forestier de 1965 ("fait de couper, enlever, mutiler, arracher" des produits forestiers) ${ }^{613}$, l'article 75 de la loi de 2002 sur les $\mathrm{PNR}^{614}$ in fine rajoute et

\footnotetext{
${ }^{613}$ Aux termes de l'article 50 alinéa $1^{\mathrm{er}}$ du Code forestier de 1965, est puni : «...d'une peine de deux mois à deux ans d'emprisonnement et d'une amende de 10.000 à 1.000 .000 de francs CFA ou de l'une de ces deux peines seulement...Quiconque coupe, enlève, mutile, écorche ou arrache des arbres ou exploite des produits forestiers accessoires, dans un but commercial ou non, sans y être autorisé...» (souligné par nous).

${ }^{614} \mathrm{~L}$ 'article 75 de la loi précitée de 2002 sur les PNR dispose : «Est puni d'une amende de 100.000 à 1.000.000 de francs CFA et d'un emprisonnement de huit jours à deux ans ou de l'une de ces deux peines seulement,
} 
termine l'énumération des faits incriminés par des faits (nouveaux) tels que endommager d'une manière quelconque la flore" dans les aires protégées. En procédant ainsi, le législateur ambitionne de punir toutes les formes possibles d'atteinte aux aires protégées.

Également, en ce qui concerne le délit d'exploitation agricole, l'extension de la liste des éléments matériels de ce délit se fait par l'élargissement du champ de la qualité d'auteur. En effet, avec la loi de 2002 sur les PNR, en plus de celui qui exploite en personne une parcelle située dans une aire protégée à but agricole ou en vue d'une mise en culture ${ }^{615}$, le législateur punit celui sur instruction de qui ce dernier agit, contrairement au Code forestier de 1965 qui ne punissait que l'auteur matériel de cette infraction ${ }^{616}$.

D'autre part, l'enrichissement des textes d'incrimination s'est réalisé par la définition de nouvelles infractions dans la loi précitée de 2002 sur les PNR. Cela signifie que le législateur a incriminé de nouveaux comportements qu'il estime dangereux pour la conservation des aires protégées.

D'abord, il incrimine des faits (constructions ou travaux de fouilles, prospection, sondage ou terrassement) accomplis dans le cadre de l'exploitation minière et commis dans les aires protégées ${ }^{617}$. Ensuite, le fait d'exploiter les bois d'œuvre et d'ébénisterie dans les aires protégées est puni ${ }^{618}$ distinctement du délit d'exploitation forestière. Enfin, les faits constitutifs de pollution dans les aires protégées (déversements, écoulements, rejets et dépôts de substances de toute nature) sont désormais sanctionnés.

Aussi l'analyse des infractions contenues dans le droit des aires protégées conduit-elle au constat de la sévérité des peines infligées aux délinquants écologiques.

quiconque, dans un parc national ou une réserve naturelle, sans l'autorisation préalable du directeur du parc ou de la réserve naturelle:

-Prélève ou collecte la flore ;

-Récolte des plantes, fruits ou produits ;

-Coupe, arrache, enlève ou endommage d'une manière quelconque la flore;

-Tue, blesse, pêche ou capture les animaux. » (souligné par nous).

${ }^{615}$ Selon les dispositions de l'article 70 de la loi de 2002 sur les PNR : «est puni d'une amende de 1.000 .000 à 25.000.000 FCFA et d'un emprisonnement de deux mois à deux ans ou de l'une de ces deux peines seulement, quiconque entreprend ou fait entreprendre...des exploitations agricoles dans les Parcs Nationaux et les Réserves naturelles » (souligné par nous)..

${ }^{616}$ Aux termes de l'article 50 alinéa 2 du Code forestier de 1965 est puni : «...d'une peine de deux mois à deux ans d'emprisonnement et d'une amende de 10.000 à 1.000 .000 de francs CFA ou de l'une de ces deux peines seulement...Quiconque, en vue d'une mise en culture ou non, effectue sans autorisation un défrichement portant sur une partie du domaine forestier classé, des périmètres de protection et des reboisements...».

${ }^{617}$ Selon les termes de l'article 70 alinéa $1^{\text {er }}$ de la loi de 2002 sur les PNR, «Est puni d'une amende de 1.000.000 à 25.000.000 de francs CFA et d'un emprisonnement de deux mois à deux ans ou de l'une de ces deux peines seulement, quiconque entreprend ou fait entreprendre des constructions ou des travaux de fouille, prospection, sondage ou terrassement ou des exploitations agricoles dans les Parcs Nationaux et les Réserves naturelles.

La peine est portée au double si les dommages causés au milieu naturel sont irréversibles. » (souligné par nous).

${ }^{618}$ En effet, aux termes de l'article 71 de la loi précitée de 2002 sur les PNR : «Est puni d'une amende de 5.000 000 à 50.000 .000 de francs et d'un emprisonnement de six mois à cinq ans ou de l'une de ces deux peines seulement, quiconque entreprend l'exploitation de bois d'œuvre et d'ébénisterie à l'intérieur d'un parc national ou d'une réserve naturelle. La peine est portée au double s'il s'agit d'un acte volontaire ou en cas de récidive. ». 


\section{Le constat de la sévérité des peines}

Bien que la loi de 2002 sur les PNR renforce dans l'ensemble les peines applicables aux infractions contre les aires protégées ${ }^{619}$, l'analyse de ses dispositions révèle qu'elles consacrent de lourdes peines d'amende pour certaines infractions ${ }^{620}$. En effet, le législateur punit désormais le délit d'exploitation agricole dans les aires protégées d'une amende fixée de 1.000.000 à 25.000.000 FCFA ${ }^{621}$, au contraire de l'ancienne peine qui était fixée à de 10.000 à 1.000 .000 de FCFA ${ }^{622}$. La peine est double : «si les dommages causés au milieu naturel sont irréversibles ${ }^{623}{ }^{\prime}$.

En ce qui concerne les faits accomplis dans le cadre d'une exploitation minière (constructions ou travaux de fouilles, prospection, sondage ou terrassement) et qui sont bien souvent réalisés dans des aires protégées ivoiriennes ${ }^{624}$, ils sont punis d'une amende de 1.000.000 à 25.000.000 FCFA ${ }^{625}$. L'article 70 alinéa 2 portent au double ces peines, en cas de circonstances aggravantes c'est-à-dire lorsque les dommages causés aux aires protégées sont irréversibles.

\footnotetext{
${ }^{619}$ Sur ce point, voir la Première partie de cette étude, le Titre II, le Chapitre I, la Section II et précisément le "Paragraphe 1 : Le durcissement des textes de répression des atteintes contre les aires protégées".

${ }^{620}$ Cette idée est également confirmée par une étude évaluative de la législation ivoirienne des aires protégées en ces termes : «La législation ivoirienne prévoit des sanctions sévères à l'encontre des infractions commises dans les parcs et réserves. ». Voir UICN/PACO, Évaluation juridique et institutionnelle pour la mise en place des conditions d'amélioration de la gestion des aires protégées d'Afrique de l'Ouest. Ouagadougou, BF: UICN/PACO, 2010, pp. 55-56.

${ }^{621}$ Aux termes de l'article 70 alinéa $1^{\text {er }}$ de la loi de 2002 sur les PNR : « Est puni d'une amende de 1.000 .000 à 25.000.000 de francs CFA et d'un emprisonnement de deux mois à deux ans ou de l'une de ces deux peines seulement, quiconque entreprend ou fait entreprendre des constructions ou des travaux de fouille, prospection, sondage ou terrassement ou des exploitations agricoles dans les Parcs Nationaux et les Réserves naturelles.».

${ }^{622} \mathrm{~L}$ 'article $50 \mathrm{du}$ Code forestier de 1965 (anciennement applicable aux aires protégées) dispose : «Sans préjudices des confiscations, restitutions, remise en état des lieux et dommages et intérêts, sont passibles d'une peine de deux mois à deux ans d'emprisonnement et d'une amende de 10.000 à 1.000.000 de francs ou l'une de ces deux peines seulement... :

$1^{\circ}$ Quiconque coupe, enlève, mutile, écorce ou arrache des arbres ou exploite des produits forestiers accessoires, dans un but commercial ou non, sans y être autorisé ;

$2^{\circ}$ Quiconque, en vue d'une mise en culture ou non, effectue sans autorisation un défrichement portant sur une partie du domaine forestier classé, des périmètres de protection et de reboisements... ».

${ }_{623}$ Art. 70 alinéa 2, loi de 2002 sur les PNR.

${ }^{624}$ À titre d'exemples, le délit d'orpaillage occupe le $2^{\text {nd }}$ rang au nombre des délits commis dans le Parc National de Taï, après le délit de braconnage. Voir République de Côte d'Ivoire, Ministère de l'Environnement et des Eaux et Forêts/Office Ivoirien des Parcs et Réserves, Plan d'aménagement et de gestion du Parc National de Taï, ,op. cit.,p. 30.) ; voir UICN/BRAO, Évaluation de l'efficacité de la gestion des aires protégées : Parcs et Réserves de Côte d'Ivoire, 2008, p. 28. L'orpaillage est également pratiqué dans le Parc National de la Comoé car l'un des permis de prospection minière accordée à sa périphérie "couvre les sites des Monts Tingui et Gorowi et empiète sur le Parc d'environ $40 \mathrm{~km}^{2}$. ». Voir République de Côte d'Ivoire, Ministère de l'Environnement et des Eaux et Forêts/Office Ivoirien des Parcs et Réserves, État de conservation des parcs et réserves de Côte d'Ivoire, op. cit., p.11. De plus, l'orpaillage est une activité qui est exercée dans le Parc National du Mont Sangbé. En ce qui concerne ce dernier exemple, voir OIPR/ AFD, Réalisation d'une étude sur l'état de la biodiversité de quatre Parcs Nationaux de Côte d'Ivoire. Azagny, Marahoué, Péko, Sangbé, Afrique Nature International, 2012 (Rapport final) p. 44.

${ }^{625}$ Art. 70 alinéa $1^{\text {er }}$, loi de 2002 sur les PNR.
} 
Pour ce qui est de l'exploitation de bois d'œuvre et d'ébénisterie, ses auteurs sont réprimés d'une amende allant de $\mathbf{5 . 0 0 0 . 0 0 0}$ à $\mathbf{5 0 . 0 0 0 . 0 0 0}$ FCFA $^{626}$. En plus, les faits constitutifs de pollution dans les aires protégées (déversements, écoulements, rejets et dépôts de substances de toute nature) sont passibles d'une peine d'amende de $\mathbf{5 0 0 . 0 0 0}$ à 100.000.000 FCFA ${ }^{627}$. Enfin, tout auteur d'incendie des aires protégées s'expose à une amende de $\mathbf{1 0 0 . 0 0 0}$ à $\mathbf{5 0 . 0 0 0 . 0 0 0}$ FCFA $^{628}$. Ces peines sont portées au double lorsque l'incendie est volontaire ou que l'auteur est un récidiviste ${ }^{629}$.

À l'analyse, ces lourdes peines d'amende que le législateur a prévu pour les délinquants écologiques dans les aires protégées ainsi que le renforcement des sanctions, en général, ont pour objectif de les dissuader.

\section{B - L'impact de la sévérité des peines sur la baisse de la criminalité écologique dans les aires protégées, une déduction possible à partir de travaux antérieurs}

S'il existe de plus en un consensus sur l'effet dissuasif de la peine ${ }^{630}$, la question de l'efficacité de la sévérité de la sanction sur le comportement criminel reste discutée ${ }^{631}$. Cependant, il est à noter que des travaux scientifiques aboutissent à des résultats qui confirment l'effet dissuasif de la sévérité de la peine.

En Côte d'Ivoire, les recherches sur l'efficacité de la sévérité des peines, en général, et dans la protection des aires protégées, en particulier, sont quasi-inexistantes. Toutefois, les résultats de plusieurs études sur les effets dissuasifs de la sévérité des peines peuvent servir à évaluer l'impact (sur le plan théorique) des lourdes peines prévues par la loi de 2002 sur les PNR.

\footnotetext{
${ }^{626}$ Aux termes de l'article 71 de la loi précitée de 2002 sur les PNR : « Est puni d'une amende de 5.000.000 à 50.000.000 francs CFA et d'un emprisonnement de six mois à cinq ans ou de l'une de ces deux peines seulement, quiconque entreprend l'exploitation de bois d'œuvre et d'ébénisterie à l'intérieur d'un Parc National ou d'une Réserve nature.

La peine est portée au double s'il s'agit d'un acte volontaire ou en cas de récidive.».

${ }^{627} \mathrm{Cet}$ article dispose : «Sans préjudice des dispositions de l'article 98 de la loi $n^{\circ} 96-766$ du 3 octobre 1996 portant Code de l'Environnement, est puni d'une amende de 500.000 à 100.000.000 de francs CFA et d'un emprisonnement de deux mois à deux ans ou de l'une de ces deux peines seulement, quiconque procède directement ou indirectement à des déversements, écoulements, rejets et dépôts de substance de toute nature susceptibles de porter atteinte à l'intégrité du Parc National ou d'une Réserve naturelle ou aux activités de leur exploitation écotouristique.

La peine est portée au double s'il s'agit de substances toxiques ou en cas de récidive.».

${ }^{628}$ Selon l'article 72 alinéa de la loi précitée de 2002 sur les PNR : «est puni d'une amende de 100.000 à 50.000.000 de francs CFA et d'un emprisonnement de six moi à cinq ans ou de l'une de ces deux peines seulement, quiconque détruit par incendie, tout ou partie d'un Parc ou d'une réserve naturelle.

La peine est portée au double s'il s'agit d'un acte volontaire ou en cas de récidive. ».

${ }^{629}$ Art. 72 alinéa 2, loi précitée de 2002 sur les PNR.

${ }^{630}$ Voir BÉBIN (Xavier), La sanction pénale est-elle dissuasive ?, Paris, Note sur discussion, Institut Pour la Justice, avril 2009, p. 2 (disponible sur www.institutpourlajustice.com, consulté le 2 septembre 2015).

${ }^{631}$ Ibidem.
} 
Aussi présenterons-nous successivement les résultats des recherches de Kessler et Levitt (1), de Helland et Tabarrok (2) ainsi que de Drago, Vertova et Galbiati (3).

\section{Les résultats de l'étude de Kessler et Levitt}

En 1982, dans l'État de Californie (aux États-Unis d'Amérique), une loi a été votée en vue de lutter contre le phénomène criminel. Il s'agit de la loi (Proposition 8) adoptée par voie référendaire. Cette loi augmentait les peines d'emprisonnement pour les récidivistes d'infractions graves.

Les travaux de KESSLER et LEVITT à propos de son impact concluent «une diminution immédiate de $4 \%$ des crimes mentionnés dans la loi par rapport aux autres ». Trois ans plus tard, l'on nota « une diminution globale de $8 \%$ des infractions visées par cette loi. ${ }^{632}$.

Il découle de cette étude que la sévérité de la sanction a un effet dissuasif sur les délinquants potentiels. Qu'en est-il des travaux de Helland et Tabarrok?

\section{Les résultats des travaux de Helland et Tabarrok}

En 1994, toujours dans l'État de Californie, il a été adopté des lois dites «Three strikes », en français, troisième crime (notre traduction). Il s'agit d'une législation qui prévoit que le troisième crime d'un délinquant est condamné à 25 ans d'emprisonnement.

Les résultats des travaux de Helland et Tabarrok ont évalué l'impact de cette législation. Ils montrent que la crainte par les délinquants d'une condamnation pour un troisième crime a réduit de 15 à $30 \%$ la propension de ceux-ci à récidiver ${ }^{633}$.

Il apparaît dans ce cas aussi que la sévérité des peines à un impact notamment un effet dissuasif sur le délinquant. Sur la question, d'autres travaux menés par des chercheurs européens permettent d'aboutir à pareille conclusion.

\section{Les conclusions des recherches de Drago, Vertova et Galbiati}

En Italie, une loi adoptée en 2006 accordait trois (3) ans de remise de peine à tous les condamnés, à l'exception des auteurs de crimes les plus graves. Une des dispositions de ladite loi prescrivait qu'en cas de récidive pendant les 5 ans suivant sa libération, le récidiviste exécutait le reste de la peine interrompue, majoré de celle à laquelle il sera condamné.

Des recherches relatives à l'impact de cette loi sur le comportement des délinquants menées par Drago, Vertova et Galbiati ont conclu que « chaque mois de prison additionnel

\footnotetext{
${ }^{632}$ Idem, pp. 2-3.

${ }^{633}$ Voir Idem, p. 3.
} 
encouru par les condamnés réduit leur propension à récidiver de manière significative ». Par conséquent, la sévérité des peines à un effet dissuasif.

En somme, il ressort de ces trois études que quoique limitée, la sévérité de la peine a un effet dissuasif. Ainsi et par extrapolation, les lourdes peines d'amende ci-dessus mentionnées que le législateur inflige aux délinquants auteurs d'infractions dans les aires protégées sont de nature à les dissuader. Elles le sont d'autant plus que la qualité d'OPJ reconnu au personnel en charge de la répression des atteintes contre les aires protégées améliore leurs moyens de répression sur le plan juridique. Subséquemment, ces dispositions pénales de la loi de 2002 sur les PNR assurent leur protection, du moins théoriquement. C'est ce que révèle également l'analyse des règles relatives à la gestion des aires protégées.

\section{SECTION II : L'analyse des règles relatives à la gestion des aires protégées}

Les dispositions de la législation sur les aires protégées que nous analyserons en vue d'apprécier leur pertinence pour la conservation sont celles relatives à la gestion participative de ces milieux naturels (Paragraphe 1) et au financement de leur gestion durable (Paragraphe 2).

\section{Paragraphe 1 : L'intérêt de la consécration légale du principe de gestion participative des aires protégées}

La loi de 2002 sur les PNR consacre le principe de la gestion participative des aires protégées. Dès lors, l'État n'est plus le gestionnaire exclusif des aires protégées en tant que personne juridique. La gestion des aires protégées étant déléguée à un Établissement public national (OIPR) depuis 2002, les instances de gestion de cette structure se composent d'une pluralité d'acteurs. Il s'agit de représentants l'État, de collectivités publiques, des personnes morales de droit privé et des représentants des communautés locales.

Par conséquent, plusieurs personnes (juridiques) participent depuis l'année 2002 à la gestion des Parcs Nationaux et Réserves naturelles. Cette innovation législative présente-t-elle des garanties pour la conservation durable des aires protégées?

Pour répondre à cette interrogation, il convient d'analyser, d'une part, l'impact de la délégation de la gestion des aires protégées à une structure autonome de gestion sur leur conservation (A). D'autre part, il s'agira d'apprécier les apports de l'implication des communautés locales à la gestion des aires protégées (B).

\section{A - L'impact de la délégation de la gestion des aires protégées à une structure autonome de gestion sur leur conservation}

De nos jours et en matière de gestion des aires protégées, la tendance pour les États est à la délégation de la gestion de ces forêts à des structures autonomes de gestion c'est-à-dire 
dotée de la personnalité juridique ${ }^{634}$. Ce recours généralisé à cette approche de gestion des aires protégées s'explique essentiellement par les difficultés qu'éprouvent les États à mobiliser des financements ${ }^{635}$ pour leur conservation durable. Également, ce constat s'explique par d'autres problèmes d'ordre administratif que sont la lenteur des procédures administratives et la corruption. C'est par exemple le cas en Côte d'Ivoire, en Zambie et au Guatemala $^{636}$.

Sur la question de la capacité financière des structures de gestion des aires protégées, James et al. (2000) cité par Wolf KRUG affirment que des travaux attestent que la structure institutionnelle de gestion des Parcs Nationaux a « un impact significatif sur le budget de conservation ${ }^{637}$. C'est certainement une des raisons qui ont amené le législateur ivoirien à déléguer la gestion des aires protégées à une structure parapublique ${ }^{638}$ dénommée Office Ivoirien des Parcs et Réserves (OIPR). Cette initiative étatique traduit la volonté des pouvoirs publics ivoiriens de régler les problèmes de financement auxquels étaient confrontés les gestionnaires en charge des aires protégées avant 2002.

Pour James et al. (2000) cité par Wolf KRUG, "l'autonomie administrative et financière de ces organismes parapublics de gestion crée des incitations chez les gestionnaires de Parcs pour l'optimisation des recettes du tourisme. De même, elle favorise

\footnotetext{
${ }^{634}$ Cette question a été traitée dans la Première partie de cette étude, au Titre II, le Chapitre 2, la Section I, et précisément le " Paragraphe 1 : La délégation de la gestion des aires protégées à un Établissement public national".

${ }^{635}$ Voir KRUG (Wolf), Private Supply of Protected Land in Southern Africa : A Review of Markets, Approaches, Barriers and Issues, Workshop Paper, World Bank / OECD International, Workshop on Market Creation for Biodiversity Products and Services, Paris, 25 and 26 January 2001, p. 10 ; SZELL (Andrea B.), HALLETT (Lucius F.), « Attitudes and Perceptions of Local Residents and Tourists toward the Protected Area of Retezat National Park, Romania », International Journal of Humanities and Social Science, Vol. 3, n 3; 2013, p. 22.

${ }^{636}$ En plus des problèmes de financement, la Côte d'Ivoire, le Guatemala, et la Zambie, pour ne citer que ces pays, ont été confrontés aux problèmes de lourdeurs administratives lorsque la gestion des aires protégées n'était opérée qu'exclusivement par l'État. Pour ces différents exemples, voir la Première partie de cette étude, le Titre II, le Chapitre II, la Section 1, le Paragraphe 1, le point "A- L'Office Ivoirien des Parcs et Réserves: l'Établissement délégataire de la gestion des aires protégées" et précisément le point intitulé "1. L'Office Ivoirien des Parcs et Réserves : un Établissement public national ".

${ }^{637}$ Voir KRUG (Wolf), Private Supply of Protected Land in Southern Africa: A Review of Markets, Approaches, Barriers and Issues, op. cit., p. 10.

${ }^{638} \mathrm{Un}$ organisme parapublic est un organisme doté d'une autonomie et qui reçoit une subvention de l'État, mais peut augmenter et conserver les recettes. Voir KRUG (Wolf), Private Supply of Protected Land in Southern Africa: A Review of Markets, Approaches, Barriers and Issues, op. cit., p. 10. C'est le cas de l'OIPR qui est un Établissement public national doté d'une autonomie administrative et financière et dont les financements sont constitués de subventions étatiques et de fonds d'origine privés. En effet, l'article 26 de la loi 2002 sur PNR dispose : «Les ressources de l'Établissement sont constituées, notamment, par :

- les subventions de l'État;

- les taxes parafiscales affectées ;

- les transferts des Fondations mentionnées au chapitre I du titre IV ci-dessous ;

- les subventions d'organismes publics autres que l'État ou privés nationaux ou internationaux ;

- les produits de l'exploitation du domaine qui lui est affecté ;

- les produits de l'exploitation légalement autorisée de la faune ou de la flore;

- les produits de ses prestations de services ;

- les redevances des activités concédées ;

- le produit des amendes et confiscations affecté par l'État ;

- les dons et legs. ».
} 
davantage la collecte de fonds additionnels pour la gestion des Parcs et des investissements... les dons, et l'assistance étrangère (notre traduction) ${ }^{639}$. En Namibie, une étude menée par Wolf KRUG atteste que les touristes locaux et étrangers consentent à payer des droits d'entrée plus élevés pour visiter les aires protégées, si la gestion des fonds est confiée à un organisme indépendant du gouvernement (société privée, ou $\mathrm{ONG})^{640}$.

Outre la délégation de la gestion des aires protégées à un Établissement public national (OIPR), la gestion participative des aires protégées se manifeste (sur le plan juridique) par l'implication des populations locales à ladite gestion. L'adoption de cette approche de gestion semble se justifier par les incidences positives qui en résulteraient pour leur conservation.

\section{B - Les intérêts de l'implication des communautés locales à la gestion des aires protégées sur leur conservation}

En l'absence de travaux de recherche en la matière sur les aires protégées en Côte d'Ivoire, nous apprécierons l'intérêt de l'implication des communautés locales à la gestion des aires protégées sur leur conservation, en procédant de deux (2) manières. Premièrement, nous aurons recours à la doctrine relative à la gestion participative des ressources naturelles et partant des aires protégées (1). Deuxièmement, cette appréciation sera faite sur la base des expériences empiriques de gestion réalisées dans divers pays (2).

\section{La justification tirée de la doctrine}

Certes, plusieurs études attestent qu'il n'est pas démontré dans quelle mesure la participation des communautés aux prises de décision relatives à la gestion des aires protégées peut susciter l'adhésion de celles-ci à ces politiques de conservation ${ }^{641}$. Cependant, les communautés locales sont plus disposées à soutenir la conservation ressources naturelles y compris les aires protégées lorsque leurs connaissances et opinions sont prises en compte dans le processus de prise de décision pour leur gestion ${ }^{642}$.

L'idée que la conservation des forêts et notamment celles des pays tropicaux impliquent nécessairement la participation des communautés locales est soutenue par des

\footnotetext{
${ }^{639}$ Voir KRUG (Wolf), Private Supply of Protected Land in Southern Africa: A Review of Markets, Approaches, Barriers and Issues, op. cit., p. 10.

${ }^{640}$ Idem, p. 11.

${ }^{641}$ Voir WILSHUSEN (Peter R.), BRECHIN (Steven R.), FORTWANGLER (Crystal L.) et WEST (Patrick C.), «Reinventing a square wheel: critique of a resurgent "protection paradigm" in international biodiversity conservation », Society and Natural Resources, Vol. 15, n¹, 2002, p. 17 ; HAYES (Tanya M.), « Parks, people, and forest protection: an institutional assessment of the effectiveness of protected areas ", World Development, Vol. 34, n¹2, 2006, p. 2066.

${ }^{642}$ Voir GELCICH (S.) et EDWARD-JONES (G.), KAISER (M.), « Importance of attitudinal differences among artisanal fishers toward co-management and conservation of marine resources», Conservation Biology, Vol.19, n³, 2005, p. 870; FU (B.), WANG (K.), LU (Y.), LIU (S.), KEMING (M.), CHEN (L.) et LIU (G.), «Entangling the complexity of protected area management: the case of Wolong Biosphere Reserve, southwestern China», Environmental Management, Vol. 33, nº, p. 789.
} 
auteurs $^{643}$. En effet, cette participation des communautés locales les amène à comprendre et à avoir conscience du rôle qu'elles peuvent jouer dans la conservation des aires protégées ; à partager leurs connaissances et leurs préoccupations en vue de l'atteinte d'un tel objectif ${ }^{644}$. Ainsi, les politiques et projets de conservation sont oints d'une légitimité populaire et partant soutenus par les communautés $^{645}$. À l'inverse, des études démontrent qu'il est constant que les communautés locales manifestent une hostilité aux initiatives de conservation lorsqu'elles n'y sont pas associées de sorte à prendre en compte leurs intérêts ${ }^{646}$.

Outre ces justifications données par la doctrine, l'intérêt de l'implication des populations à la gestion des aires protégées a pour fondement les expériences pratiques de gestion des ressources naturelles.

\section{La justification basée sur les expériences pratiques de gestion de ressources naturelles}

En Serbie, l'implication du public dans la gestion des aires protégées et plus précisément celle de Zasavica a permis d'obtenir des résultats satisfaisants. En effet, la participation du public à la planification de la gestion de ladite aire protégée, à la prise de décision et à la gestion effective la concernant a permis de restaurer son écosystème et aux populations d'obtenir des avantages sociaux et culturels ${ }^{647}$.

Au Botswana, la gestion participative des ressources naturelles a été expérimentée sous la forme de gestion communautaire de ces ressources. Par exemple, elle a permis d'améliorer la conservation des ressources naturelles dans le Delta de l'Okavango et plus précisément dans les villages de Khwai, Mbabe et Sankoyo ${ }^{648}$.

En Zambie, également la gestion participative des ressources naturelles des aires protégées mise en œuvre par les autorités publiques sous la forme de partenariat public-privé à fait l'objet d'études évaluatives ${ }^{649}$. Ces études montrent que dans l'ensemble, la conclusion

\footnotetext{
${ }^{643}$ Voir VERMEULEN (Sonja) et SHEIL (Douglas), « Partnerships for tropical conservation », art .cit., p. 434.

${ }^{644}$ Voir STOLL-KLEEMANN (Suzanne) et O'RIORDAN (Timothy), «From Participation to Partnership in Biodiversity Protection : Experience from Germany and South Africa », art. cit., p. 163.

${ }^{645}$ Ibidem.

${ }^{646}$ Voir MBAIWA (Joseph E.) et STRONZA (Amanda L.), « Changes in resident attitudes towards tourism development and conservation in the Okavango Delta», art. cit., p. 1950 ; ADELEKE (Bola Olusola), NZAMA (Thandi), «Assessment on Community Participation in Ecotourism and Conservation at Hhuhuwe-Umfolozi Park ", art. cit., p. 27 ; SZELL (Andrea B.), HALLETT (Lucius F.), «Attitudes and Perceptions of Local Residents and Tourists toward the Protected Area of Retezat National Park, Romania ", art. cit., pp. 23-24.

${ }^{647}$ Voir TOMIĆEVIĆ (Jelena), BJEDOV (Ivana), SHANNON (Margaret A.) et OBRATOV-PETKOVIĆ (Dragica), « Understanding Linkages Between Public Participation and Management of Protected Areas - Case Study of Serbia», in Dr. ISHWARAN (Natarajan) (Eds), The Biosphere, Intech , 2012, pp. 140-141.

${ }^{648}$ Voir MBAIWA (Joseph E.), STRONZA (Amanda L.), "Changes in resident attitudes towards tourism development and conservation in the Okavango Delta», art. cit., p. 1950.

${ }^{649}$ Nous pouvons citer, entre autres, POPE (Adam), A Preliminary Examination of Public Private Partnerships in National Park Management in Zambia, 2006, WHYDAH CONSULTING LTD. Development and Natural Resource Consultants, 24 pages ; NSHIMBI (Muleba) et VINYA (Royd), " Impacts of Public-Private
} 
de telles partenariats a amélioré la conservation des ressources naturelles et notamment les aires protégées. Elle a aussi amélioré les moyens de subsistance des communautés locales ${ }^{650}$.

Au Kenya, la gestion participative de la faune sous la forme de projets de partenariats conclus entre Kimana sanctuary et $G M$ sanctuary $^{651}$ a favorisé légèrement la réduction du conflit Homme-faune. Elle a aussi augmenté les populations animales ${ }^{652}$.

$\mathrm{Au}$ vu de ces résultats de recherches menées à partir d'expériences empiriques de gestion de ressources naturelles, en général, et singulièrement d'aires protégées, il est à noter que le fait pour le législateur ivoirien d'opter pour une gestion participative des aires protégées ne s'est pas fait ex-nihilo. Par cet acte, le législateur ivoirien entend poser le cadre d'une conservation durable de ces milieux naturels; d'autant plus que les principales menaces de leur conservation demeurent les pressions anthropiques. Ce faisant, en nous basant sur ces différentes études et expériences, on pourrait conclure que c'est à bon droit que le législateur ivoirien adopte l'approche de gestion participative des aires protégées.

$\mathrm{Au}$ surplus, la gestion durable des aires protégées a conduit le législateur ivoirien à élaborer des règles juridiques destinées à leur financement.

\section{Paragraphe 2 : L'élaboration de règles juridiques de financement à des fins de protection des aires protégées}

L'une des difficultés caractéristiques de la gestion des aires protégées a longtemps été l'insuffisance de financements ; qui du reste n'émanaient que des subventions étatiques de 1960 à 2002. C'est ainsi que pour résoudre ce problème de gestion des aires protégées ivoiriennes, le législateur a dans la loi de 2002 sur les PNR consacré la diversification des sources de financements destinés à leur protection (A). Aussi prescrit-il des dispositions juridiques rigoureuses de mobilisation et de gestion de ces fonds (B).

\section{A - L'édiction de règles consacrant la diversification des sources de financement destiné aux aires protégées}

La loi de 2002 sur les aires protégées prévoit des dispositions qui instituent de nouveaux mécanismes de financement des aires protégées. Il s'agit de mécanismes extra-

\footnotetext{
Partnership on Local Livelihoods and Natural Resource Dynamics: Perceptions from Eastern Zambia », Resources, Vol. 3, n 2, 2014, pp. 471-487.

${ }^{650}$ Voir POPE (Adam), A Preliminary Examination of Public Private Partnerships in National Park Management in Zambia, op. cit., p. 1 ; NSHIMBI (Muleba) et VINYA (Royd), " Impacts of Public-Private Partnership on Local Livelihoods and Natural Resource Dynamics: Perceptions from Eastern Zambia », op. cit., p. 484.

${ }^{65}$ Les dénominations Kimana sanctuary et GM sanctuary sont respectivement les formes abrégées des ranchs de faune privés Kimana Community Wildlife Sanctuary et de Golini-Mwaluganje Elephant Sanctuary. Voir MBURU (John) et BIRNER (Regina), « Emergence, Adoption, and Implementation of Collaborative Wildlife Management or Wildlife Partnerships in Kenya: A Look at Conditions for Success », art. cit., p. 393.

${ }^{652}$ Ibidem.
} 
étatiques. Ces mécanismes de financement viennent en appoint au mécanisme de financements étatiques alloués aux aires protégées sous la forme de subventions (étatiques).

Ces mécanismes de financement additionnels que sont les Fondations de Parcs et Réserves (1) et le contrat de fiducie (2) constituent des moyens incitatifs de financement pour la protection de ces milieux naturels.

\section{Les Fondations pour les Parcs et Réserves de Côte d'Ivoire, un mécanisme incitatif de financement pour la protection des aires protégées}

Les Fondations des Parcs et Réserves prévues par la loi de 2002 sur les PNR sont juridiquement des personnes morales (de droit privé) distinctes de l'État de Côte d'Ivoire ${ }^{653}$. Ces Fondations sont de par leur objet habilitées à rechercher et à recevoir des financements pour la conservation des aires protégées ${ }^{654}$. Essentiellement d'origine privée, ces financements mobilisés par les Fondations des Parcs et Réserves serviront à compléter les subventions étatiques alloués à ce secteur. Ces financements seront inéluctablement destinés, entre autres, aux actions qui concourent à la protection des aires protégées. En effet, les problèmes de gestion de ces forêts sont largement tributaires de l'insuffisance des financements étatiques ${ }^{655}$.

Ce faisant, tout comme la délégation de la gestion des aires protégées à une personne juridique autre que l'État revêt un caractère incitatif (théoriquement) pour le financement de leur gestion ${ }^{656}$, la personnalité morale des Fondations est de nature à inciter les bailleurs de fonds à soutenir plus facilement le financement des aires protégées. De même, les dispositions qui régissent le fonctionnement du mécanisme fiduciaire constituent un moyen incitatif pour la mobilisation de financements destinés, entre autres, à la protection des aires protégées.

\footnotetext{
${ }^{653}$ En effet, l'article $1^{\text {er }}$ de la loi précitée de 2002 sur les PNR dispose que les : «..."Fondations" : désigne une personne morale créée en vue de réaliser un but d'intérêt général. L'affectation de biens, droits ou ressources, à la réalisation de ce but d'intérêt général est irrévocable. Dans le cas d'espèce, les Fondations désigneront des associations dont l'objet et les principes d'organisation sont définis au titre IV de la présente loi... ».

${ }^{654}$ Aux termes de l'article 42 alinéa $1^{\text {er }}$ de la loi ci-dessus visée : «Le financement durable de certaines des charges récurrentes des Parcs et Réserves et de la réalisation de certains investissements est assuré par le revenu des placements des Fondations exclusivement destinées au financement des Parcs et Réserves, ci-après, désignées dans la présente loi "les Fondations". ».

${ }^{655}$ Voir République de Côte d'Ivoire, Ministère de l'Environnement et des Eaux et Forêts /Direction de la Protection de la Nature, Programme-Cadre de Gestion des Aires Protégées. Document-cadre, Abidjan, Janvier 2001, p. 23.

${ }^{656}$ Sur la question, voir nos analyses antérieures à la Première partie de la présente étude, le Titre II, le Chapitre II, la Section 1, le Paragraphe 1, le point "A- L'Office Ivoirien des Parcs et Réserves : l'Établissement délégataire de la gestion des aires protégées" et précisément le point intitulé "1. L'Office Ivoirien des Parcs et Réserves : un Établissement public national".
} 


\section{Le fonctionnement du mécanisme fiduciaire, un moyen incitatif de mobilisation de financements pour la protection des aires protégées}

Le fonctionnement du mécanisme fiduciaire à travers le contrat de fiducie met en relation trois (3) personnes : le fiduciant, le fiduciaire et le bénéficiaire. Dans cette opération contractuelle par excellence, le fiduciant confie (temporairement) au fiduciaire des biens, droits ou capitaux qui représentent l'actif fiduciaire afin que ce dernier le gère au profit d'un bénéficiaire. À l'échéance du contrat, le fiduciaire retourne au fiduciant l'intégralité des capitaux reçus, sans majoration d'un intérêt quelconque.

Dans le cadre de la gestion des aires protégées, cette opération contractuelle consiste pour le fiduciant à mettre à la disposition du fiduciaire, des biens, droits ou une somme d'argent (actif fiduciaire), qui provient de son patrimoine. L'actif fiduciaire est mis à la disposition du fiduciaire qui les gère pendant la durée du contrat au profit d'un bénéficiaire qui, en l'espèce, est l'Office Ivoirien des Parcs et Réserves (OIPR). Ces biens, droits ou capitaux sont gérés dans l'optique de financer les activités de gestion de l'OIPR qui se résument à la protection des aires protégées. Au terme du contrat, l'actif fiduciaire est retourné au fiduciant en intégralité (fiducie-gestion), sauf s'il en dispose autrement dans le contrat (fiducie-libéralité ou fiducie à titre de sûreté).

Le caractère incitatif d'un tel mécanisme de financement (particulièrement pour la fiducie-gestion) découle du fait que les aires protégées ont un potentiel de valorisation touristique qui garantit des investissements rentables au plan financier. Ainsi, l'actif fiduciaire pourrait servir au fiduciaire à valoriser les aires protégées sur le plan touristique et à capter d'importantes ressources financières. De la sorte, il dégagerait des bénéfices et partant serait capable de reconstituer l'actif fiduciaire à restituer au fiduciant. Dans ces conditions, les donateurs ou bailleurs de fonds pourraient être rassurés de la restitution de l'actif fiduciaire. L'assurance pour tout fiduciant (potentiel) de rentrer en possession de l'actif fiduciaire au terme du contrat de fiducie pourrait raisonnablement inciter au financement de la gestion durable des aires protégées.

Par ailleurs, les règles rigoureuses de mobilisation et de gestion des financements des aires protégées prescrites par le législateur constituent un atout pour le financement de leur protection durable.

\section{B - La prescription de règles rigoureuses de mobilisation et de gestion des fonds destinés aux aires protégées}

La prescription de règles rigoureuses de mobilisation et de gestion des fonds destinés aux aires protégées concernent aussi bien les Fondations des Parcs et Réserves (1) que les fonds envoyés en fiducie (2). 


\section{Les règles de gouvernance des Fondations des Parcs et Réserves}

La loi de 2002 sur les PNR définit des règles de gestion des fonds qu'elle habilite les Fondations des Parcs et Réserves à rechercher et à recevoir. En effet, selon l'article 49 alinéa $1^{\mathrm{er}}$ de ladite loi, les dirigeants ou administrateurs des Fondations de Parcs et Réserves peuvent faire fructifier les fonds desdites Fondations en les confiant à des professionnels (désignés sur la base d'un appel d'offre) ${ }^{657}$. C'est en toute logique que le législateur requiert des administrateurs ou dirigeants des Fondations des Parcs et Réserves des qualités morales en vue de leur gestion durable ${ }^{658}$. De même, il prescrit une obligation de gestion transparente qui les oblige à présenter des comptes annuels qui sont contrôlés par les commissaires aux comptes $^{659}$; et aussi par les bailleurs ou donateurs ${ }^{660}$. À travers pareilles dispositions, le législateur entendrait entourer les Fonds des Fondations des Parcs et Réserves d'une certaine sécurité juridique qui assurerait une conformité entre la destination pour laquelle ils sont voués et leur utilisation par les administrateurs ou dirigeants des Fondations.

De plus, à l'instar des fonds des Fondations des Parcs et Réserves, le législateur ivoirien instaure une sécurité juridique autour des fonds fiduciaires destinés aux aires protégées.

\section{L'instauration d'une sécurité juridique autour des fonds fiduciaires alloués aux aires protégées}

Le contrat de fiducie est un contrat solennel ${ }^{661}$. À travers ce formalisme rigoureux dont la sanction est la nullité, le législateur impose aux parties dudit contrat l'obligation de

\footnotetext{
${ }^{657} \mathrm{~L}$ 'article 49 alinéa 1er de la loi de 2002 sur les PNR dispose : «Les Fondations peuvent, par une décision du conseil d'administration prise conformément à leur statuts et après avis des commissaires aux Comptes, confier la gestion de tout ou partie de leurs fonds, et de ceux qui leur sont confiés par application d'un contrat de fiducie, à une ou plusieurs personnes morales tierces nationales ou étrangères, présentant toutes les garanties professionnelles en la matière, spécialisées dans la gestion de patrimoine, sélectionnés par appel d'offres, à l'effet d'en retirer un revenu optimal ».

${ }^{658}$ En la matière, l'article 50 de la loi précitée mentionne : «Nul ne peut être dirigeant ou administrateur d'une Fondation, s'il a fait l'objet d'une mesure d'interdiction de diriger, gérer ou contrôler une entreprise ou d'une mesure de faillite personnelle, ou s'il a subi une condamnation pénale ou une sanction professionnelle pour des faits contraires à l'honneur, à la probité ou aux bonnes mœurs.».

${ }^{659} \mathrm{C}$ 'est cette idée qu'exprime l'article 53 de la loi précitée de 2002 sur les PNR en ces termes : «Les comptes annuels des Fondations comprennent, outre le bilan, le compte de résultats et les annexes prévus par les dispositions législatives et réglementaires en vigueur applicables aux sociétés commerciales et, pour chaque contrat de fiducie, les états mentionnés à l'article 51 ci-dessus.

Les Fondations procèdent de manière autonome à l'enregistrement comptable des mouvements affectant la masse fiduciaire.

Les comptes des Fondations sont soumis annuellement au contrôle d'un (1) ou plusieurs commissaires aux comptes désignés par l'assemblée générale. ".

660 À ce sujet, l'article 45 de la loi de 2002 sur les PNR dispose, entre autres : «...Tous les fonds recueillis par les Fondations, ainsi que les revenus des placements, sont des deniers privés.

Les dispositions de l'alinéa précédent ne font pas obstacle à l'exercice, par les donateurs des Fondations, des contrôles qu'ils doivent exercer conformément à leur statut, aux dispositions qui les régissent ainsi qu'au contrat de fiducie.» .

${ }^{661}$ En effet, sous peine de nullité, à travers l'article 56 de la loi de 2002 sur les PNR ci-dessus citée, le législateur prescrit des conditions strictes pour sa formation en ces termes : «Le contrat de fiducie doit comporter, à peine de nullité, les stipulations suivantes:
} 
déterminer avec précision leurs volontés. Il s'agit de la détermination de l'actif fiduciaire, des missions et pouvoirs du fiduciaire, la durée et de la nature du contrat de fiducie.

En outre, le législateur fait échec aux cas de fraude éventuels aux droits des tiers dont le constituant est l'auteur, de deux (2) manières. D'une part, en cas de fraude aux droits de ses créanciers, les biens envoyés en fiducie peuvent faire l'objet de saisie, dans les conditions définies à l'article 60 alinéa 2 de la loi de 2002 sur les $\mathrm{PNR}^{662}$. D'autre part, en cas de libéralité excessive, les héritiers réservataires disposent d'une action en réduction dirigée contre la Fondation ou l'Établissement bénéficiaire. En effet, l'article 61 de la loi de 2002 sur les PNR dispose: "le contrat de fiducie ne peut porter atteinte aux droits des héritiers réservataires ». C'est à juste titre que ledit article prescrit que si, lors du décès du constituant, il s'avère que «la valeur des biens et droits transférés au gestionnaire excède la quotité disponible, le contrat de fiducie est réductible, suivant les règles applicables aux donations entre vifs et conformément aux dispositions législatives et réglementaires en vigueur ».

À la réflexion, ce souci de sécurité juridique qui entoure la formation du contrat de fiducie a pour finalité d'en permettre une exécution paisible. En effet, la protection du droit des tiers dans la formation du contrat de fiducie a pour but d'empêcher qu'un fiduciant indélicat ruse avec les tiers, en organisant son insolvabilité vis-à-vis de ses créanciers ou en déshéritant ses héritiers réservataires. Dans ces cas, ceux-ci pourraient valablement ester en justice pour être rétablis dans leurs droits. Ainsi, à travers ces dispositions, le législateur ivoirien entend éviter les recours (intempestifs) en justice notamment pour des actions en réduction de libéralités excessives ou toute autre action en réparation.

De telles actions auraient l'inconvénient de perturber l'exécution du contrat de fiducie dont l'objectif est le financement pérenne des actions qui concourent à la protection aires protégées.

Au total, la loi de 2002 sur les PNR a diversifié les mécanismes de financement de la gestion durable des aires protégées à travers l'institution de Fondations pour les Parcs et Réserves ainsi que le contrat de fiducie. Ces mécanismes de financements extra-étatiques

$\left.1^{\circ}\right)$ la détermination des biens et droits qui en sont l'objet;

$2^{\circ}$ ) la définition de la mission du gestionnaire, ainsi que l'étendue de ses pouvoirs d'administration et de disposition;

$3^{\circ}$ ) l'indication des conditions dans lesquelles les biens et droits doivent être représentés ou transmis au bénéficiaire;

$4^{\circ}$ ) la détermination de la durée de la fiducie, qui ne peut excéder quatre-vingt-dix-neuf ans à compter de la date du contrat. Si la durée est de quatre-vingt-dix-neuf ans, les biens et matériels qui en sont l'objet sont, au terme de cette durée, définitivement la propriété du bénéficiaire.

Le contrat de fiducie est passé par écrit. Lorsqu'il est conclu à des fins de transmission à titre gratuit ou lorsqu'il porte sur un bien immobilier, ou sur une somme ou sur un bien d'un montant supérieur à un montant fixé par décret pris en Conseil des Ministres, il est, à peine de nullité, passé devant notaire.

La volonté des parties aux termes d'un contrat de fiducie doit être expresse et ne se présume pas. ».

${ }^{662}$ Selon les termes de l'article 60 alinéa $2:$ «Sans préjudice des droits des créanciers du constituant, titulaires d'un droit de suite attaché à une sûreté publiée antérieurement au contrat de fiducie, et hors le cas de fraude aux droits des créanciers du constituant, les biens transférés au gestionnaire ne peuvent être saisis que par les titulaires de créances nées de la conservation ou de la gestion de ces biens. ». 
constituent des moyens de mobilisation de financements qui viennent s'ajouter au financement classique opéré par l'État -sous la forme de subventions. Par conséquent, ces financements extra-étatiques, dont la mobilisation et la gestion obéissent à des règles rigoureuses édictées par le législateur, contribuent (théoriquement) à mettre à la disposition de l'OIPR des ressources financières encore plus considérables pour la protection des aires protégées.

Somme toute, l'analyse du droit positif des aires protégées montrent que les principales innovations qui ont été apportées depuis 2002 par le législateur ivoirien, relativement à la protection de l'intégrité des aires protégées ainsi qu'à leur gestion (désormais participative), accorde une protection suffisante à ces aires protégées. En conséquence, si ces milieux naturels continuent de subir une dégradation qui menace leur existence, les causes ne relèvent pas substantiellement du droit des aires protégées.

Dans ces conditions, les causes des difficultés de l'application du droit des aires protégées n'émaneraient-elles pas plutôt des problèmes pratiques de gestion? 


\section{CHAPITRE II : LES PROBLÈMES PRATIQUES DE GESTION DES AIRES PROTÉGÉES, CAUSES DU DÉFAUT DE L'ÉFFECTIVITÉ DU DROIT DES AIRES PROTÉGÉES}

La gestion des aires protégées ivoiriennes par 1'OIPR rencontre des difficultés qui n'émanent pas essentiellement du droit positif qui les régit. Ces problèmes de gestion résultent plutôt de réalités économiques, d'évènements sociopolitiques caractéristiques de la Côte d'Ivoire, de faits ou encore des pratiques courantes de gestion. Ceux-ci rendent difficile l'application du droit des aires protégées. Ce sont ces difficultés auxquelles sont confrontés les gestionnaires d'aires protégées que nous désignons par le groupe de mots «problèmes pratiques de gestion des aires protégées »"

Les problèmes pratiques de gestion que rencontrent les gestionnaires d'aires protégées en Côte d'Ivoire peuvent être classés en deux (2) catégories. D'une part, il y a les problèmes liés à la gestion du patrimoine naturel dans des aires protégées (Section 1). D'autre part, il s'agit des limites des institutions d'exécution et de contrôle de la mise en œuvre du droit des aires protégées (Section 2).

\section{SECTION I : Les problèmes liés à la gestion du patrimoine naturel dans les aires protégées}

À l'exception du Parc National du Banco, les aires protégées de Côte d'Ivoire sont entourées de communautés humaines rurales ${ }^{663}$. Les besoins sociaux et les activités socioéconomiques desdites populations sont substantiellement tributaires de la forêt. Il s'ensuit une utilisation non durable des ressources forestières des zones périphériques des aires protégées. Cette forme d'utilisation crée une forte dépendance des communautés locales en ressources forestières (Paragraphe 1). Aussi cette dépendance des populations locales en ressources forestières explique-t-elle l'exploitation des aires protégées par celles-ci; laquelle induit de réels problèmes de gestion de ces sites (Paragraphe 2).

\section{Paragraphe 1 : La forte dépendance des populations riveraines des aires protégées en ressources forestières}

La Côte d'Ivoire est un pays dont l'économie repose essentiellement sur l'agriculture ${ }^{664}$, depuis son accession à l'indépendance. Impulsé par l'action coloniale et encouragé par les

\footnotetext{
${ }^{663}$ Situé au cœur de la ville d'Abidjan (Côte d'Ivoire), le Parc National du Banco est : « aujourd'hui le seul Parc National urbain en Afrique ». Voir République de Côte d'Ivoire, Ministère de l'Environnement et des Eaux et Forêts/Office Ivoirien des Parcs et Réserves, État de conservation des Parcs et Réserves de Côte d'Ivoire, Abidjan, 2009, p. 8.

${ }^{664}$ Selon SAWADOGO (Abdoulaye) : «Au chapitre de l'économie, c'est le progrès de l'agriculture qui a entraîné la prospérité de la Côte d'Ivoire et lui a permis de réaliser les performances que l'on sait. ». Voir SAWADOGO (Abdoulaye), L'Agriculture en Côte d'Ivoire, Paris, PUF, 1977, p. 20.

En outre, à propos du rôle de l'agriculture dans l'économie ivoirienne, l'on peut lire dans un document de travail qui traite des orientations stratégiques des mesures riveraines autour des Parcs Nationaux de Côte d'Ivoire ceci: « La Côte d'Ivoire est avant tout et pour longtemps encore un pays à vocation agricole car $34 \%$
} 
autorités ivoiriennes, le développement d'une agriculture écocide par les populations riveraines des aires protégées a des conséquences néfastes sur les forêts du milieu rural (A). Ainsi, fortement dépendante des ressources de la forêt, les populations exploitent continuellement les aires protégées (B).

\section{A - La pratique d'une agriculture écocide dans les zones périphériques des aires protégées}

Dans le cadre de cette étude, les enquêtes menées révèlent que l'agriculture reste l'activité principale des populations riveraines des aires protégées (1). Or, pour la pratique de cette activité, qui se réalise au mieux en milieu forestier, les populations utilisent des techniques agricoles rudimentaires qui ont des effets nocifs sur la biodiversité forestière (2).

\section{L'agriculture, principale activité économique des populations riveraines des aires protégées enquêtées}

Dans les développements qui suivront, nous ne traiterons que de quatre (4) aires protégées. Il s'agit des Parcs Nationaux de Taï, de la Marahoué, d'Azagny, et de la Réserve de Faune d'Abokouamékro, à l'exception du Parc National du Banco qui est un Parc urbain $^{665}$. Il ressort des résultats de nos enquêtes que si l'ensemble des communautés riveraines enquêtées ont pour activité principale l'agriculture (cultures vivrières et cultures de rente) ${ }^{666}, 98 \%$ pratique au moins une culture de rente. En effet, l'économie des départements et sous-préfectures qui environnent les Parcs Nationaux de Taï, de la Marahoué, d'Azagny, et de la Réserve de Faune d'Abokouamékro restent fortement dominée par l'agriculture, ainsi que l'attestent plusieurs travaux ${ }^{667}$. Les autres secteurs de l'économie tels que les services et

du produit intérieur brut (PIB) et $66 \%$ des ressources d'exportation proviennent de l'agriculture. " . Voir République de Côte d'Ivoire, MINEEF/OIPR, Orientations stratégiques des mesures riveraines autour des Parcs Nationaux de Côte d'Ivoire, GTZ, août 2005, p. 2.

${ }^{665}$ Dans le plan d'aménagement du Parc National du Banco, il est écrit : "À la périphérie immédiate du parc, partout où le relief le permet, des quartiers se sont développés...Une présence humaine quasi continue caractérise donc les espaces entourant le parc. Il s'agit d'une communauté urbaine...Elle est composée d'ouvriers des structures industrielles, de retraités, d'artisans, d'acteurs du secteur informel, d'ouvriers, d'agents de maîtrise, de chômeurs. ". Voir République de Côte d'Ivoire, Ministère de 1'Environnement et des Eaux et Forêts/Office Ivoirien des Parcs et Réserves, Plan d'aménagement et de gestion du Parc National du Banco, Abidjan, 2008, pp. 26-27.

Il ressort de ces différents statuts socio-économiques des populations riveraines du Parc National du Banco que l'agriculture est une activité qui n'est pas pratiquée par les riverains dudit Parc. En effet, Abidjan est une cité urbaine, à l'instar de la zone périphérique du Parc National du Banco. Ainsi, les alentours fortement urbanisés du Banco n'abritent quasiment plus d'espaces pour l'agriculture et l'exploitation forestière. Voir également SAKO (Nakouma), BELTRANDO (Gérard), «Dynamiques spatiales récentes du Parc National du Banco (PNB) et stratégies de gestion communautaire durable de ses ressources forestières (District d'Abidjan en Côte d'Ivoire) "), EchoGéo, Vol. 30, octobre 2014/décembre 2014, pp. 6-7.

${ }^{666}$ Les cultures vivrières sont, entre autres, le maïs, le riz, l'igname le manioc, les légumes. En ce qui concerne les cultures de rente, il s'agit principalement du café, du cacao, de l'hévéa, du palmier à huile.

${ }^{667}$ Voir respectivement, République de Côte d'Ivoire, Ministère de l'Environnement et des Eaux et Forêts/Office Ivoirien des Parcs et Réserves, Plan d'aménagement et de gestion du Parc National de Taï, op. cit, p.33; LAUGINIE (Francis), Conservation de la nature et des aires protégées en Côte d'Ivoire, op. cit, p. 186; République de Côte d'Ivoire, Ministère de l'Environnement et des Eaux et Forêts/ Office Ivoirien des Parcs et 
l'industrie demeurent très peu développés. Subséquemment, ces secteurs occupent une frange négligeable de la population. À titre illustratif, considérons les tableaux 2 et 3 ci-dessous.

Tableau 2 : Répartition par département des ménages producteurs de Café et Cacao en Côte d'Ivoire de 1990 à 1999 et de 2002 à 2003

\begin{tabular}{|c|c|c|c|c|c|c|c|}
\hline \multirow[t]{2}{*}{ Zone } & \multirow[t]{2}{*}{ Régions } & \multicolumn{2}{|c|}{$\begin{array}{l}\text { Nombre de } \\
\text { ménages }\end{array}$} & \multirow[t]{2}{*}{ Zone } & \multirow[t]{2}{*}{ Régions } & \multicolumn{2}{|c|}{$\begin{array}{l}\text { Nombre de } \\
\text { ménages }\end{array}$} \\
\hline & & Café & Cacao & & & Café & Cacao \\
\hline SUD & $\begin{array}{l}\text { Région des } \\
\text { Lagunes } \\
\text { Région de } \\
\text { l'Agneby } \\
\text { Région du Sud } \\
\text { Bandaman } \\
\text { Sous-total } 1 \\
\end{array}$ & $\begin{array}{l}25.064 \\
29.628 \\
23.844 \\
78.536 \\
\end{array}$ & $\begin{array}{r}35.496 \\
32.763 \\
54.076 \\
122.335 \\
\end{array}$ & NORD & $\begin{array}{l}\text { Région du } \\
\text { Worodougou } \\
\text { Région du Denguélé } \\
\text { Région des Savanes } \\
\text { Sous-total } 5\end{array}$ & $\begin{array}{r}965 \\
59 \\
- \\
1.024 \\
\end{array}$ & $\begin{array}{r}501 \\
13 \\
- \\
514 \\
\end{array}$ \\
\hline EST & $\begin{array}{l}\text { Région du Sud } \\
\text { Comoé } \\
\text { Région du Moyen } \\
\text { Comoé } \\
\text { Région du } \\
\text { Zanzan } \\
\text { Sous-total } 2\end{array}$ & $\begin{array}{l}17.850 \\
\\
17.878 \\
11.962 \\
47.690\end{array}$ & $\begin{array}{l}17.145 \\
\\
25.933 \\
12.261 \\
55.339\end{array}$ & OUEST & $\begin{array}{l}\text { Région du Moyen } \\
\text { Cavally } \\
\text { Région des } \\
\text { Montagnes } \\
\text { Sous-total } 6\end{array}$ & $\begin{array}{r}30.918 \\
69.375 \\
100.293\end{array}$ & $\begin{array}{l}29.586 \\
41.321 \\
70.907\end{array}$ \\
\hline $\begin{array}{l}\text { CENTRE- } \\
\text { OUEST }\end{array}$ & $\begin{array}{l}\text { Région du } \\
\text { Fromager } \\
\text { Région du Haut- } \\
\text { Sassandra } \\
\text { Région de la } \\
\text { Marahoué } \\
\text { Sous-total } 3\end{array}$ & $\begin{array}{r}18.633 \\
52.903 \\
17.200 \\
88.736 \\
\end{array}$ & $\begin{array}{r}38.858 \\
67.485 \\
\\
29.552 \\
13.895 \\
\end{array}$ & $\begin{array}{l}\text { SUD- } \\
\text { OUEST }\end{array}$ & $\begin{array}{l}\text { Région du } \\
\text { Bas-Sassandra } \\
\text { Sous-total } 7\end{array}$ & $\begin{array}{r}39.406 \\
39.406 \\
\end{array}$ & $\begin{array}{r}109.572 \\
109.572 \\
\end{array}$ \\
\hline CENTRE & $\begin{array}{l}\text { Région des Lacs } \\
\text { Région du N'Zi } \\
\text { Comoé } \\
\text { Région de la } \\
\text { Vallée du } \\
\text { Bandaman } \\
\text { Sous-total } 4\end{array}$ & $\begin{array}{r}8.433 \\
14.836 \\
3.288 \\
26.557\end{array}$ & & & & & \\
\hline
\end{tabular}

Source : BONNÉHIN (Léonie), Le profil historique de la zone périphérique de Tä̈ : Quel potentiel pour la conservation du Parc National de Taï, Abidjan, GTZ/OIPR, Mars 2006, p. 29

Réserves, Plan d'aménagement et de gestion du Parc National d'Azagny op. cit, p. 42 ; LAUGINIE (Francis), Conservation de la nature et des aires protégées en Côte d'Ivoire,, op. cit, p. 279. 


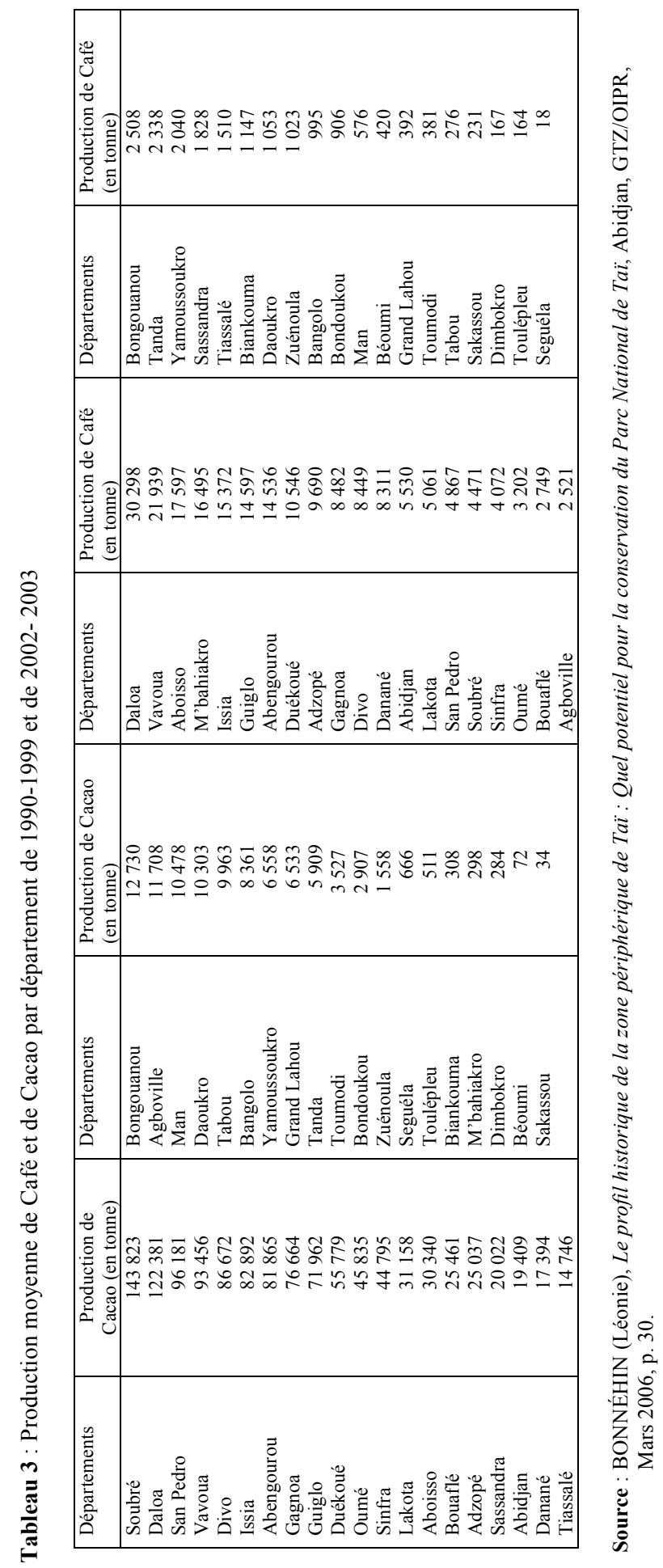




\section{$\underline{\text { Commentaire }}$}

L'examen des tableaux 1 et 2 révèle que les régions (anciennement nommées) régions du Bas-Sassandra et du Moyen Cavally dont relevaient les départements de Soubré, San Pedro, Tabou et Guiglo ${ }^{668}$ qui entourent le Parc National de Taï concentrent 139.158 ménages producteurs de cacao et 70.324 ménages producteurs de café. Elles abritent respectivement $27 \%$ et $18 \%$ des ménages producteurs de cacao et de café. De 1990 à 1999 et de 2002 à 2003, ces ménages ont produit en moyenne $311.929 \mathrm{t}$ de cacao soit $24,39 \%$ du tonnage produit en Côte d'Ivoire ; et 24.211 t de Café soit 9,35\% de la production nationale.

En outre, les régions du Haut-Sassandra et de la Marahoué ${ }^{669}$ qui entourent le Parc National de la Marahoué abritent 97.037 ménages producteurs de cacao soit $19 \%$ de ces ménages. Elles ont produit 149.400 t de cacao en moyenne, de 1990 à 1999 et de 2002-2003; soit $11,68 \%$ de la production de cacao en Côte d'Ivoire durant cette période. Ces deux (2) régions abritent également 70.103 ménages producteurs de café avec $3.070 \mathrm{t}$ produits en moyenne durant cette même période ; soit $15,02 \%$ de la production totale nationale de café.

S'agissant de l'ancienne région des lagunes ${ }^{670}$ où est située le Parc National d'Azagny, elle abrite 35.496 ménages producteurs de cacao et 25.064 de café ; soit respectivement $7 \%$ pour chaque type de ménages producteurs. Le seul département de Grand-Lahou qui fait partie intégrante de ces ménages et qui comprend en grande partie les terroirs riverains du Parc National d'Azagny produit 6.533 t de cacao et 392 t de café en moyenne, de 1990 à 1999 et de 2002 à 2003 . Ces tonnages représentent $0,51 \%$ pour le cacao et $0,17 \%$ pour le café durant cette période.

Enfin, les départements de Yamoussoukro (anciennement situé dans la région des lacs ${ }^{671}$ ) et de Dimbokro (anciennement établi dans la région du N'Zi-Comoé ${ }^{672}$ ) dont relève la Réserve de faune d'Abokouamékro abritent respectivement 22.093 et 23.269 ménages pour le cacao et le café. Ce sont respectivement $4 \%$ et $6 \%$ des ménages producteurs de cacao et de café. Ces ménages produisent $6.558 \mathrm{t}$ de cacao et $2.040 \mathrm{t}$ de café, c'est-à-dire respectivement $0,51 \%$ et $0,89 \%$ de la production moyenne nationale, de 1990 à 1999 et de 2002 à 2003.

\footnotetext{
${ }^{668}$ En effet, depuis 2011, il y a eu un nouveau découpage administratif par l'oeuvre du décret n²011-263 du 28 septembre 2011 portant organisation du territoire national en Districts et en Régions (JORCI n 3 du 20 octobre 2011, p. 37). Ce faisant, les départements de Tabou et de San-Pédro et ceux de Soubré et de Guiglo relèvent respectivement de la région de San-Pédro, Nawa et du Cavally. Voir Annexe au décret n²011-263 du 28 septembre 2011 portant organisation du territoire national en Districts et en Régions (JORCI n ${ }^{\circ} 3$ du 20 octobre 2011, pp. 38-39.

${ }^{669}$ Les dénominations de ces entités territoriales sont restées inchangées. Voir art. 4, Décret n²011-263 du 28 septembre 2011 portant organisation du territoire national en Districts et en Régions Régions (JORCI nº 3 du 20 octobre 2011, p. 37)..

${ }^{670}$ Cette région est désormais dénommée Région des Grands-Ponts. Voir www.ardci-rd.org/index.php/vie-desregions/grands-ponts (consulté le 2 septembre 2015).

${ }^{671}$ Avec le nouveau découpage administratif, ce département relève du Bélier. Voir www.ardcird.org/index.php/vie-des-regions/grands-ponts (consulté le 2 septembre 2015).

${ }^{672}$ Le département de Dimbokro fait dorénavant partie de la Région du N'zi. Voir www.ardcird.org/index.php/vie-des-regions/grands-ponts (consulté le 2 septembre 2015).
} 
$\mathrm{Au}$ total, tous les terroirs des départements dans lesquels sont établies les aires protégées sont d'importantes zones de production des cultures de rente. Ces zones concentrent des centaines de milliers de ménages producteurs de café et de cacao. Sur les 19 régions que comptait la Côte d'Ivoire ${ }^{673}$, les sept (7) qui abritaient les aires protégées enquêtées représentaient respectivement pour le cacao et le café $56 \%$ et $49 \%$ des ménages producteurs de cacao et de café. En d'autres termes, plus de la moitié des ménages producteurs de cacao (56\%) et la presque moitié (49\%) des ménages producteurs de café se concentrent dans ces sept (7) régions. Ces ménages produisent, durant la période susindiquée, plus du 1/3 de la production nationale de cacao $(37,09 \%)$ et du 1/4 de celle du café $(25,43 \%)$. Subséquemment, des superficies importantes sont défrichées pour ces cultures.

Par ailleurs, il existe d'autres spéculations agricoles dans les terroirs riverains des aires protégées. Dans la périphérie du Parc National de Taï (PNT), il est pratiqué la culture de l'hévéa et du palmier à huile. Les plantations hévéïcoles, autour du PNT, occupent une superficie de 34.000 ha. Elles se répartissent comme suit. Au Sud du PNT (dans la souspréfecture de Grand-Béréby), il y a 15.000 ha gérés par la Société des Caoutchoucs de GrandBéréby [SOGB]). Au Nord-Ouest du PNT, dans les sous-préfectures de Guiglo et Taï et zagné (géré par la Compagnie Héveïcole du Cavally [CHC]), se trouvent 14.000 ha. Au SudEst du PNT géré par la Société Africaine des Plantations d'Hévéa (SAPH), l'on a 5.000 ha. S'agissant du palmier à huile, l'on note 55.104 ha de plantations autour du PNT dont 50.104 gérés par la Société de palmier à huile de Côte d'Ivoire (PALMCI) et 5.000 ha par la SOGB). Au total, la seule aire protégée qu'est le PNT est entourée par 89.104 ha de plantations pour ce qui est seulement des deux (2) cultures que sont l'Hévéa et le Palmier à huile.

Il résulte de tout ce qui précède que l'agriculture est l'activité dominante et principale des zones périphériques des aires protégées enquêtées, à l'instar de toutes les autres localités du pays. Aussi les techniques culturales utilisées ne sont-elles pas protectrices de la forêt.

\section{Les techniques agricoles utilisées dans les zones périphériques des aires protégées et leurs conséquences sur la forêt}

L'agriculture est la principale activité des populations riveraines des aires protégées enquêtées. Ce faisant, la pratique de cette activité requiert une utilisation des surfaces cultivables des terroirs villageois qui préserve au mieux sa fertilité ; surtout dans un contexte de démographie galopante ${ }^{674}$. Or, l'agriculture itinérante sur brûlis, principale technique culturale utilisée par les populations a des conséquences néfastes sur la forêt.

\footnotetext{
${ }^{673}$ Actuellement le territoire ivoirien est : « ... organisé en deux (2) Districts Autonomes, douze (12) Districts et trente (30) Régions administratives. ». Cf. art. 2 du décret n²011-263 du 28 septembre 2011 portant organisation du territoire national en Districts et en Régions (JORCI n 3 du 20 octobre 2011, p. 37).

${ }^{674} \mathrm{La}$ Côte d'Ivoire est un pays qui a l'un des taux d'accroissement démographique les plus élevé en Afrique, avec un taux global de $2,9 \%$. La population ivoirienne "double en vingt-quatre ans et s'accroît le plus rapidement du monde ».Voir LAUGINIE (Francis), Conservation de la nature et des aires protégées en Côte d'Ivoire, op.cit., p.18). La densité moyenne de la population est de $48 \mathrm{hbts} / \mathrm{Km}^{2}$. Or, les localités enquêtées ont
} 
Ainsi, il sera présenté l'agriculture itinérante sur brûlis (a) avant d'analyser ses effets sur la forêt (b).

\section{a. L'agriculture itinérante sur brûlis}

L'agriculture itinérante sur brûlis est une technique culturale par laquelle l'agriculteur exploite le sol par rotation. Il utilise successivement des parcelles de terres vierges jusqu'à la mise en culture des terres laissées précédemment en jachère en vue du renouvellement de leur fertilité. Techniquement, l'agriculteur

débroussaille l'espace à cultiver et procède à la mise à feu des ressources végétales coupées. Ainsi, les cendres issues du brûlis des matières végétales constituent un engrais qui fertilise le sol. Ensuite, la portion de terre ainsi préparée est exploitée à but agricole, sur une période de 2 à 3 ans. Au terme de cette période, la portion de terre cultivée doit être laissée sous jachère naturelle, sur une période d'au moins 10 ans $^{675}$.

Telle que décrite, nous déduisons que, l'agriculture itinérante sur brûlis ne peut être pratiquée qu'à certaines conditions. Nous les résumons au nombre de trois (3). La première condition est l'abondance de terres cultivables et fertiles. De la sorte, il sera possible de laisser une parcelle sous jachère naturelle sur une période de 10 ans (requise pour une mise en jachère normale), avant de la remettre en exploitation. La seconde condition est que l'agriculture itinérante sur brûlis sied à l'agriculture de subsistance, en ce qu'elle demande une surface cultivable relativement peu importante. Enfin, cette technique culturale est praticable si le nombre de population est peu élevé.

quasiment une densité de population supérieure à cette moyenne. En effet, le Parc National de Taï a pour terroirs riverains les régions anciennement dénommées Bas Sassandra (actuellement district du Bas-Sassandra) et Moyen Cavally (actuellement représentées par les départements de Guiglo, Bloléquin et Toulepleu). Elles ont respectivement une densité de 53,3 et 36,1 hbts $/ \mathrm{Km}^{2}$. En ce qui concerne le Parc National de la Marahoué, il est situé sur le territoire des régions de la Marahoué et du Haut Sassandra qui ont une densité respective de 63,8\% et $71,6 \mathrm{hbts} / \mathrm{Km}^{2}$. Pour ce qui est de la Réserve de Faune d'Abokouamékro, elle est située dans la région (anciennement appelée région) des Lacs qui a une densité de 53,9 hbts/ $\mathrm{Km}^{2}$. Avec le nouveau découpage administratif de 2011, cette région correspond se trouve sur l'actuel territoire des régions du Bélier et de l'Iffou). Enfin, le Parc National d'Azagny est situé dans la région des lagunes (actuellement dénommée région des Grands Ponts) qui a une densité de $272,7 \mathrm{hbts} / \mathrm{Km}^{2}$. Pour ces données sur le Recensement Général de la Population et de l'Habitat (RGPH) de 1998 effectué par l'Institut National de la Statistique (INS), voir République de Côte d'Ivoire, l'Institut National de la Statistique, RGPH-98: Synthèse des rapports d'analyse du RGPH-98.

Il convient de noter que le nombre de populations dans les zones ci-dessus citées a augmenté, avec le recensement général de la population et de l'habitat de 2014. Cependant, les chiffres sus-cités ont été utilisés parce qu'ils reflétaient au mieux la réalité démographique de ces zones au cours de la réalisation de ces études sur les ménages de café cacao.

Quant aux informations relatives au nouveau découpage administratif de la Côte d'Ivoire de 2011 ci-dessus, voir Annexe au Décret n ${ }^{\circ} 2011-263$ du 28 septembre 2011 portant organisation du territoire national en Districts et en Régions (JORCI n³ du 20 octobre 2011, pp. 38-39.

${ }^{675}$ Ces différents développements sont inspirés par des informations sur la notion de culture itinérante sur brûlis consultées sur www.wwf.be/ media/Agriculture\%20itinerante\%20sur\%20brulis\%20en\%20RDC 122255.pdf, (consulté le 17 mars 2012). 
$\mathrm{Au}$ regard de ces conditions énumérées ci-dessus, l'agriculture itinérante sur brûlis estelle adaptée dans les zones périphériques des aires protégées ? Pour répondre à cette question, il convient d'analyser séparément ces conditions.

La Côte d'Ivoire est un pays qui enregistre une forte croissance démographique ${ }^{676}$. Par rapport à la moyenne nationale, la densité de la population est plus élevée dans les zones périphériques des aires protégées ${ }^{677}$. Il s'ensuit que la condition du nombre réduit de population que requiert l'agriculture itinérante sur brûlis n'est pas remplie ${ }^{678}$.

Également, en plus de l'agriculture de subsistance, l'agriculture d'exportation tient une place capitale dans l'économie des zones périphériques. En effet, étant la principale activité dans les zones périphériques, l'agriculture procure le plus de profit aux populations. Il en découle que, dans ce contexte d'économie de rente où la recherche du profit est largement privilégiée, l'agriculture de subsistance occupe une place secondaire. La seconde condition pour la pratique de l'agriculture itinérante n'est pas réunie.

$\mathrm{Au}$ surplus, dans les zones périphériques des aires protégées, il n'y a presque plus de friches ou de terres vierges disponibles, du fait de la forte pression démographique. Or, la condition de la disponibilité de la terre est requise pour la pratique de l'agriculture itinérante sur brûlis. En l'espèce, cette troisième condition n'est non plus remplie.

Au regard des développements qui précèdent, les trois (3) conditions qui permettent la pratique de l'agriculture itinérante sur brûlis ne sont pas réunies pour l'utilisation de cette technique culturale dans les terroirs riverains des aires protégées. Et pourtant, la pratique de cette technique y est répandue, à l'instar du reste de la Côte d'Ivoire. Par conséquent, elle a de graves conséquences dans les zones périphériques des aires protégées.

\section{b. Les conséquences de l'agriculture itinérante sur brûlis dans les zones périphériques des aires protégées}

En la matière, seuls les effets négatifs de l'agriculture itinérante sur brûlis seront abordés, pour les besoins de l'analyse. Dans un contexte de forte croissance démographique,

\footnotetext{
${ }^{676}$ Voir LAUGINIE (Francis), Conservation de la nature et des aires protégées en Côte d'Ivoire, op.cit., p.18.

${ }^{677}$ En Côte d'Ivoire, la densité moyenne nationale de la population est de $48 \mathrm{hbts} / \mathrm{Km}^{2}$. Les régions du BasSassandra et du Moyen Cavally (précédemment citées) qui entourent le Parc National de Taï ont respectivement une densité de 53,3 et 36,1 hbts $/ \mathrm{Km}^{2}$. Quant à la région des Lacs (antérieurement citée) qui abrite la Réserve de Faune d'Abokouamékro, elle a une densité moyenne de de 53,9 hbts $/ \mathrm{Km}^{2}$. Enfin, la région des lagunes (citée plus haut) sur le territoire duquel se situe le Parc National d'Azagny est densité moyenne de 272,7 hbts/Km². Pour toutes ces données relatives à la densité de la population ivoirienne. Voir République de Côte d'Ivoire, Institut National de la Statistique, Recensement Général de la Population et de l'Habitat-1998, Synthèse des rapports d'analyse.

${ }^{678} \mathrm{~A}$ ce propos, traitant des causes de la destruction de la forêt en Côte d'Ivoire en rapport avec l'agriculture itinérante sur brûlis, LAUGINIE (Francis) note : «L'accroissement démographique actuel ne permet évidemment plus le maintien d'un tel système. ». Voir LAUGINIE (Francis), Conservation de la nature et des aires protégées en Côte d'Ivoire, op.cit., p. 25.
} 
la première conséquence (négative) de la pratique de l'agriculture itinérante sur brûlis dans les zones périphériques des aires protégées est l'augmentation de la demande en terre cultivable.

L'augmentation de la demande en terres cultivables entraîne nécessairement une insuffisance des superficies exploitables à but agricole et inéluctablement le raccourcissement de la période de jachère. En effet, il ressort de nos enquêtes que $94 \%$ des agriculteurs ne laissent pas une portion de terre en jachère pendant plus de deux (2) saisons. Mieux, elles utilisent les terres jusqu'à une baisse importante de leur fertilité.

La pression foncière entraîne une surexploitation des terres cultivables et empêche de les utiliser convenablement en vue d'un meilleur profit. Il en résulte une diminution de la fertilité du sol et par contrecoup une baisse des rendements agricoles. À titre illustratif, dans les sous-préfectures de Guiglo et de Taï qui sont riveraines du Parc National de Taï, l'on note une baisse des rendements agricoles. La production du cacao enregistre $200 \mathrm{~kg} / \mathrm{ha}$ contre une moyenne nationale de $500 \mathrm{~kg}^{679}$. Ainsi, de vastes superficies sont défrichées dans le souci de faire le maximum de profit. Dans ces conditions, l'agriculture itinérante sur brûlis détruit les différents types de végétation.

Il ressort des différents développements sur l'agriculture itinérante sur brûlis qu'elle est une technique culturale dont la viabilité reste fortement influencée par des facteurs socioéconomiques et démographiques. Dans le présent cas, l'agriculture itinérante sur brûlis se révèle inadaptée car écocide. C'est ainsi que les populations des zones périphériques des aires protégées exploitent continuellement ces milieux naturels, malgré l'interdiction prescrite par la loi.

\section{B - La persistance de l'exploitation des aires protégées par les populations}

La pratique d'une agriculture non durable, c'est-à-dire destructrice de la forêt, dans les zones périphériques des aires protégées fait des aires protégées, des îles vertes, pourvoyeuses de ressources forestières (sols et biodiversité). Ainsi, elles constituent un attrait pour les populations, selon les raisons qu'elles avancent (1) pour expliquer leur exploitation (2).

\section{Les raisons évoquées par les populations}

Il ressort de nos enquêtes auprès des populations riveraines des aires protégées et des services de l'OIPR que plusieurs raisons expliquent les pressions que les populations exercent sur les aires protégées. Ces raisons tiennent substantiellement à la pauvreté pour $58 \%$ des répondants, à la baisse de la fertilité des sols et à la saturation des terres cultivables pour $66 \%$

\footnotetext{
${ }^{679}$ Voir République de Côte d'Ivoire, Ministère de l'Environnement et des Eaux et Forêts, Plan d'aménagement et de gestion du Parc National de Taï, op. cit., p. 34.
} 
et à la régression du couvert forestier et de ses ressources pour 97\%. Ces facteurs sont, de plus en plus, aggravés par la croissance rapide de la population riveraine de ces aires protégées qui est à majorité rurale et paysanne ${ }^{680}$.

À ce sujet, et en guise d'exemple, considérons le tableau 3 ci-dessous qui présentent les distances entre les villages riverains de la Réserve de Faune d'Abokouamékro (RFA) et cette aire protégée.

Tableau 4 : Villages riverains de la Réserve de Faune d'Abokouamékro

\begin{tabular}{|l|l|c|l|l|l|}
\hline $\mathbf{N}^{\circ}$ & Dénomination & $\begin{array}{l}\text { Distance par } \\
\text { rapport à la RFA } \\
\text { (en Km) }\end{array}$ & $\mathbf{N}^{\circ}$ & Dénomination & $\begin{array}{l}\text { Distance par } \\
\text { rapport à la RFA } \\
\text { (en Km) }\end{array}$ \\
\hline $\mathbf{1}$ & ABIGUI & 1,8 & $\mathbf{1 2}$ & KOMOI-DIBIKRO & 0,4 \\
\hline $\mathbf{2}$ & ABOKOUAMEKRO & 0,9 & $\mathbf{1 3}$ & KOUAKOUASSIKRO & 0,6 \\
\hline $\mathbf{3}$ & AGNEREKOFFIKRO & 1 & $\mathbf{1 4}$ & KPASSANOU & 4 \\
\hline $\mathbf{4}$ & AKIMOUYAOKRO & 1,5 & $\mathbf{1 5}$ & MOROKINKRO & 0,4 \\
\hline $\mathbf{5}$ & APANGOKRO & 0,7 & $\mathbf{1 6}$ & N'GANGRO & 4,6 \\
\hline $\mathbf{6}$ & ASSANOU & 3,9 & $\mathbf{1 7}$ & N'GBEKRO & 5,8 \\
\hline $\mathbf{7}$ & ASSE N'GATAKRO & 6,71 & $\mathbf{1 8}$ & N'GUESSANKRO & 1 \\
\hline $\mathbf{8}$ & BAKRO SAKASSOU & 6,34 & $\mathbf{1 9}$ & OUFOUEDIEKRO & 3,1 \\
\hline $\mathbf{9}$ & GOFABO & 0,7 & $\mathbf{2 0}$ & PRANOUA & 0,4 \\
\hline $\mathbf{1 0}$ & GOGOKRO & $\mathbf{2 1}$ & TOKOREYAOKRO & 0,2 \\
\hline $\mathbf{1 1}$ & KACOUBROUKRO & 4,3 & $\mathbf{2 2}$ & YEBOUEKRO & 0,3 \\
\hline
\end{tabular}

Source : OIPR/DZ Centre

\section{Commentaire}

L'analyse des données du tableau ci-dessus nous enseigne qu'en règle générale, la RFA est très proche des 22 villages situés dans ses périphéries. En effet, cinq (5) villages sont situés à moins de $500 \mathrm{~m}$ de la Réserve ; 10 à moins d' $1 \mathrm{~km}$ et 15 à moins de $3 \mathrm{~km}$. Cette proximité des villages riverains des limites de la RFA et la densité élevée des populations au $\mathrm{km}^{2}$ expliquent en partie les pressions anthropiques sur cette aire protégée. Les propos de $\mathrm{K}$. Y, habitant du village de Komoi-Dibikro (village situé à environ $400 \mathrm{~m}$ de la RFA) recueillis lors de l'enquête de terrain illustre cette réalité. En effet, il affirme : "Nous n'avons plus de terres pour l'agriculture...les terres que nous labourons pour avoir à manger sont celles que l'État a prises pour créer la Réserve».

\footnotetext{
${ }^{680}$ Voir l'Annexe 3 de la présente étude intitulé "Rapport de l'enquête sur les réalités de la gestion des aires protégées en Côte d'Ivoire", le point "II- Résultats de l'enquête auprès des communautés riveraines des aires protégées" et précisément le point "5. Les raisons des pressions sur les aires protégées". Par ailleurs, il est à noter que ces chiffres peuvent être plus importants dans la mesure où les personnes enquêtées (communautés locales) sont toujours hésitantes à donner des informations sur l'exploitation des aires protégées. En effet, elles les considèrent comme des questions sensibles, en raison de leur caractère infractionnel.
} 
En outre, l'ensemble des communautés locales enquêtées soutiennent qu'elles n'ont que l'agriculture comme activité depuis des lustres; et que sa pratique est vitale pour elle, en raison du manque d'alternatives économiques. À propos, K. R. de BLAISEKRO (village situé dans le Parc National de la Marahoué, plus précisément dans la zone agroforestière ${ }^{681}$ ) affirme ceci : "Moi Koffi, je ne sais faire que le travail de la terre. Il en est de même pour tous ceux qui vivent ici. C'est ainsi que je nourris ma famille, scolarise mes enfants. Alors, je demande pardon au gouvernement de penser à nous car si on nous chasse d'ici, c'est notre mort...».

De même, les $97 \%$ des populations enquêtées répondent que les pressions qu'elles exercent sur les aires protégées s'expliquent aussi par la disparition du couvert forestier et la rareté de certaines espèces animales et végétales dans les terroirs villageois. En effet, rares dans les terroirs villageois, les produits forestiers ligneux et non ligneux utilisés à des fins diverses ${ }^{62}$ sont prélevés dans les aires protégées par les populations. C'est ainsi que la rareté de ces ressources dans le domaine rural les conduit à s'approvisionner dans les aires protégées. Il en est de même pour la faune sauvage qui, se faisant de plus en plus rare dans les terroirs villageois, est chassée dans les aires protégées.

Enfin, 66\% des populations riveraines des aires protégées enquêtées soulignent que le manque d'emplois et d'activités génératrices de revenus pour les jeunes constituent également un facteur qui aggrave les pressions anthropiques sur les aires protégées.

\section{Illustrations des pressions anthropiques sur certaines aires protégées}

Toutes les aires protégées de Côte d'Ivoire subissent des pressions anthropiques. Cependant, des pressions relatives à quelques aires protégées seront présentées en l'espèce. Il s'agit de celles relatives aux Parcs Nationaux d'Azagny (a), de Taï (b) et de la Marahoué (c).

\section{a. Cas du Parc National d'Azagny}

Il ressort de nos enquêtes, auprès des gestionnaires, que le Parc National d'Azagny (PNA) connaît de fortes pressions anthropiques. Ces pressions sont essentiellement les défrichements, l'exploitation agricole, le braconnage et le prélèvement de produits forestiers ligneux $^{683}$ et non ligneux ${ }^{684}$.

\footnotetext{
${ }^{681}$ Dans le Parc National de la Marahoué, la zone agroforestière est une zone délimitée en 1988 par la SODEFOR à la demande du gouvernement ivoirien en vue de regrouper les populations infiltrées et disséminée dans ce Parc. Elle est évaluée à environ 9.000 ha. Sur ces différents points, voir AKOUÉ (Yao Claude), Les aspects conflictuels liés à la conservation des aires protégées de Côte d'Ivoire : le cas du Parc National de la Marahoué à Bouaflé, Mémoire de DEA en Sciences et Gestion de l'Environnement option Politique en Environnement, Université d'Abobo-Adjamé, août 2004, p. 47.

${ }^{682} \mathrm{Ces}$ produits sont utilisés, entre autres, comme source d'énergie domestique (bois de chauffe), ou matériau pour la fabrication d'outils de travail ou encore pour la construction d'habitations, pour la consommation.

${ }^{683}$ Le bois d'œuvre, le bois de chauffe, le rotin (Calamus deeratus), les bambous de raphia (Raphia hookeri), par exemple.

${ }^{684}$ Il s'agit notamment des racines, feuilles, fruits, écorces ainsi que de la faune sauvage.
} 
Aux dires des gestionnaires, bien souvent, des portions de terres à la périphérie du PNA sont défrichées par les populations pour la culture de vivriers (manioc, igname...). Cette forme de pression est plus manifeste sur la limite nord du PNA. Elle est pratiquée par les populations (riveraines) des campements de Broukro, Dely et Konankro. Ils ont également affirmé que les populations exploitent les anciennes plantations de cultures industrielles (café, cacao, hévéa, palmier à huile, et cocotier) créées sur l'assise territoriale du PNA avant son classement. Selon le Chef secteur Azagny d'alors, cette situation s'expliquerait par l'effectivité relative de "la politique de déguerpissement, de dédommagement et de réinstallation $»$ des paysans déguerpis ${ }^{685}$. En revanche, ceux-ci continuent de les exploiter clandestinement, vu la modicité des moyens de gestion dont il dispose pour éradiquer ce phénomène.

S'agissant des produits forestiers ligneux et non ligneux, ils sont prélevés dans le PNA en raison de leur rareté dans les terroirs villageois. Ils servent pour la construction d'habitations (charpentes, toitures) ; la confection de matériaux à usage domestique divers (chaises traditionnelles, pilons, mortiers) ou outils de travail (pirogue); l'alimentation et comme produits médicinaux (racines, feuilles, fruits, écorces, faune sauvage...).

Pour ce qui est particulièrement de la pêche (poissons et crustacés) et du braconnage pratiqués dans le PNA, s'ils servent à la fois à la consommation domestique, elles alimentent un commerce florissant dans de grandes villes telles qu'Abidjan, et San Pedro, aux dires des gestionnaires du Parc ${ }^{686}$. Les espèces animales principalement braconnées sont les primates, le buffle (Syncerus caffer), le guib harnaché (Tragelaphus scriptus) et les céphalophes (Cephalophus maxwelli, Cephalophus niger, Cephalophus ogilbyi).

Outre le Parc National d'Azagny, le Parc National de Taï subit également des pressions de l'homme.

\section{b. Cas du Parc National de Taï}

Le Parc National de Taï (PNT) n'est pas exempt de pressions anthropiques, à l'instar de toutes les aires protégées de Côte d'Ivoire, bien qu'étant l'aire protégée la mieux

\footnotetext{
${ }^{685} \mathrm{Au}$ début des années 80, dans le cadre de l'aménagement du PNA, les plantations et habitations qui se trouvaient à l'intérieur du Parc avaient été délogées, indemnisées et réinstallées dans une partie de la forêt classée du Gô partiellement déclassée à cet effet. Par contre, pour ce qui est des plantations qui étaient dans la zone tampon du Parc, le déguerpissement ne fut pas automatique. À l'issu d'un contrat de trois (3) ans avec le gouvernement, les populations devraient les exploiter et quitter ces parcelles à terme, sans aucune autre forme d'indemnisation. Ce sont ces contrats qui sont aujourd'hui remis en cause par les populations qui ne les perçoivent pas comme une indemnisation et continuent de récolter clandestinement les plantations. Sur ce point, voir UICN/République de Côte d'Ivoire, MINAGRA, CCE/BM/, Stratégie de conservation des Parcs Nationaux de Comoé et d'Azagny : Analyse sociologique, Gland, UICN, 1994, p.10.

${ }^{686}$ Voir UICN/BRAO, Évaluation de l'efficacité de la gestion des aires protégées : Parcs et Réserves de Côte d'Ivoire, 2008, p. 21.
} 
conservée $^{687}$. Les pressions anthropiques dont il est l'objet sont principalement le braconnage, le défrichement, l'exploitation minière, le prélèvement de produits forestiers ligneux et non ligneux. Selon le Directeur de zone Sud-Ouest de l'OIPR rencontré, l'intensification des activités de surveillance depuis 2005 a permis de constater une recrudescence des délits de chasse, d'entretien d'anciennes plantations et surtout d'orpaillage.

Le braconnage intensif dans le PNT se justifie, aux dires des gestionnaires, par le manque de protéines animales et la dépendance des populations vis-à-vis de la viande de brousse. Il en est de même du délit de défrichement qu'expliquent la saturation foncière ou le manque de terres agricoles pour des populations essentielles rurales et dont l'activité principale est l'agriculture. C'est propos sont confirmés par une étude menée sur le PNT en mars 2006 et intitulée "Le profil de la Zone Périphérique du Parc National de Taï". En effet, cette étude indique les raisons évoquées par les auteurs des délits. En effet, les délinquants justifient les délits divers dans le PNT par le déficit de développement et/ou le disfonctionnement dans le processus de développement, en général (paupérisation et disparités diverses) ${ }^{688}$. Qu'en est-il du Parc National de la Marahoué ?

\section{c. Cas du Parc National de la Marahoué}

Le Parc National de la Marahoué (PNM) est le Parc le plus infiltré de la Côte Ivoire. Les informations recueillies auprès du Chef secteur OIPR du PNM renseigne que ce Parc est occupé à plus de $75 \%$ de sa superficie. Une étude de l'UICN réalisée en 2008 et précédemment citée estimerait les populations infiltrées à 20.000 habitants ${ }^{689}$. Elles pratiquent essentiellement l'agriculture.

Les enquêtes dans certains villages et les campements de la zone agroforestière ont permis de nous rendre compte que les défrichements continuaient. À titre principal, les populations infiltrées et riveraines du PNM, en sus de l'agriculture, y pratiquent également le braconnage intensif de grands mammifères, à but commercial. Ce sont, entre autres, les antilopes, les éléphants et les primates. Subsidiairement, le braconnage est pratiqué pour une autoconsommation. Il en est de même pour la pêche.

Par ailleurs, l'exploitation forestière est également une réalité dans le PNM. Selon le Chef secteur OIPR rencontré et ses collaborateurs, elle constitue la deuxième menace en termes d'importance, après les défrichements agricoles. Ces informations sont confirmées par les populations infiltrées rencontrées. À titre illustratif, les populations du village de N'doliyaokro ont arraisonnée un camion transportant des billes de bois fraîchement abattus

\footnotetext{
${ }^{687}$ Voir République de Côte d'Ivoire, Ministère de 1'Environnement et des Eaux et Forêts/Office Ivoirien des Parcs et Réserves, État de conservation des Parcs et Réserves de Côte d'Ivoire, Abidjan, 2009, p.12.

${ }^{688}$ Voir BONNÉHIN (Léonie), Le profil de la Zone Périphérique du Parc National de Taï. Quel potentiel pour la conservation du Parc?, Abidjan, GTZ/OIPR, pp. 22-26.

${ }^{689}$ Voir République de Côte d'Ivoire, Ministère de 1'Environnement et des Eaux et Forêts/Office Ivoirien des Parcs et Réserves, État de conservation des Parcs et Réserves de Côte d'Ivoire, op.cit., p.22.
} 
de passage dans leur village (situé dans la zone agroforestière, au sud du PNM), le 27 mai $2007^{690}$.

$\mathrm{Au}$ surplus, les problèmes pratiques de gestion des aires protégées portent sur ces forêts elles-mêmes.

\section{Paragraphe 2 : Les problèmes relatifs aux aires protégées à proprement dit}

Les problèmes pratiques de gestion des aires protégées à proprement dit ont trait aux problèmes de gestion courante $(\mathrm{A})$ et aux problèmes fonciers qui perdurent $(\mathrm{B})$.

\section{A - Les problèmes de gestion courante des aires protégées}

Les problèmes de gestion courante des aires protégées sont l'insuffisance de financements et d'équipements (1) ainsi que l'insuffisance des infrastructures (2).

\section{L'insuffisance de financements et d'équipements dans la gestion des aires protégées}

La gestion des aires protégées nécessite des équipements divers. Il s'agit entre autres d'engins de déplacements (véhicule, moto, hors-bord), de communication (radio, GPS) et d'autres matériels de terrain (jumelles, bottes, uniformes, gourdes, sacs de couchage...). Dans l'ensemble des aires protégées, ce matériel est en nombre insuffisant ${ }^{691}$. Si l'ensemble des gestionnaires rencontrés (à un degré moindre ceux en charge du Parc National de Taï) soulignent cette insuffisance d'équipements, ils ne manquent pas de noter qu'elle est aggravée par leur mauvais état, en raison du manque d'entretien.

De plus, les Parcs et Réserves de Côte d'Ivoire connaissent dans l'ensemble des problèmes de financements, à l'exception également du Parc National de Taï qui bénéficie de l'aide multilatérale $(\mathrm{KFW}, \mathrm{WWF}, \mathrm{GTZ}, \mathrm{WCF})^{692}$. À la réalité, les gestionnaires d'aires protégées éprouvent des difficultés à mobiliser des financements nécessaires pour la gestion durable de ces milieux naturels ${ }^{693}$.

Par ailleurs, l'un des problèmes pratiques de gestion des aires protégées émane des insuffisances et du mauvais état des infrastructures de gestion.

\footnotetext{
${ }^{690}$ L'article de M.O intitulé «Déguerpissement du Parc de la Marahoué: les populations paniquées font des propositions», paru dans le quotidien L'INTELLIGENT D'ABIDJAN présente la photo de ce camion intercepté, le 27 mai 2007, par les populations de N'doliyaokro. Voir l'article de journal de M.O, « Déguerpissement du Parc de la Marahoué: les populations paniquées font des propositions», paru le jeudi 22 novembre 2007 dans L'INTELLIGENT D'ABIDJAN, n¹257, pp. 6-7.

${ }^{691}$ Voir UICN/BRAO, Évaluation de l'efficacité de la gestion des aires protégées : Parcs et Réserves de Côte d'Ivoire, 2008, op. cit, op. cit., p. 36.

${ }^{692}$ Idem, pp. 36-37.

${ }^{693}$ Voir République de Côte d'Ivoire, Ministère de l'Environnement et des Eaux et Forêts/Office Ivoirien des Parcs et Réserves, État de conservation des Parcs et Réserves de Côte d'Tvoire, op. cit., p. 7.
} 


\section{L'insuffisance et le mauvais état des infrastructures de gestion des aires protégées}

De manière générale, les infrastructures de gestion des aires protégées sont insuffisantes et en mauvais état, excepté le Parc National de Taï où elles sont relativement en bon état ${ }^{694}$. D'une part, il s'agit des locaux à usage administratifs, des infrastructures de surveillance qui ont été fortement dégradées durant la crise militaro-politique et/ou qui ont souffert d'un manque d'entretien, en raison des insuffisances de financements des aires protégées $^{695}$. D'autre part, en ce qui concerne les infrastructures touristiques, le constat est le même. En effet, bien que la Côte d'Ivoire n'ait pas mis en place une véritable politique touristique destinée à valoriser ses aires protégées, il reste que des infrastructures touristiques ont été établies dans quelques aires protégées ${ }^{696}$.

Ces infrastructures ont été réalisées par le biais de l'aide extérieure, à l'exception de la Réserve de Faune d'Abokouamékro. En effet, au milieu des années 1980, des actions en vue de l'exploitation touristique ont été réalisées au Parc National d'Azagny, sous financement Banque mondiale. Il s'agit, entre autres, de l'ouverture de pistes de surveillance et de visites ; d'un circuit pédestre avec des passerelles flottantes dans les zones marécageuses; d'un campement hôtel comprenant plusieurs bungalows, d'un restaurant, d'un hall d'informations ${ }^{697}$. Les passerelles flottantes ne sont plus praticables et le campement hôtel a été détruit par les braconniers ${ }^{698}$.

En ce qui concerne le Parc National de la Comoé ${ }^{699}$, il disposait d'un réseau de pistes intérieures d'environ $1000 \mathrm{~km}$ réalisées dans le cadre de plusieurs aménagements. Cependant,

\footnotetext{
${ }^{694}$ Voir UICN/BRAO, Évaluation de l'efficacité de la gestion des aires protégées : Parcs et Réserves de Côte d'Ivoire, 2008, op. cit., p .6.

${ }^{695}$ Idem, p.37.

${ }^{696}$ Par exemple, seulement cinq aires protégées, à savoir les Parcs Nationaux d'Azagny, de la Comoé, de la Marahoué, de Taï ainsi que la Réserve de Faune d'Abokouamékro ont été dotés de réceptifs hôteliers. Voir MONSAN (Ignace), Stratégie de développement de l'écotourisme : le cas du Parc National d'Azagny, Mémoire de DEA en Sciences et Gestion de 1'Environnement option Politique en Environnement, Université d'AboboAdjamé, 17 août 2004, p. 28.

${ }^{697}$ Voir LAUGINIE (Francis), Conservation de la nature et des aires protégées en Côte d'Ivoire, op. cit., pp. 93 94.

${ }^{698}$ Voir République de Côte d'Ivoire, Ministère de l'Environnement et des Eaux et Forêts/Office Ivoirien des Parcs et Réserves, État de conservation des Parcs et Réserves de Côte d'Ivoire, op. cit., p. 7.

${ }^{699}$ Crée par le décret $n^{\circ} 68-81$ du 9 février 1968 portant création du Parc National de la Comoé (JORCI n 11 du 22 février 1968, p. 556), le Parc National de la Comoé (de coordonnées $9^{\circ} 06^{\prime} 53^{\prime \prime}$ Nord $3^{\circ} 43^{\prime} 49^{\prime \prime}$ Ouest) est situé au Nord-Est de la Côte d'Ivoire, dans la Région du Bounkani. Il est inscrit sur la liste du Patrimoine mondial de l'Unesco et désigné réserve de biosphère depuis 1983. Voir UNESCO/UICN, Rapport de mission, Suivi de l'état de la conservation du Parc National de la Comoé en Côte d'Ivoire, site du Patrimoine Mondial, 10-23 juin 2006, p. 5 ; République de Côte d'Ivoire, Ministère de l'Environnement, de la Salubrité Urbaine et Développement Durable/Office Ivoirien des Parcs et Réserves, État de conservation du Parc National de la Comoé. Côte d'Ivoire, janvier 2014, p. 2.

Toutefois, il est à préciser que suite à la crise militaro-politique survenue en Côte d'Ivoire depuis 2002, le Parc National de la Comoé (PNC) a été l'objet de différentes atteintes, en raison du dysfonctionnement du dispositif de surveillance. Voir UNESCO/UICN, Rapport de mission, Suivi de l'état de la conservation du Parc National de la Comoé en Côte d'Ivoire, site du Patrimoine Mondial, 10-23 juin 2006, p. 5. Ainsi, le Comité du
} 
les $2 / 3$ de ce réseau restent impraticables à cause du manque d'entretien. Il en est de même pour le campement de Ouangofitini (au Nord du Parc) qui a été abandonné suite à la baisse de la fréquence touristique ; et de l'hôtel de Gansé (situé au Sud-Ouest du Parc) ${ }^{700}$.

En outre, le Parc National de la Marahoué a été aménagé et équipé sous un financement du Fonds Européen de Développement (FED). Au nombre des infrastructures réalisées figurent un centre d'accueil de trois bungalows, un bâtiment hangar, un restaurant, une aire de jeu, $200 \mathrm{~km}$ de pistes internes dont 150 à vocation touristique et trois (3) pistes d'accès totalisant $25 \mathrm{~km}$. Toutefois, toutes ces infrastructures sont dans un état de dégradation avancée $^{701}$.

Pour terminer, la Réserve de Faune d'Abokouamékro a été créée pour le tourisme de vision $^{702}$. À cet effet, il a fait l'objet d'investissements importants, de la part de l'État ivoirien $^{703}$. Ces investissements ont permis de la peupler avec des espèces animales capturées dans les Parcs Nationaux de la Comoé et de la Marahoué (6 éléphants) et 5 rhinocéros blancs importés d'Afrique du Sud ${ }^{704}$. Également, des infrastructures telles que des pistes intérieures et un réceptif hôtelier ont été réalisées ${ }^{705}$. Cependant, en 1999, une révolte des populations riveraines de cette aire protégée consécutive au non-respect des promesses de l'État et «des doléances soumises à l'État pour la cession de leurs terres et propriétés diverses » les a emmenés à saccager entièrement ces différentes infrastructures ${ }^{706}$.

Par ailleurs, les difficultés de la gestion des aires protégées s'expliquent, bien souvent, par la persistance de problèmes fonciers.

patrimoine mondial a inscrit cette aire protégée sur la Liste du patrimoine mondial en péril, le 10 décembre 2003. Voir décision 27 COM 7B.3 et documents WHC-03/27.COM/7B.Corr, WHC-03/27.COM/24).

${ }^{700}$ Voir LAUGINIE (Francis), Conservation de la nature et des aires protégées en Côte d'Ivoire, op. cit., p. 158.

${ }^{701}$ Voir République de Côte d'Ivoire, Ministère de l'Environnement et des Eaux et Forêts/Office Ivoirien des Parcs et Réserves, État de conservation des Parcs et Réserves de Côte d'Ivoire, Abidjan, 2009, p. 9.

${ }^{702}$ Idem, p.8.

${ }^{703}$ Sur la question LAUGINIE (Francis) affirme que la création de la Réserve de Faune d'Abokouamékro a bénéficié d'un "investissement démesuré, engloutissant l'équivalent de quinze fois le budget national consacré annuellement à la conservation de la nature ». Autrement dit, il s'agit «d'un montant de huit milliards de francs CFA, soit douze millions deux cent mille euros ». Voir LAUGINIE (Francis), Conservation de la nature et des aires protégées en Côte d'Ivoire, op. cit., p. 270.

${ }^{704}$ Voir République de Côte d'Ivoire, Ministère de l'Environnement et des Eaux et Forêts/Office Ivoirien des Parcs et Réserves, État de conservation des Parcs et Réserves de Côte d'Ivoire, op. cit., p. 8.

${ }^{705}$ Selon LAUGINIE (Francis), les travaux réalisés au titre du projet de la Réserve de Faune d'Abokouamékro « se sont traduits par :

- la réalisation de pistes périmétrales (40 kilomètres) et intérieures (126 kilomètres), de clôtures périmétrales (40 kilomètres) et internes amovibles (14 kilomètres) et d'une retenue d'eau de 350 hectares ;

- la construction de trois petits campements (...) d'une d'une paillote-avec restaurant...et deux postes de surveillance ». Voir LAUGINIE (Francis), Conservation de la nature et des aires protégées en Côte d'Ivoire, op. cit., p. 280.

${ }^{706}$ Voir République de Côte d'Ivoire, Ministère de l'Environnement et des Eaux et Forêts/Office Ivoirien des Parcs et Réserves, État de conservation des Parcs et Réserves de Côte d'Ivoire, op. cit., p. 9. 


\section{B - La survivance des problèmes relatifs au foncier dans les aires protégées}

Les problèmes pratiques de gestion des aires protégées relatifs aux fonciers perdurent depuis plusieurs années. Ces problèmes émanent de revendications d'indemnisation et de contestations de limites de celles-ci par les communautés locales (1). Ils s'expliquent également par le laxisme de l'État dans les règlements des problèmes d'occupations illégales d'aires protégées par les populations (2).

\section{La persistance des problèmes de revendications d'indemnisation et de contestation de limites d'aires protégées par les communautés locales}

La création de certaines aires protégées a affecté les droits de propriété des communautés locales. Il s'ensuit des problèmes de gestion qui se posent en termes de revendication des indemnisations ou de contestation de limites. En effet, pour ce qui est des problèmes d'indemnisation, par exemple, au Parc National du Mont Péko créé depuis $1968^{707}$, certaines populations locales revendiquent encore des indemnisations. Il s'agit des indemnisations relatives à la création dudit Parc qu'elles n'auraient pas perçues alors qu'elles ont cédés des terres pour la mise en place de cette aire protégée ${ }^{708}$. De même, des communautés riveraines de la Réserve de Faune d'Abokouamékro revendiquent des indemnisations relativement aux préjudices qu'elles auraient subis à la création de cette aire protégée. En effet, en 1993, la création de la Réserve de Faune d'Abokouamékro a provoqué le déplacement de familles; a englouti des plantations, deux (2) bois sacrés et des cimetières des villages riverains ${ }^{709}$.

Quant aux problèmes de contestations des limites, deux exemples seront fournis. Il s'agit du Parc National du Mont Péko, et de la Réserve partielle de Dahliafleur. En effet, créé en 1968, le Parc National du Mont Péko a été de nouveau délimité en 1974. Au cours de cette opération, « huit villages se sont vus lésés par empiètement sur leur terroirs pour un total de 3.500 hectares ${ }^{710}$. Également, la gestion de la Réserve partielle de Dahliafleur connaît des difficultés liées au foncier. Des promoteurs immobiliers disposent «de titre de propriété délivré par des services étatiques sur des portions de la Réserve » ${ }^{711}$.

Ces problèmes de contestations de limites et d'indemnisation qui perdurent se complexifient avec l'évolution du temps, compte tenu de la pression foncière; et surtout

\footnotetext{
${ }^{707}$ Voir Décret 68-79 du 9 février 1968 portant création du Parc National du Mont Péko, JORCI n ${ }^{\circ} 11$ du 22 février 1968 p. 307.

${ }^{708}$ Voir LAUGINIE (Francis), Conservation de la nature et des aires protégées en Côte d'Tvoire, op. cit., p.206.

${ }^{709}$ Idem, p. 279.

${ }^{710}$ Idem, p. 206.

${ }^{711}$ Voir République de Côte d'Ivoire, Ministère de 1'Environnement et des Eaux et Forêts/Office Ivoirien des Parcs et Réserves, État de conservation des Parcs et Réserves de Côte d'Ivoire, op. cit., p. 8.
} 
parce que, très souvent, les limites des aires protégées ne sont pas clairement matérialisées ${ }^{712}$. Ils constituent, par conséquent, des menaces pour la protection de l'intégrité des aires protégées, à l'instar des occupations illégales de ces espaces par les populations qui perdurent également du fait de la tolérance des pouvoirs publics.

\section{Le laxisme des pouvoirs publics dans les règlements des problèmes d'occupations illégales d'aires protégées par les populations}

En Côte d'Ivoire, les occupations illégales d'aires protégées par les populations pour la pratique d'activités agricoles uniquement ou à des fins d'habitation et d'exploitation agricole est une pratique qui perdure. En pareil cas, plutôt que régler ces problèmes au moment où ils se posent, les pouvoirs publics ou l'État ivoirien les a, bien souvent, tolérés en tergiversant. Aussi sera-t-il présenté des cas d'occupation des espaces mis en défens que sont les Parcs Nationaux de la Marahoué (a), de Taï (b), et d'Azagny (c), à travers les développements qui suivent.

\section{a. Le cas du Parc National de la Marahoué}

La forte infiltration du Parc National de la Marahoué (PNM) conduit le gouvernement à délimiter des espaces fortement dégradés dans ledit Parc, aux fins de la réinstallation (sur ces sites) de tous les planteurs et familles qui l'ont infiltré. Ce faisant, en 1988, il est délimité dans le PNM trois (3) sites couvrant une superficie de 9.000 ha dont l'ensemble ou chacune est souvent dénommé "zone agroforestière" (ZAF).

La Figure ci-dessous fournit une précision imagée sur les ZAF dans le PNM.

\footnotetext{
${ }^{712}$ Voir LAUGINIE (Francis), Conservation de la nature et des aires protégées en Côte d'Ivoire, op. cit., p.186 ; voir République de Côte d'Ivoire, Ministère de l'Environnement et des Eaux et Forêts/Office Ivoirien des Parcs et Réserves, État de conservation des Parcs et Réserves de Côte d'Ivoire, op. cit., p. 9.
} 
Figure 4 : Zonage du Parc National de la Marahoué précisant la Zone Agroforestière

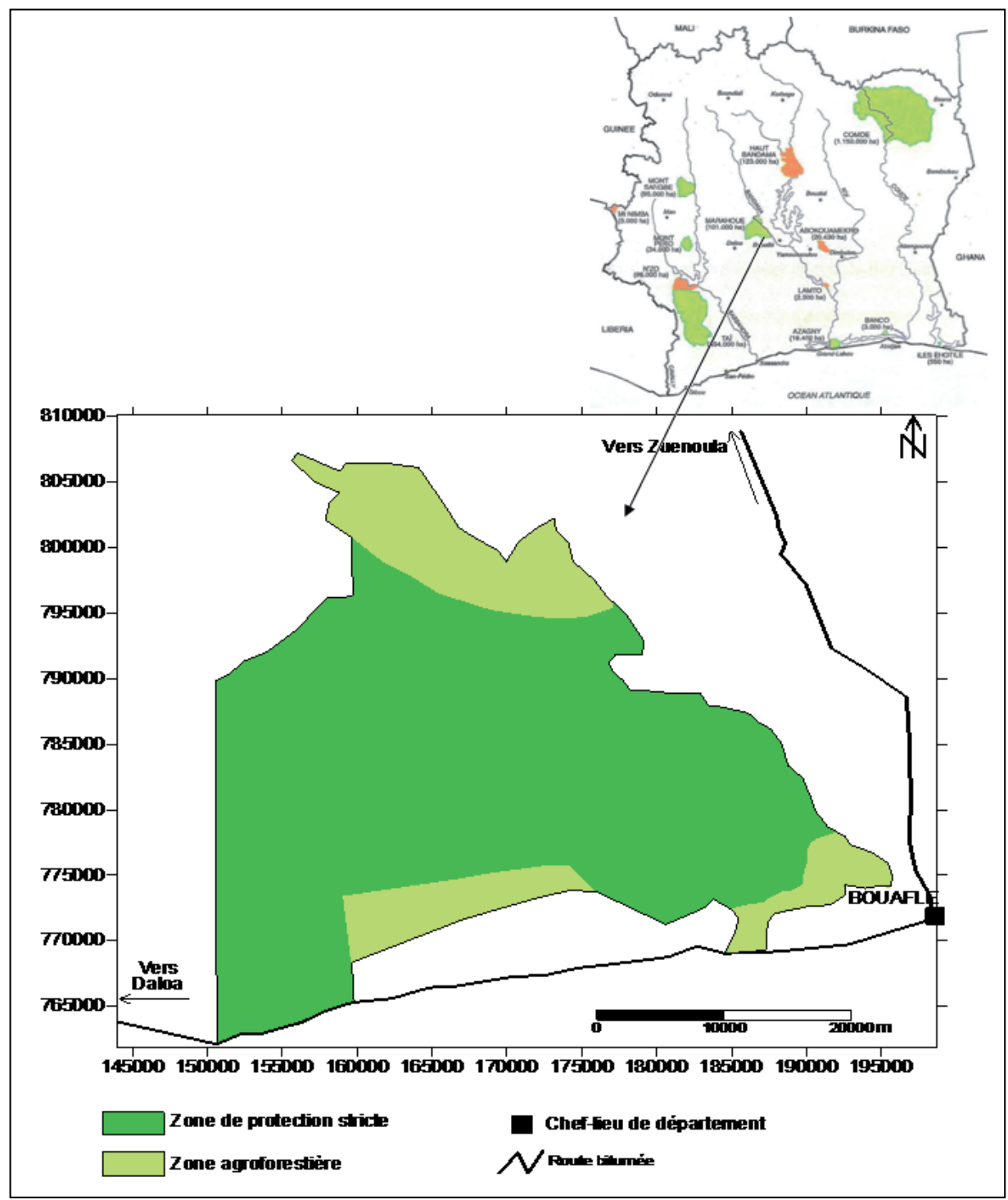

\section{Commentaires}

À la lecture de la carte du Parc National de la Marahoué ci-dessus, l'on observe qu'il y a été délimité trois (3) zones dites agroforestières. Géographiquement, il y a une zone agroforestière au Nord du Parc. Les deux (2) autres sont situées sur les axes des départements de Daloa et Bouaflé. 
La délimitation ces espaces dénommés zones agroforestières avait pour objectif de freiner la progression des pressions anthropiques dans cette aire protégée ${ }^{713}$. Cette délimitation a eu pour fondement juridique une note circulaire du ministre Vincent Pierre LOKROU $^{714}$.

À travers cette note circulaire, le ministre Vincent Pierre LOKROU a donné des instructions aux responsables de l'Administration déconcentrée ${ }^{715}$ dont il convient de présenter un extrait. En effet, sur cette note l'on peut lire: «...Ce plan vise notamment à soustraire une plus grande superficie possible du Domaine Permanent de l'État aux agressions de toute sorte tout maintenant intact le potentiel agricole productif généré dans ces domaines.

À cet égard, je tiens à vous confirmer les dispositions ci-après :

- interdiction formelle d'installer des habitations à l'intérieur des Forêts classées, des Parcs Nationaux et Réserves;

- autorisation d'accéder aux plantations en production pour les entretenir et en cueillir les produits sur une période non renouvelable de trois ans, sans toutefois procéder à de nouvelles extensions de cultures ;

- relocalisation des plantations en un seul domaine qui proviendrait en priorité de récupérations de jachères inexploitées et le cas échéant, d'un déclassement partiel du Domaine Permanent. » (Souligné par nous).

Si la note circulaire sus-citée fait obligation aux planteurs dont les plantations sont restées dans les limites du Parc (zone stricte de protection) de récolter lesdites plantations, pendant un délai de trois (3) ans, sans les entretenir ou les agrandir ${ }^{716}$, il n'est toutefois pas fait mention du sort des planteurs réinstallés dans les ZAF. En effet, sur le plan juridique, les ZAF restent des parties intégrantes du Parc National de la Marahoué puisqu'elles ne sont pas déclassées. Curieusement, cette solution transitoire a perduré de 1988 à nos jours; et l'exploitation du Parc s'est poursuivie au fil des années.

\footnotetext{
${ }^{713}$ Sur ces différents points, voir AKOUÉ (Yao Claude), Les aspects conflictuels liés à la conservation des aires protégées de Côte d'Ivoire : le cas du Parc National de la Marahoué à Bouaflé, Mémoire de DEA en Sciences et Gestion de 1'Environnement option Politique en Environnement, Université d'Abobo-Adjamé, août 2004, p. 56.

${ }^{714}$ Il s'agit de la note circulaire ${ }^{\circ}$ 1075/MINEFOR/IGEF du 21 juin 1988 qui sert de fondement à cette nouvelle zonation du PNM effectuée par la SODEFOR. Cette note circulaire avait pour objet la « Réhabilitation des Forêts classées, Parcs Nationaux et Réserve » par la lutte contre l'aliénation de la forêt et la restauration de l'ensemble des massifs forestiers du domaine permanent de l'État.

${ }^{715}$ Il s'agit des Préfets et Sous-Préfets; des Directeurs Régionaux, Départementaux et aux Chefs de Cantonnements des Eaux et Forêts.

${ }^{716} \mathrm{Il}$ est à préciser que cette autorisation d'exploitation des plantations restées dans la zone dite protégée est subordonnée à un engagement des planteurs concernés. Cet engagement est formalisé par un contrat dit Contrat d'occupation temporaire dans le Parc National de la Marahoué. Voir AKOUÉ (Yao Claude), Les aspects conflictuels liés à la conservation des aires protégées de Côte d'Ivoire: le cas du Parc National de la Marahoué à Bouaflé, Mémoire de DEA en Sciences et Gestion de l'Environnement option Politique en Environnement, Université d'Abobo-Adjamé, août 2004, Annexe 2b.
} 
Les enquêtes que nous avons effectuées dans cette aire protégée ont permis de constater que ces ZAF concentrent de gros villages ou campements et des milliers d'habitants ainsi que leurs plantations. Une étude de l'UICN de 2008 les estimait à environ 20.000 âmes $^{717}$. Elles abritent également quatre (4) écoles primaires construites par les populations. Ces écoles recevaient des instituteurs affectés par l'État jusqu'en 2004, dans la mesure où ces écoles figurent sur la carte scolaire. Les ZAF ont bénéficié d'un projet d'hydraulique villageoise de la coopération ivoiro-japonaise, en 1998 et 1999. Les planteurs des ZAF bénéficient également des prestations du ministère de l'agriculture ${ }^{718}$. En somme, dans les ZAF, il est mené une vie, à l'image de celles de toutes les autres cités rurales de Côte d'Ivoire. Une étude encore plus récente réalisée sur l'état de conservation de certaines aires protégées dont le PNM montre que les exploitations agricoles dont cette aire protégée fait l'objet menace gravement son existence ${ }^{719}$.

Cette manière de procéder de l'autorité étatique ne pourrait-elle pas s'analyser comme un déclassement? Sans toutefois l'admettre comme telle de jure, nous l'admettons de facto, dans la mesure où cette situation dure depuis 27 ans. Par conséquent, l'exploitation à but agricole et à titre d'habitation dans le Parc National de la Marahoué est une forme d'infiltration qui est tolérée par l'État.

La tolérance de l'occupation des aires protégées par l'État ivoirien concerne également le Parc National de Taï (PNT).

\section{b. Le cas du Parc National de Taï}

Le Parc National de Taï est affranchi de tout droit d'usage des populations ${ }^{720}$. Or, il se trouve que les populations riveraines exploitent certaines parties de ce Parc à des fins agricoles, depuis $1977^{721}$. Au lieu de faire cesser cette exploitation, les gestionnaires du Parc

\footnotetext{
${ }^{717}$ Voir UICN/BRAO, Évaluation de l'efficacité de la gestion des aires protégées : Parcs et Réserves de Côte d'Ivoire, 2008, op. cit, p. 22.

${ }^{718}$ Pour ces différentes actions de l'État dans la zone agroforestière du Parc National de la Marahoué, voir AKOUÉ (Yao Claude), Les aspects conflictuels liés à la conservation des aires protégées de Côte d'Ivoire: le cas du Parc National de la Marahoué à Bouaflé, Mémoire de DEA en Sciences et Gestion de l'Environnement option Politique en Environnement, Université d'Abobo-Adjamé, août 2004, op. cit., pp. 48-50.

${ }^{719}$ Voir AKINDÈS (Francis), KOUAMÉ (Kra Walter), SÉDIA (Gisèle) et SEZAN (Jules), Réalisation d'une étude sur l'état de la biodiversité des Parcs Nationaux et Réserves de Côte d'Ivoire. Aspects socio-économiques (Rapport provisoire), op. cit. p. 44.

${ }^{720} C^{\prime}$ 'est ce que mentionnent les dispositions du Décret $n^{\circ} 72-544$ du 28 août 1972 portant création du Parc National de Taï (JORCI n43 du 14 septembre 1972, p. 1451). En effet, l'article 3 dudit décret dispose : «Dans le Parc National de Taï ainsi délimité y compris le lit des rivières, l'emprise des routes et pistes formant limites, tout acte de chasse, de poursuite de capture et toute provocation du gibier quelle qu'en soit la nature, sont interdits; de même tout abattage ou mutilation d'arbres ainsi que les feux de brousse. " (souligné par nous). L'article 4 suivant indique : "Toute action de pêche quelle qu'en soit la nature, dans les rivières et mares situées tant à l'intérieur qu'en limite du Parc National, est interdite. » (souligné par nous). Quant à l'article 5 du même décret, il prescrit : "La récolte de miel, de la cire, des plantes médicinales ou alimentaires est interdite. ».

${ }^{721}$ Voir République de Côte d'Ivoire, Ministère de l'Environnement et des Eaux et Forêts/Office Ivoirien des Parcs et Réserves, État de conservation des Parcs et Réserves de Côte d'Ivoire, op. cit., p. 12.
} 
l'ont plutôt légitimée sous l'appellation de zones d'occupation contrôlée (ZOC), ainsi l'atteste la figure ci-dessous.

Figure 5 : Zonage du Parc National de Taï

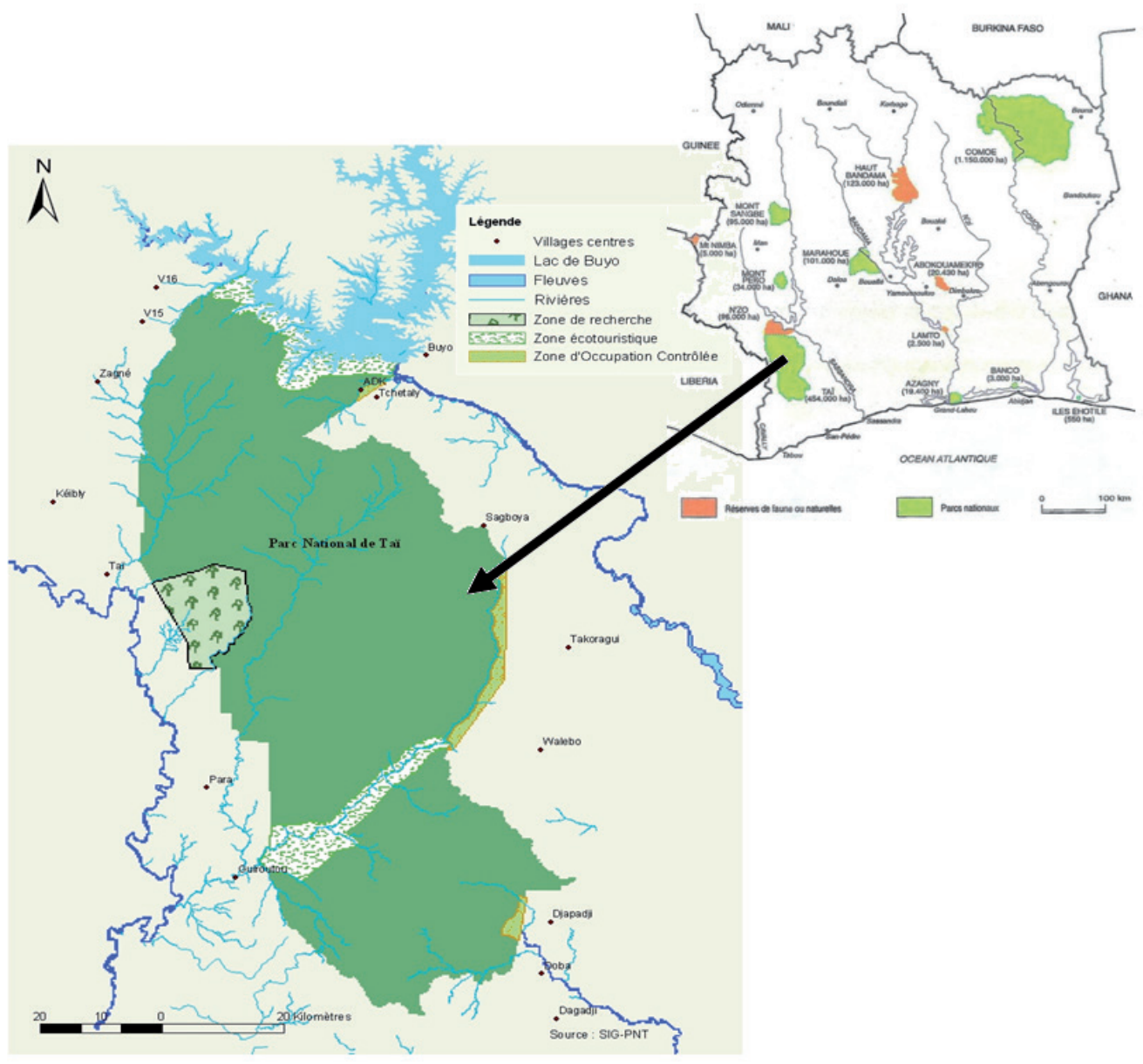

\section{Commentaires}

La figure ci-dessus relative au zonage du Parc National de Taï (PNT) présente, entres autres zones, trois (3) zones d'occupation contrôlée (ZOC). Elles se situent sur le côté Est du Parc. Au Nord-Est du PNT, l'on a une ZOC à proximité de la localité d'ADK. À l'Est, du 
PNT se trouve la plus grande des ZOC. Au Sud-Est, on peut localiser la troisième ZOC. L'ensemble de ces ZOC couvrirait une superficie d'environ $1,5 \%$ du $\mathrm{PNT}^{722}$.

À l'analyse, la justification qu'avancent les gestionnaires du Parc National de Taï pour la « création » d'une zone d'occupation contrôlée en son sein tire son fondement du fait qu'il est une réserve de biosphère ${ }^{723}$. Certes, les règles qui régissent cette catégorie d'aire de conservation peuvent admettre une zone d'exploitation réservée aux activités humaines. Ainsi, l'autorité de gestion du PNT c'est-à-dire l'OIPR justifie la présence de zones qui sont exploitées dans ledit Parc (dite zones d'occupation contrôlée), en raison de son statut de réserve de biosphère. C'est aussi en toute logique qu'il devrait exister de telles zones dans le Parc National de la Comoé (PNC) qui est également une réserve de biosphère infiltrée par les populations riveraines ${ }^{724}$. Et pourtant, contre toute attente, il n'y a pas été «crée » de zones d'occupation contrôlée (ZOC) dans le PNC. Par conséquent, il ne s'agit que d'un prétexte pour l'État de légitimer l'occupation du Parc National de Taï par les populations, en raison des difficultés que pose l'expulsion des populations en général.

Par ailleurs, le Parc National d'Azagny (PNA) n'échappe pas à ce phénomène d'occupation d'aires protégées légitimées par l'Etat ivoirien.

\section{c. Le cas du Parc National d'Azagny}

À la création du Parc National d'Azagny (PNA) en 1981, dans la zone centrale de protection et la zone périphérique existaient des plantations de café, cacao, palmiers à huile $\operatorname{etc}^{725}$. Un délai d'exploitation de trois (3) ans fut accordé aux paysans qui en étaient

\footnotetext{
${ }^{722}$ Voir UICN/BRAO, Évaluation de l'efficacité de la gestion des aires protégées : Parcs et Réserves de Côte d'Ivoire, 2008, op.cit., p. 22

${ }^{723}$ Une réserve de biosphère est un écosystème terrestre et/ou côtiers/marins crée dans le but de promouvoir des approches et des moyens qui réconcilient la conservation de la diversité biologique avec son utilisation durable. Les réserves de biosphère ne font pas l'objet d'une convention internationale. C'est un réseau de conservation de la nature initiée par l'Unesco. Elles sont régies par les lois des États sur lesquels elles sont situées ; avec un partage d'expérience entres les États membres du réseau et aussi avec l'Unesco. Chaque réserve de biosphère est organisée en trois (3) types de zones où se répartissent les objectifs de protection, d'entretien et de développement. Ainsi, dans le périmètre d'une réserve de biosphère, on a :

- des zones centrales destinées à une fonction de protection de la nature et devant être protégée par la législation nationale. Cette zone est classée aire protégée ;

- des zones tampons qui entourent ou juxtaposent les zones centrales. Dans ces zones (de développement durable), il est permis des activités de production qui sont compatibles les principes écologiques telles que la recherche scientifique, la récréation et l'éducation environnementale ;

- des zones externes de transition dans lesquels diverses activités anthropiques sont menées. Voir MANCÉRON (Stéphane), Intervenir en périphérie pour la conservation des aires protégées : réexamen du postulat. La situation du Parc $W$ et des éleveurs mobiles, Thèse Unique, Géographie, Université Paris Ouest Nanterre-La Défense, 7 octobre 2011, pp. 36-37.

${ }^{724}$ Le Parc National de la Comoé est Réserve de la Biosphère depuis 1983. Voir UNESCO/UICN, Rapport de mission, Suivi de l'état de la conservation du Parc National de la Comoé en Côte d'Ivoire, site du Patrimoine Mondial, 10-23 juin 2006, p. 5.

${ }^{725}$ Voir République de Côte d'Ivoire, Ministère de l'Environnement et des Eaux et Forêts/ Office Ivoirien des Parcs et Réserves, Plan d'aménagement et de gestion du Parc National d'Azagny, p. 15.
} 
propriétaires $^{726}$. Ils avaient été recasés dans la forêt classée du Gô partiellement déclassée ${ }^{727}$, à cet effet.

Cependant, à l'expiration de ce délai, ces paysans excipaient de problèmes d'installation pour plaider la prorogation du délai d'autorisation de récolter les plantations de la zone centrale de protection et de la zone périphérique du PNA. En effet, ils évoquaient des problèmes de production et de destructions de cultures par les éléphants ${ }^{728}$. Ce faisant, les exploitations agricoles se poursuivaient de fait dans la zone centrale de protection et dans la zone périphérique.

En 1997, intervient un arrêté ministériel qui autorise officiellement l'entretien des plantations dans ces zones. Les paysans en profitent pour agrandir leurs plantations ${ }^{729}$. Selon le Directeur de cette aire protégée, en 2006, la Direction Générale de l'OIPR a autorisé la récolte des plantations des cultures pérennes dans ces zones, suite à un homicide perpétré par un élément de la brigade mobile en patrouille de surveillance sur un "délinquant ${ }^{730 "}$. Cette autorisation qui fit l'objet d'un contrat mettait à la charge des paysans concernés, des obligations dont la principale consistait à récolter les plantations sans les entretenir, de 2006 à 2008. Ainsi, cette zone du PNA exploitée, nonobstant son statut qui l'affranchit de tout droit d'usage, était appelée «Zone d'Occupation Contrôlée » ${ }^{731}$.

En somme, la tolérance de l'occupation des aires protégées par l'État ivoirien ou les pouvoirs publics, en dépit des exigences légales en la matière, prend trois (3) formes. La première consiste en la délimitation de certaines parties de ces aires protégées, aux fins d'habitations des populations infiltrées et de la pratique de leurs activités agricoles. C'est le cas des Zones Agroforestières dans le Parc National de la Marahoué. La seconde résulte

\footnotetext{
${ }^{726}$ Voir KOFFI (Kouassi Yves), La gestion des zones humides d'importance internationale en Côte d'Ivoire et la mise en cuvre de la convention de Ramsar : cas du Parc National d'Azagny, Mémoire de $3^{\text {ème }}$ cycle en vue de l'obtention du Diplôme d'Etudes Supérieures Spécialisées en Conservation et Utilisation Durable des Zones Humides, UFR/SVT, Université de Ouagadougou, Février 2006, p. 22.

${ }^{727} \mathrm{La}$ forêt classée du Gô a été partiellement déclassée en 1982 et en 1987 aux fins de la réinstallation des planteurs qui étaient dans le PNA et sa zone tampon à son classement en 1981. En effet, il est successivement déclassé 2.150 ha (Arrêté n ${ }^{\circ}$ 63/MINEFOR/DGEF du 22/11/1982) et 2.500 ha (Décret $n^{\circ} 87-759$ du 22 juillet 1987 portant déclassement d'une partie de la forêt classée de Gô-Bodiénou [sous-préfecture de Grand-Lahou], JORCI n³2 du 20 août 1987, p. 310).

${ }^{728}$ Voir KOFFI (Kouassi Yves), La gestion des zones humides d'importance internationale en Côte d'Ivoire et la mise en ouvre de la convention de Ramsar : cas du Parc National d'Azagny, Mémoire de $3{ }^{\text {ème }}$ cycle en vue de l'obtention du Diplôme d'Etudes Supérieures Spécialisées en Conservation et Utilisation Durable des Zones Humides, op. cit. p. 22.

${ }^{729}$ Voir République de Côte d'Ivoire, Ministère de l'Environnement et des Eaux et Forêts/ Office Ivoirien des Parcs et Réserves, Plan d'aménagement et de gestion du parc national d'Azagny, Abidjan, 2008, (inédit), pp. 70 et 71 .

${ }^{730} \mathrm{Ce}$ dernier reçu une décharge une arme automatique.

${ }^{731}$ Toutefois, en sa session d'avril 2008, le Conseil de Gestion de 1'OIPR, par l'effet de sa Note de service $\mathrm{n}^{\circ} 56 / \mathrm{OIPR} / \mathrm{DZ} / \mathrm{S} / \mathrm{CE}$ du 15 octobre 2008, dénonce cette convention. Mieux, il recommande à la Direction Générale de l'OIPR qu'il en soit mis fin en raison de l'incompatibilité avec le statut de cette aire protégée. Ce faisant, depuis le 30 septembre 2008, date d'échéance des contrats d'autorisation d'exploitation dans le PNA, il n'existe plus officiellement de ZOC dans le PNA. Voir République de Côte d'Ivoire, Ministère de l'Environnement et des Eaux et Forêts/ Office Ivoirien des Parcs et Réserves, Plan d'aménagement et de gestion du Parc National d'Azagny, op.cit., pp. 76-77.
} 
d'une autorisation d'exploitation desdites aires protégées dans des zones délimitées à cet effet ou "zone d'occupation contrôlée (ZOC)". C'est le cas dans le Parc National de Taï. Enfin, la légitimation des occupations des aires protégées par l'État peut prendre la forme d'une prorogation sans cesse renouvelée des délais d'autorisation d'exploitation de plantations que les autorités publiques accordent aux populations. C'est le cas dans le Parc National d'Azagny et sa zone périphérique de protection.

Cette tergiversation des pouvoirs publics ivoiriens à régler durablement les problèmes d'occupations illégales d'aires protégées ivoiriennes pourrait s'expliquer essentiellement par la réalité de l'économie de la Côte d'Ivoire : elle repose sur l'agriculture. En témoigne la note circulaire du ministre Vincent Pierre LOKROU, précédemment présentée. En effet, bien que donnant instruction aux responsables d'Administration déconcentrée dont relève le Parc National de Bouaflé de prendre des mesures en vue de «...soustraire une plus grande superficie possible" de ce domaine de l'État aux agressions de toute sorte, cette note circulaire leur demandait curieusement de maintenir " intact le potentiel agricole productif généré dans ces domaines. " (souligné par nous). Les travaux du Professeur Michaël FAURE relatif à l'analyse économique du droit de l'environnement illustre de fort belle manière cette logique de l'État ivoirien dans laquelle les considérations économiques supplantent celles de conservation des aires protégées ${ }^{732}$.

Aussi le défaut d'effectivité du droit des aires protégées découle-t-il des limites des institutions d'exécution et de contrôle de la mise en œuvre du droit des aires protégées.

\section{SECTION II : Les limites des institutions d'exécution et de contrôle de la mise en ouvre du droit des aires protégées}

Les limites relatives aux institutions d'exécution et de contrôle seront analysées, selon qu'elles sont de nature structurelles (Paragraphe 1) ou conjoncturelles (Paragraphe 2).

\section{Paragraphe 1 : Les limites structurelles à la mise en ouvre du droit des aires protégées}

Est structurel, ce qui est déterminé par la structure ${ }^{733}$. Ainsi, les limites structurelles à la mise en œuvre du droit des aires protégées pourraient être définies comme celles relatives à l'organisation et au fonctionnement des structures ayant la charge de la mise en œuvre dudit droit. En l'espèce, ces limites sont relatives à la récurrence du défaut de détection des violations du droit des aires protégées (1) et à la réaction de l'OIPR en cas de violation dudit droit (2).

\footnotetext{
${ }^{732}$ En effet, selon FAURE (Michael), les réticences des pouvoirs publics à prendre des mesures efficaces pour faire cesser les atteintes à l'environnement pourraient s'expliquer par des raisons économiques qui sont privilégiées au détriment des considérations écologiques. Voir FAURE (Michael), L'analyse économique du droit de l'environnement, Bruylant/Bruxelles 2007, p. 32.

${ }^{733}$ Voir JEUGE-MAYNART (Isabelle) (dir.), Le Petit Larousse illustré, op. cit., p.1046.
} 


\section{A - La récurrence du défaut de détection des violations du droit des aires protégées}

Le droit des aires protégées a deux (2) volets : le volet prescriptif et le volet répressif. L'application des règles afférentes à ce second volet implique préalablement l'observation de celles relevant du volet prescriptif.

Cette relation de type causal qu'entretiennent les deux (2) volets de la norme juridique traduit l'importance de la détection de la violation de la norme juridique prescriptive. En effet, c'est la connaissance préalable de la transgression de celle-ci par l'autorité en charge de la répression qui déclenche ce processus.

Dans le cadre de la mise en œuvre du droit des aires protégées, les limites de l'OIPR découlent du manque de moyens matériels (matériels roulants, de patrouille...) et financiers. En effet, aux dires des gestionnaires rencontrées au cours de nos enquêtes de terrain, la faiblesse des budgets de fonctionnement et le manque de moyens matériels limitent considérablement les actions de surveillance. Ces insuffisances de financement et partant de moyens matériels de gestion ne permettent pas ainsi de constater les violations du droit des aires protégées, d'en rechercher les auteurs et de les punir.

À titre illustratif, les gestionnaires des aires protégées savent que, durant la saison sèche, la chasse est favorable; et connaît une recrudescence dans les aires protégées. Cependant, faute de moyens, ils ne peuvent mener des patrouilles ou autres activités de surveillance conséquentes qui permettent de constater les infractions telles que le braconnage ou d'empêcher leur commission.

En outre, même lorsqu'elle a l'information de telles violations, la réaction de l'Administration en charge des aires protégées connaît des fortunes diverses.

\section{B - La réaction inadéquate de l'Administration en charge des aires protégées en cas d'infraction au droit des aires protégées}

Lorsqu'elle est informée de la commission d'une infraction contre les aires protégées, l'Administration en charge des aires protégées se doit de réagir, en déclenchant le processus de répression des auteurs. Cependant, il ressort de nos enquêtes auprès des gestionnaires que, bien souvent, ils sont limités par deux (2) difficultés majeures.

D’une part, le manque de moyens matériels et financiers les empêche de réagir. Cette situation est aggravée lorsqu'elle est consécutive à un cas de conflit (rébellion) antérieure qui a fait des morts ou des blessés du côté des populations. En pareil cas, au-delà des difficultés liées au manque de moyens, les gestionnaires ne peuvent avoir accès à ces zones car ils y sont déclarés ennemis. Ce faisant, les populations violent à loisir les aires protégées ; manifestant ainsi leur indignation ou leur revanche. 
D'autre part, les interférences récurrentes de diverses autorités dans le règlement des litiges limitent la réaction de l'Administration en charge des aires protégées, en cas violation $\mathrm{du}$ droit des aires protégées. Selon nos enquêtes, les autorités locales qu'elles soient villageoises (rois, chefs de villages), administratives et les cadres utilisés souvent comme interlocuteurs entre l'Administration et les populations interviennent à l'occasion de ces litiges qui, en principe, doivent connaître un règlement judiciaire ou administratif. Dans ce dernier cas, la voie transactionnelle étant autorisée, les interférences des autorités sont plus considérables, par rapport à celles relatives au règlement judiciaire. Dans tous les cas, ces autorités plaident, en général, en faveur de la relaxe des prévenus, pour des raisons d'apaisement du climat social dans les localités concernées.

Dans la pratique, ces interventions entraînent un fléchissement de l'Administration en charge des aires protégées et l'on aboutit à un effritement de l'autorité des agents. Les populations jouent malheureusement sur ces différents registres pour violer la législation des aires protégées. Qu'en est-il des limites conjoncturelles à la mise en œuvre du droit des aires protégées?

\section{Paragraphe 2 : Les limites conjoncturelles à la mise en œuvre du droit des aires protégées}

La conjoncture est une situation qui résulte d'un concours de circonstances ${ }^{734}$. Les limites conjoncturelles du droit des aires protégées seront analysées sous deux (2) angles. D'une part, les limites conjoncturelles à la mise en œuvre du droit des aires protégées seront analysées, à travers le retard dans la prise des textes réglementaires (A). D'autre part, ces limites seront abordées, à travers le retard qu'accuse la mise en place et le fonctionnement des instruments et organes de gestion prévus par la loi (B) et celles relatives au conflit militaro-politique survenu en Côte d'Ivoire en septembre 2002 (C) .

\section{A - Le retard dans la prise des textes réglementaires}

Les textes réglementaires sont si importants que leur inexistence paralyse l'activité juridique et compromet l'effectivité du droit. L'inexistence de textes réglementaires ne permet pas de préciser les conditions et modalités relatives à l'application des dispositions de la loi. En l'espèce, le retard dans la prise de textes réglementaires prévus par la loi de 2002 sur les PNR est une limite conjoncturelle qui entrave fondamentalement la mise en œuvre du droit des aires protégées.

Epine dorsale du droit des aires protégées, la loi de 2002 sur les PNR a prévu plusieurs décrets en vue de sa mise en œuvre. Toutefois, la prise de certains de ces décrets s'est faite avec beaucoup de retard. Il s'agit du décret relatif à la procédure de classement des Parcs et

\footnotetext{
${ }^{734}$ Voir JEUGE-MAYNART (Isabelle) (dir.), Le Petit Larousse illustré, édition 2013, op. cit. p. 253.
} 
Réserves $^{735}$ ainsi que de celui relatif à l'habilitation des officiers de Police judiciaire de l'Office Ivoirien des Parcs et Réserves ${ }^{736}$.

Le décret relatif aux procédures de classement de Parcs et Réserves est pris en 2012, soit dix (10) ans après l'adoption de la loi de 2002 sur les PNR qui le prévoit. Or, en 2007, il a été « créé » la Réserve naturelle partielle de Dahliafleur ${ }^{737}$. Alors, nous nous interrogeons, à plus d'un titre.

Sur quelle base légale a-t-on érigée Dalhiafleur en aire protégée ? Est-ce sur la base du décret de 1966 relatif à la procédure de classement des Parcs et Réserves ? Cette aire protégée n'a-t-elle pas été classée, au mépris de l'article 6 alinéa 2 de la loi de 2002 sur les PNR qui prévoit un nouveau décret de classement des aires protégées ${ }^{738}$ ?

Le classement du site Dalhiafleur (à l'origine horticole) en Réserve naturelle du même nom, en 2007, est dépourvu de base légale. En effet, ce classement n'a pu raisonnablement se faire sur la base du décret de 1966 relatif à la procédure de classement des Parcs et Réserves, en raison de l'abrogation de ses dispositions par la loi de 2002 sur les PNR. Ce classement ne s'est non plus opéré sur le fondement du nouveau décret de classement des Parcs et Réserves prévu par la loi de 2002 sur les PNR qui n'était pas encore pris ${ }^{739}$. Le classement de la Réserve naturelle de Dalhiafleur s'est donc fait en violation du droit des aires protégées. En effet, il s'était créé un vide juridique en la matière.

Nous comprenons alors aisément que le rapport de l'OIPR de 2009 (le dernier en date) sur l'état de la conservation des aires protégées fasse état de problèmes de gestion en ce qui concerne la Réserve de Dalhiafleur. Ce sont, entre autres, les défrichements à but agricole, les grignotages de limites, les exploitations de produits secondaires et la non matérialisation des limites et surtout les contentieux sur le foncier ${ }^{740}$.

\footnotetext{
${ }^{735}$ Voir Décret n²012-163 du 9 février 2012 déterminant les procédures de classement des Parcs Nationaux et Réserves naturelles, JORCI n ${ }^{\circ} 7$ du 16 février 2012, p. 93.

${ }^{736}$ Voir Décret $n^{\circ}$ 2013-22 du 9 janvier 2013 portant modalités d'habilitation, en qualité d'officiers de Police Judiciaire, des fonctionnaires et agents des Parcs Nationaux, Réserves naturelles et des Eaux et Forêts, JORCI $\mathrm{n}^{\circ} 13$ du jeudi 28 mars 2013, p. 226.

${ }^{737}$ Le rapport sur l'état de la conservation des Parcs et Réserves de Côte d'Ivoire de 2009 indique: «Anciennement domaine horticole concédé à des expatriés Européens, la Réserve naturelle de Dahliafleur est déclarée domaine d'utilité publique par le décret $n^{\circ} 2004-566$ du 14 octobre 2014 puis érigée en Réserve naturelle partielle par l'arrêté $n^{\circ} 00895 / M I N E F / d u 17$ octobre 2007 » (souligné par nous). Voir. Ministère de l'Environnement, des Eaux et Forêts/Office Ivoirien des Parcs et Réserves, État de conservation des parcs et réserves de Côte d'Ivoire, op. cit.,p. 8.

${ }^{738}$ En effet, les articles 6 alinéa 2 et 78 de la loi de 2002 sur les PNR disposent respectivement : «les procédures de classement de nouveaux parcs et réserves seront déterminées par décret " et : "la présente loi relative à la création, à la gestion et au financement des Parcs Nationaux et Réserves naturelles abroge toutes les dispositions contraires ». Autrement dit, cette loi abroge le décret précité de 1966 relatif à la procédure de classement des Parcs et Réserves.

${ }^{739} \mathrm{C}$ 'est certainement pour corriger cette insuffisance qu'il est encore pris, en 2013, un autre texte de création de la Réserve naturelle de Dalhiafleur. Il s'agit du Décret 2013-127 du 20 février 2013 portant création de la Réserve naturelle de Dalhiafleur (JORCI n 18 du Jeudi 2 mai 2013).

${ }^{740}$ Voir Ministère de l'Environnement, des Eaux et Forêts/Office Ivoirien des Parcs et Réserves, État de conservation des Parcs et Réserves de Côte d'Ivoire, 2009, p. 21.
} 
Ces différents problèmes de gestion ne se poseraient pas, moins de deux (2) ans après le classement de cette "Réserve, si ce classement avait préalablement fait l'objet d'une procédure définie selon les standards requis; et qui assurément aurait retenu la consultation des populations. En l'espèce, l'ineffectivité du droit des aires protégées découle de la violation de celui-ci par l'Administration (la tutelle) elle-même.

En outre, elle émane de la nature de l'acte juridique qui classe la Réserve naturelle partielle de Dahliafleur. En effet, alors que l'article 13 de la loi de 2002 sur les PNR dispose que les Réserves naturelles partielles sont classées par décret pris en Conseil des ministres, la Réserve naturelle partielle de Dahliafleur est classée par arrêté ${ }^{741}$.

À l'analyse, cette seconde source d'illégalité de l'action administrative a créé une insécurité juridique. En effet, la "Réserve naturelle partielle Dalhiafleur" n'en était pas juridiquement une, au sens de la loi de 2002 sur les PNR et comme le concevait l'Administration, avant la régularisation de cette situation en $2013^{742}$.

C'est ainsi qu'en cas de litige relatif à cette aire protégée, tout demandeur ou défendeur, selon que l'initiative du procès émane de l'OIPR ou non, aurait pu facilement exciper de ce défaut de base légale pour éviter qu'on lui applique les dispositions de la loi de 2002 sur les PNR. Il se serait fondé sur l'illégalité qui entacha le statut de la Réserve naturelle partielle Dahliafleur. Le droit des aires protégées qui a été pourtant renforcé, en vue de la protection de ces milieux naturels n'aurait donc pas pu s'appliquer, à cause d'un vice de procédure grave et flagrant.

En ce qui concerne le décret relatif aux modalités d'habilitation des officiers de Police judiciaire (OPJ) de l'OIPR, il a été pris avec un retard de plus de 13 ans alors que les infractions contre les aires protégées ont continué d'être commises ${ }^{743}$. Dans ces conditions, l'efficacité de la répression qui a amené le législateur à conférer la qualité d'OPJ à certains membres du personnel de l'OIPR n'a été pas effective de 2002 à 2013.

\footnotetext{
${ }^{741}$ De même, cet arrêté de création de la Réserve naturelle de Dahliafleur (Arrêté n ${ }^{\circ} 00895 / \mathrm{MINEF} / \mathrm{du} 17$ octobre 2007) viole l'alinéa 2 de l'article 14 suivant. En effet, cet article mentionne : «Les conditions et modalités spécifiques et de gestion d'une réserve naturelle partielle, notamment l'exercice de la chasse, la capture des animaux, la collecte des végétaux, l'observation de la faune et de la flore par l'édification de bâtiments et les travaux d'aménagement, sont définies par le décret de classement ».

${ }^{742}$ L'irrégularité qui a entaché la «création » de la Réserve naturelle de Dahliafleur est corrigée en 2013 par l'autorité administrative. En effet, cette Réserve supposée créée en 2007 par un arrêté (Arrêté n00895/MINEF/du 17 octobre 2007) est de nouveau créée par le décret n²013-127 du 20 février 2013 portant création de la Réserve naturelle de Dahliafleur, JORCI n ${ }^{\circ} 18$ du jeudi 2 mai 201, p. 302.

${ }^{743}$ En effet, prévu par l'article 38 de la loi de 2002 sur les PNR, le décret relatif aux modalités d'habilitation des OPJ de l'OIPR est pris en 2013. Il s'agit du Décret $n^{\circ} 2013-22$ du 9 janvier 2013 portant modalités d'habilitation, en qualité d'officiers de Police Judiciaire, des fonctionnaires et agents des Parcs nationaux, Réserves naturelles et des Eaux et Forêts (JORCI n 13 du jeudi 28 mars 2013, p. 226).
} 
$\mathrm{Au}$ total, le retard dans la prise des instruments réglementaires prévus par la loi précitée de 2002 a constitué un frein majeur à l'effectivité du droit des aires protégées, en Côte d'Ivoire. Il en est de même pour les instruments et organes de gestion prévus par cette loi.

\section{B - Le retard dans la mise en place et le fonctionnement des instruments et organes de gestion prévus par la loi de 2002 sur les PNR}

Nous traiterons successivement $\mathrm{du}$ retard dans la mise en place des plans d'aménagement et de gestion (PAG) et des organes de gestion des aires protégées.

\section{Le retard dans la conception et l'application des plans d'aménagement et de gestion d'aire protégée}

Le plan d'aménagement et de gestion d'aire protégée est un outil important et indispensable dans la gestion des aires protégées. C'est la raison pour laquelle le législateur prescrit à l'OIPR d'en établir pour chaque Parc et Réserve ${ }^{744}$.

Cependant, force est de constater que la plupart des aires protégées ne dispose pas de plan d'aménagement et de gestion; ou si tel est le cas, il n'est pas mis en œuvre ${ }^{745}$. En effet, sur un total de quatorze (14) aires protégées gérées par l'OIPR, seulement cinq (5) possèdent chacune un plan d'aménagement et de gestion. Il s'agit des Parcs Nationaux du Banco, de la Comoé, de la Marahoué, de Taï et de la Réserve scientifique de Lamto ${ }^{746}$.

Toutefois, ces plans d'aménagement et de gestion sont caractérisés par un défaut de mesures destinées au développement des communautés locales ${ }^{747}$. Par conséquent, la conservation des Parcs et Réserves pour lesquels ils sont élaborés ne peut être que compromise. En effet, le défaut de mesures riveraines caractéristiques de ces PAG favorisera le non-respect du droit des aires protégées ${ }^{748}$.

\footnotetext{
${ }^{744}$ Art. 30 alinéa $1^{\text {er }}$, loi de 2002 sur les PNR.

${ }^{745}$ Hormis celui du Parc National de Taï.

${ }^{746}$ Voir UICN/BRAO, Évaluation de l'efficacité de la gestion des aires protégées : Parcs et Réserves de Côte d'Ivoire, 2008, op.cit., p.38.

${ }^{747}$ Idem, p. 39.

${ }^{748}$ En effet, plusieurs études et travaux de recherches menées à partir des expériences de gestion d'aires protégées en Afrique démontrent que les populations sont hostiles à la conservation des aires protégées lorsqu'elles ne tirent pas profit de leur conservation. Sur ce point, voir KRUG (Wolf), Private Supply of Protected Land in Southern Africa : A Review of Markets, Approaches, Barriers and Issues, op. cit., p. 35 ; STOLL-KLEEMANN (Suzanne), O'RIORDAN (Timothy) «From Participation to Partnership in Biodiversity Protection : Experience from Germany and South Africa ", art. cit., p. 161 ; AFD, Gestion durable de la biodiversité, coll. Parole d'acteurs, n5, 2005, p. 24 ; TESSEMA (Mekbeb E.), ASHENAFI (Zelealem T.), LILIEHOLM (Robert J.) et LEADER-WILlIAMS (Nigel), " Community Attitudes towards Wildlife Conservation in Ethiopia», Proceedings of the 2007 George Wright Society Conference, p. 290; MBAIWA (Joseph E.), STRONZA (Amanda L.), « Changes in resident attitudes towards tourism development and conservation in the Okavango Delta », Journal of Environmental Management, Vol. 92, n 8, 2011, p. 1950 ; ANDRADE (Gustavo S. M.) et RHODES (Jonathan R.), " Protected areas and local communities: an inevitable partnership toward successful conservation strategies ? », art. cit., p. 15 ; TOMIĆEVIĆ (Jelena), BJEDOV
} 
Au surplus, la violation du droit des aires protégées par l'OIPR découle de ce qu'il gère les neuf (9) autres aires protégées, sans plan d'aménagement et de gestion. Pourtant, c'est une obligation légale qui lui incombe.

Outre le retard qu'accusent la mise en place et la mise en œuvre des plans d'aménagement et de gestion d'aires protégées, la mise en place et le fonctionnement de certains organes de mise en œuvre du droit des aires protégées tardent également.

\section{Le retard dans la mise en place et le fonctionnement de certains organes de gestion des aires protégées}

Les organes de gestion des aires protégées dont la mise en place tarde ou a tardé depuis plus d'une décennie, sont le Conseil scientifique de l'OIPR (a) et les comités de gestion locale des PNR (b).

\section{a. Le retard dans la mise en place du Conseil scientifique de l'OIPR}

Le Conseil scientifique de l'OIPR est un organe essentiel de cet Établissement public national (EPN), en raison de ses missions ${ }^{749}$. Cette assertion est d'autant plus justifiée dans la mesure où dans le droit ivoirien des établissements publics nationaux, tous les EPN ne sont pas dotés d'un Conseil scientifique. En effet, l'article 13 de la loi précitée de 1998 sur les

(Ivana), SHANNON (Margaret A.) et OBRATOV-PETKOVIĆ (Dragica), «Understanding Linkages Between Public Participation and Management of Protected Areas - Case Study of Serbia » in Dr. ISHWARAN (Natarajan) (eds.), The Biosphere, 2012, Intech, pp. 140-141; ADELEKE (Bola Olusola), NZAMA (Thandi), « Assessment of Community Participation in Ecotourism and Conservation at Hhuhuwe-Umfolozi Park, South Africa ", Journal of Environment and Earth Science, Vol. 3, n³, 2013, p. 30 ; SZELL (Andrea B.), HALLETT (Lucius F.), «Attitudes and Perceptions of Local Residents and Tourists toward the Protected Area of Retezat National Park, Romania», International Journal of Humanities and Social Science, Vol. 3, n³; 2013, pp. 23-24. Également, en la matière, pour des résultats d'expériences pratiques de gestion de ressources naturelles y compris les aires protégées réalisées en Afrique de l'Est et en Afrique australe, voir des développements afférents à la deuxième partie de la présente étude, le titre II, le Chapitre I, la Section I, le Paragraphe 1 et précisément le point "B- La justification de l'interdépendance fondée sur des expériences de gestion de ressources naturelles".

${ }^{749}$ En effet, aux termes de l'article 37 de la loi précitée de 2002 sur les PNR : «L'avis du Conseil scientifique est requis:

1 Sur toute question, projet et programme ayant une incidence sur le statut des ressources et richesses de la diversité biologique des Parcs et Réserves ainsi que sur leur consistance physique;

2 Sur la pertinence des plans d'aménagement et de gestion des Parcs et Réserves en général, ainsi que de tout projet d'investissement et d'une infrastructure même situé hors d'un Parc ou d'une Réserve mais susceptible d'avoir une incidence sur sa conservation;

3 Sur toute question liée à l'introduction de nouvelles espèces de faune et de flore, au recensement des ressources naturelles et à leur conservation dans les Parcs et Réserves ainsi que leurs zones périphériques ; ${ }^{\circ}$

4 Sur la pertinence et la méthodologie des programmes de recherche scientifique effectués dans les Parcs et Réserves ou leurs zones périphériques;

5 Sur tout projet de législation ou de réglementation pouvant avoir une incidence sur la conservation de la nature et de ses ressources ainsi que sur la diversité biologique et les équilibres biologiques en Côte d'Ivoire ;

6 Sur tout projet de loi de classement ou de déclassement d'un Parc ou d'une Réserve... » (souligné par nous). 
EPN donne aux établissements publics nationaux la faculté d'en disposer ${ }^{750}$, en raison des missions de nature d'enseignement, hospitalière, technologique et scientifique. Ainsi, si l'OIPR est doté d'un Conseil scientifique, on pourrait conclure que cet établissement public national a une mission scientifique, entre autres. Qu'en est-il exactement?

Il résulte des dispositions de l'article 3 de la loi de 2002 sur les PNR qu'elle poursuit, entre autres, des objectifs essentiels que sont la conservation de la diversité biologique des aires protégées et le bien-être des populations ${ }^{751}$. C'est à juste titre que faisant allusion à l'article 3 sus-cité, l'article 34 de la loi de 2002 sur les PNR indique : "La réalisation des objectifs définis à l'article 3 ci-dessus doit également permettre le développement de la recherche scientifique répondant aux besoins de la conservation des ressources naturelles, l'exploitation des résultats de cette recherche, qu'elle soit nationale ou internationale, faite sur le territoire nationale ou dans un autre pays, et la diffusion nationale et internationale des résultats de cette recherche scientifique. » (souligné par nous). Par conséquent, l'OIPR qui a la gestion des aires protégées en Côte d'Ivoire a une mission de nature scientifique. Cela est d'autant plus vrai que l'article 37 de la loi précitée de 2002 sur les PNR dispose, entre autres, que le Conseil scientifique de l'OIPR donne son avis sur : «... Sur la pertinence et la méthodologie des programmes de recherche scientifique effectués dans les Parcs et Réserves ou leurs zones périphériques...» (souligné par nous).

C'est donc en toute logique et en application de l'article 13 de la loi précitée sur les EPN que selon l'article 35 de la loi de 2002 sur les PNR il est : «créé un Conseil scientifique des Parcs et Réserves ». Ce conseil : «...est un organe consultatif de l'Établissement » c'està-dire l'OIPR.

Au regard des dispositions de l'article 37 de la loi 2002 sur les PNR précédemment cités, les attributions de consultation du Conseil scientifique sont d'une importance capitale pour la conservation durable des aires protégées. En effet, d'une part, le Conseil scientifique donne son avis sur toutes les questions relevant de ses compétences ${ }^{752}$ de sorte à permettre au Conseil de gestion de l'OIPR -instance administrative de décisions- d'accomplir sa mission.

\footnotetext{
${ }^{750}$ Cet article dispose : «Les Établissements publics nationaux, qui ont pour objet l'exécution de missions de nature, selon les cas, scientifique, technologique, hospitalière ou d'enseignement peuvent être dotés d'un conseil scientifique chargé de définir, avec le directeur et le conseil de gestion:

- l'orientation générale des activités spécifique de l'Établissement;

- l'élaboration et la mise en æuvre de programmes de recherche ou des objectifs techniques et scientifiques qui lui sont confiés.

La composition et le fonctionnement du conseil scientifique sont fixés par le décret de création de chaque Établissement concerné. ».

${ }^{751}$ L'article 3 de la loi de 2002 sur les PNR dispose : "Les parcs et réserves sont créés et gérés aux fins de permettre la conservation des milieux naturels, de la faune et de la flore terrestres et aquatiques, ainsi que le maintien de la diversité et des processus écologiques contre toutes les causes de dégradation qui les menacent. Ils ont également pour vocation de participer, par la récréation et l'éducation du public, à l'équilibre harmonieux des populations, qu'elles soient rurales ou urbaines. ».

${ }^{752}$ Art 37, loi de 2002 sur les PNR.
} 
D'autre part, dans sa mission de gestion quotidienne des aires protégées, la Direction Générale de l'OIPR requiert l'avis du Conseil scientifique, ainsi que le dispose la loi ${ }^{753}$.

Cependant, aussi important que soit cet organe pour la conservation durable des aires protégées, nous constatons qu'il n'est pas encore mis en place. Or, en principe, l'OIPR ne peut gérer les aires protégées sans l'apport du Conseil scientifique. Il s'agit d'un cas de violation de la loi de 2002 sur les PNR. En effet, faute d'existence du Conseil scientifique, le Conseil de gestion prend seul les décisions pour lesquelles il est, en principe, astreint par la loi précitée de 2002 sur les PNR à entendre l'avis préalable dudit conseil ${ }^{754}$. Il en est de même pour la Direction Générale de l'OIPR qui gère les aires protégées au quotidien ; mais sans l'avis du Conseil scientifique.

Par conséquent, dans le présent cas, le défaut d'effectivité du droit des aires protégées résulte du fait que le Conseil scientifique n'ait pas encore été mis en place depuis l'adoption de la loi de 2002 sur les PNR qui le prévoit. Qu'en est-il du retard dans la mise en place des comités de gestion locale dans les Parcs et Réserves?

\footnotetext{
${ }^{753}$ L'article 12 du décret de 2002 portant création de l'OIPR indique : «Après avis conforme du Conseil scientifique, le Directeur Général :

- transmet au ministre de tutelle technique les propositions de la délimitation de la zone périphérique d'un parc ou d'une réserve sur la base des négociations avec les entités ou communautés concernées et des indications du Directeur du parc ou de la réserve concernée et des partenaires au développement ;

- propose au ministre de tutelle technique la classification en réserve naturelle partielle et fixe les conditions d'exploitation, d'aménagement et de gestion de ladite réserve;

- autorise les activités à l'intérieur de la Réserve ayant une incidence sur la conservation durable de l'écosystème ainsi que toute recherche scientifique entreprise dans la Réserve ;

- soumet au ministre de tutelle technique des propositions de modifications des listes d'espèces protégées de faune et de flore terrestres et aquatiques dans les parcs nationaux; les réserves naturelles et les zones périphériques;

- autorise le survol des parcs et réserves à une altitude inférieure à 200 mètres dans le cadre des activités de recherche scientifique nonobstant la réglementation de l'aviation civile. ».

${ }^{754}$ Aux termes de l'article 37 de la loi de 2002 sur les PNR : «L'avis du Conseil scientifique est requis :

1. sur toute question, projet et programme ayant une incidence sur le statut des ressources et richesses de la diversité biologique des parcs et réserves ainsi que sur leur consistance physique ,

2. sur la pertinence des plans d'aménagement et de gestion des parcs et réserves en général, ainsi que sur tout projet d'investissement et d'infrastructure même situé hors d'un parc ou d'une réserve mais susceptible d'avoir une incidence sur sa conservation ;

3. sur toute question liée à l'introduction de nouvelles espèces de faune et de flore, au recensement des ressources naturelles et à leur conservation dans les parcs et réserves ainsi que leurs zones périphériques ;

4. sur la pertinence et la méthodologie des programmes de recherche scientifique effectués dans les parcs et réserves ou leurs zones périphériques.

5. sur tout projet de législation ou de réglementation pouvant avoir une incidence sur la conservation de la nature et de ses ressources ainsi que sur la diversité et les équilibres biologiques en Côte d'Ivoire ;

6. sur tout projet de loi de classement ou de déclassement d'un parc ou d'une réserve.

Le décret mentionné à l'article 35 ci-dessus précise les matières pour lesquelles il est requis, selon le cas, un avis simple ou un avis conforme du Conseil scientifique.

Le Conseil scientifique examine les rapports annuels sur l'état de conservation des parcs et réserves et publie un rapport annuel contenant ses observations et recommandations. ».
} 


\section{b. Le retard dans la mise en place et le fonctionnement des comités de gestion locale d'aire protégée}

La gestion unilatérale des aires protégées par l'Administration en charge de ces forêts a été identifiée comme l'une des causes principales du non-respect du droit des aires protégées, en Côte d'Ivoire; et par contrecoup de leur dégradation ${ }^{755}$. C'est pour pallier cette insuffisance que le droit des aires protégées institue le comité de gestion locale (CGL) d'aire protégée. En effet, aux termes de l'article 3 du décret de 2002 portant création de l'OIPR, chaque Parc et Réserve est doté d'un comité de gestion locale.

La composition diversifiée ${ }^{756}$ du CGL répond au souci de la conservation durable des aires protégées, sur la base de décisions ou de propositions concertées, à l'échelle locale. Par conséquent, la concertation au niveau local revêt un caractère indispensable, en matière de gestion des aires protégées. En d'autres termes, l'effectivité du droit des aires protégées en son volet prescriptif est fortement influencée par l'accomplissement de la mission dévolue au CGL d'aire protégée.

Elle est un gage de l'application du droit des aires protégées, en vue de la réalisation de cet objectif. En effet, la mise en place du CGL et son fonctionnement effectif permet de jouer un rôle a priori ou a posteriori, dans la mise en œuvre du droit des aires protégées.

A priori, les décisions prises, après concertation, par les membres du CGL d'une aire protégée constituent une garantie pour le respect du droit des aires protégées. En effet, les différents membres de ce comité représentent les acteurs dont le défaut de la prise en compte des intérêts divers pourrait compromettre la sauvegarde des aires protégées. A posteriori, les difficultés de mise en œuvre du droit des aires protégées peuvent être immédiatement diagnostiquées avec précision, en vue de les purger; et de permettre, par contrecoup, son respect par les différents acteurs de l'espace PNR.

\footnotetext{
${ }^{755}$ Sur la question, voir la Première partie de cette étude, le Titre I, le Chapitre II, la Section I, le "Paragraphe 2 : Les limites des pouvoirs du personnel en charge des aires protégées".

${ }^{756}$ Aux termes de l'article 36 du décret précité portant création de l'OIPR : « La composition et les modalités de fonctionnement de chaque Comité de Gestion locale sont fixées par arrêté conjoint du Ministre chargé des Parcs et Réserves et du Ministre chargé de l'Intérieur, de la Décentralisation et des Collectivités Territoriales, dans la limite de 15 membres au maximum dont notamment:

- les Préfets des Départements ou des Régions concernées ;

- le Directeur du parc ou de la réserve;

-deux représentants élus des organisations non gouvernementales intervenant dans le parc ou la réserve concernée, ou dans sa zone périphérique de protection ;

- les représentants des collectivités territoriales et des terroirs constituant la zone périphérique de protection (Présidents des Conseils Régionaux, Présidents des Conseils Généraux, Représentants des Organisations Villageoises ...) ;

- deux représentants des opérateurs économiques dans le secteur du tourisme et de l'artisanat;

- le Directeur Général de l'Office ou son représentant;

- un membre du Conseil Scientifique. ».
} 
$\mathrm{Au}$ vu des développements qui précèdent, le CGL d'aire protégée est si important pour la conservation durable des aires protégées que leur gestion ne peut être envisagée sans ce comité. Et pourtant, la création et la mise en place de CGL, pour les aires protégées qui en possède, s'est faite avec du retard. En effet, après l'adoption de la loi de 2002 sur les PNR, ce n'est qu'en 2006 qu'il intervient un arrêté conjoint du ministre de l'Environnement et de celui de l'Intérieur qui organise la composition, les attributions et le fonctionnement des $\mathrm{CGL}^{757}$.

En 2009, au moment de la réalisation de l'enquête de terrain effectuée dans le cadre de la présente étude, soit 7 ans après l'adoption de la loi de 2002 sur les PNR qui prévoit les CGL, sur les 14 aires protégées, seulement le Parc National de Taï avait un CGL créé, mise en place et fonctionnel. Trois (3) CGL d'aires protégées (Marahoué, Lamto, Abokouamékro) ${ }^{758}$ avait été (juridiquement) créés mais n'étaient pas mis en place. Les Parcs Nationaux du Banco, d'Azagny, des Îles Ehotilé, du Mont Péko avait leur arrêté de création en cours de rédaction.

Les CGL des six (6) autres aires protégées, à savoir les Parcs Nationaux de la Comoé, du Mont Sangbé, la Réserve naturelle intégrale du Mont Nimba, et les Réserves naturelles partielles, du N'zo, de Lamto, du Haut-Bandama et de Dahliafleur n'étaient pas en voie d'être crées. Plus récemment, au cours d'un entretien ${ }^{759}$, le Directeur technique de l'OIPR nous a révélé que sur les 14 aires protégées, 12 sont dotés de CGL; lesquels fonctionnent normalement. Autrement dit, seule la Réserve du Haut-Bandama n'en dispose pas encore, 13 ans après l'adoption de la loi de 2002 sur les $\mathrm{PNR}^{760}$.

Des développements qui précèdent, nous pouvons retenir que la mise en place et le fonctionnement d'organes tels que le Conseil scientifique et les comités de gestion locale des PNR accusent un retard. Cette situation a pour inconvénient d'entraver la mise en œuvre du droit des aires protégées.

\footnotetext{
${ }^{757}$ Il s'agit de l'Arrêté n ${ }^{\circ} 00714$ du 06 septembre 2006 portant composition, attributions et fonctionnement des comités de gestion locale des Parcs Nationaux et Réserves naturelles de Côte d'Ivoire.

${ }^{758}$ Par exemple, ce n'est que le 8 novembre 2006 que le Préfet de la Région des Lacs (République de Côte d'Ivoire) a pris un arrêté (Arrêté n ${ }^{\circ} 70 / \mathrm{PY} / \mathrm{CAB}$ portant composition, attributions et fonctionnement du Comité de gestion locale de la Réserve de Faune d'Abokouamékro. Or, dans la procédure de création d'un CGL, le Préfet en charge du département sur le territoire duquel se trouve une aire protégée désigne les membres du Comité conformément à la loi ; et par la suite le ministre en charge des aires protégées prend un arrêté relatif à la création et aux modalités de fonctionnement du CGL. Cette procédure est déduite de la lettre $\left(\mathrm{n}^{\circ} 0830 /\right.$ MINEME/OIPR du 13 juillet 2005) adressée par le ministre Angèle GNONSOA au Préfet du département de Grand-Lahou (sur le territoire duquel se trouve le Parc National d'Azagny) et ayant pour objet la mise en place du comité de gestion locale du Parc National d'Azagny.

${ }^{759}$ Cet entretien que nous a accordé le Directeur technique de l'OIPR (M. N'GORAN DJE), s'est réalisé le jeudi 11 décembre 2014, au siège de l'OIPR (Abidjan, République de Côte d'Ivoire).

${ }^{760}$ Cette situation pourrait se comprendre. En effet, depuis sa création en 1973, cette Réserve n'a jamais été administrée ni disposer d'infrastructure. Voir République de Côte d'Ivoire, Ministère de l'Environnement et des Eaux et Forêts/Office Ivoirien des Parcs et Réserves, État de conservation des Parcs et Réserves de Côte d'Ivoire, op. cit., p. 9.

Par ailleurs, il est à noter que si la Réserve du N'Zo ne dispose pas de comité de gestion locale, c'est parce que dans la pratique (et en attendant la consécration légale), elle a été fusionnée avec le Parc National de Taï auquel elle est contiguë, pour une efficacité de gestion.
} 
Au surplus, en Côte d'Ivoire, l'inobservation du droit des aires protégées s'explique par la crise militaro-politique déclenchée en septembre 2002.

\section{C- Les limites relatives au conflit militaro-politique de septembre 2002}

En Côte d'Ivoire, l'effectivité de la mise en œuvre du droit des aires protégées a été contrariée par un contexte conjoncturel défavorable. Il s'agit de la crise militaro-politique de septembre 2002 dont les effets perdurent jusqu'à ce jour. L'affaiblissement ou le dépérissement de l'autorité de l'État qui en est résulté a une conséquence sur l'effectivité du droit des aires protégées.

En l'espèce, les entraves à l'effectivité du droit des aires protégées résulte de ce que la République de Côte d'Ivoire avait été divisée en deux (2) zones du fait de la crise militaropolitique sus-évoquée. D'une part, il y a eu une zone dite "zone gouvernementale" sur laquelle s'exerçait l'autorité de l'État. D'autre part, l'autre zone dite "zone sous contrôle des Forces Nouvelles" ou encore "zone Centre Nord-Ouest (CNO)" était contrôlée par les Forces Nouvelles (ex-rébellion). Ces deux (2) zones étaient séparées par une zone tampon dite "zone de confiance" placée sous l'autorité des forces impartiales (onusiennes) qui jouaient un rôle d'interposition en vue d'un retour à la paix ${ }^{761}$.

Par conséquent, l'autorité de l'État qui a la charge de veiller à l'application du droit des aires protégées ne s'exerçait plus sur cette dernière zone citée qui abritait plusieurs aires protégées $^{762}$. En d'autres termes, les Administrations en charge de leur répression ne pouvaient les constater, rechercher les auteurs et les punir du fait de la situation de crise. Par conséquent, la crise militaro-politique survenue en 2002 en Côte d'Ivoire a entravée l'effectivité du droit des aires protégées.

Toutefois, avec le retour à la paix, si la situation s'est normalisée ${ }^{763}$, il reste que certains effets de la crise ivoirienne sur la conservation des aires protégées perdurent. En effet, une

\footnotetext{
${ }^{761}$ Voir UNESCO/UICN, Rapport de mission, Suivi de l'état de la conservation du Parc National de la Comoé en Côte d'Ivoire ,op. cit., p. 9.

${ }^{762}$ À ce sujet, le Rapport annuel sur l'État de la conservation de 2009 nous renseigne que sur un total de huit (8) Parcs Nationaux et de six (6) Réserves naturelles estimés à une surface totale d'environ 2.100.000 ha, les aires protégées qui étaient situées dans la zone dite Centre Nord-Ouest (CNO) et l'ex- zone de confiance représentaient environ 1.406.150 ha. Ce sont les Réserves du Haut Bandama (123.000 ha), du Mont Nimba (5.000 ha), les Parcs Nationaux du Mont Sangbé (95.000 ha), du Mont Péko (34.000 ha), et de la Comoé $(1.149 .150 \mathrm{ha})$ dont seulement $20 \%$ sont contrôlés par l'Administration forestière. Voir UNESCO/UICN, Rapport de mission, Suivi de l'état de la conservation du Parc National de la Comoé en Côte d'Ivoire, op. cit., p.9).

${ }^{763}$ En effet, la crise militaro-politique survenue en septembre 2002 (voir République de Côte d'Ivoire, Ministère de 1'Environnement, de la Salubrité Urbaine et Développement Durable/Office Ivoirien des Parcs et Réserves, État de conservation du Parc National de la Comoé. Côte d'Ivoire, op. cit., p. 2.) a évolué jusqu'à aboutir à des élections présidentielles en 2010. Après, les violences post-électorales qui ont caractérisé lesdites élections, les institutions et Administrations ivoiriennes se sont progressivement mises en place et fonctionnent depuis l'année 2011. En octobre 2015, il y a eu de nouvelles élections présidentielles qui se sont déroulées dans la paix. Le dialogue politique et social continue entre les formations politiques sont au pouvoir et regroupées au sein du Rassemblement des Houphouétistes pour la Démocratie et la Paix(RHDP) et les partis d'opposition.
} 
étude (récente) menée dans certaines aires protégées telles que le Mont Sangbé et le Mont Péko qui étaient sous le contrôle de l'ex-rébellion montrent que celles-ci restent fortement occupées et dégradées par les populations (essentiellement burkinabè) que des chefs de guerre ont installés ${ }^{764}$. Elles s'y sont installées et sédentarisées pour la pratique de l'agriculture en occupant environ 60 à $80 \%$ du Parc National du Mont Sangbé ${ }^{765}$ et 70 à $80 \%$ du Mont Péko ${ }^{766}$.

$\mathrm{Au}$ surplus, les entraves à l'effectivité du droit des aires protégées due à la crise militaro-politique sus-citée ont été exacerbées par la suspension des procédures de financement ou le retrait des financements acquis, du fait de ladite crise ${ }^{767}$. À titre d'exemple, dès l'éclatement de la crise militaro-politique en septembre 2002, la procédure de

\footnotetext{
${ }^{764}$ Selon AKINDES (Francis) et al., dans le Parc National du Mont Sangbé (PNMS) : « La situation du foncier ... avant 2002, est celle de l'époque où certaines populations autochtones vivaient encore dans le Parc. Les terres y étaient utilisées essentiellement pour les cultures vivrières (igname, manioc, taro, etc.) et céréalières (riz, maïs, etc.) mais également pour le caféiculture (IES, 1993). Dans ces zones de superficie limitée, les activités agricoles impliquaient, quelquefois, l'usage des feux de brousse comme technique de défrichement. (...)

L'on assiste, depuis 2002, à une pression foncière beaucoup plus importante caractérisée, selon l'ensemble des acteurs interrogés, par la colonisation, tout au moins dans sa partie forestière, de la quasi-totalité du Parc essentiellement par la culture du cacao la plus économiquement rentable. Le Parc serait "devenu un vaste champ de cacao !". Un recensement partiel des exploitants dans la partie sud (du côté de Soba) a permis de dénombrer en 2011, 326 personnes essentiellement de nationalité Burkinabè...Cette pression foncière serait surtout le fait d'allogènes Burkinabè, qui comparativement aux autres groupes d'infiltrés, disposent de la possibilité de recourir à une filière burkinabè de main-d'œuvre. ». Voir AKINDÈS (Francis), KOUAMÉ (Kra Walter), SÉDIA (Gisèle) et SÉZAN (Jules), Réalisation d'une étude sur l'état de la biodiversité des parcs nationaux et réserves de Côte d'Ivoire. Aspects socio-économiques (Rapport provisoire), op. cit., p. 15.

En ce qui concerne le Parc National du Mont Péko (PNMP), ces auteurs écrivent : "L'exploitation foncière avant 2002 était essentiellement le fait d'autochtones de la périphérie du Parc, de Guéré venus d'autres terroirs du grand ouest et d'allogènes Burkinabè ayant été manœuvres chez des Guéré. Cette exploitation consistait en la création, notamment, de quelques plantations de café et de cacao à la suite des infiltrations qui se sont opérées du côté de plusieurs villages dont Gohouo Zagna, Gloplou, Goénié Tahouaké, Bouobly, Diébly, Guezon Tahouaké, Guinglo Tahouaké, Pona, Sibably, Nidrou, Bagohouo, BahéSébon (DNP, 1996). Ces infiltrations étaient, pour une bonne part, le résultat de la confusion créée par le tracé périmétral de 1974 et du mécontentement des riverains né de l'occupation, par le Préfet Kouléon Narcisse, d'une petite enclave dans le Parc (Akindès, 1996).

Après 2002, selon les informateurs, l'on a assisté à une forte ruée vers le PNMP qui s'est traduite par la création, à grande échelle, de plantations de cacao dont certaines seraient aujourd'hui âgées d'environ 10 ans. Quatre cinquièmes de la superficie totale du parc seraient colonisés par ces exploitations agricoles. Le cinquième de la superficie restant serait la partie montagneuse (vers Diébly et Bléniméouin) qui, pour des raisons évidentes de relief, n'a pu être exploitée. En plus des cultures de rente (du cacao et dans une moindre mesure du café), les infiltrés cultivent du vivrier (igname, taro) et des céréales (riz, maïs). Cette exploitation illégale serait l'ouvre de la bande armée qui, entre 2006 et 2007, aurait conquis tout le Parc après avoir combattu une autre bande armée rivale. Forts de cette victoire, les chefs de cette bande armée ont renforcé leurs rangs et accru leur rente de situation en structurant les infiltrations. Celles-ci consistent à accueillir, chaque semaine...plusieurs dizaines de Burkinabè et d'autres ressortissants de pays voisins à qui ils vendraient des parcelles ou qu'ils utiliseraient comme main-d'auvre agricole. Cette pratique qui aurait déjà commencé avant la crise postélectorale, se serait fortement accrue après ladite crise et se poursuit encore aujourd'hui avec plus d'intensité. » (souligné par nous). Voir Idem., p. 29.

${ }^{765}$ Voir Idem., p. 15.

${ }^{766}$ Voir Idem., p. 29.

${ }^{767}$ En effet, l'insuffisance des subventions étatiques allouées aux aires protégées constituait déjà une difficulté pour la mise en œuvre du droit des aires protégées. Sur ce point, voir la Première partie de cette étude, le Titre I, le Chapitre II, la Section II, le Paragraphe 1, le point intitulé "B- L'insuffisance des dotations budgétaires pour la conservation des aires protégées ".
} 
décaissement des 21 milliards prévus pour les quatre premières années du PCGAP a été annulée ${ }^{768}$. Dans cette même période, plusieurs financements acquis pour la mise en œuvre de programmes de conservation ont été retirés. Il s'agit d'un montant global de 7,3 milliards comme l'indique le tableau ci-suit.

Tableau 4 : Financements retirés aux Parcs Nationaux et Réserves dès l'éclatement de la crise militaro-poltitique de septembre 2002

\begin{tabular}{|l|c|c|l|}
\hline \multicolumn{1}{|c|}{ Intitulé du programme } & $\begin{array}{c}\text { Montant } \\
\text { (FCFA) }\end{array}$ & Bailleur & Observations \\
\hline $\begin{array}{l}\text { Programme de conservation des } \\
\text { Parcs Nationaux de la Comoé, } \\
\text { Sangbe, Péko, Nimba et Marahoué }\end{array}$ & $\mathbf{3 . 0 0 0 . 0 0 0 . 0 0 0}$ & Union Européenne & $\begin{array}{l}\text { Le programme a connu un début } \\
\text { d'exécution avant d'être } \\
\text { suspendu en mai 2003 }\end{array}$ \\
\hline $\begin{array}{l}\text { Programme Cadre de Gestion des } \\
\text { Aires Protégées }\end{array}$ & $\mathbf{4 . 0 0 0 . 0 0 0 . 0 0 0}$ & $\begin{array}{c}\text { FEM, Banque } \\
\text { Mondiale, GTZ, } \\
\text { Union Européenne }\end{array}$ & $\begin{array}{l}\text { Le fonds prévus pour la mise en } \\
\text { œuvre du programme ont été } \\
\text { retirés }\end{array}$ \\
\hline $\begin{array}{l}\text { Programme de renforcement de la } \\
\text { gestion des ressources humaines } \\
\text { des PNR }\end{array}$ & $\mathbf{3 . 0 0 0 . 0 0 0 . 0 0 0}$ & $\begin{array}{l}\text { Le programme a connu un début } \\
\text { d'exécution avant d'être } \\
\text { suspendu en 2002 }\end{array}$ \\
\hline
\end{tabular}

Source : République de Côte d'Ivoire, Ministère de l'Environnement et des Eaux et Forêts/Office Ivoirien des Parcs et Réserves, État de conservation des Parcs et Réserves de Côte d'Ivoire, op.cit., p. 7.

Somme toute, nous pouvons retenir que les entraves à l'effectivité du droit des aires protégées, en Côte d'Ivoire, ne résulte pas du droit des aires protégées. Bien au contraire, elles émanent de facteurs externes au droit des aires protégées. Aussi convient-il de faire des propositions, en guise de solutions pour une effectivité dudit droit.

\footnotetext{
${ }^{768}$ Voir République de Côte d'Ivoire, Ministère de l'Environnement et des Eaux et Forêts/Office Ivoirien des Parcs et Réserves, État de conservation des Parcs et Réserves de Côte d'Ivoire, op.cit.,p.7.
} 


\section{TITRE II : LES REMÈDES POUR UNE EFFECTIVITÉ DU DROIT DES AIRES PROTÉGÉES}

En côte d'Ivoire, le droit des aires protégées connaît des difficultés pour son application. Le défaut d'effectivité du droit des aires protégées résulte de problèmes pratiques de gestion auxquels sont confrontés les gestionnaires des aires protégées de l'État.

Cette situation explique la dégradation de ses milieux naturels dont la préservation revêt une importance capitale. Or, la conservation des forêts en Côte d'Ivoire, et partant des aires protégées, a un enjeu écologique et socio-économique.

Face à ce constat, il convient de proposer des solutions, à ce stade de l'étude, aux pouvoirs publics ivoiriens, en général, et à l'Office Ivoirien des Parcs et Réserves (OIPR), en particulier. En la matière, il est nécessaire que l'OIPR concilie conservation des aires protégées et développement local (Chapitre I). Également, l'OIPR pourrait procéder à une valorisation touristique effective des aires protégées et de leurs zones périphériques (Chapitre II). 


\section{CHAPITRE I : LA NÉCESSITÉ DE CONCILIER CONSERVATION DES AIRES PROTÉGÉES ET DÉVELOPPEMENT LOCAL}

Aux dires des communautés locales que nous avons enquêtées, les pressions subies par les aires protégées sont en partie imputables au déficit de développement ${ }^{769}$. Cela constitue un réel problème de gestion. Or, les aires protégées présentent un fort potentiel en matière de développement ${ }^{770}$.

Certains auteurs, sur la base de leurs travaux, tiennent en effet que même si développement et conservation de la biodiversité sont difficiles à concilier, il n'empêche qu'un tel objectif n'est pas forcément inattingible. Ce qu'il faut donc, c'est tout mettre en œuvre pour rendre cela possible. C'est un impératif dont la satisfaction passe par une prise en main véritable de la situation par les gestionnaires des aires protégées.

Aussi convient-il de présenter l'intérêt de cette conciliation (Section I) avant de définir les modalités afférentes (Section II).

\section{SECTION I : L'intérêt de la conciliation de la conservation des aires protégées et du développement local}

L'intérêt de concilier conservation des aires protégées et développement local découle de deux (2) idées maîtresses. D'une part, il existe une interdépendance entre la conservation des ressources naturelles et le développement (Paragraphe 1). D'autre part, les aires protégées constituent un moyen permettant d'opérer le développement au bénéfice des communautés riveraines (Paragraphe 2).

\footnotetext{
${ }^{769} \mathrm{Il}$ ressort des résultats de l'enquête menée dans le cadre de cette étude que $66 \%$ des enquêtés pensent que les pressions exercées par les communautés riveraines des aires protégées sur ces forêts sont dues, entres autres, à la pauvreté (qui elle-même est essentiellement causée par la baisse des revenus agricoles). Voir Annexe 3, le point " 5) Les raisons des pressions sur les aires protégées ". Ce résultat est confirmé par les travaux de FERRARO (Paul. J.) relatif au Ranomafana National Park, en français Parc National de Ranomafana (notre traduction), sis à Madagascar. En effet, cet auteur adhère à la thèse des gouvernements et bailleurs de fonds selon laquelle la conservation durable des aires protégées requiert le développement des communautés locales. Voir FERRARO (Paul J.), « The local costs of establishing protected areas in low-income nations : Ranomafana National Park, Madagascar ». Ecological Economics, Vol. 43, n²-3, 2002, p. 261.

${ }^{770}$ Voir le Vème Congrès mondial sur les Parcs, Recommandations sur les aires protégées et la pauvreté (Rec. 5.29). C'est également fort du potentiel des aires protégées en matière de développement local qu'en Afrique du Sud, la loi sur la gestion des aires protégées (The National Environmental Management Protected Areas Act) prône l'utilisation durable des aires protégées par l'industrie du tourisme pour le développement des communautés locales. Voir MASHALE (Christopher Modise), MOYO (Theresa), MTAPURI (Olivier), «An Evaluation of the Public-Private Partnership in the Lekgalameetse Nature Reserve in South Africa», Mediterranean Journal of Social Sciences, Vol. 5, n²0, 2014, p. 856.
} 


\section{Paragraphe 1 : L’interdépendance de la conservation des ressources naturelles et du développement local}

La conservation des ressources naturelles et du développement des communautés riveraines constituent deux (2) facettes d'une même réalité (A). De plus, sur le plan pratique, cette interdépendance se justifie par des résultats d'expériences de gestion des ressources naturelles $(\mathrm{B})$.

\section{A - Conservation des ressources naturelles et développement local, deux facettes d'une même médaille}

La conciliation de la conservation des ressources naturelles et du développement est une recommandation des initiatives internationales de protection de l'environnement (1) et une exigence des organismes de conservation et de développement (2).

\section{Une recommandation des initiatives internationales de protection de l'environnement}

Les initiatives internationales dont il sera fait mention des recommandations sont celles relatives aux rencontres internationales des Nations Unies sur l'environnement en général (a) et aux congrès mondiaux des aires protégées en particulier (b).

\section{a. Les recommandations issues des rencontres internationales des Nations Unies sur l'environnement}

Depuis la Conférence des Nations Unies de 1972 sur l'environnement ${ }^{771}$, il est constant que les instruments juridiques internationaux de protection de l'environnement consacrent l'intégration du développement et de la protection de l'environnement. En effet, le point 4 de la Déclaration de Stockholm fait le constat suivant lequel dans les pays en voie de développement, la plupart des problèmes environnementaux sont causés par le sousdéveloppement. Il tient en sus que les efforts de développement ne doivent pas se faire au détriment de l'environnement. Bien au contraire, ces efforts sont tenus de préserver et d'améliorer l'environnement. Fort de cette exigence, le principe 4 de la Déclaration précitée stipule que la conservation de la nature doit tenir une place importante dans les stratégies de développement économique.

\footnotetext{
${ }^{771}$ Face à la dégradation de l'environnement mondial, s'est tenue du 5 au 16 juin 1972, la Conférence des Nations Unies sur l'Environnement Humain (CNUEH), à Stockholm (Suède) ou Conférence de Stockholm. Voir NGAIDO (Moustapha) cité par ANDRÉ (Pierre), BEAUDET (René), BERNIER (Michel), CÔTÉ (Louis) et LANMAFANKPOTIN (Georges), SAMOURA (Karim), La participation publique dans l'évaluation environnementale en Afrique francophone, Québec, OIF/IFDD, Points de repère 23, 2013, p. 18.

Elle avait pour but d'adopter une conception commune et des principes communs qui guideront les efforts des peuples du monde entier en vue de la protection et de l'amélioration de l'environnement

Voir www.unep.org/Documents.Multilingual/Default.asp?DocumentID=97\&ArticleID=1503\&l=fr, consulté le 2 décembre 2015). La déclaration qui en est issue est la Déclaration finale des Nations Unies sur l'environnement communément appelée Déclaration de Stockholm.
} 
Le contenu de ce principe est repris au point 7 de la Charte mondiale de la nature de $1982^{772}$. En effet, il y est inscrit: «Dans la planification et l'exécution des activités de développement, il sera dûment tenu compte du fait que la conservation de la nature fait partie intégrante de ces activités.».

Dix ans plus tard, la Déclaration de Rio sur l'environnement et le développement a réaffirmé l'interdépendance de la protection de l'environnement et du développement. En effet, il peut être lu au principe 4 de ladite déclaration : "Pour parvenir à un développement durable, la protection de l'environnement doit faire partie intégrante du processus de développement et ne peut être considérée isolément. ". De même, l'article 8 de la Convention sur la Diversité Biologique qui traite de la conservation in situ ${ }^{773}$ prescrit, entre autres, la mise en place d'aires protégées et un «développement durable et écologiquement rationnel » des zones périphériques, en vue de favoriser la protection de ces sites de conservation.

Par ailleurs, en ce qui concerne spécifiquement les aires protégées, les Déclarations des congrès mondiaux sur les aires protégées recommandent d'intégrer la protection de ces espaces et le développement local.

\section{b. Les recommandations issues des Déclarations des congrès mondiaux sur les Parcs Nationaux}

Le IIIème Congrès mondial des Parcs Nationaux ${ }^{774}$ souligne le lien entre le développement des communautés et la conservation des aires protégées. La recommandation 5 de la Déclaration finale de Bali indique les communautés vivant à l'intérieur ou autour des aires protégées consentent à la protection de ces forêts à deux (2) conditions. En effet, premièrement, elles entendent tirer avantage de la conservation des espaces classés, en compensation des droits perdus. Secondement, elles demandent leur implication dans la gestion de ces milieux naturels.

\footnotetext{
${ }^{772} \mathrm{La}$ Charte mondiale de la nature $(\mathrm{CMN})$ est une résolution de l'Assemblée générale des Nations Unies (Résolution 37/7) adoptée le 28 octobre 1982. Cette charte est qualifiée de texte fondamental, d'importance mondiale et de haute valeur morale pour plusieurs raisons. En effet, ce texte proclame pour la première fois l'importance de la protection de la nature et des écosystèmes pour la survie de l'humanité (y compris implicitement les générations futures). Ce texte proclame que l’Homme, qui agit nécessairement sur la nature, doit être guidé par le souci de la conservation et de l'utilisation durable des ressources naturelles en général, des espèces (sauvages, domestiques), et leurs habitats en particulier. La Charte mondiale de la nature prône également la restauration des écosystèmes dégradés, de sorte qu'ils retrouvent leur potentiel naturel originel. Voir DOUMBÉ-BILLÉ (Stéphane), Droit international de la faune et des aires protégées : importance et implication pour l'Afrique, Étude juridique de la FAO \#/20, septembre 2001, p. 9.

${ }^{773}$ Aux termes de l'article 2 de la Convention sur la Diversité Biologique (CDB), la conservation in situ désigne : "...la conservation des écosystèmes et des habitats naturels et le maintien et la reconstitution de populations viables d'espèces dans leur milieu naturel et, dans le cas des espèces domestiquées et cultivées, dans le milieu où se sont développés leurs caractères distinctifs. ».

${ }^{774}$ Le IIIème Congrès mondial des Parcs Nationaux s'est tenu en 1982 à Bali (Indonésie). Il a eu pour thème : « Des Parcs pour le développement ». Voir FOURNIER (Anne), « Ve Congrès mondial sur les Parcs de l’Union mondiale pour la nature (UICN) : Compte rendu (Durban, Afrique du Sud, 8-18 septembre 2003 », Nature Sciences Sociétés, Vol. 12, n¹, 2014, p. 93.
} 
De même, le principe de l'équilibre entre la conservation des aires protégées et le développement des communautés a été réaffirmé dans la Déclaration de Caracas, au IVème Congrès mondial des Parcs Nationaux ${ }^{775}$. C'est à juste titre qu'au Vème Congrès mondial des Parcs Nationaux ${ }^{776}$, la recommandation 5.29 a affirmé la nécessité d'intégrer les aires protégées dans les stratégies de développement et de réduction de la pauvreté.

Par ailleurs, l'intégration de la conservation des ressources naturelles et du développement est une exigence des organismes de conservation et de développement.

\section{Une exigence des organismes de conservation et de développement}

Pour un organisme de développement tel que l'Agence Française de Développement $(\mathrm{AFD})^{777}$, il « est en effet inadmissible, sur le plan éthique et en termes d'efficacité environnementale et sociale, que la préservation de la biodiversité constitue un obstacle à la lutte contre la pauvreté. Sous peine de conflit, il est nécessaire de rechercher la convergence entre les intérêts de la planète et ceux des territoires ${ }^{778}$. En d'autres termes, pour réussir la conservation, il faudrait que les communautés en retirent des avantages qui améliorent leurs conditions d'existence.

De même, vu la perte des habitats, $1^{\prime} \mathrm{UICN}^{779}$ a suggéré que les activités dans le domaine de la conservation soient intimement liées au développement ${ }^{780}$. Le soutien financier

\footnotetext{
${ }^{775}$ Le IVème Congrès mondial des Parcs Nationaux a eu lieu en 1992 à Caracas (Venezuela). Le thème était « Des Parcs pour la vie ». Voir PIMBERT (Michel P.) et PRETTY (Jules N.), Parks, People and Professionals: Putting Participation' into Protected Area Management, UNRISD/IIED/WWF, Discussion Paper $\mathrm{n}^{\circ}$ 57, February 1995. UNRISD, Geneva, p. 4; FOURNIER (Anne), « Vème Congrès mondial sur les Parcs de 1'Union mondiale pour la nature (UICN) : Compte rendu (Durban, Afrique du Sud, 8-18 septembre 2003 », art. cit., p. 93.

${ }^{776}$ Le Vème Congrès mondial des Parcs Nationaux a eu lieu en 2003 à Durban (Afrique du Sud). Le thème de cette rencontre a été «Bénéfices par-delà les frontières». Voir UICN, Vème Congrès mondial des Parcs Nationaux : Bénéfices par-delà les frontières, Planète Conservation (Bulletin UICN), Vol. 34, n², 2003, p. 2.

${ }^{777}$ L'Agence Française de Développement (AFD) est une institution financière de la République de la France. Elle a pour mission de mettre en œuvre la politique publique de la France en luttant contre la pauvreté et en favorisant le développement durable. Voir AFD, Gestion durable de la biodiversité, coll. Parole d'acteurs, $\mathrm{n}^{\circ} 5$, 2005 , p. 7.

${ }^{778}$ Voir AFD, Gestion durable de la biodiversité, coll. Parole d'acteurs, n5, 2005, p.1.

${ }^{779}$ Créée en 1948, l'Union Internationale pour la Conservation de la Nature (UICN) est la plus grande et la plus ancienne des organisations de conservation de la nature. À ce titre, l'UICN s'est donnée trois (3) missions. Premièrement, dans son effort de conservation de la nature à l'échelle mondiale, elle influence les politiques des gouvernements, des ONG, les organisations des Nations Unies, en vue de développer des lois, des politiques et des meilleurs pratiques. Deuxièmement, 1'UICN conduit des projets dans tout le monde entier dont l'objectif est la gestion durable des ressources naturelles. Enfin, cette organisation développe et soutient des activités scientifiques de conservation de la nature (espèces, écosystèmes et biodiversité) et leur impact sur les moyens de subsistance de l'homme. Voir www.iucn.org/fr (consulté le 2 décembre 2015).

${ }^{780}$ Voir UICN, World Conservation Strategy: Living Resource Conservation for Sustainable Development, Gland (Switzerland) : IUCN-The World Conservation Union, United Nations Environmental Programme, World Wildlife Fund, 1980 (document non paginé), les points 7 et 20 intitulés respectivement "Priority requirements : sustainable utilization" et "Towards sustainable development". Ce document est disponible sur www.portals.iucn.org/library/efiles/edocs/WCS-004.pdf, consulté le 02 février 2015.
} 
que le Fonds Mondial pour l'Environnement (FEM) ${ }^{781}$ accorde aux gouvernements menant des politiques de conservation soucieuses du développement local ${ }^{782}$ coïncide bien avec cette exhortation. Il en est de même pour l'UICN dont les observations sont fort à propos. Cet organisme fait en effet remarquer que pour mettre en adéquation la conservation de la biodiversité et le partage équitable de ses avantages entre les populations locales, il est indispensable de définir clairement les droits des parties prenantes sur les terres et les ressources $^{783}$.

Il ressort des développements précédents que les organismes de conservation de la nature et de développement prônent la mise en convergence des politiques de conservation des sites protégés et de développement local. C'est là un gage véritable pour opérer le développement local et, concomitamment, protéger les richesses de la faune et de la flore.

$\mathrm{Au}$ surplus, des expériences de gestion de ressources naturelles confirment cette approche de gestion selon laquelle pour réussir les projets de conservation, il faudrait que la conservation des ressources protégées contribue au développement des communautés locales.

\section{B - La justification de l'interdépendance fondée sur des résultats d'expériences de gestion des ressources naturelles}

Divers travaux de recherche attestent qu'il est impérieux de concilier conservation de la biodiversité et développement local, si l'on veut emmener les communautés locales à être moins rétives à la conservation des ressources naturelles ${ }^{784}$. En ce qui concerne spécifiquement les aires protégées, pour des auteurs comme Jelena TOMIĆEVIĆ et al., les populations riveraines s'engageront véritablement pour la conservation de ces milieux

\footnotetext{
${ }^{781}$ Le Fonds pour l'Environnement Mondial (FEM) est un mécanisme financier international. Ce fonds est destiné à s'attaquer aux problèmes environnementaux à l'échelle mondiale en encourageant le développement durable dans les États. À cet effet, le FEM soutient, entre autres, des projets des projets relatifs à la diversité biologique, aux changements climatiques, à la dégradation des sols. Voir http://www.unep.org/Documents.multilingual/Default.asp?DocumentID=340\&ArticleID=4173\&l=fr, $\quad$ consulté le 25 mai 2016.

${ }^{782}$ Voir STOLL-KLEEMANN (Suzanne), O'RIORDAN (Timothy) «From Participation to Partnership in Biodiversity Protection : Experience from Germany and South Africa », Society \& Natural Resources: An International Journal, Vol. 15, n², 2002, p. 163.

${ }^{783}$ Cette position est adoptée par l'UICN à l'occasion du Vème Congrès mondial des Parcs Nationaux qui s'est tenu en 1997 à Albany (Australie). Voir SCHERL (Lea M.), WILSON (Alison), WILD (Robert) et al., Can Protected Areas Contribute to Poverty Reduction? Opportunities and Limitations, Gland, Switzerland and Cambridge, UK, IUCN, 2004, p. 38.

${ }^{784}$ Voir STOLL-KLEEMANN (Suzanne), O'RIORDAN (Timothy) «From Participation to Partnership in Biodiversity Protection : Experience from Germany and South Africa », art. cit., p. 161 ; MBAIWA (Joseph E.), STRONZA (Amanda L.), « Changes in resident attitudes towards tourism development and conservation in the Okavango Delta », Journal of Environmental Management, Vol. 92, n 8, 2011, p. 1950; ANDRADE (Gustavo S. M.) et RHODES (Jonathan R.), «Protected areas and local communities: an inevitable partnership toward successful conservation strategies ? », Ecology and Society, Vol.17, n4, 2012, p. 15 ; SZELL (Andrea B.), HALlETT (Lucius F.), «Attitudes and Perceptions of Local Residents and Tourists toward the Protected Area of Retezat National Park, Romania», International Journal of Humanities and Social Science, Vol. 3, n³, 2013, pp. 23-24.
} 
naturels si leur gestion est inclusive et impacte sensiblement leur niveau de développement ${ }^{785}$.

Selon des auteurs, de plus en plus, les gestionnaires africains d'aires protégées cherchent à définir de nouvelles approches de gestion de ces milieux naturels qui concilient conservation et développement local $^{786}$. Cette situation pourrait, sur le plan pratique, expliquer la généralisation de la mise en œuvre de projets intégrés de conservation et de développement en Afrique, à partir des années $1980^{787}$.

Ces projets ont enregistré des résultats qu'il convient de présenter à travers quelques exemples, notamment en Afrique orientale et australe.

\section{Des résultats de gestion d'aires protégées en Afrique de l'Est}

Traitant des aires protégées du Rwanda, des auteurs soutiennent que la conservation de ces milieux naturels est inséparable du développement local, dans un environnement caractérisé par la pauvreté des populations ${ }^{788}$.

Une étude réalisée en Éthiopie, a montré que les populations sont en grande partie favorables à la conservation des ressources naturelles des aires protégées. Cependant, le fait qu'elles ne bénéficient pas des avantages de la conservation limite cette volonté locale ${ }^{789}$. L'on comprend dès lors que, dans le cadre de la gestion du Parc National du Balé ${ }^{790}$, l'amélioration des conditions de vie des communautés locales ait incité celles-ci à participer activement à sa conservation ${ }^{791}$. En effet, l'exploitation touristique du Parc a contribué à créer

\footnotetext{
${ }^{785}$ Voir TOMIĆEVIĆ (Jelena), BJEDOV (Ivana), SHANNON (Margaret A.) et OBRATOV-PETKOVIĆ (Dragica), «Understanding Linkages Between Public Participation and Management of Protected Areas - Case Study of Serbia » in Dr. ISHWARAN (Natarajan) (eds.), The Biosphere, Intech, pp. 140-141. Ce point de vue est partagé par plusieurs auteurs. Voir notamment KRUG (Wolf), Private Supply of Protected Land in Southern Africa : A Review of Markets, Approaches, Barriers and Issues. Workshop on Market Creation for Biodiversity Products and Services, 25 and 26 January 2001, Paris, Workshop Paper, World Bank / OECD International, p. 35 ; AFD, Gestion durable de la biodiversité, coll. Parole d'acteurs, n5, 2005, p. 24 ; TESSEMA (Mekbeb E.), ASHENAFI (Zelealem T.), LILIEHOLM (Robert J.) et LEADER-WILLIAMS (Nigel), « Community Attitudes towards Wildlife Conservation in Ethiopia», Proceedings of the 2007 George Wright Society Conference, $\mathrm{p}$. 290; ADELEKE (Bola Olusola), NZAMA (Thandi), « Assessment of Community Participation in Ecotourism and Conservation at Hhuhuwe-Umfolozi Park, South Africa », Journal of Environment and Earth Science, Vol. $3, \mathrm{n}^{\circ} 3,2013$, p. 30 .

${ }^{786}$ Voir NEWMARK (William D.), HOUGH (John L.), " Conserving Wildlife in Africa: Integrated Conservation and Development Projects and Beyond », BioScience, Vol. 50, n7, 2000, p. 585.

${ }^{787}$ Ibidem.

${ }^{788}$ Voir RUTAGARAMA (Eugène), MARTIN (Adrian), « Partnerships for protected area conservation in Rwanda », The Geographical Journal, Vol. 172, n4, 2006, p. 293.

${ }^{789}$ Voir TESSEMA (Mekbeb E.), ASHENAFI (Zelealem T.), LILIEHOLM (Robert J.) et LEADER-WILLIAMS (Nigel), «Community Attitudes towards Wildlife Conservation in Ethiopia », op. cit., p. 291.

${ }^{790}$ Le Parc National du Balé est situé dans la partie méridionale de l'Éthiopie. C'est un Parc de catégorie II de l'UICN crée en 1969 et qui couvre une superficie de $1.675 \mathrm{~km}^{2}$. Voir Unesco, Convention concernant la protection du patrimoine mondial culturel et naturel/ liste du Patrimoine mondial/ Demande d'Assistance Technique présentée par l'Éthiope : , Parc National du Mont Balé, 26/04/1979, nº d'ordre 111-1, p. 1.

${ }^{791}$ Idem, p. 290.
} 
des emplois pour les populations locales ; le partage des revenus du tourisme a développé l'économie locale et aidé à réaliser des infrastructures de base (centre de santé, expansion du réseau électrique). Fort de tous ces avantages, les populations soutiennent et encouragent les efforts de conservation du Parc National du Balé ${ }^{792}$. De plus, une étude menée dans quatre (4) aires protégées d'Éthiopie ${ }^{793}$ a démontré que $94 \%$ des communautés locales sont favorables à la conservation des aires protégées, en raison des revenus qu'elles retirent de leur valorisation touristique ${ }^{794}$.

En outre, au Kenya, il ressort des conclusions de travaux de recherche plus récents que la constitution d'aires protégées ${ }^{795}$ a réduit les pâturages, les surfaces agricoles et les ressources naturelles utilisables par les populations. Cette situation a suscité la désapprobation des populations maasaï. Éleveurs par excellence, ces populations sont hostiles aux projets de conservation de la faune et ce, pour plusieurs raisons. La première est qu'elles ne bénéficient pas des retombées du tourisme, en dépit de la réduction des pâturages ${ }^{796}$. La seconde est qu'elles enregistrent des pertes en vies humaines, des destructions de cultures ou des maladies qui menacent la santé du bétail du fait de la faune sauvage ${ }^{797}$. Selon Roselyne N. OKECK, pour emmener les communautés locales à soutenir la conservation, il faut leur permettre d'en tirer profit à travers des initiatives communautaires ${ }^{798}$.

Qu'en est-il des résultats de gestion des aires protégées en Afrique australe?

\section{Des résultats de gestion d'aires protégées en Afrique australe}

En Afrique australe, la politique de gestion des aires protégées a aussi généré des résultats probants ${ }^{799}$. Cela a également contribué à l'amélioration du niveau de vie des populations $^{800}$.

À titre d'exemples, au Mozambique, pourtant pays pauvre, lorsque les autorités, soutenues par les populations locales, ont décidé de valoriser le patrimoine du Parc National

\footnotetext{
${ }^{792}$ Ibidem.

${ }^{793}$ Il s'agit du Parc National Abijata-Shalla Lakes, du Parc National Awash, du Parc National du Balé Mountains et du sanctuaire Hartebeest de Senkelle Swayne.

${ }^{794}$ Voir TESSEMA (Mekbeb E.), ASHENAFI (Zelealem T.), LILIEHOLM (Robert J.) et LEADER-WILLIAMS (Nigel), « Community Attitudes towards Wildlife Conservation in Ethiopia », op. cit., p. 288.

${ }^{795}$ Il s'agit de Parcs Nationaux ou d'aires de conservation de faune privées.

${ }^{796}$ Sur ces points, voir OKECK (Roselyne N.), «Wildlife-community conflicts in conservation areas in Kenya », African Journal of Conflict Resolution, Vol. 10, n², 2010, p. 67.

${ }^{797}$ Ibidem, p. 67.

${ }^{798}$ Idem, p. 69.

${ }^{799}$ Voir KRUG (Wolf), Private Supply of Protected Land in Southern Africa : A Review of Markets, Approaches, Barriers and Issues, Workshop Paper, World Bank / OECD International, Workshop on Market Creation forBiodiversity Products and Services, Paris , 25 and 26 January 2001, p. 35.

${ }^{800}$ Voir KRUG (Wolf), Private Supply of Protected Land in Southern Africa : A Review of Markets, Approaches, Barriers and Issues, op.cit., p. 35 ; AFD, Gestion durable de la biodiversité, coll. Parole d'acteurs, n5, 2005, p. 24 ; ADELEKE (Bola Olusola), NZAMA (Thandi), " Assessment of Community Participation in Ecotourism and Conservation at Hhuhuwe-Umfolozi Park, South Africa », Journal of Environment and Earth Science, Vol. $3, \mathrm{n}^{\circ} 3,2013$, p. 30 .
} 
des Quirimbas, de nouvelles activités génératrices de revenus ont ainsi pu être créées ${ }^{801}$. Les communautés riveraines en ont été les premières bénéficiaires. Cette situation donna encore lieu à une augmentation des captures halieutiques et à la réduction significative des destructions d'exploitations agricoles causées par la faune sauvage ${ }^{802}$. Subséquemment, les revenus des populations ont augmenté et leur qualité de vie s'est améliorée (en matière de sécurité alimentaire). Dans l'Est de la Zambie, les résultats de l'étude de Muleba et al. montrent qu'avec l'amélioration des moyens de subsistance des communautés locales vivant autour de la Lupande Game Management Area $(\mathrm{GMA})^{803}$, la conservation de l'environnement s'est considérablement améliorée ${ }^{804}$.

Il ressort de ces expériences de gestion que lorsque les communautés locales tirent avantage de la conservation des aires protégées, elles soutiennent plus fermement les efforts des gestionnaires desdits sites. Autrement dit, le succès des stratégies de conservation des aires protégées requiert des gestionnaires qu'ils concilient les objectifs de conservation et les préoccupations sociales et économiques des communautés locales ${ }^{805}$.

Par conséquent, pour que les populations soutiennent la conservation des aires protégées en Côte d'Ivoire, elles doivent en retirer des avantages socio-économiques conséquents. Ce faisant, l'OIPR et/ou éventuellement les personnes morales de droit privé ayant la charge de la gestion d'aires protégées sont invités à entreprendre le partage des avantages de la conservation de ces ressources avec les communautés locales.

Cet objectif demeure réalisable d'autant plus que les aires protégées elles-mêmes constituent un moyen pour opérer le développement des communautés locales.

\section{Paragraphe 2 : Les aires protégées, un moyen pour le développement des communautés locales}

Selon CHAVIS, la notion de développement des communautés locales renvoie à : " un processus de coopération volontaire, d'entraide et de construction de liens sociaux entre les résidents et les institutions d'un milieu local visant l'amélioration des conditions de vie

\footnotetext{
${ }^{801}$ Il s'agit notamment de petite aquaculture, de projets agricoles à vocation protectrice de l'environnement, d'activités de diversification agricole etc. Voir AFD, Gestion durable de la biodiversité, coll. Parole d'acteurs, n 5 , 2005, p. 24.

${ }^{802}$ Ibidem.

${ }^{803}$ En français Réserve de chasse de Lupande (notre traduction).

${ }^{804}$ Voir LEWIS (D.), BELL (S.D.), FAY (J.), BOTHI (K.L.), GATERE (L.), KABILA (M.), MUKAMBA (M.), MATOKWANI (E.), MUSHIMBALUME (M.) MORARU (C.I.) et al., " Community markets for conservation (COMACO) links biodiversity conservation with sustainable improvements in livelihoods and food production", Proceedings of the National Academy of Sciences of the United States of America, Vol. 108, $\mathrm{n}^{\circ} 40$, 2011, pp. 13958-13959.

${ }^{805}$ Voir ANDRADE (Gustavo S. M.) et RHODES (Jonathan R.), « Protected areas and local communities: an inevitable partnership toward successful conservation strategies ? », Ecology and Society, Vol.17, n4, 2012, p.14.
} 
sur le plan physique, social et économique ${ }^{806}$. De cette définition, il se déduit que se développer induit la réduction de la pauvreté ${ }^{807}$.

En la matière, il ressort de certaines expériences de gestion des ressources naturelles que celles-ci ont permis d'augmenter les moyens de subsistance des communautés locales (A) et de développer les capacités d'organisation sociale desdites communautés (B).

\section{A - Les aires protégées, un outil d'amélioration des moyens de subsistance des communautés locales}

L'appréciation de la capacité des aires protégées à améliorer les moyens d'existence des communautés locales se fera sur la base des expériences de gestion desdites ressources en Afrique de l'Est (1) et en Afrique australe (2).

\section{L'exposé de quelques expériences de gestion d'aires protégées en Afrique de l'Est}

En Afrique de l'Est et plus précisément en Éthiopie, la gestion du Parc National du Balé a permis d'améliorer les conditions de vie des populations riveraines et de les inciter à participer activement à sa conservation ${ }^{808}$. En effet, l'exploitation touristique dudit Parc a contribué à créer des emplois pour les populations locales. De plus, le partage des revenus du tourisme a développé l'économie locale et aidé à réaliser des infrastructures de base (centre de santé, expansion du réseau électrique). Fort de tous ses avantages, les populations soutiennent et encouragent les efforts de conservation du Parc National du Balé ${ }^{809}$.

$\mathrm{Au}$ Kenya, The International Coral Reef Action Network (ICRAN) ${ }^{810}$, aide au développement des communautés riveraines du Parc marin Malindi-Watamu, en vue d'obtenir leur soutien pour la conservation de cette aire protégée. Ainsi, ICRAN appuie les communautés locales en améliorant la réparation et la maintenance des bateaux appartenant

\footnotetext{
${ }^{806}$ Voir CHAVIS (2000) cité dans INSPQ INSTITUT NATIONAL DE SANTÉ PUBLIQUE DU QUÉBEC, La santé des communautés : perspectives pour la contribution de la santé publique au développement social et au développement des communautés, 2002, p. 16.

${ }^{807}$ Fonction des perceptions des peuples et le plus souvent défini en termes économiques, la pauvreté a des dimensions économique et sociale qui intègrent notamment :

- le manque d'actifs, de revenu et de possibilités de mener des activités productives qui procurent des moyens de subsistance ;

- l'absence de liberté d'expression, la non-autonomisation de groupes sociaux, de peuples et leur exclusion des processus de prise de décision, des systèmes de gouvernance et des voies de recours ;

- la vulnérabilité de ceux-ci aux crises économiques, catastrophes naturelles et aux problèmes de santé ;

- et enfin le manque de capacité à promouvoir et à défendre leurs intérêts ${ }^{807}$. Voir SCHERL (Lea M.), WILSON (Alison), WILD (Robert) et al., Can Protected Areas Contribute to Poverty Reduction? Opportunities and Limitations, Gland, Switzerland and Cambridge, UK. , IUCN, 2004, pp. 15-16.

${ }^{808}$ Voir TESSEMA (Mekbeb E.), ASHENAFI (Zelealem T.), LILIEHOLM (Robert J.) et LEADER-WILLIAMS (Nigel), « Community Attitudes towards Wildlife Conservation in Ethiopia», op. cit., p. 290.

${ }^{809}$ Ibidem.

${ }^{810}$ En français, il faudrait lire le Réseau International d'Action pour la protection des Récifs Coralliens (notre traduction).
} 
aux opérateurs locaux. Il en est de même pour les infrastructures d'hébergement pour les visiteurs. De plus, l'ICRAN œuvre au renforcement des capacités des opérateurs de bateaux. C'est dans ce contexte que les projets d'écotourisme ont généré des fonds pour les frais d'écolage des enfants des communautés locales ${ }^{811}$.

Ces exemples qui enseignent que les aires protégées peuvent améliorer les moyens de subsistance des communautés locales se rencontrent également en Afrique australe.

\section{La présentation d'exemples de gestion d'aires protégées en Afrique australe}

En 2001, un rapport d'activités sur les aires de conservation privées en Afrique du Sud révélait que les propriétés privées de faune Mala Mala, situées dans la Réserve de SabiSands, qui opéraient dans le secteur du tourisme employaient 220 personnes dans les années 1990. Parmi ceux-ci, 190 provenaient des communautés locales. Ce rapport indiquait aussi que ces travailleurs avaient 2000 personnes à leur charge. Dans l'ancienne province du Transvaal, le nombre de personnes employées dans l'industrie de la faune privée était de 12.000 avec 100.000 personnes à charge ${ }^{812}$. De même, les populations riveraines du Parc National Hluhluwe-Umfolozi ${ }^{813}$ se sont vues accorder un droit de préemption pour les emplois dans ledit Parc par les gestionnaires. Ces communautés avaient l'opportunité de commercialiser des produits locaux grâce au programme d'écotourisme et de conservation ${ }^{814}$. Ainsi, les revenus des communautés riveraines du Parc National Hluhluwe-Umfolozi avaient augmenté grâce au programme d'écotourisme et de conservation ${ }^{815}$.

En Zambie, la Réserve de chasse de Lupande, contigüe au Sud du Parc National de Luangwa, soutien 50.000 personnes. En effet, si 80\% des revenus issus de la chasse revient aux villageois, deux (2) concessions de chasse rapportent environ 230.000 US\$ aux communautés locales. Une partie de ces revenus est accordée aux villageois. Une autre est destinée à la réalisation de projets décidés par les communautés ${ }^{816}$.

\footnotetext{
${ }^{811}$ Pour ces développements sur les actions de l'ICRAN en ce qui concerne les aires mairines protégées, voir SCHERL (Lea M.), WILSON (Alison), WILD (Robert) et al., Can Protected Areas Contribute to Poverty Reduction? Opportunities and Limitations, Gland, Switzerland and Cambridge, UK., IUCN, 2004, p. 28.

${ }^{812}$ Sur ces différents points, voir KRUG (Wolf), Private Supply of Protected Land in Southern Africa : A Review of Markets, Approaches, Barriers and Issues, Workshop Paper, World Bank / OECD International, Workshop on Market Creation for Biodiversity Products and Services, Paris , 25 and 26 January 2001, p. 35.

${ }^{813}$ En anglais Hluhluwe-Umfolozi Park, le Parc National Hluhluwe-Umfolozi est situé dans la ville de Durban (Afrique du Sud), dans la province du KwaZulu-Natal. Voir FOLIO (Fabrice) et DERROISNE (Anaëlle), « Écotourisme et Parcs naturels : innovations et contradictions sud-africaines, le cas de HIP (Hluhluwe Imfolozi Park) », EchoGéo, Vol. 13, 2010, p. 2.

${ }^{814}$ Sur ces différents points, voir ADELEKE (Bola Olusola), NZAMA (Thandi), « Assessment of Community Participation in Ecotourism and Conservation at Hluhluwe-Umfolozi Park, South Africa », art. cit., p.30.

${ }^{815}$ Ibidem.

${ }^{816}$ Voir CHILD (Brian) et DALAL-CLAYTON (Barry), «Transforming Approached to CBNRM: Learning from the Luangwa Experience, Zambia », in McSHANE (T.O.) et WELLS (M.P.) (eds), Getting Biodiversity Projects to Work : Towards More Effective Conservation and Development, NY, Columbia University Press, 2004, p. 262.
} 
Au total, ces différents exemples attestent que, d'une part, la gestion d'aires protégées permet aux communautés locales de bénéficier d'emplois. D'autre part, elle leur procure des retombées financières qui leur sont directement reversées et/ou qui sont investies dans des projets collectifs identifiées par les communautés elles-mêmes.

Par ailleurs, en termes de développement, les aires protégées ont permis également de développer les capacités des populations en matière d'organisation sociale.

\section{B - Les aires protégées, un moyen pour le développement des capacités d'organisation sociale des communautés locales}

En Afrique, les ressources naturelles, en général, et les aires protégées, en particulier, ont d'abord fait l'objet d'un accaparement par l'État; lequel a centralisé leur gestion ${ }^{817}$. Il s'en est suivi une importante dégradation desdites ressources ${ }^{818}$. C'est ainsi que pour aboutir à de meilleurs résultats en matière de conservation de la nature, il a été adopté la Gestion Communautaire des Ressources Naturelles (GCRN) ${ }^{819}$.

Un bilan des expériences de GCRN en Afrique montre qu'en plus des impacts économiques (amélioration des revenus des communautés) et environnementaux (amélioration de la conservation des ressources naturelles), l'un des impacts importants demeure l'autonomisation des populations locales $^{820}$. Par exemple, en Zambie, dans la vallée

\footnotetext{
${ }^{817}$ Voir ROE (Dilys), NELSON (Fred), «Origines et évolution de la gestion communautaire des ressources naturelles en Afrique », in ROE (Dilys), NELSON (Fred) et SANDBROOK (Chris) (eds), Gestion communautaire des ressources naturelles en Afrique - Impacts, expériences et orientations futures, Londres, Royaume-Uni, Institut International pour l'Environnement et le Développement, Série Ressources Naturelles no. 18,2009 , p. 6.

${ }^{818}$ Voir la première partie de cette thèse, le titre I et précisément le "Chapitre II : Les insuffisances du cadre juridique des aires protégées de 1960 à 2002".

${ }^{819} \mathrm{~L}$ 'accaparement des ressources naturelles et la centralisation de leur gestion par les États africains, après leur accession à l'indépendance, ont été déboutés par les populations et partant eu un impact négatif sur la conservation desdites ressources. C'est fort de l'échec de cette politique de gestion des ressources naturelles que des États adoptent, depuis les années 1980, une stratégie de gestion décentralisée desdites ressources. Elle consiste à accorder aux communautés ou aux groupes d'utilisateurs locaux des droits sur les terres et les ressources naturelles qu'elles regorgent de sorte à en retirer des bénéfices économiques et sociaux. Voir BINOT (Aurélie), BLOMLEY (Tom), COAD (Lauren), ROE (Dilys), NELSON (Fred) et SANDBROOK (Chris), « Participation communautaire à la gestion des ressources naturelles en Afrique - vues régionales d'ensemble ", in ROE (Dilys), NELSON (Fred) et SANDBROOK (Chris), (eds), Gestion communautaire des ressources naturelles en Afrique - Impacts, expériences et orientations futures, Londres, Royaume-Uni, Institut International pour l'Environnement et le Développement, Série Ressources Naturelles no. 18, 2009, p. 41. C'est cette approche de gestion aux formes variées, mais dont l'objectif principal commun vise à intégrer conservation et développement, que l'on a désigné sous le vocable de Gestion Communautaire des Ressources Naturelles (GCRN). Voir ROE (Dilys), NELSON (Fred), « Origines et évolution de la gestion communautaire des ressources naturelles en Afrique », in ROE (Dilys), NELSON (Fred) et SANDBROOK (Chris) (eds), Gestion communautaire des ressources naturelles en Afrique - Impacts, expériences et orientations futures, Londres, Royaume-Uni, Institut International pour 1'Environnement et le Développement, Série Ressources Naturelles no. 18, 2009, pp. 6-7.

${ }^{820} \mathrm{C}$ 'est cette thèse que soutiennent BINOT (Aurélie), BLOMLEY (Tom), COAD (Lauren) et al. lorsqu'ils affirment que «De nombreux analystes soutiennent que le renforcement de l'autonomie est l'un des impacts les plus importants de la GCRN (Cf. p. ex. Arntzenet al., 2003 ; WRI, 2005) - un impact qui dépasse largement tout bénéfice économique ou environnemental. ». Voir BINOT (Aurélie), BLOMLEY (Tom), COAD (Lauren),
} 
de Luangwa, la redistribution des revenus issus de la GCRN a développé chez les communautés locales une capacité d'organisation et renforcé leur autonomie. En effet, il y a été noté un niveau élevé de participation desdites communautés en matière de prise de décision relative à la redistribution des revenus générés par les projets GCRN. De même, ces communautés ont pu développer des capacités de gestion de ces revenus. Il s'agit, entre autres, de l'ouverture de compte bancaire, de l'organisation d'audits de gestion et d'élections régulières pour les différents organes de gestion établis par les communautés ${ }^{821}$.

En Tanzanie, le village d'Ololosokwan situé dans le district de Ngorongoro est doté d'un Conseil villageois qui dispose d'un budget mis en place grâce à la gestion communautaire des ressources naturelles ${ }^{822}$. Ce projet a consisté, pour le village d'Ololosokwan, à conclure des contrats avec le secteur privé touristique.

Ces partenariats ont permis d'augmenter l'enveloppe budgétaire du Conseil villageois $\mathrm{d}^{\prime} O$ lolosokwan ${ }^{823}$. Ces revenus ont contribué à réaliser d'importants investissements dans les services sociaux et de fournir d'autres bénéfices aux villageois ${ }^{824}$. Aussi ces avantages procurés par la gestion des ressources naturelles ont-ils «accru la capacité du village à défendre ses droits sur les terres et les ressources, en utilisant le capital financier du tourisme pour développer le capital politique dans la lutte pour obtenir le contrôle des droits sur les terres et les ressources. $\gg{ }^{825}$.

NELSON (Fred), ROE (Dilys) et SANDBROOK (Chris), " Quels ont été les résultats de la GCRN en Afrique ? Renforcement de l'autonomie, économie, environnement » in ROE (Dilys), NELSON (Fred) et SANDBROOK (Chris) (eds), Gestion communautaire des ressources naturelles en Afrique - Impacts, expériences et orientations futures, Londres, Royaume-Uni, Institut International pour l'Environnement et le Développement, Série Ressources Naturelles no. 18, 2009, p. 65.

${ }^{821}$ Sur ces points, voir CHILD (Brian) et DALAL-CLAYTON (Barry), "Transforming Approached to CBNRM: Learning from the Luangwa Experience, Zambia », in McSHANE (T.O.) et WELLS (M.P.) (eds), Getting Biodiversity Projects to Work : Towards More Effective Conservation and Development, op. cit., pp. 262-263.

${ }^{822}$ Voir NELSON (Fred), OLE MAKKO (S.), Communities, conservation, and conflicts in the Tanzanian Serengeti. Third Annual Community-based conservation network seminar: turning natural resources into assets, Savannah Georgia, forthcoming, 2005 (www.sandcounty.net/assets/chapters/assets_chapter_5.pdf). Accessed 27/6/2006) cités par BINOT (Aurélie), BLOMLEY (Tom), COAD (Lauren), NELSŌN (Fred), ROE (Dilys) et SANDBROOK (Chris), «Quels ont été les résultats de la GCRN en Afrique ? Renforcement de l'autonomie, économie, environnement " in ROE (Dilys), NELSON (Fred) et SANDBROOK (Chris) (eds), Gestion communautaire des ressources naturelles en Afrique - Impacts, expériences et orientations futures, op.cit., p. 66.

${ }^{823}$ Selon NELSON (Fred) et OLE MAKKO (S.), le budget du Conseil villageois du village d'Ololosokwan qui était de 2.500 dollars US entre 1995 et 1997 a atteint environ 60.000 dollars US en 2003, grâce à la conclusion d'accords touristiques entre ledit village et le secteur privé. Voir NELSON (Fred), OLE MAKKO (S.), Communities, conservation, and conflicts in the Tanzanian Serengeti. Third Annual Community-based conservation network seminar: turning natural resources into assets, Savannah Georgia, forthcoming, 2005 (www.sandcounty.net/assets/chapters/assets_chapter_5.pdf). Accessed 27/6/2006) cités par BINOT (Aurélie), BLOMLEY (Tom), COAD (Lauren), NELSON (Fred), ROE (Dilys) et SANDBROOK (Chris), " Quels ont été les résultats de la GCRN en Afrique ? Renforcement de l'autonomie, économie, environnement » in ROE (Dilys), NELSON (Fred) et SANDBROOK (Chris) (eds), Gestion communautaire des ressources naturelles en Afrique-Impacts, expériences et orientations futures, op. cit., p. 66.

${ }^{824}$ Ibidem.

${ }^{825}$ Ibidem. 
Il ressort de ces différentes expériences de gestion d'aires protégées que celles-ci ont contribué au développement des communautés locales. Premièrement, elles ont vu leurs revenus augmenter par la création d'emplois et le versement de bénéfices monétaires découlant des activités développées autour des aires protégées. Deuxièmement, l'implication de ces communautés dans la gestion des aires protégées, des bénéfices et des intérêts en résultant a également permis de développer, en leur sein, des capacités d'organisation sociale.

En définitive, les développements antérieurs permettent d'aboutir à deux (2) conclusions majeures. D'une part, les communautés riveraines des aires protégées soutiennent leur conservation lorsqu'elles en retirent des bénéfices en termes de développement. D'autre part, la gestion des aires protégées peut permettre d'atteindre cet objectif de développement.

C'est à juste titre qu'il y a lieu d'intégrer conservation des aires protégées ivoiriennes et développement des zones périphériques. Toutefois, comment peut-on opérationnaliser cet objectif?

\section{SECTION II : Les modalités de mise en ouvre de l'intégration de la conservation des aires protégées et du développement local}

La mise en œuvre de l'intégration de la conservation des aires protégées ivoiriennes et $\mathrm{du}$ développement local requiert diverses actions. À cet effet, il serait judicieux d'entreprendre des actions de conservation et de développement (Paragraphe 1). Aussi le recours au secteur privé pour la gestion des aires protégées est-il à envisager par l'OIPR (Paragraphe 2).

\section{Paragraphe 1 : Les actions de conservation et de développement à entreprendre}

Les actions de conservation et de développement qui pourraient être entreprises sont de deux (2) ordres. Ils se rapportent aux actions relevant de la responsabilité directe de l'État (A). À l'échelle locale, ces actions consisteront à encourager les communautés locales à mettre en place des projets communautaires de gestion des ressources naturelles dans les zones périphériques des aires protégées $(\mathrm{B})$.

\section{A - Les actions relevant de la responsabilité directe de l'État}

$\mathrm{Au}$ nombre des actions de conservation et de développement à initier par l'État figurent deux (2) catégories d'actions. Il s'agit des actions urgentes et préalables de sauvegarde des aires protégées (1) ainsi que la coordination des actions de développement autour de la conservation des aires protégées (2). 


\section{Les actions urgentes et préalables de sauvegarde des aires protégées}

Les aires protégées de Côte d'Ivoire sont actuellement soumises à de fortes pressions anthropiques qui menacent gravement l'existence de certaines d'entre elles ${ }^{826}$. Par conséquent, il importe de mener des actions urgentes en vue d'inverser cette tendance.

Dans un premier temps, l'OIPR devrait s'atteler à régler les différends fonciers relatifs aux aires protégées. Nous faisons allusion aux contestations nées de modifications des limites d'aires protégées au détriment des terroirs villageois ainsi qu'aux indemnisations liées à la création d'aires protégées qu'elles n'auraient pas perçues ${ }^{827}$.

Sur la question, il est souhaitable que les limites contestées soient redéfinies ou reprécisées. Cette solution d'ordre pratique destinée à protéger les aires protégées est facilitée par une loi modificative de la loi de 2002 sur les $\mathrm{PNR}^{828}$. En effet, l'article 9 alinéa 2 nouveau de la loi de 2002 sur les PNR indique : "Pendant une durée de cinq ans, à compter de la date d'entrée en vigueur de la présente loi, le Gouvernement est autorisé à modifier, par décret, les limites administratives existantes des Parcs Nationaux et des Réserves naturelles, sans qu'il puisse en résulter une diminution supérieure à deux pour cent de la superficie de chaque Parc ou Réserve, et sans permettre l'enclavement de zone habitées ou cultivées. ».

Ce faisant, pour les cas de limites d'aires protégées contestées, de nouvelles délimitations devraient être faites selon les modalités suivantes. Il s'agira de consulter les documents dont dispose l'Administration; d'examiner les documents ou le cas échéant les témoignages émanant des populations. Dans tous les cas, en fonction des intérêts de conservation et de ceux des communautés, des limites consensuelles, acceptées par tous et définitives devraient être établies dans ces cas, conformément à la loi. Dès lors, elles devraient être constatées par des documents administratifs.

À cet effet, un comité tripartite (OIPR, Communautés locales et Administration locale) pourrait être mise en place. De plus, ces limites redéfinies ainsi que les limites non contestées devraient être dûment matérialisées afin d'éviter tout risque de confusion, à l'avenir.

Dans un deuxième temps, pour ce qui est des occupants illégaux des aires protégées, l'État de Côte d'Ivoire est invité à démontrer une volonté ferme d'apurement de ces forêts.

\footnotetext{
${ }^{826}$ Voir en Introduction (de cette étude), le point "I- Enjeux de la protection des forêts en Côte d'Ivoire", " Cl'état des pressions anthropiques sur la biodiversité en Côte d'Ivoire", et précisément le " Paragraphe 2 : Les pressions anthropiques sur les aires protégées en particulier ".

${ }^{827}$ Voir la Deuxième partie de la présente étude, le Titre I, le Chapitre II, la Section I, le Paragraphe 2, le point B (La survivance des problèmes relatifs au foncier dans les aires protégées) et précisément le point "1. La persistance des problèmes de revendications d'indemnisation et de contestation de limites d'aires protégées par les communautés locales".

${ }_{828}$ Il s'agit de la loi n²013-864 du 23 décembre 2013 modifiant l'alinéa 2 de l'article 9 de la loi n²002-102 du 11 février 2002 relative à la création, à la gestion et au financement des Parcs Nationaux et des Réserves naturelles (JORCI ${ }^{\circ} 1$ du vendredi 3 janvier 2014, Numéro Spécial, p. 5).
} 
Ces occupants illégaux sont pour la plupart des migrants économiques qui arrivent de l'Afrique de l'Ouest, en général, et du Burkina Faso, en particulier ${ }^{829}$; surtout depuis la crise militaro-politique de 2002. Certes, l'exploitation agricole des aires protégées en Côte d'Ivoire remonte à une date plus ancienne. Cependant, il ne s'agissait que de grignotages de certaines limites d'aires protégées par les communautés locales pour la pratique de cultures vivrières. Ces grignotages n'étaient pas de nature à remettre en cause l'existence de ces massifs forestiers. Toutefois, l'infiltration massive et incontrôlée des aires protégées ivoiriennes par ces migrants économiques pour la culture du cacao et d'autres cultures de rente date de depuis $2002^{830}$. Ce faisant, ceux-ci n'arrêterons pas de coloniser les aires protégées pour la pratique de l'agriculture de rente et à des fins d'habitation, si des mesures vigoureuses ne sont pas prises à leur encontre.

Ainsi, en premier lieu, l'OIPR est appelé à informer les occupants illégaux d'arrêter les nouveaux défrichements car les jeunes plantations seront systématiquement détruites. De plus, ils devraient être informés qu'ils seront invités à se faire identifier sur déclaration; et qu'il sera procédé à une vérification sur le terrain, en présence des occupants voisins. De la sorte, ils pourront bénéficier des avantages d'un déguerpissement à caractère humanitaire ou sociale.

En second lieu, il s'agira de recenser ces occupants illégaux afin d'établir un fichier numérique fiable. Ce fichier devrait renseigner sur leur identité, les superficies exploitées, les cultures pratiquées ainsi que le niveau de développement desdites cultures (jeunes plants, plantations en production...). Également, la destruction systématique de toutes nouvelles plantations et des plantations voisines telle qu'annoncée aux populations, sauf dénonciation des contrevenants par les voisins, est conseillée. Ainsi, les exploitants d'un périmètre n'agrandiront pas leurs plantations ou n'accepterons pas de voisins dont l'activité menacerait leurs intérêts.

Pour le cas des plantations en production ou sur le point de produire, l'OIPR est exhorté à signer, avec les occupants illégaux de cette catégorie, un contrat d'exploitation temporaire. Ce contrat accordera des droits aux occupants illégaux et mettra à leur charge des obligations. En effet, le contrat d'exploitation temporaire aura pour objet de permettre l'accès de l'occupant aux plantations existant dans les aires protégées en vue de leur récolte. Cette récolte pourrait se faire sur une période deux (2) ans renouvelables, si l'exploitant illégal respecte scrupuleusement les stipulations du contrat. À titre indicatif, il devra dans le trimestre de la signature du contrat d'exploitation temporaire solliciter, auprès des services de l'OIPR ou d'un partenaire technique qui lui sera indiqué, un nombre de plants d'arbres que

\footnotetext{
${ }^{829}$ Voir la Deuxième partie de la présente thèse, le Titre I, le Chapitre II, la Section II, le Paragraphe 2, le point intitulé "C- Les limites relatives au conflit au conflit militaro-politique de septembre 2002".

${ }^{830} \mathrm{Cf}$. les références ci-dessus et également AKINDÈS (Francis), KOUAMÉ (Kra Walter), SÉDIA (Gisèle) et SÉZAN (Jules), Réalisation d'une étude sur l'état de la biodiversité des parcs nationaux et réserves de Côte d'Ivoire. Aspects socio-économiques (Rapport provisoire), op. cit., pp. 91-92.
} 
devra contenir la parcelle qu'il exploite ${ }^{831}$. Il en sera de même chaque année. De la sorte, au bout d'un certain nombre d'années, les arbres plantés auront occupé les surfaces cultivées, au terme du contrat d'exploitation temporaire.

Ces arbres, une fois plantés, sont sous la responsabilité de l'occupant illégal cocontractant. Il bénéficiera du soutien technique des services compétents désignés à cet effet. Leur bon entretien par l'occupant illégal cocontractant lui permettra de voir son contrat d'exploitation temporaire renouvelé. Cependant, en cas de destruction desdits arbres, la plantation de l'exploitant illégal cocontractant est détruite. De plus, son nom sera rayé de la base des occupants illégaux d'aires protégée bénéficiant du programme de déguerpissement négocié.

En troisième lieu, le plan d'apurement des aires protégées ci-dessus devra, dans sa mise en œuvre, faire l'objet d'un suivi par l'OIPR qui responsabilisera ses personnels (en général et de terrain en particulier). Pour éviter la corruption dont ils sont souvent accusés ${ }^{832}$, ceux-ci seront évalués sur la base de leurs résultats, en fonction des périmètres dont ils sont responsables. Les agents compétents et honnêtes pourraient être félicités et encouragés dans l'accomplissement de leurs missions. À l'inverse, la responsabilité (civile, pénale et administrative) des agents corrompus ainsi que leur carrière devraient être engagées.

En quatrième et dernier lieu, ces différentes mesures devraient être accompagnées par une politique de communication qui vise à informer, sensibiliser les communautés locales sur l'importance de la conservation des aires protégées. L'accent pourrait, par exemple, être mis surtout sur le fait qu'elles favorisent les conditions climatiques propices (température, pluviométrie...) pour la pratique de l'agriculture, entres autres.

Par ailleurs, pour soutenir et pérenniser les résultats des actions préalables et urgentes de sauvegarde des aires protégées, l'OIPR et le gouvernement ivoirien sont invités à œuvrer à la coordination des actions de développement dans les zones périphériques d'aires protégées, autour des aires protégées.

\footnotetext{
${ }^{831} \mathrm{Ce}$ nombre de plants d'arbres ainsi que les espèces requises seront déterminés par les services techniquement compétents afin de favoriser le reboisement progressif des aires protégées dégradées par les exploitations agricoles.

${ }^{832}$ Les agents en charge de la surveillance des Parcs Nationaux et Réserves sont accusés de corruption dans le cadre de leur mission. En effet, ils laisseraient des individus piller les ressources naturelles des Parcs Nationaux du Mont Sangbé, du Mont Péko, de la Marahoué et d'Azagny. Voir AKINDĖS (Francis), KOUAMÉ (Kra Walter), SÉDIA (Gisèle) et SÉZAN (Jules), Réalisation d'une étude sur l'état de la biodiversité des parcs nationaux et réserves de Côte d'Ivoire : Aspects socio-économiques (Rapport provisoire), op. cit., pp. 19, 33, 45 et 98 .

De plus, traitant de l'implication des agents en charge de la surveillance du patrimoine forestier dans l'exploitation frauduleuse du Parc National du Mont Sangbé (PNMS), le Professeur AKINDÈS (Francis) et al. mentionnent: "Cette complicité, de l'avis des divers acteurs rencontrés, serait également le fait de certains agents des Eaux et Forêts qui sembleraient en tirer profit : "Aujourd'hui, l'exploitation de bois est un commerce international à Biankouma, Touba et Man. Elle est devenue la source d'argent PMU de quelques agents des Eaux et Forêts. Lorsqu'ils voient de l'argent, ils ne font plus de la protection mais de la contrebande ..." confiait l'un des interlocuteurs de la mission sur le terrain. ». Idem, p. 19.
} 


\section{La coordination des actions de développement local autour de la conservation des aires protégées}

Plusieurs ministères interviennent dans les zones périphériques des aires protégées en vue de l'accomplissement de leurs missions respectives. Il s'agit à titre indicatif des ministères de l'Agriculture, du Commerce, des Eaux et Forêts, de l'Intérieur, de la Sécurité et de l'Éducation etc. Les actions de ses différents ministères dans ces zones ont pour objectifs de concourir au bien-être des populations c'est-à-dire au développement.

Il a été antérieurement indiqué que les enquêtes réalisées dans le cadre de cette étude attestent que les causes des pressions sur les aires protégées sont diverses. Ce sont essentiellement la baisse de fertilité des sols et par voie de conséquence la baisse des rendements agricoles qui accentue la pauvreté et la disparition du couvert forestier dans les terroirs villageois $^{833}$.

Aussi l'OIPR devrait-elle établir une cartographie des exploitations agricoles opérant dans les zones périphériques des aires protégées. Ces agriculteurs bénéficieront d'un programme de pratique d'agriculture durable. À travers ce programme, d'une part, les agriculteurs verront leur capacité renforcer sur l'utilisation de techniques culturales qui améliorent la conservation et la fertilité du sol. D'autre part, il pourrait être promu une agriculture durable par le renforcement des capacités et l'incitation des communautés à la pratique de l'agroforesterie ; l'encouragement et la facilitation de l'obtention de plants à fort taux de rendement.

De même, les revenus des communautés devraient être assurés par l'organisation de la collecte et de la commercialisation des produits agricoles aux meilleurs prix. Ainsi, cette politique incitative à la rentabilité agricole augmentera les revenus des planteurs. Par contrecoup, elle diminuera l'extension des périmètres agricoles fait dans le souci de compenser les pertes de rendements et partant l'exploitation agricole des aires protégées.

En outre, les capacités des populations locales devraient être renforcées à travers un programme d'éducation à l'utilisation et à la conservation durable des ressources naturelles dans les zones périphériques des aires protégées. L'accent pourrait être mis, par exemple, sur l'utilisation rationnelle du bois d'énergie par la vulgarisation de foyers améliorés et d'autres sources d'énergie (gaz butane).

La mise en œuvre des recommandations ci-dessus requiert l'intervention de plusieurs ministères. À titre indicatif, il y a le ministère de l'Environnement, de l'Agriculture, des Eaux et Forêts, du Commerce et celui en charge du plan et du développement. C'est à juste titre que la mise en place d'un Comité Interministériel de Coordination des Actions de

\footnotetext{
${ }^{833}$ Voir l'Annexe 3 de la présente étude intitulé "Rapport de l'enquête sur les réalités de la gestion des aires protégées en côte d'ivoire", le point "II- Résultats de l'enquête auprès des communautés riveraines des aires protégées" et précisément le point "5. Les raisons des pressions sur les aires protégées".
} 
Développement dans les Zones Périphériques d'aires protégées (CICAD-ZP) faciliterait cette synergie d'action attendue de ces différents ministères. Ce comité sera représenté à un échelon inférieur par des cellules locales là où se trouve une aire protégée. Le CICAD-ZP et partant ses cellules locales auront pour objet de coordonner les actions de développement autour des aires protégées.

Dans ce comité interministériel, le ministère de l'Environnement qui assure la tutelle technique des aires protégées assurera le secrétariat et le suivi des décisions par le canal de l'OIPR. Le ministère de l'Agriculture aura la responsabilité de l'encadrement technique du monde paysan en vue de l'utilisation de techniques et de matériel végétal qui garantit un bon rendement à l'hectare ainsi que la pratique de l'agroforesterie. Le ministère du commerce œuvrera à l'organisation et la collecte ainsi que la commercialisation des produits agricoles à un juste prix qui garantit un bon revenu à l'hectare. En ce qui concerne le ministère des Eaux et Forêts, il renforcera les capacités du monde paysan sur le choix des essences à croissance rapide et à fort pouvoir calorifique. À cet effet, les ONG et autres compétences techniques pourront être sollicités afin d'accompagner ces initiatives. Elles devront surtout vulgariser des techniques d'utilisation durable de la biodiversité ; et du bois d'énergie à travers les foyers améliorés par exemples. S'agissant du ministère en charge du plan et du développement, il aura la responsabilité technique de la coordination et de l'efficacité de ces actions de développement. Enfin, le ministère de l'intérieur et de la sécurité interviendra pour la mise en œuvre des déguerpissements et du suivi de cette politique.

Au-delà de ces actions à entreprendre par l'État, les pouvoirs publics et leurs partenaires sont exhortés à encourager les communautés locales à mettre en place des projets communautaires de gestion durable des ressources naturelles.

\section{B - L'encouragement des communautés locales à la mise en place de projets communautaires de gestion des ressources naturelles dans les zones périphériques des aires protégées}

La mise en place de projets communautaires de gestion des ressources naturelles revêt un intérêt (1) qu'il convient de présenter avant de proposer des exemples de projets à initier (2). Aussi des recommandations relatives à la gestion de ces projets communautaires de gestion des ressources naturelles seront-elles formulées (3).

\section{L'intérêt de la mise en place de projets communautaires de gestion des} ressources naturelles dans les zones périphériques des aires protégées

En Côte d'Ivoire, le constat est que les populations rurales (essentiellement pauvres) sont dépendantes des ressources naturelles, compte tenu de leur faible pouvoir économique. C'est ainsi que dans un contexte de démographie galopante, elles exercent des pressions sur 
les ressources naturelles des terroirs villageois et aussi des aires protégées lorsque ces ressources se raréfient dans l'espace rural $^{834}$.

Dans des cas similaires de pressions anthropiques sur les ressources naturelles, il a été adopté comme solution la mise en place de projets de gestion communautaire des ressources naturelles (GCRN). Ces projets ont eu pour objectif, à la fois, de conserver la biodiversité desdites ressources y compris celles des aires protégées et de réaliser le développement des communautés locales ${ }^{835}$.

Un bilan de la gestion communautaire des ressources naturelles (GCRN) en Afrique enseigne que cette approche de gestion a permis, au-delà des critiques ${ }^{836}$, d'enregistrer des résultats satisfaisants tant en matière de développement local qu'en matière de conservation. C'est à juste titre que traitant du cas du Botswana, Joseph MBAIWA et Amanda STRONZA

${ }^{834}$ Sur la question, dans leur rapport sur l'état de la biodiversité des Parcs Nationaux et Réserves de Côte d'Ivoire, le Professeur AKINDÈS (Francis) et al. indiquent : «...l'Ouest ivoirien souffrait déjà, comme dans tout le milieu rural, d'une aggravation de la pauvreté. Selon les résultats de l'enquête sur le niveau de vie $(E N V)$ de 2008, trois pauvres sur quatre vivaient alors en milieu rural (source, PND 2012-2015). Certaines de ces populations voient donc logiquement, dans l'exploitation des PNR, une source de revenus. Dans un tel contexte de croissance de la pauvreté et de raréfaction de ressources foncières dans le domaine rural, l'on peut comprendre le sens et la logique des pressions sur les ressources des parcs et réserves, soit pour tirer rente de leur mise en exploitation, soit pour les exploiter directement. ". Voir AKINDÈS (Francis), KOUAMÉ (Kra Walter), SÉDIA (Gisèle) et SÉZAN (Jules), Réalisation d'une étude sur l'état de la biodiversité des parcs nationaux et réserves de Côte d'Ivoire : Aspects socio-économiques (Rapport provisoire), op. cit., p. 19.

Selon le Cinquième rapport national sur la diversité biologique de la Côte d'Ivoire : "La pauvreté accrue des populations et surtout celle des populations vulnérables, les conduisent à prélever considérablement les espèces de faune et de flore. ». Voir République de Côte d'Ivoire, Cinquième rapport national sur la diversité biologique, Abidjan, mars 2014, p. 4

${ }^{835}$ Voir NEWMARK (W.), HOUGH (J.), « Conserving wildlife in Africa: Integrated conservation and development projects and beyond », BioScience, Vol. 50, n 7, 2000, p. 585 ; BARAL (Nabin), STERN (Marc J.), HEINEN (Joël T.), « Integrated conservation and development project life cycles in the Annapurna conservation area, Nepal: is development overpowering conservation ? ", Biodiversity Conservation, Vol. 16, $\mathrm{n}^{\circ} 10,2007$, p. 2903; BERKES (F.), « Rethinking community-based conservation », Conservation Biology, Vol. $18, \mathrm{n}^{\circ} 3,2004$, p. 621.

${ }^{836} \mathrm{Au}$ Botswana par exemple, des sociétés de gestion de projets communautaires de ressources naturelles y compris des élites locales ont détourné ou mal géré les revenus touristiques issus de l'exploitation des aires de conservation de la faune. Voir BINOT (Aurélie), BLOMLEY (Tom), COAD (Lauren), NELSON (Fred), ROE (Dilys), SANDBROOK (Chris), "Quels ont été les résultats de la GCRN en Afrique ? Renforcement de l'autonomie, économie, environnement » in ROE (Dilys), NELSON (Fred), SANDBROOK (Chris) (eds), Gestion communautaire des ressources naturelles en Afrique - Impacts, expériences et orientations futures, op.cit., pp. 66 et 71.

En Tanzanie, dans certains villages, des revenus touristiques ont été détournés. Au Kenya, l'échec de la gestion de ranchs collectifs a conduit les pasteurs à individualiser les pâturages (voir Idem, p. 66). Pour NEWMARK (William D.) et HOUGH (John L.), en Afrique, de nombreux projets communautaires n'ont pas eu le succès escompté à cause des hypothèses erronées faites par les concepteurs de tels projets. NEWMARK (William D.) et HOUGH (John L.), « Conserving Wildlife in Africa: Integrated Conservation and Development Projects and Beyond », art. cit, pp. 588-589. Sur l'impact limité des projets intégrés de conservation et de développement, voir également BARRETT (CB), ARCESE (P.), « Are integrated conservation-development projects (ICDPs) sustainable? On the conservation of large mammals in sub-Saharan Africa ", World Development, Vol. 23, $\mathrm{n}^{\circ}$, 1995, p. 1073; GIBSON (Clark C.), MARKS (Stuart A.) « Transforming rural hunters into conservationists: An assessment of community-based wildlife management programs in Africa ", World Development, Vol. $23, \mathrm{n}^{\circ} 6$, 1995, p. 941. OATES (John F.), «The dangers of conservation by rural development -a case study from the forests of Nigeria», Oryx Vol. 29, n², 1995, p. 115. 
soutiennent que pour réaliser les objectifs de conservation et de développement local, les décideurs devraient encourager la gestion communautaire des ressources naturelles ${ }^{837}$.

En effet, une étude menée par ces auteurs sur la gestion communautaire des ressources naturelles dans le delta de l'Okavango (Botswana) conclut que cette approche de gestion a contribué significativement à l'amélioration des moyens de subsistance des communautés locales ${ }^{838}$. D'autres travaux de recherches ont été conduits par ces auteurs dans la même zone d'étude et plus précisément dans les villages de Sankoyo, Mababe et Khwai. Ceux-ci révèlent que la gestion communautaire des ressources naturelles a créé des opportunités d'emplois au profit des communautés locales. Elle a aussi généré d'importants revenus qui ont servi à soutenir des activités communautaires (aides pour l'organisation des funérailles; d'activités sportives...). Ces revenus ont été également utilisés pour la réalisation d'équipements communautaires (construction de borne-fontaines, télévision, radios) ainsi que l'assistance de couches vulnérables de la population (construction de maisons pour les personnes âgées et les nécessiteux ; aide aux orphelins et handicapés $)^{839}$. De plus, ces auteurs notent que fort de ces retombées économiques, la conservation des ressources naturelles (telles que l'herbe de chaume, la girafe et d'autres ressources) s'est améliorée ${ }^{840}$.

En Namibie, la gestion communautaire des ressources naturelles dans le cadre des conservancies (en français concessions de conservation-notre traduction) ${ }^{841}$ a généré

\footnotetext{
${ }^{837}$ Voir MBAIWA (Joseph E.), STRONZA (Amanda L.), « Changes in resident attitudes towards tourism development and conservation in the Okavango Delta », Journal of Environmental Management, Vol. 92, , n ${ }^{\circ}$, 2011, p. 1950.

${ }^{838}$ Voir MBAIWA (Joseph E.) et STRONZA (Amanda L.), « The effects of tourism on rural livelihoods in the Okavango Delta, Botswana», Journal of Sustainable Tourism, Vol. 18, n5, 2010, p. 635.

${ }^{839}$ Voir MBAIWA (Joseph E.), STRONZA (Amanda L.), « Changes in resident attitudes towards tourism development and conservation in the Okavango Delta », art. cit., p. 1957. D'autres études confirment que la GCRN a contribué à améliorer de manière significative les moyens de subsistance des populations des villages de Sankoyo, (au Botswana). Voir ARNTZEN (J.), MOLOKOMME (D.L.), TERRY (E.M.), MOLEELE (N.), TSHOSA (O.) et MAZAMBANI (D.), Final Report of the Review of Community-Based Natural Resource Management in Botswana Volume 1 : Main findings A study carried out for the National CBNRM Forum, UICN, Departement for International Development, September 2003, pp. 51-52. NB: ce document (consulté le 5 février 2016, sur www.the-eis.com/data/literature/FinalReport CBNRMReview.pdf) n'est pas paginé à l'origine. Ces numéros et nombres de pages qui figurent ci-dessous sont déduits du nombre de pages que contient le rapport dont il fait l'objet.

${ }^{840}$ Voir MBAIWA (Joseph E.), STRONZA (Amanda L.), « Changes in resident attitudes towards tourism development and conservation in the Okavango Delta », art. cit., p. 1956.

${ }^{841}$ Prévues par la loi namibienne de 1996 sur la gestion de la faune sauvage, les Conservancies ou au singulier Conservancy sont des entités juridiques ou institutions communautaires de gestion des ressources naturelles par le truchement desquelles la gestion de ressources naturelles sur des terres communales géographiquement délimitées est transférée par l'État aux communautés locales. Une fois constituée, selon la législation en vigueur, les communautés locales ont pour obligation la gestion durable des ressources naturelles du ressort de la Conservancy. En contrepartie, il leur est reconnu un droit de propriété sur lesdites ressources. Les communautés locales peuvent donc faire une utilisation consommatrice et non consommatrice desdites ressources. C'est ainsi qu'elles les utilisent à la fois pour des besoins de subsistance et de développement de l'écotourisme, en fonction d'une délimitation qui affecte chaque partie de la concession de conservation à une fonction précise. Voir MASSONNIE (Karine), Le cas des Conservancies en Namibie (www.kinoutour.overblog.com/article 6403177.html, (document non paginé), publié le 16 avril 2007 et consulté le 2 août 2015 ; voir SCHERL (Lea M.) et al., Can Protected Areas Contribute to Poverty Reduction ? Opportunities and Limitations. IUCN, Gland, Switzerland and Cambridge, UK., p. 38.
} 
d'importants revenus aux communautés locales ${ }^{842}$. Ces revenus ont permis de réaliser des investissements collectifs à caractère communautaire (centre de santé, écoles...) et/ou été distribués selon les décisions des instances de gestion aux communautés. C'est fort de ces retombées de la conservation en terme de développement que par exemple, dans le Nord de la Namibie, ce type de GCRN a contribué à rétablir les populations de faune y compris les espèces en voie disparition (zèbre de Hartmann, rhinocéros noir, éléphant...) ${ }^{843}$.

Au Kenya, dans le district Laikipia, des terres appartenant à des propriétaires privés, des communes ou des communautés locales sont constituées en aires de conservation de la faune et gérées collectivement à des fins touristiques. Il s'en est suivi un rétablissement et une augmentation des populations de faune (zèbre, lions, hyènes) dans cette zone ${ }^{844}$.

En République centrafricaine, dans le cadre des activités des Zones Cynégétiques Villageoises (ZCV), il a été mis en place des projets communautaires que sont les zones de chasse autour des aires protégées. Ces projets communautaires ont permis d'augmenter certaines populations de faune que sont, entre autres, les éléphants, le léopard, le phacochère, le sanglier de brousse et l'hylochère ${ }^{845}$.

Enfin, le bilan sur la GCRN en Afrique de l'Ouest révèle que le Projet pilote de gestion communautaire des ressources naturelles et de la faune en Afrique de l'Ouest et précisément en Côte d'Ivoire et au Burkina a contribué à la conservation des ressources naturelles. En effet, il a été enregistré une réduction des empiètements agricoles dans les zones de conservation que le projet a créées ${ }^{846}$.

Il découle de tous ces exemples de GCRN que cette approche de gestion a permis d'améliorer la conservation de l'environnement en général et des aires protégées en particulier, en raison des retombées financières que les communautés en retirent ${ }^{847}$. Par conséquent, la mise en place de projets communautaires de gestion des ressources naturelles autour des aires protégées étatiques en Côte d'Ivoire pourrait permettre de concilier la conservation des aires protégées et le développement local. Ces aires de conservation communautaires auront un double avantage. D'une part, elles fourniront aux communautés locales des ressources naturelles, selon les besoins. Ainsi, elles constitueraient des boucliers

\footnotetext{
${ }^{842}$ Voir MASSONNIE (Karine), Le cas des Conservancies en Namibie (disponible sur www.kinoutour.overblog.com/article 6403177.html, (document non paginé), publié le 16 avril 2007 et consulté le 2 août 2015.

${ }^{843}$ Voir BINOT (Aurélie), BLOMLEY (Tom), COAD (Lauren), NELSON (Fred), ROE (Dilys), SANDBROOK (Chris), «Quels ont été les résultats de la GCRN en Afrique ? Renforcement de l'autonomie, économie, environnement » in ROE (Dilys), NELSON (Fred), SANDBROOK (Chris) (eds), Gestion communautaire des ressources naturelles en Afrique - Impacts, expériences et orientations futures, op. cit., , p. 98.

${ }^{844}$ Idem, p. 99.

${ }^{845}$ Idem, p. 100.

${ }^{846}$ Ibidem.

${ }^{847}$ C'est fort de l'importance de ces aires de conservation communautaire pour la conservation de la biodiversité au service du développement que le Vème congrès mondial sur les Parcs de Durban de 2003 les a recommandées pour une conservation durable de la biodiversité mondiale. Voir Recommandation $5.26 \mathrm{du} \mathrm{Ve}$ Congrès mondial sur les Parcs tenue du 8 au 17 septembre 2003, à Durban (Afrique du Sud).
} 
écologiques pour les aires protégées. D'autres part, elles pourraient leurs procurer d'autres moyens financiers de subsistance, en raison du mode d'exploitation qu'elles auront adopté. Aussi convient-il de proposer quelques exemples de projets communautaires à initier en Côte d'Ivoire.

\section{Quelques exemples de projets communautaires de gestion des ressources naturelles à initier}

Les projets communautaires dont nous proposons la mise en place sont la constitution de forêts communautaires autour des aires protégées sous la forme de Réserve naturelle ou de Réserves naturelles volontaires. La législation ivoirienne permet la mise en place de telles aires de conservation. En effet, d'une part, la loi de 2002 sur les aires protégées en son article $1^{\text {er }}$ prévoit une catégorie d'aire protégée qu'est la Réserve naturelle volontaire ${ }^{848}$. De la sorte, les communautés riveraines des aires protégées pourraient être encouragées à dégager des terres sur lesquelles elles détiennent des droits fonciers en vue de la création de Réserves naturelles volontaires communautaires (RNVC).

Les RNVC pourrait faire l'objet d'un découpage en trois (3) zones ayant chacune une fonction donnée. Il s'agit d'abord d'une zone non exploitable ou zone A. Ensuite, il y a une zone exploitable sur le plan commercial ou zone B; et enfin une zone d'exploitable durable ou zone $\mathrm{C}$. La zone non exploitée ou zone $\mathrm{A}$, contigüe à une aire protégée étatique, est une réserve de biodiversité pour les communautés et un bouclier contre les pressions sur l'aire protégée étatique. En ce qui concerne la zone exploitable sur plan commerciale ou zone B, elle pourrait notamment être affectée à des projets écotouristiques; la culture d'espèces végétales qui pourraient rapporter aux communautés une forte valeur ajoutée. Quant à la zone d'exploitation durable ou zone C, elle est celle qui peut être exploitée par la communauté pour ses besoins en ressources naturelles selon des règles définies par elle et qui en assure la viabilité.

D'autre part, le Code forestier de 2014 permet aux communautés rurales de créer des forêts communautaires. En effet, aux termes de l'article 40 dudit code, "Les forêts des communautés rurales sont des forêts protégées appartenant à une ou plusieurs communautés rurales. Elles sont de catégories suivantes :

- les forêts naturelles situées sur des terres sur lesquelles les communautés rurales jouissent d'un droit de propriété ou de droits coutumiers conformes à la législation domaniale et foncière ;

- Les plantations forestières créées sur des terres immatriculées au nom des communautés rurales ou sur des terres occupées par celles-ci en vertu de la coutume ou d'un bail...».

\footnotetext{
${ }^{848}$ La Réserve naturelle volontaire est une innovation de la loi de 2002 sur PNR. L'article $1{ }^{\text {er }}$ ladite loi la définit comme étant: "la réserve naturelle partielle créée à l'initiative d'une Collectivité territoriale, d'un Établissement public ou d'une personne de Droit privé, sur un terrain lui appartenant et pour la préservation d'un écosystème ou d'un paysage remarquable...».
} 
Par ailleurs, nos recommandations portent également sur la gestion des projets communautaires de gestion des ressources naturelles.

\section{Les recommandations relatives à la gestion des projets communautaires de gestion des ressources naturelles}

Les recommandations relatives à la gestion des projets communautaires de gestion des ressources porteront sur les droits des communautés locales (a) ainsi que la gestion courante de ces projets (b).

\section{a. Les recommandations relatives aux droits des communautés locales}

La question des droits des communautés locales relative aux projets de gestion communautaire des ressources naturelles est largement discutée dans plusieurs travaux de recherche en la matière ${ }^{849}$. Il ressort de ces études que le fait pour les communautés locales de détenir un droit de propriété sur les terres et les ressources naturelles qui s'y trouvent demeure une des conditions pour mener à bien les projets communautaires ${ }^{850}$. Ce droit de propriété implique la latitude des communautés de déterminer au niveau local des règles de gestion et d'utilisation des ressources naturelles ainsi que des dividendes qui résultent de leur exploitation $^{851}$. En d'autres termes, la réussite des projets de conservation communautaire commande que l'autorité de la gestion des ressources naturelles soit déléguée aux communautés locales.

Selon Wolf KRUG, les expériences de gestion communautaire des ressources naturelles en Afrique australe enseignent que pour réussir la conservation de la biodiversité, il faudrait que les particuliers possèdent un droit de propriété sur les terres qui constituent l'assise des aires de conservation et la faune sauvage qui la peuple ${ }^{852}$. On comprend dès-lors que traitant singulièrement de l'expérience botswanaise, Joseph MBAIWA et Amanda STRONZA aboutissent à une conclusion similaire. En effet, en étudiant la conservation de la biodiversité dans les localités de Khwai, Mababe et Sankoyo situées dans le Delta de

\footnotetext{
${ }^{849}$ Voir ROE (Dilys) et NELSON (Fred), « Origines et évolution de la gestion communautaire des ressources naturelles en Afrique» in ROE (Dilys), NELSON (Fred) et SANDBROOK (Chris) (eds), Gestion communautaire des ressources naturelles en Afrique - Impacts, expériences et orientations futures, Londres, Royaume-Uni, Institut International pour l'Environnement et le Développement, Série Ressources Naturelles, $n^{\circ}$ 18, 2009, p. 10 ; BOUDREAUX (Karol C.), Community Conservation in Namibia : Devolution a Tool for the Legal Empowerment of the Poor, Working Paper n 10-64, Mercatus Center at Georges Mason University, 2010, p. 21; MBAIWA (Joseph E.), STRONZA (Amanda L.), "Changes in resident attitudes towards tourism development and conservation in the Okavango Delta », art. cit., p.1958.

${ }^{850}$ Voir ROE (Dilys) et NELSON (Fred), « Origines et évolution de la gestion communautaire des ressources naturelles en Afrique»" in ROE (Dilys), NELSON (Fred) et SANDBROOK (Chris) (eds), Gestion communautaire des ressources naturelles en Afrique - Impacts, expériences et orientations futures, op.cit., p. 10 .

${ }^{851}$ Ibidem.

${ }^{852}$ Voir KRUG (Wolf), Private Supply of Protected Land in Southern Africa: A Review of Markets, Approaches, Barriers and Issues, Workshop Paper, World Bank / OECD International, Workshop on Market Creation for Biodiversity Products and Services, Paris, 25 and 26 January 2001, p. 2.
} 
l'Okavango (Botswana), ces auteurs concluent que le succès de la gestion communautaire des ressources naturelles requiert, entres autres conditions ${ }^{853}$, la reconnaissance de droit tant sur le foncier que les ressources naturelles aux communautés locales ${ }^{854}$.

Par conséquent, pour réussir les projets de conservation communautaire tels que les RNVC ou les forêts communautaires à mettre en place autour des aires protégées ivoiriennes, il est nécessaire que les communautés locales disposent d'un droit de propriété sur les terres qui en constituent l'assise. Il en est de même pour la biodiversité qu'elles regorgent.

En la matière, en l'état actuel de la législation en Côte d'Ivoire, il n'y a pas d'obstacle juridique pour l'accession des personnes (ivoiriennes) autres que l'État à la propriété foncière tant en milieu urbain que rural. En effet, la loi de 98-750 du 23 décembre 1998 relative au foncier rural consacre l'accès à la propriété des personnes physiques ${ }^{855}$. Il en résulte que par ce moyen, les populations rurales peuvent créer des forêts communautaires.

La possibilité de créer des forêts communautaires dont elles sont propriétaires est également offerte par le Code forestier de 2014 aux communautés rurales. En effet, aux termes de l'article 19 dudit code : "L'ensemble des forêts, sur toute l'étendue du territoire national, fait partie du patrimoine national auquel toute personne physique ou personne morale peut accéder.

\footnotetext{
${ }^{853}$ En plus du droit de propriété sur la terre et les ressources naturelles qui doit être reconnu aux communautés, il doit leur être également reconnu le droit de définir leurs propres règles et mécanismes de gestion dont elles peuvent assurer la mise en œuvre. Voir MBAIWA (Joseph E.), STRONZA (Amanda L.), « Changes in resident attitudes towards tourism development and conservation in the Okavango Delta », op. cit., p.1958.

${ }^{854}$ Ibidem.

${ }^{855}$ Les aires protégées ivoiriennes sont situées hors des périmètres urbains, c'est-à-dire dans le milieu rural, à l'exception du Parc National du Banco qui est établi dans la capitale économique ivoirienne (Abidjan). Ainsi, concernant nos suggestions sur la constitution de forêts communautaires autour des aires protégées, nous n'évoquerons que les règles relatives à l'accès à la propriété des terres qui composent le domaine foncier rural, d'une part. De plus, des dispositions du Code forestier relatives à la propriété forestière seront visées. C'est ainsi qu'il convient de savoir, dans un premier temps, la composition de ce domaine foncier rural. Aux termes de 1'article 2 de la loi ${ }^{\circ}$ 98-750 du 23 décembre 1998 relative au domaine foncier rural (JORCI n² du 14 janvier 1999, p. 23), modifiée par la loi n 2004-412 du 14 août 2004 portant amendement de l'article 26 de la loi n 98 750 du 23 décembre 1998 relative au domaine foncier rural (JORCI n49 du 2 décembre 2004, p. 863) : «Le Domaine Foncier Rural est à la fois :

- Hors du domaine public;

- Hors des périmètres urbains;

- Hors des zones d'aménagement différé officiellement constituées;

- Hors du domaine forestier classé.

Le Domaine Foncier Rural est composé :

à titre permanent :

- des terres propriété de l'État;

- des terres propriété des collectivités publiques et des particuliers;

- des terres sans maître.

à titre transitoire :

- des terres du domaine coutumier,

- des terres du domaine concédé par l'État à des collectivités publiques et des particuliers. ».

Dans un deuxième temps, traitant (évidemment) du domaine foncier rural, l'article 2 de la loi précitée dispose : «...seuls l'État, les collectivités publiques et les personnes physiques ivoiriennes sont admis à en être propriétaires. ».
} 
Toutefois, seul l'État, les Collectivités territoriales, les communautés rurales et les personnes physiques ivoiriennes sont admis à en être propriétaires. » (souligné par nous).

De même, l'article 40 du Code forestier de 2014 consacre la création de type de forêts dénommées "forêts des communautés rurales». En effet, selon les termes dudit article : « Les forêts des communautés rurales sont des forêts protégées appartenant à une ou plusieurs communautés rurales. Elles sont composées de catégories suivantes:

- les forêts naturelles situées sur des terres sur lesquelles les communautés rurales jouissent d'un droit de propriété ou de droits coutumiers conformes à la législation domaniale et foncière ;

- les plantations forestières créées sur des terres immatriculées au nom des communautés rurales ou sur des terres occupées par celles-ci en vertu de la coutume locale ou d'un bail ; - les forêts cédées aux communautés rurales par l'État, les Collectivités territoriales ou les personnes physiques ou morales de droit privé ;

- les forêts acquises. ».

En outre, s'agissant des droits de propriété des communautés rurales sur la biodiversité des aires communautaires de conservation à mettre en place, il est à noter que la législation ivoirienne reconnaît désormais la propriété de l'arbre au propriétaire foncier, dans le milieu rural. En effet, l'article 21 de la loi n²014-427 du 14 juillet 2014 portant Code forestier prescrit : «Les arbres situés soit dans un village, soit dans son environnement immédiat, soit dans un champ collectif ou individuel, sont la propriété collective du village ou celle de la personne à laquelle appartient le champ. ». Cependant, il demeure que les communautés et autres personnes de droit privé ne disposent toujours pas de droit de propriété sur la faune sauvage en Côte d'Ivoire. En d'autres termes, c'est l'État qui est demeure propriétaire de la faune sauvage ${ }^{856}$.

Par conséquent, la loi sur la protection de la faune et l'exercice de la chasse de 1965 devrait être modifiée, à l'instar du nouveau Code forestier précité de 2014 qui reconnaît dorénavant la propriété de l'arbre à celui qui est propriétaire du sol forestier ${ }^{857}$, de sorte qu'il en soit autant concernant la faune sauvage.

\footnotetext{
${ }^{856}$ En effet, si l'article 3 de la loi précitée sur la faune et l'exercice de la chasse de 1965 dispose : « La protection de la faune tend à assurer la conservation et l'enrichissement quantitatif et qualitatif des animaux, des espèces vivant naturellement dans le pays, tant sur les surfaces relevant du Domaine de l'État que sur les terrains des particuliers. ", l'article 2 prescrit: "Les animaux tenus en captivité ou les dépouilles des animaux sauvages ne deviennent la propriété des particuliers que si ces animaux ont été capturés ou tués conformément aux règlements pris pour l'exercice de la capture ou de la chasse, ou pour des éliminations ou destructions dûment autorisées. " (souligné par nous). Il résulte de ces textes que les particuliers ne sont pas originairement propriétaires de la faune sauvage vivant sur leurs terres. Ce droit de propriété sur la faune sauvage dont l'État ivoirien est le détenteur exclusif peut être reconnu exceptionnellement pour des captures ou abattages effectuées conformément à la loi. C'est certainement l'une des raisons pour lesquelles la chasse est officiellement fermée en Côte d'Ivoire depuis 1974. Voir sur ce dernier point l'Arrêté n003/ SEPN/ CAB du 20 février 1974 portant fermeture de la chasse, JORCI n 13 du 28 mars 1974, p. 487.

${ }^{857}$ Sous l'empire du Code forestier ivoirien de 1965, la propriété de l'arbre qui se situe sur une parcelle de forêt dont un particulier à la propriété demeure une celle de l'État. Certes, aucune disposition du Code forestier ne l'affirme expressément. Toutefois, les articles 26 et 27 du Code forestier de 1965 permettent de faire pareille
} 
C'est solution a été adoptée par des États qui font figure d'exemples en matière protection de la nature. En effet, au Botswana bien que la faune appartienne à l'État, la loi sur la conservation de la faune de 1982 reconnaît aux propriétaires fonciers privés le droit d'avoir la faune sauvage sur les aires de conservation privée, de l'utiliser et d'en tirer profit. Au Zimbabwe, la loi sur les Parcs et la faune de 1975 a donné aux agriculteurs privés le droit d'utiliser et tirer pleinement profit de leurs ressources fauniques. En Namibie, l'Ordonnance (4 de 1975) sur la conservation de la nature a accordé aux propriétaires des exploitations commerciales un droit de propriété sur la faune sauvage se trouvant sur leurs terres. En 1996, le Décret (5 de 1996) qui amende ladite ordonnance étend ce droit de propriété sur la faune sauvage aux propriétaires des terres communautaires. Pour en bénéficier, ils doivent se constituer en association de gestion collective. D'une part, ce droit de propriété sur la faune sauvage permet aux communautés d'en faire une utilisation consommatrice (chasse aux trophées, capture, vente de gibier...). D’autre part, la possibilité leur est donnée de faire une utilisation non consommatrice (c'est-à-dire à des fins de récréation, éducationnelles, culturelles...) de ladite faune ${ }^{858}$. Ce faisant, cette loi a permis aux communautés de créer des entreprises touristiques sur leurs terres.

Qu'en est-il des recommandations relatives à la gestion courante des projets communautaires de gestion des ressources naturelles?

déduction. En effet, l'article 26 précité dispose : «Les particuliers et les collectivités propriétaires de forêts immatriculées en leurs noms, y exerceront les droits résultant de leur titre de propriété. Ils ne pourront toutefois en pratiquer le défrichement qu'en vertu d'une autorisation administrative » (souligné par nous). Quant à l'article 27 suivant, il prescrit : «En cas d'infraction à l'article précédent, les propriétaires pourront être mis en demeure de rétablir en nature de bois les lieux défrichés...» (souligné par nous). Il s'en déduit que les particuliers propriétaires de forêts et les collectivités ne disposent de pas la propriété des végétaux qui s'y trouvent et partant des arbres, de sorte à en disposer avec discrétion. Une récente étude comparative portant sur les réformes opérées dans le nouveau Code forestier, à la lumière de celui de 1965, confirme le fait que seul l'État ivoirien disposait de la propriété des arbres avant l'entrée en vigueur du Code forestier de 2014. En effet, il y est écrit: "Les articles 21 et 32 du nouveau code forestier précisent que les arbres appartiennent au propriétaire du terrain sur lequel les arbres sont situés. Ceci abroge la disposition de l'ancien code forestier, selon laquelle tous les arbres naturels appartenaient à l'État. Les communautés ont maintenant, pour la première fois, la possibilité de posséder les arbres. ».

En tout état de cause, avec l'entrée en vigueur du Code forestier de 2014, la propriété du sol forestier emporte celle de l'arbre pour le propriétaire. C'est ce que disposent respectivement les articles 21 et 32 du Code forestier de 2014 en ces termes : "Les arbres situés soit dans un village, soit dans son environnement immédiat, soit dans un champ collectif ou individuel, sont la propriété collective du village ou celle de la personne à laquelle appartient le champ.

Ces arbres peuvent faire l'objet d'une cession en faveur des tiers. »; et "Les produits forestiers non situés dans le domaine forestier national, notamment, les arbres hors forêts, appartiennent aux personnes physiques ou morales à qui la législation domaniale et foncière reconnaît un droit de propriété ou des droits coutumiers sur la terre.

La propriété des produits forestiers prévus à l'alinéa précédent et situés sur une terre sans maître revient à l'État. ».

${ }^{858}$ Pour ces différentes informations, voir JONES (Brian T. B.), La gestion communautaire des ressources naturelles en Namibie, pp. 19-20 (article en ligne, disponible sur www.researchgate.net/puplication/228591248 et consulté le 2 septembre 2016). 


\section{b. Les recommandations portant sur la gestion courante des projets communautaires de gestion des ressources naturelles}

Plusieurs enseignements peuvent être tirés des expériences de GCRN en Afrique. Premièrement, la viabilité des projets de GCRN requiert que les communautés locales en tirent des retombées économiques qui supplantent les coûts de gestion ${ }^{859}$. Deuxièmement, les communautés locales à qui la loi délègue la gestion des ressources naturelles doivent être autonomes pour assumer cette responsabilité pour la bonne gestion des projets de GCRN ${ }^{860}$. Troisièmement, pour certains auteurs, une des limites qui compromet les projets communautaires de gestion des ressources naturelles est l'accaparement de ceux-ci par des élites ${ }^{861}$. Enfin, des auteurs conseillent le renforcement des capacités des gestionnaires d'aires protégées et des populations locales pour la réussite des projets de conservation ${ }^{862}$. En ce qui concerne le renforcement des capacités à l'endroit des communautés locales, des auteurs pensent qu'il peut porter sur la relation entre la biodiversité et l'agro-écologie ${ }^{863}$ ainsi que les implications socio-économiques de sa conservation ${ }^{864}$. À la réalité, il s'agit d'une éducation environnementale qui porte sur la conservation durable de la biodiversité et ses aspects de développement social.

Pour ce qui est des gestionnaires des aires protégées, au-delà des renforcements des capacités techniques dans le domaine de la conservation, elles peuvent être instruites sur les techniques en matière de résolution des conflits et de diplomatie. En effet, bien souvent, les communautés locales développent une hostilité à l'encontre des projets de conservation ${ }^{865}$. Il s'ensuit un rapport conflictuel entre celles-ci et les gestionnaires de ressources naturelles.

\footnotetext{
${ }^{859}$ Voir MBAIWA (Joseph E.), STRONZA (Amanda L.), « Changes in resident attitudes towards tourism development and conservation in the Okavango Delta », art. cit., p. 1957.

${ }^{860}$ Voir ROE (Dilys) et NELSON (Fred), "Origines et évolution de la gestion communautaire des ressources naturelles en Afrique»" in ROE (Dilys), NELSON (Fred) et SANDBROOK (Chris) (eds), Gestion communautaire des ressources naturelles en Afrique - Impacts, expériences et orientations futures, op. cit., p. 10 .

${ }^{861}$ Voir par exemple MOMBESHORA et al. (2001), cités par WOLMER (William) et ASHLEY (Caroline), «Part II : Resources and Policies. 3. Wild resources management in southern Africa : Participation, partnerships, eco regions and redistribution », IDS Bulletin, Vol. 34, n³, 2003, p. 32. C'est cette position que soutiennent également VERMEULEN (Sonja) et SHEIL (Douglas) lorsqu'ils affirment : "la grande majorité des initiatives de conservation continue d'être conçue et contrôlé par de puissantes voix externes. " (notre traduction). Voir VERMEULEN (Sonja) et SHEIL (Douglas), "Partnerships for tropical conservation », art. cit., p. 434.

${ }^{862}$ Voir ANDRADE (Gustavo S. M.) et RHODES (Jonathan. R.), « Protected areas and local communities: an inevitable partnership toward successful conservation strategies ? », Ecology and Society, Vol. 17, n ${ }^{\circ}, 2012 .$, p. 18.

${ }^{863}$ Voir PRETTY (Jules) et SMITH (David), « Social capital in biodiversity conservation and management », Conservation Biology, Vol. 18, n³, 2004, p. 631.

${ }^{864}$ Voir FU (B.), WANG (K.), LU Y., LIU (S.), KEMING (M.), CHEN (L.) et LIU (G.), « Entangling the complexity of protected area management : the case of Wolong Biosphere Reserve, southwestern China ", Environmental Management, Vol. 33, nº6, 2004, p. 798.

${ }^{865}$ Voir AKAMA (John S.), LANT (Christopher L.) et BURNETT (G.), « Conflicting attitudes towards state wildlife conservation programs in Kenya », Society and Natural Resources, Vol. 8, n³, pp. 133-144. p. 133; FIALLO (Elba A.) et JACOBSON (Susan K.), « Local communities and protected areas: attitude of rural residents towards conservation and Machalilla National Park, Ecuador », Environmental Conservation, Vol. 22, n³, 1995, p. 241.
} 
Par conséquent, pour la gestion des projets de conservation communautaire à réaliser en vue de la conservation des aires protégées, l'OIPR est exhortée à œuvrer au renforcement de capacité de son personnel et des communautés locales. Les programmes de formation peuvent, à titre indicatif, porter sur des méthodes de résolution pacifique des conflits (nés de la conservation). Ils pourraient également porter sur les techniques d'utilisation durable des sols, de la biodiversité, les techniques d'agroforesterie, d'intensification agricole, de commercialisation de produits agricoles, de comptabilité simplifié, de gestion rationnelle des ressources financières. De plus, la gestion des projets communautaires devrait se faire sur la base de la transparence, de l'équité et de la juste distribution des bénéfices entre les membres de la communauté ou les parties prenantes au projet.

Par ailleurs, vu que les moyens de l'Administration publique ivoirienne en charge des aires protégées demeurent limités, le recours au secteur privé pour la réalisation de ces projets communautaires s'avère nécessaire.

\section{Paragraphe 2 : La nécessité de recourir au secteur privé pour la gestion durable des aires protégées}

La nécessité de recourir au secteur privé pour la gestion durable des aires protégées ivoiriennes témoigne d'un intérêt en la matière pour la mise en place de projets intégrant conservation et développement autour des aires protégées (A). Cette relation pourrait s'établir dans le cadre de la conclusion de contrat de partenariat public-privé (B).

\section{A - L'intérêt du recours au secteur privé pour la mise en place de projets intégrant conservation et développement autour des aires protégées}

L'intérêt pour la Côte d'Ivoire de recourir au secteur privé en vue de la mise en place de projets intégrant conservation et développement autour des aires protégées se situe à deux (2) niveaux. D'une part, cet intérêt découle de l'importance du recours au secteur privé dans la gestion des aires protégées (1). D'autre part, il existe un cadre juridique favorable en la matière (2).

\section{L'importance du recours au secteur privé dans la gestion des aires protégées}

Le secteur privé a joué et continue de jouer un rôle considérable dans la conservation de la diversité biologique ${ }^{866}$ dans le monde ${ }^{867}$, en général, et particulièrement en Afrique

\footnotetext{
${ }^{866}$ Selon le Ve Congrès mondial sur les Parcs : « une partie considérable de la biodiversité de la terre survit sur les territoires relevant de la propriété, le contrôle ou la gestion des peuples autochtones et les
} 
australe $^{868}$ ainsi qu'en Afrique de l'Est ${ }^{869}$. Dans de nombreux pays qui composent ces régions de l'Afrique subsaharienne, le réseau d'aires protégées mis en place comprend aussi bien les aires protégées étatiques que celles créées et exploitées par des personnes de droit privée ${ }^{870}$.

À titre d'exemple, en Afrique du Sud, environ $25 \%$ de la population de rhinocéros blanc soit $83 \%$ de la population mondiale de cette espèce faunique (en danger parce que fortement braconnée ${ }^{871}$ ) se trouve sur les aires de conservation ou Réserve privée ${ }^{872}$. Ces aires de conservation privée prennent des formes diverses dont les Réserves de faune destinée à la chasse touristique ou autre activité touristique. C'est ainsi que dans ce pays, l'on dénombre environ 9.000 ranchs de faune (sauvages) c'est-à-dire des Réserves de chasse touristique ; et 15.000 ranchs de faune et de bétail ${ }^{873}$. En Namibie, environ $80 \%$ des gros mammifères se trouvent dans les aires de conservation privée ${ }^{874}$. La Réserve de chasse de Niassa (Niassa Game Reserve) située au Nord du Mozambique et celle du Masaï Mara (Masai Mara Reserve Game) au Kenya sont gérées chacune par une société privée ${ }^{875}$.

Par conséquent, ces aires protégées privées constituent des outils importants de conservation de la diversité biologique à l'échelle du continent et de développement local.

communautés locales. » (notre traduction). Voir SCHERL (Lea M.) et al., Can Protected Areas Contribute to Poverty Reduction? Opportunities and Limitations., op. cit., p. 35.

${ }^{867}$ Par exemple, le Professeur SADELEER (Nicolas de), fait remarquer qu'avec la redéfinition du rôle de l'État au sein de l'Union européenne, les États et le secteur privé nouent, de plus en plus, des partenariats pour la protection ou la conservation de l'environnement. Voir SADELEER (Nicolas de), «Particularités de la subsidiarité dans le domaine de l'environnement», Droit et Société, Vol. 1, n80, p. 76.

${ }^{868}$ Selon KRUG (Wolf), en Afrique australe, l'on assiste à la conversion des terres agricoles en aires de conservation de la faune sauvage ou d'aires communautaires de gestion de faune. Voir KRUG (Wolf), Private Supply of Protected Land in Southern Africa: A Review of Markets, Approaches, Barriers and Issues, op. cit., pp.18- 19. De plus, pour COUSINS et al., les Réserves privées : «jouent un rôle de plus en plus important dans l'avenir de la conservation en Afrique du Sud» (notre traduction). Voir COUSINS (Jenny A.), SADLER (Jon P.) et EVANS (James), «Exploring the Role of Private Wildlife Ranching As a Conservation Tool in South Africa : Stakeholder Perspectives », Ecology and Society, Vol. 3, n², 2008, pp. 1-34 (document non paginé sur internet, disponible sur www.ecologyandsociety.org/vol13/iss2/art43/ (consulté, le 15 août 2015), la première phrase du point intitulé "Wildlife ranching and conservation benefits".

${ }^{869}$ Voir VEIT (Peter G.), NSHALA (Rugemeleza), ODHIAMBO (Michael Ochieng'), MANYINDO (Jacob), Protected Areas and Property Rights. Democratizing Eminent Domain in East Africa, WRI Report, World Resources Institute, p. 8.

${ }^{870}$ Voir KRUG (Wolf), Private Supply of Protected Land in Southern Africa: A Review of Markets, Approaches, Barriers and Issues, op. cit., p. 18.

${ }^{871} \mathrm{En}$ effet, la forte demande asiatique a contribué au braconnage de 1000 rhinocéros blanc seulement en 2013 sur un total estimé à environ 20.404 en 2013, soit trois (3) rhinocéros par jour. À cette allure, l'on prévoit la disparition de cette espèce dans le Parc National de Kruger qui en concentre la plus grande population en 2016. Voir PERNETTA (Angelo P.), «A disappearing drylands icon ? White rhinoceros conservation and the need for public-private partnerships », Biodiversity, Vol. 15, n²-3, 2014, p. 231.

${ }^{872}$ Voir BIGGS (Duan), COURCHAMP (Franck.), MARTIN (Rowan.) et POSSINGHAM (Hugh. P.), « Legal Trade of Africa's Rhino Horns », Science, Vol. 339, 2013, p. 1038.

${ }^{873}$ Voir COUSINS (Jenny A.), SADLER (Jon P.) et EVANS (James), « Exploring the Role of Private Wildlife Ranching As a Conservation Tool in South Africa : Stakeholder Perspectives », Ecology and Society, Vol. 3, $\mathrm{n}^{\circ} 2,2008$, la première phrase du point intitulé "Introduction". (document non paginé sur internet, disponible sur www.ecologyandsociety.org/vol13/iss2/art43/ (consulté, le 15 août 2015).

${ }^{874}$ Voir RICHARDSON (Julie A.), «Wildlife utilization and biodiversity conservation in Namibia: conflicting or complementary objectives? », Biodiversity and Conservation, Vol. 7, n4,1998, p. 554.

${ }^{875}$ Voir KRUG (Wolf), Private Supply of Protected Land in Southern Africa: A Review of Markets, Approaches, Barriers and Issues, Workshop Paper, op. cit., p. 19. 
C'est juste titre que le recours au secteur privé pour la création et la gestion des aires protégées en Côte d'Ivoire paraît nécessaire ; d'autant plus qu'il existe un cadre juridique favorable à cet effet.

\section{L'existence en Côte d'Ivoire d'un cadre juridique favorable au recours au secteur privé pour la création et la gestion des aires protégées}

La participation du secteur privé en matière de protection des aires protégées en Côte d'Ivoire est légalement permise par plusieurs dispositions contenues dans la législation ivoirienne, en général, et celle relative à la protection de la nature, en particulier. En effet, il ressort des dispositions des articles $1^{\text {er }}$ et 13 alinéa 4 de la loi de 2002 sur les PNR que les personnes de droit privé c'est-à-dire les particuliers et les personnes morales de droit privé peuvent créer des Réserves naturelles volontaires sur les terrains dont elles sont propriétaires $^{876}$.

En outre, les dispositions qui régissent les mécanismes participatifs de gestion des aires protégées ivoiriennes ${ }^{877}$ servent également de fondement au recours au secteur privé pour la gestion durable des aires protégées en Côte d'Ivoire. En effet, aux termes de l'article 1er de la loi précitée de 2002 sur les PNR, le contrat de gestion de terroir est : "le contrat passé entre le gestionnaire d'un parc ou d'une réserve et les populations rurales de la zone périphérique représentées par des structures associatives, privées ou administratives...» (souligné par nous). Il s'en déduit que le secteur privé (en l'espèce une société commerciale) qui représente, par exemple, les intérêts des communautés peut participer à la gestion durable des aires protégées en accomplissant des actions qui contribuent à la fois à la protection de ces forêts et au développement des communautés locales. Il en est de même pour les concessions d'exploitation des aires protégées dans le cadre desquels les conventions afférentes peuvent être établies entre l'autorité en charge de la gestion des aires protégées et une personne morale de droit privé ${ }^{878}$.

Au total, la législation ivoirienne permet de recourir au secteur privé pour la gestion durable des aires protégées. Dès lors, les gestionnaires d'aires protégées sont exhortés à

\footnotetext{
${ }^{876}$ Selon les termes de ces articles ci-dessus respectivement visés, la Réserve naturelle volontaire est: «la Réserve naturelle partielle créée à l'initiative d'une collectivité territoriale, d'un Établissement public ou d'une personne de droit privé, sur un terrain lui appartenant et pour la préservation d'un écosystème ou d'un paysage remarquable...» et Les Réserves naturelles volontaires «...sont gérées par la Collectivité territoriale, l'Établissement public ou la personne de droit privé , propriétaire du terrain constituant la Réserve...».

${ }^{877} \mathrm{Il}$ est fait, en l'espèce, allusion au contrat de gestion de terroir et au contrat de concession d'exploitation des Parcs et Réserves. Sur ces deux (2) contrats, voir nos développement $\mathrm{s}$ à la Première partie de cette thèse, le Titre II, le Chapitre II, la Section II, le Paragraphe 2 et précisément le point intitulé " B- La contractualisation de la gestion des aires protégées ".

${ }^{878}$ Aux termes de l'article 32 alinéa de la loi de 2002 sur les PNR : «Tout ou partie des missions d'exploitation d'un Parc ou d'une Réserve, selon la nature de ce Parc ou de cette Réserve, peuvent être concédées par l'Établissement à une personne morale de Droit privé dans le cadre d'une Convention de concession d'exploitation...» (souligné par nous).
} 
s'orienter de plus en plus vers l'établissement de contrats de partenariat public-privé à cet effet.

\section{B - La conclusion de contrats de partenariat public-privé, une solution de gestion durable des aires protégées en Côte d'Ivoire}

Le partenariat public-privé (PPP) ne fait pas, à l'heure actuelle, l'objet d'une définition universellement admise ${ }^{879}$. Toutefois, des essais de définitions existent. Selon WILSON et $a l$., qui formulent une définition à caractère général, les PPP s'entendent de tout accord dans lequel les secteurs public et privé travaillent ensemble en vue de fournir un service $^{880}$. En matière de conservation de la nature, pour NSHIMBI et VINYA, les PPP pourraient être appréhendés comme des partenariats dont l'objectif est de faire des communautés riveraines des ressources naturelles des acteurs clés pour leur conservation, en contrepartie des retombées financières qu'elles retirent de leur gestion ${ }^{881}$.

L'idée que la conclusion de partenariats public-privé est une solution pour la gestion durable des aires protégées ivoiriennes repose sur une thèse essentielle. Elle est celle qui postule qu'il y a un intérêt à conclure ces partenariats pour la gestion durable des aires protégées (1). Toutefois, des précautions qui garantissent leurs succès doivent être prises par les différentes parties prenantes (2).

\section{L'intérêt de la conclusion de contrats de partenariat public-privé pour la gestion des aires protégées ivoiriennes}

Hormis le scepticisme de certains auteurs sur le caractère bénéfique des PPP en matière de conservation ${ }^{882}$, l'intérêt pour les pouvoirs publics ivoiriens de conclure de tels

\footnotetext{
${ }^{879}$ Voir NSHIMBI (Muleba), VINYA (Royd), « Impacts of Public-Private Partnership on Local Livelihoods and Natural Resource Dynamics : Perceptions from Eastern Zambia », art. cit., p. 472.

${ }^{880}$ Voir WILSON (Erica), Noah et BUULTJENS (Jeremy), « From lessees to partners: exploring tourism public-private partnerships within the New South Walesnational parks and wildlife service ", Journal of Sustainable Tourism, Vol. 17, n², 2009, p. 271.

${ }^{881}$ Voir NSHIMBI (Muleba), VINYA (Royd), «Impacts of Public-Private Partnership on Local Livelihoods and Natural Resource Dynamics: Perceptions from Eastern Zambia », art. cit., p. 473.

${ }^{882} \mathrm{La}$ question de la conclusion de partenariats avec le secteur privé pour la gestion des aires protégées est controversée malgré les avantages précédemment évoquées. En effet, des auteurs révèlent que, bien souvent, les gestionnaires d'aires protégées, les écologistes et les communautés locales craignent que les considérations financières ne l'emportent sur les objectifs de conservation, dans le cadre de tels partenariats. À ce sujet, EAGLES (Paul J.) notent que lorsque que le partenaire privé est très puissant que le gouvernement ou l'agence gouvernementale avec qui elle conclut le partenariat de gestion des ressources naturelles, il y a un risque qu'il surexploite lesdites ressources de sorte à faire plus de profit.[ voir EAGLES (Paul F.J.), International trends in park tourism, Paper presented at EUROPARC Conference, 3-7 October, Hohe Tauern National Park, Matrei, Austria, 2001, p. 31]. Également, les recherches d'un auteur tel BUCKLEY (Ralf) qui a réfléchi sur les mécanismes de financement en matière touristique des Parcs Nationaux dans l'État de New South Wales (en français Nouvelle-Gale du Sud) en Australie établissent que ces accords peuvent présenter un risque. En effet, cet auteur affirme : "Le financement de la conservation des Parcs Nationaux par le tourisme est faisable. Cependant cette approche présente un risque considérable de sorte que l'alternative de financement publique est nécessaire. ». Voir BUCKLEY (Ralf), " Innovative funding mechanisms for visitor infrastructure in
} 
partenariats s'explique par diverses raisons. D'une part, cet intérêt se justifie par le fait que de tels partenariats constituent un moyen pour la gestion durable des aires protégées (a). D'autre part, des expériences de gestion confirment l'idée précédente (b).

\section{a. Les partenariats public-privé, un moyen pour la gestion durable des aires protégées}

En matière de gestion des forêts, il est de constat que pour inverser la tendance de la déforestation et de la dégradation, il se développe de plus en plus de nombreux partenariats public-privé (entre les gouvernements, le secteur privé et la société civile) ${ }^{883}$. Les raisons de cet engouement vers les partenariats public-privé sont diversement appréhendées par la doctrine relative à la conservation des aires protégées. En effet, certains auteurs affirment qu'en matière de gestion des ressources naturelles, les partenariats corrigent les insuffisances des approches communautaires de gestion ${ }^{884}$ ainsi que de gestion exclusive alors opérés par les États ${ }^{885}$. Pour d'autres, la popularité des PPP en matière de gestion des aires protégées s'explique en ce qu'ils ont un potentiel de mobilisation d'importantes ressources financières et de réduire le niveau de pauvreté des communautés locales ${ }^{886}$. D'autres encore, pour

Australian National Parks. In A natural partnership: Making national parks a tourism priority. Sydney: TTF Australia and CRC for Sustainable Tourism, Juin 2004, p. 17.

${ }^{883}$ Voir PATNEY (Atjun), « Saving Lands and Wildlife : Corporations and Conservation Groups in Partnership ", Corporate Environmental Strategy, n7, n4, 2000, p. 363; STOLL-KLEEMANN (Suzanne), O'RIORDAN (Timothy) «From Participation to Partnership in Biodiversity Protection : Experience from Germany and South Africa », art. cit., p. 161; POPE (Adam), A Preliminary Examination of Public Private Partnerships in National Park Management in Zambia, op. cit., p. 3 ; RUTAGARAMA (Eugène), MARTIN (Adrian), " Partnerships for protected area conservation in Rwanda », art. cit., p. 291; MBURU (John), BIRNER(Regina), " Emergence, Adoption, and Implementation of Collaborative Wildlife Management or Wildlife Partnerships in Kenya: A Look at Conditions for Success ", Society and Natural Resources, Vol. 20 n $^{\circ}$, 2007, p. 379; VERMEULEN (Sonja), SHEIL (Douglas), " Partnerships for tropical conservation », Oryx Vol. 41 n $^{\circ} 4$ October 2007, p. 434; VISSEREN-HAMAKERS (Ingrid J.), GLASBERGEN (Pieter), « Partnerships in forest governance », Global Environmental Change, Vol. 17, 2007, p. 408 ; WILSON (Erica), NIELSEN (Noah), BUULTJENS (Jeremy), « From lessees to partners: exploring tourism public-private partnerships within the New South Wales national parks and wildlife service», art. cit., p. 269 ; AHEBWA (Wilber Manyisa), DUIM (V. René Van der), SANDBROOK (Chris G), " Private-community Partnerships: Investigating a New Approach to Conservation and Development in Uganda ", art. cit., p. 305; MASHALE (Christopher Modise), MOYO (Theresa), MTAPURI (Olivier), «Evaluation of the Public-Private Partnership in the Lekgalameetse Nature Reserve in South Africa », art. cit., p. 855; NSHIMBI (Muleba), VINYA (Royd), « Impacts of Public-Private Partnership on Local Livelihoods andNatural Resource Dynamics: Perceptions from Eastern Zambia », art. cit., p. 473.

${ }^{884}$ Voir MBURU (John), BIRNER (Regina), « Emergence, Adoption, and Implementation of Collaborative Wildlife Management or Wildlife Partnerships in Kenya: A Look at Conditions for Success », p. 380.

${ }^{885}$ Contrairement à l'approche préservationniste de conservation de la nature et partant des aires protégées (des États) qui ne se limitaient qu'à conserver ces milieux naturels uniquement pour cette fin, la gestion de ces forêts au moyen de partenariat public-privé concilie, à la fois, conservation des ressources naturelles et développement local. C'est cette idée qu'expriment WOLWER et al. à travers la phrase suivante : «les partenariats visent à équilibrer l'objectif de conservation de la biodiversité et à améliorer le développement économique local et lutte contre la pauvreté. » Voir WOLMER (William) et ASHLEY (Caroline), " Part II: Resources and Policies. 3. Wild resources management in southern Africa: Participation, partnerships, eco regions and redistribution », art. cit., p. 31

${ }^{886} \mathrm{C}$ 'est ce point de vue que défendent CHRISTOPHER et al. lorsqu'ils écrivent: «Au cours de ces dernières années, les partenariats public-privé (PPP) dans les zones protégées sont de plus en plus populaires car perçus comme des moyens de générer des revenus et de réduire la pauvreté » (notre traduction). Voir MASHALE (Christopher Modise), MOYO (Theresa) et MTAPURI (Olivier), « Evaluation of the Public-Private Partnership 
montrer l'intérêt des PPP en matière de gestion des aires protégées soutiennent qu'ils sont plus rentables ; qu'ils fournissent de meilleurs services, en raison d'un personnel efficace et que les risques sont à la charge du secteur privé ${ }^{887}$.

Dès lors, les PPP sont un moyen efficace pour la conservation des aires protégées, en général, et celles de l'Afrique, en particulier -où les exigences (surtout financières) de la conservation de ces forêts dépassent largement les capacités des États ${ }^{888}$. C'est à juste titre que traitant de la conservation des forêts tropicales, Sonja VERMEULEN et al. s'expriment en ces termes : «...les partenariats ne sont pas la panacée, mais un véritable engagement envers le partenariat offre des résultats de conservation qui sont plus éthiques et souvent plus possibles que les modèles actuels. » (notre traduction) ${ }^{889}$.

Par ailleurs, les expériences de gestion de ressources naturelles et notamment d'aires protégées à travers les PPP enseignent que ces partenariats représentent un moyen efficace pour la gestion durable des aires protégées ivoiriennes.

\section{b. La présentation de partenariats public-privé de gestion d'aires protégées réussis}

Plusieurs exemples de partenariats public-privé en matière de gestion des forêts, en général, et des aires protégées, en particulier, existent. En effet, à l'échelle mondiale, l'alliance Banque mondiale-WWF pour la conservation et l'utilisation durable des forêts, créée en 1998, est un partenariat public-privé qui opère selon une approche intégrée de conservation et de développement local. Ce partenariat œuvre, entres autres, à la création et à la gestion durable des aires protégées, à la lutte contre les exploitations illégales des forêts ainsi qu'à l'augmentation des forêts certifiées ${ }^{890}$.

$\mathrm{Au}$ niveau régional, il peut être cité le Partenariat pour les forêts du Bassin du Congo $^{891}$. Le partenariat pour les forêts du Bassin du Congo a plusieurs objectifs. Ils

in the Lekgalameetse Nature Reserve in South Africa », op. cit., p. 855; VISSEREN-HAMAKERS (Ingrid J.) et GLASBERGEN (Peter), «Partnerships in forest governance », art. cit., p. 415.

${ }^{887}$ Voir EDWARDS (Pamela) et SHAOUL (Jean), " Partnerships: For better or worse? », Accounting, Auditing and Accountability, Vol. 16, n³, 2002, p. 397.

${ }^{888}$ Voir la Première partie de cette étude, le Titre I, le Chapitre II, la Section II, le Paragraphe 1, le point intitulé "B- L’insuffisance des dotations budgétaires pour la conservation des aires protégées ".

${ }^{889}$ Voir VERMEULEN (Sonja) SHEIL (Douglas), « Partnerships for tropical conservation », art.cit. , p. 434.

${ }^{890}$ Voir VISSEREN-HAMAKERS (Ingrid J.) et GLASBERGEN (Peter), « Partnerships in forest governance », art. cit., p. 415.

${ }^{891}$ Le partenariat pour les forêts du Bassin du Congo (PFBC) est une initiative de conservation des forêts du Bassin du Congo fondée sur la synergie des efforts de la communauté internationale. Elle est initiée, depuis septembre 2002, par le Secrétaire d'État américain (Colin Powell) en charge du Sommet mondial sur le développement durable qui s'est tenu à Johannesburg (Afrique du Sud).

Le Partenariat pour les Forêts du Bassin du Congo (PFBC) est un partenariat non-contraignant enregistré auprès de la Commission pour le Développement Durable des Nations Unies. Il rassemble environ 70 partenaires (des organisations internationales, des États africains, des bailleurs de fonds et pays donateurs, des représentants d'institutions de recherche, le secteur privé et des ONG. Voir www.bi.chm-cbd.net/cooperation/organismesregionaux/partenariat-pour-les-foret-du-bassin-du-congo-pfbc (consulté le 6 décembre 2015). 
consistent à améliorer la gouvernance forestière et des ressources naturelles ; à développer un réseau d'aires protégées ; et à assurer des moyens de subsistance durables aux populations locales $^{892}$.

$\mathrm{Au}$ Guatemala, fort des mauvais résultats de la gestion exclusive des forêts étatiques par l'État lui-même, celle-ci a été déléguée à une structure autonome dénommée Istituto Nacional de Bosques (INAB), -en français Institut National des Forêts. Cette institution est dotée d'un Conseil de gestion composé de membres du secteur public, du secteur privé (industrie du bois...), des Organisations Non Gouvernementales (ONG) ${ }^{893}$. Autrement dit, il s'agit d'une gestion des forêts guatémaltèques à travers un partenariat public-privé. Cette réforme de gestion des forêts du Guatemala sous fond de PPP a permis de lutter significativement contre la corruption et partant d'améliorer la conservation des forêts. En effet, à travers un processus de décentralisation, l'INAB a renforcé les capacités des communes et s'est engagé dans le développement de participation communautaire à la gestion durable des forêts. C'est ainsi qu'elle a entrepris des reboisements incitatifs qui consiste à encourager les reboisements et à faire bénéficier les paysans de semis. Ces initiatives de l'INAB ont permis de passer de 20.000 ha de forêts en 20 ans à 70.000 ha au terme des sept premières années d'existence de l'INAB ${ }^{894}$.

Au Kenya, la gestion des aires protégées fait l'objet de partenariats public-privé entre la Kenyan Wildlife Service (KWS) et les entreprises privées. Dans ces partenariats, une place importante est accordée au développement des communautés rurales en vue de la conservation durable des aires protégées ${ }^{895}$. Il ressort d'une étude que ces PPP au Kenya ont permis d'augmenter considérablement le nombre des populations de faune ${ }^{896}$; et d'améliorer les revenus des communautés locales ${ }^{897}$.

Ce partenariat a deux objectifs spécifiques :

- contribuer à la gestion durable des ressources naturelles du Bassin du Congo. D'une part, l'opérationnalisation de cet objectif consiste à créer un réseau de près de 10 millions d'hectares de Parcs Nationaux et d'aires protégées ainsi que près de 20 millions d'hectares de concessions forestières sous aménagement forestier. D'autre part, il s'agit de lutter contre l'exploitation illégale des bois, d'appliquer des lois contre le braconnage.

- promouvoir le développement économique, la réduction de la pauvreté et des inégalités ainsi que l'amélioration de la gouvernance en faveur des populations dépendantes des ressources naturelles. Sur ces différents points voir www.riddac.org/index2.php?option=com content\&do pdf=1\&id=67 (consulté le 6 décembre 2015).

${ }^{892}$ Voir VISSEREN-HAMAKERS (Ingrid J.) et GLASBERGEN (Peter), « Partnerships in forest governance », art. cit., p. 415.

${ }^{893}$ Voir BIRNER (Regina) et WITTMER (Heidi), « Better public sector governance through partnership with the private sector and civil society: the case of Guatemala's forest administration », art. cit., p. 459.

${ }^{894}$ Voir CASTANEDA (Luis) and UMANS (Laurent) (2004), cités par BIRNER (Regina) et WITTMER (Heidi), «Better public sector governance through partnership with the private sector and civil society: the case of Guatemala's forest administration », art. cit., p. 463.

${ }^{895}$ Voir MBURU (John) et BIRNER (Regina), " Emergence, Adoption, and Implementation of Collaborative Wildlife Management or Wildlife Partnerships in Kenya: A Look at Conditions for Success », art. cit., p . 380.

${ }^{896} \mathrm{En}$ effet, par exemple, les partenariats public-privé qui portent sur les aires de conservation privés telles que G M Sanctuary et Kinama ont respectivement permis d'augmenter la population d'animaux, en général, et d'éléphants, en particulier. Ce constat pourrait s'expliquer par l'engouement des communautés locales à la protection de la faune avec l'avènement des PPP qui leur permet de bénéficier des avantages de la conservation (revenus et autres avantages). Pour ce qui est des ressources financières générées par la conservation et 
En outre, il ressort de l'évaluation des impacts des PPP conclus pour la gestion des ressources naturelles dans l'Est de la Zambie qu'ils ont permis d'atteindre des résultats satisfaisants en matière de conservation et de développement des communautés locales. En effet, D'une part, selon NSHIMBI et VINYA, le succès de ces PPP émane de la réalisation d'activités diversifiées relatives aux moyens de subsistances des communautés locales. D'autre part, ils ont favorisé l'accès des produits locaux aux marchés urbains. De la sorte, ces PPP ont atteint à la fois la sécurité alimentaire; réduit la pauvreté rurale parmi les communautés qui participaient à ces programmes et par contrecoup contribué à la protection de la nature ${ }^{898}$.

Par exemple, les travaux d'Adam Pope attestent que le recours aux PPP pour la gestion la gestion des Parcs Nationaux tels que Kasanka Case et South Luangwa est avantageux pour leur conservation. En effet, sous exploitation PPP, au Parc National Kasanka :

- les recettes touristiques ont augmenté jusqu'à 272,000 US\$ en 2005;

- 1'application de la loi s'est améliorée de 2000 à 2005 ;

- le personnel a augmenté de 1990 à 2005;

- les investissements en matière d'infrastructures (routes, pistes...) se sont accrus;

- la population de la faune sauvage (éléphant, phacochère, antilope...) a augmentét ${ }^{899}$.

En ce qui concerne le Parc National South Luangwa, la gestion sous PPP a permis :

- une meilleure gestion financière de cette aire protégée de sorte que contrairement au passé (c'est-à-dire avant le recours au PPP), les recettes dépassent les dépenses. En 2005 par exemple, les recettes du Parc ont dépassé de 26\% ses dépenses;

- les revenus propres du Parc couvrent l'ensemble de son budget ${ }^{900}$.

En somme, au-delà du scepticisme de certains auteurs sur le caractère bénéfique des PPP en matière de conservation de la nature, il ressort de ces différentes expériences que le partenariat permet la gestion durable des ressources naturelles. En effet, les PPP sont un moyen qui aide le gestionnaire d'aire protégée à mobiliser d'importantes ressources financières; à disposer des capacités techniques appropriées pour la conservation et le développement.

l'utilisation de la faune, au-delà des critiques de corruption et de manque de transparence et d'équité dans leur partage dans certains cas, des sommes en espèce ont été distribués aux membres de GM Sanctuary. En ce qui concerne Kinama, les bénéfices de la conservation sont partagés en cas de besoin. Voir MBURU (John) et BIRNER (Regina), « Emergence, Adoption, and Implementation of Collaborative Wildlife Management or Wildlife Partnerships in Kenya: A Look at Conditions for Success », art. cit., p. 387.

${ }^{897}$ Ibidem.

${ }^{898}$ Pour ces développements sur les impacts écologiques et sociaux des PPP conclus dans 1'Est de la Zambie, voir NSHIMBI (Muleba) et VINYA (Royd), «Impacts of Public-Private Partnership on Local Livelihoods and Natural Resource Dynamics: Perceptions from Eastern Zambia », art. cit., p. 484.

${ }^{899}$ Voir POPE (Adam), A Preliminary Examination of Public Private Partnerships in National Park Management in Zambia, op. cit., p. 16.

${ }^{900}$ Ibidem. 
Par conséquent, l'OIPR est encouragé à opter pour la conclusion de partenariats public-privé dans la gestion des aires protégées ivoiriennes. Ainsi, ces PPP pourraient permettre la mobilisation de ressources financières conséquentes pour le financement des activités de conservation des aires protégées y compris le développement local. Ce faisant, l'OIPR pourrait, par le canal des PPP, encourager, développer et appuyer techniquement la création et la gestion de forêts communautaires à vocation de conservation et de développement autour des aires protégées de Côte d'Ivoire ou RNVC.

Toutefois, pour atteindre ces objectifs, les PPP conclus devraient éviter certains travers qui ont constitué des insuffisances pour certaines expériences de PPP.

\section{Les précautions à prendre pour le succès des partenariats public-privé} dans le cadre de la gestion des aires protégées ivoiriennes

Selon Eugène RUTAGARAMA, il est possible que les partenariats conclus pour la gestion des aires protégées rencontrent des difficultés, en dépit de la volonté des parties de s'impliquer pour la réalisation des objectifs poursuivis ${ }^{901}$. C'est par exemple le cas du PPP conclu pour gestion de la Réserve naturelle de Lekgalameetse (en Afrique du Sud). En effet, en 1913, la politique de l'apartheid ${ }^{902}$ a entraîné la spoliation des terres des communautés noires. En 1994, à la faveur de l'abolition de cette politique ségrégationniste, la possibilité légale avait été donnée aux communautés de revendiquer la restitution des terres. Or, la plupart d'entre elles abritaient des aires protégées appartenant à des personnes de droit privé.

Dans ce nouveau contexte politico-juridique, les gouvernements successifs ont opté pour la conclusion de partenariats dans l'optique de réaliser l'application de la loi tout en préservant les différents intérêts. Il s'est agi de conserver les aires protégées en raison de leur importance (écologique et potentiel financier) et de préserver les intérêts fonciers des communautés. En d'autres termes, la conservation de la biodiversité et le développement local en vue de réduire la pauvreté étaient les buts de ces partenariats.

Cependant, dans ce contexte de revendication foncière, les communautés n'ont pas compris les implications de cette volonté de gestion des aires protégées et de l'application de la législation qui ont motivé ces partenariats. En effet, elles estiment que les possibilités d'emplois (qui sont temporaires et occasionnels), de collecte de bois de chauffe, de pâturage

\footnotetext{
${ }^{901}$ Voir RUTAGARAMA (Eugène) et MARTIN (Adrian), «Partnerships for protected area conservation in Rwanda», art. cit., p. 291.

${ }^{902}$ L'apartheid est une politique ségrégationniste développée en Afrique du Sud en 1913 et abolit en 1991. Elle consistait à opérer un développement séparé entre la minorité blanche (Afrikaners ) et le reste de la population composée essentiellement de Noirs. Voir www.un.org/fr/events/mandeladay/apartheid.shtml et www.larousse.fr/encyclopedie/divers/apartheid/22047 (consultés le 24 mai 2016).
} 
n'ont pas été largement satisfaites. Autrement dit, ces communautés jugent que les avantages dont elles bénéficient sont moins que ceux escomptés ${ }^{903}$.

$\mathrm{Au}$ Kenya, une recherche menée sur les facteurs qui contribuent à l'adoption et à l'émergence des partenariats en matière de conservation de la faune, sur la base de deux (2) études de cas, conclu que :

- les parties prenantes au partenariat notamment les communautés devraient avoir des capacités en matière d'organisation ;

- elles devraient bénéficier des retombées de la conservation de la faune, en raison de leurs droits de propriété foncière sur les terres qui abritent les aires protégées ${ }^{904}$.

En Australie, des travaux de recherche qui examinent les processus à travers lesquels des partenariats efficaces sont formés concluent qu'il faut pour ce faire un dialogue constructif entre les parties prenantes ${ }^{905}$. Également, elles doivent faire preuve de souplesse et de créativité pour solutionner les problèmes qui se posent ${ }^{906}$. Dans le même ordre d'idée, selon une étude conduite au Rwanda : «La conservation des Parcs Nationaux peut être mieux faite à travers des partenariats souples et inclusifs qui cherchent à intégrer les activités de conservation et de développement $\gg$ (notre traduction) ${ }^{907}$.

Il ressort d'autres recherches effectuées sur des partenariats en matière de conservation de la nature que les relations de pouvoirs déséquilibrées entre les parties peuvent créer des conditions de conflits qui sont susceptibles de compromettre les objectifs de conservation et de développement poursuivis ${ }^{908}$. De plus, ces partenariats doivent prendre

\footnotetext{
${ }^{903}$ Pour ces développements sur la Réserve naturelle de Lekgalameetse, voir MASHALE (Christopher Modise), MOYO (Theresa) et MTAPURI (Olivier), «Evaluation of the Public-Private Partnership in the Lekgalameetse Nature Reserve in South Africa », art. cit., p. 855.

${ }^{904}$ Voir MBURU (John) et BIRNER (Regina), « Emergence, Adoption, and Implementation of Collaborative Wildlife Management or Wildlife Partnerships in Kenya: A Look at Conditions for Success ", art. cit., p. 393. Cette position qui consiste à intégrer conservation et développement local pour réussir les projets de conservation est également soutenue par RUTAGARAMA (Eugène) et MARTIN (Adrian) lorsqu'ils écrivent que l'échec des PPP en matière de conservation de la nature est dû au fait qu'ils n'ont pu concilier conservation de la biodiversité et développement des communautés locales. Voir RUTAGARAMA (Eugène), et MARTIN (Adrian), «Partnerships for protected area conservation in Rwanda», art. cit., p. 303. Il en est de même pour DODSON Giles qui soutient que pour réussir la conservation, il faut un processus juridique qui intègre conservation et développement. Voir DODSON (Giles), «Co-Governance and Local Empowerment? Conservation Partnership Frameworks and Marine Protection at Mimiwhangata, New Zealand», art. cit., p. 522.

${ }^{905}$ Voir Idem, p. 539. Ce point de vue est partagée par Adam cité RUTAGARAMA (Eugène), et MARTIN (Adrian) qui affirment: "Les partenariats ne peuvent être conçues et imposés. " (notre traduction). Voir RUTAGARAMA (Eugène), et MARTIN (Adrian), " Partnerships for protected area conservation in Rwanda», art. cit., p. 303.

${ }^{906}$ Voir DODSON (Giles), « Co-Governance and Local Empowerment? ConservationPartnership Frameworks and Marine Protection at Mimiwhangata, New Zealand », art. cit., p. 539.

${ }^{907}$ Voir RUTAGARAMA (Eugène), et MARTIN (Adrian), « Partnerships for protected area conservation in Rwanda», art. cit., p. 291.

${ }^{908}$ Voir AHEBWA (Wilber Manyisa), DUIM (V. René Van der) et SANDBROOK (Chris G.), « Privatecommunity Partnerships: Investigating a New Approach to Conservation and Development in Uganda ", Conservation and Society, Vol. 10, n4, 2012, p. 305.
} 
en compte les réalités locales ${ }^{909}$. Enfin, pour Patrick TRIPLET, pour atteindre leurs objectifs, les partenariats doivent reposer sur la transparence, l'équité et les bénéfices mutuels ${ }^{910}$.

Fort des développements qui précèdent, les recommandations suivantes pour le succès des partenariats public-privé en vue de la conservation durable des aires protégées peuvent être faites. En effet, les partenariats public-privé conclus dans le cadre de la gestion durable des aires protégées ivoiriennes devraient établir un équilibre entre la protection de ces sites et le développement des communautés locales. Dans cet optique, les communautés locales devront bénéficier des retombées financières (et autres avantages) de la conservation des aires protégées communautaires et surtout bénéficier d'un droit de propriété sur la faune et les terres sur lesquelles elles sont établies. Cette redistribution des avantages de la conservation devrait se faire sur la base de la transparence et de l'équité.

En outre, il est souhaitable que les parties au partenariat connaissent avec précision leurs droits et obligations respectifs et développent chacune des capacités en matière d'organisation. Enfin, elles sont exhortées à développer des relations empreintes d'égalité, rechercher le consensus, être souples et créatives lorsque surgissent des difficultés dans leurs rapports.

Par ailleurs, la valorisation touristique des aires protégées et de leurs zones périphériques pourrait contribuer à l'effectivité du droit des aires protégées.

\footnotetext{
${ }^{909}$ Voir RUTAGARAMA (Eugène), et MARTIN (Adrian), « Partnerships for protected area conservation in Rwanda», art. cit., p. 303.

${ }^{910}$ Voir TRIPLET (Patrick) et VERMEULEN (Cédric), « Comprendre la gouvernance », in TRIPLET (Patrick) (dir.), Manuel de gestion des aires protégées d'Afrique francophone, Paris, Awely, 2009, p. 248.
} 


\section{CHAPITRE II : LA VALORISATON TOURISTIQUE DES AIRES PROTÉGÉES ET DE LEURS ZONES PÉRIPHÉRIQUES}

Selon l'UICN, pour réaliser la gestion durable des aires protégées, il importe de les valoriser sur le plan économique ${ }^{911}$. De plus, cette organisation a identifié le tourisme comme un moyen de conservation durable des aires protégées ${ }^{912}$. Il en découle que la valorisation touristique des aires protégées et par extension de leurs zones périphériques pourrait être une solution pour la conservation durable de ces milieux naturels.

Dans cette perspective, la valorisation touristique des aires protégées ivoiriennes ainsi que de leurs zones périphériques revêt un intérêt pour leur conservation qu'il convient de présenter (Section I) avant d'analyser son opérationnalisation (Section II).

\section{SECTION I : L'intérêt de la valorisation touristique des aires protégées ivoiriennes et de leurs zones périphériques}

L'intérêt de la valorisation touristique des aires protégées ivoiriennes et de leurs zones périphériques découle de deux (2) faits. Le tourisme et notamment le tourisme axé sur les aires protégées est une activité qui pourrait permettre de générer d'importantes ressources financières en vue de leur conservation durable et du développement des communautés riveraines (Paragraphe 1). Également, les aires protégées ainsi que leurs zones périphériques disposent respectivement d'attraits naturels et culturels à fort potentiel de valorisation (Paragraphe 2).

\section{Paragraphe 1 : Le tourisme, une activité financièrement rentable}

Diverses études attestent que le tourisme est un moyen qui permet, à la fois, de financer les actions de conservation des ressources naturelles notamment les aires protégées $^{913}$ et d'opérer le développement des communautés riveraines desdites forêts ${ }^{914}$. Il découle de ce qui précède que le tourisme constitue une source de financement pérenne pour

\footnotetext{
${ }^{911}$ Voir UICN-PAPACO, La lettre des Aires Protégées en Afrique de l'Ouest, UICN-PAPACO, n49, Janvier 2012, pp. 5-6.

${ }^{912}$ Voir UICN/PACO, Le tourisme dans les aires protégées d'Afrique de l'Ouest : quelle contribution à la conservation ?, Ouagadougou, BF: UICN/PACO, 2010, p. 19.

${ }^{913}$ Voir NTOUGOU (Omer), « Développer les ressources financières par le tourisme », in TRIPLET (Patrick) (dir.), Manuel de gestion des aires protégées d'Afrique francophone, Paris, Awely, 2009, p. 806 ; TRIPLET (Patrick), "Diversifier et augmenter les ressources financières des aires protégées », in TRIPLET (Patrick) (dir.), Manuel de gestion des aires protégées d'Afrique francophone, Paris, Awely, 2009, pp. 800 et 804.

${ }^{914}$ Voir NEWMARK (William. D), LEONARD (Nancy L.), SARIKO (Hashim I.), GAMASSA (Deo-Gratias M.), «Conservation attitudes of local people living adjacent to Five protected areas in Tanzania», Biological Conservation, Vol. 63, $\mathrm{n}^{\circ}$ 2, 1993, p. 177; SEKHAR (Nagothu Udaya), « Local people's attitudes towards conservation and wildlife tourism around Sariska Tiger Reserve, India ", Journal of Environmental Management, Vol. 69, $\mathrm{n}^{\circ}$ 4, 2003, p. 339 ; ASMAMAW (Demeke), VERMA (Ashok), « Local attitudes towards environmental conservation and ecotourism around Bale Mountains national park, Ethiopia», Scholarly Journal of Agricultural Science, Vol. 3, n¹1, 2013, p. 512.
} 
la conservation des aires protégées (A) et un levier pour le développement des communautés riveraines (B).

\section{A - Une source de financement pérenne pour la conservation des aires protégées}

Le tourisme est la première industrie mondiale ${ }^{915}$. Ce secteur d'activité totalise 200 millions d'emplois directs et environ 900 millions d'arrivées internationales en 2007, 1 milliard en 2011 et 1, 6 milliard en $2020^{916}$. Sur le plan régional, en Europe et notamment en Allemagne, le tourisme représente un secteur important de l'économie. Son apport est estimé à 164,2 milliards US\$ (de dollars américain) en 2013 soit 4,7\% de son Produit Intérieur Brut $(\mathrm{PIB})^{917}$. En Espagne, le tourisme constitue un des piliers du développement économique ${ }^{918}$. Il rapporte à ce pays 203,8 milliards US\$ en 2013 soit $15,7 \%$ du $\mathrm{PIB}^{919}$. Également, le tourisme joue un rôle important dans l'économie française. En effet, ce secteur d'activité génère 246,2 milliards US\$ en 2013 soit 9,5\% du $\mathrm{PIB}^{920}$.

Cette importance du secteur touristique pour l'économie des États n'est pas spécifique au continent européen. En effet, sur le continent américain, le tourisme demeure un secteur important pour l'économie des pays. En guise d'exemples, en Amérique du Nord et plus précisément aux États-Unis d'Amérique, les recettes touristiques s'élèvent à 1.415,8 milliards US\$ en 2013 soit $8,4 \%$ du PIB $^{921}$. Au Canada, les dépenses des touristes internationaux se chiffraient à 81,8 milliards US\$ en 2013 soit $8,4 \%$ du $\mathrm{PIB}^{922}$. En ce qui concerne l'Amérique du Sud et notamment le Chili, le tourisme a contribué pour 24,3 milliards US\$ à l'économie en 2013 soit 8,6\% du $\mathrm{PIB}^{923}$. En Amérique centrale et plus précisément au Mexique, le tourisme est un secteur d'activité important. Il représente 13,3\% du PIB pour des recettes de 168 milliards US\$ en 2013.

En Afrique et partant dans les pays en voie de développement, le tourisme constitue également une source importante de ressources financières. Par exemple, en Afrique subsaharienne et notamment au Kenya, selon le ministère du tourisme et de la faune, les ressources fauniques contribuent directement et indirectement à l'économie locale et nationale par la production de recettes et la création de richesse ${ }^{924}$. Par exemple, en juin 2006, la valorisation de la faune représentait $70 \%$ du revenu brut du tourisme et $25 \%$ du produit

\footnotetext{
${ }^{915}$ Voir NTOUGOU (Omer), « Développer les ressources financières par le tourisme », in TRIPLET (Patrick) (dir.), Manuel de gestion des aires protégées d'Afrique francophone, op. cit., p. 806.; UICN/PACO, Le tourisme dans les aires protégées d'Afrique de l'Ouest : quelle contribution à la conservation ?, op. cit., 2010, p. 14..

${ }^{916}$ Ibidem.

${ }^{917}$ Voir www.wttc-infographic.org/compare/germany/united-states (consulté le 29/12/2015).

${ }^{918}$ Voir OCDE, Tendances et politiques du Tourisme de l'OCDE 2012, Éditions OCDE, 2013, p. 188.

${ }^{919}$ Voir www.wttc-infographic.org/compare/spain/united-states (consulté le 29/12/2015).

${ }^{920}$ Voir www.wttc-infographic.org/compare/france/united-states (consulté le 29/12/2015).

${ }^{921}$ Voir www.wttc-infographic.org/compare/united-states/united-states (consulté le 29/12/2015).

${ }^{922}$ Voir www.wttc-infographic.org/compare/canada/united-states (consulté le 29/12/15).

${ }^{923}$ Voir www.wttc-infographic.org/compare/chile/united-states (consulté le 29/12/15).

${ }^{924}$ Voir Republic of Kenya, Ministry of Tourism and Wildlife. Draft Wildlife Policy 2007, 2007, p.7.
} 
intérieur brut $(\mathrm{PIB})^{925}$. À l'instar du Kenya, le tourisme axé sur les aires protégées procure d'importantes ressources financières à l'État botswanais. Sur le plan économique, le secteur de l'industrie touristique occupe la deuxième place, après l'exploitation minière ${ }^{926}$. En effet, en 2013, le tourisme a rapporté 1,7 milliards US\$ à l'économie du Botswana soit, 8,4\% du PIB $^{927}$. Il a offert 67.000 emplois soit 9,9\% du total des emplois ${ }^{928}$. En Afrique du Sud, le tourisme a permis de récolter 33,4 milliards US\$ en $2013^{929}$ et sa part dans le PIB était de $9,5 \%{ }^{930}$.

De même en Afrique du Nord, le tourisme demeure une activité économique importante. En effet, en Égypte, malgré la baisse de l'activité touristique en raison l'instabilité du climat sociopolitique qui y a prévalu ces dernières années, le revenu du tourisme en 2014 est estimé à 2,7 milliards de dollars. Par ailleurs, ce secteur offre plus de 4 millions d'emplois (directs ou indirects) soit $6,12 \%$ de $6,12 \%$ de la population de l'Égypte $^{931}$. En outre, en Tunisie, l'industrie touristique a contribué pour 7 milliards US\$ à l'économie en 2013 soit $15,2 \%$ du PIB $^{932}$.

Il ressort de ces quelques exemples susmentionnés que le tourisme et subséquemment l'écotourisme constitue un secteur d'activité qui permet aux États de capter d'importantes ressources financières. Par conséquent, l'OIPR est invitée à accorder une place de choix à l'exploitation écotouristique des aires protégées. Elle lui permettra de mobiliser des ressources financières conséquentes en vue du financement de la gestion durable de ces milieux naturels.

Au-delà de procurer des ressources financières pour la conservation des aires protégées, l'exploitation touristique des aires protégées et/ou de leurs zones périphériques pourrait contribuer au développement des communautés locales.

\section{B - Un levier pour le développement des communautés riveraines des aires protégées}

L'intérêt du tourisme en matière de développement local est double. En effet, le tourisme permet de capter des ressources financières plus ou moins importantes et de créer

\footnotetext{
${ }^{925}$ Voir OKECK (Roselyne N.), « Wildlife-community conflicts in conservation areas in Kenya », African Journal of Conflit Resolution, Vol. 10, n², 2010, p. 66.

${ }^{926}$ Voir MBAIWA (Joseph E.), STRONZA (Amanda L.), « Changes in resident attitudes towards tourism development and conservation in the Okavango Delta, Botswana », op. cit., p. 1951.

${ }^{927}$ Voir www.wttc-infographic.org/compare/botswana/united-states (consulté le 29/12/15).

${ }^{928}$ Voir www.wttc-infographic.org/compare/botswana/united-states (consulté le 29/12/15).

${ }^{929}$ Voir www.wttc-infographic.org/compare/south-africa/united-states (consulté le 29/12/15).

${ }^{930}$ Voir www.wttc-infographic.org/compare/south-africa/united-states (consulté le 29/12/15).

${ }^{931}$ Voir www.sis.gov.eg/Fr/Templates/Articles/tmpArticles.aspx?CatID=328\#.V1MFvTXJxdg (consulté le 24 mai 2016).

${ }^{932}$ Voir www.wttc-infographic.org/compare/tunisia/united-states (consulté le 29/12/15).
} 
des emplois qui contribuent à réduire la pauvreté ; qui elle-même demeure la cause principale des pressions anthropiques sur les aires protégées ${ }^{933}$.

Dans le cadre de cette étude, le potentiel du tourisme à contribuer au développement local peut être perçu à travers des expériences de gestion d'aires protégées réalisées dans plusieurs régions d'Afrique. Il s'agit notamment de l'Afrique de l'Ouest (1), de l'Afrique de l'Est (2) et de l'Afrique australe (3).

\section{L'exposé d'expériences de gestion d'aires protégées en Afrique de l'Ouest et en Afrique centrale}

Au Bénin, au Parc National de la Pendjari ${ }^{934}$, 30\% des recettes produites par les activités cynégétiques sont alloués au développement local ${ }^{935}$. En 2005, les comités villageois dudit Parc ont perçu $70 \%$ des bénéfices de l'écotourisme et de la chasse. Ces revenus se chiffrent à 70.000 dollars US $^{936}$. De plus, les communautés locales perçoivent des revenus issus des circuits touristiques villageois, des frais de guidage, entres autres ${ }^{937}$.

Au Sénégal, l'organisation de l'exploitation touristique du Parc National du Djoudj donne aux communautés des attributions en matière touristique. En effet, le Comité intervillageois des sept villages périphériques du Parc a la charge de l'accueil, du guidage, des activités d'observation des oiseaux d'eau en pirogue, des balades en calèches, de l'hébergement, de la restauration et de la vente d'objet d'art ${ }^{938}$. C'est dans ce cadre que le Comité Inter Villageois du Djoudj a pu bénéficier de richesses et opportunités d'emplois ${ }^{939}$. De même, Cédric VERMEULEN et OUEDRAOGO écrivait, en 2003, qu'au Burkina, la chasse au buffle dans la Réserve de Nazinga a rapporté 1.300 dollars US soit l'équivalent de 21 salaires locaux ${ }^{940}$.

\footnotetext{
${ }^{933}$ Voir UICN/PACO, Le tourisme dans les aires protégées d'Afrique de l'Ouest : quelle contribution à la conservation?, op. cit., p. 19.

${ }^{934}$ Le Parc national de la Pendjari est situé au Nord-Ouest du Bénin, dans le département de l'Atacora, à la frontière du Burkina-Faso. Mieux, ses limites géographiques sont comprises entre $10^{\circ} 30^{\prime}$ et $11^{\circ} 30^{\prime}$ de latitude Nord et $0^{\circ} 50^{\prime}$ et $2^{\circ} 00^{\prime}$ de longitude Est. Voir www.pendjari.net/ (consultés le 14 août 2015).

${ }^{935}$ Voir UICN/PACO, Le tourisme dans les aires protégées d'Afrique de l'Ouest : quelle contribution à la conservation?, op. cit., p. 19.

${ }^{936}$ Voir PNUE (2008) Cité par BINOT (Aurélie), BLOMLEY (Tom), COAD (Lauren), NELSON (Fred), ROE (Dilys) et SANDBROOK (Chris), " Quels ont été les résultats de la GCRN en Afrique ? Renforcement de l'autonomie, économie, environnement » in ROE (Dilys), NELSON (Fred) et SANDBROOK (Chris) (eds), Gestion communautaire des ressources naturelles en Afrique - Impacts, expériences et orientations futures, op. cit., p. 82.

${ }^{937}$ Voir UICN/PACO, Le tourisme dans les aires protégées d'Afrique de l'Ouest : quelle contribution à la conservation?, op. cit., p. 18.

${ }^{938}$ Idem, p. 41.

${ }^{939}$ Idem, p. 20.

${ }^{940}$ Voir VERMEULEN et OUEDRAOGO cité par BINOT (Aurélie), BLOMLEY (Tom), COAD (Lauren), NELSON (Fred), ROE (Dilys) et SANDBROOK (Chris), " Quels ont été les résultats de la GCRN en Afrique ? Renforcement de l'autonomie, économie, environnement » in ROE (Dilys), NELSON (Fred) et SANDBROOK (Chris) (eds), Gestion communautaire des ressources naturelles en Afrique - Impacts, expériences et orientations futures, op. cit., p. 82.
} 
En République centrafricaine, les zones cynégétiques villageoises (ZCV) rapportent des revenus aux populations locales. En 1999 et 2000, elles ont récolté environ 110.000 dollars US ; et $82 \%$ de ces revenus ont été répartis entre les communautés ${ }^{941}$. Selon MMANG-KANGA, le total de ces revenus s'élevait à 1.641.376 d'Euro de 1992 à 2008; et 6.000 Euro l'an par village, en $2004^{942}$. Qu'en est-il des expériences de gestion d'aires protégées en Afrique de l'Est?

\section{La présentation d'expériences de gestion d'aires protégées en Afrique de l'Est}

En dépit des critiques relatives à la modicité des revenus que les GCRN rapportent aux ménages, en raison de facteurs tels que le taux démographique de la communauté ou la faible valeur faunique, des projets de GCRN ont atteints des résultats satisfaisants ${ }^{943}$. Au Kenya, au milieu des années 1970, les honoraires payés par les chasseurs rapportaient au Ranch collectif Mbirikana (situé à proximité du Parc National Amboseli) s'élevaient à 35.000 dollars US ${ }^{944}$. De même, dans le Sud du Kenya, le Ranch collectif et d'écotourisme de Shompole a rapporté 115.000 US en cinq ans. Ces revenus ont été utilisés dans des investissements communautaires tels que l'octroi de bourses d'études aux enfants des pauvres, la modernisation du système d'adduction en eau ${ }^{945}$.

Dans le Nord de la Tanzanie, depuis plusieurs années, des villages riverains de Parcs Nationaux tels que ceux de Serengeti et Tarangire ont conclu des accords touristiques avec les investisseurs privés. Les populations ont adopté une telle démarche dans le but de se voir verser des redevances touristiques dérivés de l'exploitation de la faune qui se trouvent sur leurs terres ${ }^{946}$.

\footnotetext{
${ }^{941}$ Voir MBITIKON (R.) (2004) cité par BINOT (Aurélie), BLOMLEY (Tom), COAD (Lauren), NELSON (Fred), ROE (Dilys) et SANDBROOK (Chris), " Quels ont été les résultats de la GCRN en Afrique ? Renforcement de l'autonomie, économie, environnement » in ROE (Dilys), NELSON (Fred) et SANDBROOK (Chris) (eds), Gestion communautaire des ressources naturelles en Afrique - Impacts, expériences et orientations futures, op. cit., p. 82.

${ }^{942}$ Voir MAMANG-KANGA, 2008 cité par BINOT (Aurélie), BLOMLEY (Tom), COAD (Lauren), NELSON (Fred), ROE (Dilys) et SANDBROOK (Chris), " Quels ont été les résultats de la GCRN en Afrique ? Renforcement de l'autonomie, économie, environnement » in ROE (Dilys), NELSON (Fred) et SANDBROOK (Chris) (eds), Gestion communautaire des ressources naturelles en Afrique - Impacts, expériences et orientations futures, op. cit., p. 82.

${ }^{943}$ Par exemple, pour ROE et al. (2006) : «...en général, les programmes formels de GCRN en Afrique australe n'ont pas obtenu de bons résultats pour ce qui est de la génération de revenus au niveau des ménages...». Ces auteurs sont cités par BINOT (Aurélie), BLOMLEY (Tom), COAD (Lauren), NELSON (Fred), ROE (Dilys) et SANDBROOK (Chris), «Quels ont été les résultats de la GCRN en Afrique ? Renforcement de l'autonomie, économie, environnement " in ROE (Dilys), NELSON (Fred) et SANDBROOK (Chris) (eds), Gestion communautaire des ressources naturelles en Afrique - Impacts, expériences et orientations futures, op. cit., $\mathrm{p}$. 86.

${ }^{944}$ Idem, p. 94 .

${ }^{945}$ Idem, p. 85

${ }^{946}$ Idem, p. 93.
} 
Ces initiatives qui constituent des opportunités économiques pour les communautés locales sont soutenues par les autorités gouvernementales. À titre d'exemples, la structure de gestion des Parcs Nationaux de la Tanzanie ou Tanzania National Parks (TANAPA) a aidé le village d'Ololosokwan à construire un camping afin d'avoir des revenus touristiques supplémentaires.

C'est dans ce contexte que chaque année les revenus tirés du tourisme par le village d'Ololosokwan et d'autres villages sis dans le district de Ngorongoro se sont élevés à plus de 300.000 dollars US, depuis la fin des années 1990. Pour ce qui est de la chasse touristique, les revenus des zones de gestion touristique (en anglais Wildlife Management Areas) sont versés à la division de la faune et une partie est reversée aux communautés locales. L'on estime à $61.850,75$ dollars US et $69.883,44$ dollars US les droits de chasse touristiques versés aux communautés pour la faune chassée sur leurs terres respectivement en 2006 et $2007^{947}$.

Ces exemples de développement des communautés locales à partir des revenus provenant de la faune s'étendent à l'Afrique australe.

\section{Des exemples d'expériences de gestion d'aires protégées en Afrique australe}

En Namibie, la politique de conservation de la nature dans le cadre des concessions de conservation a permis aux communautés de conserver la totalité des revenus dans la mesure où ils ne sont pas taxés. Autrement dit, les communautés perçoivent $100 \%$ des revenus issus de la faune sauvage ${ }^{948}$. Au Botswana, par exemple, des auteurs notent que dans certaines zones, les revenus dérivés des concessions communautaires pour la chasse au trophée peuvent s'élever à 45 dollars US par mois soit $87 \%$ des revenus moyens des ménages ${ }^{949}$.

Au Zimbabwe, en 2006, le ward de Masoka a reçu 132.522 dollars US comme droit dérivé de la faune, dans le cadre du programme de GCRN dénommée CAMPFIRE soit environ 390 dollars US par ménage ${ }^{950}$. Selon TAYLOR et MURPHREE (2007), ces revenus découlant de la faune constituent la principale source de revenu à Masoka suivi du coton ${ }^{951}$.

\footnotetext{
${ }^{947}$ Voir BINOT (Aurélie), BLOMLEY (Tom), COAD (Lauren), NELSON (Fred), ROE (Dilys) et SANDBROOK (Chris), "Quels ont été les résultats de la GCRN en Afrique ? Renforcement de l'autonomie, économie, environnement " in ROE (Dilys), NELSON (Fred) et SANDBROOK (Chris) (eds), Gestion communautaire des ressources naturelles en Afrique - Impacts, expériences et orientations futures, op. cit., $\mathrm{p}$. 94.

${ }^{948}$ Ibidem.

${ }^{949}$ Voir Arntzen et al. (2003) cité par BINOT (Aurélie), BLOMLEY (Tom), COAD (Lauren), NELSON (Fred), ROE (Dilys) et SANDBROOK (Chris), « Quels ont été les résultats de la GCRN en Afrique ? Renforcement de l'autonomie, économie, environnement » in ROE (Dilys), NELSON (Fred) et SANDBROOK (Chris) (eds), Gestion communautaire des ressources naturelles en Afrique - Impacts, expériences et orientations futures, op. cit., p. 87.

${ }^{950}$ Idem, p. 86.

${ }^{951}$ Voir TAYLOR et MURPHREE (2007) cité par BINOT (Aurélie), BLOMLEY (Tom), COAD (Lauren), NELSON (Fred), ROE (Dilys) et SANDBROOK (Chris), " Quels ont été les résultats de la GCRN en Afrique ? Renforcement de l'autonomie, économie, environnement » in ROE (Dilys), NELSON (Fred) et SANDBROOK
} 
Il ressort de ces différents exemples que la valorisation touristique des aires protégées a permis de générer des ressources aux communautés locales. Elle a permis de créer des emplois ainsi que de renforcer les capacités des populations en matière de gestion des projets communautaires. En d'autres termes, elle a contribué à leur développement. Par conséquent, le tourisme et notamment l'écotourisme constitue un moyen qui permet d'opérer le développement local.

Or, la Côte d'Ivoire dispose de plusieurs aires protégées ainsi que d'un riche patrimoine culturel y compris celui des communautés riveraines des aires protégées qui ont un fort potentiel touristique (dont la valorisation pourrait contribuer au développement économique des communautés locales) ${ }^{952}$.

\section{Paragraphe 2 : L'existence d'attraits naturels et culturels dans les aires protégées et leurs zones périphériques}

L'étude des potentialités écotouristiques des aires protégées (A) précédera celle relative à la diversité culturelle des communautés des zones périphériques des aires protégées (B).

\section{A - Les potentialités écotouristiques des aires protégées}

La Côte d'Ivoire possède plusieurs aires protégées qui offrent un important potentiel écotouristique de nature sauvage représentative de l'ensemble de ses écosystèmes ${ }^{953}$. Dans l'optique d'une étude de ces potentialités touristiques, ces aires protégées peuvent être réparties en plusieurs catégories. Ce sont les aires protégées de forêts (1), celles situées en zone de transition Forêt-Savane (2), en milieu urbain (3) et sur la façade littorale atlantique (4).

\section{Les atouts touristiques des aires protégées situées en zone forestière}

Sous ce vocable, nous faisons allusion aux aires protégées couvertes de forêts denses humides ou forêts ombrophiles sempervirentes. Dans cette catégorie d'aires protégées se

(Chris) (eds), Gestion communautaire des ressources naturelles en Afrique - Impacts, expériences et orientations futures, op. cit., p. 86.

${ }^{952}$ Voir République de Côte d'Ivoire, Ministère du Tourisme, La relance touristique en Côte d'Ivoire (Dossier de presse), p. 2.

${ }^{953}$ C'est cette idée que souligne LAUGINIE (Francis) en ces termes : « La Côte d'Ivoire a la chance de posséder un remarquable réseau...de huit Parcs Nationaux et cinq Réserves. Bien réparti selon les grands écosystèmes du pays et couvrant plus de $6 \%$ du territoire national, il abrite des richesses souvent méconnues...». Voir LAUGINIE (Francis), Conservation de la nature et des aires protégées en Côte d'Ivoire, Abidjan, NEI/Hachette et Afrique Nature, 2007, p. 3.

De même, cet auteur écrit: "Les Parcs Nationaux et Réserves, judicieusement répartis sur l'ensemble du territoire, représentent un large échantillonnage des différents écosystèmes de Côte d'Ivoire. ». Voir Idem, p. 32. 
trouvent le Parc National du Mont Péko (PNMP) ${ }^{954}$, le Parc National de Taï (PNT) ${ }^{955}$, et la Réserve de Faune du N'Zo (RFN) qui en est le prolongement par le Nord ${ }^{956}$ et qui de fait forme un seul complexe, en raison de l'unicité des structures de gestion ${ }^{957}$. Le climat au Parc National du Mont Péko est de Type équatorial ${ }^{958}$ et subéquatorial dans le Parc National de Taï et la Réserve partielle de Faune du N'Zo ${ }^{959}$.

Les attraits naturels que constituent ces aires protégées et qui sont de nature à susciter et encourager leur valorisation sur le plan écotouristique peuvent s'apprécier à divers égards. En effet, le Parc National de Taï revêt plusieurs spécificités qui en font un site écotouristique particulier $^{960}$. Cette aire protégée est l'un des derniers blocs forestiers originels des forêts denses de l'Afrique occidentale ${ }^{961}$. Également le Parc National de Taï est la plus grande forêt tropicale primaire mise en défens en Afrique de l'Ouest ${ }^{962}$. Ces différentes caractéristiques lui valent d'être inscrit sur la liste des Réserves de biosphère en 1978 et du patrimoine mondial de l'humanité en $1982^{963}$. De plus, le Parc National de Taï et la Réserve de Faune du N'Zo représente plus de $50 \%$ de la superficie totale des zones forestières sous statut de protection stricte en Afrique de l'Ouest ${ }^{964}$.

Ce vestige de forêt tropicale (Parc National de Taï) dispose d'une riche diversité biologique animale et végétale ${ }^{965}$. En règle générale, la faune présente un attrait touristique constitué d'environ 140 espèces de mammifères (ongulés, primates, carnivores, buffles,

\footnotetext{
${ }^{954}$ Selon LAUGINIE (Francis) le Parc National du Mont Péko : «...est, dans sa totalité, couvert d'une forêt dense humide semi-décidue. ». Voir LAUGINIE (Francis), Conservation de la nature et des aires protégées en Côte d'Tvoire, op. cit., p. 196.

${ }^{955}$ Le Parc Nation de Taï est la plus grande forêt tropicale d'Afrique de l'Ouest placée sous statut de protection. Voir République de Côte d'Ivoire, Ministère de l'Environnement et des Eaux et Forêts/Office Ivoirien des Parcs et Réserves, Plan d'Aménagement du Parc National de Taï, Abidjan, mars 2006, p. 1.

${ }^{956}$ LAUGINIE (Francis) note : « La Réserve partielle de faune du N’Zo prolonge le Parc National de Taï vers le Nord. ». Il écrit également : «La Réserve du N'Zo est recouverte d'une forêt dense humide sempervirente... ». Voir respectivement Voir LAUGINIE (Francis), Conservation de la nature et des aires protégées en Côte d'Ivoire, op. cit., pp. 343 et 346.

${ }^{957}$ Voir République de Côte d'Ivoire, Ministère de l'Environnement et des Eaux et Forêts/Office Ivoirien des Parcs et Réserves, Plan d'Aménagement du Parc National de Taï, op. cit., p. 1.

${ }^{958}$ Voir LAUGINIE (Francis), Conservation de la nature et des aires protégées en Côte d'Ivoire, op. cit., pp. 242 et 344.

${ }^{959}$ Voir République de Côte d'Ivoire, Ministère de l'Environnement et des Eaux et Forêts/ Office Ivoirien des Parcs et Réserves, Plan d'aménagement et de gestion du Parc National d'Azagny, Abidjan, 2008, p. IV. Cette référence sur le climat qui prévaut dans le Parc National de Taï est valable pour la Réserve du N'Zo dans la mesure où ces deux (2) aires protégées (contiguës) sont issues du même bloc forestier.

${ }^{960}$ Il est à noter que dans le présent cas, les développements relatifs aux attraits naturels du Parc National de Taï sont essentiellement les mêmes que ceux de la Réserve du N'Zo dans la mesure où il s'agit du même bloc de forêt qui a été baptisé différemment, en raison du statut de chacune de ces aires protégées.

${ }^{961}$ Voir LAUGINIE (Francis), Conservation de la nature et des aires protégées en Côte d'Ivoire, op. cit., p. 241.

${ }^{962}$ Voir République de Côte d'Ivoire, Ministère de l'Environnement et des Eaux et Forêts/Office Ivoirien des Parcs et Réserves, Plan d'Aménagement du Parc National de Taï, op. cit., p.1.

${ }^{963}$ Voir www.whc.unesco.org/fr/list/195 (consulté le 6 janvier 2016).

${ }^{964}$ Voir République de Côte d'Ivoire, Ministère de l'Environnement et des Eaux et Forêts/Office Ivoirien des Parcs et Réserves, Plan d'Aménagement du Parc National de Taï, op. cit., p.1.

${ }^{965}$ Voir www.whc.unesco.org/fr/list/195 (consulté le 6 janvier 2016).
} 
éléphants de forêt...), 235 à 240 espèces d'oiseaux, 56 espèces d'amphibiens et 43 espèces de serpents $^{966}$.

Parmi ces espèces, il y a des espèces en grand danger d'extinction, à savoir les céphalophes zébrés (Cephalophus zebra) et de Jentink (Cephalophus jentiki). Également, on y rencontre les oiseaux menacés d'extinction ou dont la protection est d'intérêt mondial tels que la Chouette-pêcheuse rousse (Scotopelia ussheri), le Bulbul à barbe jaune (Criniger olivaceus), le Bulbul à queue verte (Bleda eximius), la Pintade à poitrine blanche (Agelastes meleagrides), le Picatharte chauve de Guinée (Picathartes gymno cephalus). Les espèces endémiques sont l'Antilope royale (Neotragus pygmaeus), le Céphalophe d'Ogilby (Cephalophus ogilbyi brookei), l'Hippopotame pygmée (Hexaprotodon liberiensis liberiensis $)^{967}$. En outre, l'endémisme s'étend à des espèces telles que les primates ${ }^{968}$, les oiseaux $^{969}$.

Le Parc National du Mont Péko a la particularité d'être une aire protégée de forêt faite de domes granitiques, de collines ou monts dont le principal est le mont Péko. Dans ce Parc, il y a des Éléphants (Loxodonta africana cyclotis), des primates, des ongulés (antilopes), des carnivores tels que la panthère (Pantheras pardus), le chat doré, et des oiseaux $(257 \text { espèces })^{970}$.

Qu'en est-il des attraits écotouristiques des aires protégées situées en zone de transition Forêt-Savane?

\section{Les attraits écotouristiques des aires protégées localisées en zone de transition Forêt-Savane}

Les aires protégées situées dans la zone de transition Forêt-Savane sont des espaces mis en défens couverts par deux végétations : la forêt et le savane. Il s'agit des Parcs Nationaux de la Comoé ${ }^{971}$, de la Marahoué ${ }^{972}$, du Mont Sangbé ${ }^{973}$ et de la Réserve de Faune

\footnotetext{
${ }^{966}$ Voir KOUADIO (Konan Kan Fulbert), Analyse du système de biomonitoring du Parc National de Taï, Mémoire on line, Ingénieur des Eaux et Forêts, Institut National Polytechnique Houphouet-Boigny, 2006, non paginé; disponible sur www.memoireonline.com/06/09/2139/m Analyse-du-systeme-de-biomonitoring-duParc-National-de-Tai3.html (consulté, le 15 août 2015).

${ }^{967}$ Voir LAUGINIE (Francis), Conservation de la nature et des aires protégées en Côte d'Ivoire, op. cit., pp. 242-243.

${ }^{968}$ Voir LAUGINIE (Francis) fait remarquer que chez les primates, huit espèces et sous-espèces se révèlent endémiques. Ce sont le Colobe noir et blanc d'Afrique occidentale (Colobus polykomos polykomos), le Colobe bai (Colobus badius badius), le Colobe de Van Beneden (Colobus verus), les cercopithèques Diane (Cercopithecus diana diana), Mone (Cercopithecus mona campbelli), Pétauriste (Cercopithecus petaurista buettikoferi) et le Cercocèbe fuligineux (Cercocebus torquatus atys). Voir Idem, p. 243.

${ }^{969}$ En ce qui concerne les espèces d'oiseaux, 28 sont endémiques. Voir LAUGINIE (Francis), Conservation de la nature et des aires protégées en Côte d'Ivoire, op. cit., pp. 242-243.

${ }^{970}$ Voir LAUGINIE (Francis), Conservation de la nature et des aires protégées en Côte d'Ivoire, op. cit., pp. 203-204.

${ }^{971}$ Voir LAUGINIE (Francis) affirme : «La végétation du Parc National de la Comoé appartient au domaine soudanais dont les principales formations sont la forêt claire et la savane. ». Voir LAUGINIE (Francis), Conservation de la nature et des aires protégées en Côte d'Ivoire, op. cit., p. 125.
} 
d'Abokouamékro ${ }^{974}$. Les climats dans ces zones sont les climats subéquatoriaux ou équatoriaux et les climats tropicaux ${ }^{975}$, à l'exception du Parc National de la Comoé qui se trouve dans une zone de transition climatique climat tropical humide-climat tropical subaride $^{976}$.

Ces aires protégées situées dans la zone de transition forêt-savane disposent de plusieurs attraits touristiques. Premièrement, la spécificité climatique de ces aires protégées permet d'avoir sur leur territoire des espèces fauniques et floristiques à la fois de forêt et de savane. Toutefois, à travers les lignes à venir, nous ne traiterons que des espèces ou éléments de ces aires protégées qui ont un potentiel écotouristique. Ainsi, si dans toutes les aires protégées établies dans la zone de transition forêt-savane il existe des espèces fauniques telles que les primates, l'Antilope bubale, l'Hippotrague, le Cob de Buffon, le Buffle, l'Éléphant ${ }^{977}$, d'autres espèces sont spécifiques à certaines aires protégées. Par exemple, dans le Parc National de la Comoé, l'on rencontre des crocodiles, des hippopotames et des oiseaux migrateurs ${ }^{978}$. Au Parc National du Mont Sangbé il existe une espèce en danger telle que le léopard ${ }^{979}$,. Dans la Réserve de faune d'Abokouamékro, se retrouve une espèce telle que le rhinocéros ${ }^{980}$.

La Réserve de faune d'Abokouamékro et le Parc National du Mont Sangbé (PNMS) ont un relief accidenté ${ }^{981}$. À Abokouamékro, l'on a des plateaux et des collines ${ }^{982}$ tandis que dans le PNMS il est à noter la présence de montagnes, d'inselbergs et de collines. Pour ROUGERIE, ces reliefs comptent parmi les plus spectaculaires d'Afrique de l'Ouest ${ }^{983}$. Ces

\footnotetext{
972 Selon LAUGINE Francis : «Le Parc de la Marahoué est très exactement situé à cheval sur les deux grands biomes du pays, environ le quart de sa superficie étant recouvert de savanes et les trois quarts de forêts semidécidue... ».Voir LAUGINIE (Francis), Conservation de la nature et des aires protégées en Côte d'Ivoire, op. cit., p. 178.

${ }^{973}$ En ce qui concerne la végétation du Parc National du Mont Sangbé, LAUGINIE (Francis) écrit : «La partie $d u$ Sud du Parc, plus humide, permet le développement d'une végétation de type guinéen tandis que la partie septentrionale est couverte de savanes sub-soudaniennes. ». Voir LAUGINIE (Francis), Conservation de la nature et des aires protégées en Côte d'Ivoire, op. cit., pp. 213-214.

${ }^{974} \mathrm{La}$ végétation d'Abokouamékro est faite de formations forestières (galeries forestières) et de formations de savane. Voir LAUGINIE (Francis), Conservation de la nature et des aires protégées en Côte d'Ivoire, op. cit., pp. 275-276.

${ }_{975}$ Idem, pp. $177-178 ; 212 ; 272$

${ }^{976}$ Idem, pp. 123-124.

${ }^{977}$ Voir www.oipr.ci/index.php/reseau/parcs-nationaux/parc-national-de-la-Comoé (consulté le 26 mai 2016) ; www.oipr.ci/index.php/reseau/parcs-nationaux/parc-national-de-la-Marahoué (consulté le 26 mai 2016); www.oipr.ci/index.php/reseau/reserves-naturelles/reserve-de-faune-d-abokouamekro (consulté le 2 août 2015) ; www.oipr.ci/index.php/reseau/parcs-nationaux/parc-national-du-mont-sangbé (consulté le 2 août 2015).

${ }^{978}$ Voir www.oipr.ci/index.php/reseau/parcs-nationaux/parc-national-de-la-Comoé (consulté le 26 mai 2016).

${ }^{979}$ Voir www.oipr.ci/index.php/reseau/reserves-naturelles/reserve-de-faune-d-abokouamekro (consulté le 2 août 2015); www.oipr.ci/index.php/reseau/parcs-nationaux/parc-national-du-mont-sangbé (consulté le 2 août 2015).

${ }^{980}$ Voir www.oipr.ci/index.php/reseau/reserves-naturelles/reserve-de-faune-d-abokouamekro (consulté le 2 août 2015).

${ }_{981}$ Voir LAUGINIE (Francis), Conservation de la nature et des aires protégées en Côte d'Ivoire, op. cit., pp. 212 et 272.

${ }^{982}$ Idem, p. 272.

983 Voir ROUGERIE (1960), cité par Voir LAUGINIE (Francis), Conservation de la nature et des aires protégées en Côte d'Ivoire, op. cit., p. 211.
} 
aires protégées sont adaptées au tourisme de montagne. En ce qui concerne le PNC, il est le plus vaste parc de l'Afrique de l'Ouest. On y des plateaux, des roches, de grandes vallées, de vastes plaines $^{984}$. Ces étendues de végétations sont favorables au tourisme de vision.

Au surplus, certaines aires protégées situées en milieu urbain possèdent également des atouts écotouristiques.

\section{Les atouts écotouristiques des aires protégées situées en milieu urbain}

Les aires protégées situées en milieu urbain sont le Parc National du Banco ${ }^{985}$ et la Réserve partielle de flore Dahliafleur ${ }^{986}$. La Réserve partielle de flore Dahliafleur, à l'origine réserve horticole ${ }^{987}$, est une étendue de 95 hectares de forêts biens conservée et 8 hectares de forêts secondaires $^{988}$. Elle est dotée d'une diversité floristique et est bordée par la lagune Ébrié $^{989}$. Cette aire protégée renferme également 15 espèces de mammifères et 43 espèces d'oiseaux ${ }^{990}$.

En ce qui concerne le Parc National du Banco, c'est une forêt dense humide dont la biodiversité floristique est similaire à celle des aires protégées de forêts. Cette biodiversité floristique est estimée à plus de 622 espèces dont 10 à 15 espèces sont classées rares. Sept espèces sont en voie de disparition. Ce sont: Bowringia discolor, Dorstenia embergeri, Zanthoxylum mesoneurospinosa, Hugonia rufipilis, Pararistolochia mannii et Solanum terminale, lodes liberica ${ }^{991}$.

En outre, le Parc National du Banco est traversée par une rivière (rivière du Banco). La faune se caractérise essentiellement par les primates, en général, y compris des groupes de chimpanzés, des carnivores (civette, Mangouste rouge), des antilopes (Antilopes royales, céphalophes...) et des rongeurs ${ }^{992}$.

Par ailleurs, la Côte d'Ivoire possède des aires protégées situées sur la façade littorale atlantique qui renferment également des attraits.

\footnotetext{
${ }^{984}$ Voir LAUGINIE (Francis), Conservation de la nature et des aires protégées en Côte d'Ivoire, op. cit., p. 123.

${ }^{985}$ Le Parc National du Banco est située au cœur de la ville d'Abidjan (c'est-à-dire la capitale économique de la Côte d'Ivoire). Voir LAUGINIE (Francis), Conservation de la nature et des aires protégées en Côte d'Ivoire, op. cit., p. 99.

${ }_{986}$ La Réserve partielle de flore Dahliafleur est située à la périphérie de la ville d'Abidjan (en Côte d'Ivoire). Voir www.ci.chm-cbd.net/links/geopoints/dahlia-fleur (consulté le 17 janvier 2016).

${ }^{987}$ Voir www.ci.chm-cbd.net/links/geopoints/dahlia-fleur (consulté le 17 janvier 2016).

${ }^{988}$ Voir www.oipr.ci/index.php/reseau/reserves-naturelles/reserve-naturelle-partielle-de-dahliafleur (consulté le 17 janvier 2016).

${ }^{989}$ Voir www.oipr.ci/index.php/reseau/reserves-naturelles/reserve-naturelle-partielle-de-dahliafleur (consulté le 17 janvier 2016).

${ }^{990}$ Voir www.oipr.ci/index.php/reseau/reserves-naturelles/reserve-naturelle-partielle-de-dahliafleur (consulté le 17 janvier 2016).

${ }_{991}^{991}$ Voir LAUGINIE (Francis), Conservation de la nature et des aires protégées en Côte d'Ivoire, op. cit., p. 108.

${ }^{992}$ Idem, pp. 108-109.
} 


\section{Les attraits écotouristiques des aires protégées localisées sur la façade littorale atlantique}

Les aires protégées ivoiriennes situées sur la façade littorale atlantique sont les Parcs Nationaux d'Azagny ${ }^{993}$ et des Îles Éhotilé ${ }^{994}$. Les atouts écotouristiques de ces aires protégées relèvent de plusieurs caractéristiques biologiques, physiques et naturelles.

En effet, ces deux (2) aires protégées appartiennent au réseau des zones humides d'importance internationale. En effet, le Parc National d'Azagny a été inscrit sur la liste Ramsar 1996 et le Parc National des Îles Éhotilé en 2005 ${ }^{995}$. En outre, le Parc National des Îles Éhotilé (PNIE) est inscrit sur la liste indicative du patrimoine mondial de l'Unesco en $2006^{996}$. Ce faisant, ces zones disposent d'une riche diversité biologique dont l'intérêt de la conservation leur a valu d'être inscrits sur la liste des sites Ramsar, d'une part, et sur celle précitée de l'Unesco (pour ce qui du PNIE), d'autre part.

Le Parc National d'Azagny regorge de nombreux plans d'eau et plus de $60 \%$ de la superficie du Parc est inondée ${ }^{997}$. La végétation faite de forêts denses $(48 \%)$, de savanes côtières, de marécages de mangrove et autres formations aquatiques ${ }^{998}$ dote cette aire protégée en une faune riche et variée. Ainsi, l'on y retrouve, à titre indicatif, des mammifères tels que les primates y compris des chimpanzés sur une île du Parc, l'éléphant de forêt, le Buffle, les antilopes, le potamochère, des carnivores (Civette, Panthère noire...) et les oiseaux migrateurs, des crocodiles (Crocodile du Nil, Crocodile à front large...) ${ }^{999}$. Certaines espèces rares et menacées telles que l'hippopotame pygmée, chevrotain aquatique, le lamantin ouest africain s'y retrouvent également ${ }^{1000}$.

En ce qui concerne le Parc National des Îles Éhotilé, composée de six îles, cette aire protégée est d'une richesse faunique relativement pauvre en mammifères, en raison du braconnage. Néanmoins, on y trouve des carnivores (Civette), des ongulés (le Guib harnaché, le Céphalophe de Maxwell, l'Antilope royale, le Potamochère) ${ }^{1001}$. La particularité de cette aire protégée demeure son caractère insulaire, de site archéologique, la présence du lamantin

\footnotetext{
${ }^{993}$ En effet, traitant de la végétation du Parc National d'Azagny, LAUGINIE (Francis) écrit : « La proximité de l'océan, l'étendue des zones hydromorphes et les caractéristiques des sols ont rassemblé, sur ces quelques milliers d'hectares, une multiplicité de formations végétales du domaine guinéen qui s'imbriquent en une mosaïque du plus bel effet, avec des végétations ombrophiles, littorale, marécageuse, aquatique et de fourrés. ". Voir LAUGINIE (Francis), Conservation de la nature et des aires protégées en Côte d'Ivoire, op. cit., p. 83. ${ }^{994}$ Idem, p. 164.

${ }^{995}$ Voir TIEBLEY (Yves-Didier), La Côte d'Ivoire et la gestion des ressources naturelles marines, Thèse unique, Droit public, Université de Maastricht, op. cit., p. 60.

${ }^{996}$ Voir www.oipr.ci/images/stories/pdf/depliant_2011_iles-ehotile.pdf, consulté le17 janvier 2016.

${ }_{997}^{9}$ Voir LAUGINIE (Francis), Conservation de la nature et des aires protégées en Côte d'Tvoire, op. cit., p. 82

${ }^{998}$ Idem, pp. 83-87.

${ }^{999}$ Voir LAUGINIE (Francis), Conservation de la nature et des aires protégées en Côte d'Ivoire, op. cit., pp. 8991.

${ }^{1000}$ Ibidem.

${ }^{1001}$ Voir LAUGINIE (Francis), Conservation de la nature et des aires protégées en Côte d'Ivoire, op. cit., p. 188 .
} 
ouest-africain (menacés d'extinction), des chauves-souris, une avifaune mal connue et surtout les oiseaux migrateurs ${ }^{1002}$.

En somme, les aires protégées de Côte d'Ivoire renferment des attraits biologiques (diversité fauniques et floristiques, des espèces endémiques), physiques (montagnes, collines, savanes, forêts, zones humides...) à fort potentiel écotouristique, à l'instar de leurs zones périphériques.

\section{B - La diversité culturelle des communautés riveraines des aires protégées}

La Côte d'Ivoire compte environ 60 ethnies réparties en quatre (4) grands groupes culturels : les Akans, Les Gours, les Krous et les Mandé ${ }^{1003}$. Les ethnies qui composent le groupe Akan sont installées au Sud, au Centre et à l'Est ${ }^{1004}$. Les peuples du groupe Akan installés au Sud (Abbey, Abouré, Ahizi, Adjoukrou, Alladian, Appolo ou N'Zima, Attié ou Akyé, Avikam, Brignan, Ébrié, Éga, Éhotilé, Élomouin, Éssouma, Gwa,M’batto), sont majoritairement des agriculteurs et de tradition pêcheurs, en raison des plans d'eaux (mers, lagunes...) qui drainent leurs terres. Ce sont des peuples dont l'organisation sociale repose pour la plupart sur des systèmes de classe d'âge formant des générations ; et à travers lesquels le pouvoir politique se transmet de génération en génération.

En ce qui concerne ceux du centre (Baoulé, Ayaou ) et de l'Est (Abron, Agni), ils ont un mode d'organisation sociale stratifié de manière ascendante (en chefferie, canton, royaume) dans lequel la dévolution du pouvoir politique est, par principe, matrilinéaire. L'or, les attributs royaux, le port de grands pagnes traditionnels et la fête d'igname sont communs à ces peuples et constituent des attraits touristiques.

Les peuples qui forment le groupe Gour sont établis au Nord de la Côte d'Ivoire ${ }^{1005}$. En ce qui concerne le groupe Krou, ces ethnies composantes ${ }^{1006}$ sont géographiquement

\footnotetext{
${ }^{1002}$ Ibidem.

${ }^{1003}$ Voir République de Côte d'Ivoire, Institut National de la Statistique, Langues officielles et langues nationales, disponible sur

www.ins.ci/gene/LANGUES\%20OFFICIELLES\%20ET\%20LANGUES\%20NATIONALES.html (consulté le 27 mai 2016).

${ }^{1004}$ Il s'agit des ethnies Abbey, Abouré, Adjoukrou, Alladian, Appolo ou N'Zima, Attié ou Akyé, Avikam, Ayaou, Baoulé, Brignan, Ébrié, Éga, Éhotilé, Élomouin, Éssouma, Gwa, M’batto. Voir BOTAU (Blay Adrienne), Intégration et autonomie des minorités en côte d'ivoire, Commission des Droits de l'Homme/ Sous-Commission de la promotion et de la protection des droits de l'homme/Groupe de travail sur les minorités/Dixième session, 1-5 mars 2004, p. 3.

${ }^{1005}$ Ce sont les ethnies Birifor, Camara, Degha, Djafolo, Djimini, Djamala, Gbin, Koulango, Lobi, Lohon, Lohron, Tagbana ou Tagouana, Ténéwéré, Tiembara, Nafara, Niarafolo, Samassogo, Sénoufo. Voir BOTAU (Blay Adrienne), Intégration et autonomie des minorités en côte d'ivoire, op.cit., pp. 3-4.

${ }^{1006}$ Nous faisons allusion au Ahizi, Bakwe, Bété, Dida, Gagou, Godié, Guéré, Kouzié, Kroumen, Neyo, Niaboua, Wini, Wobè. Voir BOTAU (Blay Adrienne), Intégration et autonomie des minorités en côte d'ivoire, op. cit., p. 4.
} 
réparties au Sud-Ouest ainsi qu'au Centre-Ouest de la Côte d'Ivoire ${ }^{1007}$. Quant au groupe Mandé, les ethnies qui le forment ${ }^{1008}$ sont originaires de la Guinée, du Libéra, du Mali et sont implantées au Nord-Ouest ainsi qu'à l'Ouest de la Côte d'Ivoire. Les Krou et les Mandés sont des sociétés caractérisés par l'univers des maques et les danses traditionnelles. Cette diversité ethnique est expressive d'une mosaïque de cultures.

C'est ainsi pour les Parcs Nationaux du Sud-Ouest que sont le Parc National de Taï et la Réserve du N'Zo, et de l'Ouest tels que le Mont Péko, le Mont Sangbé et du Centre ouest (le Parc National de la Marahoué), les visiteurs pourraient bénéficier dans les zones périphériques des attraits culturels des circuits de masques traditionnelles y compris celui que représente les fillettes qui s'élancent sur des poignards tendus des danseurs ${ }^{1009}$.

Les visiteurs des Parcs Nationaux du centre (Abokouaméko, Lamto) situées en pays Baoulé pourraient découvrir la culture de ce peuple akan (baoulé) à travers son histoire, les statuettes, le savoir-faire dans l'exploitation aurifère ainsi que les objets de mesures traditionnelles y relatifs et les produits dérivés. Egalement, il y a les pagnes richement tissées.

Enfin, les visiteurs du Parc National de la Comoé pourraient au-delà de ces attraits naturels précédemment analysés, découvrir la culture du peuple Gour à travers le circuit du Poro $^{1010}$, la mosquée sahélo-soudanaise de Kong (qui date du 17 siècle-la plus ancienne de la Côte d'Ivoire ${ }^{1011}$ ), les villages artisanaux notamment de tisserands (Waraniéné), de forgerons (Koni), de colliers en perles (Kapelé) ${ }^{1012}$. De plus, les danses, les arts culinaires, l'univers des chasseurs traditionnels $\left(\operatorname{dozos}^{1013}\right)$ aux pouvoirs surnaturels, les pratiques et expressions

\footnotetext{
${ }^{1007}$ Ibidem.

${ }^{1008}$ Le groupe ethnique Mandé se compose de Gouro, Koyata, Mahou, Malinké, Mangoro, Nomou, Toura, Wan, Yacouba ou Dan. Ibidem.

${ }^{1009}$ Voir www.tourismeci.org/circuits.htm (Consulté le 19 décembre 2015).

${ }^{1010}$ Le Poro est société d'initiation secrète [voir BASSET (J. Thomas), Le coton des paysans : une révolution agricole (Côte d'Ivoire 1880-1999), IRD Éditions, Collection À travers champs, 2002, p. 92.] commune à la majorité des populations établies dans les forêts guinéennes de l'Afrique de l'Ouest. Il s'agit de la Sierra Leone, de la Guinée, du Libéria, du Sud du Mali et au Nord de la Côte d’Ivoire. Voir LITTLE (K.), « The political functions of the Poro, part 1 », Africa, vol. 35, 1965, p. 349-365 et «The political functions of the Poro, part 2», Africa, vol. 36, 1966, p. 62-72 cité par BØAS (Morten), «Funérailles pour un ami : des luttes de citoyenneté dans la guerre civile libérienne », Politique africaine, $n^{\circ}$ 112, décembre 2008, p. 43. et KOUAMÉ (Y. Lucien), KOUAMÉ (I. Roland), KOUASSI (K. M. Colombe) et KOUASSI (Y. Cyrus), Le Poro des Senoufos de Côte d'Ivoire, une véritable école de formation traditionnelle, Exposé dirigé par Dr. Diomandé, UFR Communication Milieu Société, L1, Université Alassane Ouattara (Côte d'Ivoire), 2014, pp. 5-8.

Chez les peuples Sénoufos du Nord de la Côte d'Ivoire, l'initiation qui est principalement destinée aux jeunes garçons commence à l'âge de 7 ans (jusqu'à 12 ans) et se déroule à l'extérieur du village (dans le bois sacré) en trois (3) étapes de 7 ans. Les jeunes y effectuent périodiquement des retraites d'un mois environ. En ce qui concerne les femmes, elles peuvent être initiées à la première étape. Voir KOUAMÉ (Y. Lucien), KOUAMÉ (I. Roland), KOUASSI (K. M. Colombe) et KOUASSI (Y. Cyrus), Le Poro des Senoufos de Côte d'Ivoire, une véritable école de formation traditionnelle, op. cit., pp. 5-8.

${ }^{1011}$ Voir www.whc.unesco.org/fr/listesindicatives/5088/ (consulté le 26 mai 2016).

${ }^{1012}$ Voir AUZIAS (Dominique) et LABOURDETTE (Jean-Paul), Côte d'Ivoire 2015, Petit Futé, 2015, p. 407.

${ }^{1013}$ Les Dozos sont des chasseurs traditionnels aux pouvoirs surnaturels appartenant à des confréries africaines de chasseurs qui sont des sociétés plus ou moins sécrètes. Répandus dans toute l'Afrique subsaharienne, les Dozos ont l'art de la chasse qui les caractérise, une bonne connaissance de la faune sauvage, de la flore, des
} 
culturelles liées au balafon inscrites sur la liste du patrimoine culturel immatériel de l'humanité de l'Unesco ${ }^{1014}$ constituent d'autres attraits culturels.

Il ressort de tous les développements précédents que la Côte d'Ivoire regorge d'attraits touristiques qu'elle peut valoriser. D'une part, il s'agit de son réseau d'aires protégées présentant des sites exceptionnels; représentatifs d'un écosystème variée (Foret et savane, milieux aquatique). Il est de même pour les espèces de faune et de flore que l'on y retrouve ; y compris des espèces rares, en danger ou menacées d'extinction. D'autre part, ces atouts touristiques sont une mosaïque culturelle tributaire de la diversité ethnique des peuples de ce pays d'Afrique de l'Ouest.

La valorisation touristique des potentialités (touristiques) des aires protégées ivoiriennes et de leurs zones périphériques est susceptible de générer des ressources financières importantes à l'OIPR et aux communautés locales. C'est ainsi que ces ressources pourraient permettre au gestionnaire d'accroître les investissements relatifs aux moyens matériels et opérationnels requis pour la conservation durable de ces forêts mis en défens. Mieux, les ressources générées par l'exploitation touristique des aires protégées pourraient contribuer à la réduction de la pauvreté des communautés locales et logiquement permettre d'espérer une réduction significative des pressions anthropiques. En effet, les communautés locales identifient la pauvreté comme une cause essentielle des pressions qu'elles exercent sur les aires protégées.

Fort de ces conclusions, l'OIPR est invité à œuvrer de manière effective et efficace à la valorisation touristique des aires protégées en vue d'une conservation durable de ces milieux naturels. Autrement dit, il s'agira de promouvoir l'écotourisme ${ }^{1015}$ dans les aires protégées et leurs zones périphériques. Cette solution se justifie par le fait, qu'au-delà de son caractère d'utilité pratique, elle a aussi un fondement légal. En effet, il découle de la lecture des articles 19, 20 alinéa 4 de la loi de 2002 sur les aires protégées et $1^{\text {er }}$ du décret portant création de l'OIPR que l'OIPR a, dans le cadre de sa mission, la valorisation touristique des Parcs Nationaux et des Réserves naturelles partielles ainsi que de leurs zones périphériques $^{1016}$. C'est à juste titre qu'il sied d'analyser les modalités de la valorisation

plantes médicinales et de la cosmogonie. Voir ONUCI, Rapport sur les abus des droits de l'homme commis par les dozos en République de Côte d'Tvoire, Juin 2013, pp. 10-11.

${ }^{1014}$ Le balafon des communautés Sénoufo (réparties sur les territoires) de la Côte d'Ivoire, du Burkina-Faso et du Mali un xylophone pentatonique qui a été inscrit, en 2012 à Paris, sur la liste représentative du patrimoine culturel immatériel de l'humanité (Unesco), par la Décision 7. COM 1121 (Paris 2012). Voir www.unesco.org/culture/ich/fr/RL/les-pratiques-et-expressions-culturelles-liees-au-balafon-des-communautessenoufo-du-mali-du-burkina-faso-et-de-cote-divoire-00849 (consulté le 24 mai 2016).

${ }^{1015}$ Certes, il n'y a pas, à ce jour, une définition universelle de l'écotourisme (voir UICN/PACO, Le tourisme dans les aires protégées d'Afrique de l'Ouest : quelle contribution à la conservation ?, op. cit., p.12). Toutefois, depuis 1991, la Société Internationale d'Écotourisme a formulé une définition qui semble aujourd'hui la définition de référence. En effet, elle définit l'écotourisme comme : «...une forme de voyage responsable dans des espaces naturels, qui contribue à la préservation de l'environnement et au bien-être des populations locales. ». Voir www.ecotourism.org (consulté le 10 août 2015).

${ }^{1016} \mathrm{Ces}$ articles disposent respectivement : «La gestion des parcs et réserves relève d'un établissement public national de type particulier, doté de la personnalité morale et de l'autonomie financière...» et « Les missions de 
écotouristique des aires protégées et de leurs zones périphériques afin de réaliser l'objectif de conservation durable des aires protégées ivoiriennes.

\section{SECTION II : Les modalités de la valorisation écotouristique des aires protégées et de leurs zones périphériques}

$\mathrm{Au}$ chapitre des modalités de la valorisation écotouristique des aires protégées ivoiriennes et de leurs zones périphériques, il est souhaitable que l'exploitation écotouristique de ces zones soit concédée à des opérateurs touristiques privés (Paragraphe 1). En outre, des projets communautaires écotouristiques devraient être développés à l'intérieur des aires protégées ainsi que dans leurs zones périphériques (Paragraphe 2).

\section{Paragraphe 1 : La nécessité de concéder l'exploitation écotouristique des aires protégées à des opérateurs touristiques privés}

La nécessité de concéder l'exploitation écotouristique des aires protégées à des opérateurs touristiques privés suppose que cette approche de gestion revêt un intérêt qu'il convient de présenter (A), avant de réfléchir sur les aspects juridiques de ladite concession (B).

\section{A - De l'intérêt de la concession de l'exploitation écotouristique des aires protégées à des opérateurs touristiques privés}

Les Parcs Nationaux et Réserves naturelles partielles de la Côte d'Ivoire sont insuffisamment valorisés sur le plan touristique ${ }^{1017}$. Ce constat est valable pour les pays de l'Afrique de 1 'Ouest ${ }^{1018}$. En effet, pour Omer NTOUGOU, les aires protégées : «...offrent souvent la part la plus importante de telles activités récréatives mais elles ne génèrent qu'une petite partie du total des bénéfices économiques produits par l'écotourisme. ». Ce constat est

l'Établissement, sur l'ensemble des parcs et réserves sur lesquels il exerce son autorité, comprennent, notamment et obligatoirement : ... 4. la mise en œuvre d'une politique de gestion durable par la promotion des activités légalement permises en fonction de la nature juridique du parc ou de la réserve considérée et de sa zone périphérique; ...». En ce qui concerne l'article 3 du décret précité de 2002 portant création de l'OIPR qui traite des missions de cet EPN, il indique, entre autres : "L'Office a pour mission, sur l'ensemble des parcs et réserves sur lesquels il exerce son autorité, notamment : ...

11. assurer le développement touristique des parcs et réserves par la promotion du secteur. » (souligné par nous). Par ailleurs, aux termes de l'article 3 alinéa 8 du décret précité portant création de l'OIPR, l'une de ses missions est de : "négocier et suivre des contrats de terroir définissant les modalités de collaboration avec les populations riveraines des parcs pour la mise en ouvre de programmes d'aménagement de la zone périphérique...». Sur ces points relatifs aux attributions de l'OIPR en matière de valorisation touristique, voir également République de Côte D’Ivoire, Ministère de l'Environnement, des Eaux et Forêts/ Office Ivoirien des Parcs et Réserves, Procédures de concession de l'exploitation touristique des Parcs Nationaux et Réserves, Abidjan, avril 2010, p. 2.

${ }^{1017}$ Voir LAUGINIE (Francis), Conservation de la nature et des aires protégées en Côte d'Ivoire, op. cit., p. 54.

${ }^{1018}$ Voir NTOUGOU (Omer), « Développer les ressources financières par le tourisme », in TRIPLET (Patrick) (dir.), Manuel de gestion des aires protégées d'Afrique francophone, Paris, Awely, 2009, p. 806. 
également fait dans le cadre d'une étude sur la capacité du tourisme à réduire la pauvreté au sein des communautés locales dans les aires protégées ouest-africaines ${ }^{1019}$.

C'est ainsi qu'il convient de s'interroger sur les raisons qui expliquent cette situation. La raison principale de cette insuffisance de valorisation des aires protégées est essentiellement la gestion étatique de ces milieux naturels. Ainsi, la valorisation touristique des aires protégées est limitée par des facteurs tels que l'insuffisance, le manque d'entretien des aménagements réalisés pour l'exploitation touristique (pistes d'accès, et infrastructures...) et la qualité médiocre du service ${ }^{1020}$. Cette défaillance des États à gérer de manière durable les aires protégées étatiques s'expliquerait par des difficultés de financement auxquelles ils sont confrontés ${ }^{1021}$. En outre, bien souvent, les gestionnaires (étatiques) des aires protégées ne disposent pas de compétences requises pour tous les aspects de leur gestion notamment en matière de valorisation écotouristique ${ }^{1022}$.

De plus, même si la tendance de la gestion des aires protégées est à la cogestion (État/communauté) ou la gestion par le privé, l'UICN fait le constat selon lequel les systèmes de gestion se révèlent défaillants, à quelques exceptions près ${ }^{1023}$. Or, selon une étude de l'UICN sur la contribution du tourisme à la conservation des aires protégées, le recours au secteur privé en matière de développement du tourisme est indispensable parce qu'il permet de rapporter des recettes pour la valorisation des aires protégées ${ }^{1024}$. Au surplus et en guise d'exemple, pour faire face aux difficultés de financements auxquelles ils sont confrontés dans la mise en œuvre des politiques touristiques, les États membres de $1^{\prime} \mathrm{OCDE}^{1025}$ ont recours au secteur privé ${ }^{1026}$. Également, selon l'OCDE, en matière touristique, les opérateurs du privé sont les principaux prestataires de services ${ }^{1027}$.

\footnotetext{
${ }^{1019}$ Voir UICN/PACO, Le tourisme dans les aires protégées d'Afrique de l'Ouest : quelle contribution à la conservation?, Ouagadougou, BF: UICN/PACO, 2010, p. 8.

${ }^{1020}$ Ibidem.

${ }^{1021}$ Voir les développements faits à la Première partie de cette étude, le Titre I, le Chapitre II, la Section 2, le Paragraphe 1 et précisément le point intitulé " B- L'insuffisance des dotations budgétaires pour la conservation des aires protégées".

${ }^{1022}$ Voir la Première partie de cette thèse, le Titre I, le Chapitre II, la Section 1, le Paragraphe 2 : Les limites des pouvoirs du personnel en charge des aires protégées et précisément le point "A- Les limites relatives aux pouvoirs d'administration des aires protégées"; voir UICN/PACO, Le tourisme dans les aires protégées d'Afrique de l'Ouest : quelle contribution à la conservation ?, op. cit., p. 8.

${ }^{1023}$ À titre d'exemples, une Réserve privée telle que Makasutu Cultural Forest en Gambie ou une aire protégée comme AMPC de Bamboung au Sénégal qui bénéficient de financements extérieurs se révèlent efficaces en matière d'implication des communautés locales à la gestion durable desdites aires protégées, de répartition équitable des bénéfices. Voir UICN/PACO, Le tourisme dans les aires protégées d'Afrique de l'Ouest : quelle contribution à la conservation ?, op. cit., p. 8.

${ }^{1024}$ Idem, p. 21.

${ }^{1025}$ L'Organisation de Coopération et de Développement Économiques (OCDE) est une organisation économique créée en 1960 par dix-huit (18) pays européens, le Canada et les États-Unis. Cette organisation regroupe aujourd'hui trente-quatre (34) pays membres dont majoritairement des pays les plus avancés et quelques pays émergents tels que la Turquie, le Mexique et le Chili. En tant que plateforme de développement économique, la mission de l'OCDE est de promouvoir les politiques qui amélioreront le bien-être dans le monde. À cet effet, l'OCDE permet à des gouvernements d'agir en synergie, de partager leurs expériences et de chercher à trouver des solutions à des problèmes communs. Voir www.oecd.org/fr/apropos/.

${ }^{1026}$ Voir OCDE, Tendances et politiques du Tourisme de l'OCDE 2012, Éditions OCDE, 2013, pp. 35-36 et 48.

${ }^{1027}$ Idem, p. 52.
} 
Il s'ensuit que, d'une part, cette catégorie professionnelle dispose d'une expertise en la matière qui pourrait développer le secteur de la valorisation touristique des aires protégées. D'autre part, elle détient des moyens financiers conséquents en la matière. Par conséquent, il est utile que l'OIPR concède l'exploitation touristique de ces aires protégées au secteur privé, vu que cet organisme dispose de moyens financiers et techniques limités pour assurer leur valorisation écotouristique et partant impacter positivement le développement local.

Par ailleurs, par quel mécanisme juridique pourrait se faire la concession de la valorisation écotouristique des aires protégées au secteur privé?

\section{B - De l'environnement juridique de la concession de l'exploitation écotouristique des aires protégées à des opérateurs touristiques privés}

Il s'agira, en l'espèce, d'étudier la forme juridique de l'accord de concession de l'exploitation écotouristique des aires protégées : le contrat de partenariat public-privé ou PPP (1) et de ses clauses (2).

\section{De la forme juridique de l'accord de concession de l'exploitation écotouristique des aires protégées : le contrat de partenariat public- privé}

La réalisation des investissements relatifs à la gestion des aires protégées notamment la valorisation de ces milieux naturels nécessite d'importants moyens financiers dont ne dispose pas l'État ivoirien ou l'OIPR ${ }^{1028}$. Un rapport récent de l'OCDE révèle que cette situation n'est pas spécifique à la Côte d'Ivoire. En effet, traitant des tendances et politiques du tourisme de l'OCDE en 2012, ce rapport indique qu'une fois les stratégies touristiques élaborées, les États éprouvent des difficultés à les mettre en œuvre compte tenu des problèmes de financement ${ }^{1029}$.

C'est à juste titre qu'en guise de solution l'exploitation écotouristique des aires protégées pourrait être concédée au secteur privé, par le mécanisme des contrats de partenariat public-privé (PPP) ${ }^{1030}$. La conclusion de tels contrats de partenariat permettrait de mobiliser des ressources financières conséquentes, par le truchement des investisseurs privés. Elles serviront à réaliser les investissements importants requis en la matière. Il s'agit, entre

\footnotetext{
${ }^{1028}$ Voir AKINDÈS (Francis), KOUAMÉ (Kra Walter), SÉDIA (Gisèle) et SÉAN (Jules), Réalisation d'une étude sur l'état de la biodiversité des parcs nationaux et réserves de Côte d'Ivoire. Aspects socio-économiques (Rapport provisoire), Abidjan, août 2012, p. 98.

${ }^{1029}$ Voir OCDE, Tendances et politiques du Tourisme de l'OCDE 2012, op. cit., p. 32.

${ }^{1030}$ Les développements faits au chapitre précédent sur la notion de PPP, l'intérêt à les conclure sont valables pour le présent chapitre. Voir la deuxième partie de la présente étude, le Titre II, le Chapitre I, la Section II, le Paragraphe 2 et précisément le point intitulé "B- La conclusion de partenariat public-privé, une solution de gestion durable des aires protégées en Côte d'Ivoire".
} 
autres, de la réalisation de réceptifs hôteliers, d'infrastructures touristiques, d'acquisition de matériels de gestion.

À titre illustratif, en Afrique australe et orientale, les PPP en matière touristique ont permis de générer des ressources aux communautés locales et d'opérer par contrecoup le développement local ${ }^{1031}$. Par ailleurs, les PPP en matière touristique sont si importants qu'au New South Wales(Australie) ${ }^{1032}$, toute activité commerciale ou affaire qui porte sur les aires protégées doit faire l'objet d'une concession ${ }^{1033}$.

La proposition qui consiste à conclure des PPP pour l'exploitation écotouristique des aires protégées est aisée à mettre en œuvre en ce sens que l'article 32 de la loi sur les PNR de 2002 peut lui servir de base légale. En effet, aux termes de l'article 32 alinéa $1^{\mathrm{er}}$ de ladite loi : «Tout ou partie des missions d'exploitation d'un parc ou d'une réserve, selon la nature de ce parc ou de cette réserve, peuvent être concédées par l'Établissement à une personne morale de droit privé dans le cadre d'une convention de concession d'exploitation ».

Qu'en est-il des clauses du contrat de PPP touristique pour l'exploitation écotouristique des aires protégées?

\section{Des orientations relatives aux clauses du contrat de partenariat} public-privé d'exploitation écotouristique des aires protégées

Sous réserve du respect des dispositions légales qui régissent les contrats en Côte d'Ivoire, à titre indicatif et d'orientation, le contrat de PPP portant sur l'exploitation écotouristique des aires protégées devrait prévoir les clauses suivantes :

1. l'exploitation écotouristique d'une aire protégée dans une optique d'équilibre des intérêts de rentabilité financière des parties et de la conservation durable du patrimoine (naturel et culturel) à valoriser ${ }^{1034}$;

\footnotetext{
${ }^{1031}$ Sur ces points, des exemples sont disponibles au chapitre précédent. Voir la deuxième partie de la présente étude, le Chapitre I, la Section II, le Paragraphe 2, le point B, le sous point 1 et précisément le point intitulé "b. La présentation de partenariats public-privé de gestion d'aires protégées réussis".

${ }^{1032}$ Le New South Wales (en français Nouvelle-Galles du Sud) est l'un des six (6) États (de l'État fédéral) d'Australie. Il est situé au Sud-Est de ce pays. Voir BOSA (Bastien), « La (non)-assimilation des Aborigènes dans la nouvelles-Galles du Sud », Le Mouvement Social, Vol. 1, n²30, p. 99.

${ }^{1033}$ Voir WILSON (Erica), NIELSEN (Noah) et BUULTJENS (Jeremy), « From lessees to partners: exploring tourism public-private partnerships within the New South Wales national parks and wildlife service », art. cit, p. 273.

${ }^{1034}$ Par exemple, dans le cadre du contrat de partenariat public-privé conclu pour la gestion de la Réserve naturelle de Lekgalameetse en Afrique du Sud, les avantages dont ont pu bénéficier les communautés ont été moindres que ceux prévus. Voir MASHALE (Modise Christopher), MOYO (Theresa), MPUTARI (Olivier), "An Evaluation of the Public-Private Partnership in the Lekgalameetse Nature Reserve in South Africa ", Mediterranean Journal of Social Sciences, Vol. 5, n²0, 2014, p. 855. Par ailleurs, sur la question de la controverse relative à la conclusion de partenariats avec le secteur privé pour la gestion des aires protégées, voir la deuxième partie de la présente étude, le Chapitre I, la Section II, le Paragraphe 2, le point B, et précisément la note de bas de page de la première phrase du sous point intitulé "1. L'intérêt de la conclusion de partenariat public-privé pour la gestion des aires protégées ivoiriennes".
} 
2. L'opérateur touristique privé s'engage à aider les communautés locales à mettre en place de petites entreprises touristiques ainsi que leurs organes de gestion et à les assister dans la gestion. Il s'oblige également à développer le tourisme communautaire dans les aires protégées et leurs zones périphériques en soutenant ces initiatives communautaires.

3. De préférence, les offres touristiques dans une aire protégée et ses zones périphériques devraient être, autant que possible, complémentaires afin de préserver les intérêts des parties et d'éviter tout conflit susceptible d'en émaner.

Par ailleurs, le tourisme communautaire pourrait être développé dans les aires protégées et leurs zones périphériques ${ }^{1035}$.

\section{Paragraphe 2 : La nécessité de développer le tourisme communautaire à l'intérieur et dans les zones périphériques des aires protégées}

Nécessaire à la conservation durable des aires protégées, il faudrait que l'OIPR et les pouvoirs publics ivoiriens, en général, encouragent et encadrent les populations à la mise en place de projets touristiques (A). Ainsi, des propositions relatives à la vie juridique de l'entreprise touristique communautaire seront faites (B).

\section{A - L'encouragement et l'encadrement des populations à la mise en place de projets touristiques communautaires}

Selon THERIAULT (Gabrielle), la notion de tourisme communautaire n'a pas de définition universelle. Toutefois, une définition donnée par TREJOS et CHIANG, à laquelle se réfèrent plusieurs auteurs, la conçoit comme étant un tourisme «local et géré par la communauté, qui, par conséquent, reçoit une bonne partie des bénéfices ${ }^{1036}$. Autrement dit, l'une des caractéristiques fondamentales des projets touristiques communautaires est qu'ils sont entièrement portés par les communautés tant dans la conception que la mise en œuvre ainsi que l'utilisation des bénéfices qu'ils génèrent.

Aussi sera-t-il présenter, en premier lieu, l'intérêt des projets touristiques (1). En second lieu, la conception et l'exécution des projets touristiques communautaires (2) ainsi que des activités touristiques à proposer en la matière (3) retiendront notre attention.

\footnotetext{
${ }^{1035}$ Ces projets touristiques communautaires seront gérés selon une approche écotouristique.

${ }^{1036}$ Voir TREJOS et CHIANG (2009) cité par THERIAULT (Gabrielle), « le tourisme communautaire : quand le développement touristique est une affaire de communauté », Bulletin de l'observatoire québécois du loisir, Vol. $12, \mathrm{n}^{\circ} 8,2015$, p. 1 ; UICN/PACO, Le tourisme dans les aires protégées d'Afrique de l'Ouest : quelle contribution à la conservation ?, op. cit.,, p. 12.
} 


\section{L'intérêt des projets touristiques communautaires}

Le tourisme communautaire constitue un moyen pour le développement des communautés où cette activité est menée ${ }^{1037}$. De même, l'écotourisme peut contribuer à la conservation des aires protégées ${ }^{1038}$, à la condition que les communautés locales en retirent des revenus et que ceux-ci soit distribués de façon équitable et transparente ${ }^{1039}$. Par exemple, les projets d'exploitation écotouristique et de conservation de Hhuhuwe-Umfolozi Park ont permis à certaines membres des communautés locales d'augmenter leurs revenus ${ }^{1040}$.

Il en découle que le tourisme et en l'espèce le tourisme communautaire est susceptible de contribuer à la conservation durable des aires protégées ivoiriennes. C'est à juste titre que l'OIPR et ses partenaires devraient encourager le développement de tels projets dans les zones périphériques des aires protégées ; d'autant plus que la loi leur en donne la possibilité. En effet, l'article $1^{\mathrm{er}}$ de la loi de 2002 sur les aires protégées prévoit la Réserve naturelle volontaire. Cet article dispose que la Réserve naturelle volontaire : " est créée à l'initiative d'une Collectivité territoriale, d'un Établissement public ou d'une personne de Droit privé, sur un terrain lui appartenant et pour la préservation d'un écosystème ou d'un paysage remarquable...». Il en résulte que par ce mécanisme et constituées sous la forme associative ou de toute autre entité dotée de la personnalité morale, les communautés locales peuvent créer des Réserves naturelles communautaires volontaires (RNCV) à exploiter à but touristique.

De plus, l'article 40 du Code forestier ivoirien de 2014 permet la création de forêts communautaires par les communautés rurales ${ }^{1041}$. C'est ainsi qu'il constitue un moyen pour

\footnotetext{
${ }^{1037}$ Voir TREJOS et CHIANG (2009) cité par THERIAULT (Gabrielle), « Le tourisme communautaire : quand le développement touristique est une affaire de communauté », art. cit., p. 1. ; voir également PARENT (Sylvie) KLEIN (Juan-Luis), JOLIN (Louis), « Le développement communautaire local et le tourisme communautaire : une analyse conceptuelle comparative ", in BARNÈCHE-MIQUEU (Laurence) (dir.), Tourisme et économie résidentielle, hybridations des pratiques et des espaces, publié dans ESSACHESS-Journal for Communication Studies, Vol 2, n 4, 2009, p. 75.

${ }^{1038}$ Voir HE (Guangming), CHEN (X.) LIU (W.), BEARER (S.), ZHOU (S.), CHENG (L.Y.), ZHANG (H.), OUYANG (Z.) and LIU (J.), « Distribution of Economic Benefits from Ecotourism: A Case study of Wolong Nature Reserve for Giant Pandas in China», Environmental Management. 42, n¹, 2008, p. 1017.

${ }^{1039}$ Voir ARCHABALD (Karen) et NAUGHTON-TREVES Lisa, "Tourism revenue-sharing around National Parks in Western Uganda : Early efforts to identify and reward local communities», Environmental Conservation, Vol. 28, n², 2001, p. 135 ; ADELEKE (Bola Olusola), NZAMA (Thandi), « Assessment on Community Participation in Ecotourism and Conservation at Hhuhuwe-Umfolozi Park», Journal of Environment and Earth Science, Vol. 3, n³, 2013, pp. 27-37, p. 28.

${ }^{1040}$ Voir ADELEKE (Bola Olusola), NZAMA (Thandi), "Assessment on Community Participation in Ecotourism and Conservation at Hhuhuwe-Umfolozi Park», art. cit., p. 37.

${ }^{1041}$ En effet, cet article dispose : «Les forêts des communautés rurales sont des forêts protégées appartenant à une ou plusieurs communautés rurales. Elles sont composées de catégories suivantes :

- les forêts naturelles situées sur des terres sur lesquelles les communautés rurales jouissent d'un droit de propriété ou de droits coutumiers conformes à la législation domaniale et foncière ;

- les plantations forestières créées sur des terres immatriculées au nom des communautés rurales ou sur des terres occupées par celles-ci en vertu de la coutume locale ou d'un bail ;

- les forêts cédées aux communautés rurales par l'État, les Collectivités territoriales ou les personnes physiques ou morales de droit privé ;

- les forêts acquises. ».
} 
la mise en place de projets touristiques communautaires dans les zones périphériques des aires protégées par les communautés rurales riveraines.

Enfin, par le canal du contrat de gestion de terroir ${ }^{1042}$, les populations peuvent être encouragées à mettre en place des projets touristiques communautaires. En effet, aux termes de l'article 33 alinéa 2 de la loi sur les PNR, les contrats de gestion de terroir : «...ont pour objet de définir les conditions et modalités de l'association de ces populations à la conservation du parc ou de la réserve et de favoriser ainsi les retombées économiques pour ces populations. ».

Par ailleurs, comment concevoir et exécuter les projets touristiques communautaires afin d'éviter les écueils du passé caractéristiques de certaines expériences de gestion en la matière?

\section{De la conception à l'exécution des projets touristiques communautaires}

En Côte d'Ivoire, les expériences des communautés locales en matière de projets touristiques communautaires sont quasi-inexistantes. Compte tenu du caractère étranger de ce type de projets pour les communautés locales, elles devraient être techniquement appuyées en la matière. Cette démarche garantira la réussite de ces projets et renforcera les capacités des communautés à porter ces projets de manière autonome à terme. Ainsi, les communautés riveraines d'une aire protégée donnée seront exhortées par l'opérateur touristique en charge de l'exploitation touristique de ladite aire protégée à identifier des projets écotouristiques, à choisir des dirigeants compétents pour leur gestion. L'utilisation des retombées financières devrait être décidée par les instances habilitées par la communauté.

La raison essentielle qui fonde ces propositions s'expliquent par le fait que lorsque les populations ne sont pas véritablement impliquées aux différentes étapes de la gestion des projets de conservation, ceux-ci n'ont pas le succès escompté. Par exemple, Au Gabon, dans le cadre de la gestion du Parc National de Loango, un projet pilote d'écotourisme communautaire dénommé Programme Sectoriel de Valorisation des Aires Protégées (PSVAP) a été mis en place de 2000 à 2006. Ce projet a connu un échec parce que la conservation a été privilégiée au détriment du développement des communautés locales. Les populations n'ont pas été associées aux prises de décision, en dépit de la volonté de leur implication qui s'est matérialisée par des réunions. En outre, il n'a pas été suffisamment tenu compte des réalités culturelles locales ; et la communication développée autour du projet a été

\footnotetext{
${ }^{1042} \mathrm{~L}$ 'article $1^{\mathrm{er}}$ de la loi de 2002 sur les PNR le définit comme « le contrat passé entre le gestionnaire d'un Parc ou d'une Réserve et les populations rurales de la zone périphérique représentées par des structures associatives, privées ou administratives. Ce contrat définit, notamment, les modalités d'intervention des populations contractantes dans la surveillance, la gestion, l'entretien et, le cas échéant, l'animation culturelle et touristique d'un Parc, d'une Réserve ou de leur zone périphérique... ». Pour plus d'informations sur le contrat de gestion de terroir, voir la première partie de cette étude, le Titre II, le Chapitre II, la Section II, le Paragraphe 2, le point B et précisément le sous point intitulé "Le contrat de gestion de terroir".
} 
peu performante. Enfin, les organismes responsables de la mise en tourisme de cette aire protégée que sont l'Agence Nationale des Parcs Nationaux et le WWF n'étaient pas compétents en la matière ${ }^{1043}$.

De même, en Afrique du Sud, les leçons tirées de la gestion du Hhuhuwe-Umfolozi Park (Parc Hhuhuwe-Umfolozi) enseignent qu'il est important pour les gestionnaires des aires protégées de recueillir l'avis des communautés locales pendant la conception des projets écotouristiques. De la sorte, elles pourront dire comment ces projets leurs seront le plus bénéfiques ${ }^{1044}$.

C'est ainsi que sous la supervision des services publics compétents, cet opérateur devra les aider à identifier des projets économiquement rentables et viables, complémentaires aux prestations qu'il offre; à en organiser techniquement la gestion, en formant les animateurs sur la qualité des services à offrir. De plus, il devra les assister aussi longtemps que possible pour le développement du projet. Gage de succès des projets touristiques communautaires, ce processus de gestion desdits projets sous assistance de l'opérateur touristique professionnel permettra aux communautés de disposer à long terme d'une expérience technique et professionnelle en la matière et de s'approprier les projets.

Dans cet élan d'implication des populations à la gestion touristique des aires protégées, nous invitons les gestionnaires de ces milieux naturels à établir des partenariats avec des structures telles que la Fondation ST-EP (Sustainable Tourism-Eliminating Poverty) ${ }^{1045}$. Également, ils peuvent également nouer des partenariats avec l'organisation néerlandaise pour le développement (SNV). En effet, entre autres missions, cette organisation assure une assistance technique et financière aux communautés locales en matière de tourisme en vue d'augmenter leurs revenus et de développer l'économie locale ${ }^{1046}$.

Par ailleurs, quelles sont les activités touristiques que les communautés locales pourraient proposer dans le cadre du tourisme communautaire.

\footnotetext{
${ }^{1043}$ Voir PAYEN (Ariane), Tourisme communautaire dans les Suds : quelle implication des populations locales dans les aires protégées? Cas du Parc National de Loango, Gabon, Mémoire de Master professionnel en Développement et Aménagement Touristique des Territoires, Université de Paris 1-Panthéon Sorbonne, Septembre 2012, p. 5.

${ }^{1044}$ Voir ADELEKE (Bola Olusola), NZAMA (Thandi), « Assessment on Community Participation in Ecotourism and Conservation at Hhuhuwe-Umfolozi Park», art. cit., p. 37.

${ }^{1045}$ En français, Tourisme Durable-Élimination de la pauvreté (notre traduction), SustainableTourismEliminating Poverty est une initiative de la Fondation ST-EP mise en place par l'Organisation Mondiale du Tourisme (OMT). Elle développe des programmes d'appui d'intégration des communautés dans la gestion des activités touristiques. Voir www.snv.org/ (consulté le 7 août 2015).

${ }^{1046}$ Voir UICN/PACO, Le tourisme dans les aires protégées d'Afrique de l'Ouest : quelle contribution à la conservation ?, op. cit., p. 17.
} 


\section{Des activités touristiques à proposer dans le cadre du tourisme communautaire}

Dans tous les Parcs Nationaux et les Réserves naturelles partielles ainsi que leurs zones périphériques, il est préférable de développer l'écotourisme ou tourisme vert. Plus explicitement, il s'agira de proposer aux visiteurs des activités touristiques diverses qui peuvent être menées dans toute aire protégée. En outre, des activités touristiques seront proposées en fonction de la spécificité des attraits touristiques de chaque aire protégée et de sa zone périphérique.

Dans les Parcs Nationaux et Réserves naturelles partielles, des activités écotouristiques telles que les safaris (de nuit, de jour, photographiques et à pied ou en voiture), l'observation des végétaux pourraient être développées. Toutefois, compte tenu de la spécificité des attraits touristiques de certaines aires protégées, les activités suivantes pourraient être proposées en fonction de ces sites.

Dans les Parcs Nationaux d'Azagny, des Îles Éhotilé et de la Comoé, il serait indiqué de développer le tourisme ornithologue. En effet, les deux premières aires protégées citées sont des zones humides d'importance internationale situées sur la façade littorale atlantique. À cet effet, elles reçoivent à des périodes de l'année des oiseaux d'eau (migrateurs). Également, le Parc national de la Comoé qui est une Réserve de biosphère et inscrit sur la liste du patrimoine mondial de l'humanité est traversé par le fleuve Comoé ${ }^{1047}$. Il reçoit aussi de façon périodique des oiseaux d'eau. En dehors des oiseaux d'eau, l'avifaune est très importante dans ces aires protégées. Au Parc National des Îles Éhotilé, l'un des attraits touristiques demeure les chauves-souris.

En outre, au Parc National d'Azagny et dans le Parc National des Îles Éhotilé qui ont une superficie importante couverte de plans d'eau ainsi que leurs zones périphériques qui sont drainées par l'océan atlantique et des lagunes, il peut être développées le tourisme fluvial et balnéaire. Des activités sportives (pêche sportive, ski nautique), la plongée sous-marine, la pêche, la baignage, les ballades à pirogue de visite de mangroves, entre autres, peuvent être proposées aux touristes. Dans les Parcs Nationaux du Mont Péko, du Mont Sangbé, de la Comoé et dans la Réserve d'Abokouamékro qui ont des reliefs accidentés (montagnes, collines, inselbergs), il pourrait être développé le tourisme de montagne.

L'offre touristique dans les aires protégées devrait être complétée par la découverte des attraits touristiques des zones périphériques. Il s'agit du patrimoine culturel des peuples installés dans ces zones (danses, jeux, cuisines, habitats, histoires, anthropologie et la sociologie des peuples), de leurs us et coutumes etc. Également, la visite des sites archéologiques, anciens et de lieux à caractère particulier devrait être prise en compte dans

${ }^{1047} \mathrm{La}$ Cote d'Ivoire est arrosée par quatre (4) grands fleuves dont le fleuve Comoé qui prendre sa source au Burkina-Faso. Le fleuve comoé traverse la Côte d'Ivoire du Nord au Sud. Voir HALLE (Birgit), Dr. BRUZON (Véronique), Profil Environnemental de la Côte d'Ivoire, op. cit., p. 18. 
les offres touristiques. La découverte de ces attraits culturels pourrait être organisée autour des thématiques suivantes :

- Vie quotidienne et festivités dans un village Baoulé, par exemple (Danses, jeux, cuisine et tenues traditionnels...);

- Origine et histoires du peuple Baoulé ;

- Sites historiques et activités économiques (Visites de plantations de cacao, d'anacarde, coton, café, villages de tisserands, forgerons, de pêcheurs, de chasseurs traditionnels, maisons coloniales et autres...).

À cet effet, les touristes pourraient être informés des attraits touristiques des aires protégées et de leurs zones périphériques à partir de support papier et électronique, au niveau des centres d'accueil des aires protégées. C'est ainsi que des fiches ethnobotaniques devraient être réalisées pour différentes espèces de flore ${ }^{1048}$ que l'on trouve dans une aire protégée ainsi que leur localisation. Également, des films documentaires sur les espèces de flore, de faune qu'on rencontre dans chaque aire protégée ainsi que leurs habitats devraient être réalisés. Il en est de même pour les cultures des peuples des zones périphériques (danses traditionnelles, art vestimentaire et autres attraits). Ces actions requièrent une bonne connaissance de la biodiversité des aires protégées et un inventaire du patrimoine culturel matériel et immatériel des communautés locales des zones périphériques des aires protégées. Les visiteurs des Parcs pourraient par exemple être entretenus en salle sur ces attraits avant de les découvrir physiquement.

$\mathrm{Au}$ surplus, quelle pourrait être la structuration juridique de ces projets touristiques communautaires?

\section{B - La structuration juridique des projets touristiques communautaires}

Sur le plan juridique, les projets touristiques communautaires pourraient se réaliser dans le cadre d'une entité juridique dénommée : Organisation Touristique Communautaire (1). Cette structure juridique devrait être dotée d'organes de gestion et de dirigeants aux pouvoirs clairement définis (2).

\section{De la forme juridique de l'organisation touristique communautaire}

Les projets communautaires précédemment proposés en vue du développement local devraient se réaliser dans le cadre de structures communautaires dotées de la personnalité juridique. Cette approche aurait pour avantages, entre autres, de permettre aux communautés membres de tisser elles-mêmes des partenariats selon leurs intérêts, même en étant assistées ou encadrées.

${ }^{1048}$ Par cette tâche peut être confiée à des étudiants en botaniques qui font des stages dans les aires protégées. 
Toutefois, vue l'inexpérience des populations en matière de gestion de projets communautaires, ces projets pourraient être menés dans le cadre d'associations officiellement déclarées. Ces structures associatives pourraient évoluer progressivement vers des formes juridiques de type entreprises telles que consacrées par le droit des sociétés. De la sorte, les communautés locales pourraient apprendre à maîtriser la gestion de type entreprise, à travers des structures aux modes de fonctionnement simples sur le plan juridique qui se complexifieront avec l'évolution de la forme juridique des projets.

Aussi qu'en est-il des organes de gestion et pouvoirs des dirigeants ?

\section{Des organes de gestion et pouvoirs des dirigeants}

Les organes de gestion susceptibles de figurer dans l'organigramme des organisations touristiques communautaires (OTC) sont l'Assemblée générale, le Conseil de gestion et le Comité Exécutif. Ces trois (3) organes ont des pouvoirs qui les distinguent les uns des autres.

En premier lieu, il est important que toute organisation touristique communautaire (OTC) soit dotée d'une Assemblée générale composée de tous les membres de la communauté partie au projet communautaire. L'Assemblée générale de l'OTC devrait être présidée par un Conseil de sage dont la mission est limitée dans le temps et définie par l'Assemblée générale elle-même. L'Assemblée générale de l'OTC est l'instance habilitée à prendre les plus hautes décisions relatives à la vie du projet communautaires telles que le renouvellement du Conseil de gestion, du Comité Exécutif ou de l'identification de types d'infrastructures à réaliser pour les besoins de la communauté.

En second lieu, l'organisation touristique communautaire (OTC) devraient disposer d'un Conseil de gestion. Ce conseil est composé d'un nombre limité de personnalités représentant toutes les couches sociales (hommes, femmes, jeunes) et un expert par branche d'activités menées dans le cadre du projet communautaire.

Ce conseil aura pour mission de prendre toutes les décisions de gestion, dans le sens des orientations de gestion de l'Assemblée générale, de l'intérêt et de l'aspiration des membres de la communauté pour la gestion d'un projet communautaire. Également, il déterminera le mode d'utilisation des bénéfices générés par le projet communautaire, dans le sens des orientations définies par l'Assemblée générale.

En dernier lieu, les projets communautaires initiés dans les zones périphériques des aires protégées devraient être géré(s) au quotidien par un Comité Exécutif. Il pourrait être composé d'un (1) Directeur et d'adjoints, d'un (1) secrétaire comptable nommés par le Conseil de gestion; et d'un (1) commissaire aux compte ainsi que de leurs adjoints élus par l'Assemblée générale. Cette composition du Comité Exécutif de gestion de projet communautaire pourrait être complétée par d'autres postes, eu égard à la nature des activités 
des projets. Dans tous les cas, ce comité doit être le plus restreint possible afin de garantir l'efficacité de son fonctionnement.

$\mathrm{Au}$ surplus, les titulaires des postes des différents organes ci-dessus mentionnées devraient être choisis sur la base de qualités telles que la probité, l'humilité, l'esprit de cohésion sociale et l'expérience ainsi que l'expertise technique en matière de gestion d'intérêts collectifs. 


\section{CONCLUSION DE LA DEUXIÈME PARTIE}

La gestion durable des aires protégées est loin d'être une réalité en Côte d'Ivoire. Ces milieux naturels subissent, dans l'ensemble, diverses pressions anthropiques qui menacent l'existence de certaines d'entre elles. Et pourtant, les aires protégées de l'État de Côte d'Ivoire sont régies par des règles de droit qui les affranchissent de tout droit d'usage. Il s'ensuit que l'application du droit des aires protégées n'est pas effective.

Les causes de ce défaut d'effectivité du droit des aires protégées ont été recherchées à travers une analyse des dispositions qu'il consacre. Cette analyse a été menée à la lumière de législations et d'expériences de conservation de pays étrangers qui constituent des exemples en matière de conservation de la nature. Elle a abouti à la conclusion selon laquelle le droit ivoirien des aires protégées est adapté à une gestion durable de ces milieux naturels. En d'autres termes, le renforcement de la protection juridique de l'intégrité physique des aires protégées à travers leur incorporation au domaine public de l'État, la consolidation des dispositions pénales qui leur sont applicables concourent à leur gestion durable. Il en est de même pour la délégation de la gestion des aires protégées à une structure (OIPR) dotée de l'autonomie sur les plans administratif et financier; de la consécration de leur gestion participative ainsi que la diversification des sources de financements destinés à leur gestion.

Dès lors, il paraît évident que la dégradation continue des aires protégées et partant l'inobservation de la législation qui leur est applicable par tout le corps social, en général, et les communautés locales, en particulier, est tributaire de problèmes pratiques de gestion. Ceux-ci peuvent être classés en deux (2) catégories. Dans un premier temps, il s'agit de problèmes liés à l'exploitation du patrimoine naturel des aires protégées. De manière plus explicite, d'une part, il y a la forte dépendance des populations riveraines des aires protégées en ressources forestières. Cette situation s'explique essentiellement par la pratique de l'agriculture itinérante sur brûlis qui détruit, à un rythme accéléré, le couvert forestier et la biodiversité associée, dans un contexte rural d'économie de rente, de croissance démographique et de pauvreté généralisée. Dans ces conditions, les aires protégées sont des lieux privilégiés vers lesquels s'orientent les populations pour la satisfaction de leur besoin. D'autre part, les problèmes pratiques de gestion des aires protégées concernent l'insuffisance des financements, des moyens matériels et infrastructurels pour la gestion de ces milieux naturels. À ces problèmes s'ajoutent la négligence des pouvoirs publics ivoiriens dans le règlement de problèmes fonciers liés aux aires protégées ainsi que le laxisme de ces autorités face aux cas d'occupations illégales de ces espaces naturels.

Dans un deuxième temps, le défaut d'effectivité du droit des aires protégées résulte des limites des institutions d'exécution et de contrôle dudit droit. À ce niveau, il est à retenir premièrement que la modicité des moyens de gestion des aires protégées limite les gestionnaires dans leur mission de prévention et d'arrêt des pressions anthropiques sur ces forêts dont ils ne sont pas, le plus souvent, informés à temps. En outre, le défaut d'effectivité 
du droit des aires protégées résulte du retard de la prise de textes d'application de la loi précitée de 2002 sur les PNR. Il en est de même pour le retard dans la mise en place et le fonctionnement d'organes de gestion de ces milieux naturels. Enfin, la crise militaropolitique de septembre 2002, qui a occasionné une perturbation du système de surveillance des aires protégées et le retrait ou la suspension de l'aide financière bilatérale ou multilatérale destinée aux aires protégées, a été préjudiciable à la conservation desdites forêts.

En guise de solutions à ces problèmes pratiques de gestion des aires protégées, la conciliation de la gestion des aires protégées et le développement de leurs zones périphériques s'avère nécessaire. De même, l'OIPR est appelée à œuvrer à la valorisation touristique des aires protégées et de leurs zones périphériques. Ainsi, les fonds mobilisés serviront à financer la conservation et les actions de développement qui auront pour conséquences de réduire les pressions sur les aires protégées. À cette fin, l'OIPR devrait aider à la mise en place de projets de gestion communautaires de ressources naturelles ainsi que de projets touristiques communautaires dans et autour des aires protégées. Les communautés locales devraient détenir un droit de propriété sur les ressources (terres, flore et faune) concernés par lesdits projets. Aussi est-il nécessaire pour l'OIPR de conclure des contrats de partenariat public privé afin de faciliter l'opérationnalisation de ces recommandations. 


\section{CONCLUSION GÉNÉRALE}

Dans l'étude du droit des aires protégées en Côte d'Ivoire, il s'est agi pour nous de comprendre les raisons pour lesquelles ces milieux naturels subissent des pressions anthropiques qui menacent même l'existence de certains d'entre eux, malgré la mise en place d'une armature juridique destinée à leur protection. Dans cette entreprise, il nous a paru utile de procéder à un examen de la législation ivoirienne des aires protégées. De la sorte, il serait aisé de savoir si l'application difficile du droit des aires protégées et partant la dégradation de ce patrimoine naturel est tributaire des insuffisances dudit droit ou si celle-ci résulte de facteurs externes.

Pour ce faire, l'étude de l'évolution du droit des aires protégées en Côte d'Ivoire a scruté le cadre juridique originaire des aires protégées qui a servi de base à leur gestion de 1960 à 2002, afin de comprendre la portée des changements opérés par le législateur dans ledit droit depuis l'année 2002. En effet, après l'accession de la Côte d'Ivoire à l'indépendance (le 7 août 1960), les autorités ivoiriennes ont opté pour la préservation de ses milieux naturels. Ce faisant, elles ont constitué, entre autres, des espaces forestiers mis en défens tels que les aires protégées. Subséquemment, il a été adopté des lois (lato sensu) en vue de leur conservation durable.

Cependant, les aires protégées subissent une dégradation continue du fait de l'homme, en dépit de l'interdiction des droits d'usage des populations sur ces espaces. Fort de ce constat, le législateur ivoirien a, en 2002, remanié substantiellement la législation applicable aux aires protégées. Les innovations dont elle fait l'objet et qui ont pour objectif de les protéger contre les pressions anthropiques sont de deux (2) ordres.

D'une part, il s'agit d'un enrichissement des règles (juridiques) destinées à la protection de l'intégrité physique des aires protégées. À ce sujet, les innovations opérées par le législateur concernent l'évolution des règles domaniales de protection de l'intégrité des aires protégées. En effet, le législateur renforce la protection juridique des aires protégées, en les faisant passer de la catégorie de biens du domaine privé de l'État à celle de biens de son domaine public, en 2002. Par conséquent, les aires protégées étant désormais inaliénables, imprescriptibles et leurs occupants sans titre s'exposant à une expulsion, elles ne peuvent plus entrer dans le patrimoine des tiers, suite à des décisions de l'autorité exécutive, contrairement au passé. En outre, la protection de l'intégrité de ces sites de conservation émane de la consolidation des dispositions pénales qui leur sont applicables. À ce niveau, le législateur a élargi le champ des éléments matériels des infractions prévues par la législation des aires protégées en vigueur avant l'année 2002 et alourdit également leurs peines. De même, il a incriminé de nouveaux faits. Enfin, le législateur a renforcé les compétences en matière pénale du personnel de l'Office Ivoirien des Parcs et Réserves (OIPR) en charge de la répression des atteintes contre les aires protégées. En d'autres termes, il leur a conféré la qualité d'officier de Police judiciaire. Par ces actes, il entend œuvrer à l'efficacité de la 
répression des infractions contre les aires protégées en permettant de les constater avec plus de célérité, de rechercher et appréhender leurs auteurs qui seront lourdement sanctionnés.

D'autre part, l'enrichissement des règles relatives aux aires protégées porte sur les règles de gestion de ces forêts. En effet, dans un premier temps, le législateur procède à un renforcement des moyens de l'Administration en charge de la gestion des aires protégées. À cet effet, en 2002, il délègue la gestion des aires protégées à un établissement public national: l'Office Ivoirien des Parcs et Réserves (OIPR) et qui partant est doté de l'autonomie administrative et financière.

De la sorte, l'autonomie administrative de l'OIPR permet de purger les lourdeurs administratives caractéristiques de la gestion des aires, par le passé. Quant à l'autonomie financière de l'OIPR, elle permet (sur le plan juridique) de palier les problèmes d'insuffisances des budgets alloués à leur gestion par l'État. Dorénavant, l'OIPR a la possibilité de recourir aux financements extra-étatiques, en général, ou à des mécanismes de financements prévus par la loi de 2002 sur les PNR, en particulier. Il s'agit des Fondations des Parcs et Réserves et du contrat de fiducie. Aussi le législateur prescrit-il la gestion rigoureuse et transparente des fonds (subventions étatiques et fonds d'origine privée) destinés à la conservation des aires protégées. Ces règles assurent la pérennité des financements pour les actions nécessaires à la conservation des aires protégées.

De plus, depuis l'année 2002, le législateur ivoirien consacre la gestion participative des aires protégées. Cette nouvelle approche de gestion se met en œuvre de deux (2) manières. D'une part, elle est symbolisée par l'implication d'une pluralité d'acteurs dans la gestion des aires protégées au niveau local, à travers l'institution d'un comité de gestion locale pour chaque Parc et Réserve. Ainsi, en plus de l'État, les autres acteurs qui participent dorénavant à la gestion des aires protégées sont : les communautés locales, les collectivités locales, les ONG, les opérateurs économiques. D'autre part, la loi de 2002 sur les PNR consacre la technique de la contractualisation de la gestion durable des aires protégées, à travers le contrat de gestion de terroir et la convention de concession d'exploitation. Cette nouvelle approche de gestion vise à impliquer les populations à la gestion des aires protégées en vue de développer davantage chez elle une conscience écologique et d'obtenir leur soutien pour la gestion durable de ces massifs forestiers.

Dans l'ensemble, les changements apportés à la législation des aires protégées en 2002 ont pour finalité la gestion durable de ces forêts. Cependant, la dégradation des aires protégées a toujours cours. Cette situation suscite une interrogation: le droit des aires protégées assure-t-il une protection juridique suffisante à ces milieux naturels ? Une analyse $\mathrm{du}$ droit positif des aires protégées, à la lumière des législations et expériences de conservation réussies d'aires protégées de pays étrangers, permet de répondre à cette interrogation par l'affirmative. Par conséquent, il apparaît que la difficile application du droit des aires protégées résulte de problèmes pratiques de gestion auxquels sont confrontés les gestionnaires de ces catégories de forêts. 
Premièrement, il s'agit du problème de l'insuffisance de financements qui persiste malgré la diversification des sources de financement destinées à la conservation des aires protégées. Certes, sur le plan juridique, l'OIPR peut solliciter ou recevoir des financements pour l'accomplissement de ses missions. Toutefois, dans la pratique, les financements dont dispose l'OIPR demeurent toujours insuffisants. Cette situation a pour conséquence l'insuffisance et le défaut d'entretien des infrastructures et des équipements de gestion. Subséquemment, le personnel en charge de la gestion durable des aires protégées est limité dans sa mission alors que les pressions anthropiques sur ces forêts s'accentuent, en raison de la rareté de certaines ressources naturelles dans les domaines villageois qu'expliquent un certain nombre de facteurs. Il s'agit de l'évolution de la croissance démographique, dans un contexte de paupérisation des communautés locales rurales fortement dépendantes des ressources naturelles. Elles s'orientent, par conséquent, vers les aires protégées qui en regorgent.

Deuxièmement, la crise militaro-politique de septembre 2002 a entravé la gestion durable des aires protégées. En effet, le dysfonctionnement du système de surveillance dû à l'inexistence de l'Administration en charge des aires protégées dans les zones anciennement sous contrôle de la rébellion armée a entraîné le pillage de ce patrimoine naturel et leur infiltration massive par les populations. Elles s'y sont sédentarisées et pratiquent, en autres, l'agriculture de rente et principalement la culture du binôme cacao-café qui constituent le pilier de l'économie ivoirienne. Cette réalité économique pourrait, entre autres raisons, expliquer les tergiversations des pouvoirs publics ivoiriens à apporter une solution à l'occupation de ces espaces forestiers mis en défens.

Enfin, le retard dans la prise d'instruments réglementaires de mise en œuvre de la loi de 2002 sur les PNR constitue un frein à la gestion durable des aires protégées ivoiriennes. Il en est de même pour le retard lié à la mise en place et au fonctionnement d'organes de gestion (comité de gestion locale de Parc ou Réserve, Conseil Scientifique de l'OIPR).

C'est à juste titre que les solutions suivantes pour une meilleure appropriation du droit des aires protégées par tout le corps social, en général, et les communautés locales, en particulier, s'imposent. En effet, la gestion durable des aires protégées ivoiriennes en dépend.

En premier lieu, l'État de Côte d'Ivoire devrait entreprendre et/ou faciliter des actions urgentes, fermes et courageuses de sauvegarde des aires protégées ivoiriennes. D'une part, il s'agit des actions consistant à l'arrêt immédiat de la colonisation des aires protégées pour la pratique de l'agriculture, par la destruction de nouvelles plantations, l'expulsion et la poursuite systématique des contrevenants. D'autre part, il s'agit d'initier un programme d'apurement écologiquement viable, à caractère humanitaire et social négocié et progressif avec les communautés qui acceptent le principe du départ volontaire ; et d'expulser les récalcitrants. Au surplus, l'État de Côte d'Ivoire est invité à coordonner ses actions de développement dans les zones périphériques des aires protégées autour de la conservation durable de celles-ci. À titre d'exemples, l'encadrement agricole du monde paysan et la 
commercialisation des produits agricoles dont est tributaire l'économie locale et qui relèvent des ministères de l'agriculture et du commerce devront se faire avec pour objectif principal la conservation des aires protégées. Cette première catégorie d'actions permettra de restaurer progressivement le couvert forestier et la biodiversité associée des aires protégées; surtout celles dont l'existence est menacée.

En second lieu, il est impérieux que l'Office Ivoirien des Parcs et Réserves s'attèle à développer une politique et des actions effectives qui concilient conservation des aires protégées et développement des communautés locales pour tous les Parcs Nationaux et Réserves. Également, l'OIPR devrait œuvrer à la valorisation touristique effective des aires protégées ainsi que de leurs zones périphériques aux richesses (naturelles et/ou culturelles) diverses et variées. Ces solutions visent à permettre à l'OIPR de mobiliser d'importantes ressources financières à partir des potentialités écotouristiques des aires protégées et de leurs zones périphériques, à l'instar de ce qui se fait par les structures de gestion d'aires protégées en Afrique australe et orientale. Ces fonds mobilisés pourraient servir à financer les actions de conservation et de développement local, à l'image des expériences réussies en la matière, dans les régions de l'Afrique précédemment citées.

À cet effet, l'OIPR est exhorté à soutenir les communautés locales dans la mise en place et la gestion de projets communautaires destinées à la réduction de la pauvreté, à la conservation et à l'utilisation durable des ressources naturelles dans les terroirs villageois. De plus, les communautés locales devraient bénéficier d'un droit de propriété sur les terres qui constituent l'assise de ses projets communautaires ainsi que sur la biodiversité floristique et faunistique associée. Sur ce dernier point, les pouvoirs publics ivoiriens sont invités à modifier la loi précitée de 1965 sur la protection de la faune et l'exercice de la chasse de sorte que les particuliers aient un droit de propriété sur la faune sauvage vivant sur leurs terres.

Pour l'opérationnalisation de cette deuxième catégorie de recommandations, l'OIPR pourrait utilement recourir au secteur privé au moyen de la conclusion de contrats de partenariat public-privé. Ce recours au secteur privé permettrait à l'OIPR de bénéficier de l'expertise dudit secteur et de mobiliser des financements importants pour les investissements nécessaires à la conservation durable des aires protégées. Des pays d'Afrique australe et orientale qui ont réalisé des succès en matière de conservation, dans un environnement rural caractérisé par la pauvreté des populations, l'ont expérimenté.

En troisième et dernier lieu, l'OIPR pourrait encourager, soutenir, initier, développer des programmes et actions d'éducation à la culture de la conservation et de l'utilisation durable de la biodiversité dans les terroirs villageois ainsi qu'à la culture du respect de l'intégrité des aires protégées. À cet effet, l'OIPR devra développer la dynamique associative autour des aires protégées en renforçant, en cas de besoin, les capacités des structures "existantes". En effet, les rares associations de conservation opérant dans le secteur des aires protégées sont inactives. C'est ainsi que l'OIPR devrait aider à les réactiver et susciter la 
création d'autres associations. Ces structures constitueront, à termes, des partenaires qui mèneront des actions de relais auprès des communautés et de l'OIPR.

Au total, il apparaît que l'effectivité du droit des aires protégées en Côte d'Ivoire et partant la conservation durable de ces milieux naturels requiert la synergie des actions des différentes parties prenantes, à travers un engagement empreint de responsabilité et d'éthique. 


\section{SUMMARY OF THE DISSERTATION}

(According to Article $24 \xi 3$ of the Regulation Governing the Attainment of Doctoral Degrees - Maastricht University 2013)

\section{DOES PROTECTED AREAS LAW PROVIDE SUSTAINABLE PROTECTION TO THESE FORESTS?}

Republic of Côte d'Ivoire endeavours to achieve the sustainable management of its protected areas. This means that the management approach of protected areas aims at conciliating ecology and economy and the needs of present generations and also those of the future one.

For this purpose, in one hand, Ivorian authorities have developed different legal instruments that establish an institutional and legal framework for protected areas management. In other hand, Ivorian authorities adhered to international legal instruments related to the preservation of biodiversity.

A close analysis of the rules into force reveals that protected areas law ensures sustainable protection of protected areas. But these rules cannot effectively apply because of the protected areas' management realities. These are adjacent areas of protected areas deficit of development and inadequate financial resources for their management.

Therefore, the sustainable management of protected areas in Republic of Côte d'Ivoire requires conciliating the conservation of these forests and the development of local communities. Then, the Ivorian National Parks and Reserves Office have to promote tourism development in and around protected areas. We suggest involving effectively local communities in conservation projects by promoting community-based natural resources management and also recognizing their property rights over lands and natural resources subject of these conservation projects

The application of the above mentioned propositions by Ivorian authorities will probably contribute to facilitate protected areas law enforcement and consequently ensure their sustainable management. 


\section{BREF CURRICULUM VITAE}

(According to Article 26 of the Regulation Governing the Attainment of Doctoral Degrees Maastricht University 2013)

a. Date et lieu de naissance : 19 Mai 1975 à Adjamé (Abidjan, République de Côte d'Ivoire)

b. Formation pré-universitaire : achevée en juin 1997 avec l'obtention du Baccalauréat, série A2 obtenu au Lycée classique d'Abidjan, République de Côte d'Ivoire.

c. Diplômes universitaires : 2009, Master en Droit International et Comparé de l'Environnement, Université de Limoges, fdse, République Française.

2006 : DESS en Conservation et Utilisation Durable des Zones Humides, Université de Ouagadougou, ufr SVT, Burkina Faso.

2005 : DEA en Politiques Environnementales, Université Abobo-Adjamé, UFR SGE, République de Côte d'Ivoire.

2003-2004 : (Diplôme préparé) DEA en Droit privé fondamental, Université de Cocody, UFR SJAP, République de Côte d'Ivoire ${ }^{1049}$.

Juillet 2004 : Diplômé de l'Académie Internationale de Droit Constitutionnel de Tunis, Académie Internationale de Droit Constitutionnel, République tunisienne.

2002-2003 : Licence d'Anthropologie, Université de Cocody, UFR SHS, République de Côte d'Ivoire.

2001-2002 : Maîtrise en Droit privé, Université de Cocody, UFR SJAP, République de Côte d'Ivoire.

d. Pratique professionnelle après l'obtention du diplôme de DEA (essentiellement dans des universités privées et un Centre de Recherche) :

2014-2016 : Chargé de travaux dirigés en Droit Pénal et Droit Judiciaire Privé à l’Université Nord-Sud, Abidjan, République de Côte d'Ivoire ;

2012-2015 : Chargé de travaux dirigés en Droit Civil et en Droit Pénal des Affaires à l'Université Nouvelle de Côte d'Ivoire, Abidjan, République de Côte d'Ivoire ;

2012-2014 : Chargé de travaux dirigés en Droit Civil et en Droit Commercial à l’Université Charles Louis de Montesquieu, Abidjan, République de Côte d'Ivoire ;

Janvier 2006-Septembre 2007 : Juriste-Formateur et Chargé de mission à l'Institut de la Dignité et des Droits Humains (IDDH) du Centre de Recherche et d'Action pour la Paix (CERAP), Abidjan, République de Côte d'Ivoire.

\footnotetext{
${ }^{1049}$ Après les cours et les évaluations aux différents séminaires, nous n'avons pu terminer la formation pour des
} raisons de santé qui nécessitaient un départ sur l'étranger. 
ANNEXES 


\section{LISTES DES ANNEXES}

- Annexe 1 : Questionnaire adressé aux populations des zones périphériques ou infiltrées des aires protégées.

- Annexe 2 : Guide d'entretien à l'endroit des gestionnaires et autorités de tutelle technique des aires protégées.

- Annexe 3 : Rapport de l'enquête sur les réalités de la gestion des aires protégées en Côte d'Ivoire.

- Annexe 4 : Tableau récapitulatif des enquêtés.

- Annexe 5 : Loi n²002-102 du 11 février 2002 relative à la création, à la gestion et au financement des Parcs Nationaux et des Réserves naturelles (JORCI n ${ }^{\circ} 20$ du 16 mai 2002, p. 338).

- Annexe 6 : Décret n²002-359 du 24 juillet 2002, portant création, organisation et fonctionnement de l'Office Ivoirien des Parcs et Réserves (JORCI n ${ }^{\circ} 40$ du 2 octobre 2003, p. 588).

- Annexe 7 : Addendum relatif à la valorisation de la thèse. 
ANNEXE 1 :

\section{QUESTIONNAIRE ADRESSÉ AUX POPULATIONS DES ZONES PÉRIPHERIQUES OU INFILTRÉES DES AIRES PROTÉGÉES}

(Enquête sur les réalités de la gestion des aires protégées en Côte d'Ivoire)

Fiche $n^{\circ}$ :

Date :

1. Localisation de la zone périphérique ou de l'aire protégée

Région : Département

Commune Sous-préfecture : Village

2. Information sur l'enquêté

Nom et Prénoms de l'enquêté :

Contact téléphonique

3. Quelles les activités économiques que vous exercez ?

- Agriculture

Oui : $\square$

Non : $\square$

- Industrie

Oui :

Non : $\square$

- Commerce

Oui :

Non :

- Autres

Oui :

Non :

Si oui, précisez :

4. Quelles sont les techniques culturales que vous utilisez ?

- Agriculture itinérante sur brûlis

- Culture hors sol

$\begin{array}{ll}\text { Oui : } \square & \text { Non }: \square \\ \text { Oui : } \square & \text { Non }: \square \\ \text { Oui : } \square & \text { Non }: \square\end{array}$

- Autres

Oui :

Non : $\square$

Si oui, précisez :

5. Avez-vous constaté des changements dans votre environnement naturel ? Si oui précisez

- Climat,

Oui : $\square$

Non :

Si oui, précisez :

- Végétation

Oui : $\square$

Non : $\square$

Si oui, précisez :

- Faune

Oui : $\square$

Non :

Si oui, précisez :

- Autres (à préciser) Oui : $\quad$ Non : $\square$

Si oui, précisez :

6) La conservation des aires protégées est-elle importante ?

Oui : $\square$

Non : $\square$

Si oui, pourquoi selon vous?

7) Tirez-vous des bénéfices de la conservation des aires protégées ?

Oui :

Non :

Si oui, lesquels

6) Qu'est ce qui explique les pressions anthropiques sur les aires protégées selon vous ?

- Pauvreté

Oui : $\square$

Non : $\square$

- Infertilité des sols

Oui : $\square$

Non : $\square$

- Saturation foncière

Oui : $\square$

Non : $\square$

- Autres

Oui :

Non : $\square$

Si oui, précisez

Merci pour votre coopération ! 
ANNEXE 2 :

GUIDE D'ENTRETIEN À L'ENDROIT DES GESTIONNAIRES ET AUTORITÉS DE TUTELLE TECHNIQUE DES AIRES PROTÉGÉES

(Enquête sur les réalités de la gestion des aires protégées en Côte d'Ivoire)

Fiche $\mathbf{n}^{\circ}:$

Date :

Nom :

Fonction

Structure ou Administration :

Adresse :

Téléphone :

Fax

E-mail :

Site internet

1-Les pressions anthropiques exercées sur l'aire ou les aires protégées.

2- Le budget, les équipements et le matériel de travail dont dispose l'Administration en charge de l'aire ou des aires protégées.

3-Les infrastructures dont dispose l’aire ou les aires protégées.

4-L'état de la gestion participative de l'aire ou des aires protégées.

5-Les difficultés rencontrées par les gestionnaires d'aire protégée.

Merci pour votre coopération ! 


\section{ANNEXE 3 :}

\section{RAPPORT DE L'ENQUÊTE SUR LES RÉALITÉS DE LA GESTION DES AIRES PROTÉGÉES EN CÔTE D'IVOIRE}

\section{Introduction}

Le droit des aires protégées connaît de sérieuses difficultés d'application. L'objectif de cette enquête est de savoir si des réalités de gestion expliquent ces entraves à l'effectivité du droit des aires protégées.

\section{I- MÉTHODOLOGIE}

Une enquête de terrain a été effectuée dans quatre (4) Parcs Nationaux (Azagny, Banco, Marahoué Taï) et une Réserve naturelle partielle (Réserve de Faune d'Abokouamékro), les Administrations dont relèvent ces aires protégées et leurs zones périphériques en vue de collecter des informations ${ }^{1050}$.

\section{Méthodologie de collecte des données}

Deux approches méthodologiques ont été adoptées pour la collecte des informations. D'une part, il s'agit de la méthode quantitative. Elle a consisté à administrer des questionnaires aux populations des zones périphériques des aires protégées et celles infiltrées dans ces forêts. D'autre part, en ce qui concerne la méthode qualitative, un guide d'entretien a été soumis aux personnels de la Direction Générale et des Administrations locales de l'établissement en charge de la gestion des aires protégées (Office Ivoirien des Parcs et Réserves). Également, quelques (rares) ONG opérant dans les aires protégées ${ }^{1051}$ ainsi que la Direction de la Protection de la Nature (DPN) ont été enquêtées.

La taille de l'échantillon a été déterminée sur la base de l'échantillonnage à choix raisonné. Au total, 384 individus ont été interrogés.

L'approche méthodologique adoptée se veut complémentaire afin de croiser les informations collectées auprès des communautés locales à la phase quantitative avec celles obtenues auprès des structures publiques et parapubliques de gestion des aires protégées. $\left.2009^{1052}\right)$.

L'enquête s'est déroulée sur une période de deux (2) mois (août et septembre

\footnotetext{
${ }^{1050}$ Voir Annexe 4 : Tableau récapitulatif des enquêtés.

${ }^{1051}$ En Côte d'Ivoire, les ONG qui opèrent dans les aires protégées sont pas en nombres. Voir AKINDÈS (Francis), KOUAMÉ (Kra Walter), SÉDIA (Gisèle) et SÉZAN (Jules), Réalisation d'une étude sur l'état de la biodiversité des Parcs Nationaux et Réserves de Côte d'Ivoire: Aspects socio-économiques (Rapport provisoire), op. cit., p. 92. De plus, les entretiens avec les deux responsables d'ONG rencontrées ont servi à comprendre certaines préoccupations abordées avec les communautés locales et les gestionnaires. Compte tenu de la petite taille de cette catégorie, ils n'ont pu faire l'objet d'une présentation de résultats, contrairement aux communautés locales et aux gestionnaires des aires protégées.

${ }^{1052}$ Toutefois, certaines informations recueillies ont été actualisées à l'aide d'entretiens réalisés avec les gestionnaires des aires protégées et de rapports sur l'état de leur conservation, compte tenu du contexte de crise post-électorale de 2011 qui a fragilisé toutes les institutions ivoiriennes.
} 


\section{Méthodologie de traitement des données}

L'analyse des données quantitatives a été menée, sous Sphinx plus ${ }^{2}$ version $4.5^{1053}$. Un formulaire conçu à partir des différentes rubriques dudit questionnaire a donc servi à la saisie des données collectées auprès des populations lors de l'enquête de terrain.

Après l'opération de saisie, des corrections (apurements) ont été effectuées dans la base des données afin de rectifier les incohérences possibles constatées lors de la saisie. Ensuite, il a été procédé à une analyse univariée qui a consisté à faire des tris à plat ${ }^{1054}$.

Pour ce qui est de la méthode d'analyse des données qualitatives, c'est de l'analyse de l'analyse de contenu ${ }^{1055}$ qui a été retenue parce qu'elle est l'une des plus utilisées dans le traitement des données qualitatives ${ }^{1056}$.

Au total, les résultats obtenus sont présentées selon qu'ils résultent de l'enquête avec les populations et les autorités administratives en charge des aires protégées.

\footnotetext{
${ }^{1053}$ Sphinx est un logiciel d'enquête et d'analyse des données. Il permet d'aider à la réalisation d'une enquête dans ses quatre (4) grandes étapes (hors phase de collecte des données) :

- la réalisation du questionnaire ;

- la saisie des réponses ;

- les traitements quantitatifs des données et l'analyse des données qualitatives ;

- la rédaction du rapport d'étude (www.enset-media.ac.ma/cpa/Fixe/Sphinx.pdf).

Sphinx est composé d'une suite de 3 logiciels :

- le Sphinx Primo : il permet de concevoir un questionnaire, de saisir et dépouiller les réponses et d'explorer les données enregistrées.

- le Sphinx Plus ${ }^{2}$ : il offre les mêmes fonctionnalités que "Primo" mais intègre des techniques d'analyse multidimensionnelle plus avancées (analyse factorielle, typologie, etc.) et permet d'analyser tout fichier contenant des nombres et/ou du texte, quel que soit son origine.

- le Sphinx Lexica: il permet d'enrichir les possibilités d'analyse lexicale et de contenu existantes dans "Primo" ou "Plus"2" (traitement des questions ouvertes, des interviews non directives ou de tout autre texte quelle que soit son origine).

Si chacun de ces logiciels permet d'accomplir toutes les étapes d'une étude (élaboration du questionnaire, saisie des réponses, traitements quantitatifs et qualitatifs), il reste qu'ils se distinguent par leur capacité de traitement. En effet, Sphinx Lexica a des compétences plus étendues en analyse lexicale et Sphinx Plus ${ }^{2}$ en analyse quantitative. C'est pour cette dernière raison que le Sphinx Plus ${ }^{2}$ version 4.5 (ftp://gestec2000.com/BDC/SphinxME/.../Fr/NouveautesV4.5.pdf) a été utilisé pour le traitement des données (quantitatives) collectés auprès des communautés locales.

${ }^{1054} \mathrm{Un}$ tri à plat restitue la distribution des différentes réponses obtenues à une question unique dans le cadre d'un questionnaire d'étude (www.definitions-marketing.com/Definition-Tri-a-plat : consulté le 30/10/2014).

${ }^{1055}$ L'analyse de contenu peut être définie comme un ensemble de technique dont l'objectif est de comprendre un texte c'est-à-dire des écrits ou des paroles enregistrées et transcrites, en faire la synthèse, en extraire des idées. Voir HENRY (P.), MOSCOVICI (S.), « Problèmes de l'analyse de contenu », Langages, vol. 2, $\mathrm{n}^{\circ} 11$, 1968, pp. 36-60.

${ }^{1056}$ Voir KRIPPENDORFF (K.), Content analysis : an introduction to its methodology, $3{ }^{\text {rd }}, 2012$, Edition, Sage Publications, Thousand Oaks, CA, 441 pages; PAILLE (P.), MUCCHIELLI (A.), L'analyse qualitative en sciences humaines et sociales, $3^{\text {ème }}$ édition, Paris, Armand Colin, Collection U, 2012, 424 pages.
} 


\section{II- RÉSULTATS DE L'ENQUÊTE AUPRÈS DES COMMUNAUTÉS RIVERAINES DES AIRES PROTÉGÉES}

\section{L'agriculture est la principale activité des localités riveraines des aires protégées enquêtées (à l'exception du Parc National du Banco ${ }^{1057}$ )}

Si $100 \%$ des personnes enquêtées affirment qu'elles pratiquent l'agriculture, 98\% d'entre elles pratiquent au moins une culture de rente. Les produits vivriers cultivés sont le maïs, le riz, la banane plantain, l'igname, le manioc, les légumes. Les cultures de rentes sont le café, le cacao, l'hévéa, le palmier à huile.

L'industrie (peu développée) et les services sont principalement basés sur les produits agricoles y compris le bois.

\section{Les techniques culturales utilisées par les communautés riveraines ou infiltrées dans les aires protégées sont l'agriculture itinérante sur brûlis}

Les techniques culturales utilisées par l'ensemble des communautés locales enquêtées sont l'agriculture itinérante sur brûlis. Les populations enquêtées (94\%) affirment qu'elles ne laissent pas une portion de terre en jachère pendant plus de deux (2) saisons; et qu'elles les utilisent jusqu'à une baisse importante de leur fertilité.

\section{Les communautés riveraines des aires protégées observent des changements environnementaux}

\section{a. Sur le plan climatique}

Les populations enquêtées (97\%) ont répondu qu'elles constatent un changement au niveau du paysage et du climat. Selon elles, les saisons des pluies et les saisons sèches sont instables; et les saisons des pluies sont de plus en plus courtes.

\section{b. Au niveau de la diversité biologique}

Les populations interrogées (97\%) affirment qu'au niveau de la végétation, il y a un recul de la forêt. Elles constatent aussi la rareté de certaines espèces animales et végétales. Dans l'ensemble, les populations lient ces différents changements au recul des surfaces forestières.

\section{Les populations estiment qu'il est important de conserver les aires protégées}

À un taux de $78 \%$, les répondants pensent que les aires protégées sont importantes parce qu'elles favorisent la tombée des pluies grâce aux gros arbres qu'elles contiennent. Hormis certaines populations du Parc National de Taï, l'ensemble des personnes enquêtées disent qu'elles ne retirent pas d'avantages financiers ou matériels de la conservation des aires protégées.

\footnotetext{
${ }^{1057}$ Le Parc National du Banco est un Parc urbain. Situé dans la capitale économique ivoirienne, les personnes qui vivent à sa périphérie n'ont pas pour activité principale l'agriculture. Ce sont plutôt des fonctionnaires, des commerçants, des ouvriers etc. Voir République de Côte d'Ivoire, Ministère de l'Environnement et des Eaux et Forêts/Office Ivoirien des Parcs et Réserves, Plan d'aménagement et de gestion du Parc National du Banco, Abidjan, 2008, pp. 26-27.
} 
Dans l'ensemble, les populations affirment être favorables à leur conservation. Toutefois, elles pensent que les aires protégées seront mieux conservées si elles en retirent des avantages en termes de développement.

\section{Les raisons des pressions sur les aires protégées}

Si $66 \%$ des personnes enquêtées pensent que les pressions sur les aires protégées sont dues à la pauvreté causée par la baisse des revenus agricoles et le manque d'alternative économique, 58\% affirment qu'elle résulte de l'infertilité des sols et de la saturation foncière dans les zones périphériques des aires protégées. Ces chiffres peuvent être plus importants dans la mesure où les enquêtés (communautés locales) sont, le plus souvent, hésitants à donner des informations sur l'exploitation des aires protégées. En effet, elles les considèrent comme des questions sensibles, en raison de leur caractère infractionnel.

En outre, les communautés locales enquêtées affirment à $97 \%$ qu'étant donné que certains produits forestiers (bois de chauffe, plantes alimentaires, médicinales, faune sauvage etc.) se font de plus en plus rares dans les terroirs villageois, la probabilité et la tentation de s'en approvisionner dans les aires protégées est plus grande.

Les différents types de pressions sur les aires protégées en Côte d'Ivoire sont, en général, l'exploitation agricole, le braconnage, le prélèvement de produits ligneux et non ligneux (plantes alimentaires, médicinales...). Dans certains cas, il y a l'exploitation minière (cas du Parc National de Taï). Les communautés riveraines de la Réserve de Faune d'Abokouamékro (RFA) expliquent les pressions foncières sur cette aire protégée par le fait qu'elles n'ont pas été dédommagées pour les terres qu'elles ont cédées à l'État en vue de la création de ladite Réserve. En d'autres termes, l'État n'ayant pas tenu ses promesses, la création de cette aire protégée n'a pas amélioré les conditions de vie des communautés locales alors qu'elles ont perdu des terres agricoles.

\section{Verbatim}

K. Y, habitant du village de Komoi-Dibikro (village situé à environ $400 \mathrm{~m}$ de la RFA) : «Nous n'avons plus de terres pour l'agriculture...les terres que nous labourons pour avoir à manger sont celles que l'État a prises pour créer la Réserve ».

K. R. de BLAISEKRO (village situé dans le Parc National de la Marahoué, plus précisément dans la zone agroforestière $\left.{ }^{1058}\right)$ : «Moi Koffi, je ne sais faire que le travail de la terre. Il en est de même pour tous ceux qui vivent ici. C'est ainsi que je nourris ma famille, scolarise mes enfants. Alors, je demande pardon au gouvernement de penser à nous car si on nous chasse d'ici, c'est notre mort...».

\footnotetext{
${ }^{1058}$ Dans le Parc National de la Marahoué, la zone dite agroforestière est une zone composée de trois (sites) délimitée en 1988 par la SODEFOR à la demande du gouvernement ivoirien. L'objectif de cette décision était de regrouper les populations infiltrées et disséminée dans ce Parc. Évaluée à 11.000 ha et faisant toujours partie intégrante (juridiquement) du Parc, la zone agroforestière (ZAF) donne l'impression d'être déclassée de fait, en raison des activités humaines qui s'y développent. En effet, c'est une cité humaine, à l'instar de toutes celles des zones rurales. Sur ces points, voir les développements faits dans la Deuxième partie de cette étude, le Titre I, le Chapitre II, la Section I, le Paragraphe 2, le point B, le sous-point 2 (le laxisme dans les règlements des problèmes d'occupations illégales d'aires protégées par les populations) et précisément le sous-point intitulé "Le cas du Parc National de la Marahoué ".
} 


\section{III- RÉSULTATS DE L'ENQUÊTE AUPRÈS DES GESTIONNAIRES D'AIRES PROTÉGÉES}

\section{Les aires protégées enquêtées subissent diverses formes de pressions anthropiques}

\section{a. Exploitation agricole}

Des portions de terres à la périphérie du Parc National d'Azagny (PNA) sont défrichées par les populations pour la culture de vivriers (manioc, igname...). Cette forme de pression est plus manifeste sur la limite nord du PNA. Elle pratiquée par les populations (riveraines) des campements de Broukro, Dely et Konankro. De plus, les populations exploitent clandestinement les anciennes plantations de cultures industrielles (café, cacao, hévéa, palmier à huile, et cocotier) et situées dans l'ex-zone tampon du Parc. Une autorisation fut accordée officiellement aux populations par la Direction du Parc de récolter (uniquement) ces plantations sans les entretenir et sans les étendre de 2006 à 2008.

En outre, bien qu'étant l'aire protégée la mieux conservée en Côte d'Ivoire, le Parc National Taï (PNT) fait également l'objet d'exploitation agricole. Il s'agit de plantations créées en 1977 et cantonnées dans un périmètre que les gestionnaires ont convenu d'appelée zone d'occupation contrôlée (ZOC).

S'agissant du Parc National de la Marahoué (PNM), c'est le Parc le plus infiltré de la Côte Ivoire. Occupé à plus de $75 \%$, les populations s'y sont sédentarisées pour la pratique de l'agriculture. Il y existe des écoles construites par les populations dans lesquels étaient affectés des enseignants par l'État de Côte d'Ivoire, jusqu'en 2004.

Enfin, la Réserve de Faune d'Abokouamékro fait l'objet d'exploitation agricole (à l'intérieur de la Réserve) surtout dans sa zone non aménagée.

\section{b. Exploitation forestière}

Dans le Parc National d'Azagny, des produits forestiers ligneux et non ligneux sont prélevés pour la construction d'habitations (charpentes, toitures) ; la confection de matériaux à usage domestique divers (chaises traditionnelles, pilons, mortiers) ou outils de travail (pirogue) ; l'alimentation et comme produits médicinaux (racines, feuilles, fruits, écorces, faune sauvage...).

$\mathrm{Au}$ Parc National de la Marahoué, l'exploitation forestière constitue la deuxième menace, après les défrichements agricoles.

Dans la Réserve de Faune d'Abokouamékro, des clandestins se livrent à l'exploitation forestière, en raison de l'attribution de périmètres forestiers à exploiter contigus à la Réserve. Il s'ensuit souvent des incursions dans la Réserve, à cause de la défaillance du système de surveillance dû au manque de moyens matériels et financiers des organes de gestion.

\section{c. Exploitation minière (orpaillage)}

Depuis 2005, l'intensification des actions de surveillance dans le Parc National de Taï a permis de constater que l'orpaillage se pratique, de plus en plus, dans cette aire protégée. Cette forme de pression est l'œuvre de clandestins, essentiellement d'allogènes et allochtones. 


\section{d. Le braconnage}

Dans le Parc National d'Azagny (PNA), les communautés riveraines prélèvent des ressources halieutiques, à savoir les poissons et les crustacés (généralement dénommés au niveau local Crabes poilus). Quant à la faune sauvage, il s'agit principalement des primates, $\mathrm{du}$ buffle (Syncerus caffer), du guib harnaché (Tragelaphus scriptus), du céphalophe (Cephalophus maxwelli, Cephalophus niger, Cephalophus ogilbyi).

Il en est de même pour le Parc National de la Marahoué (PNM) où les populations infiltrées et riveraines pratiquent la pêche (des poissons) et le braconnage de faunes sauvages notamment l'éléphant (loxondata africana) les primates, le buffle (Syncerus caffer), le guib harnaché (Tragelaphus scriptus), le céphalophe (Cephalophus maxwelli, Cephalophus niger, Cephalophus ogilbyi).

Dans le Parc National de Taï (PNT), il se pratique un braconnage intensif, depuis 2005. Les délinquants justifient cette pression par le manque de protéines animales et leur dépendance vis-à-vis de la viande de brousse.

En ce qui concerne la Réserve de Faune d'Abokouamékro (RFA), la chasse y est également pratiquée nonobstant le fait que depuis son saccage par les communautés révoltées (en 1999), la population de faune y a considérablement diminué.

\section{L'insuffisance de financements, d'équipements et de matériels de travail pour la gestion durable des Parcs Nationaux et Réserves}

La quasi-totalité des Parcs et Réserves enquêtées (Parcs Nationaux du Banco, de la Marahoué, d'Azagny; Réserve de Faune d'Abokouamékro RFA) souffre d'un manque de financements, d'équipements et de matériel de travail. Seul le Parc National de Taï, qui bénéficie de l'aide financière et technique multilatérale (KFW, WWF, GTZ, WCF), dispose, dans le domaine du raisonnable, d'équipements et de moyens financiers de gestion.

Selon les différents gestionnaires, les problèmes de financement expliquent l'absence de projets de développement en faveur des zones périphérique, à l'exception du Parc National de Taï.

\section{L'insuffisance et la dégradation des infrastructures de gestion des aires protégées}

À l'exception du Parc National de Taï (PNT), les gestionnaires des aires de la quasitotalité des Parcs et Réserves enquêtées (Parcs Nationaux du Banco, de la Marahoué, d'Azagny et la Réserve de Faune d'Abokouamékro) nous ont révélé que les bâtiments administratifs, les infrastructures de surveillance et touristiques sont insuffisants et fortement dégradées.

Il en est de même des pistes et circuits touristiques qui, pour la plupart, sont impraticables parce qu'ils ne sont pas entretenus à cause de l'insuffisance des budgets de gestion. C'est par exemple le cas des passerelles flottantes [du circuit touristique du Parc National d'Azagny (PNA)] installées dans les zones marécageuses de ladite aire protégée. 


\section{Les interférences répétées des autorités locales et des cadres dans le règlement des litiges écologiques}

Selon les gestionnaires, bien souvent, certaines autorités locales (administratives, traditionnelles) et cadres interviennent en faveur des délinquants écologiques pris dans les aires protégées, en vue d'un règlement à l'amiable. Ils justifient ces interventions par des objectifs de recherche de cohésion sociale entre communautés riveraines et gestionnaires des aires protégées.

\section{Le retard dans la mise en place des outils de gestion participative}

En 2009, au moment de la réalisation de cette enquête de terrain, aux dires des gestionnaires, sur les quatorze (14) aires protégées, seulement le Parc National de Taï avait un comité de gestion locale (CGL) créé, mise en place et était fonctionnel. Trois (3) CGL d'aires protégées (Marahoué, Lamto, Abokouamékro) ${ }^{1059}$ avaient été (juridiquement) créés mais n'étaient pas mis en place. Les Parcs Nationaux du Banco, d'Azagny, des Iles Ehotilé, du Mont Péko avait leur arrêté de création en cours de rédaction.

Les CGL des six (6) autres aires protégées, à savoir les Parcs Nationaux de la Comoé, du Mont Sangbé, de la Réserve naturelle intégrale du Mont Nimba, et des Réserves naturelles partielles du N'zo, de Lamto, du Haut-Bandama et de Dahliafleur n'étaient pas en voie d'être crées.

En 2014, au cours d'un entretien ${ }^{1060}$, le Directeur technique de l'OIPR nous a révélé que sur les 14 aires protégées, 12 sont dotés de CGL; lesquels fonctionnent normalement. La Réserve du N'zo (qui dans la gestion pratique est rattachée au Parc National de Taï et la Réserve du Haut-Bandama n'en disposent pas.

\footnotetext{
${ }^{1059}$ Par exemple, ce n'est que le 08 novembre 2006 que le Préfet de la Région des Lacs (République de Côte d'Ivoire) a pris un arrêté (Arrêté n $70 / \mathrm{PY} / \mathrm{CAB}$ ) portant composition, attributions et fonctionnement du Comité de gestion locale de la Réserve de Faune d'Abokouamékro. Or, dans la procédure de création d'un CGL, le Préfet en charge du département sur le territoire duquel se trouve une aire protégée désigne les membres du comité conformément à la loi ; et par la suite le ministre en charge des aires protégées prend un arrêté relatif à la création et aux modalités de fonctionnement du CGL. Cette procédure est déduite de la lettre $\left(\mathrm{n}^{\circ} 0830 /\right.$ MINEME/OIPR du 13 juillet 2005) adressée par le ministre Angèle GNONSOA au Préfet du département de Grand-Lahou (sur le territoire duquel se trouve le Parc National d'Azagny) et ayant pour objet la mise en place du Parc National d'Azagny.

${ }^{1060}$ Cet entretien que nous a accordé le Directeur technique de l'OIPR (M. N'GORAN DJE), s'est réalisé le jeudi 11 décembre 2014, au siège de l'OIPR (Abidjan, République de Côte d'Ivoire).
} 


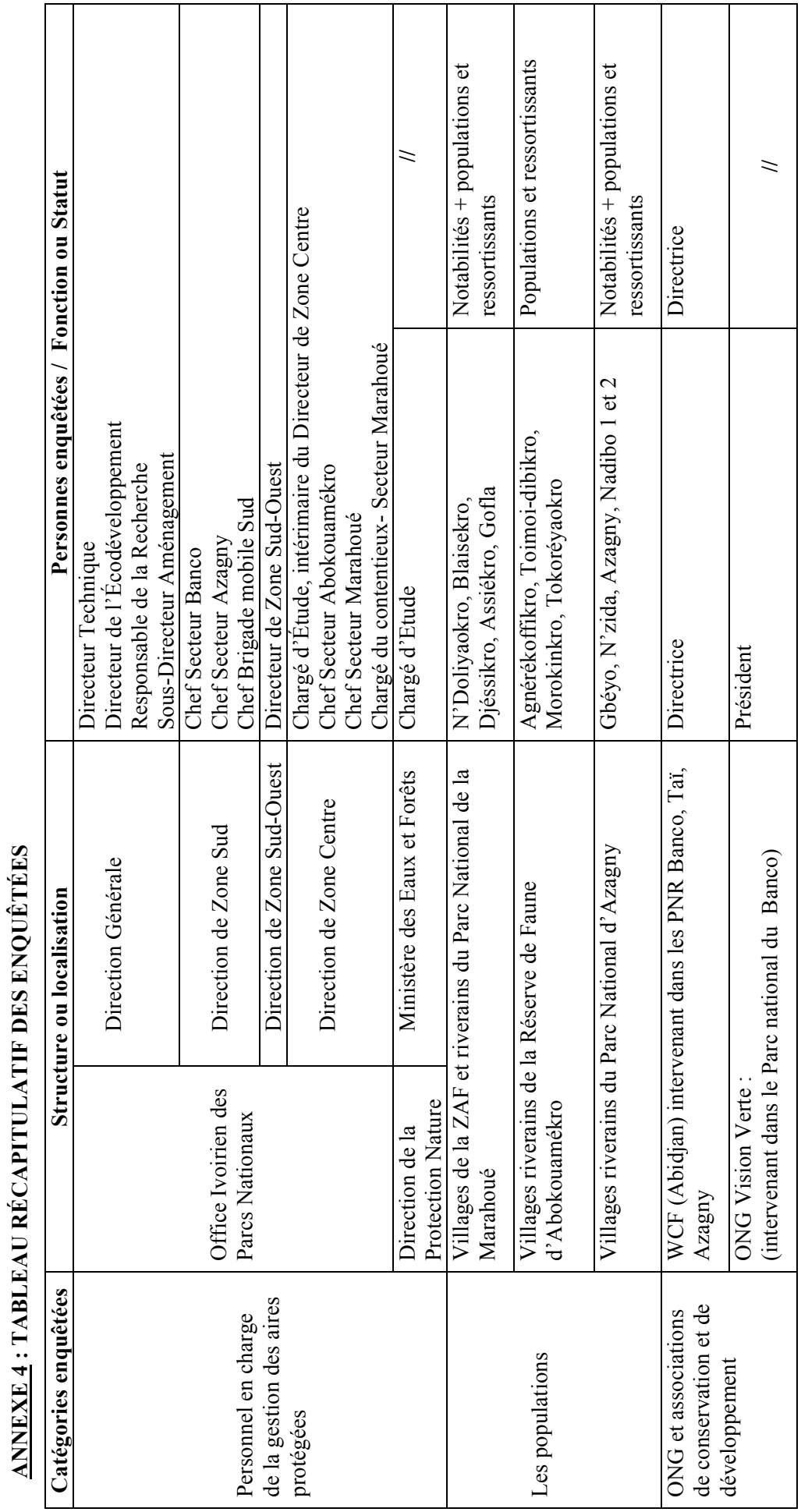




\section{ANNEXE 5 :}

\section{République de Côte d'Ivoire \\ Union-Discipline-Travail}

Loi $n^{\circ}$ 2002-102 du 11 février 2002 relative à la création, à la gestion et au financement des Parcs Nationaux et des Réserves naturelles (JORCI nº 20 du 16 mai 2002, p. 338)

$* * * *$

L'ASSEMBLÉ NATIONALE a adopté,

LE PRÉSIDENT DE LA RÉPUBLIQUE promulgue la loi dont la teneur suit : 


\section{TITRE PREMIER - DISPOSITIONS GÉNÉRALES}

\section{Article Premier.}

Au sens de la présente loi, les expressions ci-après ont les définitions suivantes :

"aire protégée" : désigne une portion de terre, de mer, de rivière et/ou de lagune géographiquement délimitée qui est définie, réglementée et gérée pour la protection et le maintien du patrimoine naturel et culturel ;

"conseil scientifique" : désigne le conseil dont la composition et les attributions sont définies aux articles 36 et suivants de la présente loi ;

"conservation" : Le Conseil Scientifique est un organe consultatif dont l'avis est requis dans les domaines énumérés à l'article 37 de la présente loi ;

"conservation in-situ" : désigne la conservation des écosystèmes et des habitats naturels et le maintien et la reconstitution des populations viables d'espèces dans leur milieu naturel et, dans le cas des espèces domestiques et cultivées, dans le milieu où se sont développés leurs caractères distinctifs;

"contrat de fiducie" : désigne la convention par laquelle un constituant ou mandant transfère tout ou partie de ses biens et droits à un gestionnaire qui, tenant ces biens et droits séparés de son patrimoine personnel, agit dans un but déterminé au profit d'un ou de plusieurs bénéficiaires ;

"contrat de gestion de terroir" : désigne le contrat passé entre le gestionnaire d'un parc ou d'une réserve et les populations rurales de la zone périphérique représentées par des structures associatives, privées ou administratives. Ce contrat définit notamment les modalités d'intervention des populations contractantes dans la surveillance, la gestion, l'entretien et, le cas échéant, l'animation culturelle et touristique d'un parc, d'une réserve ou de leur zone périphérique ;

"diversité biologique" : désigne au sens de la Convention sur la diversité biologique adoptée à Rio de Janeiro (Brésil) en 1992, la variabilité des organismes vivants de toute origine y compris, entre autres, les écosystèmes terrestres, marins et autres écosystèmes aquatiques, et les complexes écologiques dont ils font partie ; cela comprend la diversité au sein des espèces, et entre espèces, ainsi que celle des écosystèmes ;

"Fondations" : désigne une personne morale créée en vue de réaliser un but d'intérêt général. L'affectation de biens, droits ou ressources, à la réalisation de ce but d'intérêt général est irrévocable. Dans le cas d'espèce, les fondations désigneront des associations dont l'objet et les principes d'organisation sont définis au titre IV de la présente loi ;

"gestion durable des parcs et réserves" : désigne l'ensemble des mesures et des modalités de conservation des milieux et paysages naturels ainsi que de leurs ressources, dans un parc ou une réserve, à l'effet de maintenir l'équilibre et la stabilité des écosystèmes, au profit des générations présentes et aux fins de leur transmission dans les meilleures conditions aux générations futures ;

"Etablissement " : désigne une personne morale de droit public gérant un service public. Dans le cas d'espèce, l'établissement est chargé de la gestion des parcs et réserves "parcs et réserves": désignent, sans distinction, une réserve naturelle intégrale, un parc national, une réserve naturelle partielle, ou une réserve naturelle volontaire;

"Parc national" désigne une aire :

- placée sous le contrôle de l'Etat et dont les limites ne peuvent être changées, ni aucune partie aliénée, sauf par l'autorité législative compétente ;

- exclusivement destinée à la propagation, la protection, la conservation et l'aménagement de la végétation et les populations d'animaux sauvages, ainsi qu'à la protection des sites, des paysages, ou des formations géologiques d'une valeur scientifique ou esthétique particulière, dans l'intérêt et pour la récréation du public ;

- dans laquelle l'abattage, la chasse, la capture d'animaux et la destruction ou la collecte des plantes sont interdits, sauf pour des raisons scientifiques ou pour les besoins de l'aménagement et à condition que de telles opérations aient lieu sous la direction et le contrôle de l'autorité compétente ; 
- comportant tout milieu aquatique auquel s'appliquent toutes ou l'une quelconque des dispositions des alinéas 1 et 3 de la présente définition.

"Réserve naturelle intégrale" désigne une aire :

- Placée sous le contrôle de l'Etat et dont les limites ne peuvent être changées, ni aucune partie aliénée, sauf par l'autorité législative compétente ;

- Sur l'étendue de laquelle toute espèce de chasse ou de pêche, toute exploitation forestière, agricole ou minière, tout pâturage, toute fouille ou prospection, tout sondage ou terrassement, toute construction, tous travaux tendant à modifier l'aspect du terrain ou de la végétation, toute pollution des eaux et, de manière générale, tout acte de nature à apporter des perturbations à la faune ou à la flore, toute introduction d'espèces zoologiques ou botaniques, soit indigènes soit importées, sauvages ou domestiquées seront strictement interdits ;

- où il sera défendu de résider, de pénétrer, de circuler ou de camper et qu'il sera interdit de survoler à basse altitude, sans autorisation spéciale écrite de l'autorité compétente et dans laquelle les recherches scientifiques (y compris les éliminations d'animaux et de végétaux en vue de maintenir un écosystème) ne pourront être effectuées qu'avec la permission de cette autorité.

"Réserve naturelle partielle" désigne une aire protégée gérée dans le but de conservation in situ des écosystèmes naturels ou d'espèces ou peuplements ou biotopes spécifiques au profit et à l'avantage et pour l'utilisation durable, la récréation et l'éducation du public.

"Réserve partielle de faune" désigne une aire :

- mise à part pour la conservation, l'aménagement et la propagation de la vie animale sauvage, ainsi que pour la protection et l'aménagement de son habitat ;

- dans laquelle la chasse, l'abattage, ou la capture de la faune sont interdits, sauf par les autorités de la réserve ou sous leur direction ou leur contrôle pour l'équilibre de l'écosystème, après avis du Conseil Scientifique;

- où l'habitation et les autres activités humaines sont réglementées ou interdites.

"plan d'aménagement et de gestion" : désigne le document, ou l'ensemble des documents, décrivant de manière détaillée les éléments constitutifs physiques et biologiques, d'un parc ou d'une réserve, son environnement socio-économique, les objectifs de mise en valeur à court et moyen terme, les stratégies et modalités d'aménagement et de gestion, la planification sur une base décennale des mesures envisagées, leurs indicateurs d'impact et le budget de mise en œuvre dudit plan.

"réserve naturelle volontaire " : désigne la réserve naturelle partielle créée à l'initiative d'une collectivité territoriale, d'un établissement public ou d'une personne de droit privé, sur un terrain lui appartenant et pour la préservation d'un écosystème ou d'un paysage remarquable.

"terroir" : désigne une zone géographique homogène au regard de sa population, de son histoire et de son organisation ;

"utilisation durable" : désigne l'utilisation des ressources naturelles renouvelables d'une manière et à un rythme qui n'entraînent pas leur appauvrissement à long terme et sauvegardent, ainsi, leur potentiel pour satisfaire les besoins et aspirations des générations présentes et futures ;

"zone périphérique" : désigne la zone géographique environnante des parcs et réserves constituée de l'entière superficie des terroirs, des sous-préfectures et, le cas échéant, des forêts classées, aux fins de circonscrire une communauté avec laquelle l'autorité chargée de la gestion de l'aire protégée peut établir et formaliser des relations en vue de la réalisation des objectifs de la présente loi.

\section{Article 2.}

L'objectif général de la présente loi est de marquer la volonté de l'Etat de Côte d'Ivoire d'agir dans le secteur des parcs et réserves, et permettre le renforcement de la politique globale de conservation de la nature. Les objectifs spécifiques sont de :

1. adapter le service public en charge des parcs et réserves aux impératifs actuels d'une gestion rationnelle ;

2. conférer aux biens fonciers des parcs nationaux et réserves naturelles intégrales la domanialité publique afin de les rendre inaliénables ; 
3. définir la catégorie d'établissement la mieux adaptée pour gérer les parcs et réserves ;

4. préciser les modalités d'intervention contractuelle du secteur privé dans la gestion des parcs et réserves;

5. définir un mécanisme de financement à long terme des parcs et réserves.

La réalisation de ces objectifs et, d'une façon générale, la sauvegarde du patrimoine naturel sont d'intérêt général et constituent des objectifs prioritaires de la Nation.

\section{Article 3.}

Les parcs et réserves sont créés et gérés aux fins de permettre la conservation des milieux naturels, de la faune et de la flore terrestres et aquatiques, ainsi que le maintien de la diversité biologique et des processus écologiques contre toutes les causes de dégradation qui les menacent. Ils ont également pour vocation de participer, par la récréation et l'éducation du public, à l'équilibre harmonieux des populations, qu'elles soient rurales ou urbaines.

\section{Article 4.}

A l'effet de la réalisation des objectifs définis à l'article 2 ci-dessus, l'Etat prendra, chaque fois que nécessaire, toutes les mesures appropriées visant à promouvoir et à renforcer la collaboration et la coopération sous régionales et internationales, conformément aux conventions internationales auxquelles il est partie.

Cette coopération doit notamment porter sur les questions relatives :

(a) à la prévention et à la répression des infractions à la législation sur les parcs et réserves et à la protection des ressources naturelles ;

(b) à l'harmonisation des politiques et des législations relatives aux parcs et réserves et aux questions qui s'y rapportent ;

(c) à la recherche scientifique et aux inventaires des éléments constitutifs de la diversité biologique ;

(d) à la promotion des activités de protection, d'aménagement et d'utilisation durable des parcs et réserves, y compris la promotion du tourisme à vocation environnementale.

\section{TITRE II - RÉGIME JURIDIQUE DES PARCS NATIONAUX ET RESERVES NATURELLES}

\section{Article 5.}

En vue de préserver les milieux naturels et de promouvoir la mise en valeur de la faune et de la flore sauvages, il peut être créé, dans le respect des dispositions de la présente loi, des parcs nationaux, des réserves naturelles intégrales, des réserves naturelles partielles, des réserves naturelles volontaires et des zones périphériques.

\section{Article 6.}

Par effet de la présente loi :

- les aires protégées dont la liste constitue l'annexe 1 ci-jointe sont classés parc nationaux ;

- les aires protégées dont la liste constitue l'annexe 2 ci-jointe sont classées réserves naturelles.

Les annexes 1 et 2 , ci-jointes, précisent la dénomination, la localisation et la superficie à la date d'entrée en vigueur de la présente loi, de chaque réserve naturelle et de chaque parc national.

Les procédures de classement de nouveaux parcs et réserves seront déterminées par décret.

\section{Section I. Les réserves naturelles intégrales et les parcs nationaux}

\section{Article 7.}

Les réserves naturelles intégrales et les parcs nationaux font partie du domaine public inaliénable de l'État, à compter de la date de leur classement dans l'une ou l'autre de ces catégories.A cet effet, les réserves naturelles intégrales et les parcs nationaux font l'objet d'une procédure de classement, conformément aux dispositions des articles 6 ci-dessus et 8 ci- dessous.

Le domaine public des réserves naturelles intégrales et des parcs nationaux comprend, selon les cas, indistinctement, le domaine public terrestre, maritime, lagunaire, fluvial ou aérien. 


\section{Article 8.}

La loi porte classement et déclassement total ou partiel des réserves naturelles intégrales et des parcs nationaux.

La loi portant création d'une réserve naturelle intégrale ou d'un parc national en précise la dénomination, la localisation et la superficie.

Un décret d'application en détermine les limites administratives par points géodésiques et par limites naturelles.

Sans préjudice des dispositions de l'article 9 ci-dessous, ces limites peuvent être modifiées par décret pris en Conseil des Ministres, après autorisation de la loi.

\section{Article 9.}

A titre transitoire, les limites administratives existantes des parcs nationaux et des réserves naturelles intégrales, telles qu'elles résultent des dispositions réglementaires en vigueur à la date de promulgation de la présente loi, valent délimitation légale desdits parcs nationaux et réserves naturelles intégrales.

Pendant une durée de cinq ans à compter de la date d'entrée en vigueur de la présente loi, le gouvernement est autorisé à modifier, par décret, les limites administratives existantes, sans qu'il puisse en résulter une diminution supérieure à $2 \%$ de la superficie de chaque parc ou réserve et sans permettre l'enclavement de zones habitées ou cultivées.

$\mathrm{Au}$ cours de cette période transitoire, toute modification de limites conduisant à une diminution supérieure à $2 \%$ de la superficie d'un parc ou d'une réserve doit être autorisée par la loi.

A l'issue de ce délai de cinq ans, les limites administratives sont réputées intangibles et ne peuvent être modifiées que par la loi.

\section{Article 10.}

Sur toute l'étendue de la réserve naturelle intégrale, toute forme de chasse ou de pêche, d'abattage ou de capture de la faune, de destruction ou de collection de la flore, toute exploitation forestière, agricole ou minière, tout pâturage, toute fouille ou prospection, tout sondage, terrassement ou construction, tous travaux tendant à modifier l'aspect du terrain ou de la végétation, tout acte de nature à nuire ou à apporter des perturbations à la faune ou à la flore, toute introduction d'espèces zoologiques ou botaniques, soit indigènes, soit importées, sauvages ou domestiquées, sont strictement interdits.

Les recherches scientifiques ne peuvent se faire dans la réserve naturelle intégrale qu'avec la permission de l'autorité chargée de la gestion des parcs nationaux et réserves naturelles, après avis du Conseil scientifique.

La circulation, le camping et le survol à une altitude inférieure à 200 mètres ne peuvent se faire dans ou au-dessus de la réserve naturelle intégrale qu'avec la permission de l'autorité en charge de sa gestion et dans le cadre des activités de gestion ou de recherche scientifique autorisées.

\section{Article 11.}

Sur toute l'étendue du parc national, toute forme de chasse, de pêche et d'abattage, toute forme d'exploitation forestière, agricole ou minière, tout pâturage, toute fouille ou prospection, tout sondage, terrassement ou construction, tout travail tendant à modifier l'aspect du terrain ou de la végétation, tout acte de nature à nuire ou à apporter des perturbations à la faune ou à la flore, toute introduction d'espèces zoologiques ou botaniques, soit indigènes, soit importées, sauvages ou domestiquées sont strictement interdits.

Sur toute l'étendue du parc national, toute forme de capture de la faune, de destruction ou de collection de la flore, de récolte de plantes, fruits ou produits, sont interdites, sauf exceptionnellement par l'entremise de, ou sous la direction ou le contrôle de l'autorité chargée de sa gestion et dans des conditions fixées par décret pris en Conseil des Ministres.

Les recherches scientifiques ne peuvent se faire dans le parc national qu'avec la permission de l'autorité chargée de sa gestion, après avis du Conseil scientifique. 
Les conditions et modalités de l'exploitation touristique du parc national sont fixées par décret pris en Conseil des Ministres.

La circulation, le camping, l'atterrissage d'aéronefs ou d'engins à moteur ne peuvent se faire dans le parc national qu'avec la permission de l'autorité chargée de sa gestion, et dans le cadre des activités de gestion ou de recréation, d'éducation ou de recherche scientifique autorisées.

\section{$\underline{\text { Article } 12 .}$}

Pour l'application des dispositions du présent titre et notamment de ses articles 9, 10, 11 et 14, sont autorisés, par décision administrative du directeur du parc national ou de la réserve intégrale, l'arrachage des plantations ainsi que la destruction des installations et impenses privées, non autorisées, sises à l'intérieur des limites d'un parc national ou d'une réserve naturelle intégrale.

La décision administrative, prise conformément aux dispositions de l'alinéa précédent, n'est pas suspensive en cas de recours devant la juridiction compétente.

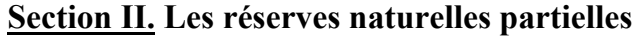

\section{Article 13.}

Les réserves naturelles partielles sont classées par décret pris en Conseil des ministres.

Les aires protégées de ces catégories peuvent appartenir indifféremment au domaine public ou privé de l'État ou des collectivités territoriales, ou au domaine privé des particuliers.

La création d'une réserve naturelle partielle n'affecte pas les droits fonciers, selon le cas, de l'Etat, des personnes morales de droit public, des communautés ou des personnes privées sur les portions de territoire qui la composent. Toutefois, les droits fonciers ainsi détenus par les personnes mentionnées au présent article peuvent être réduits des suites des servitudes liées à l'application de la présente loi

Les réserves naturelles volontaires sont gérées par la collectivité territoriale, l'établissement public ou la personne de droit privé, propriétaire du terrain constituant la réserve, avec l'appui le cas échéant, de l'autorité chargée de la surveillance des parcs et réserves.

\section{Article 13.}

Sur toute l'étendue d'une réserve naturelle partielle, toute forme de chasse, de pêche, d'abattage ou de capture de la faune, de destruction ou de collection de la flore, de récolte de plantes, fruits ou produits, toute exploitation forestière, agricole ou minière, tout pâturage, toute fouille ou prospection, tout sondage, terrassement ou construction, tout travail tendant à modifier l'aspect du terrain ou de la végétation, tout acte de nature à nuire ou à apporter des perturbations à la faune ou à la flore, toute introduction d'espèces zoologiques ou botaniques, soit indigènes, soit importées, sauvages ou domestiquées sont interdits, sauf par l'entremise, ou sous la direction ou le contrôle de l'autorité chargée de sa gestion et dans des conditions et modalités fixées par décret pris en Conseil des Ministres.

Les conditions et modalités spécifiques d'exploitation et de gestion d'une réserve naturelle partielle, notamment l'exercice de la chasse, la capture des animaux, la collecte des végétaux, l'observation de la faune et de la flore par l'édification de bâtiments et les travaux d'aménagement, sont définies par le décret de classement.

Les recherches scientifiques ne peuvent se faire dans la réserve naturelle partielle qu'avec la permission de l'autorité chargée de sa gestion, après avis du Conseil scientifique.

Les conditions et modalités de l'exploitation touristique ou cynégétique d'une réserve naturelle partielle sont fixées par le décret de classement.

La circulation, le camping, l'atterrissage d'aéronefs ou d'engins à moteur ne peuvent se faire dans la réserve naturelle partielle qu'avec la permission de l'autorité chargée de sa gestion et dans le cadre des activités de gestion ou de recréation, d'éducation ou de recherche scientifique ou d'utilisation durable autorisées. 


\section{Section 3 : Les zones périphériques}

\section{$\underline{\text { Article } 15}$.}

En application des dispositions de la présente loi, il peut être constitué des zones périphériques.

La pêche, la chasse, l'abattage et la capture de faune sauvage, les activités agricoles et forestières, la cueillette de plantes, la collecte de minéraux ou fossiles peuvent se faire dans la zone périphérique, dans le respect des dispositions législatives et réglementaires régissant ces activités et, le cas échéant, des stipulations plus contraignantes des contrats de gestion de terroir mentionnés à l'article 33 cidessous.

Les zones périphériques sont régies par le droit commun, y compris le droit foncier, sans préjudice des privilèges ou des servitudes qui peuvent résulter de l'application de la présente loi.

\section{Article 16:}

L'étendue de la zone périphérique d'un parc national ou d'une réserve naturelle est définie en concordance avec les limites administratives, selon le cas, des terroirs, des collectivités territoriales ou des forêts classées.

\section{Article 17}

Les conditions et modalités d'association, d'une part, des populations des zones périphériques à la gestion d'un parc national ou d'une réserve naturelle et, d'autre part, de l'autorité chargée de la gestion d'une telle aire protégée au développement de la zone périphérique, font l'objet d'un contrat de gestion de terroir mentionné à l'article 33 ci- dessous.

Tout projet industriel, minier, de carrière, de lotissement ou d'équipement touristique, de fouille archéologique, ainsi que toute réalisation d'infrastructures linéaires tels que routes, lignes électriques, oléoducs, gazoducs, dans la zone périphérique, qu'ils soient situés intégralement ou partiellement dans celle-ci, sont soumis à l'avis préalable de l'Etablissement.

Ces projets font l'objet d'une étude d'impact environnemental, conformément aux dispositions législatives et réglementaires en vigueur.

\section{Section 4 : Modification des limites}

\section{Article 18.}

Toute modification des limites administratives existantes, à la date d'entrée en vigueur de la présente loi, d'un parc national, d'une réserve naturelle intégrale ou d'une réserve naturelle partielle ainsi que, le cas échéant, de la zone périphérique, est obligatoirement précédée d'une étude d'impact environnemental, conformément aux dispositions législatives et réglementaires en vigueur.

\section{TITRE III - DE LA GESTION DES PARCS NATIONAUX ET RÉSERVES}

\section{CHAPITRE PREMIER - DISPOSITIONS GÉNÉRALES}

\section{Article 19}

La gestion des parcs et réserves relève d'un établissement public national de type particulier, doté de la personnalité morale et de l'autonomie financière, dont les missions et les principes d'organisation et de fonctionnement sont définis par la présente loi.

L'Etablissement est régi, à titre principal, par les dispositions de la présente loi et des décrets pris pour son application. Il est également régi, à titre subsidiaire, par les dispositions législatives et réglementaires applicables aux établissements publics nationaux, en ce que ces dispositions ne sont pas contraires à celles de la présente loi et des décrets pris pour son application.

\section{Article 20.}

Les missions de l'Établissement, sur l'ensemble des parcs et réserves sur lesquels il exerce son autorité, comprennent, notamment et obligatoirement :

1. la gestion du patrimoine foncier qui en constitue l'assise ; 
2. l'exercice de la police administrative ;

3. l'exercice de la police judiciaire dans les conditions définies au chapitre IV ci-dessous ;

4. la mise en œuvre d'une politique de gestion durable par la promotion des activités légalement permises en fonction de la nature juridique du parc ou de la réserve considérée et de sa zone périphérique ;

5. le cas échéant, la coordination ou la réalisation des études nécessaires à la création, à l'extension, ou à l'aménagement d'un parc, d'une réserve ou de sa zone périphérique ;

6. l'information, l'éducation et la communication.

\section{Article 21}

A l'effet de l'exécution de ses missions, l'Établissement est chargé de :

1. Mettre en œuvre les orientations de la politique nationale de protection et de gestion durable des ressources des parcs et réserves ;

2. Définir les modalités de protection et d'utilisation rationnelle des ressources naturelles des parcs et réserves, dans le respect de leur pérennité, y compris les ressources en faune et flore sauvages, terrestres et aquatiques ;

3. Définir les conditions de préservation des paysages naturels qui leur sont attachés, en tenant compte de l'équilibre et de la stabilité des écosystèmes ;

4. Veiller, avec l'appui du comité de gestion de chaque parc et réserve, à l'élaboration et à la mise en œuvre des plans de gestion durable des parcs et réserves ;

5. Mettre en place les moyens de protection des habitats naturels et de la vie sauvage, notamment des espèces de faune et de flore rares ou en danger de disparition, dans les zones où se développent les activités de visite et de tourisme écologique ;

6. Coordonner ses activités avec celles des institutions scientifiques, techniques et des associations de protection de la nature dont les programmes sont liés aux objectifs de la politique de conservation des parcs et réserves ;

7. Planifier et mettre en œuvre la formation continue des personnels chargés de la gestion, de la protection des parcs et réserves et de leurs ressources en faune et en flore ;

8. Assurer la centralisation, le traitement et la diffusion des informations relatives aux aires protégées afin d'assurer un suivi national des indicateurs de conservation des parcs et réserves ;

9. Promouvoir l'information générale sur les parcs et réserves ;

10. Promouvoir la conservation de la diversité biologique.

\section{Article 22.}

Il est exercé, dans des conditions fixées par décret pris en Conseil des Ministres, une tutelle administrative et financière sur l'Établissement.

Par l'exercice de cette tutelle, l'État veille au respect de sa politique en matière de protection de la nature et des ressources naturelles, de surveillance, de sécurité et de valorisation des parcs et réserves, ainsi qu'à l'exécution par l'Établissement de ses missions et à l'équilibre économique et financier de sa gestion.

\section{Article 23.}

Les personnels de l'Établissement sont :

- des fonctionnaires, agents des Eaux et Forêts, ainsi qu'éventuellement d'autres corps de la Fonction Publique, mis en position de détachement auprès de l'Établissement,

- des agents contractuels, régis par le Code du travail.

\section{Article 24.}

Les personnels de l'Établissement, fonctionnaires et agents de l'État, perçoivent les mêmes traitements et indemnités que ceux de la Fonction Publique. Ils reçoivent également des indemnités et primes spécifiques dans des conditions fixées par décret pris en Conseil des Ministres.

\section{Article 25.}

L'Établissement est affectataire du domaine public de l'État ou d'une collectivité territoriale constituant le ou les parcs et réserves dont il a la gestion. Il peut disposer d'un patrimoine propre. 


\section{CHAPITRE II - ADMINISTRATION ET GESTION}

\section{Section 1 : La gestion financière}

\section{Article 26.}

Les ressources de l'Établissement sont constituées, notamment, par :

- les subventions de l'État ;

- les taxes parafiscales affectées;

- les transferts des Fondations mentionnées au chapitre I du titre IV ci-dessous ;

- les subventions d'organismes publics autres que l'État ou privés nationaux ou internationaux ;

- les produits de l'exploitation du domaine qui lui est affecté ;

- les produits de l'exploitation légalement autorisée de la faune ou de la flore ;

- les produits de ses prestations de services ;

- les redevances des activités concédées ;

- le produit des amendes et confiscations affecté par l’État;

- les dons et legs.

\section{Article 27.}

Les dépenses de l'Établissement sont constituées, notamment, par :

- les charges de fonctionnement :

- les indemnités et primes des agents ;

- les rémunérations servies aux populations rurales au titre des vacations ;

- la rémunération éventuelle des conventions d'exploitation, des prestations de service et des contrats de

gestion de terroir ;

- les autres charges de fonctionnement

- les travaux d'aménagement et d'investissement.

\section{Article 28.}

Le régime financier et comptable de l'Établissement est le même que celui des établissements publics nationaux.

Les ressources de l'Établissement, provenant des Fondations mentionnées au chapitre I du titre IV cidessous ne sont pas des deniers publics et sont gérées conformément aux règles de la comptabilité privée.

\section{Article 29.}

L'Établissement peut ouvrir des comptes dans des établissements bancaires pour recevoir des fonds provenant des fondations et autres sources privées.

\section{Section 2 : Dispositions particulières à chacun des parcs et réserves}

\section{Article 30.}

L'Établissement établit, pour chaque parc et réserve, un plan d'aménagement et de gestion, dans un délai maximum de cinq ans à partir de sa création ou de l'entrée en vigueur de la présente loi.

Le plan d'aménagement et de gestion est approuvé par le Ministre de tutelle des parcs et réserves après avis du conseil scientifique. Il est mis à jour chaque fois que nécessaire et, au moins, tous les dix ans.

\section{Article 31.}

Les modalités de la gestion décentralisée des parcs nationaux et des réserves naturelles et, notamment, les attributions et la composition des comités de gestion, ainsi que les attributions des directeurs, sont définies par décret pris en Conseil des Ministres. 


\section{Section 3 : Délégation de concession d'exploitation}

\section{Article 32.}

Tout ou partie des missions d'exploitation d'un parc ou d'une réserve, selon la nature de ce parc ou de cette

réserve, peuvent être concédées par l'Établissement à une personne morale de droit privé dans le cadre d'une convention de concession d'exploitation :

Ladite convention d'exploitation conclue conformément au présent article entre l'Établissement et le concessionnaire, est approuvée, avant son entrée en vigueur, par le Ministre de tutelle des parcs et réserves et les Ministres concernés par les activités concédées, selon des modalités fixées par décret pris en Conseil des Ministres.

\section{Section 4 : Les contrats de gestion de terroir}

\section{Article 33.}

Les contrats de gestion de terroir sont passés au bénéfice réciproque d'un parc ou d'une réserve et de ses populations environnantes. Ces contrats ont pour objet de définir les conditions et modalités de l'association de ces populations à la conservation du parc ou de la réserve et favoriser ainsi les retombées économiques pour ces populations.

Ces contrats de gestion de terroir peuvent porter notamment, selon qu'il s'agit d'un parc ou d'une réserveet en considération des espaces concernés de la zone périphérique, sur la gestion des ressources naturelles, sur les activités d'éducation, de loisir, de formation de guides, d'hôtellerie et d'aménagement.

Les contrats de gestion de terroir conclus conformément au présent article entre l'autorité chargée d'une aire protégée et les représentants des populations environnantes sont, préalablement à leur entrée en vigueur, approuvés par l'autorité compétente dont relève le parc ou la réserve.

\section{CHAPITRE III - L'ORGANE CONSULTATIF SCIENTIFIQUE}

\section{Article 34.}

La réalisation des objectifs définis à l'article 3 ci-dessus doit également permettre le développement de la recherche scientifique répondant aux besoins de la conservation des ressources naturelles, l'exploitation des résultats de cette recherche, qu'elle soit nationale ou internationale, faite sur le territoire national ou dans d'autres pays, et la diffusion nationale et internationale des résultats de cette recherche scientifique.

\section{Article 35.}

Il est créé un Conseil scientifique des parcs et réserves, dont la composition, les attributions et le fonctionnement sont définis par décret pris en Conseil des Ministres, dans le respect des dispositions du présent titre.

Le Conseil scientifique est un organe consultatif de l'Établissement.

\section{Article 36.}

Le Conseil scientifique est constitué de personnalités issues des milieux scientifiques et de la recherche, de nationalité ivoirienne ou étrangère, choisies pour leur compétence et leur expérience, ainsi que leur complémentarité, en matière de conservation de la nature.

Les membres du conseil scientifique sont nommés par décret pris en Conseil des Ministres. Ils élisent en leur sein un Président.

\section{Article 37.}

L'avis du Conseil scientifique est requis :

1. sur toute question, projet et programme ayant une incidence sur le statut des ressources et richesses de la diversité biologique des parcs et réserves ainsi que sur leur consistance physique; 
2. sur la pertinence des plans d'aménagement et de gestion des parcs et réserves en général, ainsi que sur tout projet d'investissement et d'infrastructure même situé hors d'un parc ou d'une réserve mais susceptible d'avoir une incidence sur sa conservation ;

3. sur toute question liée à l'introduction de nouvelles espèces de faune et de flore, au recensement des ressources naturelles et à leur conservation dans les parcs et réserves ainsi que leurs zones périphériques;

4. sur la pertinence et la méthodologie des programmes de recherche scientifique effectués dans les parcs et réserves ou leurs zones périphériques.

5. sur tout projet de législation ou de réglementation pouvant avoir une incidence sur la conservation de la nature et de ses ressources ainsi que sur la diversité et les équilibres biologiques en Côte d'Ivoire;

6. sur tout projet de loi de classement ou de déclassement d'un parc ou d'une réserve.

Le décret mentionné à l'article 35 ci-dessus précise les matières pour lesquelles il est requis, selon le cas, un avis simple ou un avis conforme du Conseil scientifique.

Le Conseil scientifique examine les rapports annuels sur l'état de conservation des parcs et réserves et publie un rapport annuel contenant ses observations et recommandations.

\section{CHAPITRE IV - LE POUVOIR DE POLICE}

\section{Article 38.}

Le responsable de l'Établissement, les directeurs des parcs et réserves ou groupements de parcs et réserves, ainsi que les agents des Eaux et Forêts appartenant au corps des ingénieurs, détachés auprès d'un parc ou d'une réserve, ont la qualité d'officier de police judiciaire.

Les modalités de leur habilitation sont fixées par décret pris en Conseil des Ministres.

\section{Article 39.}

Le pouvoir de contrôle et de police exercé par l'Établissement s'étend sur le domaine dont il est affectataire.

\section{Article 40.}

Les infractions sont constatées et réprimées selon les dispositions pénales prévues au titre $\mathrm{V}$ de la présente loi.

\section{Article 41.}

L'affectation, entre l'Établissement et l'État, du produit des amendes, confiscations et transactions est définie par décret.

\section{TITRE IV - DU FINANCEMENT DES PARCS NATIONAUX ET RESERVES NATURELLES}

\section{CHAPITRE PREMIER - LES FONDATIONS}

\section{Article 42.}

Le financement durable de certaines des charges récurrentes des parcs et réserves et de la réalisation de certains investissements est assuré par le revenu des placements des Fondations exclusivement destinées au financement des parcs et réserves, ci-après, désignées dans la présente loi "les Fondations".

Les Fondations sont régies par les dispositions de la présente loi et, à titre subsidiaire, par les dispositions régissant les associations reconnues d'utilité publique.

Les statuts des Fondations, élaborés conformément aux dispositions de la présente loi, des décrets pris pour son application, ainsi que des dispositions législatives et réglementaires régissant les associations reconnues d'utilité publique, déterminent les conditions et modalités d'admission et de retrait de ses membres. 
Les statuts des Fondations peuvent prévoir plusieurs catégories de membres et une représentation dans des collèges distincts au sein des organes délibérant, en fonction des financements auxquels ils contribuent.

\section{Article 43.}

Par dérogation aux dispositions de la loi sur les associations, les Fondations sont considérées comme nationales, quel que soit le nombre des personnes physiques ou morales étrangères qui en sont membres ou administrateurs.

\section{Article 44.}

Les Fondations sont dotées d'un conseil d'administration investi des pouvoirs les plus étendus pour agir en toute circonstance en leurs noms.

Les statuts des Fondations fixent, notamment, les modalités de détermination de la composition du conseil d'administration, de telle sorte que l'expression de la représentation des intérêts privés soit toujours majoritaire. Les statuts précisent également les pouvoirs que le conseil d'administration ne peut déléguer et les décisions qui relèvent de sa compétence exclusive.

Le décret de reconnaissance d'utilité publique des Fondations peut soumettre la modification de certaines des stipulations des statuts qui ont justifié de ladite reconnaissance d'utilité publique à une approbation par décret pris en Conseil des Ministres.

Le conseil d'administration accepte les dons et legs, sans préjudice de leur affectation, le cas échéant, à un parc ou à une réserve ou à un objet déterminé entrant dans le champ d'application de la présente loi.

L'avis de constitution et les statuts d'une Fondation, ainsi que toutes modifications ultérieures de ses statuts sont publiés au Journal Officiel.

\section{Article 45.}

À l'effet de la réalisation de leur objet, les Fondations reçoivent, notamment, des dons et legs de personnes physiques ou morales, nationales ou internationales, des dotations, subventions et contreparties financières de l'État, les produits du mécénat en faveur de l'environnement, les affectations de taxes parafiscales, la conversion de dettes, le produit de campagnes publicitaires et médiatiques organisées pour leur compte, ainsi que tout autre produit financier.

Les Fondations peuvent recueillir des fonds, identifiés sur des comptes, spécialement et exclusivement affectés à un parc, une réserve, une activité, ou à un objet particulier entrant dans le champ d'application de la présente loi.

L'exécution des dépenses financées par ces comptes se fait dans le cadre de contrats de projet conclus entre une Fondation et un parc ou une réserve bénéficiaire, dans le respect des conditions et modalités fixées par le donateur et acceptées par la Fondation.

Tous les fonds recueillis par les Fondations, ainsi que les revenus des placements, sont des deniers privés.

Les dispositions de l'alinéa précédent ne font pas obstacle à l'exercice, par les donateurs des Fondations, des contrôles qu'ils doivent exercer conformément à leur statut, aux dispositions qui les régissent ainsi qu'au contrat de fiducie.

Les Fondations peuvent, dans le cadre de leur mission, acquérir ou financer l'acquisition de terrains privés destinés à devenir des parcs ou réserves.

Les biens ainsi acquis sont transmis à l'Établissement qui en assure la gestion, sans frais pour la Fondation.

\section{$\underline{\text { Article } 46 .}$}

Les fonds recueillis par les Fondations sont exonérés de tous impôts et taxes, et notamment de l'impôt sur les successions. Ils sont déductibles des revenus des donateurs nationaux dans des conditions fixées par la loi. 


\section{Article 47.}

Les revenus des placements des Fondations sont exonérés de tous impôts et taxes.

\section{Article 48.}

Les Fondations peuvent agir en qualité de fonds fiduciaire à l'effet de l'exécution des contrats de fiducie définis au chapitre II du titre IV ci-après.

\section{Article 49.}

Les Fondations peuvent, par une décision du conseil d'administration prise conformément à leurs statuts et après avis des commissaires aux comptes, confier la gestion de tout ou partie de leurs fonds, et de ceux qui leur sont confiés par application d'un contrat de fiducie, à une ou plusieurs personnes morales tierces, nationales ou étrangères, présentant toutes les garanties professionnelles en la matière, spécialisées dans la gestion de patrimoine, sélectionnées par appel d'offres, à l'effet d'en retirer un revenu optimal.

Il est rendu compte chaque année, en assemblée générale, des résultats obtenus par chacun des gestionnaires.

\section{Article 50.}

Nul ne peut être dirigeant ou administrateur d'une Fondation, s'il a fait l'objet d'une mesure d'interdiction de diriger, gérer ou contrôler une entreprise ou d'une mesure de faillite personnelle, ou s'il a subi une condamnation pénale ou une sanction professionnelle pour des faits contraires à l'honneur, à la probité ou aux bonnes mœurs.

\section{Article 51.}

Chaque Fondation établit pour chaque contrat de fiducie :

1. Un état des biens et droits ainsi que des créances et des dettes, concernant l'exécution du contrat. Cet état décrit séparément les éléments actifs et passifs de la masse fiduciaire ;

2. Un état des produits et des charges afférents au contrat de fiducie sans qu'il soit tenu compte de leur date d'encaissement ou de paiement ; cet état fait apparaître, par différence après déduction des amortissements et des provisions, le résultat de la masse fiduciaire.

Il ne peut être procédé à aucune réévaluation des éléments de la masse fiduciaire.

Un décret pris en Conseil des Ministre précisera les modalités d'établissement des états fiduciaires.

\section{Article 52.}

Les états prévus à l'article 51, ci-dessus, sont communiqués à l'Etablissement bénéficiaire à sa demande.

\section{Article 53.}

Les comptes annuels des Fondations comprennent, outre le bilan, le compte de résultats et les annexes prévus par les dispositions législatives et réglementaires en vigueur applicables aux sociétés commerciales et, pour chaque contrat de fiducie, les états mentionnés à l'article 51 ci-dessus.

Les Fondations procèdent de manière autonome à l'enregistrement comptable des mouvements affectant la masse fiduciaire.

Les comptes des Fondations sont soumis annuellement au contrôle d'un (1) ou plusieurs commissaires aux comptes désignés par l'assemblée générale.

\section{Article 54.}

Les délibérations de l'assemblée générale extraordinaire portant dissolution des Fondations et fixant les modalités de leur liquidation sont approuvées par décret pris en Conseil des Ministres. 
En cas de dissolution d'une Fondation, la délibération des organes compétents pour la prononcer et fixer les conditions et modalités de la dévolution de son patrimoine est entérinée par un décret pris en Conseil des Ministres.

En cas de dissolution, les biens et droits objets d'un contrat de fiducie ne font pas partie de l'actif partageable ou transmissible à titre universel.

\section{CHAPITRE II - DU CONTRAT DE FIDUCIE}

\section{Article 55.}

En vue du financement d'une activité ou d'un investissement à réaliser conformément aux objectifs définis par la présente loi, par ou pour le compte de l'Etablissement, il peut être conclu un contrat de fiducie par lequel un constituant transfère tout ou partie de ses biens et droits à un gestionnaire qui, tenant ces biens et droits séparés de son patrimoine personnel, agit dans un but déterminé au profit d'un ou plusieurs bénéficiaires conformément aux stipulations du contrat.

Une Fondation peut être constituant, bénéficiaire ou gestionnaire de tels contrats. L'Etablissement peut être bénéficiaire de tels contrats.

Le contrat de fiducie est soumis aux règles ci-après énoncées et, à titre subsidiaire, aux dispositions du code civil.

\section{Article 56.}

Le contrat de fiducie doit comporter, à peine de nullité, les stipulations suivantes :

$\mathbf{1}^{\circ}$ ) la détermination des biens et droits qui en sont l'objet ;

$\mathbf{2}^{\circ}$ ) la définition de la mission du gestionnaire, ainsi que l'étendue de ses pouvoirs d'administration et de disposition ;

$3^{\circ}$ ) l'indication des conditions dans lesquelles les biens et droits doivent être représentés ou transmis au bénéficiaire ;

$4^{\circ}$ ) la détermination de la durée de la fiducie, qui ne peut excéder quatre-vingt-dix-neuf ans à compter de la date du contrat. Si la durée est de quatre-vingt-dix-neuf ans, les biens et matériels qui en sont l'objet sont, au terme de cette durée, définitivement la propriété du bénéficiaire.

Le contrat de fiducie est passé par écrit. Lorsqu'il est conclu à des fins de transmission à titre gratuit ou lorsqu'il porte sur un bien immobilier, ou sur une somme ou sur un bien d'un montant supérieur à un montant fixé par décret pris en Conseil des Ministres, il est, à peine de nullité, passé devant notaire.

La volonté des parties aux termes d'un contrat de fiducie doit être expresse et ne se présume pas.

\section{Article 57.}

Dans ses rapports avec les tiers, le gestionnaire est réputé disposer des pouvoirs les plus étendus sur les biens et droits, objet du contrat de fiducie, à moins qu'il ne soit démontré que les tiers avaient connaissance de la limitation de ses pouvoirs.

\section{Article 58.}

Lorsque la mutation des droits et biens d'un contrat de fiducie est soumis à publicité, celle-ci doit mentionner le nom et la qualité du gestionnaire.

\section{Article 59.}

Le gestionnaire exerce sa mission dans le respect de la confiance du constituant.

Si le gestionnaire manque gravement à ses devoirs, ou met en péril les intérêts qui lui sont confiés, le constituant ou le bénéficiaire peut demander en justice la nomination d'un administrateur provisoire ou le remplacement du gestionnaire contesté.

L'un ou l'autre peut demander qu'il soit mis fin au contrat de fiducie. La décision judiciaire faisant droit à la demande emporte de plein droit le dessaisissement du gestionnaire. 


\section{Article 60.}

Le gestionnaire doit prendre toutes mesures propres à éviter la confusion des biens et droits transférés ainsi que des dettes s'y rapportant en application d'un contrat de fiducie, soit avec ses biens personnels, soit avec d'autres biens fiduciaires.

Sans préjudice des droits des créanciers du constituant, titulaires d'un droit de suite attaché à une sûreté publiée antérieurement au contrat de fiducie, et hors le cas de fraude aux droits des créanciers du constituant, les biens transférés au gestionnaire ne peuvent être saisis que par les titulaires de créances nées de la conservation ou de la gestion de ces biens.

\section{Article 61.}

Le contrat de fiducie ne peut porter atteinte aux droits des héritiers réservataires. Si, lors du décès du constituant, la valeur des biens et droits transférés au gestionnaire excède la quotité disponible, le contrat de fiducie est réductible suivant les règles applicables aux donations entre vifs, conformément aux dispositions législatives et réglementaires en vigueur.

\section{Article 62.}

La valeur des biens et droits transférés au gestionnaire s'impute sur la quotité disponible de la succession du constituant conformément aux dispositions législatives et réglementaires en vigueur.

\section{$\underline{\text { Article } 63 .}$}

L'action en réduction est exercée contre la Fondation ou l'Etablissement, bénéficiaire, selon le cas lorsque les biens et droits leur ont été transmis.

Lorsque le contrat de fiducie porte sur une entreprise à caractère industriel, commercial, artisanal, agricole ou libéral ou sur la majorité des parts ou actions d'une société ayant l'un de ces objets, la réduction peut toujours être faite en valeur.

\section{Article 64.}

Lorsque le contrat de fiducie prévoit la transmission de biens et droits à titre gratuit à une Fondation ou à l'Etablissement, bénéficiaire, il peut être stipulé, par dérogation aux articles 1er et 4 de la loi ${ }^{\circ}$ 64-380 du 7 octobre 1964, relative aux donations entre vifs et testaments et à l'article 1130 du code civil, que la transmission prendra effet au décès du constituant.

\section{Article 65.}

Pour l'application de l'article 17 de la loi n 64-380 du 7 octobre 1964, susmentionnée, il est tenu compte, après en avoir déduit les dettes, de la valeur et de l'état des biens fiduciaires au jour du décès du constituant, s'ils n'ont pas été transmis à l'Etablissement ou à une Fondation, bénéficiaire. Pour les biens transmis à l'Etablissement ou à la Fondation, bénéficiaire, il est tenu compte de leur état au jour de cette transmission et de leur valeur au jour du décès du constituant. Si les biens ont été aliénés par une Fondation ou l'Etablissement, bénéficiaire, il est tenu compte de leur valeur à l'époque de l'aliénation et, s'il y a eu novation ou subrogation, de la valeur des nouveaux biens au jour du décès du constituant.

\section{Article 66.}

Pour l'application de l'article 18 de la loi n64-380 du 7 octobre 1964 susmentionnée, il est tenu compte, pour les biens transmis à une Fondation ou à l'Etablissement, bénéficiaire, de la date à laquelle la désignation de ces derniers ne peut plus être modifiée et de la date du décès du constituant pour les biens non encore transmis au bénéficiaire.

\section{Article 67.}

Une Fondation peut demander la révocation ou la révision du contrat de fiducie dans les conditions prévues par le contrat de fiducie sans préjudice de l'application des dispositions, le cas échéant, du code civil. 


\section{Article 68.}

Le contrat de fiducie prend fin par la survenance du terme fixé ou la réalisation du but poursuivi, quand celle-ci a lieu avant ce terme. La fiducie prend également fin par une décision de justice lorsque, en l'absence de stipulations prévoyant les conditions dans lesquelles le contrat se poursuivra, se produit l'un des événements ci-après :

1. La renonciation du bénéficiaire à tout ou partie des biens transmis;

2. La dissolution de la Fondation bénéficiaire, le contrat pouvant cependant se poursuivre jusqu'à la clôture des opérations de liquidation ;

3. La liquidation judiciaire de la Fondation bénéficiaire ;

4. La disparition de la Fondation, par suite d'une absorption ou d'une cession prononcée dans le cadre d'un redressement ou d'une liquidation judiciaire.

Toutefois, dans les cas prévus à l'alinéa précédent, le juge peut, à la demande du constituant ou de l'Etablissement bénéficiaire, prendre toutes mesures permettant la poursuite du contrat.

\section{Article 69.}

Lorsque la fiducie prend fin, pour quelque cause que ce soit, et en l'absence de bénéficiaire, les biens et droits subsistants font retour au constituant ou à ses ayants droit, sauf stipulations contraires du contrat de fiducie.

\section{TITRE V - DISPOSITIONS PENALES}

\section{Article 70.}

Est puni d'une amende de 1.000 .000 à 25.000 .000 de francs et d'un emprisonnement de deux mois à deux ans ou de l'une de ces deux peines seulement, quiconque entreprend ou fait entreprendre des constructions ou des travaux de fouille, prospection, sondage ou terrassement ou des exploitations agricoles dans les parcs nationaux et les réserves naturelles.

La peine est portée au double si les dommages causés au milieu naturel sont irréversibles.

\section{Article 71.}

Est puni d'une amende de 5000000 à 50000000 de francs et d'un emprisonnement de six mois à cinq ans ou de l'une de ces deux peines seulement, quiconque entreprend l'exploitation de bois d'œuvre et d'ébénisterie à l'intérieur d'un parc national ou d'une réserve naturelle.

La peine est portée au double s'il s'agit d'un acte volontaire ou en cas de récidive.

\section{Article 72.}

Est puni d'une amende de 100.000 à 50.000 .000 de francs et d'un emprisonnement de six mois à cinq ans ou de l'une de ces deux peines seulement, quiconque détruit par incendie, tout ou partie d'un parc ou d'une réserve naturelle.

La peine est portée au double s'il s'agit d'un acte volontaire ou en cas de récidive.

\section{Article 73.}

Sans préjudice des dispositions de l'article 98 de la loi n ${ }^{\circ} 96-766$ du 3 octobre 1996, portant code de l'environnement, est puni d'une amende de 500.000 à 100.000 .000 de francs et d'un emprisonnement de deux mois à deux ans ou de l'une de ces deux peines seulement, quiconque procède directement ou indirectement à des déversements, écoulements, rejets et dépôts de substance de toute nature susceptibles de porter atteinte à l'intégrité du parc national ou d'une réserve naturelle ou aux activités de leur exploitation éco-touristique.

La peine est portée au double s'il s'agit de substances toxiques ou en cas de récidive.

\section{Article 74.}

Est puni d'une amende de 20.000 à 250.000 francs et d'un emprisonnement de huit jours à un an, ou de l'une de ces deux peines seulement, quiconque nuit ou apporte des perturbations graves à la faune ou à la flore ou s'introduit de manière frauduleuse dans un parc national ou une réserve naturelle. 


\section{Article 75.}

Est puni d'une amende de 100.000 à 1.000 .000 de francs et d'un emprisonnement de huit jours à deux ans ou de l'une de ces deux peines seulement, quiconque, dans un parc national ou une réserve naturelle, sans l'autorisation préalable du directeur du parc ou de la réserve naturelle:

- Prélève ou collecte la flore,

- Récolte des plantes, fruits ou produits,

- Coupe, arrache, enlève ou endommage d'une manière quelconque la flore,

- Tue, blesse, pêche ou capture les animaux.

\section{Article 76.}

Est passible des peines prévues à l'article 88 de la loi no $96-766$ du 3 octobre 1996 portant code de l'environnement, toute personne physique ou morale qui omet de demander l'autorisation préalable du gestionnaire d'un parc national ou d'une réserve naturelle pour tous travaux entrepris dans la zone périphérique, nécessitant une étude d'impact environnemental.

\section{Article 77.}

Sans préjudice de l'application des dispositions du présent titre, l'Etat, l'Etablissement, les Fondations, les collectivités territoriales signataires d'un contrat de gestion de terroir et les associations dont l'objet spécifique est la défense de l'environnement et la protection de la nature peuvent se constituer partie civile à l'effet de demander réparation du préjudice subi du fait des actes commis par l'auteur de l'infraction.

\section{TITRE VI - DISPOSITIONS FINALES}

\section{Article 78.}

La présente loi relative à la création, à la gestion et au financement des parcs nationaux et réserves naturelles abroge toutes les dispositions antérieures contraires.

\section{Article 79.}

La présente loi sera publiée au Journal Officiel de la République de Côte d'Ivoire et exécutée comme loi de l'Etat.

Fait et adopté en séance publique

Abidjan, le 11 février 2002

\section{Laurent GBAGBO}

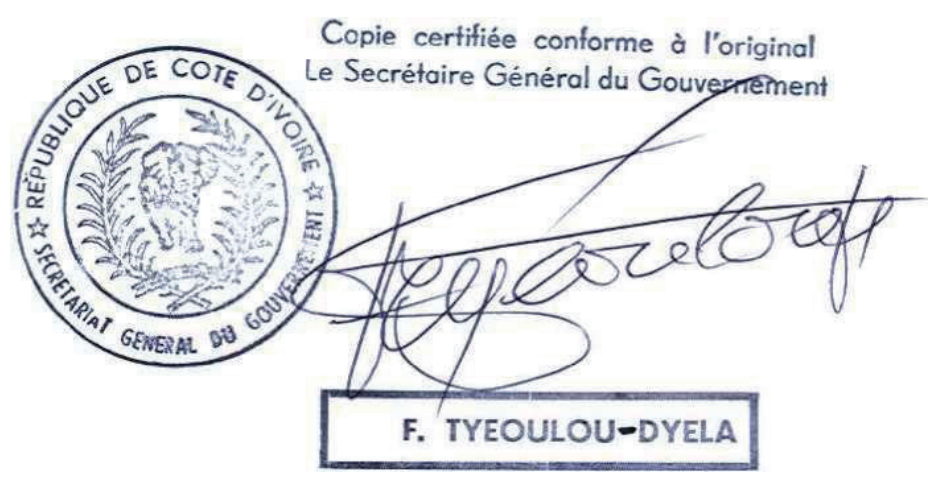


ANNEXE 1 : LISTE DES PARCS NATIONAUX

\begin{tabular}{|c|c|c|c|}
\hline Dénomination & Préfecture & Date et $\mathrm{N}^{\circ}$ Décret de création & $\begin{array}{l}\text { Superficie } \\
\text { (en ha) }\end{array}$ \\
\hline Parc National d'AZAGNY & Grand-Lahou & $\begin{array}{l}\text { Arrêté } n^{\circ} 536 \text { du } 25 / 06 / 1960 \text { puis } \\
\text { Décret } n^{\circ} 81-218 \text { du } 2 / 4 / 1981\end{array}$ & 19400 \\
\hline Parc National du BANCO & Abidjan & Décret du 31/10/1953 & 3000 \\
\hline Parc National de la COMOE & Bouna & Décret n ${ }^{\circ} 68-81 \mathrm{du}$ 09/02/1968 & 1149150 \\
\hline $\begin{array}{l}\text { Parc National des ÎLES } \\
\text { ÉHOTILE }\end{array}$ & Adiaké & Décret n $74-179$ du 25/04/1974 & 550 \\
\hline $\begin{array}{l}\text { Parc National de la } \\
\text { MARAHOUÉ }\end{array}$ & Bouaflé & Décret n ${ }^{\circ} 68-80$ du 09/02/1968 & 101000 \\
\hline $\begin{array}{l}\text { Parc National du Mont } \\
\text { PÉKO }\end{array}$ & Duékoué & Décret nº68-79 du 09/02/1968 & 34000 \\
\hline $\begin{array}{l}\text { Parc National du Mont } \\
\text { SANGBÉ }\end{array}$ & Biankouma & Décret nº 76-215 du 19/02/1976 & 95000 \\
\hline Parc National de TAI & Guiglo, Soubré & $\begin{array}{c}\text { Décret } \mathrm{n}^{\circ} 72-544 \mathrm{du} 28 / 08 / 72 \text { puis } \\
\mathrm{n}^{\circ} 77-348 \mathrm{du} 03 / 06 / 1977\end{array}$ & 330000 \\
\hline TOTAL & & & 1732100 \\
\hline
\end{tabular}

ANNEXE 2 : LISTE DES RÉSERVES NATURELLES

\begin{tabular}{|l|l|l|c|}
\hline \multicolumn{1}{|c|}{ Dénomination } & Préfecture & Date et $\mathbf{n}^{\circ}$ de Décret création & $\begin{array}{c}\text { Superficie } \\
\text { (en ha) }\end{array}$ \\
\hline $\begin{array}{l}\text { Réserve de faune } \\
\text { d'ABOKOUAMEKRO }\end{array}$ & Yamoussoukro & Décret n 93-695 du19/08/93 & 20430 \\
\hline $\begin{array}{l}\text { Réserve de faune du HAUT } \\
\text { BANDAMA }\end{array}$ & Katiola & Décret $n^{\circ} 73-133 \mathrm{du} 21 / 03 / 73$ & 123000 \\
\hline $\begin{array}{l}\text { Réserve scientifique de } \\
\text { LAMTO } \\
\text { Réserve intégrale du Mont } \\
\text { NIMBA }\end{array}$ & $\begin{array}{l}\text { Toumodi } \\
\text { Tiassalé }\end{array}$ & $\begin{array}{l}\text { Arrêté } \mathrm{n}^{\circ} \text { 857/AGRI/DOM } \\
\text { du } 12 / 07 / 1968\end{array}$ & 2500 \\
\hline $\begin{array}{l}\text { Réserve intégrale du Mont } \\
\text { NIMBA }\end{array}$ & Danané & Décret du 05/07/1944 & 5000 \\
\hline Réserve de faune du N'ZO & Taï & $\begin{array}{l}\text { Décret } n^{\circ} 72-545 \mathrm{du} 28 / 08 / 1972 \\
\text { puis } \mathrm{n}^{\circ} 73-132 \mathrm{du} 21 / 03 / 73\end{array}$ & 92700 \\
\hline $\begin{array}{l}\text { Zone périphérique de } \\
\text { Protection du Parc de TAI }\end{array}$ & Soubré & Décret $n^{\circ} 77-348 \mathrm{du} 03 / 06 / 77$ & 96000 \\
\hline TOTAL & & & $\mathbf{3 3 9 6 3 0}$ \\
\hline
\end{tabular}




\section{ANNEXES 6 :}

\section{DÉCRET No 2002-359 DU 24 JUILLET 2002, PORTANT CREATION, ORGANISATION ET FONCTIONNEMENT DE L'OFFICE IVOIRIEN DES PARCS ET RESERVES (JORCI n ${ }^{\circ} 40$ du 2 octobre 2003, p. 588)}

\section{Sur rapport conjoint du Ministre de l'environnement et du Ministre de I 'économie et des finances ;}

Vu la Constitution ;

Vu la loi n $2002-102$ du 11 février 2002 relative à la création, à la gestion et au financement des parcs nationaux et des réserves naturelles ;

Vu la loi n98-388 du 2 juillet 1998 fixant les règles générales relatives aux établissements publics nationaux et portant création de catégories d'établissements publics, et abrogeant la loi 80-1070 du 13 septembre 1980 ;

$\mathrm{Vu}$ le décret $\mathrm{n}^{\circ} 66-427 \mathrm{du} 15$ septembre 1966 portant répartition du produit net des amendes, confiscations, restitutions, dommages-intérêts, contraintes et transactions en matière de police forestière ;

Vu le décret $n^{\circ} 98-716$ du 16 décembre 1998 portant réforme des circuits et des procédures d'exécution des dépenses et des recettes du budget général de l'État, des comptes spéciaux du Trésor, et mise en œuvre du système intégré de gestion des finances publiques ;

Vu le décret $\mathrm{n}^{\circ} 2001-42$ du 24 janvier 2001 portant nomination des membres du Gouvernement tel que modifié par le décret $n^{\circ} 2002-116$ du 25 février 2002 ;

Vu le décret $n^{\circ}$ 2001-91 du 11 février 2001 portant attributions des membres du Gouvernement;

\section{LE CONSEIL DES MINISTRES ENTENDU,}

\section{D É C R È T E :}

\section{TITRE I. - DISPOSITIONS GENERALES}

\section{Article 1.}

Il est créé un établissement public de type particulier dénommé "Office Ivoirien des Parcs et Réserves » en abrégé O.I.P.R., ci-après dénommé dans le présent décret "l'Office". L'Office est régi par les dispositions législatives et réglementaires en vigueur.

\section{Article 2.}

Le siège de l'Office est fixé à Abidjan.

\section{Article 3.}

L'Office a pour mission, sur l'ensemble des parcs et réserves sur lesquels il exerce son autorité, notamment :

1. la gestion de la faune, de la flore, et de leur biotope qui en constitue le fondement ; 2.la gestion du patrimoine foncier qui constitue l'assise de la faune, la flore et les plans d'eau ;

2. la gestion du patrimoine foncier qui constitue l'assise de la faune, la flore et les plans d'eau ;

3. 1'exercice de la police administrative et judiciaire conformément à la loi $n^{\circ} 2002-102$ du 11 février 2002 sus visée ; 
4. la mise en œuvre d'une politique de gestion durable par la promotion des activités légalement permises en fonction de la nature juridique du parc ou de la réserve considérée et de sa zone périphérique ;

5. le cas échéant, la coordination ou la réalisation des études nécessaires à la création, à l'extension ou à l'aménagement d'un parc, d'une réserve ou de sa zone périphérique ;

6. l'information, l'éducation et la communication.

\section{Article 4.}

Pour l'exécution de ses missions définies à l'article 3 ci-dessus, l'Office est chargé de :

1. mettre en œuvre les orientations de la politique nationale en matière de conservation et de gestion durable des ressources des parcs et réserves ;

2. définir les modalités de conservation et d'utilisation rationnelle des ressources naturelles des parcs et réserves, dans le respect de leur diversité biologique (milieux naturels, faune et flore sauvages tant terrestres qu'aquatiques) ;

3. définir les conditions de préservation des paysages naturels et des éléments culturels qui leur sont attachés, en tenant compte de l'équilibre et de la stabilité des écosystèmes;

4. veiller avec l'appui des comités de gestion de chaque parc et réserve à l'élaboration, au développement et à la mise en œuvre des plans de développement durable des parcs et réserves ;

5. mettre en place les moyens de protection des habitats naturels et de la vie sauvage, notamment des espèces de faune et de flore rares ou en danger de disparition, dans les zones où se développent les activités de visite et de tourisme écologique ;

6.coordonner ses activités avec celles des institutions scientifiques, techniques et des associations de protection de la nature dont les programmes sont liés aux objectifs de la politique de conservation des parcs et réserves ;

7. planifier et mettre en œuvre la formation continue des personnels chargés de la gestion, de la protection des parcs et réserves et de leurs ressources en faune et en flore ;

8. négocier et suivre des contrats de terroir définissant les modalités de collaboration avec les populations riveraines des parcs pour la mise en œuvre de programmes d'aménagement de la zone périphérique ;

9.assurer la centralisation, le traitement et la diffusion des informations relatives aux aires protégées afin d'assurer un suivi national des indicateurs de conservation des parcs et réserves ;

10. promouvoir le cas échéant, l'information générale sur les parcs et réserves et la conservation de la diversité biologique.

11. assurer le développement touristique des parcs et réserves par la promotion du secteur.

\section{TITRE II - ORGANISATION ET FONCTIONNEMENT}

\section{Article 5.}

L'Office Ivoirien des Parcs et Réserves est composé des organes suivants : le Conseil de Gestion, la Direction générale et le Conseil Scientifique.

\section{Chapitre 1 : Le Conseil de Gestion}

\section{Article 6.}

L'Office est administré par un Conseil de gestion conformément à la loi nº 98-388 du 2 juillet 1998 sus visée.

Le Conseil de Gestion est investi des pouvoirs les plus étendus pour agir en toutes circonstances au nom de l'Office et sous réserve des attributions propres du Directeur Général et des règles de tutelle.

Le Conseil de Gestion, notamment : 
-vote le budget et arrête les comptes annuels ;

-approuve la nomination et la révocation des Directeurs des parcs et réserves proposées par le Directeur Général ;

-approuve les mesures de gestion des parcs et réserves proposées par le Directeur Général pour la conservation durable de l'écosystème naturel et, notamment, l'aménagement des parcs et réserves et leur ouverture au public ;

-approuve les règles de gestion du personnel élaborées par le Directeur Général ;

-approuve le règlement intérieur des parcs ou réserves proposés par le Directeur Général ;

-approuve le projet de convention de concession d'exploitation d'un parc ou d'une réserve partielle.

\section{Article 7.}

Le Conseil de Gestion est composé de douze membres nommés pour une durée de trois ans renouvelable une fois.

La composition du Conseil de Gestion doit permettre l'association de compétences diversifiées en matière, de conservation de la nature, de gestion administrative et financière et de représentation des intérêts professionnels, sectoriels et locaux. A cet effet, les membres du Conseil de Gestion peuvent être choisis indistinctement au sein et en dehors de l'administration publique.

\section{Article 8.}

Le Conseil de Gestion est composé comme suit :

-un Représentant du Ministère chargé des Parcs et Réserves, Président du Conseil de Gestion;

-un Représentant du Ministère chargé de l'Agriculture et des Ressources Animales ;

-un Représentant du Ministère chargé de l'Économie et des Finances ;

-un Représentant du Ministère chargé des Eaux et Forêts ;

- un Représentant du Ministère chargé de l'Enseignement Supérieur et de la Recherche Scientifique ;

-un Représentant du Ministère chargé du Tourisme et de l'Artisanat ;

-un Représentant du Ministère chargé de l'Intérieur, de la Décentralisation et de l'Administration

Territoriale ;

-un Représentant des Fondations ayant pour objet le financement des parcs et Réserves ;

-trois Représentants des populations des zones périphériques des parcs et réserves ;

-un Représentant des Organisations non Gouvernementales nationales en charge de la protection de la nature en Côte d'Ivoire ;

\section{Article 9.}

Les membres du Conseil de Gestion représentant un département ministériel sont nommés par arrêté des Ministres concernés. Les membres du Conseil de Gestion, choisis en dehors de l'administration afin d'assurer la représentation d'intérêts collectifs, sectoriels, financiers, locaux, ou techniques, sont désignés par les instances compétentes des organismes qu'ils représentent.

\section{Article 10.}

Le Conseil de Gestion se réunit en session ordinaire, au moins une fois par trimestre sur convocation du Président. Il délibère sur le rapport d'activités et financier de l'Office, sur les objectifs et prévissions d'activités et sur les adaptations qui paraissent nécessaires. Il peut se réunir en sessions extraordinaires autant de fois que de besoin.

Le Conseil de Gestion peut inviter aux réunions du Conseil, avec voix consultative, toute personne dont il estime utile d'entendre les avis. Le Directeur Général assiste aux réunions du Conseil et en assure le secrétariat.

L'Agent Comptable de l'Office, le Contrôleur Budgétaire ainsi que les Commissaires aux comptes participent, avec voix consultative, aux réunions du Conseil de Gestion. Le Conseil de Gestion ne 
peut délibérer que si la majorité de ses membres est présente ou représentée. Il est tenu une feuille de présence qui est émargée par chaque membre du Conseil.

Tout membre absent à la réunion du Conseil de Gestion est tenu de se faire représenter par un autre membre du Conseil qui ne peut disposer que d'un seul pouvoir.

Les décisions du Conseil de Gestion sont adoptées à la majorité des voix des membres présents ou représentés. En cas de partage des voix, la voix du président est prépondérante.

Les délibérations du Conseil de Gestion sont constatées par des procès-verbaux, réunis en un registre spécial, signés par le président et deux membres du Conseil.

\section{Chapitre 2 : la Direction Générale}

\section{Article 11.}

La Direction Générale comporte :

- une Direction Technique ;

-une Direction de l'Administration et des Ressources Humaines;

-une Direction des Finances et de la Comptabilité;

-deux cellules techniques autonomes.

\section{SECTION 1. LE DIRECTEUR GÉNÉRAL}

\section{Article 12.}

L'Office est dirigé par un Directeur nommé par décret, sur proposition du Ministre de tutelle technique et après avis du Conseil de Gestion. Il a rang de Directeur Général d'Administration Centrale.

Le Directeur Général de l'Office en est l'ordonnateur principal. Il autorise les recherches scientifiques dans les parcs et réserves après avis conforme du conseil scientifique ainsi que toute forme de capture de la faune, de destruction ou de collection de la flore, de récolte de plantes, fruits ou produits sur toute l'étendue des parcs et réserves partielles, dans les conditions fixées par décret.

Le Directeur Général établit obligatoirement, chaque année, un rapport détaillé et quantifié sur l'état de conservation de chacun des parcs et réserves gérés par l'Office.

Les conventions d'exploitation et les contrats de terroir sont signés par le Directeur Général de l'Office, après avis du Conseil de Gestion, sur proposition d'un Directeur de parc ou d'une réserve. Il propose à l'approbation du Conseil de Gestion les règles générales de l'exploitation touristique des parcs et réserves partielles après consultation des acteurs du secteur concerné.

Il est habilité à signer, dans des conditions définies par le Conseil de Gestion et le cas échéant, après consultation des Directeurs de parcs ou de réserves concernés, des contrats de conventions particulières.

Après avis conforme du Conseil scientifique, le Directeur Général :

- transmet au Ministre de tutelle technique les propositions de délimitation de la zone périphérique d'un parc ou d'une réserve sur la base des négociations avec les entités ou les communautés concernées et des indications du Directeur du parc ou de la réserve concernée et des partenaires au développement;

-propose au Ministre de tutelle technique, la classification en réserve naturelle partielle et fixe les conditions d'exploitation, d'aménagement et de gestion de ladite réserve ;

-autorise les activités à l'intérieur de la réserve ayant une incidence sur la conservation durable de l'écosystème ainsi que toute recherche scientifique entreprise dans la réserve ;

-soumet au Ministre de tutelle technique des propositions de modification des listes d'espèces protégées de faune et de flore terrestres et aquatiques dans les parcs nationaux, les réserves naturelles et les zones périphériques;

-autorise le survol des parcs et réserves à une altitude inférieure à 200 mètres dans le cadre d'activités de recherche scientifique nonobstant la réglementation de l'aviation civile. 


\section{SECTION 2 : LE DIRECTEUR TECHNIQUE}

\section{Article 13.}

Le Directeur Technique est chargé de la programmation, de la coordination et du suivi des activités techniques et de recherche au sein de l'Office. Il est chargé notamment de :

- constituer la base de données géographiques de l'Office et de coordonner l'ensemble des actions à mener en matière de système d'information géographique (SIG) ;

-concevoir des méthodes efficaces de suivi écologique ;

-superviser et coordonner les activités de suivi écologique de l'ensemble de l'Office ;

-élaborer le programme annuel d'aménagement adapté aux objectifs de conservation et de protection de la diversité biologique;

-concevoir des stratégies de surveillance en prenant en compte les données de terrain de chaque parc et réserve ;

-établir le canevas de programme annuel d'activités pour les structures de terrain en matière d'écotourisme;

-assurer la promotion de l'éco-tourisme ;

-élaborer un plan directeur annuel d'aménagement de la zone périphérique des parcs.

Le directeur technique est nommé par le Directeur Général après avis du Conseil de Gestion. Il a rang de Directeur de l'Administration centrale.

\section{Article 14.}

La Direction Technique comporte :

-la cellule d'appui technique;

-la cellule d'appui à l'écodéveloppement La cellule est équivalente à la Sous-Direction dans l'Administration centrale.

Les Sous-Directeurs sont nommés par le Directeur général.

\section{SECTION 3 : LE DIRECTEUR FINANCIER ET DE LA COMPTABILITÉ}

\section{Article 15.}

Le Directeur Financier et de la comptabilité est chargé de :

- mettre en place les outils de comptabilité générale et analytique de l'établissement ; -assurer le suivi de la trésorerie de l'Office, ainsi que les opérations financières à moyen et long terme; -assurer la vérification et le contrôle de l'ensemble des pièces comptables de l'Office ; -établir les comptes annuels cumulés de l'Office ; -établir et de suivre les demandes de retrait de fonds ; -tenir les différents journaux comptable des projets ; -élaborer le bilan financier et comptable consolidé de l'établissement en fin d'année ; -élaborer le plan de trésorerie de l'Office.

Le Directeur Financier est nommé par le Directeur Général après avis du Conseil de Gestion. Il a rang de Directeur d'Administration centrale.

\section{Article 16.}

La Direction Financière comprend la cellule de comptabilité. Le responsable de la cellule est nommé par le Directeur Général. Il a rang de sous-directeur de l'Administration Centrale.

\section{SECTION 4 : LE DIRECTEUR DE L'ADMINISTRATION ET DES RESSOURCES HUMAINES}

\section{Article 17.}

Le Directeur de l'Administration et des Ressources Humaines est chargé de : - définir la politique de gestion des ressources humaines de l'établissement ; -évaluation périodique des performances du personnel de l'Office : -élaborer et mettre en œuvre le plan de formation pour le personnel de l'Office ; 
-concevoir, de mettre en place et de contrôler les procédures administratives de gestion des Ressources Humaines ;

-définir et suivre la politique médicale (prévention, hygiène et sécurité au travail) ;

-définir les moyens matériels et humains nécessaires à l'atteinte des objectifs de l'Office ;

-veiller au respect de la discipline, des règlements et procédures administratives et de proposer des sanctions.

Le Directeur de l'Administration et des Ressources Humaines est nommé par le Directeur Général après avis du Conseil de Gestion. Il a rang de Directeur de l'Administration Centrale.

\section{$\underline{\text { Article } 18 .}$}

La Direction de l'Administration et des Ressources Humaines comprend : -la cellule d'appui à la logistique ; -la cellule d'appui aux ressources humaines. Les responsables de ces cellules sont nommés par le Directeur Général. Ils ont rang de Sous- directeur de l'Administration Centrale.

\section{SECTION 5 : LES CHARGES DU CONTRÔLE ET DE LA PLANIFICATION}

\section{$\underline{\text { Article } 19 .}$}

La cellule du Contrôle et de la Planification est chargée de : -planifier l'ensemble des activités de l'Office ;

-rédiger les rapports périodiques et annuels ; -mettre en place les outils nécessaires à un suivi optimal des programmes ;

-évaluer l'état d'avancement des différents projets ;

-formaliser les procédures administratives, commerciales et comptables et de veiller à leur application;

-procéder à la révision des comptes ; -préparer et suivre l'exécution du budget.

\section{Article 20.}

Cette cellule est composée de :

-l'audit interne ;

-le contrôle de gestion

et -la planification.

Le responsable de la cellule est nommé par le Directeur Général de l'Office. Il a rang de sousdirecteur d'Administration centrale.

\section{SECTION 6: LA CELLULE DU DÉVELOPPEMENT INFORMATIQUE}

\section{Article 21.}

La cellule du développement informatique est chargée de : -assurer la gestion des ressources informatiques de l'Office ;

-conduire les études et projets de développement informatique ; -assurer la maintenance des équipements informatiques ;

-élaborer le plan directeur informatique de l'Office et de suivre sa mise en œuvre ;

-assurer la maintenance des applications (paie, approvisionnement, gestion des ressources humaines, gestion budgétaire etc...);

Le responsable de cette cellule est nommé par le Directeur Général de l'Office. Le coordonnateur de la cellule a rang de sous-directeur d'administration centrale. 


\section{Chapitre 3 : Le Conseil Scientifique}

\section{Article 22.}

Le Conseil Scientifique des parcs et réserves est un organe consultatif de l'Office. Il est constitué de personnalités issues du milieu scientifique et de la recherche ; de nationalité ivoirienne ou étrangère, choisies pour leur compétence et leur expérience ainsi que leur complémentarité en matière de conservation de la nature.

\section{Article 23.}

La composition, les attributions et le fonctionnement du Conseil Scientifique sont définis par arrêté du Ministre chargé des parcs et réserves. Les membres du Conseil scientifique sont nommés par arrêté conjoint du Ministre chargé de la recherche scientifique et du Ministre chargé des parcs et réserves.

\section{Article 24.}

L'avis du Conseil Scientifique est requis pour toutes les questions relatives à la gestion des parcs et réserves conformément à l'article 37 de la loi n²002-102 du 11 février 2002 sus visée.

\section{TITRE III - DISPOSITIONS FINANCIERES ET COMPTABLES SECTION 1 : LE BUDGET}

\section{Article 25.}

Les ressources et les dépenses de l'Office sont celles définies respectivement aux articles 27 et 28 de la loi $\mathrm{n}^{\circ} 2002-102$ du 11 février 2002.

Les ressources et dépenses d'origine publique sont ordonnancées et payées sous forme de subventions ou de dotations conformément aux dispositions applicables aux établissements publics nationaux.

Les ressources d'origine privée sont gérées conformément aux règles de la comptabilité privée, par l'ordonnateur principal et chaque ordonnateur délégué sous l'autorité du Directeur Général et le contrôle du Conseil de Gestion. Elles peuvent être placées, sur autorisation du Conseil de Gestion, sur des comptes ouverts dans des banques commerciales.

Chaque catégorie de ressources est affectée au financement de catégories de dépenses également identifiées dans le budget de l'Office et dans chacun des budgets secondaires.

\section{Article 26.}

26.1 Le projet de budget général annuel de l'Office est élaboré par le Directeur Général à partir des projets de budgets secondaires établis par chaque parc et réserve ainsi que par le Conseil scientifique. Il est arrêté par le Conseil de Gestion, conformément aux dispositions législatives et réglementaires applicables aux établissements publics nationaux. Le budget général de l'Office, ainsi que chaque budget secondaire, distingue obligatoirement les ressources d'origine publique de celles d'origine privée et l'affectation des financements en résultant.

26.2 La partie du budget général de l'Office et celle de chacun de ses budgets secondaires dont les recettes sont constituées par des deniers publics sont approuvées conformément aux dispositions législatives et réglementaires applicables en la matière aux établissements publics nationaux.

26.3 La partie du budget général de l'Office et celle de chacun de ses budgets secondaires dont les recettes sont constituées par des ressources d'origine privée sont arrêtées par le Conseil de Gestion en concertation avec les Fondations qui concourent à son financement. A la demande des Fondations et des bailleurs de fonds privés, il est obligatoirement fourni par l'Office, les justificatifs des dépenses afférentes aux projets dont ils ont totalement ou partiellement assuré le financement.

\section{Article 27.}

27.1. Les budgets secondaires incluent obligatoirement toutes les dépenses de fonctionnement et d'investissement du parc ou de la réserve considérée ainsi que celles du Conseil scientifique. 
27.2. Le projet de budget secondaire de chaque parc national et de chaque réserve naturelle est établi par son Directeur et soumis à l'avis préalable du comité de gestion locale du parc ou de la réserve concernée, mentionné à l'article 38 ci-dessous, avant transmission au Directeur Général de l'Office pour intégration dans le projet de budget général de l'Office. L'avis du comité de gestion locale est joint au projet de budget lors de son examen par le Conseil de Gestion.

27.3. Le budget secondaire de chaque parc et de chaque réserve est ordonnancé par son Directeur qui a la qualité d'ordonnateur délégué. Les ressources financières sont mises à la disposition de l'Agent Comptable Secondaire de chaque parc et réserve selon un calendrier, fixé par le Conseil de Gestion de l'Office, dont l'exécution s'impose au Directeur Général et à l'Agent Comptable Principal.

27.4. Par dérogation à l'article 27.3 ci-dessus, les Directeurs de parc (s) ou réserve (s) peuvent être ordonnateurs principaux des fonds d'origine privée mis à la disposition de l'Office dans le cadre des conventions particulières visées à l'article 12 ci-dessus. 27.5Le Conseil de Gestion veille à la réalité et à la sincérité des budgets secondaires.

\section{Article 28.}

28.1 Les ressources d'origine publique sont affectées, au financement :

-des indemnités et primes des agents en service dans les parcs et réserves ; -des dépenses en équipement des personnels en service dans les parcs et réserves ;

-des investissements inscrits au budget de l'État ; -des travaux d'équipement et d'aménagement des parcs et réserves ;

-des dépenses de fonctionnement de l'Office ;

28.2 Les ressources d'origine privée provenant des Fondations sont affectées, notamment:

-au financement d'indemnités et de primes des agents en fonction dans les parcs et réserves qui ne sont pas déjà prises en charge par le budget mentionné à l'article 26.2 ci-dessus ou par un autre budget de l'État.;

-à la rémunération des prestations effectuées par les populations rurales et au financement des obligations des parcs et réserves au titre des contrats de terroirs ;

-aux dépenses d'investissement qui ne sont pas financées par les ressources d'origine publique ; -aux dépenses de fonctionnement de l'Office qui ne sont pas financées par les ressources d'origine publique.

28. 3 Le projet de budget de l'Office est soumis par le Directeur Général à l'examen du Conseil de Gestion dans un délai permettant son approbation conformément aux dispositions de l'article 20 de la loi n⿳908-388 du 2 juillet 1998. A cet effet, le Directeur Général transmet au Conseil de Gestion le projet de budget au moins 15 jours avant la date de la réunion du Conseil de Gestion pour approbation.

\section{Article 29.}

Les comptes financiers annuels de chaque parc et réserve et du Conseil scientifique sont centralisés et consolidés dans le compte financier de l'Office par le Directeur Général. Le Directeur Général rend compte de l'utilisation du budget et de chaque budget secondaire au Conseil de Gestion.

Le Directeur Général de l'Office adresse aux Fondations qui ont concouru au financement des parcs et réserves, un rapport financier annuel. Le rapport financier doit expressément préciser, pour chaque projet financé sur les fonds de Fondation, l'utilisation des fonds qui lui ont été transférés à cet effet et les résultats obtenus.

\section{SECTION 2 - LES MESURES SOCIALES}

\section{Article 30.}

30. 1 L'ensemble du personnel de l'Office peut bénéficier de primes et d'indemnités dont les modalités et les montants sont fixés par arrêtés conjoint du Ministre chargé des parcs et réserves et du Ministre chargé de l'Économie et des Finances

30. 2 Il bénéficie d'un régime de protection et d'assurance sociale dans des conditions approuvées par le Conseil de Gestion. 


\section{TITRE IV. - DISPOSITIONS PARTICULIÈRES À CHACUN DES PARCS ET RESERVES}

\section{Chapitre 1. Le Directeur des Parcs ou Réserves}

\section{Article 31.}

Chaque parc et réserve ou groupement de parcs et réserves est dirigé par un Directeur. Il est nommé par le Directeur Général de l'Office après avis du Conseil de Gestion. Il a rang de Directeur d'Administration centrale. Sans préjudice des dispositions de l'article 27.4 ci-dessus, il est ordonnateur délégué du budget de ce parc ou cette réserve. Il coordonne les activités des Cellules et des Secteurs dont les responsables nommés par le Directeur Général ont rang de sous-directeur d'Administration centrale

\section{Article 32.}

Le Directeur du parc, de la réserve met en œuvre toutes les mesures nécessaires pour faire observer les interdictions prévues par la loi. A cet effet, le Directeur du parc ou de la réserve fait ordonner, par application des articles 10,11, 12 et 15 de la loi n 2002-102 du 11 février 2002, l'arrachage des plantations ainsi que la destruction des installations et impenses privées, non autorisées, à l'intérieur des limites du parc ou de la réserve considérées.

Le Directeur du parc ou de la réserve :

-élabore le règlement intérieur du parc ou de la réserve dont il assure la gestion et le soumet, préalablement à son entrée en vigueur, au Directeur Général pour avis ;

-fixe les conditions et modalités de circulation, d'hébergement et, le cas échéant, de survol au-delà de 200 mètres d'altitude nonobstant la réglementation de l'aviation civile, dans le cadre des activités de gestion ou de récréation ou d'éducation ;

-surveille toute l'étendue du parc ou de la réserve, dans des conditions fixées par décret, de toute forme de capture de la faune, de destruction ou de collection de la flore, de récolte de plantes, fruits ou produits ; -soumet à la direction générale, les règles générales de l'exploitation touristique du parc ou de la réserve dont il a la charge.

Le Directeur d'un parc, d'une réserve, ou d'un groupement de parcs et réserves :

-assure la préparation et l'exécution du ou des plan (s) d'aménagement et de gestion des parcs ou réserves dont il a la charge ;

- négocie les contrats de terroir ; -met en œuvre la Stratégie de gestion des populations infiltrées dans les parcs ou réserves ; - sélectionne les concessionnaires et suit les contrats de concession.

\section{Article 33.}

Le Directeur du parc ou de la réserve établit chaque année, en même temps que l'état d'exécution de son budget, un rapport détaillé et quantifié sur l'état de conservation du parc ou de la réserve qu'il gère.

\section{Article 34.}

Le Directeur Général de l'Office et les Directeurs des parcs et des réserves de l'Office constituent un comité de direction dont le fonctionnement est défini par un arrêté du Ministre de tutelle technique.

\section{Chapitre 2. Le Comité de Gestion locale du Parc ou de la Réserve}

\section{Article 35.}

Chaque parc et chaque réserve sont dotés d'un Comité de Gestion locale présidé par le Préfet de Département ou de Région dont dépend la plus grande superficie du parc et de la réserve. Le secrétariat du Comité de Gestion est assuré par le Directeur du parc ou de la réserve.

\section{Article 36.}


La composition et les modalités de fonctionnement de chaque Comité de Gestion locale sont fixées par arrêté conjoint du Ministre chargé des Parcs et Réserves et du Ministre chargé de l'Intérieur, de la Décentralisation et des Collectivités Territoriales, dans la limite de 15 membres au maximum dont notamment:

-les Préfets des Départements ou des Régions concernées ; -le Directeur du parc ou de la réserve ;

-deux représentants élus des organisations non gouvernementales intervenant dans le parc ou la réserve concernée, ou dans sa zone périphérique de protection;

-les représentants des collectivités territoriales et des terroirs constituant la zone périphérique de protection (Présidents des Conseils Régionaux, Présidents des Conseils Généraux, Représentants des Organisations Villageoises ...);

-deux représentants des opérateurs économiques dans le secteur du tourisme et de l'artisanat;

-le Directeur Général de l'Office ou son représentant ; -un membre du Conseil Scientifique.

\section{Article 37.}

Les membres du comité de gestion locale sont choisis selon les modalités définies par les organisations qu'ils représentent.

\section{Article 38.}

Le comité de gestion locale se réunit au moins une fois par trimestre. Le comité de gestion locale donne son avis sur toutes les questions d'ordre technique, culturel ou budgétaires qui lui sont soumises, à titre consultatif, par le Directeur du parc ou de la réserve considérée. Les avis du comité de gestion locale sont joints aux rapports transmis par le Directeur du parc ou de la réserve concerné au Directeur Général de l’Office.

\section{TITRE V. - LA TUTELLE ET LE CONTROLE}

\section{Article 39.}

L'Office est placé sous la tutelle économique et financière du Ministre chargé de l'Économie et des Finances et sous la tutelle technique du Ministre chargé des Parcs et Réserves.

\section{Article 40.}

La vérification des comptes de l'Office est effectuée par la Cour des Comptes dans les conditions prévues par les dispositions législatives et réglementaires en vigueur.

\section{Article 41.}

Outre les contrôles et vérifications de la Cour des Comptes et des Services spécialisés de l'État, le contrôle de l'utilisation des ressources d'origine privée est effectué par deux commissaires aux comptes nommés par arrêté du Ministre chargé de l'Économie et des Finances.

Les commissaires aux comptes ont l'obligation de saisir le Ministre de l'économie et des finances par un rapport circonstancié et motivé chaque fois qu'ils constatent la survenance d'une difficulté résultant, notamment, de l'inobservation d'une disposition législative ou réglementaire susceptible d'affecter le fonctionnement normal de l'Office et de compromettre la réalisation de ses missions. Ce rapport doit également indiquer, sous forme de recommandation, les solutions d'ordre comptable qui pourraient être prises pour pallier les conséquences des irrégularités constatées.

Le rapport mentionné à l'alinéa précédent doit obligatoirement être adressé au Ministre chargé de l'Économie et des Finances dans le mois qui suit la survenance des irrégularités, si l'un ou l'autre des commissaires aux comptes en est informé à cette période, ou à défaut, dans le mois qui suit sa constatation, lors des diligences effectuées postérieurement. Le premier des commissaires aux comptes qui constate les irrégularités en informe le second. Le rapport des commissaires aux comptes, prévu au présent article, peut être conjoint ou séparé.

Dans le mois qui suit la transmission du rapport mentionné à l'alinéa ci-dessus, et après avoir reçu, si nécessaire, l'avis du Conseil de Gestion ou du Directeur Général, le Ministre chargé de l'Économie et des Finances fait toute recommandation et donne toute instruction utile au Conseil de Gestion, au 
Directeur Général ou aux commissaires aux comptes, selon le cas, pour pallier les conséquences des irrégularités constatées.

\section{TITRE VI. - RÉPARTITION DU PRODUIT DES AMENDES}

\section{Article 42.}

La répartition du produit net des amendes, confiscations, restitutions, dommages et intérêts, contraintes et transactions sera fixée par arrêté conjoint du Ministre chargé de l'Économie et Finances et du Ministre chargé des Parcs et Réserves.

\section{TITRE VII. - DISPOSITIONS FINALES}

\section{Article 43.}

Le Ministre de l'Environnement et le Ministre de l'Économie et des Finances sont chargés, chacun en ce qui le concerne, de l'exécution du présent décret qui abroge toute disposition contraire et qui sera publié au Journal Officiel de la République de Côte d'Ivoire.

Fait à Abidjan, le 24 juillet 2002

Laurent GBAGBO 


\section{ANNEXE 7 :}

\section{ADDENDUM RELATIF À LA VALORISATION DE LA THÈSE}

La valorisation des travaux de recherches universitaires revêt un caractère d'importance capitale pour le développement des États en ce sens que ceux-ci traitent, bien souvent, des problèmes de la société. L'université de Maastricht fait sienne cette position. C'est à juste titre que le candidat au Doctorat à l'obligation de faire figurer dans sa thèse, un addendum relatif à la valorisation de celle-ci (art. $23 \S 5$ du règlement des thèses de l'université de Maastricht -notre traduction ${ }^{1061}$ ). Le présent addendum exposera l'intérêt des résultats de cette thèse (I) ainsi que les modalités de sa valorisation (II).

\section{I- Intérêts des résultats de la thèse}

Les résultats de cette thèse "Le droit des aires protégées en Côte d'Ivoire" ont un intérêt scientifique, économique et social que nous présenterons successivement.

Les aires protégées de la République de Côte d'Ivoire subissent dans l'ensemble des pressions anthropiques, depuis de longues années ${ }^{1062}$. Celles-ci sont, en bien des cas, de nature à compromettre l'existence de certains de ces milieux naturels ${ }^{1063}$. La question est d'autant plus préoccupante que malgré l'évolution des règles qui forment le droit des aires protégées en Côte d'Ivoire, les interdictions des droits d'usage qu'elles prescrivent ne sont pas observées par les populations alors qu'il est indispensable qu'elles le soient. À l'analyse, le droit des aires protégées n'assure pas sa fonction de protection de ces espaces naturels. Plusieurs hypothèses sont donc envisageables pour expliquer cette situation. L'inobservation $\mathrm{du}$ droit des aires protégées par les populations pourrait résulter d'insuffisances dudit droit et/ou émaner de facteurs externes. Certes, les problèmes de conservation des aires protégées de Côte d'Ivoire ont fait l'objet de plusieurs études. Cependant, rares sont celles qui analysent principalement le droit des aires protégées afin de savoir les raisons pour lesquelles il peine à remplir sa fonction de conservation de ces milieux naturels. C'est ce doute que cette thèse s'est employée à dissiper avec l'objectif final de proposer des solutions pour la conservation durable des aires protégées de Côte d'Ivoire.

La présente thèse a démontré, à travers une approche comparative faite avec les expériences de gestion d'aires protégées de pays qui ont plus de succès en la matière, que le

\footnotetext{
${ }^{1061}$ Originellement, le titre de ce document (en anglais) est "Regulation governing attainment of doctoral degrees. Maastricht University 2013 ".

${ }^{1062}$ Voir UICN/BRAO, Évaluation de l'efficacité de la gestion des aires protégées : Parcs et Réserves de Côte d'Ivoire, 2008, pp. 5 et 20-27; République de Côte d'Ivoire, Ministère de l'Environnement et des Eaux et Forêts/OIPR, État de conservation des Parcs et Réserves de Côte d'Ivoire, Abidjan, 2009, pp. 6-9 ; AKINDÈS (Francis), KOUAMÉ (Kra Walter), SÉDIA (Gisèle) et SÉZAN (Jules), Réalisation d'une étude sur l'état de la biodiversité des parcs nationaux et réserves de Côte d'Ivoire. Aspects socio-économiques (Rapport provisoire), Abidjan, août 2012, pp. 91-92.

${ }^{1063}$ Voir AKINDĖS (Francis), KOUAMÉ (Kra Walter), SÉDIA (Gisèle) et SÉZAN (Jules), Réalisation d'une étude sur l'état de la biodiversité des parcs nationaux et réserves de Côte d'Ivoire. Aspects socio-économiques, op. cit., pp. 28 et 89-90.
} 
droit ivoirien des aires protégées assure une protection juridique adéquate à ces milieux naturels. En effet, d'une part, les règles domaniales destinées à la protection de l'intégrité de ces milieux naturels ont été affermies. Plus explicitement, depuis 2002, les aires protégées appartiennent au domaine public de l'État ${ }^{1064}$ et sont, par conséquent, inaliénables et imprescriptibles. Aussi les dispositions pénales qui leur sont applicables ont-elles été renforcées. D'autre part, les règles juridiques de gestion des aires protégées ont été renforcées. Il s'agit du renforcement des moyens juridiques de l'Administration des aires protégées par la délégation de la gestion de ces milieux naturels à un établissement publique national et de l'institution de mécanismes de financement extra-étatiques pour leur gestion. En outre, ces règles de gestion instaurent un cadre participatif de gestion des aires protégées au niveau local. Toutes ces réformes dont le droit des aires protégées a fait l'objet, depuis 2002 par l'œuvre de la loi précitée, ont produit des résultats satisfaisants pour la conservation de la nature dans les pays anglophones d'Afrique de l'Est et d'Afrique australe.

En conséquence, les problèmes de conservation des aires protégées ivoiriennes procèdent de facteurs externes audit droit. Des enquêtes de terrain effectuées dans le cadre de cette thèse révèlent qu'il s'agit essentiellement de problèmes pratiques de gestion; lesquelles expliquent le défaut d'effectivité du droit des aires protégées. Premièrement, l'étude démontre que l'agriculture demeure l'activité principale des communautés locales. Or, les techniques agricoles utilisées (agriculture itinérante sur brulis) appauvrissent le sol et détruisent considérablement la biodiversité des zones périphériques, dans un contexte de démographie galopante et d'économie de rente. Il s'ensuit un manque de ressources naturelles dans les terroirs villageois pour la satisfaction des besoins des communautés ; qui malheureusement en sont dépendantes. Subséquemment, elles exploitent les aires protégées à des fins agricoles et aussi leur biodiversité.

Cette situation est exacerbée par l'insuffisance des moyens infrastructurels, financiers et des équipements dont disposent les gestionnaires des aires protégées. Il en est de même pour le laxisme des autorités ivoiriennes dans le règlement des problèmes d'occupations des aires protégées par les communautés locales consécutifs à des conflits fonciers. En outre, il y a la crise militaro-politique ivoirienne survenue en Côte d'Ivoire en septembre 2002 et qui a duré environ une décennie. Elle a perturbé le dispositif de surveillance de certains de ces espaces naturels. C'est ainsi qu'ils ont été envahis par des migrants économiques venus, pour la plupart, de la sous-région ouest-africaine. Enfin, les retards dans la prise des textes réglementaires de la loi précitée de 2002 sur les Parcs Nationaux et Réserves naturelles ont considérablement empêché la mise en œuvre du droit des aires protégées et partant entravé la gestion durable des aires protégées. De plus, les retards dans la mise en place et le fonctionnement des instruments et organes de gestion prévus par ladite loi ont eu pour inconvénients de produire le même résultat.

Ces difficultés de mise en œuvre du droit des aires protégées identifiées, l'étude a suggéré des solutions en vue d'inverser la tendance. Premièrement, elle propose d'intégrer

\footnotetext{
${ }^{1064}$ Voir les articles 7 et 13 de la loi n²002-102 du 11 février 2002 relative à la création, à la gestion et au financement des Parcs Nationaux et Réserves naturelles (JORCI n²0 du 16 mai 2002, p. 338).
} 
conservation des aires protégées et développement des communautés locales, à travers une politique globale de conservation et de développement autour des aires protégées. Cette politique sera exécutée par un comité interministériel à mettre en place, à savoir le Comité Interministériel de Coordination des Actions de Développement dans les Zones Périphériques d'aires protégées (CICAD-ZP). La mission du CICAD-ZP sera de coordonner les actions de développement autour de celles de la conservation des aires protégées. Concrètement, il s'agira de poursuivre la conservation des aires protégées mais en développant corrélativement, cette fois-ci, une agriculture durable dans leurs zones périphériques. L'agriculture durable s'entend, en l'espèce, d'une agriculture à forte rentabilité économique et qui protège l'environnement. D'une part, elle sous-entend l'adoption de nouvelles techniques culturales qui augmentent les rendements à l'hectare et protègent la fertilité des sols (l'agroforesterie par exemple). D'autre part, elle implique la mise en place d'une stratégie d'achat des cultures aux producteurs à un meilleur prix. De plus, les capacités des communautés locales devront être renforcées dans le sens de l'utilisation et la conservation durable des ressources naturelles de leurs terroirs. La mise en œuvre de cet ensemble de mesures permettrait de réduire les pressions que les communautés locales exercent sur les aires protégées, au mépris de la loi.

Deuxièmement, la thèse recommande la valorisation des aires protégées et des atouts touristiques de leurs zones périphériques comme une alternative à l'insuffisance des financements destinés à la conservation de ces forêts et du développement local. D'autres modalités telles que la mise en place de projets communautaires et leur gestion sont formulées à cette fin. Enfin, l'étude recommande aux pouvoirs publics ivoiriens de régler de manière durable et définitive les conflits fonciers relatifs aux aires protégées par la recherche de solutions consensuelles avec les communautés locales. Elle recommande également aux autorités ivoiriennes de démonter une volonté ferme d'apurement (progressif ou systématique) des aires protégées de leurs occupants illégaux.

Il se déduit, de tout ce qui précède, que les résultats de cette thèse ont un intérêt scientifique. En outre, leur intérêt économique convient d'être exposé.

L'intérêt économique des résultats de cette étude réside en ce qu'ils proposent des modalités pour la valorisation touristiques des aires protégées et de leurs zones périphériques susceptibles de mobiliser des financements importants. Ainsi, les problèmes d'insuffisances de financement pour la conservation des aires protégées seront résorbés et des actions de développement économique pourraient être entreprises en faveur des communautés locales. De plus, les propositions formulées encouragent, orientent les pouvoirs publics et les communautés locales d'ordinaire agricultrices quant à la mise en place de projets communautaires. Ces projets à vocation de conservation et exploitables sur le plan écotouristique sont petites entreprises communautaires capables de générer des revenus importants aux communautés. Enfin, la Côte d'Ivoire est un pays dont l'économie repose sur l'agriculture. Cette agriculture est essentiellement pluviale. Les suggestions de l'étude relatives à la conservation et à l'utilisation durable des ressources naturelles y compris des forêts sont de nature à garantir les conditions bioclimatiques (pluies, humidité...) 
indispensables pour sa pratique. La mise en œuvre de ces différentes propositions permettra à l'économie ivoirienne essentiellement agricole d'être toujours compétitive.

En ce qui concerne l'intérêt social des résultats de cette étude, il peut être appréhendé à divers égards. D'une part, les recommandations de cette étude qui exhortent à intégrer conservation des aires protégées et développement local ont pour objectif final de réduire les conflits entre gestionnaires et communautés locales. D'autre part, les propositions relatives à la gestion des projets communautaires visent à développer au sein des communautés des capacités de gestion d'intérêts collectifs. Les responsabilités qui en résultent exigent des communautés, la recherche du consensus dans les prises de décisions, la transparence dans la gestion et la reddition des comptes. Cette manière de procéder contribue à fraterniser, à consolider les rapports sociaux et à développer les capacités d'organisation sociale des communautés locales.

Aussi comment envisageons-nous la valorisation des résultats de cette thèse?

\section{II- Modalités de la valorisation des résultats de la thèse}

Outre la communauté universitaire, cette thèse présente un intérêt pour plusieurs acteurs de droit interne et international. Il s'agit notamment de l'État ivoirien, en général, et particulièrement du ministère en charge des aires protégées et de l'Office Ivoirien des Parcs et Réserves (OIPR), des ministères en charge des Eaux et Forêts, du Tourisme et de l'Artisanat, de l'Emploi, du Plan et du Développement. Elle intéresse également les ONG, les cabinets d'études nationaux et internationaux; les institutions techniques et de développement ainsi que les organisations internationales et régionales environnementales.

Cette thèse apporte des réponses concrètes et innovantes aux problèmes d'effectivité de l'application du droit des aires protégées et partant de leur conservation durable. En effet, c'est pour la première que dans la recherche de solutions pour la conservation des aires protégées ivoiriennes, une étude analyse le droit des aires protégées au soutien d'enquêtes de terrain, afin de savoir s'il accorde une protection juridique suffisante à ces milieux naturels. De plus, des études précédentes ne se limitent qu'à proposer d'intégrer conservation des aires protégées et développement local ainsi que la valorisation des aires protégées pour leur conservation durable, sans en préciser les modalités. Toutefois, tout en reconduisant ces propositions, cette étude en fait d'autres et innove par la définition de modalités pratiques pour leur mise en application.

Ce faisant, l'application des modalités des recommandations relatives à la pratique d'une agriculture durable dans les zones périphériques des aires protégées ainsi que la conservation et l'utilisation durable de la biodiversité par les pouvoirs publics ivoiriens (OIPR, ministère des Eaux et Forêt...) permettrait de réduire considérablement les pressions anthropiques sur les aires protégées et autres forêts mises en défens. De même, les modalités relatives à la valorisation touristique des aires protégées et de leurs zones périphériques pourraient servir au ministère du tourisme qui œuvre actuellement à la relance du tourisme en Côte d'Ivoire. Les résultats de cette thèse pourraient contribuer au développement d'une 
industrie touristique autour des aires protégées ivoiriennes et de leurs zones périphériques. Cela offrirait des revenus substantiels aux communautés locales et des emplois aux jeunes qui sont, pour la plupart, confrontés à la crise de l'emploi.

$\mathrm{Au}$ surplus, cette thèse met à la disposition des $\mathrm{ONG}$ de conservation nationales et internationales des solutions pratiques qui les aiderait à capitaliser. En effet, les différentes recommandations assorties de modalités antérieurement présentées contribueront à les orienter davantage dans leurs missions et actions de sensibilisation, de développement en faveur des communautés et de plaidoyer auprès des décideurs. Il en est de même pour les institutions techniques et de développement à vocation internationale qui soutiennent la conservation et le développement.

Par ailleurs, les résultats de cette étude feront l'objet de publications dans des revues académiques. Nous les présenterons également à l'occasion de manifestions à caractère scientifique d'envergure nationale et internationale (colloques, conférences etc.) auxquelles nous participerons. Au surplus, ces résultats serviront à formuler des recommandations pour la gestion et l'exploitation des forêts ivoiriennes, en général, et des aires protégées, en particulier. Celles-ci seront adressées à l'Office Ivoirien des Parcs et Réserves (OIPR) et au ministère de l'Environnement, à la Société de développement des Forêts (SODEFOR) et au ministère des Eaux et Forêts au ministère du Tourisme, au ministère de l'Emploi ainsi qu'au ministère du Plan et du Développement. 


\section{BIBLIOGRAPHIE}

\section{I - OUVRAGES GÉNÉRAUX}

ASSI-ESSO (Anne-Marie Hortense), DIOUF (N'diaw), OHADA. Recouvrement des créances, Bruxelles, Bruylant, Collection UNIDA, 2002, 252 pages.

BAYLE (Jean-Louis Loubel del), Initiation aux méthodes des sciences sociales, Paris, L'Harmattan, 2000, 272 pages.

BECQUE-ICKOWICZ (Solange), Le parallélisme des formes en droit privé, Paris, Éditions Panthéon Assas, Coll. "Thèses", 490 pages.

BLÉOU (Djézou Martin), Les grands arrêts de la jurisprudence administrative ivoirienne, Abidjan, CNDJ/GIZ, 2012, 484 pages.

BOULOC (Bernard), Droit pénal général, 19e édition, Paris, Dalloz, 2005, 702 pages.

CABRILlAC (Rémi), Droit des obligations, $7^{\text {ème }}$ édition, Paris, Dalloz, 2006, 377 pages.

CHAPUS (René), Droit administratif général (t. 1), $11^{\text {ème }}$ édition, Paris, Montchrestien, 1997, 1283 pages.

CHAPUS (René), Droit administratif général (t.3), $11^{\text {ème }}$ éd., Paris, Montchrestien, 1998, 763 pages.

CORNU (Gérard) (dir.), Vocabulaire juridique, $4^{\text {ème }}$ éd. (Association Henri Capitant), Paris, PUF, 2003, 951 pages.

DÉGNI-SÉGUI (René), Droit administratif général : L'action administrative (t. 2), 4 ème édition, Abidjan, NEI-CEDA, 2012, 446 pages.

DÉGNI-SÉGUI (René), Droit administratif général : L'organisation administrative (t.1), $4^{\text {ème }}$ édition, Abidjan, éditions Omniprésence, 2013, 331 pages.

DÉGNI-SÉGUI (René), Introduction au Droit, Abidjan, EDUCI, Collections Sciences Juridiques, 2009, 383 pages.

DESPORTES (Frédéric), GUNEHEC (Francis Le), Droit pénal général, 14 édition, Paris, Economica, 2007, 1120 pages.

DUPUIS (Georges), GUÉDON (Marie-José), CHRÉTIEN (Patrice), Droit administratif, $9^{\text {ème }}$ éd., Paris, 2004, 682 pages.

ELDIN (Michel), MILLEVILLE (Pierre), Le risque en agriculture, IRD Éditons, 1989, 619 pages.

GEORGES (Phillipe), Droit public: concours administratifs, 8 édition, Paris, Sirey, 1992, 472 pages.

GRAWITZ (Madeleine), Lexique des sciences sociales, $8^{\text {ème }}$ édition, Paris, Dalloz, 2004, 421 pages. 
GRAVITZ (Madeleine), Méthodes des sciences sociales, $11^{\text {ème }}$ édition, Paris, Dalloz, 2001, 1019.

GUGLIÉLMI (Gilles J.), KOUBI (Geneviève), Droit $d u$ service public, Paris, Montchrestien, 2000, 585 pages.

GUINCHARD (Serges), DEBARD (Thierry) (dir.), Lexiques des termes juridiques, $19^{\mathrm{e}}$ édition, Paris, Dalloz, 2012, 912 pages.

JEUGE-MAYNART (Isabelle) (dir.), Le Petit Larousse illustré, édition 2013, Paris, Larousse, 2013, 1927 pages.

KIPRÉ (Pierre), Côte d'Ivoire : La formation d'un peuple, Paris, SIDES-IMA, Collection L'AFRIQUE " dans tous ses états", 2005, 295 pages.

KRIPPENDORFF (K.), Content analysis : an introduction to its methodology, $3^{\text {rd }}, 2012$, Edition Sage Publications, Thousand Oaks, CA, 441 pages.

LAUBADÈRE (André de), VENEZIA (Jean-Claude), GAUDEMET (Yves), Traité de droit administratif (t. 2), 10 éd., Paris, LGDJ, 1995, 557 pages.

LAUBADÈRE (André de), GAUDEMET (Yves), Traité de Droit Administratif, Droit Administratif des Biens. Les domaines administratifs. L'expropriation: la réquisition, les travaux publics (t. 2), $11^{\text {ème }}$ édition, Paris, LGDJ, 1998, 477 pages.

LEY (Albert), Le régime domanial et foncier et le développement économique de la Côte d'Ivoire, Tome XVIII, Paris, LGDJ, 1972, 746 pages.

LOUCOU (Jean-Noël), La Côte d'Ivoire coloniale. 1893-1960, Abidjan, Les Éditions FHB \& Les Éditions du CERAP, 2012, 365 pages.

MAHIEU (François Régis), Introduction aux finances publiques de la Côte d'Ivoire, Abidjan, Les Nouvelles Éditions Africaines, 1983, 128 pages.

MERLE (Roger), VITU (André), Traité de droit criminel (t. 1), $7^{\mathrm{e}}$ édition, Paris, Cujas, 1997, 1068 pages.

MORAND-DEVILLER (Jacqueline), Droit administratif des biens, Paris, Montchrestien, 1999, 909 pages.

N'DA (Paul), Méthodologie de la recherche: de la problématique à la discussion des résultats. Comment réaliser un mémoire, une thèse en Sciences Sociales et en Éducation, $2^{\text {ème }}$ éd., Abidjan : EDUCI, 2002, 144 pages.

PAILLÉ (P.), MUCCHIELLI (A.), L'analyse qualitative en sciences humaines et sociales, $3^{\text {ème }}$ édition, Paris, Armand Colin, Collection U, 2012, 424 pages.

PAULAIS (Thierry), Le développement urbain en Côte d'Ivoire, 1979-1990: les projets de la Banque mondiale, Paris, KARTHALA, 1995, 183 pages.

PEISER (Gustave), Droit administratif général, 21 édition, Paris, Dalloz-Sirey, 2002, 295 pages.

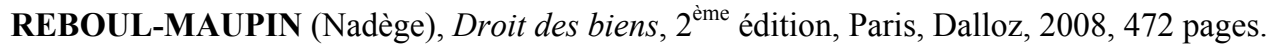




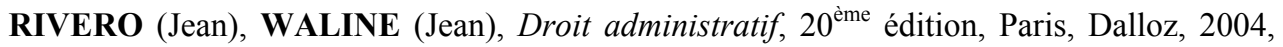
623 pages.

ROUGEVIN-BAVILLE (Michel), DENOIX de SAINTMARC (Renaud), LABETOULLE (Daniel), Leçons de droit administratif, Paris, Éditions Hachette, 1989, 639 pages.

SAWADOGO (Abdoulaye), L'Agriculture en Côte d'Ivoire, Paris, PUF, 1977, 368 pages.

SEUBE (Jean-Baptiste), Droit des biens, $3^{\text {ème }}$ édition, Paris, Litec/Lexis Neris, 2006, 164 pages.

THEUX (Axel de), KOVALOVSKY (Imre), BERNARD (Nicolas), Précis de méthodologie juridique: les sources documentaires du droit ( $2^{\mathrm{eme}}$ édition revue et mise à jour), Bruxelles, Publications des Facultés universitaires Saintes-Louis, 2000, 749 pages.

TOULET (Valérie), Droit civil: Obligations, Responsabilité civile, Paris, Centre de publication universitaires, 1998, 415 pages.

\section{II - OUVRAGES SPECIALISÉS}

ADAMS (Williams M.), "Nature and the colonial mind », in ADAMS (Williams M.) et MULLIGAN (Martin) (eds), Decolonizing Nature: Strategies for Conservation in the Postcolonial Era. , London: Earthscan, 2003, pp.16-50.

ANDERSON (David) et GROVE (Richard H.), « The scramble for Eden : Past, present and future in African conservation " in ANDERSON (David) et GROVE (Richard H.) (eds), Conservation in Africa: People, Policies and Practice, Cambridge University Press, 1988, pp.1-12.

ANDRÉ (Pierre), BEAUDET (René), BERNIER (Michel), CÔTÉ (Louis) et LANMAFANKPOTIN (Georges), SAMOURA (Karim), La participation publique dans l'évaluation environnementale en Afrique francophone, Québec, IFDD, Points de repère 23, 2013, 162 pages.

BINOT (Aurélie), BLOMLEY (Tom), COAD (Lauren), NELSON (Fred), ROE (Dilys) et SANDBROOK (Chris), " Quels ont été les résultats de la GCRN en Afrique ? Renforcement de l'autonomie, économie, environnement » in ROE (Dilys), NELSON (Fred) et SANDBROOK (Chris) (eds), Gestion communautaire des ressources naturelles en Afrique Impacts, expériences et orientations futures, Londres, Royaume-Uni, Institut International pour l'Environnement et le Développement, Série Ressources Naturelles no. 18, 2009, pp. 65-112.

BINOT (Aurélie), BLOMLEY (Tom), COAD (Lauren), ROE (Dilys), NELSON (Fred) et SANDBROOK (Chris), « Participation communautaire à la gestion des ressources naturelles en Afrique - vues régionales d'ensemble », in ROE (Dilys), NELSON (Fred) et SANDBROOK (Chris), (eds), Gestion communautaire des ressources naturelles en Afrique - Impacts, expériences et orientations futures, Londres, Royaume-Uni, Institut International pour l'Environnement et le Développement, Série Ressources Naturelles no. 18, 2009, pp. $15-64$.

CHILD (Brian) et DALAL-CLAYTON (Barry), « Transforming Approached to CBNRM: Learning from the Luangwa Experience, Zambia », in McSHANE (T.O.) et WELLS (M.P.) 
(eds), Getting Biodiversity Projects to Work : Towards More Effective Conservation and Development, NY, Columbia University Press, 2004, pp. 256-289.

FAURE (Michael), L'analyse économique du droit de l'environnement, Bruylant/Bruxelles 2007, 362 pages.

GIROD (Jacques), L'énergie en Afrique : la situation énergétique de 34 pays de l'Afrique subsaharienne et du Nord, France, Karthala Éditions, 1994, 467 pages.

GNANGUI (Adon), « Côte d'Ivoire : Évolution du droit de l'environnement depuis 1992 », in Michel PRIEUR (dir.), Vers un nouveau droit de l'environnement ?, Limoges, Centre international de droit comparé de l'environnement, 2003/05, pp. 361-364.

GRANIER (Laurent), «Introduction», in GRANIER (Laurent) (dir), Aspects contemporains du droit de l'environnement en Afrique de l'Ouest et centrale, UICN, Gland, Suisse, 2008, pp.13-34.

IBO (Guéhi Jonas), « Les nouvelles orientations de la gestion du patrimoine naturel en Côte d'Ivoire », in CORMIER-SALEM (Marie-Christine), JUHÉ-BEAULATON (Dominique), BOUTRAIS (Jean) et ROUSSEL (Bernard) (dir.), Patrimoine naturels au Sud: Territoires, identités et stratégies locales, IRD Éditions, 2005, pp. 71-96.

KAMTO (Maurice), Droit de l'environnement en Afrique, Vanves, EDICEF, 1996, 416 pages.

LAUGINIE (Francis), Conservation de la nature et des aires protégées en Côte d'Ivoire, Abidjan, NEI/Hachette et Afrique Nature, 2007, 668 pages.

LEROY (Maya), DERROIRE (Géraldine), VENDÉ (Jeremy) et LEMÉNAGER (Tiphaine), La gestion durable des forêts tropicales. De l'analyse critique du concept à l'évaluation environnementale des dispositifs de gestion, AFD, À savoir nº 18, mai 2013, 235 pages.

MEKOUAR (Mohamed Ali), «Les forêts de Rio à Johannesburg et la perspective d'une convention forestière mondiale ", in PRIEUR (Michel) (dir.), Vers un nouveau droit de $l^{\prime}$ environnement?, Limoges, Centre international de droit comparé de l'environnement, 2003/05, pp.69-83.

NTOUGOU (Omer), « Développer les ressources financières par le tourisme », in TRIPLET (Patrick) (dir.), Manuel de gestion des aires protégées d'Afrique francophone, Paris, Awely, 2009, pp. 806-816.

PRIEUR (Michel), Droit de l'environnement, 4ème édition, Paris, éditions Dalloz, 2001, 944 pages.

ROE (Dilys), NELSON (Fred), «Origines et évolution de la gestion communautaire des ressources naturelles en Afrique ", in ROE (Dilys), NELSON (Fred) et SANDBROOK (Chris) (eds), Gestion communautaire des ressources naturelles en Afrique - Impacts, expériences et orientations futures, Londres, Royaume-Uni, Institut International pour l'Environnement et le Développement, Série Ressources Naturelles no. 18, 2009, pp. 5-14.

SADELEER (Nicolas de), « Les enjeux de la temporalité dans le droit de l'environnement », in GÉRARD (Philippe), OST (François) et KERCHOVE (Michel van de) (dir.), 
L'accélération du temps juridique, Bruxelles, Publications des Facultés universitaires SantLouis, 2000, pp. 893-910.

SCHERL (Lea M.), WILSON (Alison), WILD (Robert) et al., Can Protected Areas Contribute to Poverty Reduction? Opportunities and Limitations, Gland, Switzerland and Cambridge, UK., IUCN, 2004, viii + 60 pages.

SCOONES (Ian), MELNYK (Mary), PRETTY (Jules N.), The Hidden Harvest. Wild Foods Agricultural Systems. A literature Review and Annotated Bibliography, WWF/ IIED, 1992, 256 pages.

THUNIS (Xavier), «La recherche juridique à contretemps », in GÉRARD Philippe, OST François et KERCHOVE (Michel van de) (dir.), L'accélération du temps juridique, Bruxelles, Publications des Facultés universitaires Sant-Louis, 2000, pp. 911-920.

TOMIĆEVIĆ (Jelena), BJEDOV (Ivana), SHANNON (Margaret A.) et OBRATOVPETKOVIĆ (Dragica), «Understanding Linkages Between Public Participation and Management of Protected Areas - Case Study of Serbia», in Dr. ISHWARAN (Natarajan) (Eds), The Biosphere, 2012, Intech, pp. 131-142.

TRIPLET (Patrick), «Diversifier et augmenter les ressources financières des aires protégées ", in TRIPLET (Patrick) (dir.), Manuel de gestion des aires protégées d'Afrique francophone, Paris, Awely, 2009, pp. 800-805.

TRIPLET (Patrick), LANGRAND (Olivier), « Définir les aires protégées d'Afrique », in TRIPLET (Patrick) (dir.), Manuel de gestion des aires protégées d'Afrique francophone, Paris, Awely, 2009, pp. 18-24.

TRIPLET (Patrick), VERMEULEN (Cédric), « Améliorer la participation des populations à la gestion des aires protégées » in TRIPLET (Patrick) (dir.), Manuel de gestion des aires protégées d'Afrique francophone, Paris, Awely, 2009, pp. 228-231.

TRIPLET (Patrick) et VERMEULEN (Cédric), "Comprendre la gouvernance », in TRIPLET (Patrick) (dir.), Manuel de gestion des aires protégées d'Afrique francophone, Paris, Awely, 2009, pp. 246-251.

VEIT (Peter G.), NSHALA (Rugemeleza), ODHIAMBO (Michael Ochieng'), MANYINDO (Jacob), Protected Areas and Property Rights. Democratizing Eminent Domain in East Africa, WRI Report, World Resources Institute, 2008, 30 pages.

\section{III - ARTICLES DE DOCTRINE}

ADELEKE (Bola Olusola) et NZAMA (Thandi), « Assessment of Community Participation in Ecotourism and Conservation at Hhuhuwe-Umfolozi Park, South Africa », Journal of Environment and Earth Science, Vol. 3, n³, 2013, pp. 27-37.

AGRAWAL (Arun) et GIBSON (Clark C.), « Enchantment and Disenchantment: The Role of Community in Natural Resource Conservation »,World Development, Vol. 27, $\mathrm{n}^{\circ} 4$, 1999, pp. 629-649.

AHEBWA (Wilber Manyisa), DUIM (V. René Van der) et SANDBROOK (Chris G.), « Private-community Partnerships: Investigating a New Approach to Conservation and Development in Uganda », Conservation and Society, Vol. 10, n 4, 2012, pp. 305-317. 
AKAMA (John S.), LANT (Christopher L.) et BURNETT (G.), « Conflicting attitudes towards state wildlife conservation programs in Kenya », Society and Natural Resources, Vol. 8, n³, pp. 133-144.

AMANI (Célestin Yao), " Production agricole et changement climatique: vers une tragédie des comportements paysans à Tiassalé?, European Scientific Journal, Vol. 8, n¹6, 2012, pp. 227-244.

ANDRADE (Gustavo S. M.) et RHODES (Jonathan R.), "Protected areas and local communities: an inevitable partnership toward successful conservation strategies ? ", Ecology and Society, Vol.17, $\mathrm{n}^{\circ} 4,2012,14-30$.

ARCHABALD (Karen) et NAUGHTON-TREVES Lisa, «Tourism revenue-sharing around National Parks in Western Uganda : Early efforts to identify and reward local communities», Environmental Conservation, Vol. 28, n², 2001, pp. 135-149.

ASMAMAW (Demeke) et VERMA (Ashok), « Local attitudes towards environmental conservation and ecotourism around Bale Mountains national park, Ethiopia, Scholarly Journal of Agricultural Science, Vol. 3, n¹1, 2013, pp. 506-514.

AUBY (Jean-Marie), « L'action domaniale », A.J.D.A, octobre 1983, pp. 507-513.

BARAL (Nabin), STERN (Marc J.) et HEINEN (Joël T.), « Integrated conservation and development project life cycles in the Annapurna conservation area, Nepal: is development overpowering conservation ? ", Biodiversity Conservation, Vol. 16, n ${ }^{\circ}$, 2007, pp. 29032917.

BARRETT (C. B.) et ARCESE (P.), « Are integrated conservation-development projects (ICDPs) sustainable? On the conservation of large mammals in sub-Saharan Africa ", World Development, Vol. 23, nº 7, 1995, pp. 1073-1084.

BARRETT (Christopher B.), TRAVIS (Alexander J.) et DASGUPTAD (Partha), " On biodiversity conservation and poverty traps ", Proceedings of the National Academy of Sciences, Vol. 108, n³4, 2011, pp. 13907-13912.

BERKES (F.), « Rethinking community-based conservation », Conservation Biology, Vol. $18, n^{\circ} 3,2004$, pp. 621-630.

BIGGS (Duan), COURCHAMP (Franck.), MARTIN (Rowan.) et POSSINGHAM (Hugh. P.),. « Legal Trade of Africa's Rhino Horns », Science, Vol. 339, 2013, pp. 1038-1039.

BIRNER (Regina) et WITTMER (Heidi), « Better public sector governance through partnership with the private sector and civil society: the case of Guatemala's forest administration ", International Review of Administrative Sciences, Vol 72, n4, 2006, pp. 459-472.

BROU (Télesphore Yao), "Modification du régime des précipitations et risque de stress hydrique des formations végétales en zone de forêt dense humide ivoirienne », Cahiers Nantais, $\mathrm{n}^{\circ} 51,1999$, pp. 105-115.

BROU (Télesphore Yao), SERVAT (Eric) et PATUREL (Jean-Emmanuel), « Contribution à l'analyse des interrelations entre activités humaines et variabilité climatique : cas du sud forestier ivoirien », Académie des sciences, Vol. 327, 1998, pp. 833-838. 
BRUNER (Aaron G.), GULLISON (Raymond E.) et BALMFORD (Andrew), « Financial cost and shortfalls managing and expanding protected-area systems in developing countries », BioScience, Vol. 54, n¹2, 2004, pp. 1119-1126.

CHAPMAN (Duane) et BARKER (Randolph), " Environmental protection, resource depletion, and the sustainability of developing country agriculture », Economic Development and Cultural Change, Vol. 39, $\mathrm{n}^{\circ} 4,1991, \mathrm{pp} .723-737$.

COOMES (Oliver T.), TAKASAKI (Yoshito) et RHEMTULLA (Jeanine M.), Land-use poverty traps identified in shifting cultivation systems shape long-term tropical forest cover, Proceedings of the National Academy of Sciences, Vol. 108, n³4, 2011, pp. 13925-13930.

COUSINS (Jenny A.), SADLER (Jon P.) et EVANS (James), « Exploring the Role of Private Wildlife Ranching As a Conservation Tool in South Africa : Stakeholder Perspectives ", Ecology and Society, Vol. 3, n², 2008, la première phrase du point intitulé "Introduction" (document non paginé sur internet, disponible sur www.ecologyandsociety.org/vol13/iss2/art43/.

DODSON (Giles), «Co-Governance and Local Empowerment? Conservation Partnership Frameworks and Marine Protection at Mimiwhangata, New Zealand», Society and Natural Resources, Vol. 27, n5, 2014, pp. 521-539.

DURAIAPPAH (Anantha K.), « Poverty and environmental degradation: A review and analysis of the nexus ", World Development, Vol. 26, $\mathrm{n}^{\circ} 12,1998,2169-2179$.

EDWARDS (Pamela) et SHAOUL (Jean), "Partnerships: For better or worse? ", Accounting, Auditing and Accountability, Vol. 16, n³, 2002, pp. 397-421.

FAURE (Michaël), GOODWIN (Morag) et WEBER (Franziska), «Bucking the Kuznets Curve: Designing Effective Environmental Regulation in Developing Countries ", Virginia journal of international law, Vol. 51, 2010, pp. 95-157.

FERRARO (Paul J.), «The local costs of establishing protected areas in low-income nations: Ranomafana National Park, Madagascar », Ecological Economics, Vol. 43, n²-3, 2002, pp. 261-275.

FIALLO (Elba A.) et JACOBSON (Susan K.), « Local communities and protected areas: attitude of rural residents towards conservation and Machalilla National Park, Ecuador », Environmental Conservation, Vol. 22, n³, 1995, pp. 241-249.

FIERENS (Marie), «'Il était une fois l'indépendance de la Côte d'Ivoire...' Récits médiatiques d'hier et d'aujourd'hui », Le Temps des Médias, Vol. 1, n²6, 2016, pp. 144-162.

FOLIO (Fabrice) et DERROISNE (Anaëlle), «Écotourisme et Parcs naturels : innovations et contradictions sud-africaines, le cas de HIP (Hluhluwe Imfolozi Park) », EchoGéo, Vol. 13, 2010, pp. 1-22.

FOURNIER (Anne), «Vème Congrès mondial sur les Parcs de l'Union mondiale pour la nature (UICN) : Compte rendu (Durban, Afrique du Sud, 8-18 septembre 2003 », Nature Sciences Sociétés, Vol. 12, n¹, 2014 pp. 93-96.

FU (B.), WANG (K.), LU (Y.), LIU (S.), KEMING (M.), CHEN (L.) et LIU (G.), « Entangling the complexity of protected area management : the case of Wolong Biosphere Reserve, southwestern China », Environmental Management, Vol. 33, n6, 2004, pp. 788798. 
GBADEGESIN (Adeniyi) et AYILEKA (Olatubosum), « Avoiding the mistakes of the past: Towards a community oriented management strategy for the proposed National Park in Abuja-Nigeria », Land Use Policy, Vol. 17, n², 2000, pp. 89-100.

GELCICH (S.), EDWARD-JONES (G.) et KAISER (M.), « Importance of attitudinal differences among artisanal fishers toward co-management and conservation of marine resources», Conservation Biology, Vol.19, n³, 2005, pp. 865-875.

GIBSON (Clark C.) et MARKS (Stuart A.) « Transforming rural hunters into conservationists: An assessment of community-based wildlife management programs in Africa », World Development, Vol. 23, nº, 1995, pp. 941-957.

HATCHWELL (Matthew), « Public-private partnerships as a management option for protected areas», Animal Conservation, Vol. 17, n²1, 2014, pp. 3-4.

HAYES (Tanya. M.), « Parks, people, and forest protection: an institutional assessment of the effectiveness of protected areas », World Development, Vol. 34, n 12 , 2006, pp. 20642075 .

HE (Guangming), CHEN (X.) LIU (W.), BEARER (S.), ZHOU (S.), CHENG (L.Y.), ZHANG (H.), OUYANG (Z.) et LIU (J.), " Distribution of Economic Benefits from Ecotourism: A Case study of Wolong Nature Reserve for Giant Pandas in China», Environmental Management, Vol. 42, $\mathrm{n}^{\circ} 1,2008$, pp. 1017-1025.

HEGLAND (Joar Stein), RYDGREN (Knut) et SELDAL (Tarald), « The response of Vaccinium to variations in grazing intensity in a Scandinavian pine forest on the island of Svanøy », Canadian journal of botany, vol. 83, n²12, 2005, pp. 1638-1644.

HENRY (P.) et MOSCOVICI (S.), « Problèmes de l'analyse de contenu », Langages, Vol. $2, \mathrm{n}^{\circ} 11,1968$, pp. 36-60.

HOROWITZ (Leah Sophie), " Integrating indigenous resource management with wildlife conservation: a case study of Batang Ai National Park, Sarawak, Malaysia », Human Ecology, Vol. 26, n³, 1998, pp. 371-403.

HOVIK (Sissel) et EDVARDSEN (Morten), « Private-public partnership: An exceptional solution in nature conservation in Norway », Local Environment, Vol. 11, n 4, 2006, pp. 361372.

IBO (Guéhi Jonas), «La politique coloniale de protection de la nature en Côte d'Ivoire (1900-1958) », Revue Française d'Histoire d'Outre-mer, Vol. 80, n²98, 1993, pp. 83-104 pages.

INAMDAR (Amar), JODE (Helen de), LINDSAY (Keith) et COBB (Stephen), «Capitalizing on Nature Protected Area Management », Science, Vol. 283, n5409, 1999, pp. 1856-1857.

KEPE (Thembela), « Land claims and co-management of protected areas in South Africa: Exploring the challenges », Environmental Management, Vol. 41, n³, 2008, pp. 311-321.

LAVIALLE (Christian), "L'imprescriptibilité du domaine public », Revue Française de Droit, Administratif, $\mathrm{n}^{\circ} 1,1985$, pp. 27-44 pages.

LEADER-WILLIAMS (N.) et ALBON (S. D.), « Allocation of resources for conservation», Nature, Vol. 336, n 6199, 1988, pp. 533-535. 
LEWIS (D.), BELL (S.D.), FAY (J.), BOTHI (K.L.), GATERE (L.), KABILA (M.), MUKAMBA (M.), MATOKWANI (E.), MUSHIMBALUME (M.) MORARU (C.I.) et al., «Community markets for conservation (COMACO) links biodiversity conservation with sustainable improvements in livelihoods and food production», Proceedings of the National Academy of Sciences of the United States of America, Vol. 108, n40, 2011, pp. 1395713962.

MASHALE (Christopher Modise), MOYO (Theresa) et MTAPURI (Olivier), « Evaluation of the Public-Private Partnership in the Lekgalameetse Nature Reserve in South Africa », Mediterranean Journal of Social Sciences, Vol. 5, n²0, 2014, pp. 855-862.

MBAIWA (Joseph E.) et STRONZA (Amanda L.), " The effects of tourism on rural livelihoods in the Okavango Delta, Botswana», Journal of Sustainable Tourism, Vol. $18, \mathrm{n}^{\circ} 5$, 2010, pp. 635-656.

MBAIWA (Joseph E.), STRONZA (Amanda L.), « Changes in resident attitudes towards tourism development and conservation in the Okavango Delta ", Journal of Environmental Management, Vol. 92, n8, 2011, pp. 1950-1959.

MBURU (John) et BIRNER (Regina), « Emergence, Adoption, and Implementation of Collaborative Wildlife Management or Wildlife Partnerships in Kenya: A Look at Conditions for Success », Society and Natural Resources, Vol. 20, n 5, 2007, pp. 379-395.

MYERS (Norman), MITTERMEIER (Russel A.), MITTERMEIER (Cristine G.), FONSECA (Gustavo A. B. da) et KENT (Jennifer), " Biodiversity hotspots for conservation priorities », Nature, Vol. 403, 2000, pp. 853-858.

NAUGHTON-TREVESA (Lisa), ALIX-GARCIAB (Jennifer), et CHAPMAN ( Colin A.), «Lessons about parks and poverty from a decade of forest loss and economic growth around Kibale National Park, Uganda», Proceedings of the National Academy of Sciences, Vol. 108, n³4, 2011, pp. 13919-13924.

NEWMARK (William D.), HOUGH (John L.), « Conserving Wildlife in Africa: Integrated Conservation and Development Projects and Beyond », BioScience, Vol. 50, n7, 2000, pp. 595-592.

NEWMARK (William D), LEONARD (Nancy L.), SARIKO (Hashim I.) et GAMASSA (Deo-Gratias M.), « Conservation attitudes of local people living adjacent to Five protected areas in Tanzania», Biological Conservation, Vol. 63, n², 1993, pp. 177-183.

NSHIMBI (Muleba) et VINYA (Royd), « Impacts of Public-Private Partnership on Local Livelihoods and Natural Resource Dynamics: Perceptions from Eastern Zambia », Resources, Vol. 3, n 2, 2014, pp. 471-487.

OATES (John F.), «The dangers of conservation by rural development -a case study from the forests of Nigeria», Oryx, Vol. 29, n², 1995, pp. 115-122.

OGLE (Britta Mathilda) et GRIVETTI (Louis Evan), « Legacy of the Chameleon: Edible Wild Plants in the Kingdom of Swaziland, Southern Africa: A Cultural, Ecological, Nutritional Study, Part II -- Demographics, Species Availability and Dietary Use, Analysis by Ecological Zone.», Ecology of Food and Nutrition, Vol. 17, n¹, 1985, pp. 1-30.

OKECK (Roselyne N.), «Wildlife-community conflicts in conservation areas in Kenya», African Journal of Conflit Resolution, Vol. 10, n², 2010, pp. 65-80. 
PARENT (Sylvie), KLEIN (Juan-Luis) et JOLIN (Louis), « Le développement communautaire local et le tourisme communautaire : une analyse conceptuelle comparative ", ESSACHESS-Journal for Communication Studies, Vol 2, n 4, 2009, pp. 73-89.

PATNEY (Atjun), « Saving Lands and Wildlife : Corporations and Conservation Groups in Partnership », Corporate Environmental Strategy, nº ${ }^{\circ}{ }^{\circ} 4,2000$, pp. 362-370.

PERNETTA (Angelo P.), «A disappearing drylands icon? White rhinoceros conservation and the need for public-private partnerships », Biodiversity, Vol. 15, n²-3, 2014, pp. 231233.

PIPER (Jake M.), « Partnership and Participation in Planning and Management of River Corridors », Planning, Practice \& Research, Vol. 20 (1), 2005, pp. 1-22.

PRETTY (Jules) et SMITH (David), " Social capital in biodiversity conservation and management », Conservation Biology, Vol. 18, n³, 2004, pp. 631-638.

PIMM (Stuart L.) et RAVEN (Peter), « Extinction by numbers », Nature, Vol. 403, 2000, pp. $843-845$, p. 844 .

RICHARDSON (Julie A.), «Wildlife utilization and biodiversity conservation in Namibia: conflicting or complementary objectives? », Biodiversity and Conservation, Vol. 7, $\mathrm{n}^{\circ} 4$, 1998, pp. 549-559.

RUTAGARAMA (Eugène), et MARTIN (Adrian), " Partnerships for protected area conservation in Rwanda », The Geographical Journal, Vol. 172, n 4, 2006, pp. 291-305.

SADELEER (Nicolas de), «Particularités de la subsidiarité dans le domaine de l'environnement», Droit et Société, Vol. 1, n80, pp. 73-90.

SAKO (Nakouma), BELTRANDO (Gérard), «Dynamiques spatiales récentes du Parc National du Banco (PNB) et stratégies de gestion communautaire durable de ses ressources forestières (District d'Abidjan en Côte d'Ivoire) », EchoGéo, Vol. 30, octobre 2014/décembre 2014, pp. 1-17.

SEKHAR (Nagothu Udaya), «Local people's attitudes towards conservation and wildlife tourism around Sariska Tiger Reserve, India », Journal of Environmental Management, Vol. $69, \mathrm{n}^{\circ} 4,2003$, pp. $339-447$.

STOLL-KLEEMANN (Suzanne) et O'RIORDAN (Timothy), «From Participation to Partnership in Biodiversity Protection : Experience from Germany and South Africa ", Society and Natural Resources : An International Journal, Vol. 15, n 2, 2002, pp. 161-177.

SZELL (Andrea B.) et HALLETT (Lucius F.), "Attitudes and Perceptions of Local Residents and Tourists toward the Protected Area of Retezat National Park, Romania », International Journal of Humanities and Social Science, Vol. 3, n 3; 2013, pp. 18-34.

THACKWAY (R.) et OLSSON (K.), «Public/private partnerships and protected areas : Selected Australian case studies », Landscape and urban planning, vol. 44, n²-3, 1999, pp. 87-97.

THOMAS-SLAYTER (Barbara), « Implementing Effective Local Management of Natural Resources: New Roles for NGOs in Africa », Human Organization, Vol. 51, n², 1992, pp. 136-143. 
THOMAS (R.T.) et VOSTI (S.A), « Links between rural poverty and the environment in developing countries: Asset categories and investment poverty ", World Development, Vol.23, n9, 1995, pp. 1495-1506.

VERMEULEN (Sonja) et SHEIL (Douglas), "Partnerships for tropical conservation », Oryx, 41, n 4, 2007, pp. 434-440.

VISSEREN-HAMAKERS (Ingrid J.) et GLASBERGEN (Peter), « Partnerships in forest governance », Global Environmental Change, Vol. 17, n³-4, 2007, pp. 408-419.

WILSHUSEN (Peter R.), BRECHIN (Steven R.), FORTWANGLER (Crystal L.) et WEST (Patrick C.), "Reinventing a square wheel: critique of a resurgent "protection paradigm" in international biodiversity conservation ", Society and Natural Resources, Vol.15, n 1,2002 , pp. 17-40.

WILSON (Erica), NIELSEN (Noah) et BUULTJENS (Jeremy), « From lessees to partners: exploring tourism public-private partnerships within the New South Wales national parks and wildlife service », Journal of Sustainable Tourism, Vol. 17, n², 2009, pp. 269-285.

WOLMER (William) et ASHLEY (Caroline), « Part II : Resources and Policies. 3. Wild resources management in southern Africa : Participation, partnerships, eco regions and redistribution », IDS Bulletin, Vol. 34, n³, 2003, pp. 31-40.

\section{IV - THÈSES, MÉMOIRES ET COURS}

\section{Thèses}

BINOT (Aurélie), La conservation de la nature en Afrique centrale entre théorie et pratique. Des espaces protégés à géométrie variable, Thèse de Géographie, Paris, Université Paris 1 Panthéon-Sorbonne, 2010, 442 pages.

DIALLO (Mamadou Saïdou), Évolution de la gestion des aires protégées en Guinée : La difficile cohabitation des politiques publiques et des systèmes traditionnels. Cas du Parc National du Haut Niger, Thèse unique, Géographie, Université du Maine, 14 avril 2011, 268 pages.

GOH (Denis), Les approches participatives dans la gestion des Aires Protégées en Côte d'Ivoire : l'expérience du Projet Autonome pour la Conservation du Parc National de Taï (PACPNT), Thèse unique, Environnement, Université d'Abobo-Adjamé, 21 mai 2005, pp. 307.

KRAGBÉ (Gilles A.), Les politiques de gestion de l'environnement à l'aune du droit traditionnel africain: cas des peuples des régions forestières de la Côte d'Ivoire, Thèse unique, Environnement, Université d'Abobo-Adjamé, 17 février 2011, 414 pages.

MANCÉRON (Stéphane), Intervenir en périphérie pour la conservation des aires protégées : réexamen du postulat. La situation du Parc $W$ et des éleveurs mobiles, Thèse Unique, Géographie, Université Paris Ouest Nanterre-La Défense, 7 octobre 2011, 586 pages.

TIEBLEY (Yves-Didier), La Côte d'Ivoire et la gestion des ressources naturelles marines, Thèse unique, Droit public, Université de Maastricht, 20 mai 2010, 369 pages. 


\section{Mémoires}

AKOUÉ (Yao Claude), Les aspects conflictuels liés à la conservation des aires protégées de Côte d'Ivoire: le cas du Parc National de la Marahoué à Bouaflé, Mémoire de DEA en Sciences et Gestion de l'Environnement option Politique en Environnement, Université d'Abobo-Adjamé, août 2004, 81 pages.

KOFFI (Kouassi Yves), La gestion des zones humides d'importance internationale en Côte d'Ivoire et la mise en œuvre de la convention de Ramsar: cas du Parc National d'Azagny, Mémoire de $3^{\text {ème }}$ cycle en vue de l'obtention du Diplôme d'Etudes Supérieures Spécialisées en Conservation et Utilisation Durable des Zones Humides, UFR/SVT, Université de Ouagadougou, Février 2006, 68 pages.

KOFFI (Kouassi Yves), La politique de gestion des Parcs Nationaux et Réserves naturelles en Côte d'Ivoire et la conservation de la diversité biologique de 1965 à 2002 : Aspects juridiques, Mémoire de DEA en Sciences et Gestion de l'Environnement option Politique en Environnement, Université d'Abobo-Adjamé, Septembre 2004, 51 pages + annexes.

MONSAN (Ignace), Stratégie de développement de l'écotourisme : le cas du Parc National d'Azagny, Mémoire de DEA en Sciences et Gestion de l'Environnement option Politique en Environnement, Université d'Abobo-Adjamé, 17 août 2004, 56 pages.

PAYEN (Ariane), Tourisme communautaire dans les Suds: quelle implication des populations locales dans les aires protégées? Cas du Parc National de Loango, Gabon, Mémoire de Master professionnel en Développement et Aménagement Touristique des Territoires, Université de Paris 1-Panthéon Sorbonne, Session de Septembre 2012, 99 pages.

\section{Cours}

KOBO (Pierre-Claver), Droit Administratif des Biens (D.A.B.), Abidjan, A.B.C édition, 2004, 162 pages.

\section{V - DOCUMENTS DIVERS}

AFD, Gestion durable de la biodiversité, coll. Parole d’acteurs, n5, 2005, 42 pages.

AFD, Gestion durable de la forêt et lutte contre la désertification, coll. Paroles d'acteurs, n 1, 2002, 34 pages.

AFD, Note de communication publique d'opération. Côte d'Ivoire. C2D/ Conservation des ressources naturelles, disponible sur:

www.afd.fr/base projets/downloadDocument.action?idDocument=1470 et consulté le 12 juin 2015).

AGRAWAL (Arun), VARUGHESE (George), Conservation's Visions: Poverty, Participation, and Protected Area Management in Nepal's Terai, Conference Paper: Constituting the Commons: Crafting Sustainable Commons in the New Millennium, the Eighth Biennial Conference of the International Association for the Study of Common Property, (May 31-June 4) 2000, 44 pages. 
AKINDÈS (Francis), KOUAMÉ (Kra Walter), SÉDIA (Gisèle) et SÉZAN (Jules), Réalisation d'une étude sur l'état de la biodiversité des Parcs Nationaux et Réserves de Côte d'Ivoire : Aspects socio-économiques (Rapport provisoire), Abidjan, août 2012, 103 pages.

ARNTZEN (J.), MOLOKOMME (D.L.), TERRY (E.M.), MOLEELE (N.), TSHOSA (O.) et MAZAMBANI (D.), Final Report of the Review of Community-Based Natural Resource Management in Botswana Volume 1 : Main findings A study carried out for the National CBNRM Forum, UICN, Departement for International Development, September 2003, pp. 51-52. (NB: ce document consulté le 5 février 2016, sur www.theeis.com/data/literature/FinalReport CBNRMReview.pdf, n'est pas paginé à l'origine. Ces numéros et nombres de pages qui figurent ci-dessous sont déduits du nombre de pages que contient le rapport dont il fait l'objet).

BONNÉHIN (Léonie), Le profil historique de la zone périphérique de Taï: Quel potentiel pour la conservation du Parc National de Taï, Abidjan, GTZ/OIPR, Mars 2006, 82 pages.

BOUDREAUX (Karol C.), Community Conservation in Namibia : Devolution a Tool for the Legal Empowerment of the Poor, Working Paper $\mathrm{n}^{\circ} 10-64$, Mercatus Center at Georges Mason University, 2010, 26 pages.

BUCKLEY (Ralf), « Innovative funding mechanisms for visitor infrastructure in Australian National Parks. In A natural partnership: Making National Parks a tourism priority. Sydney: TTF Australia and CRC for Sustainable Tourism, Juin 2004, 43 pages.

COUNSELL (Simon), Forest Governance in Africa, Johannesburg, South African Institute of International Affairs, Occasional paper $\mathrm{n}^{\circ} 50$, Governance of Africa's Resources Programme, 2009, 40 pages.

DOUMBÉ-BILLÉ (Stéphane), Droit international de la faune et des aires protégées: importance et implication pour l'Afrique, Étude juridique de la FAO \#/20, septembre 2001, 30 pages.

FAO, L'Étude prospective du secteur forestier en Afrique (FOSA) : Côte d'Ivoire, 34 pages, disponible sur www.fao.org/docrep/003/X6780F/X6780F05.htm, consulté le 04 juin 2016.

EAGLES (Paul F.J.), International trends in park tourism, Paper presented at EUROPARC Conference, 3-7 October 2001, Hohe Tauern National Park, Matrei, Austria, 2001, 143 pages.

FAO, Évaluation des ressources forestières mondiales 2015. Répertoire des données de FRA 2015, Rome Organisation des Nations Unies pour l'Alimentation et l'Agriculture, 2015, 245 pages.

FAO, Situation des forêts du monde: Mieux tirer parti des avantages socioéconomiques des forêts, Rome, Organisation des Nations Unies pour l'Alimentation et l'Agriculture, 2014, 102 pages + Annexes.

GIEC, 2007: Résumé à l'intention des décideurs. In: Changements climatiques 2007 : Les éléments scientifiques. Contribution du Groupe de travail I au quatrième Rapport d'évaluation du Groupe d'experts intergouvernemental sur l'évolution du climat. [Solomon, S., D. Qin, M. Manning, Z. Chen, M. Marquis, K.B. Averyt, M.Tignor et H.L. Miller (éds.)]. Cambridge University Press, Cambridge, UK et New York, NY, USA, 2007, 18 pages. 
HALLE (Birgit), Dr. BRUZON (Véronique), Profil Environnemental de la Côte d'Ivoire, Rapport Final, Août 2006, 128 pages.

IBO (Guéhi Jonas), Histoire de la protection de la nature en Côte D'Ivoire par les textes, IRD/UAA, 2004, pp. 29-34.

INSTITUT NATIONAL DE SANTÉ PUBLIQUE DU QUÉBEC, La santé des communautés : perspectives pour la contribution de la santé publique au développement social et au développement des communautés, 2002, 50 pages.

JOHANNESEN (Anne Borge), Protected areas, wildlife conservation and local welfare, Working Paper Series, $\mathrm{n}^{\circ}$ 13/2005 (Department of Economics, Norwegian University of Science and Technology), 40 pages.

JONES (Brian T. B.), La gestion communautaire des ressources naturelles en Namibie, 25 pages (article en ligne, disponible sur www.researchgate.net/puplication/228591248 et consulté le 2 septembre 2016).

KRUG (Wolf), Private Supply of Protected Land in Southern Africa: A Review of Markets, Approaches, Barriers and Issues, Workshop Paper, World Bank / OECD International, Workshop on Market Creation for Biodiversity Products and Services, Paris, 25 and 26 January 2001, 42 pages.

LOUPPE (Dominique), OUATTARA (N'Klo), Étude sur l'exploitation forestière et les contraintes d'une gestion durable des forêts dans le domaine rural en Côte d'Ivoire, Abidjan, CIRAD, GTZ, décembre 2013, 216 pages.

MAKOMBE (Kudzai), Sharing the land: Wildlife, People, and Development in Africa, , Harare, Zimbabwe, IUCN/SUWP? Washington, USA, IUCN/ROSA Environmental Issues Series $n^{\circ} 1$, IUCN/ROSA, 1994, 36 pages.

NELSON (John) et GAMI (Norbert), Enhancing equity in the relationship between protected areas and indigenous and local communities in Central Africa, in the context of global change, CEESP-WCPA-IUCN Theme on Indigenous and Local Communities, Equity and Protected Areas, (Final Report), 2003, 20 pages.

OCDE, Tendances et politiques du Tourisme de l'OCDE 2012, Éditions OCDE, 2013, 456 pages.

OIPR/AFD, Réalisation d'une étude sur l'état de la biodiversité de quatre Parcs Nationaux de Côte d'Ivoire. Azagny, Marahoué, Péko, Sangbé, Afrique Nature International, 2012 (Rapport final), 129 pages.

PIMBERT (Michel P.) et PRETTY (Jules N.), Parks, People and Professionals: Putting Participation' into Protected Area Management, UNRISD/IIED/WWF, Discussion Paper $n^{\circ}$ 57, February 1995. UNRISD, Geneva, 65 pages.

POPE (Adam), A Preliminary Examination of Public Private Partnerships in National Park Management in Zambia, 2006, WHYDAH CONSULTING LTD. Development and Natural Resource Consultants, 24 pages.

QUEBEC, Ministère des Ressources Naturelles de la Faune et des Parcs, Direction du développement de l'industrie des produits forestiers, Direction du développement de 
l'industrie des produits forestiers, Aperçu des produits forestiers non ligneux (PFNL), juin 2003, 11 pages.

Republic of Zambia, Ministry of Tourism, Environment and Natural Resources, Reclassification and effective management of protected areas system project : Current protected area categories and the institutions responsible for their management in Zambia, UNDP/GEF, 44 pages.

Republic of Kenya, Ministry of Tourism and Wildlife, Draft Wildlife Policy 2007, 2007, 38 pages.

République de Côte d'Ivoire, Cinquième rapport national sur la diversité biologique, mars 2014, 106 pages.

République de Côte d'Ivoire, Institut National de la Statistique, RGPH-98: Synthèse des rapports d'analyse du RGPH-98.

République de Côte d'Ivoire, Ministère d'État, Ministère du Plan et du Développement, Document Stratégique de Réduction de la Pauvreté: Stratégique de Relance du Développement et de Réduction de la Pauvreté, Janvier 2009, Abidjan, 2009, 198 pages.

République de Côte d'Ivoire, Ministère de l'Économie et des Finances, La Côte d'Ivoire en chiffres, édition 96-97, Abidjan, Dialogue Production, 1997, 232 pages.

République de Côte d'Ivoire, Ministère de l'Économie, des Finances et du Plan, La Côte d'Ivoire en chiffres, édition 86-87, Paris, Inter Afrique Presse, 1988, 220 pages.

République de Côte d'Ivoire, Ministère de l'Environnement, de la Salubrité Urbaine et Développement Durable/Office Ivoirien des Parcs et Réserves, État de conservation du Parc National de la Comoé. Côte d'Ivoire, janvier 2014, 7 pages.

République de Côte d'Ivoire, Ministère de l'Environnement et des Eaux et Forêts /Direction de la Protection de la Nature, Programme Cadre de Gestion des Aires Protégées. Documentcadre, Abidjan, Janvier 2001, 129 pages.

République de Côte d'Ivoire, Ministère de l'Environnement et des Eaux et Forêts/Office Ivoirien des Parcs et Réserves, État de conservation des Parcs et Réserves de Côte d'Ivoire, Abidjan, 2009, 29 pages.

République de Côte d'Ivoire, MINEEF/OIPR, Orientations stratégiques des mesures riveraines autour des Parcs Nationaux de Côte d'Ivoire, GTZ, août 2005, 15 pages.

République de Côte d'Ivoire, Ministère de l'Environnement et des Eaux et Forêts/ Office Ivoirien des Parcs et Réserves, Plan d'aménagement et de gestion du Parc National d'Azagny, Abidjan, 2008, (inédit), 99 pages.

République de Côte d'Ivoire, Ministère de l'Environnement et des Eaux et Forêts/Office Ivoirien des Parcs et Réserves, Plan d'aménagement et de gestion du Parc National du Banco, Abidjan, 2008, 95 pages.

République de Côte d'Ivoire, Ministère de l'Environnement et des Eaux et Forêts/Office Ivoirien des Parcs et Réserves, Plan d'Aménagement du Parc National de Tä̈, Abidjan, mars 2006, 99 pages. 
République de Côte d'Ivoire, Ministère de l'Environnement, des Eaux et Forêts/ Office Ivoirien des Parcs et Réserves, Procédures de concession de l'exploitation touristique des Parcs Nationaux et Réserves, Abidjan, avril 2010, 9 pages.

République de Côte d'Ivoire, Ministère des Eaux et Forêts, Plan Directeur Forestier 19882015, Abidjan, décembre 1988, 88 pages.

République de Côte d'Ivoire, Ministère du Plan, La Côte d'Ivoire en chiffres, éd. 1975, Abidjan, 1975, 227 pages.

République de Côte d'Ivoire, Ministère du Plan et du Développement, Document Stratégique de Réduction de la Pauvreté, Stratégique de Relance du Développement et de Réduction de la Pauvreté, Janvier 2009, 180 pages.

République de Côte d'Ivoire, Ministère du Plan et du Développement, Plan national de développement PND 2016-2020 : Diagnostic stratégique de la Côte d'Ivoire sur la trajectoire de l'émergence (tome 1), Abidjan, 110 pages.

République de Côte d'Ivoire, Ministère du Tourisme, La relance touristique en Côte d'Ivoire (Dossier de presse), 17 pages.

SANDIA (Mohamed), Rapport de l'Exercice de Consultation des Populations Riveraines aux Aires Protégées, Abidjan, PCGAP/BM, mai 1998, pp. 10-14 et 23 ; GTZ/OIPR, Le profil de la Zone Périphérique du Parc National de Taï, Quel potentiel pour la conservation du Parc?, Abidjan, mars 2006, 50 pages.

TESSEMA (Mekbeb E.), ASHENAFI (Zelealem T.), LILIEHOLM (Robert J.) et LEADER-WILLIAMS (Nigel), "Community Attitudes towards Wildlife Conservation in Ethiopia », Proceedings of the 2007 George Wright Society Conference, 2007, pp. 287-292.

IUCN, Parks for Life : Report of the IVth World Congress on National Parks and Protected Areas, Gland, Switzerland, , IUCN ,1993, viii+ 260 pages.

UICN, Renforcer la conservation des aires protégées d'Afrique... : synthèse de la rencontre Weotenga, UICN-PAPACO - Ouagadougou, janvier 2012, 58 pages.

UICN, Vème Congrès mondial des Parcs Nationaux: Bénéfices par-delà les frontières, Planète Conservation (Bulletin UICN), Vol. 34, n², 2003, 32 pages.

UICN-PACO, Bâtir l'avenir par des solutions naturelles et novatrices : Rapport annuel 2013, Ouagadougou, Burkina Faso : UICN, 2014, 36 pages.

UICN/BRAO, Évaluation de l'efficacité de la gestion des aires protégées : Parcs et Réserves de Côte d'Ivoire, 2008, 48 pages.

UICN/PACO, Évaluation de l'efficacité de la gestion des aires protégées : aires protégées du Togo, 2008, 41 pages.

UICN/PACO, Évaluation juridique et institutionnelle pour la mise en place des conditions d'amélioration de la gestion des aires protégées d'Afrique de l'Ouest, Ouagadougou, BF: UICN/PACO, 2010, 92 pages.

UICN/PACO, Le tourisme dans les aires protégées d'Afrique de l'Ouest : quelle contribution à la conservation ?, Ouagadougou, BF: UICN/PACO, 2010, 101 pages + annexes. 
UICN/PACO, Parks and reserves of Ghana : Management effectiveness assessment of protected areas. Ouagadougou, Burkina Faso : UICN/PACO, 2010, 54 pages.

UICN/PACO, Retombées économiques des aires protégées d'Afrique de l'Ouest, Ouagadougou, BF, 2011, 52 pages.

UICN-PAPACO, La lettre des Aires Protégées en Afrique de l'Ouest, UICN-PAPACO, n49, Janvier 2012, 7 pages.

UICN/République de Côte d'Ivoire, MINAGRA, CCE/BM/, Stratégie de conservation des Parcs Nationaux de Comoé et d'Azagny: Analyse sociologique, Gland, UICN, 1994, 14 pages.

UNESCO/UICN, Rapport de mission, Suivi de l'état de la conservation du Parc National de la Comoé en Côte d'Ivoire, site du Patrimoine Mondial, 10-23 juin 2006, 25 pages.

USAID, Issues in Poverty Reduction and Natural Resource Management, United States Agency for International Development, Washington, DC., 2006, 47 pages + annexes.

VEIT (Peter G.), NSHALA (Rugemeleza), ODHIAMBO (Michael Ochieng'), MANYINDO (Jacob), Protected Areas and Property Rights. Democratizing Eminent Domain in East Africa, WRI Report, World Resources Institute, 30 pages.

\section{VI - TEXTES JURIDIQUES}

\section{A- Textes juridiques internationaux}

Textes de portée universelle:

Conventions internationales :

Convention de Ramsar du 2 février 1971 relative aux zones humides d'importance internationale particulièrement comme habitats des oiseaux d'eau.

Convention de Washington du 3 mars 1973 sur le commerce international des espèces de faune et de flore menacées d'extinction.

Convention-cadre des Nations Unies sur les Changements Climatiques, 4 juin 1992.

Convention de Rio sur la diversité biologique, 5 juin 1992.

Protocole de Kyoyo à la Convention-Cadre des Nations Unies sur les Changements Climatiques, 1998.

Textes déclaratoires internationaux :

Agenda 21.

Charte mondiale de la nature (CMN) est une résolution de l'Assemblée générale des Nations Unies (Résolution 37/7) adoptée le 28 octobre 1982.

Rec. 5.26 du Vème Congrès mondial sur les Parcs (Durban, Afrique du Sud du 8-17 septembre 2003). 
Rec. $5.29 \mathrm{du}$ Vème Congrès mondial sur les Parcs(Durban, Afrique du Sud du 8-17 septembre 2003).

Textes de portée régionale:

Acte Uniforme portant organisation des procédures simplifiées de recouvrement et des voies d'exécution» [JO OHADA, $\mathrm{n}^{\circ} 6$, du $1^{\mathrm{er}}$ juin 1998] in OHADA, Traités et Actes Uniformes commentés et annotés, Juriscope, 2011, 1072 pages + annexes.

\section{B - Textes juridiques nationaux :}

République de Côte d'Ivoire :

\section{Lois et ordonnance :}

Loi 60-356 du 3 novembre 1960 portant Constitution de la République de Côte d'Ivoire, JORCI n58 Numéro Spécial du 4 novembre 1960, p. 1271.

Loi 2000-513 du $1^{\text {er }}$ août 2000 portant Constitution de la République de Côte d'Ivoire, JORCI n³0 du 3 août 2000 , p. 529.

Loi 59-249 du 31 décembre 1959 organisant les finances publiques en Côte d'Ivoire.Cette loi est modifiée et complétée par les lois 62-53 du 12 février 1962 organisant la gestion des Finances publiques (JORCI n ${ }^{\circ} 9$ Numéro Spécial du 23 février 1962, p. 213); loi n67-588 du 31 décembre 1967 portant loi de Finances pour l'exercice 1968 (JORCI n³ Numéro Spécial du 16 janvier 1968); par la loi 70-214 du 24 mars 1970.

Loi $\mathrm{n}^{\circ}$ 60-366 du 14 novembre 1960 portant Code de procédure pénale (JORCI n 12 Numéro Spécial du 24 février 1961, p. 207) telle que modifiée par les lois $n^{\circ} 62-231$ du 29 juin $1962, \mathrm{n}^{\circ} 63-526$ du 11 janvier 1963, n 69-371 du 12 août 1969, n $81-640$ du 31 juillet 1981, $\mathrm{n}^{\circ}$ 96-673 du 29 août 1996 et $\mathrm{n}^{\circ}$ 98-747 du 23 décembre.

Loi $\mathrm{n}^{\circ}$ 65-255 du 4 août 1965 relative à la protection de la faune et à l'exercice de la chasse, JORCI n4 41 du 26 août 1965 , p. 898

Loi $\mathrm{n}^{\circ} 65-425$ du 20 décembre 1965 portant Code forestier, JORCI n³ du 13 janvier 1966, p. 38 .

Loi n81-640 du 31 juillet 1981 instituant le Code pénal (JORCI n 1 Numéro Spécial du lundi 4 janvier 1982, p. 1), ) modifiée par modifiée la loi n95-522 du 6 juillet 1995 et la loi $\mathrm{n}^{\circ} 2015-134 \mathrm{du} 9$ mars 2015.

Loi n94-442 du 16 août 1994 portant modification de la loi $n^{\circ}$ 65-255 du 4 août 1965 relative à la protection de la faune et à l'exercice de la chasse, JORCI $n^{\circ} 42 \mathrm{du} 13$ octobre 1994, p. 778.

Loi $\mathrm{n}^{\circ} 96-766$ du 3 octobre 1996 portant Code de l'Environnement, JORCI nº du 6 février 1997, p. 114.

Loi ${ }^{\circ} 98-338$ du 2 juillet 1998 fixant les règles générales relatives aux Établissements publics nationaux et portant création de catégories d'établissements publics et abrogeant la loi $\mathrm{n}^{\circ} 80$ 1070 du 13 septembre 1980, JORCI, n³0, du jeudi 23 juillet 1998, p. 707. 
Loi $\mathrm{n}^{\circ}$ 98-750 du 23 décembre 1998 relative au domaine foncier rural, JORCI $\mathrm{n}^{\circ} 2 \mathrm{du} 14$ janvier 1999, p. 23.

Loi n²001-476 du 9 août 2001 d'orientation sur l'organisation générale de l'Administration territoriale, JORCI ${ }^{\circ} 36$ du jeudi 6 septembre 2001, p. 746.

Loi n²002-102 du 11 février 2002 relative à la création, à la gestion et au financement des parcs nationaux et réserves naturelles, JORCI n²0 du 16 mai 2002, p. 338.

Loi n ${ }^{\circ} 2004-412$ du 14 août 2004 portant amendement de l'article 26 de la loi n ${ }^{\circ}$ 98-750 du 23 décembre 1998 relative au domaine foncier rural, JORCI n 49 du 2 décembre 2004, p. 863.

Loi n²013-864 du 23 décembre 2013 modifiant l'alinéa 2 de l'article 9 de la loi n²002-102 du 11 février 2002 relative à la création, à la gestion et au financement des Parcs Nationaux et des Réserves naturelles, JORCI n 1 du vendredi 3 janvier 2014, Numéro Spécial, p. 5.

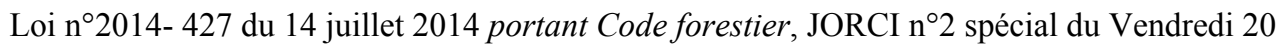
Mars 2015, page 17.

Loi n²014-451 du 05 août 2014 portant orientation de l'organisation générale de l'Administration territoriale, JORCI n³9 du 21 août 2014, p. 877.

Ordonnance $\mathrm{n}^{\circ}$ 2011-262 du 28 septembre 2011 portant orientation de l'organisation générale de l'Administration territoriale de l'Etat JORCI n 3 du 20 octobre 2011, p. 33.

\section{Décrets et arrêtés :}

Décret du 20 juillet 1900 organisant le régime forestier de la Côte d'Ivoire, JOCI du 15 octobre 1900, p. 1.

Décret du 18 juin 1912 réglementant le régime forestier de la Côte d'Ivoire, JOCI du 31 août 1912, p. 457.

Décret du 29 septembre 1928 modifiant le décret du 9 juillet 1900 portant réglementation du domaine public et des servitudes d'utilité publique en Afrique Occidentale Française, Bulletin Officiel des Colonies n 10,1928 .

Décret du 4 juillet 1935 sur le régime forestier de l'Afrique occidentale française, JOAOF du 3 août 1935, p. 611.

Décret du 15 juin 1939 portant réglementation des mariages entre indigènes en Afrique Occidentale française ou décret Mandel, JOAOF, $35^{\text {ème }}$ année, $\mathrm{n}^{\circ} 1837$ du 24 juin 1939, p. 842.

Décret du 5 juillet 1944 portant création de la Réserve naturelle intégrale du mont Nimba, JORF du 15 juillet 1944, p. 609.

Décret $\mathrm{n}^{\circ}$ 66-433 du 15 septembre 1966 portant statut et réglementation de la procédure de classement des réserves naturelles intégrales ou partielles et des Parcs Nationaux, JORCI n50 du 27 octobre 1966, p. 1419.

Décret 68-79 du 9 février 1968 portant création du Parc National du Mont Péko, JORCI n¹1 du 22 février 1968 p. 307. 
Décret 68-80 du 9 février 1968 portant création du Parc National de la Marahoué, JORCI n¹1 du 22 février 1968 p. 309.

Décret 68-81 du 9 février 1968 portant création du Parc National de la Comoé, JORCI n 11 du 22 février 1968, p. 310.

Décret n ${ }^{\circ} 72-545$ du 28 août 1972, portant création de la Réserve partielle de Faune du N'Zo, JORCI n43 du 14 septembre 1972, p. 1451.

Décret n $\mathrm{n}^{\circ} 2-545$ du 28 août 1972 portant création de la Réserve partielle de faune du N'Zo, JORCI n ${ }^{\circ} 43$ du 14 septembre 1972, p. 1452

Décret $\mathrm{n}^{\circ} 72-546$ du 28 août 1972, portant autorisation exceptionnelle de coupes de bois dans la Réserve de Faune du N'Zo ; JORCI n43 du 14 septembre 1972, p. 1452.

Décret $\mathrm{n}^{\circ} 73-27 \mathrm{du} 17$ janvier 1973, fixant les conditions de fonctionnement et de gestion du Fonds spécial des Parcs Nationaux, JORCI n8 du 8 février 1973, p. 245.

Décret 73-132 du 21 mars 1973, portant modification de la limite séparant le Parc National de Taï de la Réserve partielle de Faune du N’Zo, JORCI n¹ 18 du 19 avril 1973, p. 603.

Décret 73-133 portant création de la Réserve de Faune et de Flore du Haut-Bandama, JORCI n¹8 du 10 avril 1973, p.603.

Décret n76-215 du 19 février 1976 portant création du Parc National du Mont Sangbé (JORCI n 13 du jeudi 18 mars 1976, p. 515),

Décret 77-116 du 25 février 1977 portant déclassement d'une parcelle du Parc National de la Comoé, JORCI n 13 du 31 mars 1977 p. 521.

Décret 77-348 du 3 juin 1977, portant redéfinition des limites du Parc National de Taï et création d'une zone périphérique de protection, JORCI n ${ }^{\circ} 30$ du 28 juillet 1977, p. 1438.

Décret n ${ }^{\circ}$ 81-218 du 2 avril 1981 portant création du Parc National d'Azagny avec une zone de protection périphérique, JORCI n²1 du Jeudi 14 mai 1981, p. 241.

Décret n ${ }^{\circ} 87-759$ du 22 juillet 1987 portant déclassement d'une partie de la forêt classée de Gô-Bodiénou [sous-préfecture de Grand-Lahou], JORCI n³2 du 20 août 1987, p. 310

Décret $n^{\circ} 94-448$ du 25 août 1994 portant adhésion de la République de Côte d'Ivoire à la Convention sur le commerce international des espèces de faune et de flore sauvages menacées de disparition, signée à Washington le 3 mars 1973, JORCI n47 du jeudi 17 novembre 1994, p. 892.

Décret n ${ }^{\circ} 94-449$ du 14 novembre 1994 portant publication de la Convention sur le commerce international des espèces de faune et de flore sauvages menacées de disparition, signée à Washington le 3 mars 1973, JORCI n ${ }^{\circ} 47$ du jeudi 17 novembre 1994, p. 892.

Décret $\mathrm{n}^{\circ} 94-450$ du 25 août 1994 portant adhésion de la République de Côte d'Ivoire à la Convention relative aux zones humides d'importance internationale particulièrement comme habitats des oiseaux d'eau, adoptée à Ramsar (Iran), le 2 février 1971, JORCI n48 du jeudi 24 novembre 1994, p. 913.

Décret $n^{\circ} 94-451$ du 25 août 1994 portant publication de la Convention relative aux zones humides d'importance internationale particulièrement comme habitats des oiseaux d'eau, JORCI n48 du jeudi 24 novembre 1994, p. 913. 
Décret n94-614 du 14 novembre 1994 portant ratification de la Convention sur la diversité biologique, faite à Rio de Janeiro, le 5 juin 1992, JORCI n52 du jeudi 22 décembre 1994, p. 1004.

Décret n94-615 du 14 novembre 1994 portant publication de la Convention sur la diversité biologique, faite à Rio de Janeiro, le 5 juin 1992, JORCI n52 du jeudi 22 décembre 1994, p. 1004.

Décret n94-616 du 14 novembre 1994 portant ratification de la Convention sur les changements climatiques, faite à New York, le 9 mai 1992, JORCI n51 du jeudi 15 décembre 1994, p. 980.

Décret n94-617 du 14 novembre 1994 portant publication de la Convention sur les changements climatiques, faite à New York, le 9 mai 1992, JORCI n51 du jeudi 15 décembre 1994, p. 980.

Décret 2002-359 du 24 juillet 2002 portant création, organisation et fonctionnement de l'OIPR, JORCI n 40 du 2 octobre 2003, p. 588)

Décret $\mathrm{n}^{\circ}$ 2004-566 du 14 octobre portant déclaration d'utilité publique du domaine Dahliafleur, JORCI n ${ }^{\circ} 4$ du 27 janvier 2005, p. 60.

Décret 2008-137 du 14 avril 2008 portant création d'une Société d'État dénommée Société de Développement Touristique de la Région des Lacs en agrégé « SODETOUR-LACS », JORCI $\mathrm{n}^{\circ} 4$ du jeudi 27 mars 2011, p. 38.

Décret n²011-263 du 28 septembre 2011 portant organisation du territoire national en Districts et en Régions (JORCI n`3 du 20 octobre 2011, p. 37)

Décret n²012-163 du 9 février 2012 déterminant les procédures de classement des Parcs Nationaux et Réserves naturelles, JORCI $n^{\circ} 7$ du 16 février 2012, p. 93.

Décret n ${ }^{\circ} 2013-22$ du 9 janvier 2013 portant modalités d'habilitation, en qualité d'officiers de Police Judiciaire, des fonctionnaires et agents des Parcs nationaux, Réserves naturelles et des Eaux et Forêts, JORCI n ${ }^{\circ} 13$ du jeudi 28 mars 2013, p. 226.

Décret n ${ }^{\circ} 2013-127$ du 20 février 2013 portant création de la Réserve naturelle de Dahliafleur, JORCI ${ }^{\circ} 18$ du jeudi 2 mai 2013, p. 302.

Décret n²014-427 du 14 juillet 2014 portant Code forestier, JORCI n² Numéro Spécial du Vendredi 20 Mars 2015, page 17.

Arrêté $n^{\circ} 003 / \mathrm{SEPN} / \mathrm{CAB}$ du 20 février 1974 portant fermeture de la chasse, JORCI ${ }^{\circ} 13 \mathrm{du}$ 28 mars 1974, p. 487.

Arrêté $n^{\circ}$ 63/ MINEFOR /DGEF du 22/11/1982 portant déclassement partiel de la forêt $d u$ Gô et le Décret n87-759 du 22 juillet 1987 portant déclassement partiel de la forêt du Gô, JORCI n 32 du 20 août 1987, p. 310.

Arrêté ministériel $\mathrm{n}^{\circ} 00714$ du 06 septembre 2006 portant composition, attributions et fonctionnement des Comités de gestion locale des Parcs Nationaux et Réserves naturelles de Côte d'Ivoire.

Arrêté préfectoral $\mathrm{n}^{\circ} 70 / \mathrm{PY} / \mathrm{CAB}$ du 08 novembre 2006 portant composition, attributions et fonctionnement du Comité de gestion locale de la Réserve de Faune d'Abokouamékro. 
Arrêté n00895/MINEEF/du 17 octobre 2007 confiant la gestion du domaine d'utilité publique Dahliafleur à l'Office Ivoirien des Parcs et Réserves avec la dénomination Réserve naturelle partielle de Dahliafleur.

Note circulaire $n^{\circ} 1075 / \mathrm{MINEFOR} / \mathrm{IGEF}$ du 21 juin 1988 ayant pour objet la réhabilitation des Forêts Classées, des Parcs Nationaux et Réserves.

Lettre $n^{\circ} 0830 /$ MINEME/OIPR du 13 juillet 2005 adressée par le ministre Angèle GNONSOA au Préfet du département de Grand-Lahou (sur le territoire duquel se trouve le Parc National d'Azagny) et ayant pour objet la mise en place du comité de gestion locale du Parc National d'Azagny.

Note de service ${ }^{\circ} 56 / \mathrm{OIPR} / \mathrm{DZ} / \mathrm{S} / \mathrm{CE}$ du 15 octobre 2008 (relative à l'arrêt de la délivrance des autorisations d'exploitation de plantations dans le Parc National d'Azagny -ainsi libellé par nous dans la mesure où en dehors des références nous n'avons pu avoir accès une copie de la note de service elle-même).

\section{Droit étranger}

Loi française $\mathrm{n}^{\circ}$ 60-708 du 22 juillet 1960 relative à la création de Parcs Nationaux, JORF du 23 juillet 1960, p. 6751.

Loi $\mathrm{n}^{\circ} 78-190$ du 5 mai 1978 portant statut d'une entreprise publique dénommée Institut Congolais pour la Conservation de la Nature.

Loi zambienne $\mathrm{n}^{\circ} 12$ de 1998 relative à la gestion et la conservation de la faune.

Ordonnance $\mathrm{n}^{\circ} 75-023$ du 22 juillet 1975 portant création de l'Institut Zaïrois pour la Conservation de la Nature.

Ordonnance $\mathrm{n}^{\circ} 06 / 2002 \mathrm{du} 22 / 08 / 2002$, portant modification de certaines dispositions de la Loi ${ }^{\circ}$ 016/2001 du 31/12/2001, portant Code forestier en République gabonaise.

Décret $\mathrm{n}^{\circ}$ 10/15 du 10 avril 2010 fixant les statuts d'un établissement public dénommé Institut Congolais pour la Conservation de la Nature en sigle «I.C.C.N. » (Journal officiel de la République démocratique du Congo $\mathrm{n}^{\circ} 11 \mathrm{du} 1^{\mathrm{er}}$ juin 2010).

\section{VI- JURISPRUDENCE}

\section{Jurisprudence ivoirienne}

CSCA, arrêt nº2 du 28 avril 1976, François-Xavier SANTUCCI c/ l'Université d'Abidjan.

\section{Jurisprudence française}

C.E. français. 10 déc. 1954, Commune de Champigny, Rec. p. 658.

Cass civ. $1^{\mathrm{er}}, 2$ avril 1963, Montagne c/ Réunion des musées de France, A.J.D.A. 1963, p. 486 , note Jean DUFAU.

C.E. français 6 mai 1985, Assoc. Eurolat, R.F.D.A. 1986, p. 21, concl. B. Genevois.

Cass. civ. 3" 12 fév. 1986, Société Notre Dame des Fleurs c/ S.A.R.L. Montlaur-Grasse, A.J.D.A. 1986, p. 391, observ. J.C. 
Cass. civ. 21 déc. 1987 Bureau de recherches géologiques et minières c./ Société Lloyd Continental, Bull. civ. I, n 348, p. 249.

Cass. civ. $1^{\mathrm{er}}, 3$ mai 1988, Consorts Renaults c/ E.D.F., A.J.D.A. 1988, p. 676, note Jean DUFAU; et supra, p.12, note 30 .

\section{VIII - PÉRIODIQUES}

Académie des sciences

Accounting, Auditing and Accountability

African Journal of Conflit Resolution

Animal Conservation

Biodiversity

Biodiversity Conservation

Biological Conservation

BioScience

Cahiers Nantais

Canadian journal of botany

Conservation and Society

Conservation Biology

Corporate Environmental Strategy

EchoGéo

Ecological Economics

Ecology and Society

Ecology of Food and Nutrition

Economic Development and Cultural Change

Environmental Conservation

Environmental Management

ESSACHESS-Journal for Communication Studies

European Scientific Journal

Global Environmental Change

Human Ecology

Human Organization

IDS Bulletin

International Journal of Humanities and Social Science

International Review of Administrative Sciences

Journal of Environment and Earth Science

Journal of Environmental Management

Journal of Sustainable Tourism

Land Use Policy

Landscape and urban planning

Local Environment

Le Temps des Médias

Mediterranean Journal of Social Sciences

Nature

Oryx

Planning, Practice and Research

Proceedings of the National Academy of Sciences

Resources 
Revue Française d'Histoire d'Outre-mer

Revue Française de Droit, Administratif

Scholarly Journal of Agricultural Science

Science

Society and Natural Resources

The Geographical Journal

Virginia journal of international law

World Development

\section{VIII - DOCUMENTATION ÉLECTRONIQUE}

www.afd.fr/base projets/downloadDocument.action?idDocument=1470/

www.ardci-rd.org/index.php/vie-des-regions/bounkani

www.ardci-rd.org/index.php/vie-des-regions/grands-ponts

www.bf.undp.org/content/burkina faso/fr/home/operations/projects/poverty reduction/PAPE.html/

www.bi.chm-cbd.net/cooperation/organismes-regionaux/partenariat-pour-les-foret-du-bassin-du-congo-pfbc

www.ci.chm-cbd.net/links/geopoints/dahlia-fleur

www.cites.org/fra/disc/what.php

www.cons-dev.org/consdev/madagascar/MANANARA/Mananet/TEXTE/annexes/angap.html

www.cop21.gouv.fr/quest-ce-que-les-gaz-a-effet-de-serre/

www.developpement-durable.gouv.fr/-Effet-de-serre-et-changement-.html

www.dictionnaire.reverso.net/francaisdefinition/principeduparallismedesformes

www.donnees.banquemondiale.org/pays/cote-d'ivoire

www.dlc.dlib.indiana.edu/dlc/bitstream/handle/10535/1106/agrawala041000.pdf?sequence $=1$

www.ecologyandsociety.org/vol13/iss2/art43/

www.ecotourism.org/

www.europarl.europa.eu/workingpapers/agri/s5-7-2 fr.htm

www.evb.lacsq.org/fileadmin/user upload/microsites/eav-evb internet/documents/trousses-et-activites/forets-

du-monde/fiche 3 2.pdf

www.fao.org/about/what-we-do/fr/

www.fao.org/docrep/003/X6780F/X6780F05.htm

Www.fondationparc.ci

www.gouv.ci/actualite 1.php?recordID $=5525$

www.institutpourlajustice.com

www.iucn.org/fr

www.larousse.fr/encyclopedie/pays/Botswana/109606

www.larousse.fr/encyclopedie/pays/Ghana/121314

www.larousse.fr/encyclopedie/pays/Guatemala/122493

www.larousse.fr/encyclopedie/pays/Guinée-Bissau/122719

www.larousse.fr/encyclopedie/pays/Kenya/127291

www.larousse.fr/encyclopedie/pays/Madagascar/130983

www.larousse.fr/encyclopedie/pays/Népal/134965

www.larousse.fr/encyclopedie/pays/Ouganda/136566

www.larousse.fr/encyclopedie/pays/Royaume-Uni de Grande

Bretagne et dIrlande du Nord institutions/122086

www.larousse.fr/encyclopedie/pays/Rwanda/141732)

www.larousse.fr/encyclopedie/pays/Serbie/143964

www.larousse.fr/encyclopedie/pays/Zambie/150467

www.larousse.fr/encyclopedie/rechercher?q=problématique \& $\mathrm{t}$

www.madagascar-tribune.com/Angap-devient-Madagascar-National,9937.html

www.memoireonline.com/06/09/2139/m Analyse-du-systeme-de-biomonitoring-du-Parc-National-de-Tai3.html

www.oecd.org/fr/apropos/

www.oipr.ci/images/stories/pdf/depliant 2011 iles-ehotile.pdf

www.oipr.ci/index.php/reseau/parcs-nationaux/parc-national-du-mont-sangbé

www.oipr.ci/index.php/reseau/reserves-naturelles/reserve-de-faune-d-abokouamekro

www.oipr.ci/index.php/reseau/reserves-naturelles/reserve-naturelle-partielle-de-dahliafleur

www.parcsgabon.org/l-anpn/l-agence 
www.parcs-madagascar.com/madagascar-national-parks.php?Navigation=25

www.pendjari.net/

www.portals.iucn.org/library/efiles/edocs/WCS-004.pdf

www.recherche.afd.fr

www.riddac.org/index 2 .php?option $=$ com content $\&$ do $p d f=1 \& \mathrm{id}=67$

www.snv.org/

www.the-eis.com/data/literature/FinalReport CBNRMReview.pdf

www.tourismeci.org/circuits.htm

www.ugandawildlife.org/

www.uicn.fr/-Aires-protégées-.html

www.unep.org/Documents.Multilingual/Default.asp?DocumentID=97\&ArticleID=1503\&l=fr

www.vertigo.revues.org/4126

www.wttc-infographic.org/compare/botswana/united-states

www.wttc-infographic.org/compare/canada/united-states

wttc-infographic.org/compare/chile/united-states

www.wttc-infographic.org/compare/france/united-states

www.wttc-infographic.org/compare/germany/united-states

www.wttc-infographic.org/compare/south-africa/united-states

www.wttc-infographic.org/compare/spain/united-states

www.wttc-infographic.org/compare/tunisia/united-states

www.wttc-infographic.org/compare/united-states/united-states

www.wwf.be/_media/Agriculture itinerante_sur_brulis_en_RDC_122255.pdf 